Reinald Koch

Die Aufkommens- und Belastungswirkungen alternativer Vorschläge zur Reform der

Konzernbesteuerung in Europa 


\section{Die Aufkommens- und Belastungswirkungen alternativer Vorschläge zur Reform der Konzernbesteuerung in Europa}

Die fehlende Harmonisierung der Körperschaftsbesteuerung in Europa führt zu einer Verzerrung von Standort-, Investitions- und Finanzierungsentscheidungen multinationaler Konzerne. Für die Steuerverwaltungen begründet das Nebeneinander von 27 separaten Steuersystemen die zunehmende Notwendigkeit, das inländische Steueraufkommen zu schützen. Um diesen Problemen zu begegnen, werden von der Europäischen Kommission gegenwärtig unterschiedliche Reformvorschläge diskutiert. Hierzu zählen insbesondere die Einführung einer Gemeinsamen Konsolidierten Körperschaftsteuerbemessungsgrundlage (GKKB) sowie die Vereinheitlichung der Regelungen zur konzerninternen Verlustverrechnung. Ziel der Arbeit ist es, mögliche Auswirkungen dieser Reformvorschläge auf das Steueraufkommen der Mitgliedsstaaten sowie die Steuerbelastung der betroffenen Konzerne zu quantifizieren.

Reinald Koch, 1978 in Gifhorn geboren; 1998-2004 Studium der Betriebswirtschaftslehre an der Universität Göttingen, 2001-2002 an der Warwick Business School (Großbritannien); 2004-2010 Wissenschaftlicher Mitarbeiter am Institut für deutsche und internationale Besteuerung der Universität Göttingen. 
Die Aufkommens- und Belastungswirkungen alternativer Vorschläge zur Reform der Konzernbesteuerung in Europa 


\title{
cege-Schriften
}

Centrum für Europa-, Governance- und Entwicklungsforschung (cege)

Georg-August-Universitöt Göttingen

Center for European, Governance and Economic Development Research (cege)

\section{Band 18}

\author{
Herausgegeben von \\ Kilian Bizer, Stephan von Cramon-Taubadel, \\ Andreas Oestreicher, Renate Ohr, \\ Gerhard Rübel, Robert Schwager, \\ Verantwortlicher Herausgeber für diesen Band: \\ Andreas Oestreicher
}

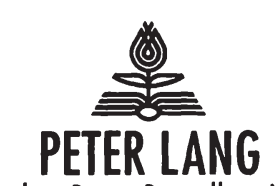

Frankfurt am Main - Berlin - Bern - Bruxelles - New York - Oxford - Wien 


\section{Reinald Koch}

\section{Die Aufkommens- und Belastungswirkungen alternativer Vorschläge zur Reform der Konzernbesteuerung in Europa}

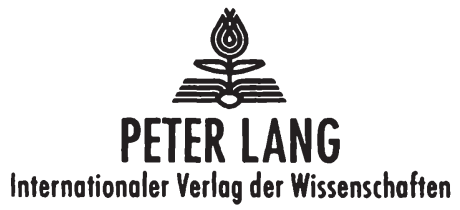




\title{
Bibliografische Information der Deutschen Nationalbibliothek
}

Die Deutsche Nationalbibliothek verzeichnet diese Publikation in der Deutschen Nationalbibliografie; detaillierte bibliografische Daten sind im Internet über http://dnb.d-nb.de abrufbar.

Open Access: The online version of this publication is published on www.peterlang.com and www.econstor.eu under the international Creative Commons License CC-BY 4.0. Learn more on how you can use and share this work: http://creativecommons.org/ licenses/by/4.0.

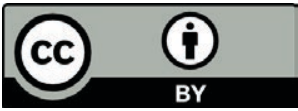

This book is available Open Access thanks to the kind support of ZBW - Leibniz-Informationszentrum Wirtschaft.

Zugl.: Göttingen, Univ., Diss., 2010

\author{
Umschlaggestaltung: \\ Olaf Glöckler, Atelier Platen, Friedberg
}
Gedruckt auf alterungsbeständigem, säurefreiem Papier.

\author{
D 7
}

ISSN 1617-741X

ISBN 978-3-631-60884-5

ISBN 978-3-631-75683-6 (eBook)

(c) Peter Lang GmbH

Internationaler Verlag der Wissenschaften

Frankfurt am Main 2010

Alle Rechte vorbehalten.

Das Werk einschließlich aller seiner Teile ist urheberrechtlich geschützt. Jede Verwertung außerhalb der engen Grenzen des

Urheberrechtsgesetzes ist ohne Zustimmung des Verlages unzulässig und strafbar. Das gilt insbesondere für

Vervielfältigungen, Übersetzungen, Mikroverfilmungen und die Einspeicherung und Verarbeitung in elektronischen Systemen.

www.peterlang.de 


\section{Vorwort}

Der europäische Binnenmarkt kann nur dann reibungslos funktionieren, wenn die für diesen Markt angestrebte Mobilität wirtschaftlicher Ressourcen durch steuerliche Vorschriften nicht behindert wird. Während die Harmonisierung der indirekten Steuern in diesem Sinne fortgeschritten ist, sind die Koordinierungserfolge auf dem Gebiet der direkten Steuern jedoch bescheiden. Zwar war die Harmonisierung der Unternehmensbesteuerung in Europa schon vielfach Gegenstand zahlreicher Initiativen auf der europäischen Ebene. Da für den Bereich der direkten Steuern alle Maßnahmen der EU einstimmig verabschiedet werden müssen, konnten diese Initiativen aber nicht immer gegen die Interesse Mitgliedstaaten, die die Sicherung ihrer nationalen Besteuerungs- und Ertragshoheit im Blick haben müssen, durchgesetzt werden. Daher kamen wichtige Maßnahmen der Europäischen Kommission auf dem Gebiet der Unternehmensbesteuerung (ein Beispiel ist die steuerliche Behandlung von Verlusten bei grenzübergreifenden Sachverhalten), bisher nicht über das Stadium einer Mitteilung hinaus. Vergleichbar damit ist nicht auszuschließen, dass auch das ehrgeizige Projekt einer gemeinsamen konsolidierten Körperschaftsteuerbemessungsgrundlage (CCCTB), das die Europäische Kommission in ihrer Studie zur Unternehmensbesteuerung in Europa 2001 anregte und seit dem Jahr 2004 aktiv verfolgt, am Einigungswillen der Mitgliedstaaten scheitert.

Dieser Einigungswille der Mitgliedstaaten ist nicht zuletzt deshalb schwach ausgeprägt, weil neben technischen und administrativen Fragen vor allem auch die finanziellen Konsequenzen offen sind, die mit der Einführung einer CCCTB für die Haushalte und Steuerbelastungen der betroffenen Mitgliedstaaten und Unternehmen verbunden sein dürften. Zwar liegen hierzu bereits erste Studien vor. Die Komplexität der Fragestellung setzt aber eine Untersuchung in allen 27 Mitgliedstaaten voraus, die auf Unternehmensdaten beruhen. Vor diesem Hintergrund besteht das Ziel der Arbeit von Herrn Koch darin, die möglichen Wirkungen herauszuarbeiten, die alternative Vorschläge zur Reform der Konzernbesteuerung auf das Steueraufkommen der Mitgliedsstaaten sowie die Steuerbelastung der Konzerne haben. Dabei beschränkt er sich nicht auf die Überlegungen der Europäischen Kommission zur Einführung einer CCCTB, sondern bezieht auch die gezielten Vorschläge zur steuerlichen Behandlung von Verlusten bei grenzüberreifenden Sachverhalten ein.

Die Anfertigung dieser Arbeit erfolgte im Rahmen eines breiter angelegten Forschungsprojekts zur „Besteuerung von Konzernen in Europa“, das am Institut für deutsche und internationale Besteuerung der Universität Göttingen in Kooperation mit dem Zentrum für Europäische Wirtschaftsforschung, Mannheim, durchgeführt wird. Auf der Grundlage des hier entwickelten Mikrosimulationsmodells werden differenzierte Aussagen zu den finanziellen Auswirkungen ge- 
wonnen, die mit der Einführung einer CCCTB oder einer Umsetzung der verschiedenen Vorschläge zur grenzübergreifenden Verlustverrechnungen verbunden wären. So zeigt sich zum Beispiel, dass Aufkommensverluste, die mir der verpflichtenden Einführung einer CCCTB verbunden wären, vor allem jene Länder treffen, die sich im gegenwärtigen System durch attraktive steuerliche Rahmenbedingungen auszeichnen. Eine verpflichtende CCCTB dürfte aber auch die Bandbreite durchschnittlicher Steuerbelastungen in Europa reduzieren und sich durch eine im Vergleich zu den gezielten Maßnahmen wirksamere Verlustverrechnung auszeichnen.

Die Ergebnisse der vorliegenden Arbeit sind nicht nur für Entscheidungsträger interessant, die im politischen Prozess einer Herstellung der Rahmenbedingungen für einen wettbewerbsfähigen Binnenmarkt Verantwortung tragen. Sie sind auch für die im Binnenmarkt aktive Unternehmen von Bedeutung, die sich auf neue Belastungs- und Verteilungsfolgen einzurichten hätten. Schließlich leistet die vorliegende Arbeit aber auch einen Beitrag zur Entwicklung von Mikrosimulationsmodellen im Unternehmensbereich, so dass sie auch von den Vertretern dieses Forschungszweigs mit Gewinn zu lesen ist. Vor diesem Hintergrund wünsche ich der Arbeit von Herrn Koch eine gute Aufnahme im Markt.

Prof. Dr. Andreas Oestreicher 


\section{Danksagung}

Die vorliegende Arbeit entstand während meiner Tätigkeit als wissenschaftlicher Mitarbeiter am Institut für deutsche und internationale Besteuerung der Universität Göttingen. Aus diesem Grund gilt an erster Stelle mein Dank dem Direktor dieses Instituts und meinem Doktorvater Professor Dr. Andreas Oestreicher, der frühzeitig mein Interesse für eine empirisch ausgerichtete Forschungstätigkeit geweckt hat und mir die Möglichkeit gegeben hat, während meiner Tätigkeit an interessanten Drittmittelprojekten sowohl für das Bundesfinanzministerium und die Europäische Kommission als auch gefördert durch die Deutsche Forschungsgemeinschaft mitzuarbeiten. Ferner habe ich von wertvollen DenkanstöBen im Rahmen zahlreicher gemeinsamer Gespräche über meine Forschungstätigkeit profitiert. Ebenfalls danken möchte ich Herrn Professor Dr. Robert Schwager sowie Herrn Professor Dr. Olaf Korn für die Übernahme des Zweitgutachtens und die Leitung der Disputation.

Ferner gilt mein Dank meinen Kollegen Christian Hundeshagen, Melanie Klett und Jens Prassel für die stets vorhandene Bereitschaft zu inhaltlichen Diskussionen über meine Arbeit sowie das wiederholte Anspornen und die Unterstützung in der Endphase. Insbesondere möchte ich mich bei ihnen aber auch für die angenehme Zusammenarbeit am Lehrstuhl sowie schöne gemeinsame Abende außerhalb des Lehrstuhls beim Pokern oder Fussballschauen bedanken. In diesem Sinne gilt mein Dank auch den Kollegen in meiner frühen Zeit am Lehrstuhl, Dr. Andreas Poppe und Heike Riesselmann, unserer Sekretärin Monika Rudolph sowie den studentischen Hilfskräften, die ich aufgrund ihrer großen Anzahl nicht alle namentlich erwähnen kann. Jens Prassel danke ich insbesondere auch dafür, dass er im Rahmen regelmäßiger Squash-Matches dafür gesorgt hat, dass meine körperliche Fitness in der Endphase meiner Arbeit nicht vollständig verloren ging.

Ebenfalls bedanken möchte ich mich bei meinen Freunden Dr. Kléber Djadjaglo, Stephan Eippert, Tung Lam Thanh, Claudia Prang, Christoph Reich, Christian Reise, Kilian Sartor und Christoph Voges zunächst einmal für das entgegengebrachte Verständnis für die wenige Zeit, die ich in der Endphase meiner Arbeit hatte sowie für die Unterstützung in dieser Zeit. Insbesondere aber danke ich ihnen auch für die stets angenehmen Ablenkungen vom wissenschaftlichen Arbeiten.

Zuletzt, aber in ganz besonderem Maße, möchte ich meinen Eltern, meinem Bruder Michael und meiner Oma für den Rückhalt und das Verständnis während meiner Promotionszeit sowie in den Jahren davor danken. Ohne sie wäre die Anfertigung einer solchen Arbeit um vieles schwierieger gewesen. Ihnen ist diese Arbeit gewidmet.

Reinald Koch 
Reinald Koch - 978-3-631-75683-6

Downloaded from PubFactory at 01/11/2019 03:01:46AM

via free access 


\section{Inhaltsverzeichnis}

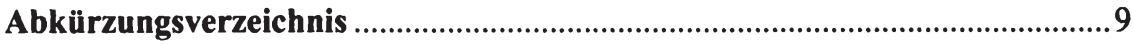

Abbildungsverzeichnis............................................................................... 17

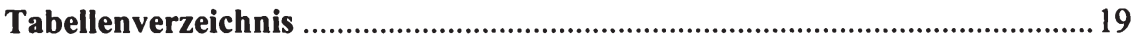

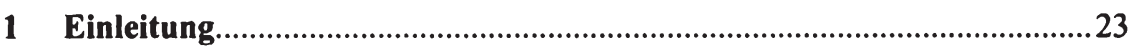

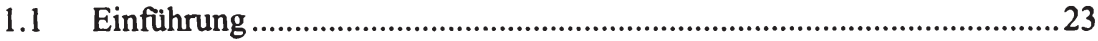

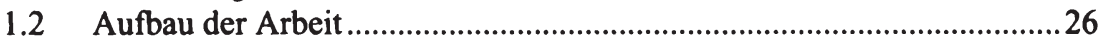

2 Konzeptionelle Grundlagen der Konzernbesteuerung..............................29

2.1 Die Konzernunternehmung im Spannungsfeld zwischen rechtlicher Vielheit und wirtschaftlicher Einheit....................................................29

2.2 Einheitstheorie und Trennungstheorie als grundlegende Konzepte

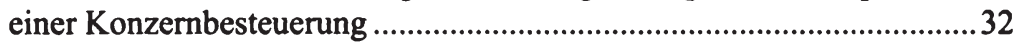

2.3 Qualitative Beurteilung der Einheits- und Trennungstheorie ....................34

3 Konzernbesteuerung in Europa de lege lata .............................................4 41

3.1 Überblick ........................................................................................

3.2 Ertragsbesteuerung der Kapitalgesellschaft als selbständiges

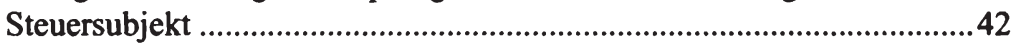

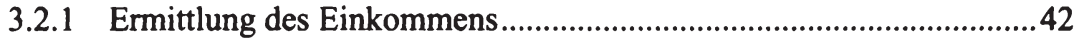

3.2.1.1 Steuerliche Gewinnermittlung ...............................................42

3.2.1.2 Behandlung ausländischer Einkünfte .........................................44

3.2.1.3 Intertemporale Verlustverrechnung .........................................45

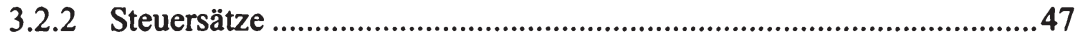

3.3 Einheitstheoretische Elemente bei der Besteuerung von verbundenen Kapitalgesellschaften ..........................................................48

3.3.1 Vermeidung einer Doppelbesteuerung ausgeschütteter Gewinne und einer doppelten Verlustberücksichtigung .....................................49

3.3.2 Gruppenbesteuerungssysteme......................................................51

3.4 Vorschriften zur Sicherung des inländischen Steueraufkommens ..........56

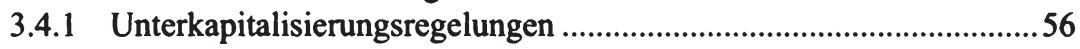

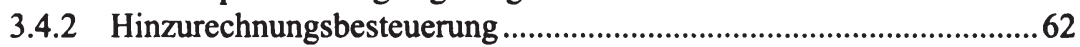

3.4.3 Gewinnabgrenzung im Konzern .......................................................65

3.5 Probleme der geltenden Konzernbesteuerung ........................................68

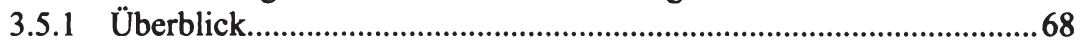

3.5.2 Steuern als Hindernis der grenzüberschreitenden Geschäftstätigkeit?

3.5.2.1 Steuerliche Nachteile der grenzüberschreitenden Geschäftstätigkeit. 
3.5.2.2 Steuerliche Vorteile der grenzüberschreitenden Geschäftstätigkeit aus Unternehmenssicht .................................73

3.5.3 Steuern als Ursache von Entscheidungs- und

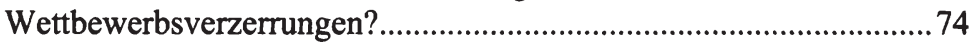

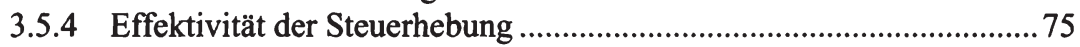

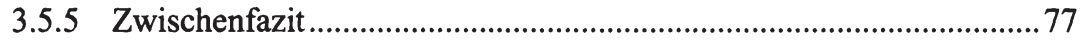

4 Konzernbesteuerung in Europa de lege ferenda.....................................79

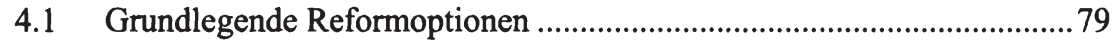

4.2 . Einführung einer „Gemeinsamen konsolidierten

Körperschaftsteuerbemessungsgrundlage “ “......................................... 84

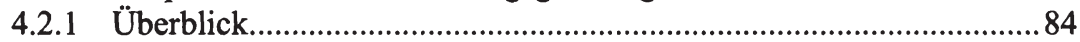

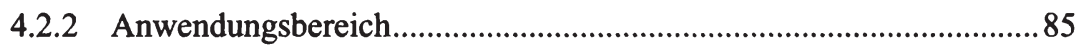

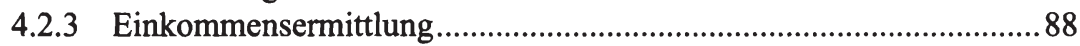

4.2.3.1 Gemeinsame steuerliche Gewinnermittlung ................................88

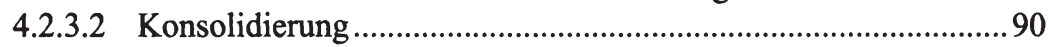

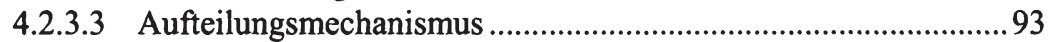

4.2.3.3.1 Alternative Ansätze ........................................................93

4.2.3.3.2 Makroökonomische oder industrieweite Faktoren ................95

4.2.3.3.3 Traditionelle Formelzerlegung ............................................96

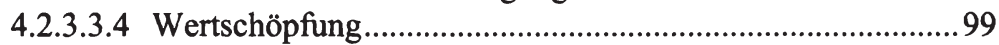

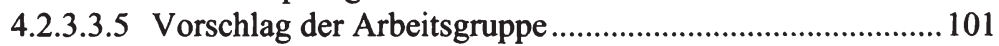

4.2.4 Administration und institutionelle Fragen ........................................ 103

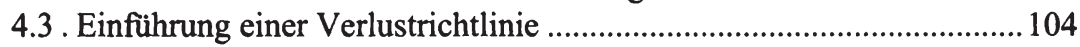

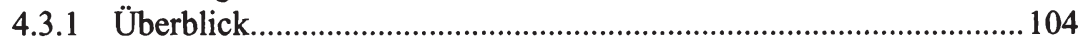

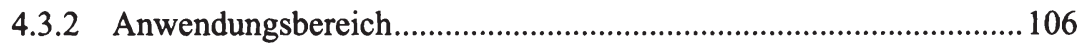

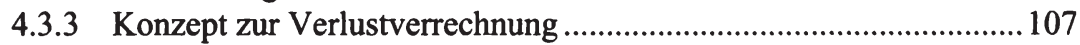

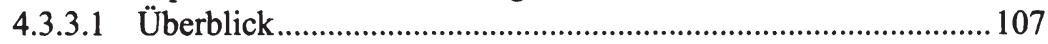

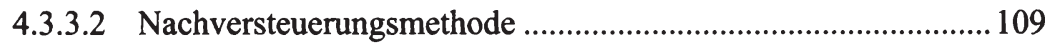

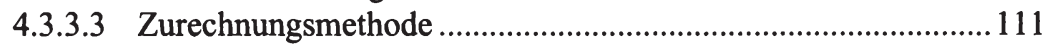

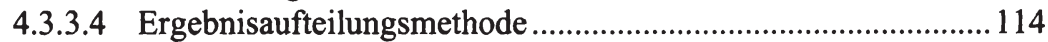

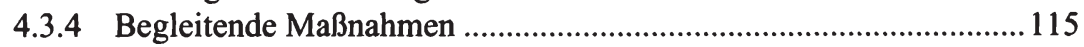

5 Datenbasis und Methodik der empirischen Analyse ............................117

5.1 Überblick ......................................................................................117

5.2 Inhalt und Umfang der verwendeten Datenbasis.................................118

5.3 Aufbereitung der Mikrodaten ..........................................................120

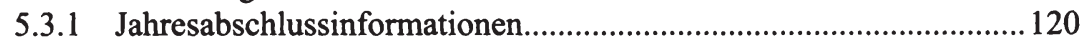

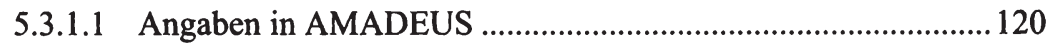

5.3.1.2 Bereinigung und Aufbereitung............................................... 122

5.3.1.3 Ergänzung fehlender Angaben für die Formelfaktoren .............. 125

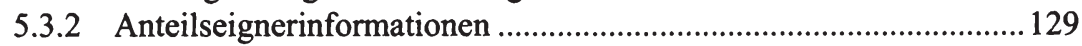

5.3.2.1 Angaben in AMADEUS ..................................................... 129 
5.3.2.2 Bereinigung und Aufbereitung................................................130

5.3.2.3 Abbildung der Konzernkreise und Auswahl der Unternehmen.

5.3.2.3.1 Datenbasis für Zwecke der Aufkommensberechnungen ....132

5.3.2.3.2 Datenbasis für Zwecke der Belastungsrechnungen ............. 135

5.4 Ermittlung der Steuerzahlungen ........................................................137

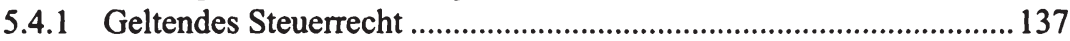

5.4.2 Gemeinsame konsolidierte Körperschaftsteuerbemessungsgrundlage ........................................ 144

5.4.3 Alternative Konzepte für eine Verlustrichtlinie................................. 148

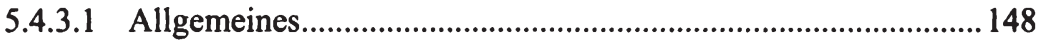

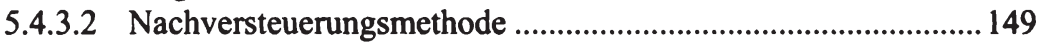

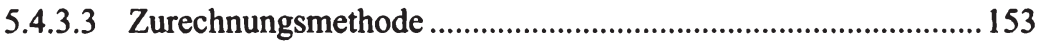

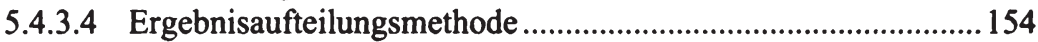

5.5 Ermittlung des Steueraufkommens..................................................156

5.6 Ermittlung der durchschnittlichen Steuerbelastung............................. 158

6 Empirische Ergebnisse: Auswirkungen auf die Steuerbelastung der

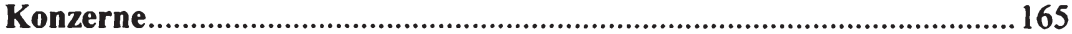

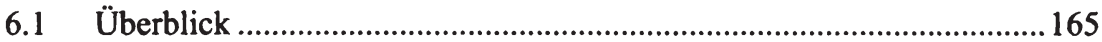

6.2 Auswirkungen auf die Attraktivität der Mitgliedsstaaten als

Steuerstandort und die Wettbewerbsfähigkeit europäischer

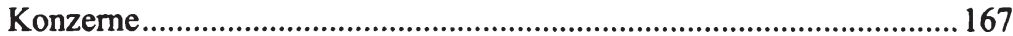

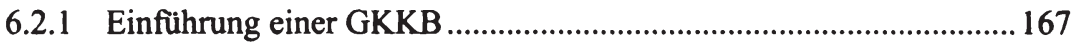

6.2.2 Einführung einer Verlustrichtlinie ...............................................173

6.3 Auswirkungen auf steuerliche Wettbewerbsverzerrungen im

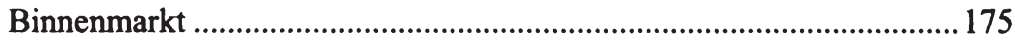

6.3.1 Auswirkungen auf die Schwankung der Steuerbelastung in

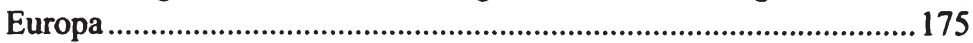

6.3.2 Auswirkungen auf Belastungsunterschiede zwischen nationalen und multinationalen Konzernen..................................................... 177

6.3.3 Auswirkungen auf intersektorale Belastungsunterschiede ...............180

6.3.4 Auswirkungen auf größenabhängige Belastungsunterschiede...........182

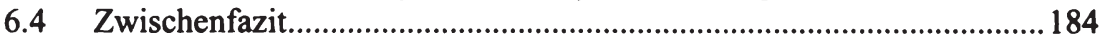

7 Empirische Ergebnisse: Auswirkungen auf das Steueraufkommen

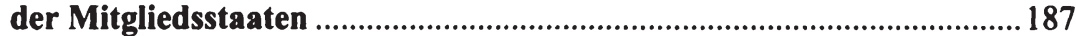

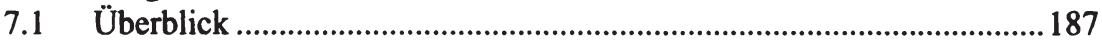

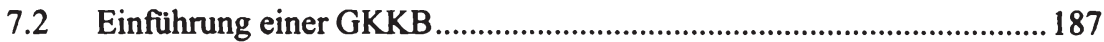

7.2.1 Basisszenario: Verpflichtende GKKB ........................................... 187

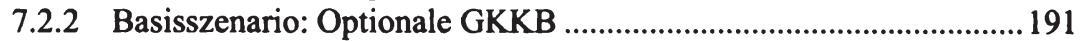

7.3 Einführung einer GKKB: Alternativszenarien ....................................193

7.3.1 Überblick....................................................................................... 193 


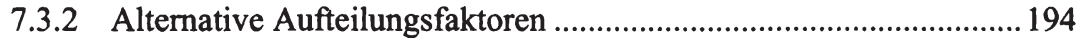

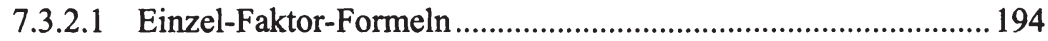

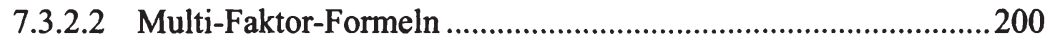

7.3.3 Einfluss der Ebene des Verlustvortrags ...........................................203

7.3.4 Verstärkte Zusammenarbeit mehrerer Mitgliedsstaaten ...................204

7.4 Einführung einer Verlustrichtlinie ...................................................205

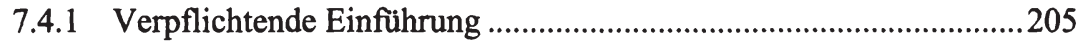

7.4.2 Optionale Einführung......................................................................210

7.5 Analyse der Einflussfaktoren der Aufkommenseffekte.........................2213

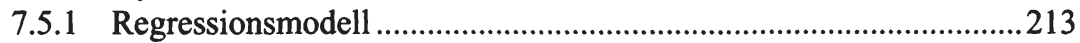

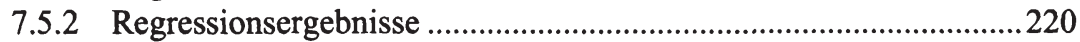

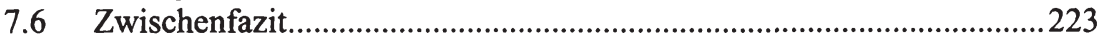

8 Unternehmerische Verhaltensreaktionen als Folge einer Reform der Konzernbesteuerung ...........................................................................222

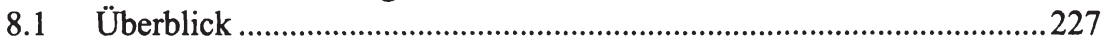

8.2 Unternehmerische Verhaltensreaktionen bei Einführung einer

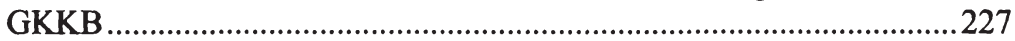

8.2.1 Steuerplanungsgestaltungen...........................................................227

8.2.2 Realwirtschaftliche Entscheidungen .................................................229

8.3 Unternehmerische Verhaltensreaktionen bei Einführung einer

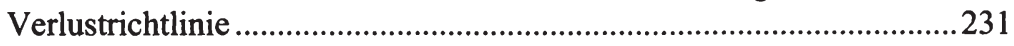

9 Zusammenfassende Beurteilung der Reformvorschläge ........................233

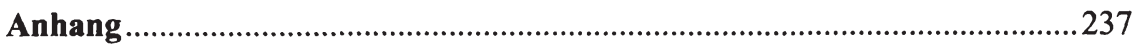

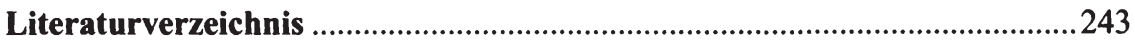

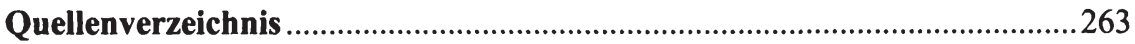

Rechtsprechungsverzeichnis .........................................................................2264 


\section{Abkürzungsverzeichnis}

A.A.

a.F.

Abl.

Abs.

AktG

APA

Art.

ATR

$\mathrm{ATR}^{+}$

ATR $^{-}$

$\mathrm{ATR}^{\text {mult }}$

$\mathrm{ATR}^{\text {nat }}$

bzw.

$\mathrm{CF}$

CFC

DBA

d.h.

DCF

DIECOFIS

DIW

EEA

EBITDA

EDV

EG

EK

endg.

EStG anderer Auffassung

alte Fassung

Amtsblatt

Absatz

Aktiengesetz vom 6. September 1965 (BGBl. I S. 1089), das durch Artikel 1 des Gesetzes vom 31. Juli 2009(BGB1. I 2009 S. 2509) zuletzt geändert worden ist

Advance Pricing Agreements

Artikel

durchschnittliche Steuerbelastung (average tax rate) durchschnittliche Steuerbelastung rentabler Konzerne durchschnittliche Steuerbelastung verlusterzielender Konzerne

durchschnittliche Steuerbelastung multinationaler Konzerne

durchschnittliche Steuerbelastung nationaler Konzerne

beziehungsweise

Cashflow to Equity

Controlled Foreign Company

Abkommen zur Vermeidung der Doppelbesteuerung

das heißt

Cashflow aus Fremdfinanzierung

Development of a System of Indicators on Economic

Competitiveness and Fiscal Impact on Enterprise Performance

Deutsches Institut für Wirtschaftsforschung

Europäischer Wirtschaftsraum (European Economic Area)

Earnings before interest, taxes, depreciation, and amortization

elektronische Datenverarbeitung

Europäische Gemeinschaft

Eigenkapital

endgültig

Einkommensteuergesetz in der Fassung der Bekanntmachung vom 19. Oktober 2002 (BGBl. I S. 3210; 
2003 I S. 179), das durch Artikel 1 des Gesetzes vom

et al. 29. Juli 2009 (BGBl. I S. 2302) geändert worden ist

EU und andere

EU15

Europäische Union

EU25

Mitgliedsstaaten der EU vor dem 1.5.2004

EuGH Mitgliedsstaaten der EU seit dem 1.5.2004

EuGHE

FK

Europäischer Gerichtshof

Entscheidungssammlung des EuGH

Fremdkapital

Fn.

GA

GKKB

$\mathrm{GmbH}$

$\mathrm{GuV}$

Fußnote

Gewinnabgrenzung

Gemeinsame konsolidierte Körperschaftsteuerbemessungsgrundlage

Gesellschaft mit beschränkter Haftung

Gewinn- und Verlustrechnung

HGB

Hrsg. Handelsgesetzbuch in der im Bundesgesetzblatt Teil III, Gliederungsnummer 4100-1 veröffentlichten bereinigten Fassung, das durch Artikel 6a des Gesetzes vom 31. Juli 2009 (BGBl. I S. 2512) geändert worden ist

IAS

Herausgeber

IBFD

ICF

IFRS

IFSC

k.A.

K'umen

Mio.

MEUR

MW

mwN.

n.a.

NACE

OCF

OECD

OECD-MA

PLN

Rn.

International Accounting Standards

International Bureau of Fiscal Documentation

Investitionscashflow

International Financial Reporting Standards

International Financial Services Centre

keine Angabe

Konzernunternehmen

Millionen

Millionen Euro

Mittelwert

mit weiteren Nachweisen

nicht anwendbar (not applicable)

Nomenclature statistique des activités économiques dans la Communauté européenne

Operativer Cashflow

Organisation for Economic Co-operation and Development

OECD-Musterabkommen in der Fassung vom 15. Juli 2005

polnische Zloty

Randnummer 
S.

SE

Sp.

Stabw

Tz.

u.a.

UNICE

v.

VBA

v.H.

vgl.

z.B.

ZEW

ZEW TaxCoMM

Zsfg.
Seite

Societas Europaea (Europäische Gesellschaft)

Spalte

Standardabweichung

Textziffer

unter anderem

Union of Industrial and Employers' Confederations of Europe

vom

Visual Basic

vom Hundert

vergleiche

zum Beispiel

Zentrum für Europäische Wirtschaftsforschung ZEW Corporate Taxation Microsimulation-Model Zusammenfassung 
Reinald Koch - 978-3-631-75683-6

Downloaded from PubFactory at 01/11/2019 03:01:46AM

via free access 


\section{Abbildungsverzeichnis}

Abbildung 1: Nominelle Steuersätze auf Gewinne von Kapitalgesellschaften in Europa

Abbildung 2: Entwicklung der nominellen Steuersätze auf Gewinne von Kapitalgesellschaften in Europa.

Abbildung 3: Mögliche Ansätze für eine abgestimmte Weiterentwicklung der Konzernbesteuerung in Europa

Abbildung 4: Durchschnittliche Höhe der Bemessungsgrundlagenänderung in Abhängigkeit von der Gewichtung des Arbeitsfaktors

Abbildung 5: Zusammenhang von Bruttobetriebsgewinnen (gesamtwirtschaftlich, 2004) und dem Arbeitsfaktor (gesamtwirtschaftlich, 2004) in den Mitgliedsstaaten

Abbildung 6: Korrelation zwischen den Bruttounternehmensgewinnen (gesamtwirtschaftlich) und dem Arbeitsfaktor (gesamtwirtschaftlich) im Zeitablauf 
Reinald Koch - 978-3-631-75683-6

Downloaded from PubFactory at 01/11/2019 03:01:46AM

via free access 


\section{Tabellenverzeichnis}

Tabelle 1: Methode zur Vermeidung der Doppelbesteuerung bei ausländischen Betriebsstätteneinkünften .......................................................45

Tabelle 2: Zeitliche Verlustvortrags- und Verlustrücktragsbegrenzungen........ 46

Tabelle 3: Behandlung von Gewinnen und Verlusten aus dem Verkauf von Beteiligungen bei der Körperschaftsteuer ....................................51

Tabelle 4: Gruppenbesteuerungssysteme (Beteiligungsanforderungen) ............54

Tabelle 5: Gruppenbesteuerungssysteme (Berücksichtigung der wirtschaftlichen Einheit).

Tabelle 6: Gruppenbesteuerungssysteme (Verlustverrechnungsmechanismus) 56

Tabelle 7: Gesellschafterfremdfinanzierung (Rechtsgrundlage) 58

Tabelle 8: Gesellschafterfremdfinanzierung (Anwendungsvoraussetzungen) 58

Tabelle 9: Gesellschafterfremdfinanzierung (Rechtsfolgen)

Tabelle 10: CFC-Besteuerungsregime in den EU-Mitgliedsstaaten.

Tabelle 11: Ausgestaltung der CFC-Besteuerungsregime in den EU Mitgliedsstaaten.....

Tabelle 12: Erfassungsgrad europäischer Kapitalgesellschaften in AMADEUS

Tabelle 13: Struktur der Jahresabschlussinformationen in AMADEUS

Tabelle 14: Aufbereitung der Jahresabschlussinformationen (Bilanz).

Tabelle 15: Aufbereitung der Jahresabschlussinformationen (Gewinn- und Verlustrechnung)

Tabelle 16: Datenverfügbarkeit bei Unternehmen in der Stichprobe 126

Tabelle 17: Aufbereitung der Anteilseignerinformationen

Tabelle 18: Geografische Zusammensetzung der Stichprobe (Aufkommensberechnungen)

Tabelle 19: Geografische Zusammensetzung der Stichprobe (Belastungsberechnungen).

Tabelle 20: Simulation der Wirkungen von Gruppenbesteuerungssystemen im geltenden Steuerrecht 
Tabelle 21: Ermittlung des Steueraufkommens................................................ 157

Tabelle 22: Durchschnittliche Steuerbelastung bei Einführung einer GKKB ... 168

Tabelle 23: Zusammenhang von Steuerbelastungsänderung (GKKB) und relevanten Steuerparametern bei nationalen Konzernen .

Tabelle 24: Zusammenhang von Steuerbelastungsänderung (GKKB) und relevanten Steuerparametern bei multinationalen Konzernen........ 170

Tabelle 25: Durchschnittliche Steuerbelastung multinationaler Konzerne bei Einführung einer Verlustrichtlinie

Tabelle 26: Zusammenhang von Steuerbelastungsänderung

(Verlustrichtlinie) und relevanten Steuerparametern bei multinationalen Konzernen

Tabelle 27: Variationskoeffizient der durchschnittlichen Steuerbelastung im Vergleich

Tabelle 28: Relative Abweichung der Steuerbelastung bei nationalen und multinationalen Konzernen

Tabelle 29: Relative Abweichung der Steuerbelastung bei profitablen und verlusterzielenden Konzernen

Tabelle 30: Abhängigkeit der Steuerbelastung multinationaler Konzerne von der Konzerngröße (GKKB) ....

Tabelle 31: Abhängigkeit der Steuerbelastung multinationaler Konzerne von der Konzerngröße (Verlustrichtlinie)

Tabelle 32: Wirkung der verpflichtenden Einführung einer GKKB auf das EU-Gesamtsteueraufkommen.

Tabelle 33: Wirkung der verpflichtenden Einführung einer GKKB auf das Steueraufkommen der einzelnen Mitgliedsstaaten

Tabelle 34: Wirkung der optionalen Einführung einer GKKB auf das EUGesamtsteueraufkommen

Tabelle 35: Wirkung der optionalen Einführung einer GKKB auf das Steueraufkommen (brutto) der einzelnen Mitgliedsstaaten

Tabelle 36: Relative Änderung des EU-Gesamtsteueraufkommens (brutto) bei alternativen Aufteilungsfaktoren (verpflichtende Einführung).

Tabelle 37: Relative Änderung der Bemessungsgrundlage (netto) der einzelnen Mitgliedsstaaten bei alternativen Aufteilungsfaktoren (verpflichtende Einführung) 196 
Tabelle 38: Relative Änderung der Bemessungsgrundlage (netto) der einzelnen Mitgliedsstaaten bei alternativen Aufteilungsfaktoren (optionale Einführung)

Tabelle 39: Relative Änderung des EU-Gesamtsteueraufkommens (brutto) bei alternativen Aufteilungsfaktoren (optionale Einführung) ........200

Tabelle 40: Wirkung alternativer einheitlicher Multi-Faktor-Formeln auf die durchschnittliche Höhe der Änderung der Bemessungsgrundlage (netto) der einzelnen Mitgliedsstaaten.......201

Tabelle 41: Wirkung branchenspezifischer Multi-Faktor-Formeln....................202

Tabelle 42: Wirkung der verpflichtenden Einführung einer GKKB mit Verlustvortrag auf Konzernebene auf das EUGesamtsteueraufkommen 203

Tabelle 43: Wirkung der Einführung einer GKKB im Wege der verstärkten Zusammenarbeit 205

Tabelle 44: Wirkung der verpflichtenden Einführung einer Verlustrichtlinie auf das EU-Gesamtsteueraufkommen 206

Tabelle 45: Wirkung der verpflichtenden Einführung einer Verlustrichtlinie auf das Bruttosteueraufkommen der einzelnen Mitgliedsstaaten.....

Tabelle 46: Wirkung der optionalen Einführung einer Verlustrichtlinie auf das EU-Gesamtsteueraufkommen

Tabelle 47: Wirkung der optionalen Einführung einer Verlustrichtlinie auf das Bruttosteueraufkommen der einzelnen Mitgliedsstaaten

Tabelle 48: Unabhängige Variablen - Deskriptive Statistik

Tabelle 49: Regressionsergebnisse

Tabelle 50: Steuerrechtliche Grundlage der Simulationsberechnungen (1)......237

Tabelle 51: Steuerrechtliche Grundlage der Simulationsberechnungen (2)......238

Tabelle 52: Steuerrechtliche Grundlage der Simulationsberechnungen (3)......239

Tabelle 53: Steuerrechtliche Grundlage der Simulationsberechnungen (4) ......240

Tabelle 54: Korrelationen zwischen den unabhängigen Variablen 
Reinald Koch - 978-3-631-75683-6

Downloaded from PubFactory at 01/11/2019 03:01:46AM

via free access 


\section{Einleitung}

\subsection{Einführung}

„Business goes global, taxes stay local.“" Dieser vielzitierte Ausspruch liefert eine treffende Umschreibung des sich aufbauenden Spannungsverhältnisses, dem gleichsam die europäischen Unternehmen und die europäischen Steuergesetzgeber in zunehmendem Maße ausgesetzt sind. Während europäische Unternehmen mittlerweile regelmäßig den ganzen Binnenmarkt als ihren Heimatmarkt verstehen², macht die Steuergesetzgebung - zumindest in Bezug auf die direkte Besteuerung von Unternehmen - an den Landesgrenzen halt. ${ }^{3}$ Dieses bedeutet für grenzüberschreitend tätige Konzerne, dass sie innerhalb der EU der Befolgung von bis zu 27 verschiedenen Steuersystemen ausgesetzt sind, während die Steuerhoheiten im Gegenzug auf den Schutz des inländischen Steueraufkommens bedacht sein müssen, da multinationale Konzerne durch Finanzierungsgestaltungen und/oder die Gestaltung konzerninterner Verrechnungspreise das internationale Steuerlastgefälle ausnutzen können. Zusätzliche steuerliche Hemmnisse für die grenzüberschreitende Geschäftstätigkeit sind die Konsequenz erforderlicher Vorschriften zum Schutz des inländischen Steueraufkommens.

Für die Beseitigung dieser Probleme können unterschiedliche Ansätze in Erwägung gezogen werden. Diese reichen von einer unilateralen Weiterentwicklung der Konzernbesteuerung, über die Beseitigung einzelner Probleme durch gezielte koordinative Maßnahmen bis hin zu einer vollständigen Harmonisierung der Konzernbesteuerung in Europa. Eine jüngste Initiative der Europäischen Kommission zur Harmonisierung der Konzernbesteuerung geht zurück auf eine Mitteilung aus dem Jahr 2001, in der die Kommission unter anderem die Einführung einer „Gemeinsamen konsolidierten Körperschaftsteuerbemessungsgrundlage" (GKKB) als langfristiges Ziel für die Konzernbesteuerung in Europa vorschlägt. ${ }^{4}$

Zielsetzung der vorliegenden Arbeit ist vor diesem Hintergrund die Beurteilung der Folgen alternativer Vorschläge zur Reform der Konzernbesteuerung für das Steueraufkommen der Mitgliedsstaaten sowie die Steuerbelastung der Konzerne. Die Anfertigung dieser Arbeit erfolgt im Rahmen eines breiter angelegten Forschungsprojekts zur „Besteuerung von Konzernen in Europa“, welches gemeinsam von der Universität Göttingen und dem Zentrum für Europäische Wirt-

1 Vgl. beispielsweise Rödder (2007), S. 381; Oestreicher (2000), S. 5.

2 Vgl. Kommission der Europäischen Gemeinschaften (2001a), S. 5.

3 Vgl. Oestreicher (2000), S. 5.

4 Vgl. Kommission der Europäischen Gemeinschaften (2001a). 
schaftsforschung, Mannheim durchgeführt wird. ${ }^{5}$ Intention dieses Projekts ist die Entwicklung eines Mikrosimulationsmodells auf Basis empirischer Unternehmensdaten, welches eine Abbildung der Besteuerung europäischer Konzerne und dabei auch eine Berücksichtigung von Anpassungsreaktionen der Konzerne auf zu beurteilende Steuerreformen erlaubt. Hierzu wurde in einem ersten Projektabschnitt ein komparativ-statisches Grundmodell entwickelt, welches von derartigen Verhaltensreaktionen abstrahiert. Die Ergebnisse dieses Modells werden im Rahmen der vorliegenden Arbeit dargestellt. In einem zweiten, aktuell laufenden Projektabschnitt werden eine Verfeinerung der methodischen Vorgehensweise, Erweiterung der abgebildeten steuerlichen Vorschriften sowie eine Einbindung der Verhaltensreaktionen angestrebt.

Die Abschätzung der quantitativen Wirkungen der untersuchten Reformvorschläge ist aus verschiedenen Gründen von Relevanz, wie auch die Europäische Kommission in entsprechenden Verlautbarungen unterstreicht. ${ }^{6}$ Eine Analyse der Steuerbelastung kann sowohl Aufschluss geben über die steuerliche Standortattraktivität eines Landes, soweit auf ihre durchschnittliche Höhe abgestellt wird, als auch über die Effizienzwirkungen des Steuersystems, wenn auf bestehende Unterschiede oder die Schwankung der Steuerbelastung im Ländervergleich Bezug genommen wird. In diesem Sinne sollen mit einer Analyse der Steuerbelastung von Konzernen de lege lata bestehende Entscheidungsverzerrungen und damit die tatsächliche Notwendigkeit zur Reform aufgezeigt werden. In welchem Ausma $ß$ verschiedene Reformvorschläge zur Beseitigung dieser Ineffizienzen beitragen können und mit ihnen gleichzeitig eine Verbesserung der steuerlichen Standortattraktivität sowie der Wettbewerbsfähigkeit europäischer Unternehmen erreicht werden kann, soll durch eine Untersuchung der Steuerbelastung de lege ferenda abgeleitet werden. Diese Untersuchung ist deshalb von besonderem Interesse, weil die Verbesserung der Wettbewerbsfähigkeit europäischer Unternehmen und die Beseitigung steuerlicher Ineffizienzen zu den wesentlichen Zielsetzungen gehört, welche die Kommission mit den gemachten Vorschlägen verfolgt. ${ }^{7}$ Bei der Analyse darf allerdings nicht übersehen werden, dass die EU-weite Umsetzung eines Reformvorschlags einen einstimmigen Beschluss sämtlicher Mitgliedsstaaten voraussetzt, deren Zustimmung allerdings in nicht unerheblichem $\mathrm{Maße}$ von den Auswirkungen auf das inländische Steueraufkommen abhängen sollte. Eine Analyse der Aufkommenswirkungen der Reformvorschläge liefert

5 An einem ersten Projektabschnitt war zusätzlich das International Tax Institute der Universität Hamburg beteiligt.

6 Vgl. Kommission der Europäischen Gemeinschaften (2002), S. 543; Kommission der Europäischen Gemeinschaften (2003), S. 6. So ist eine Untergruppe der von der Kommission eingesetzten Arbeitsgruppe mit der Folgenabschätzung der Reform beschäftigt. Vgl. hierzu auch Arbeitsgruppe GKKB (2007b).

7 Kommission der Europäischen Gemeinschaften $(2006), S_{12} 2-3$, 
somit insbesondere Anhaltspunkte für die Aussichten auf eine Umsetzung der verschiedenen Vorschläge.

Trotz dieser Bedeutung derartiger Analysen fehlt es bis zum heutigen Zeitpunkt an geeigneten Untersuchungen. Mit Blick auf die Ermittlung effektiver Steuerbelastungen dürften hierfür insbesondere restriktive Annahmen der üblicherweise verwendeten Modelle („Devereux/Griffith-Modell“"8, „European Tax Analyzer" ${ }^{(9)}$ ) ursächlich sein. So liegen diesen Modellen hypothetische Investitionsvorhaben (,Devereux/Griffith“) oder ein repräsentatives einzelnes Modellunternehmen (,European Tax Analyzer") zugrunde, während Konzernbeziehungen nur eingeschränkt erfasst werden können. Eine entsprechende Erweiterung dieser Modelle um Konzernsachverhalte würde insbesondere aufgrund der Heterogenität multinationaler Konzerne erhebliche Schwierigkeiten bereiten. Ferner können Verlustsituationen in diesen Modellen - wenn überhaupt - nur eingeschränkt erfasst werden ${ }^{10}$, was die Eignung dieser Modelle zur Beurteilung von Reformvorschlägen, die im Kern eine Erweiterung der Möglichkeiten zur steuerlichen Verlustverrechnung vorsehen, zusätzlich limitiert. So wurde dieser zentrale Aspekt derartiger Reformvorschläge in entsprechenden Untersuchungen bisher ausgeblendet oder nur unter stark vereinfachen Annahmen abgebildet. ${ }^{11}$ Auch die diesen Modellen zugrunde liegende Annahme ökonomisch identischer Investitionsvorhaben und Modellunternehmen bei der Durchführung des internationalen Vergleichs schränkt die Aussagefähigkeit der ermittelten Steuerbelastung ein. Steuergestaltungen grenzüberschreitend tätiger Konzerne, welche einen maßgebenden Einfluss auf die Höhe der Steuerbelastung nehmen sollten, können mit dieser Vorgehensweise nicht berücksichtigt werden. ${ }^{12}$

Die Aufkommenswirkungen entsprechender Reformvorschläge sind hingegen bereits teilweise Gegenstand empirischer Untersuchungen gewesen. Poppe (2008), Devereux/Loretz (2008) und Fuest/Hemmelgarn/Ramb (2007) berichten entsprechende Ergebnisse für die Einführung einer GKKB, während Oestreicher/Scheffler/Spengel et al. (2008) und Fuest/Hemmelgarn/Ramb (2005) die Aufkommenswirkungen zumindest der unilateralen Einführung einer grenzüberschreitenden Verlustverrechnung ohne Formelaufteilung untersuchen. Grenzen

8 Vgl. zu diesem Ansatz Devereux/Griffith (1999).

9 Vgl. grundlegend zum European Tax Analyzer Jacobs/Spengel (1996).

$10 \mathrm{Vgl}$. Mintz/Weiner (2003), S. 700.

11 So untersuchen Oestreicher/Reister/Spengel (2009) mithilfe des „European Tax Analyzers“ die Wirkungen einer Harmonisierung der Gewinnermittlung allerdings ohne Ausdehnung der Verlustverrechnungsmöglichkeiten über die Grenze. Devereux/Loretz (2008a) berücksichtigen diesen Aspekt zwar, gehen bei der Simulation der Verlustverrechnung allerdings davon aus, dass ein einmaliger Verlust zur Unternehmensbeendigung und damit einem endgultigen Verlustuntergang fuhrt. Die Wirkung einer Ausdehnung der Möglichkeiten zur phasengleichen Verlustverrechnung im Konzern wird unter diesen Annahmen uberschătzt.

12 Vgl. so beispielsweise Oestreicher/Reister/Spengel (2009): Devereyux-_L_retz-(2908a) 
dieser Berechnungen, die sich entweder aus den zugrundeliegenden Daten ${ }^{13}$ oder der methodischen Vorgehensweise ${ }^{14}$ ergeben, rechtfertigen allerdings eine erneute Untersuchung auch in diesem Bereich.

\subsection{Aufbau der Arbeit}

Zur Bearbeitung der genannten Fragestellung werden im Anschluss an diese Einführung zunächst die konzeptionellen Grundlagen der Konzernbesteuerung dargestellt (Kapitel 2). Hierbei wird insbesondere auf die Eignung der Trennungstheorie und der Einheitstheorie als sich gegenüberstehende theoretische Ansätze für eine Ausgestaltung der Konzernbesteuerung eingegangen. Hieran anschließend zeigt Kapitel 3 den Reformbedarf auf, der für die aktuell vorherrschenden steuerlichen Vorschriften aus der Perspektive der Unternehmen und der Mitgliedsstaaten besteht. Hierzu wird zunächst ein Überblick über die Vorschriften zur Konzernbesteuerung im geltenden Recht der Mitgliedsstaaten gegeben und hierauf aufbauend die Probleme der aktuellen Konzernbesteuerung herausgearbeitet. In Kapitel 4 erfolgt eine begründete Auswahl und Beschreibung der im weiteren Verlauf zu beurteilenden Reformvorschläge. Nach einem Überblick über mögliche Reformansätze werden im Anschluss die Vorschläge zur Einführung einer GKKB und einer Verlustrichtlinie im Detail erläutert.

Die verbleibenden Abschnitte sind der empirischen Analyse gewidmet. Kapitel 5 beinhaltet eine Beschreibung der Datenbasis und der methodischen Vorgehensweise. Hierbei wird im Einzelnen auf die Inhalte der Datenbank AMADEUS, welche den Berechnungen zugrundegelegt wird, die Schritte zur Aufbereitung der Datenbasis sowie das Vorgehen bei der Ermittlung der Steuerzahlungen, des Steueraufkommens und der Steuerbelastung eingegangen. Die Ergebnisse dieser Berechnungen werden ausführlich in den Kapiteln 6 und 7 diskutiert, wobei Kapitel 6 sich mit den Auswirkungen der Reformvorschläge auf die steuerliche Standortattraktivität der Mitgliedsstaaten und die Effizienz der Konzernbesteuerung beschäftigt, während in Kapitel 1 die Auswirkungen auf das Steueraufkommen der Mitgliedsstaaten untersucht werden. Bei der Interpretation dieser Ergebnisse ist zu berücksichtigen, dass die Simulationsrechnungen auf die statischen Wirkungen der verschiedenen Reformvorschläge beschränkt sind. Verhaltensanpassungen der Unternehmen werden nicht einbezogen. Zur Abschätzung der hierdurch entstehenden Ungenauigkeiten sowie als Ausblick auf zukünftige Weiter-

13 So beschränken Oestreicher/Scheffler/Spengel et al. (2008), Fuest/Hemmelgarn/Ramb (2007) und Fuest/Hemmelgarn/Ramb (2005) ihre Untersuchengen auf deutsche Outbound-Konzerne, während Poppe (2008) einperiodige Daten zugrundelegt.

14 Devereux/Loretz (2008) ermitteln das zu versteuernde Einkommen retrograd ausgehend von dem im handelsrechtlichen Jahresabschluss ausgewiesenen Steueraufwand. Dieser Ansatz führt zu Ungenauigkeiten, soweit dieser Aufwand auch latente Steuern beinhaltet oder Unternehmen Verluste erzielen. 
entwicklungen des Modells beinhaltet Kapitel 8 eine Darstellung zu erwartender unternehmerischer Verhaltensreaktionen. Die Arbeit schließt mit einer zusammenfassenden Beurteilung der Reformvorschläge in Kapitel 9. 
Reinald Koch - 978-3-631-75683-6

Downloaded from PubFactory at 01/11/2019 03:01:46AM

via free access 


\section{Konzeptionelle Grundlagen der Konzernbesteuerung}

\subsection{Die Konzernunternehmung im Spannungsfeld zwischen rechtlicher Vielheit und wirtschaftlicher Einheit}

Bis zum heutigen Zeitpunkt fehlt es in den Rechts- und Wirtschaftswissenschaften an einer einheitlichen Begriffsdefinition für die Organisationsform Konzern. Grundsätzlich anerkannt ist lediglich, dass Konzerne aus Unternehmen bestehen, die rechtlich selbständig, wirtschaftlich aber voneinander abhängig sind.$^{15}$ Daran anknüpfend kann ein Konzern in allgemeiner Form definiert werden als Verbindung von rechtlich selbständigen Unternehmen zu einer wirtschaftlichen Einheit. Diese Definition verdeutlicht das Spannungsfeld zwischen rechtlicher Vielheit und wirtschaftlicher Einheit, in dem sich der Konzern befindet.

Im Zivilrecht wird der wirtschaftlichen Verbundenheit der Konzerngesellschaften nur eingeschränkt Rechnung getragen. Rechtssubjekte im Sinne des Zivilrechts sind natürliche Personen, juristische Personen und - mit Einschränkungen - Personengesellschaften. Zwar knüpfen verschiedene Rechtsgebiete spezielle Anforderungen an Unternehmen, die zu einem Konzern zusammengefasst sind ${ }^{16}$, Rechtspersönlichkeiten im Sinne des Zivilrechts verbleiben allerdings die einzelnen Gesellschaften und ihre Anteilseigner. ${ }^{17}$ Vielmehr sollen durch das aktienrechtliche Konzernrecht, welches in den $\S \S 15-22$ und 291-328 des Aktiengesetzes geregelt ist, insbesondere die Interessen der an den Konzerngesellschaften beteiligten Stakeholder (z.B. Gläubiger, Arbeitnehmer, Minderheitsgesellschafter) geschützt werden. ${ }^{18}$ Hiermit wird der erhöhten Gefahr einer nachteilhaftigen Einflussnahme durch einen beherrschenden Gesellschafter Rechnung getragen. ${ }^{19}$ Der Umfang der Pflichten der herrschenden Gesellschaft steigt dabei mit zunehmender Einflussnahmemöglichkeit. ${ }^{20}$

$15 \mathrm{Vgl}$. Baetge/Kirsch/Thiele (2004), S. 1.

16 Hierzu zählen insbesondere das gesellschaftsrechtliche Konzemrecht im Aktiengesetz und GmbH-Gesetz, das Konzernsteuerrecht, das Konzernbilanzrecht, das Kartellrecht, das Kapitalmarktrecht, das Mitbestimmungsrecht und das Arbeitsrecht. Vgl. Emmerich/Habersack (2005), S. 1-2.

$17 \mathrm{Vgl}$. Oestreicher (2005b), S. 2.

$18 \mathrm{Vgl}$. Emmerich/Habersack (2005), S. 7; Baetge/Kirsch/Thiele (2004), S. 1. Scheuchzer bezeichnet diese Sichtweise des Konzernrechts als Schutzrecht für Minderheitsgesellschafter und Gläubiger als traditionelles, aber immer noch vorherrschendes Verstăndnis des Konzernrechts. Er sieht nur eine langsame Bewegung hin zu einem Konzernorganisationsrecht. Vgl. Scheuchzer (1994), S. 25.

19 Vgl. hierzu Emmerich/Habersack (2005), S. 9-11.

20 So besteht im faktischen Konzern lediglich eine Ausgleichspflicht bei nachteilhafter Einflussnahme durch das herrschende Unternehmen (§311 AktG), wăhrend im Vertragskonzern eine generelle Pflicht zum Verlustausgleich (§302 AktG) und im Eingliederungskonzern eine ge- 
Das Konzernbilanzrecht knüpft mit der Pflicht zur Aufstellung eines Konzernabschlusses als zweites Rechtsgebiet wesentliche Rechtsfolgen an das Vorliegen eines Konzerns. In diesem Abschluss ist zwar „die Vermögens-, Finanz- und Ertragslage so darzustellen, als ob diese Unternehmen insgesamt ein einziges Unternehmen wären"21, die Veröffentlichung dieses Abschlusses dient allerdings insbesondere Informationszwecken. ${ }^{22}$ Weitergehende Rechtsfolgen, wie die Bemessung der Ausschüttungen oder die Maßgeblichkeit für die Steuerbilanz, gehen von diesem Abschluss nicht aus, sondern ergeben sich ausschließlich aus dem handelsrechtlichen Einzelabschluss. ${ }^{23}$

In der betriebswirtschaftlichen Literatur wird für die Organisationsform Konzern hingegen häufig der Begriff der „Konzernunternehmung“ verwendet. ${ }^{24}$ Dieser Begriffsgebung liegt die Vorstellung des Konzerns als eine „einheitliche wirtschaftliche Planungs-, Koordinierungs- und Entscheidungseinheit" zugrunde. ${ }^{25}$ Die einzelnen Konzerngesellschaften werden als wirtschaftlich weitgehend unselbständige Betriebsabteilungen des Konzerns aufgefasst und verlieren zugunsten der wirtschaftlichen Einheit die Eigenschaften einer Unternehmung im betriebswirtschaftlichen Sinne, die von Kosiol in der ,wirtschaftlichen Selbständigkeit" ${ }^{\star 26}$ und von Gutenberg in der ,,inneren und äußeren Autonomie ${ }^{\text {“27 }}$ gesehen werden. ${ }^{28}$

Dabei wird allerdings auch in der betriebswirtschaftlichen Literatur nicht übersehen, dass Konzernunternehmen nicht nur Merkmale unselbständiger Betriebsabteilungen, sondern auch Merkmale wirtschaftlich und rechtlich autonomer Marktunternehmen besitzen. ${ }^{29}$ Sie unterscheiden sich somit zum einen durch Internalisierungseffekte wesentlich vom Extrempol der wirtschaftlich und rechtlich selbständigen Unternehmen, zum anderen aber auch durch Konzerneffekte vom anderen Extrempol des wirtschaftlich und rechtlich integrierten Einheitsunternehmens. ${ }^{30}$ Die Nähe zu einem der beiden Pole sollte dabei vor allem von der gesellschaftsrechtlichen Ausgestaltung der Unternehmensverbindung (zum Beispiel Beteiligungshöhe, Abschluss von Gewinnabführungs- und Beherrschungs-

samtschuldnerische Haftung für die Verluste der abhängigen Gesellschaft (§ $322 \mathrm{AktG}$ ) besteht.

$21 \S 297$ Abs. 3 S. 1 HGB.

22 Für eine Übersicht über die Zwecke des Konzernabschlusses siehe Baetge/Kirsch/Thiele (2004), S. 29-45.

23 Vgl. hierzu Baetge/Kirsch/Thiele (2004), S. 32.

24 Vgl. so beispielsweise Theisen (2000), S. 17.

$25 \mathrm{Vgl}$. Theisen (2000), S. 24 auch für das wörtliche Zitat.

26 Kosiol (1956), Sp. 5540-5545.

27 Gutenberg (1983), S. 457.

28 Vgl. Theisen (2000), S. 17; Scheuchzer (1994), S. 22-23.

29 Vgl. Ordelheide (1986), S. 296-297.

30 Vgl. Theisen (2000), S. 25. 
verträgen) sowie vom Ausmaß des Bestehens von Lieferungs- und Leistungsbeziehungen zwischen den Konzerngesellschaften abhängig sein. ${ }^{31}$

Internalisierungsvorteile werden in der Theorie regelmäßig als Erklärungsansätze für Unternehmenskonzentrationen herangezogen. Dabei sieht die neoklassische Theorie Verbundeffekte und Skaleneffekte als Gründe für die Integrationsentscheidung ${ }^{32}$, während die Transaktionskostentheorie und die Internalisierungstheorie geringere Transaktionskosten als Erklärung für die Konzernbildung anführen. ${ }^{33}$ In Bezug auf Transaktionskosten weist Schneider darauf hin, dass sich die Marktbedingungen für konzerninterne und konzernexterne Transaktionen schon allein deshalb unterscheiden, weil auch bei Existenz von Profit-Center Strukturen im Konzern bei Vertragsverhandlungen auf „Drohgebärden und Täuschungstricks" aus Rücksicht auf die wirtschaftliche Einheit regelmäßig verzichtet wird. ${ }^{34}$ Diese Vorteile sollten - diesen Theorien zufolge - dazu führen, dass sowohl bei vertikaler als auch bei horizontaler Integration das Ergebnis des Konzerns grundsätzlich oberhalb der Summe der Einzelergebnisse liegt, die die einzelnen Konzerngesellschaften als wirtschaftlich selbständige Unternehmen hätten erzielen können..$^{35}$

Als Nachteil der Integration führt Ordelheide an, dass Konzernunternehmen sich - im Unterschied zu unabhängigen Unternehmen - möglicherweise nur eingeschränkt durch Vertragsgestaltungen gegen bestehende Risiken absichern können. Dieses ist zum einen darauf zurückzuführen, dass sich das Risiko einer Tochtergesellschaft teilweise aus dem bestehenden Risikoverbund im Konzern ergibt, also dem Umstand, dass sich das Insolvenzrisiko einer Tochtergesellschaft bei Insolvenz der Muttergesellschaft erhöht. Zum anderen sind Tochtergesellschaften in ihren Entscheidungen nicht autonom, sondern können - je nach Stärke der Integration - von der Muttergesellschaft angehalten werden, marktunübliche Konditionen zu akzeptieren. ${ }^{36}$

Als positive und negative Konzerneffekte werden die wirtschaftlichen Unterschiede zwischen rechtlich selbständigen Konzerngesellschaften und dem wirtschaftlich und rechtlich einheitlichen Unternehmen bezeichnet. Derartige Effekte der Ausgliederung rechtlich selbständiger Teileinheiten können sich bei verschiedenen betriebswirtschaftlichen Funktionen ergeben. Ein Zusammenhang zwischen der internen und der externen Organisation der Konzernunternehmung wird zwar in der Literatur weitestgehend vernein $t^{37}$, vereinzelt wird allerdings auf engere

31 Vgl. Ordelheide (1986), S. 300; Ordelheide (1986a), S. 495.

$32 \mathrm{Vgl}$. Wendt (2009), S. 18.

$33 \mathrm{Vgl}$. ausführlich zu den beiden letztgenannten Theorien Oestreicher (2000), S. 78-101 mwN.

34 Schneider (2003), S. 55. So auch Oestreicher (2005a), S. 76.

35 Vgl. Oestreicher (2005a), S. 76-77; Schneider (2003), S. 54.

36 Vgl. Ordelheide (1986a), S. 495.

37 Vgl. Kirchner (1984), S. 226-227 mwN. 
Grenzen der Organisationsfreiheit in Konzernen ${ }^{38}$ oder organisatorische Vorteile in Zusammenhang mit rechtlich verselbständigten Tochterunternehmen ${ }^{39}$ verwiesen. Bezüglich der Führung ergibt sich bei der Konzernunternehmung die Besonderheit, dass die Leitungsorgane der Spitzeneinheit grundsätzlich in rechtlicher Hinsicht nur für die Leitung der eigenen Gesellschaft verantwortlich sind, nicht aber für die Leitung der Tochtergesellschaft. ${ }^{40}$ Etwas anderes gilt nur bei Eingliederung des Tochterunternehmens oder Abschluss eines Beherrschungsvertrags. ${ }^{41}$

Die deutlichsten Unterschiede zwischen einer Konzernunternehmung und einem selbständigen Marktunternehmen ergeben sich allerdings mit Blick auf die Haftung. So erlaubt die Ausgliederung einzelner Teileinheiten eine Haftungssegmentierung, da eine Inanspruchnahme der Muttergesellschaft auf Schadensersatz oder eine direkte Geltendmachung von Ansprüchen gegen die Muttergesellschaft („Durchgriffshaftung“) im faktischen Konzern nur in Ausnahmefällen möglich ist. ${ }^{42}$ Dieses kann im Ergebnis dazu genutzt werden, wirtschaftliche Risiken auf wirtschaftlich schwächere Parteien zu überwälzen. ${ }^{43}$ Weitergehende Verlustausgleichspflichten ergeben sich - wie bei den Leitungsbefugnissen - bei Eingliederung oder Beherrschung der Tochtergesellschaft. ${ }^{44}$

\subsection{Einheitstheorie und Trennungstheorie als grundlegende Kon- zepte einer Konzernbesteuerung}

Ausgehend von der rechtlichen Definition des Konzerns als Summe selbständiger Teileinheiten und der betriebswirtschaftlichen Sichtweise des Konzerns als wirtschaftlich weitgehend einheitliche Unternehmung haben sich mit der Trennungstheorie und der Einheitstheorie zwei Konzepte als Eckpfeiler zur Besteuerung von Konzernen herausgebildet. Innerhalb dieser Eckpunkte existiert eine Bandbreite alternativer Lösungsmöglichkeiten, bei denen die Doppelnatur des Konzerns zugunsten eines der beiden Pole aufgelöst wird. ${ }^{45}$

38 Vgl. Hübner (1980), Sp. 2020.

39 Vgl. Drumm (1982), S. 404-407; Blaschka (1982), S. 397-403.

40 Vgl. Emmerich/Habersack (2005), S. 369.

41 Vgl. § 308 AktG und $\S 323$ AktG.

42 Vgl. Kirchner (1984), S. 228-229 und 235. Zu diesen Ausnahmefällen gehören insbesondere die Tatbestände der Nachteilszufügung und qualifizierten Nachteilszufügung. Vgl. Emmerich/Habersack (2005), S. 374-388 und 404-412. Gesellschafter einer GmbH können daneben nach der Rechtsprechung von Gläubigern der $\mathrm{GmbH}$ in Fällen von materieller Unterkapitalisierung, bestandsvernichtenden Eingriffen und Vermögensvermischung in Anspruch genommen werden. Vgl. Grunewald (2005), S. 382-388.

43 Vgl. Ordelheide (1986), S. 300.

44 Vgl. § $302 \mathrm{AktG}$ und $\S 322 \mathrm{AktG}$.

45 Vgl. Rupp (1983), S. 28. Für einen Überblick über weitere Theorien zur steuerlichen Behandlung von Gruppen siehe Sievert (2006), S. 35-44 Reinald Koch - 978-3-631-75683-6 
Eine Besteuerung entsprechend der Trennungstheorie orientiert sich sowohl bei der Steuersubjektqualifikation als auch bei der Abgrenzung des zu versteuernden Einkommens an der zivilrechtlichen Rechtssubjektqualifikation; die wirtschaftliche Einheit des Konzerns wird hingegen negiert. ${ }^{46}$ Konkretisiert wird die Trennungstheorie durch den Grundsatz der Einzelgewinnermittlung („Trennungsprinzip") unter Beachtung des Fremdvergleichsgrundsatzes. ${ }^{47}$ Steuersubjekt ist die einzelne Konzerngesellschaft, die mit ihrem Einkommen (und Vermögen) als Steuerobjekt der Besteuerung unterliegt. Gewinne einer Tochtergesellschaft können erst dann bei der Muttergesellschaft steuerpflichtig werden, wenn sie ausgeschüttet werden. Laufende Verluste der Tochterunternehmen können beim Mutterunternehmen zumindest nicht direkt steuerlich berücksichtigt werden.$^{48}$ Lieferungs- und Leistungsbeziehungen zwischen Konzerngesellschaften sind dem Grunde nach anzuerkennen, Gewinne und Verluste aus diesen Transaktionen werden nicht eliminiert. Die Höhe der Entgelte für Leistungen aus derartigen schuldrechtlichen Verträgen orientiert sich an dem Entgelt, das voneinander unabhängige Vertragsparteien vereinbart hätten (,Fremdvergleichsgrundsatz"). ${ }^{49}$ Das Einkommen ist also so zu ermitteln, "als wäre jede Gesellschaft rechtlich und wirtschaftlich unabhängig. “50

Die Einheitstheorie, welche die „Antithese“ zur Trennungstheorie bildet, geht auf die Arbeiten von Isay (1910) und Haussmann (1926) zurück, die für ihre Überlegungen allerdings noch die Begriffe „Filialtheorie“ und „Einheitlichkeitstheorie" verwendeten. ${ }^{51}$ Im Gegensatz zur Trennungstheorie wird hier die rechtliche Selbständigkeit der Konzerngesellschaften bei der Definition des Steuersubjekts und Steuerobjekts zugunsten der Berücksichtigung der wirtschaftlichen Einheit missachtet und hiermit dem betriebswirtschaftlichen Verständnis der Konzernunternehmung gefolgt. ${ }^{52}$ Die beteiligten Konzerngesellschaften werden wie unselbständige Zweigniederlassungen der einheitlichen Konzernunternehmung

46 Vgl. Scheuchzer (1994), S. 27-29.

47 Vgl. Scheuchzer (1994), S. 29. Neben der direkten Gewinnabgrenzung nach dem Fremdvergleichsgrundsatz, welche sich aus dem Prinzip der selbstảndigen Einheit ergibt, ist auch eine indirekte Gewinnabgrenzung nach dem Prinzip des einheitlichen Unternehmens denkbar (vgl. zu diesen Prinzipien ausfuhrlich Kapitel 3.4.3 und Kapitel 4.2.3.3 sowie Oestreicher (2000)). Eine durchgehende Beachtung der rechtlichen Selbständigkeit der Konzerngesellschaften wird allerdings nur bei Umsetzung des Fremdvergleichsgrundsatzes gewährleistet.

48 Eine Berilcksichtigung dieser Verluste der Tochterunternehmen ist nach der Trennungstheorie allenfalls indirekt möglich, also soweit sie bei Verăußerung, Tausch oder Liquidation der Tochterkapitalgesellschaft zu einem Verlust der Muttergesellschaft fuhren oder eine Wertminderung der Beteiligung bewirken. Die Möglichkeiten zur indirekten Verlustverrechnung werden allerdings in der Besteuerungspraxis der Mitgliedsstaaten vielfach eingeschränkt.

49 Vgl. Oestreicher (2005b), S. 4-5; Scheuchzer (1994), S. 29-31.

50 Oestreicher (2005b), S. 4.

51 Vgl. Scheuchzer (1994), S. 36.

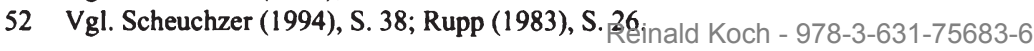


behandelt, welche als einziges Steuersubjekt der Besteuerung unterliegt. ${ }^{53}$ Dementsprechend gelten die Lieferungs- und Leistungsbeziehungen der Konzerngesellschaften direkt als Geschäftsvorfälle der Konzernunternehmung ${ }^{54}$; konzerninterne Geschäftsbeziehungen werden als steuerlich unbeachtliche innerbetriebliche Vorgänge behandelt. ${ }^{55}$

Auch wenn die Grundkonzeption der Einheitstheorie klare Vorgaben hinsichtlich des Besteuerungsergebnisses macht, kann die Herbeiführung dieses Ergebnisses nach unterschiedlichen Ansätzen erfolgen. Nach Scheuchzer können dabei alle Ansätze als Ausdruck der Einheitstheorie gewertet werden, welche die folgenden Anforderungen erfüllen, die sich konsequent aus der Vorstellung des Konzerns als auch für steuerliche Zwecke einheitliche Unternehmung ergeben: ${ }^{56}$

(1) Eine Doppelbesteuerung bei konzerninternen Gewinnausschüttungen auf Ebene der ausschüttenden und der empfangenden Konzerngesellschaft wird vermieden.

(2) Verluste einer Konzerngesellschaft können bei der steuerlichen Einkommensermittlung mit Gewinnen anderer Konzerngesellschaften ausgeglichen werden.

(3) Erfolgsbeiträge aus konzerninternen Lieferungs- und Leistungsbeziehungen werden für steuerliche Zwecke eliminiert.

\subsection{Qualitative Beurteilung der Einheits- und Trennungstheorie}

Anhaltspunkte für eine sachgemäße Ausgestaltung der Regelungen zur Konzernbesteuerung können möglicherweise aus einer qualitativen Diskussion der Einheits- und Trennungstheorie gewonnen werden. Hierbei sind sowohl steuersystematische als auch praktische Aspekte zu berücksichtigen.

Steuersystematisch ist zunächst zu fordern, dass der Ansatz zur Konzernbesteuerung grundlegenden steuerlichen Prinzipien nicht widerspricht und sich in das Steuersystem sowie das Rechtssystem als Ganzes einfügt. Zentraler Maßstab für die Ertragsbesteuerung in Deutschland ist dabei das Leistungsfähigkeitsprinzip, welches besagt, dass jedes Steuersubjekt nach Maßgabe seiner wirtschaftlichen Leistungsfähigkeit zu besteuern ist. ${ }^{57}$ Maßstab für die Ermittlung der steuerlichen Leistungsfähigkeit ist das Einkommen, welches bei Kapitalgesellschaften

53 Vgl. Sievert (2006), S. 36; Rupp (1983), S. 26. Teilweise wird die Einheitstheorie dahingehend ausgelegt, dass die Tochtergesellschaften zwar ,subjektiv steuerpflichtig bleiben, objektiv aber steuerfrei“ sind. Jurkat (1975), S. 40. Vgl. Scheuchzer (1994), S. 37.

55 Vgl. Sievert (2006), S. 37; Oestreicher (2005b), S. 20; Rupp (1983), S. 26.

56 Vgl. Scheuchzer (1994), S. 39-48.

57 Auch wenn die Anwendung des Leistungsfähigkeitsprinzips mit Unsicherheiten behaftet ist, ist die grundsätzliche Rechtfertigung dieses Prinzips auch international weitgehend anerkannt. Vgl. Endres/Oestreicher/Scheffler et al. (2007) S. S. kl kch - 978-3-631-75683-6 
unter Berücksichtigung des Realisationsprinzips zu bestimmen ist. ${ }^{58}$ Dabei sieht Leffson die Funktion des Realisationsprinzips in der Bewertung von Gütern ,vor und nach Erreichen des Absatzmarktes" oder anders ausgedrückt in der Festlegung des Zeitpunktes eines Übergang vom Unternehmensbereich in den Marktbereich. ${ }^{59}$ Damit dient das Realisationsprinzip durch den auf dem Markt bestehenden Interessengegensatz der beteiligten Parteien einer Objektivierung der Gewinnermittlung, welcher im Steuerrecht eine besondere Bedeutung zukommt. ${ }^{60}$ Befürworter der Einheitstheorie führen in diesem Zusammenhang an, dass bei Konzernen der wirtschaftliche Verbund der Konzerngesellschaften die Grenze der Unternehmung an den Konzernrand verschiebt, da bei Lieferungen und Leistungen zwischen den Konzerngesellschaften der erforderliche Interessengegensatz zwischen Angebots- und Nachfrageseite regelmäßig nicht besteht. Eine Besteuerung von Ergebnissen aus diesen Transaktionen - wie von der Trennungstheorie vorgesehen - schränke nach dieser wirtschaftlichen Sichtweise die Anwendbarkeit des Realisationsprinzips ein und beeinträchtige daher eine Besteuerung nach Maßgabe der wirtschaftlichen Leistungsfähigkeit. ${ }^{61}$ Insbesondere ist bis zum Eintreten des Außenumsatzes unklar, ob aus Sicht des Gesamtkonzerns ein Gewinn überhaupt entsteht. Aus Sicht der Trennungstheorie lässt sich diesem Argument entgegenhalten, dass auch bei konzerninternen Transaktionen zivilrechtlich durchsetzbare Ansprüche begründet werden, welche einen Anstieg der wirtschaftlichen Leistungsfähigkeit der liefernden Konzerngesellschaft bewirken. Aus dieser rechtlichen Perspektive erscheint daher die Besteuerung der Zwischengewinne aus diesen Transaktionen gerechtfertigt.

Neben die aus dem Leistungsfähigkeitsprinzip abgeleitete Forderung nach Steuergerechtigkeit tritt insbesondere aus Gründen der Allokationseffizienz die Anforderung einer wettbewerbsneutralen Besteuerung. ${ }^{62}$ Eine Besteuerung ist dann wettbewerbsneutral, wenn sie "die Wettbewerbsrelationen, die sich ohne Besteuerung ergeben würden, nicht absichtlich oder unabsichtlich...verändert."63 Diese Anforderung ist dann erfült, wenn wirtschaftlich vergleichbare Sachverhalte derselben Steuerbelastung unterliegen.

Befuirworter der Trennungstheorie stellen bei der Beurteilung der Wettbewerbswirkung einer Konzernbesteuerung auf die Einflussnahme der Besteuerung auf die Unternehmenskonzentration ab und wählen damit die Konzerngesellschaft und das unabhängige Marktunternehmen als das relevante Vergleichspaar. ${ }^{64}$ Eine

$58 \mathrm{Vgl}$. grundlegend zum Leistungsfähigkeitsprinzip und dessen Bedeutung im deutschen Steuerrecht Tipke/Lang (2002), S. 82-99; Neumark (1970), S. 121-185.

$59 \mathrm{Vgl}$. auch für das wörtliche Zitat Leffson (1976), S. 179; so auch Rupp (1983), S. 34.

60 Vgl. Rupp (1983), S. 34.

61 Vgl. Watrin/Sievert/Strohm (2004), S. 3; Herzig (2003), S. 28.

62 Vgl. so Neumark (1970), S. 262; Schön (2007), S. 415.

63 Steuerreformkommission (1971), S. 31.

64 Vgl. Rupp (1983), S. 39. 
derartige unerwünschte Konzentrationswirkung geht dann von der Besteuerung aus, wenn im Konzern verbundene Unternehmen günstiger als unabhängige Unternehmen besteuert werden. ${ }^{65}$ Befürworter der Einheitstheorie fordern demgegenüber, dass wirtschaftlich vergleichbare Tatbestände (Einheitsunternehmen und Konzern) nicht in Abhängigkeit von der rechtlichen Organisationsform unterschiedlich besteuert werden sollen. ${ }^{66}$

Beide Forderungen lassen sich allerdings nur dann rechtfertigen, wenn das gewählte Vergleichspaar zutreffend im Sinne von wirtschaftlicher Gleichheit ist. ${ }^{67}$ Auch wenn die wirtschaftliche Ähnlichkeit zwischen Konzern und unabhängigen Unternehmen im Einzelfall vom Ausmaß der finanziellen, wirtschaftlichen und organisatorischen Integration abhängig ist ${ }^{68}$, so bestehen unabhängig von der konkreten Ausgestaltung Unterschiede (z.B. Konzern als Haftungsverbund), welche die Forderung nach einer steuerlichen Gleichbehandlung der beiden Organisationsformen in Frage stellen. ${ }^{69}$ Außerdem bewirkt eine Konzernbesteuerung, die sich an der Einheitstheorie ausrichtet, keine Besserstellung des Konzerns, sondern kann lediglich eine Angleichung an die Besteuerungsfolgen, die sich beim Einheitsunternehmen ergeben, herbeiführen. ${ }^{70}$ Eine Konzentrationswirkung könnte von daher nur dann von der Besteuerung ausgehen, wenn die Besteuerung zwar nicht zwischen Einheitsunternehmen und Konzern differenziert, in der Praxis allerdings aufgrund von Schwierigkeiten bei der Implementierung eines Einheitsunternehmens diesem Weg der Unternehmenskonzentration ausgewichen wird. ${ }^{71}$

Die Forderung nach einer steuerlichen Gleichstellung von Konzern und Einheitsunternehmen lässt sich damit begründen, dass ,die Ausgliederung einzelner betrieblicher Aufgaben, Funktionen, Sparten oder regionaler Tätigkeits- und Verantwortungsbereiche bei einer Einheitsunternehmung ebenso wie bei einer Konzernunternehmung" erfolgt. ${ }^{72}$ Dennoch erscheint aufgrund der Existenz einzelner Vor- und Nachteile der Ausgliederung rechtlich selbständiger Teileinheiten (Konzerneffekte) eine vollständige steuerliche Gleichstellung auch hier nur bedingt gerechtfertigt.

65 Vgl. z.B. OECD (1995), Tz. 1.7 mit der Forderung nach einer steuerlichen Gleichstellung von Konzernunternehmen und unabhängigen Unternehmen, um ,steuerliche Vor- oder Nachteile, die die Wettbewerbspositionen zwischen beiden Unternehmenstypen verzerren würden“ zu vermeiden.

66 Vgl. stellvertretend Küting (1990), S. 490.

67 Vgl. Rupp (1983), S. 39.

68 Vgl. die Literaturstellen in Fn. 31.

69 Vgl. Rupp (1983), S. 39.

70 Vgl. Scheuchzer (1994), S. 38-39.

71 Vgl. hierzu Kirchner (1984), S. 225-226, der darauf hinweist, dass bei einer Verschmelzung das vollständige Kapital übernommen werden muss, während bei der Konzernbildung die Übernahme der Kapitalmehrheit ausreicht.

72 Scheuchzer (1994), S. 34. Vgl. ähnlich Krebühl (2003) $S_{K}$ 598R·-978-3-631-75683-6 
Von besonderer Bedeutung sind in diesem Zusammenhang die Unterschiede bei der zivilrechtlichen Haftung der Spitzeneinheit (Mutterkapitalgesellschaft oder Stammhaus) für Verluste der Grundeinheit (Tochterkapitalgesellschaft oder Zweigniederlassung). Während im Einheitsunternehmen Verluste der Zweigniederlassung das Stammhaus unmittelbar und in unbegrenzter Höhe wirtschaftlich belasten, tritt bei einem Konzern die wirtschaftliche Belastung der Muttergesellschaft durch Verluste der Tochtergesellschaft im Allgemeinen erst zeitlich verzögert und betragsmäßig begrenzt auf die Höhe der Hafteinlage ein. ${ }^{73}$ Forderungen in der Literatur oder Rechtsprechung nach einer Angleichung der Haftungsverfassung des faktischen Konzerns an das Einheitsunternehmen ${ }^{74}$, wurden bisher nicht umgesetzt. Als Gegenargument wird dabei angeführt, dass eine haftungsrechtliche Separation aufgrund der Führung von Konzernunternehmen wie Profit Center sinnvoll ist, dass diese bei Insolvenz einzelner Tochterunternehmen die Rettung anderer Konzernteile ermöglicht und zudem eine einfachere Anpassung an geänderte Marktbedingungen erlaubt. ${ }^{75}$ Unabhängig davon, ob die Haftungsbeschränkung im Konzern als gerechtfertigt eingestuft wird, stellt sie eine steuerliche Gleichstellung von Konzern und Einheitsunternehmen sowohl aufgrund der Einführung wirtschaftlicher Unterschiede als auch mit Blick auf die Einheitlichkeit der Rechtsordnung in Frage. ${ }^{76}$ Dieses gilt insbesondere für die interpersonelle Verlustverrechnung ${ }^{77}$, die sich auch an anderer Stelle im Steuerrecht an der zivilrechtlichen Haftung für bzw. wirtschaftlichen Belastung durch die Verluste orientiert ${ }^{78}$.

Gegen die Einheitstheorie spricht ferner, dass eine Nichtbeachtung der zivilrechtlichen Selbständigkeit der Kapitalgesellschaft in Form einer eigenständigen wirtschaftlichen Leistungsfähigkeit im Widerspruch zum Grundprinzip einer Anknüpfung des Steuerrechts an zivilrechtliche Wertungen steht. ${ }^{79}$ Zwar wird in der

73 Dieses gilt, wenn zusätzliche vertragliche Abreden zwischen den Konzerngesellschaften oder mit Gläubigern nicht bestehen, Verluste der Tochtergesellschaft durch die Muttergesellschaft nicht freiwillig ausgeglichen werden und die Tatbestandsmerkmale einer Durchgriffshaftung nicht erfullt sind (vgl. hierzu Fn. 42). In zeitlicher Hinsicht tritt die wirtschaftliche Belastung der Muttergesellschaft dann erst bei Veräußerung, Liquidation oder beim Anfall künftiger Gewinne der Tochtergesellschaft ein. Kritisch hierzu verweist Schön (2007) auf eine faktische Belastung der Muttergesellschaft durch Verluste einer Tochtergesellschaft. Vgl. Schön (2007), S. 416.

74 Mögliche Vorschläge sind z.B. die Nachrangigkeit von Konzerndarlehen bei Insolvenz eines Tochterunternehmens und die Bildung eines Pools von Vermögen und Ansprüchen Dritter bei Insolvenz mehrerer Tochterunternehmen. Vgl. Ordelheide (1986), S. $300 \mathrm{mwN}$.

75 Vgl. Ordelheide (1986), S. 300-301.

76 Auch Schneider bezweifelt die Rechtfertigung einer Forderung nach Gleichmäßigkeit der Besteuerung unterschiedlicher Rechtsformen bei Haftungsunterschieden. Vgl. Schneider (2002), S. 217.

77 Vgl. Oestreicher/Scheffler/Spengel et al. (2008), S. 89; Thiel (2004), S. 2605.

$78 \mathrm{Vgl}$. hierzu Sieker (1998). Dieses betrifft insbesondere die steuerliche Behandlung von Verlusten beim Kommanditisten einer Kommanditgesellschaft.

79 Vgl. zu diesem Grundprinzip Jacobs (2009), S. 91-93 
Literatur teilweise gefordert, dass das Steuerrecht dem wirtschaftlichen Gehalt eines Sachverhalts Vorrang vor der zivilrechtlichen Ausgestaltung gewähren soll, wenn die tatsächliche Ausgestaltung vom Leitbild des Gesetzgebers bei der Ausgestaltung der Vorschrift abweicht. ${ }^{80}$ Dieses wird unter anderem damit gerechtfertigt, dass auch bei der Konkretisierung des Leistungsfähigkeitsprinzips auf eine wirtschaftliche Leistungsfähigkeit abgestellt wird, weshalb der wirtschaftlichen Betrachtungsweise bei der Gesetzesauslegung im Steuerrecht höherer Stellenwert zukommen soll als im Zivilrecht. ${ }^{81}$ Diesem Argument steht allerdings entgegen, dass der Gesetzgeber auch bei vergleichbaren Fragestellungen der zivilrechtlichen Gestaltung und nicht dem wirtschaftlichen Gehalt folgt. So wird für Zwecke der Unternehmensbesteuerung nicht zwischen personalistischen und kapitalistischen Unternehmen unterschieden, sondern in Anlehnung an die zivilrechtliche Rechtsform zwischen Personenunternehmen und Kapitalgesellschaften. Ein Vorrang des wirtschaftlichen Gehalts für Zwecke der Konzernbesteuerung erscheint vor diesem Hintergrund mit Blick auf die Systematik und Einheitlichkeit des Steuerrechts problematisch.

Schließlich lassen sich auch mit Blick auf die Praktikabilität der Besteuerung Argumente sowohl für die Trennungstheorie als auch für die Einheitstheorie vorbringen. Das wesentliche praktische Problem der Trennungstheorie wird dabei in der Anwendung des Fremdvergleichsgrundsatzes bei konzerninternen Lieferungsund Leistungsbeziehungen gesehen. Für diesen Zweck müssen einzelne Geschäftsvorfälle voneinander abgegrenzt werden können, vergleichbare Transaktionen verfügbar sein und die erforderlichen Vergleichsdaten ermittelt werden können. ${ }^{82}$ Dieses ist in der praktischen Anwendung mit erheblichen Kosten sowohl für die Finanzverwaltung als auch den Steuerpflichtigen verbunden und bringt eine erhöhte Anzahl an Streitfällen mit sich. ${ }^{83}$ Bei der Einheitstheorie führt insbesondere die Beteiligung von Minderheitsgesellschaftern an der Tochtergesellschaft zu praktischen Problemen. Für diese muss ein Ausgleich gefunden werden, wenn das Einkommen dieser Tochtergesellschaft vollständig mit den Ergebnissen anderer Konzerngesellschaften zusammengefasst wird. ${ }^{84}$

Die langjährige Diskussion der Vor- und Nachteile der beiden genannten Theorien zur Konzernbesteuerung verdeutlicht, dass dieser Theorienstreit auf der Basis einer qualitativen Auseinandersetzung weder zugunsten der einen noch zugunsten der anderen Seite aufgelöst werden kann. Es bleibt somit festzuhalten, dass Aufschlüsse für eine sachgerechte Ausgestaltung der Konzernbesteuerung

80 Vgl. Jacobs (2009), S. 129-130. In diesem Sinne sind beispielsweise die Vorschläge zur Einführung einer Betriebsteuer oder Teilhabersteuer zu verstehen. Vgl. Jacobs (2009), S. 100$131 \mathrm{mwN}$.

81 Vgl. hierzu Eibelshäuser (2002), S. 1430.

82 Vgl. Oestreicher (2005a), S. 74-75.

83 Vgl. für entsprechende Nachweise Kapitel 3.5.2.1.

84 Vgl. Oestreicher (2005b), S. 20; Rupp (1983), S.R2inald Koch - 978-3-631-75683-6 
aus einer theoretischen Diskussion dieser Konzepte nicht gewonnen werden können, was die Notwendigkeit einer quantitativen Analyse unterstreicht. In die gleiche Richtung weisen die gegensätzlichen Bestrebungen, die auf politischer Ebene aktuell diskutiert werden. So wird von der OECD für die Gewinnabgrenzung im internationalen Einheitsunternehmen mit dem „Functionally separate entity approach" aktuell eine Annäherung an den zwischen verbundenen Kapitalgesellschaften zur Anwendung kommenden Fremdvergleichsgrundsatz, also der Trennungstheorie, vorgeschlagen. ${ }^{85}$ Demgegenüber fordert die EU-Kommission mit dem Vorschlag einer „Gemeinsamen konsolidierten Körperschaftsteuerbemessungsgrundlage“ eine weitgehende Umsetzung der Einheitstheorie für Kapitalgesellschaftskonzerne. ${ }^{86}$ 
Reinald Koch - 978-3-631-75683-6

Downloaded from PubFactory at 01/11/2019 03:01:46AM

via free access 


\section{Konzernbesteuerung in Europa de lege lata}

\section{1 Überblick}

Im geltenden Ertragsteuerrecht der meisten europäischen Mitgliedsstaaten wird keine der beiden in Kapitel 2.2 vorgestellten Theorien zur Besteuerung von Konzernen in Reinform umgesetzt. ${ }^{87}$ Steuersubjekt sind entsprechend der Trennungstheorie grundsätzlich die zivilrechtlich selbständigen juristischen Personen, die in ihrem Ansässigkeitsland ${ }^{88}$ mit dem nach Maßgabe des Trennungsprinzips unter Berücksichtigung des Fremdvergleichsgrundsatzes ermittelten Einkommen der Besteuerung unterliegen.

Der wirtschaftlichen Einheit des Konzerns wird allerdings in verschiedener Form durch die Berücksichtigung einheitstheoretischer Besteuerungselemente Rechnung getragen. So wird eine wirtschaftliche Doppelbesteuerung ausgeschütteter Gewinne einer Tochterkapitalgesellschaft sowie eine doppelte Berücksichtigung von Verlusten einer Tochterkapitalgesellschaft durch entsprechende Vorschriften bei der Ermittlung des Einkommens der Muttergesellschaft vermieden. Ferner gewähren zahlreiche Mitgliedsstaaten ihren nationalen (und teilweise auch grenzüberschreitend tätigen) Konzernen unter bestimmten Voraussetzungen die Besteuerung als Gruppe. Der Grad der im Rahmen dieser Gruppenbesteuerungssysteme gewährten Durchbrechungen des Trennungsprinzips reicht dabei von der Möglichkeit einer konzerninternen Übertragung von Gewinnen und/oder Verlusten, über eine Verlustverrechnung mit (eingeschränkter) Zwischenergebniseliminierung bis hin zu einer steuerlichen Vollkonsolidierung. Schließlich werden Quellensteuern auf Zins- und Lizenzzahlungen im EU-Konzernverbund weitgehend vermieden. ${ }^{89}$

Neben diesen Erleichterungen wird der wirtschaftlichen Verbundenheit von Konzerngesellschaften auch in Gestalt verschiedener Vorschriften Rechnung getragen, die der Sicherung des inländischen Steueraufkommens dienen. Das Trennungsprinzip eröffnet im Zusammenspiel mit dem internationalen Steuerlastgefälle durch die Möglichkeit zur Einkommensverlagerung in Niedrigsteuerländer einen der wesentlichen Ansätze zur internationalen Steuerplanung für multinationale Konzerne. Gebräuchliche Gestaltungen sind dabei die Gewinnverlagerung

87 Vgl. so für Deutschland Kessler (2004), S. 2.

88 Ansässigkeit wird dabei unter Bezugnahme auf rechtliche Kriterien (Sitzland bzw. Gründungsland) und/oder wirtschaftliche Kriterien (Ort der Geschäftsleitung) definiert. Kommt es zu überschneidenden Definitionen von Ansässigkeit, ist nach Artikel 4 Absatz 3 OECD. Musterabkommen der Ort der Geschäftsleitung ausschlaggebend. Vgl. Wendt (2009), S. 76$77 \mathrm{mwN}$. Im weiteren Verlauf der Arbeit wird aus Vereinfachungsgründen der Begriff „Sitzland" verwendet.

89 Vgl. Richtlinie 2003/49/EG des Rates v. 3.6.2003, Abl. EG 2003 Nr. L 157, S. 49. Zur Umsetzung dieser Richtlinie in den Mitgliedsstaaten siehe Jacobs (2007), S. 169-172. 
durch Festlegung unangemessener Verrechnungspreise oder Gestaltung der Finanzierungsstruktur sowie die Einschaltung von Basisgesellschaften..$^{90}$ Eine Erosion des inländischen Steueraufkommens durch derartige Gestaltungen wird daher in den meisten Mitgliedsstaaten durch Korrekturvorschriften vermieden.

Kapitel 3 beinhaltet einen Überblick über die Behandlung von Kapitalgesellschaftskonzernen im geltenden Steuerrecht der 27 Mitgliedsstaaten der Europäischen Union. ${ }^{91}$ Dieser dient zum einen der Erläuterung der steuerlichen Vorschriften, welche den im weiteren Verlauf der Arbeit erfolgenden Simulationsrechnungen zugrundeliegen. Insbesondere soll allerdings auch der bestehende Reformbedarf der Konzernbesteuerung aufgezeigt werden. Dazu werden zunächst in Abschnitt 3.2 die Grundzüge der Besteuerung von Kapitalgesellschaften als eigenständige Steuersubjekte dargestellt. Abschnitt 3.3 behandelt die einheitstheoretischen Elemente im geltenden Steuerrecht, während in Abschnitt 3.4 auf die Vorschriften eingegangen wird, die der Einschränkung von Steuergestaltungen dienen. Abschließend werden in Abschnitt 3.5 die Probleme der geltenden Konzernbesteuerung diskutiert. Die Rechtsdarstellungen beruhen - soweit nicht anders angegeben - auf den Angaben in Wendt (2009), Kesti (2008), Spengel (2008), Oestreicher/Scheffler/Spengel et al. (2008) sowie Endres/Oestreicher/Scheffler et al. (2007).

\subsection{Ertragsbesteuerung der Kapitalgesellschaft als selbständiges Steuersubjekt}

\subsubsection{Ermittlung des Einkommens}

\subsubsection{Steuerliche Gewinnermittlung}

Kapitalgesellschaften mit Sitz in einem Mitgliedsstaat der Europäischen Union unterliegen grundsätzlich als selbständige Steuersubjekte (Trennungstheorie) mit ihrem weltweiten Einkommen der Ertragsbesteuerung ${ }^{92}$, soweit das Besteuerungsrecht an ausländischen Einkünften nicht durch unilaterales Recht oder ein Doppelbesteuerungsabkommen beschränkt wird. Ausnahmen von diesem Grundsatz betreffen die Besteuerung dänischer und französischer Kapitalgesellschaften, die nach Maßgabe des Territorialitätsprinzips nur mit ihren inländischen Einkünften

90 Zur Steuerplanung in grenzüberschreitend tätigen Konzernen vgl. ausführlich Jacobs (2007), S. 853-1398. Weitere Möglichkeiten betreffen die Planung der Gruppenstruktur, die Nutzung von Qualifikationskonflikten und die Vermeidung konzerninterner Gewinnrealisierungen.

91 Die Darstellung beschränkt sich auf die Ebene der Konzerngesellschaften. Auf die steuerliche Behandlung der Anteilseigner der Muttergesellschaft wird nicht eingegangen. 
der Körperschaftsteuer unterliegen ${ }^{93}$, sowie die ertragsteuerliche Behandlung von Kapitalgesellschaften in Estland. Hier werden die einbehaltenen Gewinne von Kapitalgesellschaften vollständig von der Besteuerung ausgenommen, der Körperschaftsteuer unterliegen ausschließlich Gewinnausschüttungen. ${ }^{94}$ Ein derartiges Besteuerungssystem erfordert keine Einkommensermittlung im üblichen Sinne, weshalb Estland bei den meisten Aspekten der folgenden Rechtsdarstellung unberücksichtigt bleibt.

Bei der Ermittlung des Einkommens knüpfen sämtliche Mitgliedsstaaten an das Ergebnis des handelsrechtlichen Jahresabschlusses an, das in der Regel unter Beachtung spezifischer steuerlicher Vorschriften angepasst wird. Eine von der handelsbilanziellen Gewinnermittlung vollständig losgelöste steuerliche Gewinnermittlung kommt in keinem Mitgliedsstaat zur Anwendung. ${ }^{95}$ Bei der Bestimmung der Ausgangsgröße verweisen die meisten Mitgliedsstaaten auf landesspezifische Rechnungslegungsnormen. Lediglich in Zypern, Estland, Irland, Slowenien und der Slowakei bestimmt sich die Ausgangsgröße für bestimmte Gesellschaften nach Maßgabe der International Financial Reporting Standards (IFRS) oder die handelsrechtliche Gewinnermittlung kann wahlweise nach nationalen Vorschriften oder IFRS erfolgen. In Estland, Frankreich, Großbritannien und Malta bemessen sich die Handelsbilanz und damit die Ausgangsgröße für die Bestimmung der Steuerbilanz zwar nach nationalen Rechnungslegungsnormen, diese stimmen inhaltlich allerdings (weitgehend) mit den IFRS überein. ${ }^{96}$ Für die Überleitung von Handels- auf Steuerbilanz erforderliche Anpassungen betreffen sowohl die Bewertung von Vermögensgegenständen und Schulden und damit den Zeitpunkt der steuerwirksamen Berücksichtigung von Erträgen und Aufwendungen als auch die Freistellung bestimmter Erträge bzw. die Einschränkung des Abzugs bestimmter Aufwendungen. ${ }^{97}$ Der Grad der erforderlichen Anpassungen variiert dabei zwischen den Mitgliedsstaaten. ${ }^{98}$

93 In Dănemark erstreckt sich die Anwendung des Territorialitătsprinzips lediglich auf auslăndische Einkünfte aus Betriebsstätten und unbeweglichem Vermögen, in Frankreich werden ausländische Einkünfte nur besteuert, soweit es sich um passive Investmenteinkünfte handelt. Vgl. Wendt (2009), S. 77; Kesti (2008), S. 175 und 233.

$94 \mathrm{Vgl}$. Endres/Oestreicher/Scheffler et al. (2007), S. 17.

95 Vgl. Wendt (2009), S. 55; Spengel (2008), S. 3-4.

$96 \mathrm{Vgl}$. Endres/Oestreicher/Scheffler et al. (2007), S. 159-168. In Deutschland erfolgte zum 1.1.2009 eine Annäherung der handelsrechtlichen Gewinnermittlung an die IFRS-Regeln im Rahmen des Bilanzrechtsmodernisierungsgesetzes.

$97 \mathrm{Vgl}$. Endres/Oestreicher/Scheffler et al. (2007), S. 25.

98 Vgl. Wendt (2009), S. 55; Jacobs (2007), S. 103; Endres/Oestreicher/Scheffler et al. (2007), S. 25-26. Unterschiedliche Auffassungen bestehen über den Grad der Verbindung zwischen handels- und steuerrechtlicher Gewinnermittlung in einzelnen Mitgliedsstaaten. In Center for European Policy Studies (2005), S. 23-24 wird auf eine starke Verbindung in Deutschland, Österreich und Luxemburg verwiesen, während Kersting (2005), S. 351 auf eine enge Verbindung für Großbritannien und Richter (2005), S. 502-504 auf „kaum mehr zu überblickende" Durchbrechungen für Österreich hinweist. 


\subsubsection{Behandlung ausländischer Einkünfte}

Die Besteuerung ausländischer Einkünfte erfordert eine Aufteilung des Besteuerungsrechts zwischen dem inländischen und dem ausländischen Fiskus. Hierbei folgen die Mitgliedsstaaten im Wesentlichen den Vorgaben des OECD-Musterabkommens. ${ }^{99}$ Die Zuordnung des Besteuerungsrechts orientiert sich dabei grundsätzlich an der Stärke der örtlichen Verbindung der Einkunftsquelle mit dem Quellenstaat. So wird das Besteuerungsrecht bei passiven Dividenden-, Zins- und Lizenzeinnahmen grundsätzlich dem Ansässigkeitsstaat der Kapitalgesellschaft zugewiesen, während der Quellenstaat hier nur ein der Höhe nach beschränktes Besteuerungsrecht hat ${ }^{100}$, welches in Fällen europäischer Konzerne für Dividendenzahlungen aufgrund der Mutter-/Tochterrichtlinie ${ }^{101}$ und für Zins- und Lizenzzahlungen durch die Zins- und Lizenzrichtlinie ${ }^{102}$ grundsätzlich aufgehoben wird. Bei gewerblichen Einkünften besitzt der Quellenstaat hingegen regelmäßig ein der Höhe nach unbeschränktes Besteuerungsrecht, soweit diese durch eine Betriebsstätte im Quellenstaat erzielt werden. ${ }^{103}$ Die Vermeidung einer Doppelbelastung dieser Einkünfte erfolgt in diesem Fall durch Freistellung oder Anrechnung der Auslandssteuern im Ansässigkeitsstaat. Tabelle 1 gibt einen Überblick über die in den Mitgliedsstaaten zur Anwendung kommende Methode. ${ }^{104}$

Im Verlustfall unterscheiden die meisten Mitgliedsstaaten zwischen der Behandlung von negativen Einkünften aus inländischer und ausländischer $\mathrm{Ge}-$ schäftstätigkeit. Negative inländische Einkünfte können innerperiodisch überwiegend uneingeschränkt mit anderen positiven Einkünften der Kapitalgesellschaft verrechnet werden. Lediglich Irland und Großbritannien beschränken den horizontalen Verlustausgleich teilweise auf dieselbe Einkunftsart („Schedule“). ${ }^{105}$ Frankreich, Lettland und Zypern beschränken die Verrechnung von Veräußerungsverlusten (,capital losses“), welche in diesen Ländern einer gesonderten Be-

99 Vgl. Wendt (2009), S. 77.

$100 \mathrm{Vgl}$. Art. 10,11 und 12 OECD-MA.

101 Zur Umsetzung der Mutter-/Tochterrichtlinie in den Mitgliedsstaaten vgl. Jacobs (2007), S. 155-161.

102 Zur Umsetzung der Zins- und Lizenzrichtlinie in den Mitgliedsstaaten vgl. Jacobs (2007), S. 169-172. Einigen Mitgliedsstaaten wurde für die Umsetzung der Richtlinie ein Übergangszeitraum gewährt, innerhalb dessen ein der Höhe nach begrenztes Quellensteuerrecht fortbesteht.

$103 \mathrm{Vgl}$. Art. 7 OECD-MA.

104 Teilweise ist unilateral oder in Doppelbesteuerungsabkommen zwischen den Mitgliedsstaaten eine Anwendung der Anrechnungsmethode anstelle der Freistellungsmethode vorgesehen (,Switch-over Klauseln“), um eine drohende Nicht- oder Minderbesteuerung zu vermeiden. Derartige Regelungen kommen teilweise auch für passive ausländische Betriebsstätten oder Tochterkapitalgesellschaften zur Anwendung („Aktivitätsklauseln“). Vgl. hierzu Jacobs (2007), S. 87-88.

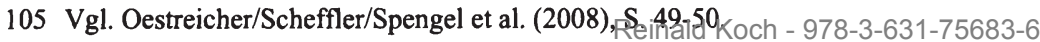


steuerung beziehungsweise einem reduziertem Steuersatz unterliegen. ${ }^{106}$ Bezüglich der Behandlung von Verlusten ausländischer Betriebsstätten folgen die meisten Mitgliedsstaaten der Behandlung von positiven Einkünften aus diesen Betriebsstätten. So knüpfen 17 Mitgliedsstaaten die Berücksichtigung dieser Auslandsverluste an die Anwendung der Anrechnungsmethode zur Vermeidung von internationaler Doppelbesteuerung. ${ }^{107}$ Nur fünf Mitgliedsstaaten (Belgien, Niederlande, Österreich, Spanien und Zypern) lassen eine Berücksichtigung von Auslandsverlusten auch bei Anwendung der Freistellungsmethode zu. ${ }^{108}$ Ein doppelter Verlustabzug wird in diesen Fällen durch eine Nachversteuerungslösung verhindert. ${ }^{109}$ Zwei Mitgliedsstaaten (Dänemark und Frankreich) untersagen eine Berücksichtigung der Auslandsverluste als Konsequenz der Besteuerung nach dem Territorialitätsprinzip generell. Allerdings ist hier eine Verlustberücksichtigung unter den Voraussetzungen der grenzüberschreitenden Gruppenbesteuerung möglich. ${ }^{110}$

Tabelle 1: Methode zur Vermeidung der Doppelbesteuerung bei ausländischen Betriebsstätteneinkünften

\begin{tabular}{|l|l|}
\hline Freistellungsmethode & $\begin{array}{l}\text { Belgien, Bulgarien, Dănemark, Deutschland, Estland, Frankreich, } \\
\text { Griechenland, Luxemburg, Niederlande, Österreich, Polen, Spanien, } \\
\text { Ungarn, Zypern }\end{array}$ \\
\hline Anrechnungsmethode & $\begin{array}{l}\text { Finnland, Großbritannien, Irland, Italien, Lettland, Litauen, Malta, } \\
\text { Portugal, Rumänien, Schweden, Slowakei, Slowenien, Tschechische } \\
\text { Republik }\end{array}$ \\
\hline
\end{tabular}

Quelle: Wendt (2009), S. 78. Die Angaben für Bulgarien und Rumänien beziehen sich ausschließlich auf das DBA mit Deutschland. Vgl. DBA Deutschland-Bulgarien vom 2.6.1987; DBA Deutschland-Rumänien vom 12.11.2003.

\subsubsection{Intertemporale Verlustverrechnung}

Ein verbleibendes negatives Einkommen kann in allen Mitgliedsstaaten ${ }^{\prime \prime \prime}$ im Wege des Verlustvortrags interperiodisch mit zukünftigen Gewinnen derselben Kapitalgesellschaft verrechnet werden, fünf Mitgliedsstaaten (Deutschland, Frankreich, Großbritannien, Italien und Niederlande) erlauben zusätzlich einen Verlust-

$106 \mathrm{Vgl}$. Endres/Oestreicher/Scheffler et al. (2007), S. 82.

$107 \mathrm{Vgl}$. Wendt (2009), S. 83. Deutschland beschränkt auch in diesen Făllen die Einbeziehung von Auslandsverlusten in den allgemeinen Verlustabzug auf Verluste aus aktiver Geschăftstătigkeit (§ 2a EStG). Die Anwendung dieser Vorschrift wurde allerdings im Rahmen des Jahressteuergesetzes 2009 auf Auslandsverluste aus Drittstaaten eingeschränkt.

$108 \mathrm{Vgl}$. Wendt (2009), S. 83.

109 Vgl. Endres/Oestreicher/Scheffler et al. (2007), S. 83-84. Vgl. zur Funktionsweise der Nachversteuenungsmethode Kapitel 4.3.3.2.

$110 \mathrm{Vgl}$. Endres/Oestreicher/Scheffler et al. (2007), S. 83-84.

111 In Estland ist eine Verlustverrechnung aufgrund der Ausschuttungsbesteuerung nicht erforderlich. 
rücktrag. Einige Mitgliedsstaaten beschränken diese Verlustverrechnungsmöglichkeiten in zeitlicher bzw. betragsmäßiger Hinsicht. Die zeitlichen Beschränkungen der Vorschriften zur intertemporalen Verlustverrechnung sind in Tabelle $2 \mathrm{zu}$ sammengefasst.

Tabelle 2: Zeitliche Verlustvortrags- und Verlustrücktragsbegrenzungen

\begin{tabular}{|c|c|c|c|}
\hline \multicolumn{2}{|c|}{ Verlustvortragsdauer } & \multicolumn{2}{|c|}{ Verlustrücktragsdauer } \\
\hline unbegrenzt & $\begin{array}{l}\text { Belgien, Dänemark, Deutschland, } \\
\text { Frankreich, Großbritannien, Lu- } \\
\text { xemburg, Malta, Niederlande, Ös- } \\
\text { terreich, Schweden, Ungarn, Zy- } \\
\text { pern }\end{array}$ & 3 Jahre & Frankreich \\
\hline 15 Jahre & Spanien & $1 \mathrm{Jahr}$ & $\begin{array}{l}\text { Deutschland, Großbritannien, } \\
\text { Niederlande }\end{array}$ \\
\hline 10 Jahre & Finnland & \multirow{4}{*}{$\begin{array}{l}\text { Nicht } \\
\text { möglich }\end{array}$} & \multirow{4}{*}{$\begin{array}{l}\text { Belgien, Bulgarien, Däne- } \\
\text { mark, Finnland, Griechenland, } \\
\text { Irland }^{112} \text {, Italien, Lettland, Li- } \\
\text { tauen, Luxemburg, Malta, } \\
\text { Österreich, Polen, Portugal, } \\
\text { Rumänien, Slowakei, Slowe- } \\
\text { nien, Spanien, Schweden, } \\
\text { Tschechische Republik, Un- } \\
\text { garn, Zypern }\end{array}$} \\
\hline 9 Jahre & Niederlande & & \\
\hline 6 Jahre & Portugal & & \\
\hline 5 Jahre & $\begin{array}{l}\text { Bulgarien, Griechenland, Italien, } \\
\text { Lettland, Litauen, Polen, Rumä- } \\
\text { nien, Slowakei, Slowenien, Tsche- } \\
\text { chische Republik }\end{array}$ & & \\
\hline
\end{tabular}

Quelle: Eigene Darstellung in Anlehnung an Endres/Oestreicher/Scheffler et al. (2007), S. 81 unter Berücksichtigung aktueller Angaben in Kesti (2008).

In Deutschland, Österreich und Polen kommen betragsmäßige Beschränkungen des Verlustvor- beziehungsweise -rücktrags zur Anwendung. So wird in Deutschland der Verlustrücktrag auf 511.500 Euro und der Verlustvortrag auf 1.000.000 Euro zuzüglich 60 Prozent des verbleibenden Gesamtbetrags der Einkünfte begrenzt. In Österreich wird der Verlustvortrag auf 75 Prozent des jeweiligen Periodengewinns beschränkt, während in Polen nur maximal die Hälfte des Verlustvortrags in einer Periode genutzt werden kann. ${ }^{13}$ Im Fall von Restrukturierungen und/oder gravierenden Änderungen im Anteilseignerkreis gehen bestehende Verlustvorträge im Regelfall unter. ${ }^{14}$

112 Für Verluste im Jahr der Unternehmensbeendigung wird ein Rücktrag über drei Perioden gewährt. Vgl. Kesti (2008), S. 369.

$113 \mathrm{Vgl}$. Oestreicher/Scheffler/Spengel et al. (2008), S. 49.

114 Vgl. hierzu Endres/Oestreicher/Scheffler et al. (2007), S. 82. Mit Ausnahme der Slowakei und Estland besitzen alle Mitgliedsstaaten eine derartige Korschrift:-8-3-631-75683-6 


\subsubsection{Steuersätze}

Kapitalgesellschaften unterliegen mit ihrem Einkommen in den meisten Mitgliedsstaaten einem linearen Körperschaftsteuertarif. Belgien, Frankreich, Großbritannien, Luxemburg, Niederlande, Spanien und Ungarn verwenden unterschiedliche lineare Steuersätze in Abhängigkeit von der Unternehmensgröße. Dabei kommen in diesen Ländern niedrigere Eingangssteuersätze für kleine und mittelgroße Unternehmen zur Anwendung, in Frankreich wird daneben ab einer bestimmten Unternehmensgröße eine zusätzliche Sozialabgabe als Zuschlag auf die Körperschaftsteuer erhoben. Andere Mitgliedsstaaten (z.B. Irland und Slowenien) sehen Sondersteuersätze für bestimmte Geschäftszweige, Einkünfte (z.B. Einkünfte aus Veräußerungsgeschäften) bzw. Regionen vor. ${ }^{115}$

Abbildung 1: Nominelle Steuersätze auf Gewinne von Kapitalgesellschaften in Europa

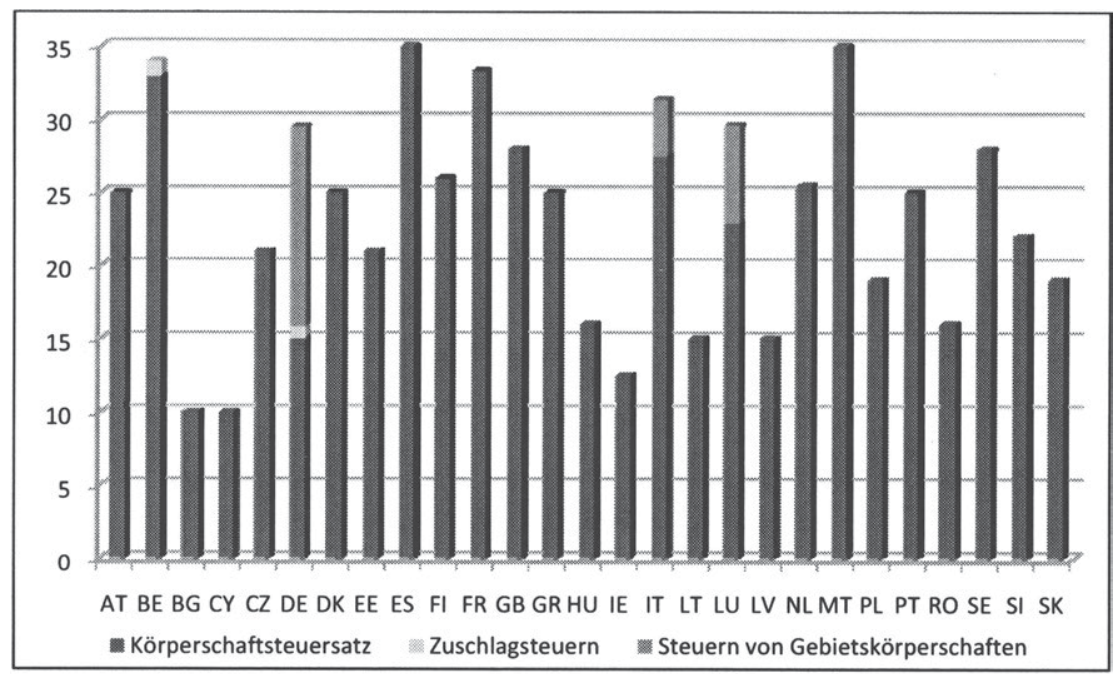

Quelle: Eigene Darstellung auf Basis der Angaben in KPMG (2008) und Kesti (2008).

In einigen Mitgliedsstaaten erhöhen sich die Körperschaftsteuersätze um Zuschläge bzw. um regionale Unternehmenssteuern, die von den jeweiligen Gebietskörperschaften erhoben werden. Zuschläge auf die Körperschaftsteuer werden in Belgien ( 0,99 Prozentpunkte) und Deutschland (0,825 Prozentpunkte) erhoben, regionale Unternehmenssteuern in Deutschland (13,69 Prozentpunkte), Italien (3,9 Prozentpunkte) und Luxemburg (6,75 Prozentpunkte). ${ }^{116}$ Die Bemessungsgrund-

$115 \mathrm{Vgl}$. Endres/Oestreicher/Scheffler et al. (2007), S. 17 sowie die Angaben in Kesti (2008).

$116 \mathrm{Vgl}$. die entsprechenden Angaben in KPMG (2008) und Kesti (2008). Die Hohe dieser regionalen Ertragsteuern auf Kapitalgesellschaftsgewinne variieren regelmäßig zwischen den Ge- 
lage dieser regionalen Unternehmenssteuern unterscheidet sich zumeist von der Bemessungsgrundlage der Körperschaftsteuer. Die (effektive) tarifliche Belastung auf Kapitalgesellschaftsgewinne in den Mitgliedsstaaten, die sich unter Berücksichtigung von Zuschlägen und regionalen Ertragsteuern ergibt, ist in Abbildung 1 dargestellt. Dabei werden Dependenzen zwischen den verschiedenen Ertragsteuern berücksichtigt, Unterschiede in der Bemessungsgrundlage zwischen Körperschaftsteuer und regionalen Unternehmenssteuern hingegen vernachlässigt.

Die effektive Tarifbelastung der Gewinne von Kapitalgesellschaften schwankt in der EU innerhalb einer Bandbreite von 25 Prozentpunkten. Die niedrigste Tarifbelastung kommt in Zypern und Bulgarien (10 Prozent) zur Anwendung, die höchste in Spanien und Malta (35 Prozent). Im EU-Durchschnitt beträgt die effektive Tarifbelastung 24,23 Prozent.

Abbildung 2: Entwicklung der nominellen Steuersätze auf Gewinne von Kapitalgesellschaften in Europa

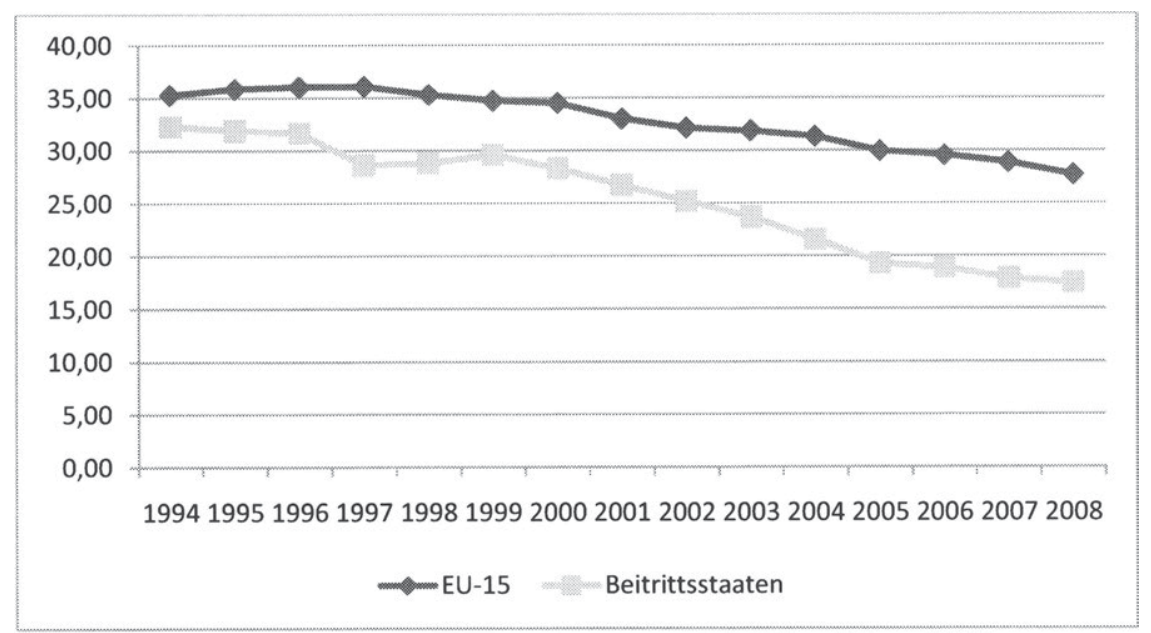

Quelle: Eigene Darstellung auf Basis der Angaben in KPMG (2008) und Kesti (2008).

Abbildung 2 verdeutlicht die Entwicklung der effektiven Tarifbelastung in den EU-Mitgliedsstaaten zwischen 1994 und 2008. Dabei zeigt sich, dass sowohl in den alten Mitgliedsstaaten als auch in den zehn Beitrittsstaaten die tariflichen Steuersätze kontinuierlich gesenkt wurden. Diese Entwicklung ist Ausdruck der

bietskörperschaften eines Mitgliedsstaats. Die Angaben in Klammern stellen typische Höhen dieser Regionalsteuern dar. Neben den vier genannten Ländern wird auch in Ungarn eine regionale Unternehmenssteuer erhoben. Diese wird allerdings bei den weiteren Betrachtungen nicht berücksichtigt, da die Bemessungsgrundlage deutlich von der Bemessungsgrundlage der Körperschaftsteuer abweicht. Vgl. Kesti (2008) $S_{\text {Sal }} 336$ 
im internationalen Steuerwettbewerb seit längerem vorherrschenden Praxis einer Senkung der Steuersätze bei gleichzeitiger Verbreiterung der Bemessungsgrundlage. ${ }^{117}$ Diese Tendenz ist insbesondere auf die „psychologische Relevanz" der tariflichen Steuersätze bei der Standortentscheidung zurückzuführen. ${ }^{118}$ Ferner zeigt sich, dass der durchschnittliche Steuersatz in den Beitrittsstaaten im Betrachtungszeitraum durchgehend unterhalb der durchschnittlichen Tarifbelastung in den alten Mitgliedsstaaten liegt. Seit der Erweiterung der EU im Jahr 2004 liegt die Differenz konstant bei etwa zehn Prozentpunkten. Die Erweiterung der EU hat somit zu einer deutlichen Verschärfung des Steuerwettbewerbs innerhalb des Binnenmarktes geführt. ${ }^{119}$ Dabei ist die Differenz zum Großteil auf eine deutlich stärkere Absenkung der Steuersätze im Betrachtungszeitraum in den Beitrittsstaaten zurückzuführen.

\subsection{Einheitstheoretische Elemente bei der Besteuerung von ver- bundenen Kapitalgesellschaften}

\subsubsection{Vermeidung einer Doppelbesteuerung ausgeschütteter Gewinne und einer doppelten Verlustberücksichtigung}

Dem Konzernverbund von Kapitalgesellschaften wird im geltenden Steuerrecht insbesondere durch zwei Formen steuerlicher Begünstigungen im geltenden Steuerrecht Rechnung getragen. Zum einen wird eine wirtschaftliche Doppelbelastung von Gewinnen der Tochterkapitalgesellschaft bei ihr und auf Ebene der Mutterkapitalgesellschaft sowie eine doppelte Berücksichtigung von Verlusten der Tochterkapitalgesellschaft vermieden. Zum anderen werden im Rahmen von Gruppenbesteuerungssystemen einheitstheoretische Besteuerungselemente umgesetzt.

Eine wirtschaftliche Doppelbesteuerung der ausgeschütteten Gewinne einer Tochterkapitalgesellschaft wird im geltenden Recht der Mitgliedsstaaten vermieden durch Dividendenfreistellung oder durch Anrechnung der auf den Gewinnen der Tochtergesellschaft lastenden Körperschaftsteuer. Aktuell verwenden in Bezug auf inländische Dividenden 25 Mitgliedsstaaten die Freistellungsmethode, wobei 21 Mitgliedsstaaten die Dividenden vollständig freistellen. Lediglich vier Mitgliedsstaaten (Belgien, Deutschland, Frankreich und Italien) machen von dem Mitgliedsstaatenwahlrecht der Mutter-Tochterrichtlinie Gebrauch, eine funfprozentige Pauschale der Dividenden als nicht-abziehbare Betriebsausgaben zu be-

117 Vgl. hierzu Oestreicher/Spengel (2003), S. 93; Chennels/Griffith (1997), S. 26-30.

$118 \mathrm{Vgl}$. Oestreicher/Spengel (2003), S. 93.

119 So auch Jacobs (2007), S. 115. 
steuern. ${ }^{120}$ In zwei Mitgliedsstaaten (Malta und Spanien) wird eine Doppelbesteuerung durch vollständige Anrechnung ${ }^{121}$ der Körperschaftsteuer, die auf den ausgeschütteten Gewinnen der Tochtergesellschaften lastet, vermieden. Ausländische Dividenden werden grundsätzlich in Übereinstimmung mit inländischen Dividenden behandelt. Lediglich Irland, Griechenland und Großbritannien verwenden für Auslandsdividenden abweichend die Anrechnungsmethode, Slowenien stellt Auslandsdividenden lediglich zu 95 Prozent steuerfrei. In Malta und Spanien kommt für Auslandsdividenden abweichend die Freistellungsmethode zur Anwendung. ${ }^{122}$

Um eine Verzerrung der Ausschüttungsentscheidung beim Tochterunternehmen durch eine unterschiedliche Behandlung von thesaurierten und ausgeschütteten Gewinnen zu vermeiden, folgt die steuerliche Behandlung von Veräußerungsgewinnen in vielen Mitgliedsstaaten der Besteuerung von Dividenden. Dementsprechend stellen 15 Mitgliedsstaaten die Gewinne aus der Veräußerung von Kapitalgesellschaften vollständig frei ${ }^{123}$, in fünf weiteren Mitgliedsstaaten kommt es zu einer teilweisen Freistellung (vgl. Tabelle 3). Dabei besteuern Deutschland, Frankreich und Italien in Übereinstimmung mit der Behandlung von Dividenden eine Pauschale in Höhe von fünf Prozent der Veräußerungsgewinne, während Slowenien und Portugal die Veräußerungsgewinne lediglich zur Hälfte freistellen. ${ }^{124}$ Insgesamt sechs Mitgliedsstaaten gewähren keine begünstigte $\mathrm{Be}-$ handlung oder eine Begünstigung nur in bestimmten Sonderfällen. ${ }^{125}$

Die Behandlung von Veräußerungsverlusten erfolgt in den meisten Mitgliedsstaaten korrespondierend zur Behandlung von Veräußerungsgewinnen. Soweit die Veräußerungsgewinne vollständig oder annähernd vollständig freigestellt sind, können Veräußerungsverluste in der Regel nicht berücksichtigt werden (vgl. Tabelle 3). Ausnahmen von diesem Grundsatz bestehen in Luxemburg und Spanien.

$120 \mathrm{Vgl}$. die entsprechenden Angaben in Kesti (2008). In Tschechien gelten fünf Prozent der Dividenden als nicht-abziehbare Gemeinkosten, es sei denn der Steuerpflichtige weist nach, dass die tatsächlichen Aufwendungen geringer ausfallen. Vgl. Kesti (2008), S. 158.

121 In Spanien ist die vollständige Anrechnung an eine Mindestbeteiligung von 5 Prozent geknüpft. Bei geringerer Beteiligungshöhe ist die Anrechnung auf 50 Prozent der Körperschaftsteuer begrenzt. Vgl. Kesti (2008), S. 722.

$122 \mathrm{Vgl}$. die entsprechenden Angaben in Kesti (2008).

123 Dabei beschränken Österreich und Spanien diese Freistellung auf ausländische Veräußerungsgewinne. Vgl. Endres/Oestreicher/Scheffler et al. (2007), S. 23.

124 Die verbleibenden 50\% können in Portugal im Wege des Roll-over-Reliefs verzögert besteuert werden. Vgl. Kesti (2008), S. 623-624.

125 Bulgarien, Griechenland und Lettland z.B. gewähren eine Freistellung nur bei börsennotierten Beteiligungen. Vgl. die entsprechenden Angaben in Kesti (2098d.-3-631-75683-6 
Tabelle 3: Behandlung von Gewinnen und Verlusten aus dem Verkauf von Beteiligungen bei der Körperschaftsteuer

\begin{tabular}{|c|c|c|c|}
\hline \multicolumn{2}{|c|}{ Veräußerungsgewinne } & \multicolumn{2}{|c|}{ Veräußerungsverluste } \\
\hline Freistellung & $\begin{array}{l}\text { Belgien, Dänemark, Finnland, } \\
\text { Großbritannien, Irland, Litau- } \\
\text { en, Luxemburg, Malta, Nie- } \\
\text { derlande, Österreich, Schwe- } \\
\text { den, Spanien }{ }^{126} \text {, Tschechische } \\
\text { Republik, Ungarn, Zypern }\end{array}$ & $\begin{array}{l}\text { Berücksich- } \\
\text { tigung nicht } \\
\text { möglich }\end{array}$ & $\begin{array}{l}\text { Belgien, Dänemark, Deutsch- } \\
\text { land, Finnland, Frankreich, } \\
\text { Griechenland, Großbritannien, } \\
\text { Irland, Italien, Litauen, Malta, } \\
\text { Niederlande }^{127} \text { Schweden, } \\
\text { Tschechische Republik, Un- } \\
\text { garn, Zypern }\end{array}$ \\
\hline $\begin{array}{l}\text { Teilweise } \\
\text { Freistellung }\end{array}$ & $\begin{array}{l}\text { Deutschland, Frankreich, Ita- } \\
\text { lien, Slowenien, Portugal }\end{array}$ & $\begin{array}{l}\text { Teilweise/ } \\
\text { einge- } \\
\text { schränkt }\end{array}$ & $\begin{array}{l}\text { Portugal, Österreich, Slowe- } \\
\text { nien }\end{array}$ \\
\hline \multirow{3}{*}{$\begin{array}{l}\text { Keine Steu- } \\
\text { erbegunsti- } \\
\text { gung oder } \\
\text { nur in Son- } \\
\text { derfallen }\end{array}$} & \multirow{3}{*}{$\begin{array}{l}\text { Bulgarien, Griechenland, Lett- } \\
\text { land, Polen, Rumänien, Slo- } \\
\text { wakei }\end{array}$} & möglich & \\
\hline & & $\begin{array}{l}\text { Verrechnung } \\
\text { gegen künf- } \\
\text { tige Veräu- } \\
\text { Berungsge- } \\
\text { winne }\end{array}$ & Lettland \\
\hline & & $\begin{array}{l}\text { Berülcksich- } \\
\text { tigung mög- } \\
\text { lich }\end{array}$ & $\begin{array}{l}\text { Bulgarien, Luxemburg, Polen, } \\
\text { Rumänien, Slowakei, Spanien }\end{array}$ \\
\hline
\end{tabular}

Quelle: Eigene Darstellung in Anlehnung an Endres/Oestreicher/Scheffler et al. (2007), S. 22-23 und 82 unter Berücksichtigung aktueller Angaben in Kesti (2008).

\subsubsection{Gruppenbesteuerungssysteme}

Gruppenbesteuerungssysteme, welche ebenfalls der wirtschaftlichen Einheit Konzern zumindest teilweise Rechnung tragen und insoweit eine Durchbrechung des Trennungsprinzips begründen, stellen neben der indirekten Berücksichtigung als Veräußerungsverluste oder Teilwertabschreibungen eine weitere Möglichkeit zur konzerninternen Verlustverrechnung dar. Derartige Systeme existieren aktuell in 17 Mitgliedsstaaten. Von den alten Mitgliedsstaaten verfügen lediglich Belgien und Griechenland nicht über ein derartiges System, bei den Beitrittsstaaten trifft dies auf mehr als die Hälfte (Bulgarien, Estland, Litauen, Rumänien, Slowakei, Slowenien, Tschechische Republik, Ungarn) zu. ${ }^{128}$ Die Ausgestaltung dieser Sys-

126 In Spanien kommt bei Auslandsbeteiligungen die Freistellungsmethode zur Anwendung, während Inlandsbeteiligungen nach der Anrechnungsmethode behandelt werden. Vgl. Kesti (2008), S. 719 und 723.

127 Eine Ausnahme besteht für ausgewählte Liquidationsverluste. Vgl. Kesti (2008), S. 571.

$128 \mathrm{Vgl}$. die entsprechenden Angaben in Kesti (2008) 
teme unterscheidet sich deutlich sowohl hinsichtlich der Anwendungsvoraussetzungen als auch der Besteuerungsfolgen. ${ }^{129}$

Mit Blick auf die Anwendungsvoraussetzungen ergeben sich Unterschiede sowohl hinsichtlich der einzubeziehenden Gesellschaften, der Anforderungen an die Beziehung zwischen diesen Gesellschaften sowie des Bestehens von Ausübungswahlrechten. Die Beschränkung hinsichtlich der einzubeziehenden Gesellschaften betrifft insbesondere die Begrenzung des räumlichen Anwendungsbereichs sowie die berücksichtigungsfähigen Rechtsformen. In räumlicher Hinsicht ist die Anwendung in den meisten Mitgliedsstaaten auf inländische Gesellschaften begrenzt. Lediglich Dänemark, Frankreich, Großbritannien, Irland, Italien und Österreich lassen eine Einbeziehung von ausländischen Untergesellschaften $\mathrm{zu}$, wobei sich die Einbeziehungsvoraussetzungen und Besteuerungsfolgen von denen bei inländischen Gruppengesellschaften unterscheiden. Dabei bestehen in Italien (nationale und internationale Gruppenbesteuerung) und Frankreich (,Integration Fiscale“ und „Regime du Benefice Consolidé“ ${ }^{\prime 130}$ ) zwei separate Gruppenbesteuerungssysteme mit weitreichenden Unterschieden, während in Dänemark und Österreich die Unterscheidung weniger strikt ist. In Dänemark beschränken sich die Unterschiede zwischen in- und ausländischen Tochtergesellschaften lediglich auf Mindestdauer und Ausübungswahlrechte, in Österreich ergeben sich gewisse Unterschiede auch beim Umfang der Verlustverrechnung. ${ }^{131}$ Großbritannien und Irland gewähren eine Verlustverrechnung nur für endgültige Auslandsverluste. ${ }^{132}$ Darüber hinaus kann in einigen Mitgliedsstaaten eine steuerliche Gruppe auch aus inländischen Schwestergesellschaften einer ausländischen Muttergesellschaft bestehen und/oder von der inländischen Betriebsstätte einer ausländischen Obergesellschaft begründet werden. ${ }^{133}$

Mit Blick auf die Rechtsform der einzubeziehenden Gesellschaften beschränken die meisten Mitgliedsstaaten die Anwendung der Gruppenbesteuerung auf Körperschaftsteuersubjekte. ${ }^{134}$ Finnland, die Niederlande, Polen, Schweden,

129 Für einen Überblick über die Gruppenbesteuerungssysteme in Europa vgl. Oestreicher/Scheffler/Spengel et al. (2008), S. 57-86; Endres/Oestreicher/Scheffler et al. (2007), S. 84-93; Bundesverband der deutschen Industrie/PriceWaterhouseCoopers (2006). Für eine detaillierte Beschreibung der Gruppenbesteuerungssysteme sämtlicher oder ausgewählter Mitgliedsstaaten vgl. Wittkowski (2008); Sievert (2006).

130 Voraussetzung für die Anwendung dieses Systems ist die Zustimmung des Ministeriums für Wirtschaft, Finanzen und Industrie. Aufgrund der restriktiven Ausübung dieser Ermessensentscheidung in der Vergangenheit ist die praktische Bedeutung dieses Systems bisher gering. Vgl. Sievert (2006), S. 160.

$131 \mathrm{Vgl}$. Oestreicher/Scheffler/Spengel et al. (2008), S. 59. So sind in Österreich ausländische Verluste nur quotal berücksichtigungsfähig. Vgl. Sievert (2006), S. 201.

132 Vgl. Kesti (2008), S. 844; Wittkowski (2008), S. 240.

133 Für die Ausführungen zum räumlichen Anwendungsbereich vgl. Oestreicher/Scheffler/Spengel et al. (2008), S. 59-61 und die Angaben zum aktuellen Rechtsstand in Kesti (2008).

134 Für die Ausführungen zu den Rechtsformvoraussetzungen vgl. Oestreicher/Scheffler/Spengel et al. (2008), S. 68-69. 
Slowenien und Spanien grenzen den Anwendungskreis weitergehend auf Kapitalgesellschaften ein. Dabei gilt in Finnland, Polen und Schweden diese zusätzliche Einschränkung nur für Untergesellschaften. In Frankreich können auch Personengesellschaften in die steuerliche Gruppe einbezogen werden, soweit diese zuvor zur Körperschaftsteuer optiert haben. Lediglich in Deutschland (Personengesellschaften und natürliche Personen) und Lettland (natürliche Personen) können auch Einkommensteuersubjekte berücksichtigt werden. Dieses betrifft allerdings nur eine Einbeziehung als Gruppenträger. ${ }^{135}$ Darüber hinaus begrenzen Großbritannien, Luxemburg und Portugal die Anwendung auf Gesellschaften, die dem regulären Körperschaftsteuertarif unterliegen; Finnland und Schweden schließen die Anwendung für bestimmte Branchen aus. ${ }^{136}$ Italien schließlich begrenzt die Anwendung der internationalen Gruppenbesteuerung auf Obergesellschaften, „die entweder an der Börse notiert sind, von in Italien ansässigen natürlichen Personen oder vom Staat oder öffentlichen Körperschaften gehalten werden." ${ }^{\text {137 }}$

Hinsichtlich der Beziehung zwischen den Gruppengesellschaften ist das dominierende Beurteilungskriterium eine direkte oder indirekte Mindestbeteiligung. ${ }^{138}$ Dabei wenden einige Mitgliedsstaaten diese Mindestbeteiligung auf die Stimmrechte, andere auf das Beteiligungskapital und wieder andere auf beide Größen an. Bei der Ermittlung der relevanten Beteiligungsquote ist eine Addition von direkt und indirekt gehaltenen Anteilen überwiegend möglich. Lediglich Lettland, Malta, Polen und Slowenien schließen dieses aus, in Österreich ist die Berücksichtigung indirekter Anteile auf nationale Tochtergesellschaften beschränkt. ${ }^{139}$

$135 \mathrm{Vgl}$. Oestreicher/Scheffler/Spengel et al. (2008), S. 68-69.

136 In Schweden ist die Anwendung für Wohnungsbaugenossenschaften und Investmentgesellschaften ausgeschlossen, in Finnland für Banken, Versicherungen und Unternehmen mit nur passiver Tätigkeit. Vgl. Oestreicher/Scheffler/Spengel et al. (2008), S. 68.

137 Sievert (2006), S. 168.

138 Für die Ausfuhrungen zu den Beteiligungsanforderungen vgl. Oestreicher/Scheffler/Spengel et al. (2008), S. 61-66.

139 In Deutschland, Frankreich, Portugal und Spanien ist die Berücksichtigung indirekter Anteile daran gebunden, dass die erforderliche Mindestbeteiligungsquote auf jeder Konzernstufe erreicht wird. In Portugal und Spanien muss das erforderliche Beteiligungsverhaltnis auch bei der indirekten Beteiligung erreicht werden. Schweden setzt für die Berücksichtigung indirekter Anteile voraus, dass die indirekte Beteiligung innerhalb der Gruppe potenziell auf die Mutter verschmolzen werden kann. Vgl. Oestreicher/Scheffler/Spengel et al-(2008)_S. 64. 
Tabelle 4 fasst die Mindestbeteiligungshöhe und die Bezugsgröße in den Mitgliedsstaaten zusammen.

Tabelle 4: Gruppenbesteuerungssysteme (Beteiligungsanforderungen)

\begin{tabular}{|c|c|c|c|}
\hline \multicolumn{2}{|c|}{ Mindestbeteiligunghöhe } & \multicolumn{2}{|l|}{ Bezugsgröße } \\
\hline$>50 \%$ & $\begin{array}{l}\text { Dänemark, Deutschland, Frankreich } \\
\text { (,Regime du Benefice Consolide“), } \\
\text { Großbritannien (Capital Gains Group), } \\
\text { Irland (Withholding Tax), Italien (nati- } \\
\text { onal und international), Malta, Öster- } \\
\text { reich }\end{array}$ & $\begin{array}{l}\text { Beteili- } \\
\text { gungs- } \\
\text { kapital }\end{array}$ & $\begin{array}{l}\text { Finnland, Großbritannien, } \\
\text { Irland, Italien (national), } \\
\text { Luxemburg, Niederlande, } \\
\text { Österreich, Polen, Schwe- } \\
\text { den, Spanien, Zypern }\end{array}$ \\
\hline$\geq 75 \%$ & $\begin{array}{l}\text { Großbritannien (Group Relief), Irland } \\
\text { (Corporate Tax Group und Capital } \\
\text { Gains Tax Group), Spanien, Zypern }\end{array}$ & & \\
\hline$\geq 90 \%$ & $\begin{array}{l}\text { Finnland, Lettland, Portugal }{ }^{140} \text {, Schwe- } \\
\text { den }\end{array}$ & Stimmrechte & $\begin{array}{l}\text { Dänemark, Deutschland, } \\
\text { Frankreich („Regime du Be- } \\
\text { nefice Consolide“), Italien } \\
\text { (international) }\end{array}$ \\
\hline$>95 \%$ & $\begin{array}{l}\text { Frankreich (,Integration Fiscale"), Lu- } \\
\text { xemburg, Niederlande, Polen }\end{array}$ & $\begin{array}{l}\text { Stimmrechte } \\
\text { und Beteili- } \\
\text { gungskapital }\end{array}$ & $\begin{array}{l}\text { Frankreich (,Integration Fis- } \\
\text { cale“), Lettland, Malta, Por- } \\
\text { tugal }\end{array}$ \\
\hline
\end{tabular}

Quelle: Eigene Darstellung in Anlehnung an Oestreicher/Scheffler/Spengel (2008), S. 62 unter Berücksichtigung aktueller Angaben in Kesti (2008).

Neben die Mindestbeteiligung beziehungsweise eine geforderte Stimmrechtsmehrheit treten in einigen Ländern zusätzliche oder alternative Anwendungsvoraussetzungen. ${ }^{141}$ Diese haben oftmals die Zielsetzung, einen Gleichlauf der steuerlichen Behandlung mit den zivilrechtlichen Konsequenzen sicherzustellen oder einem möglichen Auseinanderfallen von Beteiligungsverhältnissen und tatsächlichen Kontrollmöglichkeiten Rechnung zu tragen. ${ }^{142}$ In diesem Sinne erfordert Deutschland zusätzlich den Abschluss eines Ergebnisabführungsvertrags,

140 Die 90\%-Grenze bezieht sich auf das Beteiligungskapital. Gleichzeitig müssen mindestens $50 \%$ der Stimmrechte gehalten werden. Vgl. Sievert (2006), S. 192.

141 Für die Ausführungen zu ergänzenden Anwendungsvoraussetzungen vgl. Oestreicher/Scheffler/Spengel et al. (2008), S. 66-67.

$142 \mathrm{Vgl}$. Oestreicher/Scheffler/Spengel et al. (2008), S. 66-67. Davon abweichend verlangen Österreich und Frankreich den Abschluss eines Gruppenvertrags, während Polen eine Umsatzrentabilität der Gruppe von mindestens 3\% und ein Grundkapital von mindestens 1 Mio. PLN voraussetzt. 
während Schweden und Finnland die steuerlichen Folgen der Group Contribution an das Vorliegen entsprechender handelsrechtlich wirksamer Zahlungen knüpfen. Die Gruppenbesteuerung in Dänemark kann alternativ zum Bestehen einer Mehrheitsbeteiligung auch durch den Nachweis vertraglich abgesicherter Kontrollmöglichkeiten begründet werden. In Lettland ist die Begründung einer steuerlichen Gruppe ausgeschlossen, wenn zwar die Mindestbeteiligung erfüllt ist, die Mutter- oder Tochtergesellschaft allerdings aufgrund einer entsprechenden Vereinbarung von außenstehenden Gesellschaftern kontrolliert wird.

Für die Anwendung der Gruppenbesteuerung besteht in fast allen Mitgliedsstaaten ein Wahlrecht. ${ }^{143}$ Lediglich in Dänemark ist die Gruppenbesteuerung für nationale Tochtergesellschaften verpflichtend. Die Ausübung des Einbeziehungswahlrechts kann in den meisten Mitgliedsstaaten für jede Gruppengesellschaft separat getroffen werden. Lediglich sechs Mitgliedsstaaten folgen bei der Wahlrechtsausübung dem „All-in/all-out-Prinzip“. ${ }^{144}$

Hinsichtlich der Besteuerungsfolgen lassen sich die Gruppenbesteuerungssysteme mit Blick auf den Grad der Umsetzung der Einheitstheorie in drei Gruppen einteilen. Während zehn Mitgliedsstaaten ausschließlich einen konzerninternen Verlustausgleich zulassen, ist in sechs Mitgliedsstaaten zusätzlich zumindest eine partielle Zwischenergebniseliminierung bei konzerninternen Veräußerungsgeschäften zulässig. Die Niederlande erlaubt sogar eine steuerliche Vollkonsolidierung. Tabelle 5 gibt die Unterteilung der Länder hinsichtlich des Berücksichtigungsgrads der wirtschaftlichen Einheit wieder.

Tabelle 5: Gruppenbesteuerungssysteme (Berücksichtigung der wirtschaftlichen Einheit)

\begin{tabular}{|l|l|}
\hline Verlustausgleich & $\begin{array}{l}\text { Dänemark, Deutschland, Finnland, Italien, Lettland, Luxem- } \\
\text { burg, Österreich, Polen, Portugal, Zypern }\end{array}$ \\
\hline $\begin{array}{l}\text { Verlustausgleich und (voll- } \\
\text { ständige oder partielle) Zwi- } \\
\text { schenergebniseliminierung }\end{array}$ & Frankreich, Großbritannien, Irland, Malta, Schweden, Spanien \\
\hline Vollkonsolidierung & Niederlande \\
\hline
\end{tabular}

Quelle: Endres/Oestreicher/Scheffler et al. (2007), S. 93.

Unterschiede ergeben sich ferner im zur Anwendung kommenden Verlustverrechnungsmechanismus. ${ }^{145}$ In zehn Mitgliedsstaaten erfolgt eine Ergebniszu-

143 Für die Ausführungen zu Ausübungswahlrechten vgl. Oestreicher/Scheffler/Spengel et al. (2008), S. 70-71.

144 Dabei gilt in Frankreich und Italien diese restriktive Ausgestaltung des Wahlrechts nur für das grenzüberschreitende Gruppenbesteuerungssystem. Vgl. Oestreicher/Scheffler/Spengel et al. (2008), S. 70.

145 Für die Ausführungen zum Verlustverrechnungsmechanismus vgl. Oestreicher/Scheffler/ Spengel et al. (2008), S. 72-77. 
sammenrechnung, entweder im Wege der Konsolidierung (Niederlande) oder einer Zurechnung der Einzelergebnisse zum Gruppenträger. Dabei wird in Dänemark der Gesamtgewinn/-verlust der Gruppe anschließend auf die einbezogenen Gruppengesellschaften entsprechend ihres Beitrags zum Gesamtgewinn bzw. verlust aufgeteilt (Ergebnisaufteilungsmethode). In den verbleibenden sieben Mitgliedsstaaten können Ergebnisbeiträge flexibel zwischen den Gruppengesellschaften verschoben werden. Dieses kann entweder durch eine an eine Ausgleichszahlungen in entsprechende Höhe geknüpfte konzerninterne Zuweisung von Verlusten erfolgen (Group Contribution: Finnland und Schweden) oder durch eine $\mathrm{Zu}$ weisung von Gewinnen (Group Relief: Großbritannien, Irland, Lettland, Malta, Zypern), die regelmäßig nicht an entsprechende Zahlungsverpflichtungen gebunden ist (vgl. Tabelle 6).

Tabelle 6: Gruppenbesteuerungssysteme (Verlustverrechnungsmechanismus)

\begin{tabular}{|l|l|}
\hline Zusammenveranlagung & $\begin{array}{l}\text { Dänemark, Deutschland, Frankreich, Italien, Luxemburg, } \\
\text { Niederlande, Österreich, Polen, Portugal, Spanien }\end{array}$ \\
\hline Group Contribution & Finnland, Schweden \\
\hline Group Relief & Großbritannien, Irland, Lettland, Malta, Zypern \\
\hline
\end{tabular}

Quelle: In Anlehnung an Endres/Oestreicher/Scheffler et al. (2007), S. 91.

Für die Behandlung der Gewinne und Verluste ausländischer Gruppengesellschaften kommt in Dänemark, Frankreich und Italien die Zurechnungsmethode zur Anwendung, während Österreich die Nachversteuerungsmethode verwen$\operatorname{det}^{146}$ In Irland und Großbritannien ist die Berücksichtigung der Verluste ausländischer Tochtergesellschaften entsprechend der Vorgaben des EuGH-Urteils in der Rechtssache „Marks\&Spencer" ${ }^{\text {“147 }}$ auf im Ausland endgültig nicht verrechenbare Verluste begrenzt. ${ }^{148}$

\subsection{Vorschriften zur Sicherung des inländischen Steueraufkom- mens}

\subsubsection{Unterkapitalisierungsregelungen}

Das Trennungsprinzip bei der Besteuerung von Kapitalgesellschaften eröffnet erheblichen Gestaltungsspielraum für die Finanzierungsstruktur multinationaler

$146 \mathrm{Vgl}$. Oestreicher/Scheffler/Spengel et al. (2008), S. 75-75. Vgl. zu diesen Konzepten ausführlich Kapitel 4.3.3.

147 Vgl. EuGH v. 13.12.2005 (Marks\&Spencer), EuGHE 2005, S. I-10837. Vgl. zum Inhalt dieses Urteils auch Kapitel 4.3.3.1.

148 Vgl. Wittkowski (2008), S. 240; Kesti (2008), S 844 ald Koch - 978-3-631-75683-6 
Konzerne. Entgelte für die Fremdkapitalüberlassung an eine Tochterkapitalgesellschaft führen bei dieser grundsätzlich zum Betriebsausgabenabzug und unterliegen beim Kapitalgeber der Besteuerung (Kapitalexportneutralität). Bei Eigenfinanzierung hingegen unterliegt die Kapitalverzinsung zumindest bei Freistellung auf Ebene der ausschüttungsempfangenden Muttergesellschaft einer kapitalimportneutralen Besteuerung. Aus der Sicht eines multinationalen Konzerns ist dementsprechend die Finanzierung einer Tochtergesellschaft mit Fremdkapital insbesondere dann vorteilhaft, wenn die nominelle Steuerbelastung im Land des Fremdkapitalgebers unterhalb der Steuerbelastung im Sitzstaat des Kreditnehmers liegt. ${ }^{149}$ Insbesondere Hochsteuerländern droht durch derartige Gestaltungen eine Erosion des Körperschaftsteueraufkommens. ${ }^{150}$ Verstärkt wird dieses Problem dadurch, dass mit Implementierung der Zins- und Lizenzrichtlinie eine Erhebung von Quellensteuern auf konzerninterne Zinsentgelte durch das Sitzland der fremdfinanzierten Tochtergesellschaft grundsätzlich ausgeschlossen ist. ${ }^{151}$

Als Reaktion ist die steuerliche Abziehbarkeit von Finanzierungsaufwendungen in den meisten Mitgliedsstaaten an deren Angemessenheit sowohl der Höhe als auch dem Grunde nach geknüpft. ${ }^{152}$ Eine Korrektur von Kreditvereinbarungen mit der Höhe nach unangemessenen Zinssätzen erfolgt unter Bezugnahme auf den allgemeinen Fremdvergleichsgrundsatz (vgl. hierzu Kapitel 3.4.3). Zur Vermeidung einer dem Umfang nach exzessiven Fremdfinanzierung inländischer Tochtergesellschaften durch ausländische Gesellschafter kommen daneben in 19 Mitgliedsstaaten Unterkapitalisierungsregelungen (,Thin capitalisation rules") zur Anwendung, die entweder in Form gesetzlicher Vorschriften oder durch gängige Praxis der Finanzverwaltung Berücksichtigung finden (vgl. hierzu Tabelle 7). Nur sieben Mitgliedsstaaten sehen in ihrem Steuersystem eine entsprechende Regelung nicht vor. Dieses betrifft allerdings vor allem Niedrigsteuerländer, die eine Herausverlagerung von Bemessungsgrundlage ohnehin überwiegend nur im Verhältnis zu Steueroasen befürchten müssen. So liegt der durchschnittliche Steuersatz der Mitgliedsstaaten ohne Unterkapitalisierungsregelung mit 22,21 Prozent unter dem Durchschnittssteuersatz der Mitgliedsstaaten mit Unterkapitalisierungs-

149 Daneben kann Fremdfinanzierung auch als Gestaltungsmittel zur Nutzung steuerlicher Verlustvorträge eingesetzt werden. Vgl. Jacobs (2007), S. 954. Eine weitergehende Senkung der Konzernsteuerbelastung kann durch Einsatz von Konzernfinanzienungsgesellschaften in Niedrigsteuerländern erreicht werden. Vgl. zu derartigen komplexeren Finanzierungsgestaltungen Jacobs (2007), S. 962-964.

150 Vgl. Jacobs (2007), S. 954. Der Einfluss des Steuersatzes auf den Verschuldungsgrad grenzüberschreitender Direktinvestitionen konnte in empirischen Studien mehrfach nachgewiesen werden. Vgl. Büttner/Overesch/Schreiber et al. (2008); Mintz/Weichenrieder (2005a); Mintz/Weichenrieder (2005b). Für einen Überblick über weitere Studien vgl. de Mooij/Ederveen (2008).

151 Vgl. Jacobs (2007), S. 172-173; Schön (2007), S. 423-424.

152 Vgl. Gouthiere (2005), S. 367. 
regelung (23,95 Prozent) ${ }^{153}$ Lediglich in vier Mitgliedsstaaten (Finnland, Griechenland, Malta und Schweden), deren Steuersatz oberhalb des EU-Durchschnitts liegt, besteht keine entsprechende Vorschrift.

Tabelle 7: Gesellschafterfremdfinanzierung (Rechtsgrundlage)

\begin{tabular}{|l|l|l|}
\hline Gesetzliche Regelung & Verwaltungspraxis & Keine Regelung \\
\hline $\begin{array}{l}\text { Belgien, Bulgarien, Däne- } \\
\text { mark, Deutschland, Frank- } \\
\text { reich, Großbritannien, Italien, } \\
\text { Lettland, Litauen, Niederlan- } \\
\text { de, Polen, Portugal, Rumä- } \\
\text { nien, Slowenien, Spanien, } \\
\begin{array}{l}\text { Tschechische Republik, Un- } \\
\text { garn }\end{array}\end{array}$ & $\begin{array}{l}\text { Finnland, Griechenland, Ir- } \\
\text { land } \\
\text { Slowakei, Malta, Schweden, }\end{array}$ \\
\hline
\end{tabular}

Quelle: Eigene Darstellung auf Basis der Angaben in Kesti (2008), Jacobs (2007) und European Taxation (2005), Heft 9/10.

Trotz übereinstimmender Intention der Vorschriften bestehen deutliche Unterschiede hinsichtlich der Anwendungsvoraussetzungen und der Rechtsfolgen. In Bezug auf die Anwendungsvoraussetzungen unterscheiden sich die Regelungen insbesondere hinsichtlich der Höhe des als angemessen anerkannten Verhältnisses von Eigen- und Fremdkapital sowie der Abgrenzung des unter die Vorschrift fallenden Fremdkapitals. Abweichungen resultieren sowohl aus der erforderlichen Verbindung zwischen Kreditnehmer und Kreditgeber als auch in Bezug auf das Sitzland des Kreditgebers. Ein Überblick über die wichtigsten Anwendungsvoraussetzungen gibt Tabelle 8 .

Tabelle 8: Gesellschafterfremdfinanzierung (Anwendungsvoraussetzungen)

\begin{tabular}{|l|lll|}
\hline Land & $\begin{array}{l}\text { Kritisches } \\
\text { FK/EK-Verhältnis }\end{array}$ & Bezugsgröße bei Prüfung & $\begin{array}{l}\text { Geografischer An- } \\
\text { wendungsbereich }\end{array}$ \\
\hline Belgien & $7: 1^{155} / 1: 1^{156}$ & Gesamt-FK/Gesellschaft & In- und Ausland \\
\hline
\end{tabular}

153 Quelle: Eigene Berechnungen.

154 Zinszahlungen an beherrschende Gesellschafter (75\%) werden dennoch in bestimmten Fällen in Dividenden umqualifiziert. Vgl. Kesti (2008), S. 376; Hickson (2005), S. 402-404.

155 Das Verhältnis von 7:1 kommt zur Anwendung für Darlehen, bei denen die Gesellschaft, die der ,beneficial owner“ der Zinszahlungen ist, nicht ertragsteuerpflichtig ist oder das zur Anwendung kommende Ertragssteuersystem im Vergleich zum belgischen System als vorteilhaft eingestuft wird. Nicht erforderlich ist, dass der Darlehensgeber Anteilseigner oder nahestehende Person ist. Vgl. Clarebout/Dhaene (2005), S. 372-374.

156 Das Verhältnis von 1:1 kommt zur Anwendung für Cash-Darlehen von natürlichen Personen, die die Funktion eines Anteilseigners, Direktors Managers pdey tiquidatprs ingehaben, so- 


\begin{tabular}{|l|lll|} 
Bulgarien & $3: 1$ & Gesamt-FK & In- und Ausland \\
Dänemark & $4: 1$ & Gesamt-FK & In- und Ausland \\
Deutschland & n.a. & Gesamt-FK & In- und Ausland \\
Frankreich & $1,5: 1^{157}$ & Gesellschaft & In- und Ausland \\
Großbritannien & n.a. & n.a. & In- und Ausland \\
Italien & n.a. & Gesamt-FK & In- und Ausland \\
Lettland & $4: 1$ & Gesamt-FK & In- und Ausland \\
Litauen & $4: 1$ & Gesellschaft & In- und Ausland \\
Luxemburg & $85: 15$ & Gesamt-FK & In- und Ausland \\
Niederlande & $3: 1$ & Gesamt-FK & In- und Ausland \\
Österreich & n.a. & n.a. & In- und Ausland \\
Polen & $3: 1$ & Gesellschaft & In- und Ausland \\
Portugal & $2: 1$ & Gesellschafter & Nicht-EU Ausland \\
Rumänien & $3: 1$ & Gesamt-FK & In- und Ausland \\
Slowenien & $6: 1$ & Gesellschafter & In- und Ausland \\
Spanien & $3: 1$ & Gesellschaft & Nicht-EU Ausland \\
Tsch'e Republik & $2: 1 / 3: 1 / 6: 1^{159}$ & Gesellschaft/Gesamt-FK & In- und Ausland \\
Ungarn & $3: 1$ & Gesamt-FK & In- und Ausland \\
\hline
\end{tabular}

Quelle: Eigene Darstellung auf Basis der Angaben in Kesti (2008), Jacobs (2007) und European Taxation (2005), Heft 9/10. In der Spalte „Bezugsgröße bei Prüfung “ kennzeichnet „Gesellschafter" Regelungen, bei denen auf das überlassene Eigen- und Fremdkapital des einzelnen (qualifizierten) Gesellschafters abgestellt wird, während bei "Gesellschaft" das gesamte von qualifizierenden Gesellschaftern überlassene Eigenkapital dem entsprechenden Gesellschafter-Fremdkapital als Ganzes gegenübergestellt wird und bei "Gesamt-FK" das gesamte Eigenkapital mit dem gesamten Fremdkapital verglichen wird.

Daneben unterscheiden sich die Vorschriften auch hinsichtlich der Abgrenzung des für die Prüfung der Angemessenheit heranzuziehenden Eigen- und Fremdkapitals. Sieben Mitgliedsstaaten setzen das gesamte Fremdkapital der Tochtergesellschaft ins Verhältnis zum gesamten Eigenkapital der Gesellschaft (gekennzeichnet durch "Gesamt-FK“ in Spalte 3 von Tabelle 8), wobei nur vier dieser Mitgliedsstaaten (Bulgarien, Lettland, Rumänien und Ungarn) die Abzugsfähigkeit der Schuldzinsen auch für Darlehen von unabhängigen Dritten ein-

wie ausländischen Kapitalgesellschaften mit der Funktion eines Direktors, Managers oder Liquidators. Vgl. Kesti (2008), S. 104; Clarebout/Dhaene (2005), S. 374-375.

157 Als ergänzender Test kommt in Frankreich seit 2007 ein Vergleich der Zinsaufwendungen mit dem Unternehmensergebnis zur Anwendung. Vgl. Kesti (2008), S. 245.

158 Keine Vorschrift im Körperschaftsteuerrecht, aber bei im Branchenvergleich unangemessenem Verhältnis von Fremdkapital zu Eigenkapital werden Gesellschafterdarlehen in verdecktes Eigenkapital umqualifiziert. Vgl. Doralt/Feyl (2005), S. 370.

159 Die 2:1-Quote bezieht sich auf Darlehen von nahestehenden Personen, wobei bei Banken und Versicherungen diese Quote auf 3:1 gelockert wird. Die 6:1-Quote bezieht sich auf Fremddarlehen. Vgl. Kesti (2008), S. 165. 
schränken. Belgien, die Niederlande und Luxemburg, hingegen, nehmen nur für Darlehen von nahestehenden Personen eine Umqualifizierung vor. ${ }^{160}$ Die übrigen Mitgliedsstaaten beziehen sowohl bei der Prüfung der Angemessenheit der Kapitalstruktur als auch bei der Abgrenzung der nur eingeschränkt abzugsfähigen Fremdkapitalzinsen nur Darlehen von (qualifizierten) Anteilseignern oder nahestehenden Personen ein. Auch bei der Definition dieser relevanten Darlehensgeber bestehen deutliche Unterschiede. So reicht die Bandbreite der geforderten Beteiligungsquote von $0 \%$ (Österreich und Belgien) bis zu 50\% (Dänemark). Viele Mitgliedsstaaten erfassen Gesellschafterdarlehen ab einer Beteiligungsquote von $25 \%$. Bei der Definition nahestehender Darlehensgeber wird teilweise auf die Ausübung einer Funktion in der Gesellschaft (Direktor, Management, Liquidator) abgestellt, in anderen Mitgliedsstaaten wird hier der Kreis auf Schwestergesellschaften oder sämtliche Gesellschaften, die zu einem gemeinsamen Konzern oder derselben steuerlichen Gruppe gehören, erweitert. Einige Mitgliedsstaaten dehnen den Anwendungskreis der betroffenen Darlehen zusätzlich auf von (qualifizierten) Anteilseignern oder nahestehenden Personen garantierte Drittdarlehen und/oder Back-to-back Finanzierungen aus. ${ }^{161}$

Die Prüfung der Angemessenheit des Umfangs des von diesen Darlehensgebern überlassenen Fremdkapitals erfolgt teilweise mit Bezug auf die Kapitalüberlassung einzelner Gesellschafter (Portugal und Slowenien; gekennzeichnet durch "Gesellschafter" in Spalte 3 von Tabelle 8), überwiegend wird aber auf die Gesamtheit des von diesen Personen oder Gesellschaften bereitgestellten Eigen- und Fremdkapitals abgestellt (gekennzeichnet durch "Gesellschaft“ in Spalte 3 von Tabelle 8).

Als Konsequenz der EuGH-Rechtsprechung in der Rechtssache LankhorstHohorst ${ }^{162}$ erfassen die meisten Mitgliedsstaaten sowohl in- als auch ausländische Darlehensgeber, wobei sich der Kreis der erfassten Darlehensgeber und die Rechtsfolgen der Vorschrift teilweise unterscheiden. Lediglich Portugal und Spanien beschränken die Anwendung auf Darlehensgeber aus Drittstaaten (vgl. Tabelle 8, Spalte 4).

In einigen Mitgliedsstaaten ist ein vollständiger Abzug der Finanzierungsaufwendungen trotz Überschreiten des „Safe haven“ möglich, wenn die betreffende Gesellschaft nachweist, dass sie das Fremdkapital zu gleichen Konditionen auch von einem unabhängigen Dritten erhalten hätte können („Drittvergleich“, unter anderem möglich in Dänemark und Portugal ${ }^{163}$ ) oder dass der Verschul-

$160 \mathrm{Vgl}$. die entsprechenden Angaben in Kesti (2008) und European Taxation (2005), Heft 9/10.

161 Vgl. die entsprechenden Angaben in Kesti (2008) und European Taxation (2005), Heft 9/10.

$162 \mathrm{Vgl}$. EuGH v. 12.12.2002 (Lankhorst-Hohorst), EuGHE 2002, S. I-11779. In diesem Urteil wurde vom EuGH die frühere deutsche Regelung zur Gesellschafterfremdfinanzierung aufgrund der Schlechterstellung ausländischer Anteilseigner gegenüber reinen Inlandsfällen für europarechtswidrig erklärt.

163 Vgl. Kesti (2008), S. 184 und 635. 
dungsgrad nicht höher als der Verschuldungsgrad des Gesamtkonzerns ist („Konzernvergleich", unter anderem möglich in Deutschland und Frankreich ${ }^{164}$ ). Daneben werden kleine und mittelgroße Unternehmen oder Transaktionen durch Deminimis-Klauseln von den Rechtsfolgen dieser Vorschriften ausgenommen (unter anderem in Deutschland und Frankreich ${ }^{165}$ ).

Tabelle 9: Gesellschafterfremdfinanzierung (Rechtsfolgen)

\begin{tabular}{|l|l|}
\hline Nichtabziehbarkeit der Zinsen & Umqualifizierung in Dividenden \\
\hline $\begin{array}{l}\text { Belgien (7:1 Regime), Bulgarien, Däne- } \\
\text { mark }{ }^{166} \text {, Deutschland, Frankreich, Großbritan- }\end{array}$ & $\begin{array}{l}\text { Belgien (1:1 Regime), Luxemburg, Österreich, } \\
\text { Spanien, Tschechische Republik } \\
\text { nien }^{167} \text {, Italien, Ungarn, Lettland, Litauen, } \\
\text { Niederlande, Polen, Portugal, Rumänien, } \\
\text { Slowenien }\end{array}$ \\
\hline
\end{tabular}

Quelle: Eigene Darstellung auf Basis der Angaben in Kesti (2008), Jacobs (2007) und European Taxation (2005), Heft 9/10.

Schließlich bestehen auch Unterschiede hinsichtlich der Rechtsfolgen der Unterkapitalisierungsregeln (vgl. Tabelle 9). 14 Mitgliedsstaaten untersagen lediglich den Betriebsausgabenabzug, vier Mitgliedsstaaten nehmen eine Umqualifizierung in Dividenden vor, während in Belgien beide Rechtsfolgen in Abhängigkeit vom zur Anwendung kommenden Regime vorgesehen sind. Beide Rechtsfolgen bringen spezifische Probleme für den Steuerpflichtigen mit sich. Ist Nichtabziehbarkeit der Zinsaufwendungen die Rechtsfolge, ist eine Doppelbesteuerung zwangsläufig, aber unabhängig vom Sitzland des Kapitalgebers. ${ }^{168}$ Werden die Zinsaufwendungen als Gewinnausschüttungen angesehen, wird bei inländischen Kapitalgebern eine Doppelbesteuerung durch korrespondierende $\mathrm{Be}$ handlung beim Zahlungsempfänger vermieden. Bei ausländischen Kapitalgebern

164 Vgl. Kesti (2008), S. 245 und 269.

165 Vgl. Kesti (2008), S. 245 und 269.

166 Soweit der Empfänger der Zinsen in Dănemark ansässig ist, sind die Zinszahlungen nicht zu versteuern, wenn diese bei der zahlenden Gesellschaft nicht abziehbar sind. Dieses gilt nicht im Fall von Back-to-back-Gestaltungen. Vgl. Kesti (2008), S. 184.

167 Bei inländischen körperschaftsteuerpflichtigen Anteilseignem als Darlehensgeber sind auf Antrag nicht-abziehbare Zinszahlungen nicht zu besteuern, so dass sich bei inländischen Konzernen die Be- und Entlastungswirkungen aufgrund der Unterkapitalisierungsregel ausgleichen. Vgl. Nias/Purcell (2005), S. 435.

168 Einige Mitgliedsstaaten (z.B. Frankreich und Deutschland) erlauben zur Abmilderung dieser nachteiligen Wirkung einen Vortrag nicht-abziehbarer Zinsen. Vgl. Kesti (2008), S. 245 und 269. 
kann es demgegenüber sogar zu einer Dreifachbesteuerung kommen, wenn die Umqualifizierung im Ausland nicht nachvollzogen wird. ${ }^{169}$

\subsubsection{Hinzurechnungsbesteuerung}

Die Hinzurechnungsbesteuerung („Controlled Foreign Company Regimes“, CFC) dient der Sicherung des inländischen Steueraufkommens gegen die Verlagerung mobiler Einkommensquellen in Tochterkapitalgesellschaften im niedrig besteuernden Ausland. ${ }^{170}$ Für diesen Fall schließt die Abschirmwirkung der Kapitalgesellschaft einen Steueranspruch des Inlands aus, solange Gewinne auf Ebene der Auslandsgesellschaft thesauriert werden. Dieses würde auch dann gelten, wenn das Inland aufgrund einer „Subject-to-tax"-Klausel oder einer „Aktivitätsvorbehalts"-Klausel die Steuerfreistellung der Auslandsdividenden an eine hinreichende Vorbelastung und/oder aktive Tätigkeit im Ausland knüpft. Ein Steueranspruch des Inlands kann sich daher entsprechend der drei verbreiteten Ansätze zur CFC-Besteuerung nur durch Ausschüttungsfiktion (,deemed dividend approach"), Einkommenszurechnung (,income attribution approach") oder transparente Behandlung der Auslandsgesellschaft (,peircing the veil approach") ergeben. ${ }^{171}$

Tabelle 10: CFC-Besteuerungsregime in den EU-Mitgliedsstaaten

\begin{tabular}{|l|l|}
\hline Mitgliedsstaaten mit CFC-Regime & Mitgliedsstaaten ohne CFC-Regime \\
\hline $\begin{array}{l}\text { Dänemark, Deutschland, Finnland, Frank- } \\
\text { reich, Großbritannien, Italien, Litauen, Portu- } \\
\text { gal, Schweden, Spanien, Ungarn }\end{array}$ & $\begin{array}{l}\text { Belgien, Bulgarien, Griechenland, Irland, } \\
\text { Österreich, Polen, Rumänien, Slowakei, Slo- } \\
\text { wenien, Tschechische Republik, Zypern }\end{array}$ \\
\hline
\end{tabular}

Quelle: Eigene Darstellung auf Basis der Angaben in Kesti (2008), Maisto/Pistone (2008) und Lang/Aigner/Scheuerle et al. (2004)

In Analogie zu den Unterkapitalisierungsvorschriften lässt sich auch hier feststellen, dass CFC-Regime insbesondere in den Steuersystemen der Hochsteuerländer vorgesehen sind. So beträgt die durchschnittliche Tarifbelastung in den Mitgliedsstaaten mit CFC-Regime 26,75 Prozent, in den Mitgliedsstaaten ohne

169 Diese Dreifachbelastung ergibt sich aus der Besteuerung auf Ebene des Kapitalnehmers, der im Ausland nicht anrechenbaren „Dividenden"-Quellensteuer sowie der Besteuerung der „Zinsen“ auf Ebene des ausländischen Kapitalgebers. Vgl. Gangemi (2005), S. 406. Inwiefern eine Umqualifizierung aus diesen Gründen mit dem EU-Recht und bestehenden Doppelbesteuerungsabkommen vereinbar ist, ist in der Literatur umstritten. Vgl. Jacobs (2007), S. $955 \mathrm{mwN}$.

170 Vgl. Rust (2008), S. 492-493.

171 Vgl. Rust (2008), S. 493. 
entsprechende Vorschrift hingegen nur 21,23 Prozent. ${ }^{172}$ Tabelle 10 fasst die Mitgliedsstaaten mit und ohne CFC-Regime zusammen.

Tabelle 11: Ausgestaltung der CFC-Besteuerungsregime in den EU Mitgliedsstaaten

\begin{tabular}{|c|c|c|c|c|}
\hline \multirow[t]{2}{*}{ Land } & \multicolumn{2}{|c|}{ Mindestbeteiligung } & \multicolumn{2}{|c|}{ Sitzlandanforderungen } \\
\hline & $\begin{array}{l}\text { ohne Inlands- } \\
\text { beherrschung }\end{array}$ & $\begin{array}{l}\text { mit Inlands- } \\
\text { beherrschung }\end{array}$ & $\begin{array}{l}\text { White list/ } \\
\text { Black list }\end{array}$ & $\begin{array}{l}\text { Steuerbelas- } \\
\text { tung: absolut } \\
\text { (relativ) }\end{array}$ \\
\hline Dänemark & $50 \%$ & & & $(75 \%)$ \\
\hline Deutschland & & $0 \%{ }^{173}$ & 174 & $25 \%$ \\
\hline Finnland & & $10 \%$ & & $(75 \%)$ \\
\hline Frankreich & $50 \%$ & $5 \%$ & & $(50 \%)$ \\
\hline Großbritannien & & $25 \%$ & White List & $(75 \%)$ \\
\hline Italien & $50 \%^{175}$ & & Black List & \\
\hline Litauen & $50 \%$ & & White List & $(75 \%)$ \\
\hline Portugal & $25 \%$ & $10 \%$ & & $(60 \%)$ \\
\hline Schweden & $25 \%$ & & White List & $(55 \%)$ \\
\hline Spanien & $50 \%$ & & White List (EU) & $(75 \%)$ \\
\hline Ungarn & & & $\begin{array}{l}\text { White List (EU, } \\
\text { OECD, DBA) }\end{array}$ & $(66,67 \%)$ \\
\hline
\end{tabular}

Quelle: Eigene Darstellung auf Basis der Angaben in Kesti (2008), Maisto/Pistone (2008) und Lang/Aigner/Scheuerle et al. (2004). In der Spalte „Mindestbeteiligung ohne Inlandsbeherrschung " ist die geforderte Mindestbeteiligung angegeben, wenn nicht gleichzeitig eine Beherrschung der Auslandsgesellschaft durch inländische Anteilseigner vorausgesetzt wird. „Mindestbeteiligung mit Inlandsbeherrschung " gibt demgegenüber die Beteiligungsgrenze an, bei der als ergänzendes Kriterium eine Inländerbeherrschung der Auslandsgesellschaft vorausgesetzt wird.

Für die Anwendung werden weitgehend einheitlich eine bestimmte Mindestbeteiligung, eine bestimmte Tätigkeit sowie ein bestimmtes Sitzland der Auslandsgesellschaft vorausgesetzt. Hinsichtlich der in der Auslandsgesellschaft ausgeübten Tätigkeit werden insbesondere passive Einkünfte (zum Beispiel Zinsen,

172 Quelle: Eigene Berechnungen.

173 Erzielt die Auslandsgesellschaft uberwiegend Investment-Einkunfte ist die Beteiligung eines Steuerinländers in Höhe von mindestens einem Prozent ausreichend. Vgl. Kesti (2008), S. 270.

174 Unter bestimmten Voraussetzungen besteht die Möglichkeit zur Befreiung von der Hinzurechnungsbesteuerung bei CFC-Gesellschaften mit Sitz in einem EEA-Land. Vgl. Kesti (2008), S. 270.

175 Eingeschränkte Anwendung des CFC-Regimes erfolgt allerdings bereits ab einer Beteiligung von 20 Prozent an den Gewinnen der Auslandsgesellschaft. Ygl. Kesti (2008) $\mathbf{S}_{\mathbf{3}} \mathbf{4 1 3}$. 
Dividenden oder Mieteinkünfte) als schädlich eingestuft. ${ }^{176}$ Die Beteiligungsanforderungen und Anforderungen an das Sitzland der Auslandsgesellschaft sind in Tabelle 11 zusammengefasst.

Mit Ausnahme von Ungarn wenden alle Mitgliedsstaaten das CFC-Regime nur bei Überschreiten einer Beteiligungsgrenze an. Dabei knüpfen einige Länder ausschließlich an die Höhe der eigenen Beteiligung des jeweiligen Anteilseigners an (Spalte „ohne Inlandsbeherrschung"), während andere Länder eine geringere eigene Beteiligung bei gleichzeitiger Beherrschung - gemessen als Gesamtbeteiligung von mehr als 50 Prozent - der Auslandsgesellschaft durch Steuerinländer für ausreichend halten (Spalte ,mit Inlandsbeherrschung"). Frankreich und Portugal wenden beide Kriterien parallel an.

Für die Abgrenzung der Länder, in denen Tochterkapitalgesellschaften unter die Hinzurechnungsbesteuerungsvorschriften fallen, stellen alle Mitgliedsstaaten mit Ausnahme von Italien auf eine bestimmte Steuerbelastung ab, die überwiegend als Prozentsatz des eigenen Körperschaftsteuersatzes festgelegt wird. Diese relative Höhe schwankt dabei zwischen 50 und 75 Prozent des eigenen Körperschaftsteuersatzes (vgl. Tabelle 11, Spalte 5). Lediglich in Deutschland liegt die kritische Steuerbelastung mit 25 Prozent oberhalb des eigenen Körperschaftsteuersatzes (15 Prozent). In Italien werden die Länder, für welche die Hinzurechnungsbesteuerung zur Anwendung kommt, über eine „Black list“ festgelegt. In Litauen erfasst die Hinzurechnungsbesteuerung auch ausländische Tochtergesellschaften, die in einem auf einer „White list“ enthaltenen Land ansässig sind. In vier anderen Mitgliedsstaaten (Großbritannien, Schweden, Spanien, Ungarn) werden die auf einer „White List" aufgeführten Länder von der Hinzurechnungsbesteuerung ausgenommen (vgl. Tabelle 11, Spalte 4).

Bezüglich der Rechtsfolgen der CFC-Regime in Europa lässt sich keine klare Linie erkennen. Zwar folgen die meisten Mitgliedsstaaten bei der Abgrenzung der unter die Hinzurechnungsbesteuerung fallenden Einkünfte dem „All-in/all-out“Prinzip, bezüglich des zur Anwendung kommenden Ansatzes (,deemed dividend approach", ,income attribution approach", „peircing the vail approach") fehlt es hingegen an Einheitlichkeit. ${ }^{177}$

Aufgrund des Fehlens einer parallelen Anwendung bei Inlandssachverhalten wird die Europarechtskonformität der Hinzurechnungsbesteuerung in der Literatur schon seit längerem in Frage gestellt. ${ }^{178} \mathrm{Im}$ Jahr 2006 hat der EuGH mit der britischen CFC-Regelung erstmalig eine entsprechende Vorschrift für europarechtswidrig erklärt. ${ }^{179}$ Dieses ist nicht überraschend, da der EuGH die wesentliche Begründung für diese Vorschriften, nämlich die Missbrauchsvermeidung, nur in sehr

176 Vgl. hierzu für Deutschland Jacobs (2007), S. 473-477.

$177 \mathrm{Vgl}$. die Angaben in Lang/Aigner/Scheuerle et al. (2004).

178 Vgl. Jacobs (2007), S. 245 mwN. Vgl. hierzu auch Rust (2008), S. 496-498.

179 Vgl. EuGH, v. 12.09.2006 (Cadbury Schweppes) EuGHF,2006, \$7 -7995-731-75683-6 
engen Grenzen akzeptiert. ${ }^{180}$ Es ist daher zu erwarten, dass der Anwendungsbereich der Hinzurechnungsbesteuerung in Europa in der Zukunft durch den EuGH weiter eingeschränkt wird. ${ }^{181}$ In diese Richtung weist die Nicht-Anwendung der Hinzurechnungsbesteuerung für europäische Tochtergesellschaften in Spanien und Ungarn (vgl. Tabelle 11, Spalte 4) sowie die Möglichkeit des Nachweises einer tatsächlichen Tätigkeit für europäische Tochterkapitalgesellschaften in Deutschland ${ }^{182}$.

\subsubsection{Gewinnabgrenzung im Konzern}

Die selbständige Steuerpflicht der einzelnen Konzerngesellschaften erfordert eine Bewertung konzerninterner Lieferungs- und Leistungsbeziehungen, um die $\mathrm{Be}-$ steuerungsansprüche der beteiligten Steuerhoheiten abzugrenzen. ${ }^{183}$ Diese Abgrenzung kann grundsätzlich nach der direkten Methode oder der indirekten Methode erfolgen.

Die direkte Methode der Gewinnabgrenzung negiert in Übereinstimmung mit der Trennungstheorie die wirtschaftliche Einheit des Konzerns. Konzerninterne Transaktionen sind einzeln zu erfassen und im Rahmen der getrennten $\mathrm{Ge}$ winnermittlung der Konzerngesellschaften nach Maßgabe der Verhältnisse unabhängiger Unternehmen abzurechnen („Fremdvergleichsgrundsatz“). ${ }^{184}$ Die Angemessenheitsprüfung der vereinbarten Verrechnungspreise kann dabei transaktionsbezogen oder unternehmensbezogen erfolgen. ${ }^{185}$ International allgemein anerkannt sind dabei die transaktionsbezogenen Standardmethoden, bei welchen die für die einzelnen Geschäftsvorfälle vereinbarten Verrechnungspreise mit den Preisen, Gewinnaufschlägen oder Gewinnabschlägen verglichen werden, die sich zwischen unabhängigen Unternehmen einstellen. ${ }^{186} \mathrm{Zu}$ diesen Methoden gehört die Preisvergleichsmethode, bei der die Preise vergleichbarer Transaktionen des Konzernunternehmens mit fremden Dritten (,innerer Preisvergleich“) oder zwischen unabhängigen Dritten (,äußerer Preisvergleich“) zugrundegelegt werden. Weitere Standardmethoden sind die Wiederverkaufspreismethode sowie die Kostenaufschlagsmethode, bei denen der Wiederverkaufspreis des empfangenden Unternehmens an fremde Dritte um eine marktübliche Bruttogewinnspanne ge-

180 Vgl. Jacobs (2007), S. 204.

181 Vgl. Jacobs (2007), S. 248. Vgl. hierzu auch den Beschluss des EuGH in der Rechtssache "Test Claimants in the CFC and Dividend Group Litigation". EuGH v. 23.04.2008 (Test Claimants in the CFC and Group Litigation), Abl. EU 2008 Nr. C 209, S. 13.

182 Vgl. Kesti (2008), S. 270.

183 Dieses Problem stellt sich in gleicher Weise auch bei der Erfolgsabgrenzung zwischen Stammhaus und Betriebsstătte. Die Ausführungen hier beschränken sich allerdings auf die Erfolgsabgrenzung zwischen rechtlich selbständigen Konzernunternehmen.

$184 \mathrm{Vgl}$. Jacobs (2007), S. 584.

$185 \mathrm{Vgl}$. Jacobs (2007), S. 748-749.

186 Vgl. Oestreicher (2000), S. 35. 
kürzt wird oder die Herstellungskosten des liefernden Unternehmens um einen marktüblichen Gewinnaufschlag erhöht werden. ${ }^{187}$ Hinsichtlich der Anwendungsreihenfolge werden die transaktionsbezogenen Standardmethoden grundsätzlich als gleichrangig eingestuft. ${ }^{188}$ Die Entscheidungsfreiheit bei der Methodenwahl wird allerdings de facto dadurch eingeschränkt, dass die Steuerpflichtigen auch bei der Auswahl der Methoden an das Verhalten des unabhängigen und gewissenhaften Geschäftsführers gebunden sind. Dieses verpflichtet die Unternehmen faktisch auf die Methode, für welche preisrelevante Daten möglichst zuverlässig ermittelt werden können. Dabei sollte beim Warenverkehr häufig die Preisvergleichsmethode vorzugswürdig sein, während bei Dienstleistungsbeziehungen die anderen Methoden Vorteile mit sich bringen können. ${ }^{189}$

Neben die transaktionsbezogenen Standardmethoden treten die geschäftsfallbezogenen Gewinnmethoden, welche nach Auffassung der deutschen Finanzverwaltung und der OECD allerdings gegenüber den Standardmethoden nur nachrangig und damit lediglich in Ausnahmefällen anzuwenden sind. ${ }^{190}$ Bei diesen Methoden wird der Gewinn aus konzerninternen Transaktionen mit den Gewinnen verglichen, die sich bei voneinander unabhängigen Unternehmen ergeben. ${ }^{191} \mathrm{Im}$ Einzelnen sind hierbei die geschäftsfallbezogene Nettomargenmethode und die geschäftsfallbezogene Gewinnaufteilungsmethode zu unterscheiden, wobei letztere eine Vermischung der direkten Methode zur Gewinnabgrenzung mit Elementen der indirekten Methode vornimmt. ${ }^{192}$ Eine unternehmensbezogene Überprüfung der Angemessenheit der Verrechnungspreisfestlegung im Wege des globalen Betriebsvergleichs ist hingegen nach Auffassung der OECD abzulehnen. ${ }^{193}$ Nach diesem Konzept wird die Angemessenheit der Verrechnungspreise durch Vergleich von Renditekennzahlen der betroffenen Konzerngesellschaften mit entsprechenden Kennzahlen unabhängiger Unternehmen geprüft (,externer Betriebsvergleich"). ${ }^{194}$ Nachteile dieser Methode sind insbesondere der fehlende Bezug zur konzerninternen Einzeltransaktion und die Missachtung sonstiger Ergebnisdeterminanten. ${ }^{195}$

$187 \mathrm{Vgl}$. zu den transaktionsbezogenen Standardmethoden ausführlich Jacobs (2007), S. 750-773; Oestreicher (2000), S. 35-41.

$188 \mathrm{Vgl}$. Jacobs (2007), S. 748.

189 Vgl. Jacobs (2007), S. 748.

190 Vgl. Wendt (2009), S. 81; Jacobs (2007), S. 748-749. Der Vorrang der Standardmethoden ist seit dem 1.1.2008 in Deutschland auch gesetzlich kodifiziert ( $\$ 1$ Abs. $3 \mathrm{AStG}$ ).

191 Vgl. Jacobs (2007), S. 747-777.

192 Vgl. Jacobs (2007), S. 580 und ausführlich Oestreicher (2000), S. 65-74.

193 Vgl. Jacobs (2007), S. 193; OECD (1995), Tz. 3.1. Im Unterschied dazu erkennen die USA die „comparable profit method" als gleichberechtigte Verrechnungspreismethode an, während im Rahmen der deutschen Verwaltungsgrundsätze ein externer Betriebsvergleich zumindest in Ausnahmefällen zugelassen wird. Vgl. Jacobs (2007), S. 774.

194 Vgl. Jacobs (2007), S. 773-774.

195 Vgl. Jacobs (2007), S. 774. 
Ist die Durchführung eines tatsächlichen Fremdvergleichs mangels Datenverfügbarkeit unmöglich, kann - zumindest in Deutschland - als weitere Methode der direkten Gewinnabgrenzung ein hypothetischer Fremdvergleich durchgeführt werden. Hier wird anstelle des Rückgriffs auf beobachtbare Marktdaten der Preisbildungsprozess einer fiktiven Transaktion simuliert. ${ }^{196}$ Die Anwendung dieses hypothetischen Fremdvergleichs wurde mit Wirkung zum 1.1.2008 in Deutschland gesetzlich kodifiziert und auch auf die Verlagerung von Funktionen übertragen, bei denen der angemessene Verrechnungspreis im Rahmen einer Gesamtbewertung zu ermitteln ist. ${ }^{197}$

Die indirekte Methode (globale Gewinnaufteilung) bezieht ihre konzeptionelle Rechtfertigung insbesondere aus der Unmöglichkeit der direkten Methode zur verursachungsgerechten Lokalisation von Synergie- und Skalenvorteilen sowie praktischen Problemen bei der Abgrenzung einzelner Geschäftsvorfälle, der Identifikation vergleichbarer Transaktionen sowie der Ermittlung der erforderlichen Vergleichsdaten. ${ }^{198}$ Wird die Transaktionskostentheorie als Erklärungsansatz für die Begründung multinationaler Unternehmen zugrundegelegt, ist auch das Vorherrschen abweichender Transaktionskosten im Vergleich zu Geschäftsbeziehungen mit unabhängigen Unternehmen anzuführen. ${ }^{199}$ Nach dem Konzept der indirekten Gewinnabgrenzung wird der wirtschaftlichen Einheit des Konzerns in der Form Rechnung getragen, dass der Gesamtgewinn auf einer konsolidierten Basis ermittelt und anhand eines vorab definierten Schlüssels auf die einzelnen Gesellschaften aufgeteilt wird. ${ }^{200}$ Gegen diesen Ansatz werden insbesondere seine Pauschalität sowie die fehlende Berücksichtigung von Unterschiedenen bei den übernommenen Risiken und der Produktivität angeführt. ${ }^{201}$ Auch wenn die indirekte Methode bei der innerstaatlichen Erfolgsabgrenzung zwischen den US-Bundesstaaten, den kanadischen Provinzen, den Schweizer Kantonen und den deutschen Gemeinden zur Anwendung kommt ${ }^{202}$, haben sich die OECD-Länder für die zwischenstaatliche Erfolgsabgrenzung zwischen rechtlich selbständigen Unternehmen ausdrücklich gegen eine Anwendung dieser Methode ausgesprochen. ${ }^{203}$

196 Vgl. Jacobs (2007), S. 703.

197 Vgl. Oestreicher (2009), S. 81. Zur Rechtfertigung und Ausgestaltung der Besteuerung von Funktionsverlagerungen vgl. Oestreicher (2009). $\mathrm{Zu}$ den Bewertungsproblemen bei einer Funktionsverlagerung vgl. ausführlich Oestreicher/Hundeshagen (2009) und Oestreicher/Hundeshagen (2008). Zur Vereinbarkeit der deutschen Vorschriften mit den OECDGrundsätzen zur Gewinnabgrenzung vgl. Frotscher/Oestreicher (2009).

198 Vgl. Oestreicher (2005a), S. 74-80.

$199 \mathrm{Vgl}$. Oestreicher (2000), S. 123.

200 Zur Konzeption der indirekten Gewinnabgrenzung vgl. ausfuhrlich Kapitel 4.2.3.3.

$201 \mathrm{Vgl}$. Oestreicher (2000), S. 192. Aufgrund der Probleme beider Methoden zur konzerninternen Gewinnabgrenzung schlägt Oestreicher eine Kombination beider Verfahren im Wege einer prozessorientierten Gewinnabgrenzung vor. Vgl. Oestreicher (2000), S. 195-239.

202 Vgl. Jacobs (2007), S. 584; Oestreicher (2000), S. $126 \mathrm{mwN}$.

203 Vgl. OECD (1995), Tz. 3.58-3.74. 


\subsection{Probleme der geltenden Konzernbesteuerung}

\subsection{1 Überblick}

Die strategische Zielsetzung der Europäischen Union besteht darin, bis zum Jahr 2010 ,zum wettbewerbsfähigsten und dynamischsten wissensbasierten Wirtschaftsraum der Welt zu werden“ („Lissabon-Strategie“). ${ }^{204}$ Notwendig hierzu ist unter anderem die weitgehende Beseitigung von Barrieren des grenzüberschreitenden Geschäftsverkehrs innerhalb des Binnenmarktes. Dieses Ziel wurde mit Blick auf außersteuerliche Aspekte weitgehend erreicht, wozu auch geänderte wirtschaftliche Rahmenbedingungen seit Beginn der 90er Jahre ihren Beitrag leisteten. Hierzu zählen insbesondere die Entwicklung des elektronischen Geschäftsverkehrs, die gesteigerte Faktormobilität sowie eine hohe Anzahl internationaler Unternehmenszusammenschlüsse. Große europäische Unternehmen betrachten inzwischen mehrheitlich die gesamte Union als ihren Inlandsmarkt, weshalb der Aufbau EU-weiter Geschäftsstrukturen voranschreitet. ${ }^{205}$

Für die Unternehmensbesteuerung folgt die Europäische Kommission primär dem Leitbild einer effizienten, also entscheidungsneutralen Besteuerung. Unternehmerische Entscheidungen sollen nicht durch die Besteuerung verzerrt werden, sondern nach Maßgabe der Bruttorendite getroffen werden. Eine derartige Besteuerung führt zur Maximierung der Wohlfahrt und Steigerung des Bruttosozialprodukts im Binnenmarkt. ${ }^{206}$ Hierzu darf insbesondere auch die grenzüberschreitende Wirtschaftstätigkeit nicht durch steuerliche Hindernisse erschwert werden. ${ }^{207}$ Ferner sollte der Wettbewerb zwischen in Konkurrenz stehenden Unternehmen durch Unterschiede in der steuerlichen Belastung so wenig wie möglich verzerrt werden. Daneben ist bei der Ausgestaltung der Unternehmensbesteuerung im Binnenmarkt aber auch das berechtigte Interesse der Mitgliedsstaaten an einer Finanzierung öffentlicher Güter zu berücksichtigen. Somit tritt neben die Forderung einer entscheidungs- und wettbewerbsneutralen Besteuerung die Sicherstellung einer effektiven Steuererhebung als weitere Anforderung an das Steuersystem. In diesem Sinne ist insbesondere zu vermeiden, dass Steuerpflichtige mit grenzüberschrei-

204 Kommission der Europäischen Gemeinschaften (2003), S. 3; Kommission der Europäischen Gemeinschaften (2002), S. 61.

205 Vgl. Kommission der Europäischen Gemeinschaften (2003), S. 4; Kommission der Europäischen Gemeinschaften (2002), S. 69-75; Plasschaert (2002), S. 7-8.

206 Vgl. Jacobs (2007), S. 268-269; Kommission der Europäischen Gemeinschaften (2002), S. 78-83. Nach Homburg sind zumindest für den Produktionssektor Entscheidungsneutralität und Effizienz kompatibel. Vgl. Homburg (2007), S. 240.

$207 \mathrm{Vgl}$. Kommission der Europäischen Gemeinschaften (2002), S. 17-18. Vgl. zu diesen Anforderungen an die europäische Konzernbesteuerung auch Spengel (2008), S. 23-25; Plasschaert (1997), S. 2-3. 
tender Geschäftstätigkeit ihr Auslandsengagement in Niedrigsteuerländern zur Verlagerung von Steuersubstrat nutzen. ${ }^{208}$

Die Frage, inwieweit das geltende Recht der Mitgliedsstaaten diesen Anforderungen gerecht wird, ist Gegenstand des folgenden Abschnitts. Hierbei wird auf die drei genannten Aspekte - Steuern als Hindernis der grenzüberschreitenden Geschäftstätigkeit (Kapitel 3.5.2), Steuern als Ursache von Entscheidungs- und Wettbewerbsverzerrungen (Kapitel 3.5.3) und Effektivität der Steuererhebung (Kapitel 3.5.4) - eingegangen.

\subsubsection{Steuern als Hindernis der grenzüberschreitenden Geschäftstätig- keit?}

\subsubsection{Steuerliche Nachteile der grenzüberschreitenden Geschäftstätigkeit}

Die steuerlichen Nachteile der grenzüberschreitenden Geschäftstätigkeit im geltenden Steuerrecht, welche nach Auffassung der Europäischen Kommission die Bereitschaft der Unternehmen zur Investition im Ausland hemmen, sind in der Literatur ausgiebig diskutiert. ${ }^{209}$ Steuerbefolgungskosten stellen ein derartiges Hindernis für die grenzüberschreitende Geschäftstätigkeit dar, wenn sie ceteris paribus bei multinationalen Konzernen höher als bei nationalen Konzernen ausfallen. Dieses erscheint nicht unplausibel, da die gleichzeitige Befolgung mehrerer Steuersysteme, die erhöhte Komplexität aufgrund des Zusammenwirkens verschiedener Steuersysteme und die Angemessenheitsdokumentation der Verrechnungspreise insgesamt höhere Kosten erwarten lassen. ${ }^{210}$ Empirische Studien für europäische und nicht-europäische Unternehmen bestätigen diese Erwartungen. So ergeben auf Sekundärdaten beruhende Studien überwiegend einen höheren Anteil der Befolgungskosten an den Steuerzahlungen bei grenzüberschreitend tätigen Unternehmen. ${ }^{211}$ Diese steigen mit zunehmender Unternehmensgröße regressiv an, weshalb Befolgungskosten als Investitionshindernis insbesondere bei kleinen und mittelgroßen Unternehmen Relevanz besitzen sollten. Eine Befragung

$208 \mathrm{Vgl}$. Kommission der Europäischen Gemeinschaften (2003), S. 6.

$209 \mathrm{Vgl}$. für einen Überblick uber diese Nachteile zum Beispiel Devereux (2004), S. 73-76; Oestreicher (2002), S. 344-346. Für eine Beurteilung dieser Hindernisse aus Unternehmensperspektive vgl. UNICE (2000).

210 Vgl. Spengel (2008), S. 24; Kommission der Europäischen Gemeinschaften (2004), S. 4; Oestreicher (2002), S. 344-345; Plasschaert (2002), S. 7-8.

$211 \mathrm{Vgl}$. Kommission der Europäischen Gemeinschaften (2002), S. 799-803 mwN. Abweichend finden sich in der vom Unabhängigen Sachverständigenausschuss Ruding (1992) für europäische Unternehmen durchgeführten Befragung keine nennenswerten Abweichungen bei nationalen und grenzuberschreitend tätigen Unternehmen. Dieses kann allerdings zum einen auf die gewählte Stichprobe (uberwiegend große Unternehmen) und den relativ fruhen Erhebungszeitraum (geringere Bedeutung von Verrechnungspreisvorschriften) zuruckgefuhrt werden. Vgl. Kommission der Europäischen Gemeinschaften (2907), $\mathbf{S}_{6} \mathbf{8 0 3}_{3} \mathbf{3}_{7}$ - $683-6$ 
europäischer Unternehmen durch die Europäische Kommission („European Tax Survey") kommt zu vergleichbaren Ergebnissen. ${ }^{212}$

Ein wesentlicher Treiber der Befolgungskosten multinationaler Unternehmen wird vielfach in der Anwendung des Fremdvergleichsgrundsatzes für die Besteuerung konzerninterner Transaktionen und den hiermit verbundenen umfangreichen Dokumentationsanforderungen gesehen ${ }^{213}$, welche in Europa in den letzten Jahren weiter verschärft wurden. ${ }^{214}$ Besonders betroffen von diesen Problemen dürften erneut kleine und mittlere Unternehmen sein, da diese bei Gründung der ersten ausländischen Tochtergesellschaften kaum in der Lage sein dürften, diese Anforderungen in geeigneter Weise zu erfüllen. ${ }^{215}$

Neben Dokumentationsanforderungen bringt die Anwendung des Fremdvergleichsgrundsatzes für die betroffenen Unternehmen die Gefahr von Streitfällen und Doppelbesteuerungen bei uneinheitlicher Festlegung der Verrechnungspreise durch die beteiligten Finanzbehörden mit sich. ${ }^{216}$ Zur Vermeidung internationaler Doppelbesteuerungen bestehen mit dem abkommensrechtlichen Verständigungsverfahren (Artikel 25 OECD-Musterabkommen) sowie innerhalb von Europa dem Schiedsverfahren zwei Streitbeilegungsmechanismen, die allerdings insbesondere aufgrund der langen Verfahrensdauer ${ }^{217}$ sowie den daraus resultierenden Kosten und der Unzweckmäßigkeit für eine effektive Steuerplanung in der Praxis oftmals nicht in Anspruch genommen werden. So ergab eine von Ernst\&Young im Jahr 1999 durchgeführte Unternehmensbefragung, dass Verrechnungspreisberichtigungen durch eine beteiligte Finanzverwaltung in 42 Prozent der Fälle zu Dop-

212 Hierbei verwiesen insbesondere große multinationale Unternehmen auf Probleme bei Betriebsprüfungen und Steuerstreitfällen. 82 Prozent dieser Unternehmen verwiesen auf Probleme bei der Verrechnungspreisdokumentation. Vgl. Kommission der Europäischen Gemeinschaften (2004), S. 10-11.

213 Auch für die Finanzverwaltungen ist die Überprüfung der Angemessenheit von Verrechnungspreisen, denen aufgrund der gewährten Gestaltungsspielräume große Bedeutung zukommt, mit hohen Erhebungskosten verbunden. Vgl. Plasschaert (1997), S. 6. Die Kosten für die Befolgung der Verrechnungspreisregelungen werden in einer Studie der Europäischen Kommission für multinationale Unternehmen mittlerer Größe auf jährlich 1 bis 2 Millionen Euro geschätzt, bei großen Unternehmen auf jährlich 4 bis 5,5 Millionen Euro. Vgl. Kommission der Europäischen Gemeinschaften (2002), S. 376.

214 So haben Spanien, die Niederlande, Polen, Portugal, Ungarn, Litauen, Schweden, Finnland und Estland seit 2001 „effektive Dokumentationsvorschriften“ neu eingeführt. Vgl. Ernst\&Young (2007), S. 9.

215 Vgl. Kommission der Europäischen Gemeinschaften (2002), S. 368.

216 Vgl. Oestreicher (2002), S. 345-346.

217 Die Europäische Kommission kommt in einer Studie für die Jahre 1995 bis 1999 zum Ergebnis, dass die durchschnittliche Verfahrensdauer bei 20 Monaten lag. Eine Studie des EU-Verrechnungspreisforums aus dem Jahr 2005 ergab, dass von 107 zum 31.12.2004 nicht abgeschlossenen Verfahren für 65 Fälle (61 Prozent) die Verfahrensdauer bereits zwei Jahre überschritten hat, in 24 Fällen (22 Prozent) betrug diese sogar schon mehr als 5 Jahre. Vgl. Jacobs (2007), S. 839-840. 
pelbesteuerungen führten, obwohl die Erfolgsquote der Verständigungs- bzw. Schiedsverfahren bei etwa 90 Prozent liegt. ${ }^{218}$

Eine Alternative zur zeitaufwendigen Auseinandersetzung mit den Finanzverwaltungen bildet die Vereinbarung von bi- oder multilateralen Verrechnungspreiszusagen (,Advance Pricing Agreements“, APA). In derartigen Vereinbarungen werden die zwischen zwei Unternehmen maßgebende Verrechnungspreismethode für eine bestimmte Geschäftsart und einen bestimmten Zeitraum festgelegt sowie häufig auch eine Bandbreite für die angemessenen Ergebnisse der Unternehmen bei Anwendung der vereinbarten Verrechnungspreismethode fixiert. ${ }^{219} \mathrm{Da}$ diese Form der Streitbeilegung zum einen Planungssicherheit für die betroffenen Unternehmen gewährleistet und gleichzeitig zu einer Senkung der Erhebungskosten für die beteiligten Mitgliedsstaaten führt, empfahl die Kommission die Auflegung entsprechender bi- oder multilateraler Programme. ${ }^{220}$ Dennoch wurden derartige Programme in Europa bis 2007 lediglich in Belgien, Deutschland, Frankreich, Großbritannien und den Niederlanden umgesetzt. ${ }^{221}$ Nach einer aktuellen Umfrage von Ernst\&Young betrachten aus diesen Gründen 42 Prozent der multinationalen Konzerne in Europa Verrechnungspreise als das wichtigste Steuerthema. 72 Prozent der Konzerne sind der Meinung, dass das Thema Verrechnungspreise in den letzten zwei Jahren an Bedeutung gewonnen hat. ${ }^{222}$

Daneben wird das Fehlen einer grenzüberschreitenden Verlustverrechnung im Konzern sowohl von Unternehmen als auch von Seiten der Europäischen Kommission zu den bedeutendsten Hemmnissen der grenzüberschreitenden Geschäftstätigkeit gezählt. ${ }^{223}$ So ergab eine Umfrage des Verbands der Schwedischen Industrie, dass 96 Prozent der befragten 172 multinationalen Konzerne mit Verlusten in mindestens einem Mitgliedsstaat, diese nicht oder nur teilweise mit Gewinnen in anderen Mitgliedsstaaten verrechnen konnten. In 56 Prozent dieser Fälle kam es zu endgültigen Doppelbesteuerungen, wobei insbesondere kleine Gesellschaften Probleme bei der grenzüberschreitenden Verlustnutzung hatten. ${ }^{224}$ Trotz seiner Bedeutung konnten in diesem Bereich auf europäischer Ebene in den

218 Vgl. Jacobs (2007), S. 188-190 und 838-845; Kommission der Europäischen Gemeinschaften (2002), S. 377-383.

219 Vgl. Jacobs (2007), S. 845.

$220 \mathrm{Vgl}$. Kommission der Europäischen Gemeinschaften (2001a), S. 15.

$221 \mathrm{Vgl}$. Jacobs (2007), S. 847-848.

$222 \mathrm{Vgl}$. Emst\&Young (2007), S. 50.

223 Vgl. Kommission der Europäischen Gemeinschaften (2002), S. 347; Westberg (2002), S. 322; UNICE (1990). Die systematische Rechtfertigung einer grenzuberschreitenden Verlustverrechnung wird allerdings insbesondere aufgrund der Dividendenfreistellung im Gewinnfall teilweise in Frage gestellt. Vgl. Meussen (2003), S.148. Femer ist - ebenso wie fur den nationalen Fall - fraglich, inwiefern eine konzerninterne Verlustverrechnung ohne entsprechende wirtschaftliche Belastung der Muttergesellschaft durch die Verluste der Tochtergesellschaft gerechtfertigt ist.

$224 \mathrm{Vgl}$. Kommission der Europäischen Gemeinschaften (2002). S. 3598-360 
letzten Jahren keine nennenswerten Fortschritte erzielt werden, obwohl von der Rechtsprechung des EuGH ein gewisser Handlungsbedarf ausgehen könnte. ${ }^{225}$ Vielmehr wurde der Vorschlag zu einer Verlustrichtlinie aus dem Jahre 1991 ohne Stellungnahme des Europäischen Rates zurückgezogen ${ }^{226}$ und lediglich die Notwendigkeit einer grenzüberschreitenden konzerninternen Verlustverrechnung in einer Mitteilung der Kommission aus dem Jahr $2006^{227}$ erneut unterstrichen. Eine Verrechnung von Verlusten ist unverändert nur in einzelnen Mitgliedsstaaten aufgrund entsprechender unilateraler Vorschriften möglich. ${ }^{228}$

Inzwischen geringere Bedeutung als steuerliches Investitionshindernis kommt hingegen einer möglichen wirtschaftlichen Doppelbesteuerung bei grenzüberschreitenden Einkommensströmen und gleichzeitigen Besteuerungsansprüchen von Wohnsitz- und Quellenstaat zu. So wurde der Anwendungsbereich der Mutter-Tochter-Richtlinie durch eine Änderungsrichtlinie aus dem Jahr $2003^{229}$ hinsichtlich der unter die Richtlinie fallenden Rechtsformen sowie durch eine kontinuierliche Senkung der Mindestbeteiligungsquote von 25 Prozent auf 10 Prozent bis zum Jahr 2009 erweitert ${ }^{230}$, während Quellensteuern auf konzerninterne Zins- und Lizenzzahlungen durch die Zins- und Lizenzrichtlinie aus dem Jahr $2005 \mathrm{ab}$ einer Mindestbeteiligungsquote von 25 Prozent abgeschafft wurden. ${ }^{231}$

Auch die - insbesondere im Aufdecken stiller Reserven bestehenden - steuerlichen Nachteile in Zusammenhang mit grenzüberschreitenden Umstrukturierungsvorgängen konnten in den letzten Jahren abgemildert werden. Hierzu leisteten sowohl die Schaffung eines gesellschaftsrechtlichen Rahmens für grenzüberschreitende Fusionen oder Spaltungen durch Einführung der Europäischen Aktiengesellschaft (SE) als auch die Erweiterung des Anwendungsbereichs der Fusionsrichtlinie durch die Umsetzung einer Änderungsrichtlinie ${ }^{232}$ ihren Beitrag. ${ }^{233}$ Dennoch ist darauf hinzuweisen, dass trotz dieser Fortschritte die Umstrukturierung über die Grenze unverändert Nachteile mit sich bringen kann. So können einige steuerliche Problembereiche wie der Untergang von Verlustvorträgen bei Umwandlung durch den Verweis auf die Vorschriften bei nationalen Vorgängen nicht gelöst werden, soweit diese nationalen Vorschriften entsprechende Hindernisse für die Umstrukturierung vorsehen. ${ }^{234}$ Daneben stellt das Erfordernis einer Verhaftung der stillen Reserven in einer inländischen Betriebsstätte als Voraus-

$225 \mathrm{Vgl}$. hierzu Kapitel 4.3.1.

226 Vgl. Kommission der Europäischen Gemeinschaften (2003), S. 10.

227 Kommission der Europäischen Gemeinschaften (2006).

$228 \mathrm{Vgl}$. hierzu Kapitel 3.3.2.

229 Vgl. Richtlinie 2003/123/EG des Rates v.22.12.2003, Abl. EG 2004 Nr. L 7, S. 41

$230 \mathrm{Vgl}$. Jacobs (2007), S. 155-161.

231 Vgl. Jacobs (2007), S. 169-172.

232 Vgl. Richtlinie 2005/19/EG des Rates v. 17.2.2005, Abl. EG 2005 Nr. L 58, S. 19.

233 Vgl. Jacobs (2007), S. 161-169.

234 Vgl. Kommission der Europäischen Gemeinschatten (2002).S. 34448-3-631-75683-6 
setzung der Steuerneutralität ein weiteres verbleibendes Hindernis für grenzüberschreitende Umstrukturierungen dar. ${ }^{235}$

\subsubsection{Steuerliche Vorteile der grenzüberschreitenden Geschäftstätigkeit aus Unternehmenssicht}

Bei einer Beurteilung der Wirkung der Besteuerung als Hindernis für die grenzüberschreitende Geschäftstätigkeit im Binnenmarkt darf nicht übersehen werden, dass die Begründung einer ausländischen Tochtergesellschaft auch steuerliche Vorteile mit sich bringt. So eröffnet sich die Möglichkeit durch entsprechende Gestaltungen der konzerninternen Finanzierungsstruktur oder von Verrechnungspreisen eine Minimierung der Konzernsteuerbelastung herbeizuführen.

Inwiefern bei gleichzeitiger Berücksichtigung von Vor- und Nachteilen das Auslandsengagement insgesamt vorteilhaft besteuert wird, kann auf Basis theoretischer Überlegungen nicht beurteilt werden. Erste Anhaltspunkte hierfür kann allerdings ein Vergleich der Effektivsteuerbelastung für Inlands- und Auslandsinvestitionen liefern, wofür auf Berechnungen mit dem international verbreiteten Modellansatz von Devereux/Griffith ${ }^{236}$ Bezug genommen wird. ${ }^{237}$ Hierbei zeigt sich, dass die durchschnittliche effektive Steuerbelastung der Inbound-Investition in allen Mitgliedsstaaten leicht oberhalb der Steuerbelastung der Inlandsinvestition liegt, wenn eine durchschnittliche Kapitalstruktur zugrundegelegt wird, also Finanzierungsgestaltungen unberücksichtigt bleiben. Die Berücksichtigung der zusätzlichen Besteuerungsebene der ausländischen Muttergesellschaft führt also zu einer zusätzlichen Steuerbelastung (Mittelwert der effektiven Steuerbelastung: 24,2 Prozent gegenüber 22,3 Prozent). ${ }^{238}$ Als Erklärung für diese nur kleine Differenz muss berücksichtigt werden, dass sich die steuerlichen Nachteile der Auslandsinvestition in diesen Berechnungsergebnissen nicht vollständig widerspiegeln. So finden beispielsweise die Probleme grenzüberschreitend tätiger Konzerne bei der Verlustverrechnung in diesem Modellansatz keine Berücksichtigung. ${ }^{239}$

Wird hingegen bei der Finanzierung ausschließlich auf die steuerlich günstigste Alternative abgestellt, liegt die effektive Steuerbelastung der grenzüberschreitenden Investition mit einem Mittelwert von 22,1 Prozent leicht unterhalb des entsprechenden Wertes der inländischen Investition. ${ }^{240}$ Die Vorteilhaftigkeit zwischen der Inlandsinvestition und der Investition über die Grenze hängt somit insbesondere von der Fähigkeit des multinationalen Unternehmens ab, die Kapitalstruktur der Auslandstochter steueroptimal auszugestalten. Dabei ist zu berück-

235 Vgl. Jacobs (2007), S. 168-169.

$236 \mathrm{Vgl}$. zu diesem Ansatz grundlegend Devereux/Griffith (1999).

237 Die Berechnungsergebnisse entstammen einer Studie von Devereux/Elschner/Endres et al. (2008).

$238 \mathrm{Vgl}$. Devereux/Elschner/Endres et al. (2008), S. 50.

239 Vgl. Mintz/Weiner (2003), S. 700.

$240 \mathrm{Vgl}$. Devereux/Elschner/Endres et al. (2008), S. 51 Reinald Koch - 978-3-631-75683-6 
Konzernbesteuerung in Europa de lege lata

sichtigen, dass die Wahl der Finanzierungsform in der Realität regelmäßig durch Regelungen zur Unterkapitalisierung oder CFC-Besteuerung eingeschränkt wird, welche in den Berechnungen ebenfalls keine Berücksichtigung finden.

\subsubsection{Steuern als Ursache von Entscheidungs- und Wettbewerbsverzer- rungen?}

$\mathrm{Zu}$ einer Verzerrung des Wettbewerbs zwischen Unternehmen unterschiedlicher Mitgliedsstaaten kommt es, wenn diese in Abhängigkeit ihres Standortes einer unterschiedlichen steuerlichen Belastung unterliegen. ${ }^{241}$ Gleichzeitig kann von derartigen Belastungsunterschieden eine Beeinflussung der unternehmerischen Standortentscheidung ausgehen. ${ }^{242}$ Ein Vergleich der effektiven Steuerbelastungen nationaler Investitionen, die sich für die europäischen Mitgliedsstaaten (EU25) im Jahr 2007 nach dem Modellansatz von Devereux/Griffith ergeben ${ }^{243}$, zeigt eine deutliche Streuung. Die Höhe der effektiven Steuerbelastung bei Inlandsinvestitionen variiert in einer Bandbreite von 14,4 Prozent (Irland) und 35,5 Prozent (Deutschland), der Variationskoeffizient beträgt 29,62 Prozent. Diese Schwankung ist zu einem Großteil auf das insgesamt niedrigere Steuerniveau in den Beitrittsstaaten zurückzuführen. So beträgt der Variationskoeffizient innerhalb der EU15-Länder lediglich 21,97 Prozent.

Die große Schwankungsbreite der effektiven Steuersätze liegt nicht in einem einzelnen Element des Steuersystems begründet. Allerdings zeigt die Analyse, dass die Unterschiede im Wesentlichen auf Abweichungen in der Höhe der tariflichen Steuersätze zurückzuführen sind, während den Vorschriften zur Ermittlung der Bemessungsgrundlage nur eine untergeordnete Bedeutung zukommt. ${ }^{244}$ Gleichzeitig ergibt eine Analyse der Steuerbelastungen, dass ein niedriger Steuersatz tendenziell mit einer breiten Bemessungsgrundlage einhergeht und umgekehrt. So beträgt der Variationskoeffizient der tariflichen Steuersätze 30,48 Prozent (EU25) beziehungsweise 23,65 Prozent (EU15) und liegt somit oberhalb des entsprechenden Wertes für die effektive Steuerbelastung. Dieses unterstreicht die

241 Vgl. Kommission der Europäischen Gemeinschaften (2002), S. 192.

242 Vgl. Kommission der Europäischen Gemeinschaften (2002), S. 13. Elschner/Schwager (2007) und Elschner/Lammersen/Overesch et al. (2006) verweisen zusätzlich auf den Einfluss der Besteuerung hochqualifizierter Arbeitnehmer auf die Standortentscheidungen.

243 Die Berechnungsergebnisse entstammen auch hier der Studie von Devereux/Elschner/Endres et al. (2008).

244 Vgl. Cnossen (2004), S. 142; Kommission der Europäischen Gemeinschaften (2002), S. 192. Dabei muss allerdings berücksichtigt werden, dass der Einfluss der Gewinnermittlungsvorschriften auf die Höhe der effektiven Durchschnittssteuerbelastung im Modell von Devereux/Griffith unterschätzt wird, da diese nur unvollständig abgebildet werden. Vgl. so Mintz (2004), S. 229; Mintz/Weiner (2003), S. 700; Kommission der Europäischen Gemeinschaften (2002), S. 16. 
weit verbreitete Vermutung ${ }^{245}$, dass von den Vorschriften zur Ermittlung der Bemessungsgrundlage ein kompensatorischer Effekt zu den effektiven Steuerbelastungen ausgeht.

Stellt man dieser Analyse die Ergebnisse der Berechnungen für frühere Veranlagungszeiträume gegenüber, so zeigt sich, dass die durchschnittliche Höhe der Steuerbelastung in den EU15-Ländern (Rückgang von 30,7 Prozent auf 26,3 Prozent) und insbesondere in den Beitrittsstaaten (Rückgang von 26,3 Prozent auf 18,5 Prozent) zwischen 1998 und 2007 deutlich zurückgegangen ist. ${ }^{246}$ Die Schwankung der effektiven Steuerbelastung entwickelte sich hingegen in dem Zeitraum unterschiedlich. Während der Variationskoeffizient innerhalb der EU15Länder zwischen 1998 und 2007 leicht zurückgegangen ist (Rückgang von 24,33 Prozent auf 21,97 Prozent), ist der entsprechende Wert bei Berücksichtigung der Beitrittsstaaten hingegen deutlich angestiegen (Anstieg von 24,44 Prozent auf 29,62 Prozent). ${ }^{247}$ Das Ausmaß der steuerinduzierten Wettbewerbsverzerrung im Binnenmarkt sollte somit in Folge der Erweiterung der Europäischen Union sowie der starken Absenkung der tariflichen Steuersätze in den Beitrittsstaaten zuletzt zugenommen haben.

\subsubsection{Effektivität der Steuerhebung}

Neben den Anforderungen der Effizienz und Neutralität, die aus einer europäischen Perspektive an die Konzernbesteuenung im Binnenmarkt gestellt werden, müssen bei einer Beurteilung der Vorschriften auch die fiskalischen Interessen der Mitgliedsstaaten in hinreichendem Maße berücksichtigt werden. ${ }^{248}$ Diese Notwendigkeit, die sich insbesondere auch aus der Souveränität der Mitgliedsstaaten bei der Ausgestaltung der direkten Steuern ergibt, dokumentiert auch die Europäische Kommission in ihren Stellungnahmen zur künftigen Ausgestaltung des Steuerrechts. ${ }^{249}$ Hierbei ist insbesondere die Sicherstellung einer effektiven Erhebung der Steuern von entscheidender Bedeutung. Diese verlangt neben einer Steuererhebung, die mit möglichst geringen Kosten für die Finanzverwaltung verbunden ist, in erster Linie eine Einschränkung der Möglichkeiten zu einer Steuervermeidung durch Verlagerung von Bemessungsgrundlage in Niedrigsteuerländer. ${ }^{250}$

Derartige Gestaltungen durch Steuerpflichtige stellen die Hochsteuerländer in der jüngeren Vergangenheit vor zunehmende Probleme. Als Grund hierfür ist insbesondere auf das Fehlen eines konzeptionellen Leitbilds für die Besteuerung

245 Vgl. hierzu Oestreicher/Spengel (2003), S. 93; Chennels/Griffith (1997), S. 26-30.

246 Eigene Berechnungen auf Basis der Angaben in Devereux/Elschner/Endres et al. (2008), S. 25.

247 Eigene Berechnungen auf Basis der Angaben in Devereux/Elschner/Endres et al. (2008), S. 25.

248 Vgl. Spengel (2008), S. 23-24; Plasschaert (1997), S. 2-3.

$249 \mathrm{Vgl}$. Kommission der Europäischen Gemeinschaften (2003), S. 6.

250 Vgl. Plasschaert (1997), S. 2-3. 
Konzernbesteuerung in Europa de lege lata

der grenzüberschreitenden Unternehmenstätigkeit in Europa zu verweisen ${ }^{251}$, welches in dem Konzept einer kapitalexportneutralen Sitzlandbesteuerung oder dem Konzept einer kapitalimportneutralen Quellenbesteuerung bestehen könnte. ${ }^{252}$

Das gegenwärtige Steuerrecht ist allerdings durch eine entgegengesetzte gegenläufige Behandlung der Eigen- und die Fremdfinanzierung ausländischer Tochterkapitalgesellschaften gekennzeichnet. Rückflüsse für die Überlassung von Eigenkapital an eine ausländische Tochtergesellschaft werden überwiegend in Form der Körperschaftsteuer der Auslandstochter im Quellenstaat besteuert. Zwar wird im Falle eines europäischen Konzerns aufgrund der Mutter-Tochter-Richtlinie keine darüber hinausgehende Quellensteuer auf ausgeschüttete Gewinne erhoben, auf Ebene der Muttergesellschaft werden empfangene Dividenden allerdings in 24 der 27 Mitgliedsstaaten freigestellt. Nur in drei Mitgliedsstaaten kommt es somit bei Eigenfinanzierung zu einer Besteuerung im Sitzland des Kapitalgebers. ${ }^{253}$

Entgelte für eine konzerninterne Überlassung von Fremdkapital unterliegen demgegenüber im Land des Kapitalempfängers, wenn überhaupt, einer Quellensteuer ${ }^{254}$ Deren Erhebung wird allerdings in Europa bereits unilateral häufig an bestimmte Voraussetzungen geknüpft. ${ }^{255}$ Zum anderen wird die Höhe der Quellensteuer regelmäßig im Rahmen von Doppelbesteuerungsabkommen eingeschränkt. Für Zinszahlungen zwischen Unternehmen eines europäischen Konzerns, schließlich, sind Quellensteuern mit Umsetzung der Zins- und Lizenzrichtlinie sogar vollständig ausgeschlossen. Sie unterliegen somit im Regelfall weitgehend oder ausschließlich der Besteuerung im Sitzland des Kapitalgebers. ${ }^{256}$

Diese Ungleichbehandlung von Entgelten für Eigen- und Fremdkapitalüberlassungen eröffnet gegenwärtig erhebliche Gestaltungsspielräume für die Steuerpflichtigen, welche eine effektive Steuererhebung insbesondere für Hochsteuerländer erschweren. ${ }^{257}$ Ohne entsprechende Schutzvorschriften könnten im Extremfall die Gewinne einer in einem Hochsteuerland ansässigen Tochtergesellschaft

251 Vgl. Spengel (2008), S. 26; Jacobs (2007), S. 172-173; Devereux (2004), S. 80. Vgl. hierzu auch Schön (2007), S. 423-424.

252 Nach verbreiteter Auffassung gewährleistet nur eine kapitalexportneutrale Besteuerung eine steuerunabhängige Standortentscheidung und ist damit mit dem Grundgedanken des Binnenmarktes bestmöglich vereinbar. Vgl. Jacobs (2007), S. 273-274. Demgegenüber weist Devereux (2004), S. 82-83 darauf hin, dass sich auch bei kapitalexportneutraler Besteuerung Effizienz nur unter restriktiven Voraussetzungen einstellt.

253 Vernachlässigt werden hier die Besteuerungsansprüche aufgrund eines pauschalen Abzugsverbots fur Beteiligungsaufwendungen.

254 Etwas anderes kann sich nur ergeben, wenn Unterkapitalisierungsregeln im Quellenstaat zur Anwendung kommen.

255 Vgl. Cnossen (2004), S. 141.

256 Vgl. Schön (2007), S. 423-424.

257 Vgl. Spengel (2008), S. 26; Jacobs (2007), S. 274-275; Cnossen (2004), S. 142; Grotherr (1993), S. 6-7. 
vollständig über Zinszahlungen abgeschöpft werden, während die Gewinne von einer in einem Niedrigsteuerland ansässigen Tochtergesellschaft entweder aufgrund der Freistellungsmethode oder aufgrund von Gewinnthesaurierung von einer Besteuerung bei der Muttergesellschaft dauerhaft oder über einen langen Zeitraum ausgenommen werden können. Derartige Gestaltungen sollten aufgrund der voranschreitenden europäischen Integration und der damit ansteigenden Bedeutung grenzüberschreitender Unternehmensstrukturen in der Zukunft weiter an Relevanz gewinnen. $\mathrm{Zu}$ einer Verstärkung dieses Problems tragen ferner die ebenfalls zunehmenden Einschränkungen bei, welche sich durch die Rechtsprechung des EuGH für die Ausgestaltung der Vorschriften zum Schutz des inländischen Steuersubstrats ergeben. ${ }^{258}$ So akzeptiert der EuGH zwar die Vermeidung von Gestaltungsmissbrauch als legitime Rechtfertigung für die steuerliche Benachteiligung eines EU-Ausländers, grundsätzlich darf nach Ansicht des EuGH das Ziel der Aufkommenssicherung der Freizügigkeit innerhalb der Gemeinschaft aber nicht übergeordnet werden. ${ }^{259}$ Das Problem für die Mitgliedsstaaten besteht also in dem Erfordernis der Zielgenauigkeit entsprechender Maßnahmen. ${ }^{260}$ Gestaltungsspielräume des Gesetzgebers werden aufgrund der erheblichen Haushaltsrisiken europarechtswidriger Normen oftmals nicht mehr ausgeschöpft. ${ }^{261}$

\subsubsection{Zwischenfazit}

Insgesamt bleibt festzuhalten, dass die grenzüberschreitende Geschäftstätigkeit für Unternehmen aus steuerlicher Sicht sowohl Vor- als auch Nachteile mit sich bringt. Vorteilhaft wirkt sich die Möglichkeit zur Senkung der Steuerbelastung durch Ausnutzung des internationalen Steuergefälles aus. Dem stehen insbesondere hohe Befolgungskosten, die Gefahr von Doppelbesteuerungen sowie das Fehlen einer grenzüberschreitenden Verlustverrechnung als gravierende Nachteile gegenüber. Inwiefern grenzüberschreitende Investitionen insgesamt einer höheren oder niedrigeren Steuerbelastung unterliegen, kann auch mithilfe von Berechnungen anhand des Devereux/Griffith-Modells nicht abschließend festgestellt werden. Aus diesen Berechnungen ist allerdings zu erkennen, dass der Wettbewerb innerhalb des Binnenmarktes durch eine zunehmende Schwankung der effektiven Steuerbelastungen in immer stärkerem Maße verzerrt wird. Hierzu hat insbesondere auch die Aufnahme der Beitrittsstaaten ihren Beitrag geleistet. Es ist allerdings erneut darauf hinzuweisen, dass dieses Modell weder eine Abbildung der Nachteile grenzüberschreitender Konzerne bei der steuerlichen Verlustverrechnung noch eine realistische Abbildung einer Konzernsteuerplanung erlaubt, was

258 Vgl. Spengel/Oestreicher (2009), S. 776; Spengel (2008), 24-25; Jacobs (2005), S. 134;

Kommission der Europäischen Gemeinschaften (2003), S. 3.

$259 \mathrm{Vgl}$. Hey (2008), S. 1037 mit Verweis auf entsprechende Urteile.

$260 \mathrm{Vgl}$. Hey (2008), S. 1037.

261 Vgl. Hey (2008), S. 1039. 
die Notwendigkeit von Berechnungen unter Verwendung eines alternativen Ansatzes unterstreicht.

Eine Reform der Konzernbesteuerung in Europa erscheint allerdings insbesondere aus Sicht der Mitgliedsstaaten erforderlich. Diese werden durch das wachsende Ausmaß an Steuergestaltung vor zunehmende Probleme gestellt. Es ist davon auszugehen, dass die Schwierigkeiten bei der Steuerhebung durch den Ausbau internationaler Unternehmensstrukturen sowie die Rechtsprechung des Europäischen Gerichtshofs in Zukunft weiter verstärkt werden. 


\section{Konzernbesteuerung in Europa de lege ferenda}

\subsection{Grundlegende Reformoptionen}

Die Ausführungen in Abschnitt 3.5 haben den insbesondere aus Sicht der Mitgliedsstaaten bestehenden Bedarf für eine Reform der Vorschriften zur Konzernbesteuerung in Europa aufgezeigt. Der europarechtliche Rahmen für eine solche Reform wird durch die Regelungen des EG-Vertrags abgesteckt. Im Gegensatz zu den indirekten Steuern beinhaltet der Vertrag keinen Harmonisierungsauftrag für den Bereich der direkten Steuern, deren Ausgestaltung vielmehr in den Zuständigkeitsbereich der Mitgliedsstaaten fällt. ${ }^{262}$ Die hierbei grundsätzlich bestehende Gestaltungsfreiheit wird allerdings durch die ebenfalls im EG-Vertrag festgehaltenen Grundfreiheiten eingeschränkt, deren Einhaltung die Mitgliedsstaaten - wie bereits erwähnt - in zunehmendem Maße vor Probleme stellt. ${ }^{263}$

Trotz der Souveränität der Mitgliedsstaaten bei der Ausgestaltung der direkten Steuern schließt der EG-Vertrag koordinative Maßnahmen nicht aus. Voraussetzung für die Verabschiedung verbindlicher Rechtsakte ist dabei allerdings wie bei allen steuerlichen Maßnahmen - ein einstimmiger Beschluss des europäischen Rates. ${ }^{264}$ Die im Rahmen der Vorgaben des EG-Vertrags bestehenden Optionen für eine harmonisierte Weiterentwicklung der Konzernbesteuerung sind in Abbildung 3 zusammengefasst. ${ }^{265}$ Hierbei können insbesondere gezielte Maßnahmen, die nur einzelne, ausgewählte Investitionshindernisse beseitigen, von umfassenden Lösungsvorschlägen unterschieden werden, welche eine systematische Beseitigung sämtlicher Investitionshindernisse ermöglichen würden.

262 Vgl. Jacobs (2007), S. 96-97.

263 Weitere rechtliche Grenzen bei der Ausgestaltung der direkten Steuern können sich aus dem Verfassungsrecht und Doppelbesteuerungsabkommen ergeben.

264 Vgl. Jacobs (2007), S. 100.

265 Eine unilaterale Weiterentwicklung der Regelungen zur Konzernbesteuerung ohne entsprechende Vorgaben des EuGH wird in dieser Arbeit nicht in Betracht gezogen. Für eine sachgemäße Beseitigung der genannten Probleme erscheint dieser Ansatz nicht geeignet. 
Abbildung 3: Mögliche Ansätze für eine abgestimmte Weiterentwicklung der Konzernbesteuerung in Europa

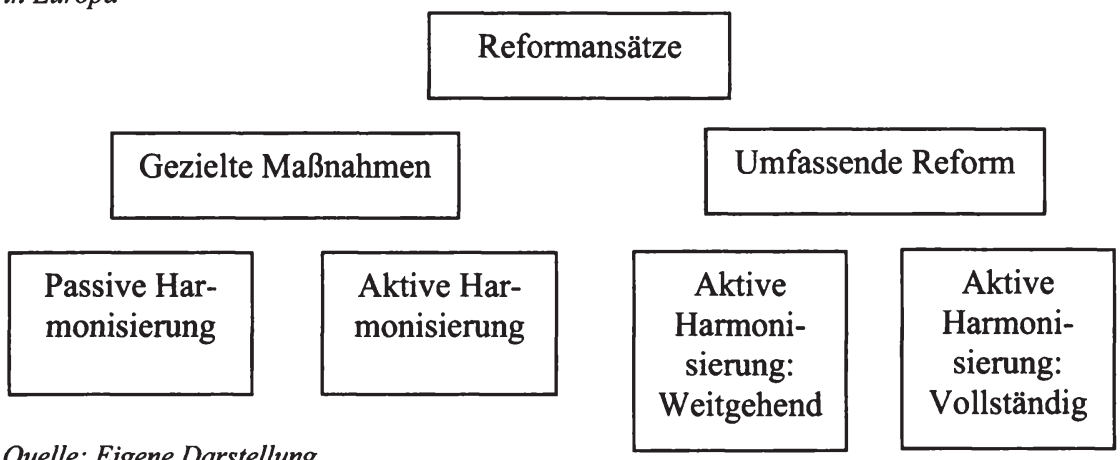

Quelle: Eigene Darstellung

Gezielte Maßnahmen bewirken einen nur geringfügigen Eingriff in die Steuersouveränität der Mitgliedsstaaten und zeichnen sich dementsprechend durch die Möglichkeit einer verhältnismäßig kurzfristigen Umsetzung aus. Die Einführung gezielter Maßnahmen kann im Wege einer aktiven oder passiven Harmonisierung erfolgen. Unter passiver Harmonisierung wird dabei eine von der Rechtssprechung des Europäischen Gerichtshofs ausgehende unilaterale Anpassung der nationalen Steuergesetze und die sich hieraus bei gleichgerichteter Umsetzung der Urteile ergebende partielle Rechtsangleichung verstanden. ${ }^{266}$ Zwar lässt dieser Weg der Harmonisierung - aufgrund der von der Rechtsprechung ausgehenden faktischen Verpflichtung zur Reform - den geringfügigsten Widerstand der Mitgliedsstaaten bei der Umsetzung erwarten, er wird allerdings mit Blick auf seine Wirkungen regelmäßig lediglich als Minimallösung angesehen. ${ }^{267}$ So kann erstens auf diesem Weg nicht die systematische Gleichbehandlung innerhalb der Mitgliedsstaaten gewährleistet werden, sondern lediglich eine Gleichbehandlung inländischer und grenzüberschreitender Sachverhalte in einem Mitgliedsstaat. ${ }^{268}$ Für Mitgliedsstaaten ohne entsprechende Diskriminierung grenzüberschreitender Sachverhalte im nationalen Recht besteht hingegen keine Notwendigkeit zur Änderung. Zweitens kann eine Harmonisierung selbst innerhalb der betroffenen Mitgliedsstaaten nur bei einheitlicher Umsetzung des entsprechenden Urteils erreicht werden. Die tatsächliche Interpretation der Auswirkungen der Urteile für die Rechtsvorschriften des jeweiligen Mitgliedsstaates ist allerdings oftmals schwierig, weshalb nicht ausgeschlossen werden kann, dass Mitgliedsstaaten ihre Vorschriften in unterschiedlicher Weise ändern, was sogar Disharmonisierung zur

266 Vgl. Jacobs (2005), S. 134. Hey (2005), S. 324 verwendet hierfür den Begriff der „stillen Harmonisierung“.

267 Vgl. Jacobs (2007), S. 275-276; Hey (2005), S. 321-324. In ähnliche Richtung zeigt auch der Kommentar von Blumenberg in Rädler (2008), S. 78.

268 Vgl. Jacobs (2007), S. 270.

Reinald Koch - 978-3-631-75683-6 
Folge haben kann. Die Europäische Kommission hat deshalb eine Koordinierung von Besteuerungselementen vorgeschlagen, die dem EU-Recht zuwiderlaufen. ${ }^{269}$ Schließlich kann im Wege der passiven Harmonisierung das Grundproblem des Fehlens einer Konzeption für die internationale Konzernbesteuerung nicht beseitigt werden. Vielmehr beinhaltet eine von der Rechtsprechung getriebene Reform die Gefahr von kurzfristigen und unsystematischen Reaktionen des Gesetzgebers. Werden als Reaktion auf ein Urteil nicht die steuerlichen Rahmenbedingungen für grenzüberschreitende Investitionen verbessert, sondern zum Schutz des Steueraufkommens vielmehr die Investitionshindernisse auf nationale Investitionen ausgedehnt (wie dieses zum Beispiel in Deutschland bei den Regelungen zur Unterkapitalisierung der Fall war), kommt es zu einer Verschlechterung der Standortattraktivität bei gleichzeitiger Beeinträchtigung der Systematik des inländischen Steuerrechts. ${ }^{270}$ Der Weg der passiven Harmonisierung wird daher überwiegend abgelehnt ${ }^{271}$, weshalb eine derartige unilaterale Weiterentwicklung der Konzernbesteuerung im Folgenden nicht in die Überlegungen einbezogen wird.

Aktive Harmonisierung dient demgegenüber als Sammelbegriff für koordinative Maßnahmen, welche regelmäßig als verbindliche Rechtsakte durch den europäischen Rat, zumeist auf Vorschlag der Europäischen Kommission ${ }^{272}$, verabschiedet werden. Verglichen mit der Möglichkeit einer passiven Harmonisierung weist diese Option den Vorteil auf, dass sie eine abgestimmte und systematische Lösung der spezifischen Probleme erlaubt. Aufgrund der nur beschränkten unilateralen Reaktionsmöglichkeiten auf die Rechtsprechung des EuGH können angemessene Antworten auf diese Urteile nur mit einem abgestimmten Vorgehen gefunden werden. ${ }^{273}$

Auch wenn durch gezielte Maßnahmen spezifische Probleme (grenzüberschreitende Verlustverrechnung, Mehrfachbelastungen aufgrund grenzüberschreitender Quellensteuern) abgemildert oder vollständig beseitigt werden können, bleibt das Grundproblem der Existenz individueller Besteuerungssysteme in einem integrierten Markt bestehen. Dieses gilt auch für die erforderliche Abgrenzung von Besteuerungsansprüchen mit der damit verbundenen Gefahr von Dop-

$269 \mathrm{Vgl}$. in diesem Sinne Kommission der Europäischen Gemeinschaften (2006a) und insbesondere Kommission der Europäischen Gemeinschaften (2007), S. 7.

270 Vgl. Spengel/Oestreicher (2009), S. 776; Hey (2008), S. 1036; Jacobs (2007), S. 275-276; Hey (2005), S. 321-324.

271 Vgl. Lang (2008), S. 25 sowie die Kommentare von Blumenberg (S. 78) und Pistone (S. 77) in Rädler (2008); Kommission der Europäischen Gemeinschaften (2003), S. 4. Wittkowski verweist auf Probleme der praktischen Umsetzung unilateraler Maßnahmen. Vgl. Wittkowski (2008), S. 265. Auch Jacobs ist der Meinung, dass die beschriebenen Probleme des geltenden Steuerrechts nur im Wege einer abgestimmten Reform sinnvoll beseitigt werden kőnnen. Vgl. Jacobs (2007), S. 270; Jacobs (2005), S. 141. Vanistendael (1996) verwendet furr diesen Tatbestand den Begriff des „Europäischen Steuerparadoxons“.

272 Vgl. Jacobs (2007), S. 100.

273 Vgl. Czakert (2006), S. 562. 
pelbesteuerungen und möglichen Gestaltungsspielräumen. Eine Beseitigung dieser Probleme kann nur im Wege einer weitgehenden oder vollständigen Harmonisierung der Konzernbesteuerung im Binnenmarkt erreicht werden. ${ }^{274}$

Versuche zu einer vollständigen Harmonisierung der Kapitalgesellschaftsbesteuerung hat die Europäische Kommission beginnend mit dem Bericht des Steuer- und Finanzausschusses (1962) („Neumark-Bericht“) in der Vergangenheit wiederholt unternommen. Letztmalig wurde in einer Kommissionsstudie aus dem Jahr 2002 eine EU-Körperschaftsteuer mit vollständiger Harmonisierung auch der Steuersätze und der Steueradministration in Erwägung gezogen. ${ }^{275}$ Hierdurch würden zwar die überwiegende Anzahl der Investitionshindernisse und Gestaltungsanreize beseitigt, die Aussicht auf Realisation muss aufgrund des weitreichenden Eingriffs in die Steuersouveränität der Mitgliedsstaaten allerdings als gering eingestuft werden. ${ }^{276}$ Gleichzeitig würde eine umfassende Harmonisierung den Steuerwettbewerb zwischen den Mitgliedsstaaten vollständig beseitigen. Während eine weitgehende Beseitigung des Steuerwettbewerbs die Motivation der frühen Vorschläge der Europäischen Kommission war, wird nach heutiger Vorstellung ein begrenzter Steuerwettbewerb von der Kommission für sinnvoll erachtet. ${ }^{277}$ Eine derart umfassende Harmonisierung wird von der Europäischen Kommission von daher nicht mehr als Ziel verfolgt ${ }^{278}$ und aus diesem Grund auch hier nicht weiter in die Betrachtungen einbezogen.

Als langfristige Zielsetzung für die Unternehmensbesteuerung im Binnenmarkt hat sich die Kommission im Rahmen der Studie vielmehr die weitgehende Harmonisierung der Kapitalgesellschaftsbesteuerung in Europa gesetzt. Zu diesem Zweck wurden im Rahmen der Studie neben der EU-Körperschaftsteuer drei Konzepte (,Sitzlandbesteuerung“, „Gemeinsame konsolidierte Körperschaftsteuerbemessungsgrundlage“, „Harmonisierte Bemessungsgrundlage ${ }^{\text {(2279) }}$ ) vorgestellt, welche hinsichtlich der Ermittlung des zu versteuernden Einkommens weitgehend der Einheitstheorie folgen und das Ziel einer konzeptionellen Beseitigung sämtlicher Investitionshindernisse verfolgen. Gleichzeitig soll durch diese Vorschläge eine effektive Erhebung der Steuern durch die Mitgliedsstaaten gewährleistet

274 Vgl. Kommission der Europäischen Gemeinschaften (2003), S. 4

275 Vgl. Kommission der Europäischen Gemeinschaften (2002), S. 509-510.

276 Sorenson (2004) und Spengel (1998) machen eigene, ähnlich weitreichende Vorschläge zur Reform der Konzernbesteuerung, äußern sich allerdings selbst kritisch bezüglich der Umsetzbarkeit ihrer eigenen Konzepte.

277 Vgl. Sorenson (2004), S. 91 und 104; Spengel (1998), S. 348. So verneinte die Kommission bereits in ihrem Bericht aus dem Jahr 1996 bestehenden Handlungsbedarf bezüglich einer Vereinheitlichung von Steuersätzen und Bemessungsgrundlage. Vgl. Kommission der Europäischen Gemeinschaften (1996), S. 6 und 14.

278 Vgl. Kommission der Europäischen Gemeinschaften (2001), S. 9; Kommission der Europäischen Gemeinschaften (2001a), S. 11.

279 Vgl. Kommission der Europäischen Gemeinschaften (2002), S. 505-511. Für eine Beurteilung dieser Vorschläge vgl. Westberg (2002). 
werden. ${ }^{280}$ Die Vorschläge sehen übereinstimmend eine Vereinheitlichung der Einkommensermittlung vor, zielen aber nicht auf eine Harmonisierung der Steuersätze und der Steuerverwaltung ab. Inzwischen konzentriert sich die Diskussion auf politischer und wissenschaftlicher Ebene ausschließlich auf den Vorschlag der Einführung einer „Gemeinsamen konsolidierten Körperschaftsteuerbemessungsgrundlage“(GKKB).

Zusammenfassend bleibt festzuhalten, dass mit der Einführung einer GKKB die Beseitigung sämtlicher Investitionshindernisse für die grenzüberschreitende Geschäftstätigkeit erreicht werden könnte. Dieses Reformszenario steht aus diesem Grund im Zentrum der folgenden Untersuchungen. Inwiefern die GKKB als umfassender Lösungsvorschlag mit dem hiermit verbundenen weitreichenden Eingriff in die Steuersouveränität der Mitgliedsstaaten Aussicht auf politische Umsetzung hat, ist zum heutigen Zeitpunkt allerdings unklar. ${ }^{281}$ Sorenson verweist darauf, dass die Kosten, die von der fehlenden Harmonisierung der Unternehmensbesteuerung ausgehen, möglicherweise erst hoch genug sein müssen, damit die Mitgliedsstaaten einem derartigen Verlust an Steuersouveränität zustimmen. ${ }^{282}$ Dieser Umstand rechtfertigt es, auch auf die spezifische Lösung einzelner Probleme ausgerichtete Koordinierungsmaßnahmen in die Betrachtung einzubeziehen. Hierbei muss allerdings berücksichtigt werden, dass die Mehrzahl der verbleibenden steuerlichen Hindernisse auf das Nebeneinander separater Besteuerungssysteme im Binnenmarkt zurückzuführen ist und demnach durch gezielte Maßnahmen nicht beseitigt werden kann. Etwas anderes gilt nur für das Fehlen einer grenzüberschreitenden Verlustverrechnung. Aus diesem Grund wird die Harmonisierung der Verlustverrechnung im Wege einer Verlustrichtlinie als zweites Reformszenario diskutiert, welche nach den Vorstellungen der europäischen Kommission insbesondere die europaweite Einführung einer grenzüberschreitenden Verlustverrechnung für ausländische Tochterkapitalgesellschaften und Betriebsstätten vorsehen soll. ${ }^{283}$

$280 \mathrm{Vgl}$. Kommission der Europäischen Gemeinschaften (2003), S. 6.

281 Vgl. so Sorenson (2002), S. 35. Andere Autoren halten nur die Einführung im Wege der verstärkten Zusammenarbeit für realistisch. Vgl. den Kommentar von Lang in Rädler (2008), S. 65; McLure (2008), S. 46; Schön (2007), S. 443.

$282 \mathrm{Vgl}$. Sorenson (2002), S. 35.

$283 \mathrm{Vgl}$. so Kommission der Europäischen Gemeinschaften (2006). 


\subsection{Einführung einer „Gemeinsamen konsolidierten Körper- schaftsteuerbemessungsgrundlage“}

\subsection{1 Überblick}

Das Konzept einer „Gemeinsamen konsolidierten Körperschaftsteuerbemessungsgrundlage“ (GKKB) wurde von der Europäischen Kommission erstmalig im Rahmen ihrer Mitteilung aus dem Jahr 2001 als langfristige Zielsetzung für die Körperschaftsbesteuerung vorgegeben ${ }^{284}$ und in wiederholten Verlautbarungen in den Folgejahren bekräftigt. ${ }^{285}$ Dabei soll die mit diesem Konzept einhergehende Ermittlung der steuerlichen Bemessungsgrundlage nach einheitlichen Vorschriften sowie auf einer konsolidierten Basis eine systematische Beseitigung der in Kapitel 3.5 aufgezeigten steuerlichen Hindernisse bewirken. Auch wenn die einzelne Konzerngesellschaft mit dem ihr zugewiesenen Anteil am konsolidierten Ergebnis selbständig steuerpflichtig bleibt, folgt dieses Konzept bei der Ermittlung des Einkommens weitgehend der Einheitstheorie. Die Einführung eines derartigen Systems würde zwar ein Novum im internationalen Steuerrecht bedeuten, bei der Ausgestaltung kann allerdings auf Erfahrungen mit vergleichbaren Regimen zur Besteuerung von Körperschaften durch Gebietskörperschaften eines Landes zurückgegriffen werden. Derartige Systeme bestehen in den USA, Kanada, der Schweiz und - mit Abstrichen - bei der deutschen Gewerbesteuer. ${ }^{286}$

Im Anschluss an die angesprochene Kommissionsmitteilung wurde im Jahr 2004 eine Arbeitsgruppe aus Sachverständigen der zuständigen Ministerien aller Mitgliedsstaaten von der Kommission mit der Ausarbeitung eines Regelungsvorschlags für die Einführung einer GKKB beauftragt. ${ }^{287}$ Die Ergebnisse dieser Arbeitsgruppe sollen die Basis für einen Richtlinienentwurf der Kommission bilden, welcher ursprünglich für die zweite Jahreshälfte 2008 angekündigt war. ${ }^{288}$ Auch wenn das Projekt entgegen früherer Skepsis gegenüber einer formelhaften Gewinnaufteilung $^{289}$ anfänglich sowohl die Unterstützung von Unternehmensseite ${ }^{290}$

284 Vgl. Kommission der Europäischen Gemeinschaften (2001a), S. 18-19. Die drei weiteren umfassenden Lösungsvorschläge, welche in dieser Studie neben der GKKB vorgestellt wurden, sind inzwischen nicht mehr oder nur nachrangig Gegenstand der politischen Diskussion. Vgl. Jacobs (2007), S. 278.

285 Vgl. Kommission der Europäischen Gemeinschaften (2007a); Kommission der Europäischen Gemeinschaften (2006b); Kommission der Europäischen Gemeinschaften (2005); Kommission der Europäischen Gemeinschaften (2003).

286 Vgl. Jacobs (2007), S. 584; Oestreicher (2000), S. $126 \mathrm{mwN}$.

287 Vgl. zur Arbeitsgruppe Czakert (2008), S. 433; Czakert (2006), S. 561-562; Kommission der Europäischen Gemeinschaften (2006b), S. 4-6.

288 Vgl. Kommission der Europäischen Gemeinschaften (2006b), S. 9.

289 Vgl. Sorenson (2004), S. 93.

$290 \mathrm{Zu}$ diesem Ergebnis kommt eine Unternehmensbefragung der KPMG aus dem Jahr 2007. Vgl. KPMG (2007). 
als auch einiger einflussreicher Mitgliedsstaaten ${ }^{291}$ genoss, wurde die Veröffentlichung des Richtlinienvorschlags nun mit Hinweis auf weiteren Forschungsbedarf bei der Abschätzung der ökonomischen Wirkungen einer Einführung bis auf Weiteres verschoben. Für die Darstellung der möglichen Ausgestaltung einer GKKB in den folgenden Abschnitten wird daher auf die von der Arbeitsgruppe veröffentlichten Arbeitspapiere zurückgegriffen. Diese Arbeitspapiere geben den letzten öffentlich verfügbaren Diskussionsstand innerhalb der Gruppe wieder.

In dieser Arbeit werden der Anwendungsbereich (Kapitel 4.2.2), die Ermittlung sowie Zerlegung des Einkommens (Kapitel 4.2.3) sowie administrative Aspekte (Kapitel 4.2.4) angesprochen. Nicht näher eingegangen wird auf die Frage der Festlegung der Steuersätze, da sich die Kommission aus den in Kapitel 4.1 genannten Gründen gegen eine Harmonisierung in diesem Bereich ausgesprochen hat. Allerdings sei an dieser Stelle darauf verwiesen, dass in der Literatur teilweise zumindest eine Eingrenzung der Bandbreite der tariflichen Steuersätze durch Einführung eines Mindeststeuersatzes als notwendige Begleitmaßnahme zur Einführung einer GKKB angesehen wird. ${ }^{292}$

\subsubsection{Anwendungsbereich}

Nach den Vorschlägen der Arbeitsgruppe erstreckt sich der Anwendungsbereich der gemeinsamen Bemessungsgrundlage auf alle europäischen Unternehmen, die nach ihrem nationalen Recht der Körperschaftsteuer (,Corporation tax") unterliegen. ${ }^{293}$ Ebenfalls in den Anwendungsbereich der GKKB fallen Drittstaatenunternehmen, die eine vergleichbare Rechtsform aufweisen und die entweder über eine Betriebsstätte in einem Mitgliedsstaat der EU tätig sind oder deren rechtlicher Sitz zwar in einem Drittstaat liegt, der steuerliche Sitz sich aber in einem Mitgliedsstaat befindet. ${ }^{294}$ Ausgeschlossen von der Einbeziehung in die GKKB-Gruppe als Spitzeneinheit sind hingegen transparent besteuerte Unternehmen. Diese Begrenzung erfolgt insbesondere aus politischen Erwägungen, da der mit einer Einbeziehung transparent besteuerter Unternehmen verbundene Eingriff in die Einkommensbesteuerung natürlicher Personen vermieden werden soll. ${ }^{295}$ Eine ausschließ-

291 Vgl. so Czakert (2008), S. 434 mit dem Verweis auf den Inhalt eines Schreibens des deutschen und französischen Finanzministers an EU-Kommissar Bolkestein vom 26. Mai 2004.

292 Vgl. Spengel/Oestreicher (2009), S. 781; Spengel (2008), S. 46; Ruding (2005), S. 2-3; Sorenson (2002), S. 35.

293 Zum Anwendungsbereich der GKKB vgl. Arbeitsgruppe GKKB (2007), S. 7-10.

294 Eine Differenzierung zwischen EU-Betriebsstätten und EU-Tochterkapitalgesellschaften würde sowohl Steuerplanungsmöglichkeiten schaffen und einen möglich Verstoß gegen die Niederlassungsfreiheit darstellen. Vgl. Spengel (2008), S. 33.

295 Vgl. Spengel (2008), S. 34. 
liche Anwendung der GKKB auf grenzüberschreitend tätige Konzerne ist von der Arbeitsgruppe nicht vorgesehen. ${ }^{296}$

Die Inanspruchnahme der gemeinsamen Bemessungsgrundlage soll optional ausgestaltet werden ${ }^{297}$, auch wenn sich die Vertreter der meisten Mitgliedsstaaten in den Diskussionen der Arbeitsgruppe gegen eine Optionalität ausgesprochen haben. ${ }^{298}$ Gegen eine verpflichtende Einführung für alle Unternehmen sprechen nach Ansicht der Europäischen Kommission zum einen mögliche Nachteile für Unternehmen, die nicht grenzüberschreitend tätig sind. Gleichzeitig sichere eine optionale Einführung die Unterstützung von Seiten der Wirtschaft und trüge eher zu dem Ziel eines funktionierenden Binnenmarktes bei. ${ }^{299}$ Auf der anderen Seite ergeben sich aus dem Nebeneinander zweier Systeme zur Ermittlung der Körperschaftsteuerbemessungsgrundlage Nachteile. So widerspricht jedes Wahlrecht dem Grundsatz der Einfachheit der Besteuerung ${ }^{300}$ und kann zu administrativen Problemen $^{301}$ führen. Ferner eröffnet die Wahl zwischen zwei Steuersystemen erhebliche Gestaltungsspielräume für die internationale Steuerplanung. Zusätzliche Kosten und eine Diskriminierung national tätiger Konzerne wären die Folge. ${ }^{302}$ Zur Einschränkung von Gestaltungsspielräumen ist das Wahlrecht einheitlich für alle unter die Richtlinie fallenden Unternehmen einer steuerlichen Gruppe auszuüben (,All-in/all-out“). Zu einer steuerlichen Gruppe zusammengefasst werden alle Unternehmen, deren Stimmrechte sich zu mindestens 50 Prozent in der Verfügungsmacht eines gemeinsamen Anteilseigners befinden. Für die Ermittlung der relevanten Beteiligungshöhen werden unmittelbar und mittelbar gehaltene Stimmrechte zusammengerechnet, wobei mittelbar gehaltene Stimmrechte multiplikativ berücksichtigt werden und nur insoweit als über mindestens 50 Prozent der Stimmrechte an der vermittelnden Gesellschaft verfügt werden kann. ${ }^{303}$ Ebenfalls zu berücksichtigen sind mittelbar über Nicht-EU-Unternehmen gehaltene Stimmrechte. ${ }^{304}$ In zeitlicher Hinsicht ist die Ausübung der Option für fünf

296 Vgl. befurwortend Schön (2007), S. 429-430; anderer Ansicht Plasschaert (2002), S. 12.

297 Vgl. Arbeitsgruppe GKKB (2007), S. 7. Vgl. kritisch zum Optionsrecht Mintz (2002), S. 6 und 8.

298 Vgl. Czakert (2008), S. 446.

299 Vgl. Mors/Rautenstrauch (2008), S. 98. Vgl. hierzu auch Czakert (2008), S. 437.

300 Vgl. Wittkowski (2008), S. 276.

301 Vgl. Czakert (2008), S. 437. So müssten die Steuererklärungen sämtlicher Gesellschaften geändert werden, wenn im Rahmen einer Betriebsprüfung zur nationalen Gewinnermittlung gewechselt wird.

302 Vgl. Blumenberg (2008), S. 16; Spengel (2008), S. 43; Mintz (2004), S. 226; Sorenson (2004), S. 102.

$303 \mathrm{Vgl}$. Mors/Rautenstrauch (2008), S.101.

304 Würde die Zwischenschaltung einer Nicht-EU-Gesellschaft zu einem Zerfallen steuerlicher Gruppen führen, könnte diese als Gestaltung bei der Ausübung des Optionsrechts genutzt werden. Vgl. Mors/Rautenstrauch (2008), S. 100 Reinald Koch - 978-3-631-75683-6 
Jahre bindend und verlängert sich ohne entsprechenden Antrag automatisch um weitere drei Jahre. ${ }^{305}$

Bei Option für die GKKB kommt es neben der Anwendung der Vorschriften zur gemeinsamen Bemessungsgrundlage zwingend zu einer Konsolidierung innerhalb eines enger definierten Konsolidierungskreises. Zur Qualifikation für die Konsolidierung müssen die Stimmrechte an der Tochtergesellschaft zu mindestens 75 Prozent unmittelbar oder mittelbar durch die Muttergesellschaft gehalten werden. Für die Berücksichtigung mittelbar gehaltener Stimmrechte gelten die im Zusammenhang mit dem Optionsrecht genannten Grundsätze. ${ }^{306}$

Mit der Vorgabe einer Mindestbeteiligungsquote hat die Arbeitsgruppe einer Abgrenzung des Konsolidierungskreises nach rechtlichen Kriterien gegenüber einer Anwendung wirtschaftlicher Kriterien den Vorrang eingeräumt. Bei einer wirtschaftlichen Abgrenzung des Konsolidierungskreises würde ausschließlich oder neben dem Vorliegen von Beherrschung eine operationale und/oder wirtschaftliche Abhängigkeit zwischen den Gruppenunternehmen gefordert. ${ }^{307}$ Mögliche Indikatoren für das Vorliegen einer wirtschaftlichen Einheit sind dabei unter anderem das Vorliegen eines zentralen Managements, die Existenz von Skaleneffekten sowie das Vorliegen von konzerninternen Transaktionen in bedeutsamem Ausmaß ${ }^{308}$ Dabei besteht in der wissenschaftlichen Diskussion weitgehende Einigkeit darüber, dass die wirtschaftliche Einheit gleichzeitig theoretische Rechtfertigung und notwendige Voraussetzung für eine sinnvolle Anwendung der Formelaufteilung bildet. ${ }^{309}$ So sind gerade Skaleneffekte und Verbundeffekte für ein Versagen des Fremdvergleichsgrundsatzes ursächlich und erfordern aus diesem Grund die Anwendung eines alternativen Aufteilungsmechanismus. ${ }^{310}$ Umgekehrt kann eine indirekte Gewinnabgrenzung zu unsachgemäßen Ergebnissen führen, wenn die Gesellschaften in unterschiedlichen Wirtschaftsbereichen tätig sind. ${ }^{311}$

Trotz seiner theoretischen Rechtfertigung bestehen gute Gründe gegen eine Abgrenzung des Konsolidierungskreises anhand wirtschaftlicher Kriterien. ${ }^{312}$ Hierbei sind insbesondere praktische Argumente anzuführen, die sich aus der Subjektivität dieses Ansatzes ergeben. Legt man die Erfahrungen der US-amerikanischen Bundesstaaten zugrunde, wo von der wissenschaftlichen Literatur und der Rechtsprechung zahlreiche Ansätze zur Definition des „unitary business“

$305 \mathrm{Vgl}$. Arbeitsgruppe GKKB (2007), S. 6-7.

$306 \mathrm{Vgl}$. Arbeitsgruppe GKKB (2007), S. 26-29.

$307 \mathrm{Vgl}$. Agundez-Garcia (2006), S. 12. In den USA wird für eine wirtschaftlich abgegrenzte Gruppe der Begriff des „Unitary business“ verwendet. Vgl. zur Abgrenzung eines „Unitary business" Oestreicher (2000), S. 136-140.

$308 \mathrm{Vgl}$. Agundez-Garcia (2006), S. 12.

309 Vgl. Spengel (2008), S. 32; Weiner (2005), S. 27-28; Sorenson (2004), S. 94; McLure (2004), S. 55; Hellerstein/McLure (2004), S. 204.

$310 \mathrm{Vgl}$. Agundez-Garcia (2006), S. 13-14.

311 Vgl. McLure (2004), S. 54.

312 Vgl. so Weiner (2005), S. 54; Hellerstein/McLure (2004) S 203-206 
entwickelt wurden ${ }^{313}$, so erscheint es fraglich, ob in Europa eine einheitliche Definition und Anwendung sichergestellt werden kann. ${ }^{314}$ Als weitere negative Konsequenz derart schwierig zu objektivierender Kriterien sind hohe Dokumentationskosten und Überwachungskosten zu erwarten. ${ }^{315}$

Auch bei der Festlegung der Beteiligungshöhe dürfte die Kommission insbesondere politische und praktische Überlegungen gegeneinander abgewogen haben. Mit der gewählten Beteiligungshöhe von 75 Prozent orientiert sich der Vorschlag der Kommission zunächst in etwa am Mittel der Beteiligungshöhen, welche von den Mitgliedsstaaten im Rahmen der nationalen Gruppenbesteuerungssysteme gefordert werden ${ }^{316}$, was eine politische Umsetzung erleichtern könnte. Für eine hohe Beteiligungsquote spricht dabei in praktischer Hinsicht insbesondere, dass die Behandlung von Minderheitsgesellschaftern an Bedeutung verliert. ${ }^{317}$ Auf der anderen Seite erleichtert eine hohe Beteiligungsquote eine gezielte Beeinflussung der Gruppenzugehörigkeit als Mittel der Steuerplanung, welches ohnehin ein grundsätzliches Problem der rechtlichen Konzernabgrenzung darstellt. ${ }^{318}$ Gleichzeitig leben bei einer hohen Mindestbeteiligung in stärkerem Ausmaß die Probleme der geltenden Besteuerung hinsichtlich der Verlagerung von Bemessungsgrundlage wieder auf. So erhöht sich mit zunehmender Mindestbeteiligungsquote die Anzahl der beherrschten Unternehmen, die nicht zum Konsolidierungskreis zählen. ${ }^{319}$

\subsubsection{Einkommensermittlung}

\subsubsection{Gemeinsame steuerliche Gewinnermittlung}

Aufgrund der Verwendung der IFRS als harmonisiertes Regelungswerk für die Konzernabschlüsse kapitalmarktorientierter Unternehmen in Europa war der Rückgriff auf die IFRS bei der Ausgestaltung einer einheitlichen steuerlichen Bemessungsgrundlage von Beginn an naheliegend und dem alternativen Ansatz

313 Vgl. für einen Überblick über verschiedene Abgrenzungsverfahren Russo (2005), S. 10-11; Weiner (2005), S. 28-32; Oestreicher (2000), S. 136-140.

314 Vgl. Spengel (2008), S. 33; Agundez-Garcia (2006), S. 14.

315 Vgl. Spengel (2008), S. 32-33; Russo (2005), S. 11. Spengel sieht einen möglichen Kompromiss in Bright-line-Tests, wie diese beispielsweise von McLure (1984) und Hellerstein (1982) vorgeschlagen werden.

316 Vgl. so auch Sievert (2006), S. 236.

317 Vgl. Agundez-Garcia (2006), S. 14. Schön spricht sich aus diesem Grund bei optionaler Einführung für eine noch höhere Mindestbeteiligungsquote aus. Vgl. Schön (2007), S. 431.

318 Vgl. Spengel (2008), S. 32.

$319 \mathrm{Vgl}$. Agundez-Garcia (2006), S. 12. Dieses wird auch als weiteres Argument gegen eine wirtschaftliche Abgrenzung des Konsolidierungskreises vorgebracht, die nach herrschender

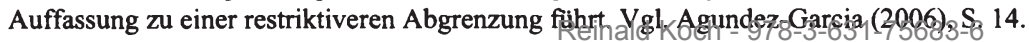


des Ausgehens von einem nationalen Regelungssystem vorzuziehen. ${ }^{320}$ Dabei wurden mit Blick auf die Art der Anknüpfung an die IFRS mit der formellen Maßgeblichkeit sowie der Entwicklung eines eigenständigen steuerlichen Gewinnermittlungsrechts mit den IFRS als inhaltlichem Ausgangspunkt alternative Konzepte angedacht. ${ }^{321}$ Eine vollständige formelle Maßgeblichkeit der IFRS, die zu Beginn der Arbeiten der Kommission diskutiert wurde, hätte den Vorteil, dass analog zum deutschen Einkommensteuergesetz - die GKKB-Richtlinie lediglich die Abweichungen von den IFRS beinhalten müsste. Diese Option wurde allerdings nicht weiterverfolgt, da zahlreiche IFRS-Vorschriften nicht vereinbar mit grundsätzlichen Besteuerungsprinzipien sind (z.B. Marktbewertung finanzieller Vermögenswerte $^{322}$ ). Ferner soll die Festlegung der steuerlichen Bemessungsgrundlage nicht in die Verantwortung eines privaten Standardsetters fallen. ${ }^{323}$ Schließlich ist in vielen Mitgliedsstaaten die Aufstellung eines befreienden IFRSEinzelabschlusses nicht möglich, weshalb die Maßgeblichkeit der IFRS nicht eine weitergehende Senkung der Befolgungskosten bewirken würde. ${ }^{324}$

Die Verwendung der IFRS als Ausgangspunkt für die Entwicklung eines steuerlichen Gewinnermittlungssystems findet hingegen weitgehende Unterstützung in der Politik und wissenschaftlichen Literatur. ${ }^{325}$ Dabei wurden für die Diskussion einzelner Aspekte des Gewinnermittlungssystems in der Arbeitsgruppe die Regelungen der IFRS als Ausgangspunkt verwendet und vor dem Hintergrund der bestehenden steuerlichen Vorschriften in den Mitgliedsstaaten sowie allgemeiner Prinzipien der steuerlichen Gewinnermittlung erörtert. ${ }^{326}$ Bei weitgehender Übereinstimmung der Regelungen in den Mitgliedsstaaten konnte die Regelung aus den IFRS für die Definition der einheitlichen Bemessungsgrundlage übernommen werden, bei größeren Abweichungen wurde eine Lösung prinzipiengestützt gesucht. ${ }^{327}$ Aufgrund weitgehender Übereinstimmung mit den Regelungen in den Mitgliedsstaaten, könnten die IFRS eine geeignete Grundlage beim Ansatz von Vermögensgegenständen und Schulden, bei der Ermittlung von Anschaffungs- und Herstellungskosten, beim Niederstwerttest sowie der Behandlung von

320 Vgl. Spengel (2008), S. 30-31; Czakert (2008), S. 436; Czakert (2006), S. 563; Schreiber (2004), S. 222.

$321 \mathrm{Vgl}$. Mors/Rautenstrauch (2008), S. 98.

322 Vgl. Oestreicher (2007), S. 582; Oestreicher/Spengel (2001), S. 893.

323 Vgl. Mors/Rautenstrauch (2008), S. 98; Spengel (2008), S. 31

324 Vgl. Mors/Rautenstrauch (2008), S. 98.

325 Vgl. Spengel (2008), S. 2 und 31; Oestreicher (2007), S. 582; Schön (2004), S. 432-440. Für Vorbehalte gegenüber einer derartigen Lösung vgl. die Nachweise bei Spengel/Oestreicher (2009).

$326 \mathrm{Vgl}$. Czakert (2008), S. 436. Hierbei wurden im Einzelnen die Prinzipien der Effizienz, Transparenz, Bestimmtheit und Einfachheit berücksichtigt. Des Weiteren soll die GKKB einer Besteuerung nach dem Leistungsfähigkeitsprinzip sowie dem Realisationsprinzip folgen. Vgl. Czakert (2008), S. 437.

327 Vgl. Czakert (2008), S. 436. 
drohenden Verlusten aus schwebenden Geschäften bilden. Eigene steuerliche Regelungen müssten insbesondere bei der Rücklagenbilanzierung und Abschreibungen entwickelt werden, da in diesen Bereichen deutliche Unterschiede zwischen den Mitgliedsstaaten bestehen. ${ }^{328}$ Ferner müssten einheitliche Regelungen zur steuerlichen Behandlung von Verlusten, Dividenden und Veräußerungsgewinnen entwickelt werden, da diese Bereiche in einem handelsrechtlichen Gewinnermittlungssystem nicht geregelt werden. ${ }^{329}$

\subsubsection{Konsolidierung}

Die wesentlichen Vorteile einer GKKB gegenüber dem gegenwärtigen Besteuerungssystem ergeben sich aus der Konsolidierung bei der Ermittlung des zu versteuernden Einkommens. ${ }^{330}$ Diese führt für die Unternehmen zu einer Senkung ihrer Befolgungskosten insbesondere aufgrund der Reduzierung des Aufwands bei der Ermittlung steuerlicher Verrechnungspreise und gleichzeitig zu einem systemimmanenten Gewinn- und Verlustausgleich im Konzern. ${ }^{331}$ Die wesentlichen Fragestellungen betreffen dabei die Abgrenzung des zu konsolidierenden und aufzuteilenden Einkommens sowie den Umfang und die Methodik der Konsolidierung.

Bei der Abgrenzung des zu konsolidierenden Einkommens steht insbesondere die Einbeziehung von Drittstaateneinkünften sowie die Unterscheidung von betrieblichen Einkommensbestandteilen (,business income") und nicht-betrieblichen Einkommensbestandteilen (,non-business income“) in Frage. Mit Blick auf die Zielsetzung einer verursachungsgerechten Erfolgsermittlung ist es sachgemäß, die Konsolidierung und Formelaufteilung nur auf die Einkommensteile zu erstrecken, deren Ursprung nicht eindeutig einem Gruppenunternehmen zugeordnet werden kann. Ist hingegen die Einkommensquelle auf direktem Wege unzweifelhaft einem Staat zuzuordnen, besteht keine Notwendigkeit für eine indirekte Gewinnabgrenzung. ${ }^{332}$ In den USA und Kanada wird für diesen Zweck zwischen betrieblichen Einkommensbestandteilen, welche in die Formelaufteilung einbezogen werden, und nicht-betrieblichen Einkommensbestandteilen, welche der empfangenden Gruppengesellschaft direkt zugeordnet werden, differenziert. ${ }^{333}$ Aus praktischer Sicht ist eine derartige Unterscheidung mit Problemen verbunden, da eine Unterscheidung zwischen unterschiedlichen Einkunftsarten gestaltungsanfäl-

328 Vgl. Spengel (2008), S. 31; Oestreicher/Spengel (2007), S. 439-446. Zum aktuellen Stand der Diskussionen der Arbeitsgruppe vgl. Arbeitsgruppe GKKB (2007), S. 10-26.

329 Vgl. Spengel (2008), S. 31.

330 Die Fragen der Konsolidierung und Verlustverrechnung sind ausfuhrlich besprochen bei Oestreicher (2008); Herzig (2008); Moreno Gonzalez/Sanz Diaz-Palacios (2008).

331 Vgl. Czakert (2008), S. 446.

332 Vgl. Spengel (2008), S. 38; Agundez-Garcia (2006), S. 17; Hellerstein/McLure (2004), S. 202.

333 Vgl. Spengel (2008), S. 38; Weiner (2005), S. 34; Hellerstein/Mcchjure(2004) So 202 
lig und streitanfällig ${ }^{34}$ ist, gleichzeitig eine Zuordnung von Ausgaben zu den verschiedenen Einkunftsarten erfordert und eine Gewinnverlagerung in Niedrigsteuerländer über Gestaltung der direkt zugeordneten Ergebnisbestandteile unverändert ermöglicht. ${ }^{335}$ Als weitere Nachteile einer direkten Zuordnung einzelner Einkommensbestandteile nennen Agundez-Garcia und Russo die Beschränkung der konzerninternen Verlustverrechnung auf konsolidierte Einkommensbestandteile. ${ }^{336}$ Hellerstein/McLure verweisen zudem auf einen zu erwartenden Wettbewerb zwischen den Steuerhoheiten innerhalb des Binnenmarktes bezüglich der direkt zuzuordnenden Einkommensbestandteile sowie die geringe praktische Relevanz der nicht- betrieblichen Einkommensbestandteile. ${ }^{337}$ Fox/Murray/Luna schließlich geben mögliche Entscheidungsverzerrungen sowie zusätzliche Befolgungskosten zu bedenken, welche sich aus dem Nebeneinander zweier Ansätze zur Einkommensabgrenzung ergeben können. ${ }^{338}$ Aufgrund dieser weitreichenden Bedenken in der Literatur hat sich auch die Arbeitsgruppe gegen eine derartige Unterscheidung ausgesprochen. ${ }^{339}$

Zur Herbeiführung einer Konsolidierung könnte ein IFRS-Konzernabschluss als Ausgangspunkt der steuerlichen Gewinnermittlung des Konzerns gewählt werden. Dieser Ansatz wurde ursprünglich von der Europäischen Kommission in Erwägung gezogen ${ }^{340}$, inzwischen allerdings verworfen, da weder die räumliche und persönliche Abgrenzung des Konsolidierungskreises noch die Konsolidierungsmethoden den steuerlichen Anforderungen entsprechen. ${ }^{341}$ Ferner wurde eine direkte Anknüpfung an einen IFRS-Abschluss oder die IFRS-Regelungen auch für die Gewinnermittlung ausgeschlossen. ${ }^{342}$ Somit verbleibt lediglich der Rückgriff auf originär steuerliche Konsolidierungsregelungen. ${ }^{343}$

Unmittelbare Folge einer steuerlichen Konsolidierung ist die Verrechnung von Gewinnen und Verlusten im Konzern. Diese kann ergänzt werden um eine Eliminierung von Zwischenergebnissen aus konzerninternen Transaktionen sowie eine Kapital- und Schuldenkonsolidierung. Die bisherigen Verlautbarungen der

$334 \mathrm{Vgl}$. zu entsprechenden Streitfallen in den USA Hellerstein (2001); Hellerstein/Hellerstein (1998).

335 Vgl. Spengel (2008), S. 38; Agundez-Garcia (2006), 17-19; Russo (2005), S. 13; Hellerstein/McLure (2004), S. 203.

$336 \mathrm{Vgl}$. Agundez-Garcia (2006), S. 18-19; Russo (2005), S. 13.

$337 \mathrm{Vgl}$. Hellerstein/McLure (2004), S. 203.

$338 \mathrm{Vgl}$. Fox/Murray/Luna (2005), S. 151-152.

$339 \mathrm{Vgl}$. Arbeitsgruppe GKKB (2007a), S. 7.

$340 \mathrm{Vgl}$. Kommission der Europäischen Gemeinschaften (2003), S. 22; so auch Spengel (2008), S. 35 .

341 Vgl. Oestreicher (2008), S. 521-522; Spengel (2008), S. 35.

342 Vgl. Kommission der Europäischen Gemeinschaften (2006b), S. 7-8.

343 Vgl. Spengel (2008), S. 35; Kommission der Europäischen Gemeinschaften (2003) S. 23. 
Arbeitsgruppe beschränken sich allerdings auf die Regelungen zum konzerninternen Verlustausgleich und zur Zwischenergebniseliminierung. ${ }^{344}$

Bezüglich der Verlustverrechnung wurden ursprünglich zwei Ansätze von der Arbeitsgruppe diskutiert. ${ }^{345}$ Zum einen könnte ein Gesamtverlust des Konzerns im Jahr der Verlustentstehung auf die Einzelgesellschaften aufgeteilt und auf deren Ebene intertemporal verrechnet werden. Dieser Ansatz gewährleistet eine symmetrische Behandlung von Gewinnen und Verlusten, da in beiden Fällen die Verhältnisse des Geschäftsjahrs der Ergebnisentstehung maßgeblich für die Aufteilung auf die Einzelgesellschaften sind. ${ }^{346}$ Gleichzeitig verlangt dieser Ansatz allerdings Regelungen zur Vermeidung einer doppelten Verlustnutzung auf Konzernebene und Ebene der Einzelgesellschaft sowie eine Harmonisierung der Vorschriften zur intertemporalen Verlustverrechnung und beinhaltet eine höhere Gefahr, dass Verluste in einem Mitgliedsstaat ungenutzt bleiben. ${ }^{347}$

Der von der Arbeitsgruppe inzwischen bevorzugte zweite Ansatz sieht demgegenüber einen Vortrag von Gesamtverlusten auf Konzernebene vor. ${ }^{348}$ Dieses führt im Ergebnis dazu, dass Verluste nicht entsprechend der Verteilung der Formelfaktoren im Jahr der Verlustentstehung sondern im Jahr der Verlustverrechnung auf Konzernebene aufgeteilt werden. ${ }^{349}$ Für die Verrechnung der Gruppenverluste schlägt die Arbeitsgruppe einen zeitlich und betragsmäßig unbegrenzten Verlustvortrag vor. Verlustvorträge, die bei Eintritt in die steuerliche Gruppe bestehen, sollen hingegen auf Ebene der Gruppengesellschaft verrechnet werden. ${ }^{350}$ Eine aus der Gruppe austretende Gesellschaft soll nach den Vorstellungen der Arbeitsgruppe keinen Anteil an auf Gruppenebene bestehenden Verlustvorträgen zugewiesen bekommen. Lediglich bei Auflösung der Gruppe ist eine Aufteilung der Verlustvorträge vorgesehen. ${ }^{351}$

Bezüglich der Methodik einer Zwischenergebniseliminierung hat sich die Arbeitsgruppe bisher nicht auf einen Ansatz festgelegt. Vielmehr werden in der Arbeitsgruppe zwei verschiedene Ansätze diskutiert. Eine erste Option besteht

344 Vgl. Arbeitsgruppe GKKB (2007), S. 26-35; so auch Herzig (2008), S. 559.

$345 \mathrm{Vgl}$. Arbeitsgruppe GKKB (2006), S. 13.

346 Vgl. Spengel (2008), S. 36.

347 Vgl. Spengel (2008), S. 36; Arbeitsgruppe GKKB (2007), S. 32; Arbeitsgruppe GKKB (2006), S. 13. Zu ungenutzten Verlusten kann es bei diesem Ansatz kommen, wenn sich die Aufteilung in Gewinn- und Verlustjahren unterscheidet.

$348 \mathrm{Vgl}$. Arbeitsgruppe GKKB (2007), S. 32.

349 Vgl. Spengel (2008), S. 36.

$350 \mathrm{Vgl}$. Arbeitsgruppe GKKB (2007), S. 31. Vgl. befürwortend zu diesem Vorschlag Oestreicher (2008), S. 537; Oestreicher/Scheffler/Spengel et al. (2008), S. 338.

351 Vgl. Arbeitsgruppe GKKB (2007), S. 32. Dieser Vorschlag der Arbeitsgruppe wurde von Seiten einiger Mitgliedsstaaten und in der Literatur kritisiert, da er eine Ungleichbehandlung des Austritts einer einzelnen Gesellschaft und der Auflösung der Gruppe vorsieht. Vgl. so die Anmerkungen in Arbeitsgruppe GKKB (2007), S. 32; Moreno Gonzalez/Sanz Diaz-Palacios (2008), S. 463. 
darin, konzerninterne Transaktionen für steuerliche Zwecke vollständig unberücksichtigt zu lassen mit der Folge, dass eine Dokumentation der Ergebnisbeiträge aus diesen Transaktionen unterbleibt. ${ }^{352}$ Bei der zweiten Option werden die Erfolgsbeiträge im Rahmen der getrennten Gewinnermittlung des liefernden und erwerbenden Unternehmens erfasst und mit dem Verrechnungspreis bewertet. Diese Zwischenergebnisse werden allerdings bis zur Realisation aus Konzernsicht $^{353}$ im Rahmen der Konsolidierung eliminiert. ${ }^{354}$ Dieser Ansatz findet zum Beispiel in Frankreich und den USA bei der nationalen Gruppenbesteuerung Berücksichtigung. ${ }^{355}$ Eine dritte Option, die bisher von der Arbeitsgruppe nicht in Erwägung gezogen wurde, sieht ebenfalls eine Bewertung der Transaktion im Rahmen der getrennten Gewinnermittlung der beiden betroffenen Unternehmen zum Verrechnungspreis vor. Im Unterschied zur zweiten Option würde eine Neutralisation der Ergebnisbeiträge allerdings durch Bildung eines Ausgleichspostens in der Einzelbilanz des verkaufenden Unternehmens herbeigeführt werden, der bei Realisation der Zwischenergebnisse aus Konzernsicht erfolgswirksam aufzulösen wäre. ${ }^{356}$

\subsubsection{Aufteilungsmechanismus}

\subsection{Alternative Ansätze}

Die Zerlegung des konsolidierten Einkommens auf die einzelnen Gruppengesellschaften ist notwendiger Bestandteil einer GKKB, um eine verursachungsgerechte Aufteilung des Besteuerungssubstrats zwischen den beteiligten Steuerhoheiten zu gewährleisten. ${ }^{357}$ Der mit der Formelzerlegung verbundene Übergang von einer

$352 \mathrm{Vgl}$. Arbeitsgruppe GKKB (2007), S. 33. Dieser Ansatz zeichnet sich durch seine Einfachheit aus, fuhrt allerdings zu Problemen, wenn fremdvergleichskonforme Daten zu einem späteren Zeitpunkt benötigt werden, wie dieses zum Beispiel bei Verlassen der Gruppe der Fall ist. Vgl. Spengel (2008), S. 37; Arbeitsgruppe GKKB (2006a), S. 9.

353 Eine Realisation aus Konzemsicht erfolgt durch Abschreibung, Verkauf außerhalb des Konzerns oder wenn das verkaufende oder erwerbende Unternehmen die steuerliche Gruppe verlässt. Vgl. Oestreicher (2008), S. 527-528.

354 Vgl. Oestreicher (2008), S. 539; Spengel (2008), S. 37; Arbeitsgruppe GKKB (2007), S. 34. Für diesen Ansatz spricht insbesondere, dass im Fall des Gruppenaustritts Informationen für eine fremdvergleichskonforme Bewertung verfügbar sind. Ferner bleibt der Einzelabschluss von der Zwischenergebniseliminierung unberuhrt und kann demzufolge weiterhin zur Bemessung der Ansprulche von Minderheitsgesellschaftern herangezogen werden. Nachteilig ist der höhere Arbeitsaufwand, wobei auch berücksichtigt werden muss, dass Anpassungen nur erforderlich sind, soweit sich die Wirtschaftsgüter zum Periodenende noch in Gruppenbesitz befinden. Vgl. zu den Vor- und Nachteilen Spengel (2008), S. 37; Oestreicher (2008), S. 527 und 539-540.

355 Vgl. Spengel (2008), S. 37.

356 Vgl. Spengel (2008), S. 37.

357 Auch die Europäische Kommission stellt heraus, dass die Formelaufteilung notwendige Kon-

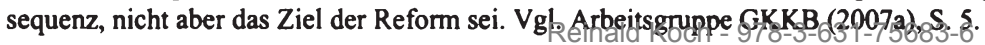


direkten zu einer indirekten Gewinnaufteilung ${ }^{358}$ bildet neben der Harmonisierung der Gewinnermittlungsvorschriften, der Einführung einer grenzüberschreitenden konzerninternen Verlustverrechnung und einer Eliminierung von Zwischenergebnissen aus konzerninternen Lieferungs- und Leistungsbeziehungen die vierte wesentliche Änderung, die die Einführung einer GKKB mit sich bringen würde. Auch aufgrund ihrer zentralen Bedeutung für die Aufkommens- und Anreizwirkungen einer $\mathrm{GKKB}^{359}$ zählt die Frage der Zusammensetzung der Aufteilungsformel $\mathrm{zu}$ den am intensivsten in Zusammenhang mit diesem Konzept diskutierten Fragestellungen. Unstrittig ist dabei lediglich, dass eine einheitliche Formeldefinition gegenüber einer dezentralen Festlegung der Aufteilungsformel deutliche Vorteile aufweist. ${ }^{360}$ Auch empirische Untersuchungen für die USA deuten auf negative externe Effekte ${ }^{361}$ und Wohlstandseinbußen ${ }^{362}$ einer uneinheitlichen Formeldefinition hin. ${ }^{363}$ Aus Sicht der Verwaltung wird gefordert, dass der Aufteilungsmechanismus einfach anzuwenden sowie schwer durch die Steuerzahler manipulierbar sein muss und zu einer gerechten Aufteilung des Steuersubstrats führt. ${ }^{364}$ Aufkommensneutralität gegenüber dem geltenden Steuerrecht ist hingegen nach Auffassung der Kommission keine explizite Anforderung an die Ausgestaltung der Aufteilungsformel. Hierbei darf allerdings die Bedeutung von Aufkommensänderungen für die Zustimmung der Mitgliedsstaaten und damit die Umsetzbarkeit einer GKKB nicht unberücksichtigt bleiben. ${ }^{365}$ Ökonomen stellen hingegen die verursachungsgerechte Erfolgslokalisation sowie mögliche Verhaltenswirkungen ins Zentrum ihrer Betrachtungen. ${ }^{366}$

Insgesamt wurden von der Arbeitsgruppe und in der Literatur ursprünglich drei verschiedene Ansätze zur Definition der Aufteilungsformel diskutiert:

(1) Aufteilung nach makroökonomischen oder industrieweiten Faktoren,

(2) Aufteilung anhand mikroökonomischer Unternehmensfaktoren (traditionelle Formelzerlegung) oder

358 Welche dieser beiden Methoden zur Gewinnabgrenzung vorzugswürdig ist, wird in der Literatur kontrovers diskutiert. Vgl. stellvertretend für diesen Theorienstreit Oestreicher (2005a) und Wassermeyer (2005).

$359 \mathrm{Vgl}$. Sorenson (2004), S. 95.

360 Vgl. Spengel (2008), S. 43; Russo (2005), S. 24; Mintz (1999), S. 409. Agundez-Garcia (2006), S. 44 weist darauf hin, dass die Komplexität der Formelzerlegung in den USA insbesondere auf die uneinheitliche Anwendung zurückzufüren ist.

$361 \mathrm{Vgl}$. Goolsbee/Maydew (2000).

$362 \mathrm{Vgl}$. Anand/Sansing (2000).

363 Niedrige Steuersätze auf Bundesstaatenebene und die Abziehbarkeit dieser Steuem von der nationalen Körperschaftsteuer mindern allerdings die negativen Konsequenzen der uneinheitlichen Formelfestlegung in den USA. Vgl. Mintz (1999), S. 408-409.

364 Vgl. Czakert (2008), S. 447; Kommission der Europäischen Gemeinschaften (2003), S. 24.

365 Vgl. Kommission der Europäischen Gemeinschaften (2003), S. 24; Arbeitsgruppe GKKB (2007a), S. 6. So auch Czakert (2008), S. 447.

366 Vgl. stellvertretend McLure (2000); Musgrave (1984)ald Koch - 978-3-631-75683-6 
(3) Aufteilung entsprechend der Wertschöpfung.

\subsection{Makroökonomische oder industrieweite Faktoren}

Bei einer Aufteilung nach makroökonomischen Faktoren ermittelt sich der Anteil der Mitgliedsstaaten an der Gesamtbemessungsgrundlage eines Konzerns anhand gesamtwirtschaftlicher Daten, wie beispielsweise dem Bruttoinlandsprodukt oder der aggregierten Umsatzsteuerbemessungsgrundlage. ${ }^{367}$ Ferner könnte eine Formelzerlegung auf Basis industrieweiter Faktoren in Erwägung gezogen werden. ${ }^{368}$ Bei beiden Ansätzen können sämtliche Mitgliedsstaaten in die Aufteilung einbezogen werden oder eine Beschränkung auf die Länder erfolgen, in denen die jeweilige Gruppe wirtschaftlich tätig ist. ${ }^{369}$

Ein wesentlicher Vorteil einer Aufteilung anhand makroökonomischer Faktoren liegt in der administrativen Einfachheit, da die erforderlichen Größen bereits für andere Zwecke erhoben werden und somit zusätzliche Befolgungskosten nur in geringem Ausmaß entstehen. ${ }^{370}$ Des Weiteren zeichnet sich dieser Ansatz durch seine Wettbewerbsneutralität und Effizienz aus. Erstreckt sich eine Aufteilung anhand makroökonomischer Größen auf sämtliche Mitgliedsstaaten (und nicht nur die, in denen die jeweilige Gruppe über einen steuerlichen Nexus verfügt), gleichen sich die Kapitalkosten aller europäischen Konzerne an. ${ }^{371}$ Bei einer Aufteilung nach industrieweiten Größen gilt dieses zumindest branchenintern. Gleichzeitig können die Größen der Aufteilungsformel nicht durch die Unternehmen beeinflusst werden, so dass eine steuerbedingte Reallokation betrieblicher Funktionen nicht zu erwarten ist. ${ }^{372}$ Diese Wirkung ist mit Blick auf den Steuerwettbewerb allerdings nicht unproblematisch, da ein ,race to the top“ bei den Körperschaftsteuersätzen zu erwarten wäre. ${ }^{373}$ Gleichwohl gehen auch von dieser Form der Ergebnisaufteilung Gestaltungsanreize für Unternehmen aus. So könnte bei einer Beschränkung der Aufteilung auf Länder mit steuerlichem Nexus die Begründung einer Niederlassung in Niedrigsteuerländern begünstigend wirken. ${ }^{374}$ Insbesondere aus Gerechtigkeitsaspekten findet dieser Ansatz allerdings in der wissenschaftlichen Literatur keine Unterstützung. ${ }^{375}$ Der fehlende Bezug von Steuerbelastung zur Verteilung der Wertschöpfung im Konzern benachteiligt

367 Vgl. Agundez-Garcia (2006), S. 39.

368 Vgl. Russo (2005), S. 23; Sorenson (2004), S. 96; Mintz (1999), S. 407.

$369 \mathrm{Vgl}$. Agundez-Garcia (2006), S. 39-40.

$370 \mathrm{Vgl}$. Russo (2005), S. 23.

$371 \mathrm{Vgl}$. Sorenson (2004), S. 96.

372 Bei einer Aufteilung anhand industrieweiter Größen gilt dieses unter der Annahme, dass die Größe der einzelnen Unternehmen/Konzerne nicht für eine Beeinflussung der Aufteilungsfaktoren ausreicht. Vgl. Sorenson (2004), S. 96.

$373 \mathrm{Vgl}$. Agundez-Garcia (2006), S.42; Sorenson (2004), S. 96.

$374 \mathrm{Vgl}$. Agundez-Garcia (2006), S.42.

375 Vgl. stellvertretend Schön (2007), S. 440 und McLure (2005), S 35 
Konzerne mit überdurchschnittlich hoher Präsenz in Niedrigsteuerländern. ${ }^{376}$ Auch wenn Agundez-Garica darauf hinweist, dass diese Ungerechtigkeit insbesondere die Steuerbelastung des einzelnen Konzerns und weniger das Steueraufkommen der Mitgliedsstaaten betreffen sollte, da verzerrende Wirkungen auf das Steueraufkommen durch die Vielzahl an Konzernen abgemildert wird ${ }^{377}$, wird diese Form der Aufteilung von der Kommission aus den genannten Gründen nicht in Erwägung gezogen.

\subsection{Traditionelle Formelzerlegung}

Diesem Ansatz liegt die Zielsetzung einer Zuordnung der Einkünfte zur Einkommensquelle zugrunde, also einer Aufteilung anhand der einkommensgenerierenden Faktoren. Diese Form der Gewinnabgrenzung hat eine lange Tradition bei der Besteuerung US-amerikanischer Gruppen auf Bundesstaatenebene und kanadischer Gruppen bei der Besteuerung durch die Provinzen. ${ }^{378}$

Bei der Festlegung der einkommensgenerierenden Faktoren unterscheidet Musgrave einen angebotsorientierten Ansatz (,supply approach“) und einen angebots-/nachfrageorientierten Ansatz (,supply/demand approach“). ${ }^{379}$ Dem angebotsorientierten Ansatz liegt das Verständnis zugrunde, dass die Erzielung von Einkommen auf den Einsatz von Produktionsfaktoren zurückzuführen ist, wodurch die Einbeziehung eines Vermögensfaktors und eines Arbeitsfaktors in die Aufteilungsformel grundsätzlich gerechtfertigt wird. ${ }^{380}$ Aufgrund des traditionellen Verständnisses der Körperschaftsteuer als eine Steuer auf die Verzinsung des eingesetzten Kapitals wird vielfach aus theoretischen Erwägungen heraus aber eine Aufteilung ausschließlich anhand des Vermögensfaktors befürwortet. ${ }^{381}$

Für eine Einbeziehung des Arbeitsfaktors spricht insbesondere die administrative Einfachheit. ${ }^{382}$ So sind die Schwierigkeiten bei der räumlichen Zuordnung und der Bewertung dieses Faktors, welche alternativ als Mitarbeiteranzahl oder als Lohnsumme erfolgen kann $^{383}$, gering. Geringe Mobilität dieses Faktors lässt ferner - verglichen mit einer Aufteilung anhand des Vermögens - geringere Ver-

$376 \mathrm{Vgl}$. Agundez-Garcia (2006), S. 41. Die gleiche Argumentation trifft mit Abstrichen auch auf industrieweite Aufteilungsfaktoren zu. Vgl. Mintz (1999), S. 407. Russo (2005), S. 24 schlägt eine Kombination mikro- und makroökonomischer Faktoren vor.

377 Vgl. Agundez-Garcia (2006), S. 41.

378 Vgl. Spengel (2008), S. 27; Agundez-Garcia (2006), S. 44.

379 Vgl. Musgrave (1984), S. 234.

380 Vgl. Spengel (2008), S. 42.

381 Vgl. Schön (2007), S. 441; Agundez-Garcia (2006), S. 49; Sorenson (2004), S. 95-96; Hellerstein/McLure (2004), S. 210; Mintz (1999), S. 408.

382 Vgl. Agundez-Garcia (2006), S. 47; Musgrave (1984), S. 238.

383 Vgl. kritisch zu einer Definition des Arbeitsfaktors als Mitarbeiteranzahl Oestreicher (2000), S. 185. 
zerrungseffekte erwarten. ${ }^{384}$ Schließlich kann für eine Berücksichtigung des Arbeitsfaktors auch angeführt werden, dass dieser als Approximation des Wertes der nur schwer $\mathrm{zu}$ erfassenden immateriellen Wirtschaftsgüter angesehen werden kann. ${ }^{385}$ Agundez-Garcia weist als möglichen Nachteil auf das Problem einer systematischen Fehlallokation durch Verwendung des Arbeitsfaktors bei bestehenden Abweichungen in der Arbeitsproduktivität hin, geht allerdings selbst davon aus, dass derartige Unterschiede mit der zunehmenden Integration im Binnenmarkt abnehmen. ${ }^{386}$

Die Verwendung des Vermögensfaktors wirft in der praktischen Umsetzung insbesondere bei der Bewertung und der Einbeziehung des immateriellen Anlagevermögens Probleme auf. Für die Bewertung des Aufteilungsfaktors Vermögen unterscheidet Musgrave einen bestandsorientierten Ansatz (,stock approach") und einen stromgrößenorientierten Ansatz (,flow approach") ${ }^{387}$ Wird der bestandsorientierte Ansatz zugrundegelegt, ist dabei eine Bewertung des Vermögens zum Marktwert sachgerecht. Eine stromgrößenorientierte Bewertung sollte zu den Kapitalnutzungskosten erfolgen, welche sich zusammensetzen aus den Zinskosten und der ökonomischen Abschreibung. ${ }^{388}$ Der letztgenannte Bewertungsansatz wird dabei insgesamt als zielführender eingestuft, da dieser keine systematische Übergewichtung langlebiger Wirtschaftsgüter bewirkt und es für die übrigen Formelfaktoren an einer bestandorientierten Definition mangelt. ${ }^{389}$ Beide theoretisch zutreffenden Bewertungsansätze zeichnen sich allerdings durch ein hohes $\mathrm{Ma} \beta$ an Subjektivität und Komplexität aus, weshalb als praktikabel nur eine Bewertung zu historischen Anschaffungs- oder Herstellungskosten oder um Abschreibungen verminderte historische Kosten angesehen wird. ${ }^{390}$

Daneben ergeben sich insbesondere Probleme bei der Erfassung, Lokalisation und Bewertung immaterieller Wirtschaftsgüter. Diesen Problemen kommt aufgrund der hohen Bedeutung dieser Vermögenswerte bei der Einkommenserzielung moderner Unternehmen besondere Relevanz zu. ${ }^{391}$ Die Erfassung immaterieller Wirtschaftsgüter führt zu Problemen, wenn für selbsterstellte immaterielle Anlagen in der Handels- und Steuerbilanz ein Aktivierungsverbot besteht. Die Lokalisation immaterieller Wirtschaftsgüter ist schwierig, da diese - soweit sie auf Forschungsaktivitäten zurückzuführen sind - häufig aus dem $\mathrm{Zu}$ -

$384 \mathrm{Vgl}$. Agundez-Garcia (2006), S. 52. Dennoch wurden in der Empirie entsprechende Verhaltensanpassungen auch furr diesen Aufteilungsfaktor nachgewiesen. Vgl. Goolsbee/Maydew (2000).

385 Vgl. Spengel (2008), S. 42.

$386 \mathrm{Vgl}$. Agundez-Garcia (2006), S. 47-48.

$387 \mathrm{Vgl}$. Musgrave (1972), S. 241.

$388 \mathrm{Vgl}$. Musgrave (1972), S. 241

$389 \mathrm{Vgl}$. Musgrave (1984), S. 241.

$390 \mathrm{Vgl}$. Agundez-Garcia (2006), S. 49.

391 Vgl. Sorenson (2004), S. 97. McLure (2000), S. 1302 bezeichnet immaterielle Wirtschaftsgüter als „Kronjuwelen“ eines Unternehmens. 
sammenwirken mehrerer Einheiten im Konzern im Rahmen gemeinsamer Forschungsprojekte entstehen. ${ }^{392}$ Schwierigkeiten bei der Bewertung immaterieller Wirtschaftsgüter ergeben sich, soweit für das übrige Vermögen historische Kosten zugrundegelegt werden. Diese machen bei immateriellen Wirtschaftsgütern, die häufig selbst erstellt werden, regelmäßig nur einen Bruchteil des tatsächlichen Werts aus. Eine gegenüber dem materiellen Vermögen abweichende Bewertung zum Marktwert - messbar über den Barwert zukünftiger Lizenzzahlungen - würde hingegen aufgrund der Ungleichbehandlung Verzerrungen mit sich bringen, beim Fehlen von Lizenzzahlungen zu Bewertungsproblemen führen und bei konzerninternen Lizenzzahlungen das Verrechnungspreisproblem wieder einführen. ${ }^{393}$ Aus diesen Gründen spricht einiges dafür, das Immaterialvermögen nicht zu berücksichtigen ${ }^{394}$, auch wenn dieses aufgrund der großen Bedeutung dieser Vermögenswerte einer verursachungsgerechten Einkommensverteilung anhand des Vermögens entgegenstehen und Verzerrungen von Investitionsentscheidungen mit sich bringen würde. ${ }^{395}$ Für eine Nichteinbeziehung des Immaterialvermögens spricht ferner die Gefahr von Steuergestaltung aufgrund der Mobilität dieses Faktors, wenn nicht alle tatsächlich abhängigen Unternehmen konsolidiert werden. ${ }^{396}$

Der angebots-/nachfrageorientierte Ansatz geht demgegenüber davon aus, dass Gewinn aus dem Zusammenwirken von Angebot und Nachfrage entsteht, weshalb sich nach diesem Ansatz beide Marktseiten in der Aufteilungsformel widerspiegeln sollten. ${ }^{397}$ Zur Abbildung der Nachfrageseite sollten dabei die Umsatzerlöse gemessen nach dem Bestimmungsortprinzip in der Formel Berücksichtigung finden, nach dem die Umsatzerlöse dem Lieferort beziehungsweise dem Ort der Leistungserbringung zugewiesen werden. ${ }^{398}$ Eine theoretische Rechtfertigung für die Einbeziehung der Umsatzerlöse kann nach Musgrave darin gesehen werden, dass der Unternehmensgewinn in eine Normalverzinsung, eine Risikoprämie und eine Monopolistenrente zerlegt werden kann. Letztere ist insbesondere auf Produkteigenschaften zurückzuführen und kann dementsprechend in vielen Fällen sachgerecht über den Umsatzfaktor zugewiesen werden ${ }^{399}$ Dem wird entgegenge-

392 Vgl. Agundez-Garcia (2006), S. 51; Sorenson (2004), S. 97.

393 Vgl. Agundez-Garcia (2006), S. 50; Sorenson (2004), S. 97.

$394 \mathrm{Vgl}$. so Oestreicher/Scheffler/Spengel et al. (2008), S. 357.

$395 \mathrm{Vgl}$. Agundez-Garcia (2006), S. 51; Sorenson (2004), S. 97.

$396 \mathrm{Vgl}$. Hellerstein/McLure (2004), S. 212.

397 Vgl. Spengel (2008), S. 42; Oestreicher (2000), S. 183; Musgrave (1984), S. 234; Musgrave (1972), S. 401.

398 Vgl. Agundez-Garcia (2006), S. 52. Alternativ könnten Umsätze auch nach dem Ursprungsort, also dem Sitz des liefernden oder leistenden Unternehmens berücksichtigt, werden. Die fehlende theoretische Rechtfertigung sowie eine erhöhte Gestaltungsanfälligkeit sprechen allerdings gegen diese Definition. Vgl. Agundez-Garcia (2006), S. 57.

399 Vgl. Musgrave (1972), S. 400. Auch Oestreicher (2000), S. 177 verweist darauf, dass der Zusammenhang zwischen der über die risikoadjustierte Verzinsung hinausgehenden Rendite und dem eingesetzten Kapital nur schwach ausgeprägt ist ${ }_{\text {Koch - 978-3-631-75683-6 }}$ 
halten, dass eine Körperschaftsbesteuerung nach dem Bestimmungsortprinzip im Widerspruch zu international üblichen Quellenregeln steht. ${ }^{400}$ Zudem muss in die Betrachtungen einbezogen werden, dass die Konsumentennachfrage mit der harmonisierten Umsatzsteuer in Europa bereits einer hinreichenden Steuerbelastung unterliegt. $^{401}$

In praktischer Hinsicht hat dieser Aufteilungsfaktor Vor- und Nachteile. Auf der einen Seite kann davon ausgegangen werden, dass er - verglichen mit den Produktionsfaktoren Vermögen und Arbeit - in nur geringem Ausmaß durch gezielte Gestaltungen verlagert werden kann, da die Verteilung dieses Faktors nicht im alleinigen Entscheidungsbereich des Unternehmens liegt. ${ }^{402}$ Auf der anderen Seite kann die Lokalisation des Umsatzortes insbesondere bei Verkäufen über das Internet Probleme bereiten. ${ }^{403}$ Des Weiteren muss eine Regelung für den Fall gefunden werden, dass die Gruppe nicht über einen steuerlichen Nexus im Bestimmungsland verfügt. ${ }^{404}$

Insgesamt bleibt festzuhalten, dass es an einem wissenschaftlich überzeugenden Konzept für die Auswahl und die Gewichtung der einkommensgenerierenden Faktoren fehlt. ${ }^{405}$ Daher erscheint es gerechtfertigt, dass sich die Kommission bei der Ausgestaltung der Aufteilungsformel insbesondere von politischen Kalkülen leiten lässt, um die Interessen der verschiedenen Mitgliedsstaaten gegeneinander abzuwägen.

\subsection{Wertschöpfung}

Der Vorschlag zur Aufteilung anhand der Wertschöpfung geht zurück auf Lodin/Gammie (2001), die eine derartige Aufteilung im Rahmen ihres Vorschlags zur Einführung einer Sitzlandbesteuerung vorsahen. ${ }^{406}$ Im Unterschied zur Definition des Umsatzfaktors im Rahmen einer Formelzerlegung wird hierbei allerdings regelmäßig nur eine ursprungsortbezogene Definition ${ }^{407}$ in Erwägung gezogen, während gegen eine bestimmungsortbezogene Erfassung der Wertschöpfung praktische und theoretische Bedenken vorgebracht werden. ${ }^{408}$

$400 \mathrm{Vgl}$. Agundez-Garcia (2006), S. 53.

401 Vgl. Schön (2007), S. 442.

402 Vgl. Mors/Rautenstrauch (2008), S. 102; Schön (2007), S. 442; Agundez-Garcia (2006), S. 52; Fox/Murray/Luna (2005), S. 149. Fox/Murray/Luna verweisen allerdings auf das Fehlen empirischer Evidenz für diese Vermutung.

403 Vgl. Hellerstein/McLure (2004), S. 213; Mintz (1999), S. 408.

404 Für die in den USA und Kanada zur Anwendung kommenden Lossungen vgl. Weiner (2005), S. 23-24.

405 Vgl. so auch Schön (2007), S. 440; Agundez-Garcia (2006), S. 46; Oestreicher (2000), S. 180.

406 Vgl. Russo (2005), S. 23.

407 Für unterschiedliche Ansätze zur Ermittlung einer ursprungsortbezogenen Wertschőpfung vgl. Agundez-Garcia (2006), S. 70-47; Sorenson (2004), S. 97.

$408 \mathrm{Vgl}$. Agundez-Garcia (2006), S. 74-75; Hellerstein/MGLure (2004) S $_{3}$ 216 
Für eine Aufteilung entsprechend einer ursprungsortbezogenen Wertschöpfung werden in der Literatur insbesondere zwei Rechtfertigungen angeführt. Zum einen wird in diesem Zusammenhang auf das Äquivalenzprinzip („,benefit principle") verwiesen, nach dem die Körperschaftsteuer als Kompensation für die Inanspruchnahme öffentlicher Güter gesehen wird. ${ }^{409}$ Hierzu wäre die Wertschöpfung ein geeigneter Maßstab, wenn von der Inanspruchnahme öffentlicher Güter eine gleichmäßige kostenreduzierende Wirkung ausgeht und die Unternehmen in einem Konzern gleichmäßig profitabel sind. ${ }^{410}$ Neben diesen restriktiven Annahmen wird das Äquivalenzprinzip allerdings auch generell als Rechtfertigung und damit als Maßstab für die Erhebung einer Ertragsteuer in Frage gestellt, da eine Inanspruchnahme öffentlicher Güter nicht auf rentable Unternehmen beschränkt sein dürfte. ${ }^{411}$

Zum anderen wird die Wertschöpfung als Maßstab für die Einkommensquelle und dementsprechend als Alternative zur Aufteilung anhand von Produktionsfaktoren nach dem angebotsorientierten Ansatz gesehen. ${ }^{412}$ Für eine Aufteilung nach der Wertschöpfung spricht in diesem Zusammenhang insbesondere die fehlende Notwendigkeit einer Bewertung und räumlichen Zuordnung des Vermögens. ${ }^{413}$ Ferner ist eine exogene Festlegung der Faktorgewichte nicht erforderlich. Diese ergeben sich vielmehr implizit. Aus diesem Grund sollte eine derartige Aufteilung als geschlossenes Konzept einheitlich für alle Branchen zugrundegelegt werden können. ${ }^{414}$ Der hohe Anteil an Personalkosten in der Wertschöpfung wird hingegen von einigen Autoren aus denselben Gründen wie bei der traditionellen Formelzerlegung als theoretischer Mangel dieses Vorschlags gesehen. Als mögliche Lösung für dieses Problem kann eine Aufteilung anhand der Wertschöpfung nach Abzug von Personalaufwendungen verstanden werden. ${ }^{415}$

Der wesentliche praktische Vorteil einer Aufteilung nach der Wertschöpfung läge in der möglichen Anknüpfung an umsatzsteuerliche Informationen, auch wenn diese in eine ursprungsortbezogene Wertschöpfung zu überführen wären. ${ }^{416}$ Diesem Vorteil stehen allerdings zwei gravierende praktische Mängel gegenüber. Russo führt an, dass eine Aufteilung nach der Wertschöpfung im Verlustfall zur Zuweisung eines negativen Anteils an der Gesamtbemessungsgrundlage führen kann.$^{417}$ Insbesondere würde eine Aufteilung nach der Wertschöpfung allerdings

409 Vgl. Musgrave (1972), S. 399-400.

410 Vgl. McLure (2000), S. 1288-1289; Musgrave (1984), S. 229-230; Musgrave (1972), S. 399400.

411 Vgl. McLure (2000), S. 1288-1289. Ähnlich auch Oestreicher (2000), S. 173-174 und Musgrave (1972), S. 399-400.

412 Vgl. Hellerstein/McLure (2004), S.214.

413 Vgl. Hellerstein/McLure (2004), S.214.

414 Vgl. Russo (2005), S. 23; Hellerstein/McLure (2004), S. 214.

415 Vgl. McLure (2005), S. 35; Sorenson (2004), S. 97-98.

416 Vgl. McLure (2005), S. 36; Russo (2005), S. 22.

$417 \mathrm{Vgl}$. Russo (2005), S. 23. 
ein Wiederaufleben der Verrechnungspreisproblematik bewirken. ${ }^{418}$ Eines der wesentlichen Ziele der Einführung einer GKKB könnte also mit dieser Form der Formelaufteilung nicht erreicht werden. Anreize zur Gestaltung der Verrechnungspreise würden durch eine Kürzung der Wertschöpfung um Arbeitskosten zusätzlich verstärkt werden. ${ }^{419}$ Insgesamt hat sich die Kommission daher gegen eine Aufteilung nach der Wertschöpfung entschieden.

\subsection{Vorschlag der Arbeitsgruppe}

Die Arbeitsgruppe hat sich bei ihrem Vorschlag - vermutlich insbesondere aus politischen Erwägungen - für eine Definition der Aufteilungsformel entschieden, welche sowohl die Angebots- als auch die Nachfrageseite berücksichtigt. ${ }^{420}$ So wird entsprechend der in den USA lange Zeit verbreiteten Massachusetts-Formel $^{421}$ eine gleichgewichtete Aufteilung nach Vermögen, Arbeit und Umsatzerlösen vorgeschlagen. ${ }^{422}$

Bei der Definition des Faktors „Arbeit“ spricht sich die Arbeitsgruppe nicht zuletzt auch aus politischen Kalkülen - für eine gleichgewichtete Berücksichtigung der Lohnsumme und der Arbeitnehmeranzahl aus. Dabei ist ein Mitarbeiter bzw. sind die Arbeitskosten dem Ort zuzuordnen, an dem der Mitarbeiter seine Leistung erbringt. Dieses wird mit Ausnahme von Mitarbeiterentsendungen regelmäßig der Ort sein, an dem das beschäftigende Unternehmen seinen Sitz hat oder eine Betriebsstätte unterhält. Arbeitnehmer, die im Rahmen von Mitarbeiterentsendungen tätig werden, sind dem Unternehmen am Leistungsort zuzuordnen, wobei eine De-minimis-Regelung für Fälle angedacht wird, in denen die Kosten für entsandte Mitarbeiter im Vergleich zur Gesamtlohnsumme nur geringfügig sind. Bei der Bewertung des Arbeitsfaktors wird für die Lohnsumme auf die Vergütung der Arbeitnehmer inklusive Lohnnebenkosten, Sozialabgaben und Mitarbeiteroptionen abgestellt. Eine pauschale Anpassung für internationale Unterschiede im Lohnniveau ist hingegen nicht vorgesehen, da eine ähnliche Wirkung bereits von der gleichzeitigen Einbeziehung der Arbeitnehmeranzahl ausgeht. Für die Arbeitnehmeranzahl werden die beschäftigten Mitarbeiter in Vollzeitäquivalente umgerechnet. ${ }^{423}$

418 Vgl. Schön (2007), S. 443; Hellerstein/McLure (2004), S. 215.

$419 \mathrm{Vgl}$. Hellerstein/McLure (2004), S. 215-216.

$420 \mathrm{Vgl}$. Mors/Rautenstrauch (2008), S. 103; Arbeitsgruppe GKKB (2007a), S. 6.

421 So ordneten im Jahr 1978 mit Ausnahme eines Staates alle US-Bundesstaaten das Aufkommen nach dieser Formel zu, während heute fast 80 Prozent aller Bundesstaaten mit Formelaufteilung den Umsatzfaktor mindestens hälftig gewichten. Vgl. McLure (2008), S. 50.

$422 \mathrm{Vgl}$. Arbeitsgruppe GKKB (2007a), S. 7. Dabei verweist die Arbeitsgruppe darauf, dass die Gleichgewichtung der Faktoren lediglich eine Arbeitshypothese bildet und die endgultige Gewichtung dem politischen Prozess überlassen wird.

423 Zur Definition, Zuordnung und Bewertung des Arbeitsfaktors vgl. Mors/Rautenstrauch (2008), S. 102; Arbeitsgruppe GKKB (2007a), S. 8-itoid Koch - 978-3-631-75683-6 
Beim Vermögensfaktor stellt die Arbeitsgruppe auf das materielle Anlagevermögen ab. Keine Berücksichtigung finden hingegen das immaterielle und finanzielle Anlagevermögen sowie das Vorratsvermögen. Ursächlich hierfür sind die in Kapitel 4.2.3.3.3 angesprochenen Probleme bei der Bewertung und örtlichen Zuordnung sowie die hohe Mobilität dieser Vermögenswerte. Der Ort der Nutzung der Wirtschaftsgüter bestimmt die örtliche Zuordnung. Analog zur Zuordnung der Mitarbeiter sollte eine Erfassung der Wirtschaftsgüter bei ihrem wirtschaftlichen Eigentümer den Regelfall darstellen. Ausnahmen ergeben sich lediglich bei Leasingverhältnissen, bei denen die betroffenen Wirtschaftsgüter nach dem Vorschlag der Arbeitsgruppe beim Leasingnehmer zu erfassen sind, soweit die Überlassung innerhalb der Gruppe erfolgt, während bei Verträgen mit Unternehmen, die nicht zur selben GKKB-Gruppe gehören, eine Zuordnung sowohl beim Leasingnehmer als auch beim Leasinggeber vorgesehen ist. Bei der Bewertung stellt die Arbeitsgruppe aus Praktikabilitätsgründen auf den steuerlichen Buchwert $a b .{ }^{424} \mathrm{Um}$ Verlagerungen zu erschweren, ist der Mittelwert zwischen Buchwert des Vermögens am Anfang und am Ende der Periode zugrunde zu legen. Als Ausnahme von der Bewertung zum Buchwert erfolgt bei Leasingverträgen außerhalb der GKKB-Gruppe eine Berücksichtigung beim Leasingnehmer zum achtfachen des jährlichen Nettomietpreises. ${ }^{425}$

Die Umsatzerlöse gehen aus den in Kapitel 4.2.3.3.3 genannten Gründen nach dem Bestimmungsortprinzip in die Formel ein. Insbesondere zur Vermeidung einer Wiedereinführung der Verrechnungspreisproblematik werden konzerninterne Umsätze nicht berücksichtigt. Die sich ergebende Minderzuweisung an reine Produktionsgesellschaften wird nach Ansicht der Arbeitsgruppe durch die beiden übrigen Faktoren kompensiert. Des Weiteren schränkt die Arbeitsgruppe die Definition der Umsatzerlöse auf Umsätze aus der Kerngeschäftstätigkeit des Unternehmens ein. Zinsen, Dividenden und Lizenzgebühren sind dementsprechend nicht zu berücksichtigen. Ebenfalls nicht berücksichtigt werden Umsatzerlöse, bei denen es an einer physischen Präsenz (Tochtergesellschaft oder Betriebsstätte) des Konzerns am Bestimmungsort fehlt. In diesen Fällen erfolgt kein reiner Rückbezug an den Ursprungsort. Vielmehr werden diese Umsätze proportional zu den übrigen Aufteilungsfaktoren unter den Gruppenunternehmen aufgeteilt (gestreute Rückbezugsregel). ${ }^{426}$

Nicht ausgeschlossen wird von der Arbeitsgruppe die Einführung branchenspezifischer Aufteilungsformeln. Die Notwendigkeit wird dabei insbesondere bei Finanzdienstleistungen, Transportdienstleistungen sowie Fernseh- und Rundfunkdienstleistungen gesehen. Dabei soll allerdings die Einbeziehung abweichender

$424 \mathrm{Vgl}$. Mors/Rautenstrauch (2008), S. 102.

425 Zur Definition, Zuordnung und Bewertung des Vermögensfaktors vgl. Mors/Rautenstrauch (2008), S. 102; Arbeitsgruppe GKKB (2007a), S. 10-14.

426 Zur Definition, Zuordnung und Bewertung des Umsatzfaktors vgl. Mors/Rautenstrauch (2008), S. 102-103; Arbeitsgruppe GKKB (20072) S Sal4-18 ch - 978-3-631-75683-6 
Formelfaktoren nach Möglichkeit vermieden werden. Vielmehr ist eine Anpassung der Definition der Aufteilungsfaktoren angedacht. ${ }^{427}$

\subsubsection{Administration und institutionelle Fragen}

Im Kern der administrativen Regelungen einer GKKB steht die Frage, welche Steuerverwaltung für die Erhebung der Körperschaftsteuer einer multinationalen Gruppe verantwortlich ist. Die Kommission zielt hierbei nicht auf die Einführung einer zentralen Verwaltungseinheit ab, sondern verfolgt in ihrem Vorschlag vielmehr das sogenannte „One Stop Shop“-Prinzip. Nach diesem Prinzip sind ein einzelnes Unternehmen (in der Regel das Mutterunternehmen) und die in dessen Ansässigkeitsstaat zuständige Finanzbehörde für die Verwaltung aller steuerlichen Einkünfte der Gruppe verantwortlich. ${ }^{428}$

Dieses Vorgehen könnte in der praktischen Umsetzung zahlreiche Probleme hervorrufen. So muss zum einen eine Lösung für europäische Schwestergesellschaften einer Nicht-EU-Muttergesellschaft gefunden werden, welche nach Vorstellung der Arbeitsgruppe für eine Teilnahme an der GKKB optieren können. Könnten diese Gruppen den Steuerverwaltungsort selbst wählen, würde Raum für zusätzliche Steuergestaltung entstehen. ${ }^{429}$ Gleichzeitig bestünde die Gefahr, dass die Durchsetzungsstärke der Steuerverwaltung als Mittel im Wettbewerb um die Ansiedlung von Konzernmuttergesellschaften verwendet wird. Dabei würde sich eine unzureichende Durchsetzung der Steuergesetze zu Lasten des Aufkommens sämtlicher Mitgliedsstaaten auswirken. ${ }^{430}$ Umgekehrt dürfte es von Seiten der Verwaltung eines Mitgliedsstaates nicht als gerechtfertigt empfunden werden, die Kosten der Steuererhebung für einen gesamten multinationalen Konzern tragen zu müssen, wenn der eigene Anteil an der Bemessungsgrundlage nur gering ist. ${ }^{431}$ Schließlich gilt es zu bedenken, dass nach dem Vorschlag der Arbeitsgruppe nicht nur die Feststellung des konsolidierten Gruppeneinkommens, sondern auch die Feststellung der Aufteilungsfaktoren und damit die Festlegung der Aufteilung der Bemessungsgrundlage zwischen den beteiligten Steuerhoheiten einseitig durch die Verwaltung eines Mitgliedsstaates erfolgen würde. Dieses Vorgehen könnte vermehrt Streitfälle hervorrufen, welche mit zusätzlichen Kosten für die Mitgliedsstaaten und die betroffenen Unternehmen verbunden wären. Das Fehlen einer übergeordneten supranationalen Verwaltungseinheit oder Gerichtsbarkeit als mög-

$427 \mathrm{Vgl}$. Arbeitsgruppe GKKB (2007a), S. 20.

428 Vgl. Mors/Rautenstrauch (2008), S. 104; Czakert (2008), S. 447-448. Vgl. befurrwortend Schön (2007), S. 444.

429 Vgl. McLure (2005), S. 36.

430 Vgl. McLure (2005), S. 36; McLure (2004), S. 58.

431 Vgl. McLure (2005), S. 36; McLure (2004), S. 58 einald Koch - 978-3-631-75683-6 
liche Schlichtungsstelle könnte sich in diesem Zusammen als problematisch erweisen. $^{432}$

In jedem Fall verlangt ein derartiges Vorgehen ein relativ hohes Maß an Vertrauen und Zusammenarbeit zwischen den Mitgliedsstaaten. ${ }^{433}$ Auch Weiner weist darauf hin, dass es bei einer Übertragung der US-amerikanischen Erfahrungen bedeutsam ist festzuhalten, dass die Bundesstaaten hier als Gebietskörperschaften eines gemeinsamen Landes fungieren und daher unter dem Dach einer übergeordneten Verwaltung tätig werden. Ferner führt sie das Funktionieren der Gewinnaufteilung zwischen den US-Bundesstaaten zu einem gewissen Grad auf die Existenz von Programmen zur Förderung der zwischenstaatlichen Kooperation bei der Steuerverwaltung zurück. ${ }^{434}$

\subsection{Einführung einer Verlustrichtlinie}

\subsection{1 Überblick}

Konkrete Anhaltspunkte für die mögliche Ausgestaltung einer Verlustrichtlinie fehlen zum heutigen Zeitpunkt, da die Überlegungen in diese Richtung bisher weder in Form eines Arbeitspapiers noch eines Richtlinienentwurfs konkretisiert worden sind. Vielmehr gibt lediglich eine Mitteilung der Kommission zur konzerninternen Verlustverrechnung aus dem Jahr $2006^{435}$ Aufschluss über eine mögliche Ausgestaltung. Ergänzend kann auf einen nicht weiter verfolgten Richtlinienentwurf aus dem Jahr $1991^{436}$, die Rechtsprechung des EuGH sowie die im geltenden Steuerrecht der Mitgliedsstaaten verankerten Gruppenbesteuerungssysteme zurückgegriffen werden.

Der EuGH hat mit seinen Urteilen insbesondere in den Rechtssachen „Marks\&Spencer“ ${ }^{437}$, „Oy AA“4438 und „Lidl“ ${ }^{439}$ seine Sichtweise bezüglich der Mindestanforderungen an eine Verlustverrechnung über die Grenze dargelegt. In diesen Urteilen wird die europarechtliche Notwendigkeit einer grundsätzlichen Berücksichtigung sämtlicher Auslandsverluste im Inland insbesondere mit Verweis auf eine ausgewogene Aufteilung des Steuersubstrats übereinstimmend verneint. Nur endgültige Auslandsverluste sind im Inland zur Verrechnung zuzulas-

432 Vgl. McLure (2005), S. 36; McLure (2004), S. 58. Vgl. so auch Czakert (2008), S. 447 mit der Forderung nach der Installation einer Schlichtungsinstanz.

433 Vgl. Czakert (2008), S. 447; Schön (2007), S. 444.

434 Vgl. Weiner (2001), S. 386.

435 Vgl. Kommission der Europäischen Gemeinschaften (2006).

436 Vgl. Kommission der Europäischen Gemeinschaften (1991).

437 Vgl. EuGH v. 13.12.2005 (Marks\&Spencer), EuGHE 2005, S. I-10837.

438 Vgl. EuGH v. 18.7.2007 (Oy AA), Abl. EU 2007 Nr. C 235, S. 3.

439 Vgl. EuGH v. 19.9.2006 (Lidl Belgium), Abl. EЩ2006 Nr.C.28 b, S. 7-631-75683-6 
sen. ${ }^{440}$ Die Definition von „Endgültigkeit“ ist bis zum heutigen Zeitpunkt allerdings umstritten. So ist zwar unstrittig, dass eine Beendigung der Auslandsgesellschaft diese Bedingung erfüllt. Unterschiedliche Auffassungen bestehen allerdings hinsichtlich der Frage, ob ein jüngeres Urteil des EuGH (,Wannsee ${ }^{، 441}$ ) so auszulegen ist, dass ein auf die zeitliche Begrenzung des Verlustvortrags im Ausland zurückgehender Verlustuntergang nicht zu endgültigen Verlusten im Sinne der früheren Urteile führt. ${ }^{42}$

Die Europäische Kommission hat im Anschluss an das Urteil des EuGH in der Rechtssache „Marks\&Spencer“ in einer unverbindlichen Mitteilung Leitlinien für eine grenzüberschreitende Verlustverrechnung dargelegt, welche über das vom EuGH verlangte Ausmaß einer grenzüberschreitenden Verrechnung hinausgehen. ${ }^{443}$ So fordert die Kommission eine phasengleiche Verrechnung für sämtliche Auslandsverluste im Jahr ihres Entstehens. ${ }^{444}$ Zur Vermeidung einer Verlagerung von Besteuerungsansprüchen sollte auch nach Auffassung der Kommission eine Verrechnung von Verlusten über die Grenze allerdings nur sekundär gegenüber einer intertemporalen und konzerninternen Verrechnung im Ausland zur Anwendung kommen. Außerdem sollte das Verrechnungssystem keine missbräuchlichen Gestaltungen (zum Beispiel gezielte Verlagerung von Verlusten in Hochsteuerländer) zulassen. Hinsichtlich des Umfangs fordert die Kommission zumindest eine vertikal aufwärts gerichtete Verlustverrechnung, also eine Verrechnung von Verlusten der Grundeinheit mit Gewinnen der Spitzeneinheit, und schlägt eine Anwendung dieser Grundsätze auch für ausländische Betriebsstätten vor. ${ }^{445}$

Offen lässt die Kommission in ihrer Mitteilung, inwiefern eine Verlustrichtlinie für das Konzept zur grenzüberschreitenden Verlustverrechnung verbindliche Vorgaben machen soll. Alternativ könnte den Mitgliedsstaaten ein Wahlrecht zwischen verschiedenen Systemen eingeräumt werden. ${ }^{446}$ Ebenfalls unklar ist, inwiefern die Richtlinie die verbindliche Einführung einer konzerninternen Verlustverrechnung im nationalen Bereich vorsehen soll. Mitgliedsstaaten ohne nationales

$440 \mathrm{Vgl}$. Jacobs (2007), S. 249-250.

441 Vgl. EuGH v.23.10.2008 (Krankenheim Ruhesitz am Wannsee-Seniorenheimstatt), Abl. EU 2008 Nr. C. 313 v. 6.12 .2008 , S. 5.

$442 \mathrm{Vgl}$. bejahend Lamprecht (2008), S. 768-769; verneinend Breuninger/Ernst (2009), S. 1986.

$443 \mathrm{Vgl}$. Kommission der Europäischen Gemeinschaften (2006).

$444 \mathrm{Vgl}$. Kommission der Europäischen Gemeinschaften (2006), S. 8.

$445 \mathrm{Vgl}$. Kommission der Europäischen Gemeinschaften (2006), S. 8; Oestreicher/Scheffler/ Spengel et al. (2008), S. 103.

446 Diese Frage war auch bereits im Richtlinienvorschlag aus dem Jahr 1991 adressiert. Hier sprach sich die Kommission bei Betriebsstätten für ein Mitgliedsstaatenwahlrecht zwischen der Nachversteuerungsmethode und der Anrechnungsmethode aus, während für Tochterkapitalgesellschaft ausschließlich die Nachversteuerungsmethode vorgesehen war. Vgl. Kom-

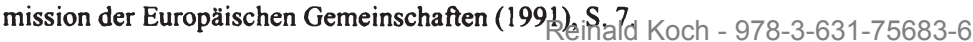


Gruppenbesteuerungssystem wären andernfalls von den Vorgaben der Richtlinie nicht betroffen. ${ }^{447}$

Dabei sprechen gute Gründe für die verbindliche Einführung einer nationalen und grenzüberschreitenden Gruppenbesteuerung in sämtlichen Mitgliedsstaaten. ${ }^{448}$ So ist nur dieser Ansatz mit dem Ziel der Schaffung neutraler Wettbewerbsbedingungen vereinbar, weshalb sich auch die Europäische Kommission in ihrem Richtlinienvorschlag aus dem Jahr 1991 für diesen Weg ausgesprochen hat. ${ }^{449}$ Ferner wären andernfalls einseitige Aufkommensverluste der Mitgliedsstaaten mit nationalem Gruppenbesteuerungssystem die Folge, welche diese zu einer Abschaffung der nationalen Systeme oder zur Ablehnung einer derartigen Reform bewegen könnten.

Die folgende Skizzierung der möglichen Ausgestaltung einer Verlustrichtlinie beginnt - analog zu den Ausführungen zur GKKB - mit einer Darstellung des möglichen Anwendungsbereichs dieser Richtlinie (Kapitel 4.3.2). Im Anschluss werden verschiedene Ansätze für eine konzerninterne Verlustverrechnung über die Grenze (Kapitel 4.3.3) sowie möglicherweise erforderliche Begleitmaßnahmen (Kapitel 4.3.4) diskutiert.

\subsubsection{Anwendungsbereich}

Bezüglich des räumlichen und persönlichen Anwendungsbereichs einer konzerninternen Verlustverrechnung kann an die entsprechenden Überlegungen zum Anwendungsbereich einer GKKB angeknüpft werden. In räumlicher Hinsicht sollten in Analogie zur GKKB sowohl grenzüberschreitend tätige als auch rein nationale Konzerne begünstigt werden. ${ }^{450}$ Soweit das Verlustverrechnungssystem ausschließlich an der Besteuerung der Muttergesellschaft ansetzt, ist im Unterschied zur GKKB hier allerdings eine Ausdehnung der Anwendung auch auf in Drittstaaten ansässige Tochtergesellschaften ohne Mitwirken der ausländischen Steuerhoheit konzeptionell möglich. Aufgrund der einseitigen Verteilung der von einer derartigen Ausdehnung ausgehenden Aufkommensverluste erscheint die Entscheidung für einen so weitreichenden Anwendungsbereich allerdings nicht wahrscheinlich. ${ }^{451}$ Umgekehrt muss der Anwendungsbereich des grenzüberschreitenden Gruppenbesteuerungssystems gegenüber der GKKB allerdings insofern eingeschränkt werden, dass europäische Schwestergesellschaften einer gemeinsamen, in einem Drittland ansässigen Muttergesellschaft nicht begünstigt werden können.

447 Vgl. Wittkowski (2008), S. 268.

448 A.A. Wittkowski (2008), S. 268.

$449 \mathrm{Vgl}$. Kommission der Europäischen Gemeinschaften (1991), S. 7.

450 Für die Vorteile einer gleichzeitigen Harmonisierung der Vorschriften zur konzerninternen Verlustverrechnung im nationalen Bereich vgl. Kapitel 4.3.1 und 4.3.4.

451 So geht auch Wittkowski in seinem Vorschlag auf eine Beschränkung der grenzüberschreitenden Verlustverrechnung auf EU-Betriebsstätten und Tochterkapitalgesellschaften aus. Vgl. Wittkowski (2008), S. 284. 
Hinsichtlich der Rechtsform der Untereinheit sollte die Anwendung nicht auf Tochterkapitalgesellschaften beschränkt bleiben, sondern auch ausländische Betriebsstätten erfassen. ${ }^{452}$ Zwar lässt sich insbesondere mit Blick auf die Verlustverrechnung aufgrund der Haftungsunterschiede eine Rechtsformneutralität zwischen diesen beiden Organisationsformen weder ökonomisch noch europarechtlich ableiten. ${ }^{453}$ Eine restriktivere Behandlung für ausländische Betriebsstättenverluste als für Tochterkapitalgesellschaftsverluste kann aufgrund dieser Haftungsunterschiede allerdings kaum gerechtfertigt werden. Um mögliche Rückwirkungen auf das Einkommensteueraufkommen zu vermeiden, sollten analog zur GKKB transparent besteuerte Personengesellschaften als Spitzeneinheit unberücksichtigt bleiben. ${ }^{454}$

Hinsichtlich der sachlichen Eingliederungsvoraussetzungen wurden im Rahmen der Ausführungen zur GKKB die Vorteile einer wirtschaftlichen und einer rechtlichen Abgrenzung der steuerlichen Gruppe diskutiert. Hierbei lag der wesentliche Vorteil des wirtschaftlichen Ansatzes in der tatsächlichen Erfassung einer wirtschaftlichen Einheit, was insbesondere für die Rechtfertigung einer indirekten Gewinnabgrenzung von Bedeutung ist. ${ }^{455}$ Diese Notwendigkeit besteht bei einem Reformvorschlag, der auf die Einführung einer Verlustverrechnung über die Grenze beschränkt ist, nicht. Aus diesem Grund spricht hier vieles dafür, aufgrund seiner administrativen Einfachheit dem rechtlichen Ansatz zu folgen. Um einen Gleichlauf mit der zivilrechtlichen Verlusthaftung zu gewährleisten, bietet sich hierbei ein Abstellen auf die Beteiligung am Kapital an.

\subsubsection{Konzept zur Verlustverrechnung}

\subsubsection{1 Überblick}

Grundsätzlich kommen für die Ausgestaltung einer Verlustverrechnung über die Grenze alle im geltenden europäischen Steuerrecht umgesetzten Konzepte für eine inländische oder grenzüberschreitende konzerninterne Verlustverrechnung in Frage. ${ }^{456}$ Bei Berücksichtigung der in Kapitel 4.3.1 genannten Anforderungen schränkt sich der Kreis möglicher Handlungsalternativen allerdings deutlich ein. So würden bei den im Rahmen nationaler Gruppenbesteuerungssysteme verwendeten Konzepten einer Zusammenveranlagung, Group Contribution oder eines Group Relief die Verluste entweder doppelt oder ausschließlich bei der Muttergesellschaft verrechnet, da diese Konzepte keinen Kompensationsmechanismus bei

452 Die Darstellungen hier beschränken sich allerdings ebenso wie die spăteren Berechnungen auf Kapitalgesellschaften.

453 Vgl. so Jacobs (2007), S. 252-254.

$454 \mathrm{Vgl}$. Oestreicher/Scheffler/Spengel et al. (2008), S. 98-99.

$455 \mathrm{Vgl}$. hierzu Kapitel 4.2.2.

$456 \mathrm{Vgl}$. Oestreicher/Scheffler/Spengel et al. (2008), S. 104. Für einen Überblick über diese Systeme in den verschiedenen Mitgliedsstaaten vgl Kapitel 3.3 .2$. 
späterer Verlustverrechnung auf Ebene der ausländischen Tochtergesellschaft vorsehen. ${ }^{457}$ Beim Group Relief oder der Group Contribution könnten Verluste zudem aufgrund der systemimmanenten Flexibilität gezielt in Hochsteuerländer verlagert werden. ${ }^{458}$ Aus diesem Grund werden im Folgenden als erste Alternative die Nachversteuerungsmethode, welche in Österreich für die grenzüberschreitende Verlustverrechnung im Rahmen des Gruppenbesteuerungssystems und in insgesamt fünf Mitgliedsstaaten für die Verlustverrechnung bei ausländischen Betriebsstätten zur Anwendung kommt, sowie als zweite Alternative die Zurechnungsmethode, welche im Rahmen der grenzüberschreitenden Gruppenbesteuerung in Dänemark, Frankreich und Italien verwendet wird, betrachtet. Beide Methoden waren auch in der Kommissionsmitteilung aus dem Jahr 2006 sowie im Richtlinienvorschlag aus dem Jahr 1991 vorgesehen. ${ }^{459}$

Als dritte Alternative wird die Ergebnisaufteilungsmethode einbezogen. Im Unterschied zu den beiden zuvor angesprochenen Konzepten ist dieses System in der bisherigen Diskussion unbeachtet geblieben. Ursächlich hierfür dürfte die Tatsache sein, dass diese Methode im geltenden Recht lediglich zur Besteuerung nationaler Gruppen in Dänemark, allerdings in keinem Mitgliedsstaat zur Besteuerung grenzüberschreitender Gruppen zur Anwendung kommt. Ferner ist diese Methode ungeeignet für eine unilaterale Reform, da sie - anders als die Nachversteuerungsmethode und die Zurechnungsmethode - sowohl zu steuerlichen Änderungen bei der Muttergesellschaft als auch den Tochtergesellschaften führt.

Dennoch wird in dieser Arbeit die Auffassung vertreten, dass auch die Ergebnisaufteilungsmethode für eine multilaterale Weiterentwicklung der Konzernbesteuerung geeignet ist. Im Unterschied zu den übrigen Konzepten einer nationalen Gruppenbesteuerung sieht diese Methode weder eine Zusammenveranlagung auf Ebene der Muttergesellschaft noch eine flexible Gewinn- oder Verlustzuordnung vor. Somit führt sie bei Anwendung über die Grenze weder zu einer ungemilderten Doppelbesteuerung (oder doppelten Verlustberücksichtigung) noch eröffnet sie übermäßige Möglichkeiten zur Steuergestaltung.

Nicht in die im Rahmen dieser Arbeit erfolgende Untersuchung einbezogen wird der in Großbritannien und Irland umgesetzte Ansatz zur grenzüberschreitenden Verlustverrechnung. In diesen Ländern wird in Übereinstimmung mit den Vorgaben des EuGH die Verlustberücksichtigung auf im Ausland endgültig nicht verrechenbare Verluste begrenzt. Dementsprechend ermöglicht dieser Vorschlag lediglich eine nachgelagerte Verlustberücksichtigung ${ }^{460}$, so dass Zins- und Liqui-

$457 \mathrm{Vgl}$. Oestreicher/Scheffler/Spengel et al. (2008), S. 105.

$458 \mathrm{Vgl}$. Oestreicher/Scheffler/Spengel et al. (2008), S. 105.

459 Vgl. Kommission der Europäischen Gemeinschaften (2006); Kommission der Europäischen Gemeinschaften (1991).

460 Vgl. Wittkowski (2008), S. 243. 
ditätsnachteile gegenüber nationalen Konzernen bestehen bleiben. ${ }^{461}$ Aus diesem Grund erscheint dieser Ansatz mit Blick auf die in der Mitteilung der Kommission formulierten Ziele einer Verbesserung der Wettbewerbsfähigkeit europäischer Konzerne und Beseitigung grenzüberschreitender Investitionshindernisse als nicht weitgehend genug.

\subsubsection{Nachversteuerungsmethode}

Die Nachversteuerungsmethode ist ein Ansatz zur Verlustverrechnung über die Grenze, der die Besteuerung der Tochtergesellschaft unberührt lässt und ausschließlich in die Besteuerung der Muttergesellschaft eingreift. ${ }^{462}$ Dabei kommt bei der Einkommensermittlung der Muttergesellschaft unverändert das Trennungsprinzip zur Anwendung, wenn die Tochtergesellschaft Gewinne erzielt. Nur bei Verlusten der Tochtergesellschaft, die in deren Ansässigkeitsstaat weder durch eine konzerninterne Verlustverrechnung noch durch einen Verlustrücktrag phasengleich verrechnet werden können, kommt es zu einer temporären Durchbrechung des Trennungsprinzips. Diese Verluste mindern das Einkommen der Muttergesellschaft im Jahr ihres Entstehens. Zur Vermeidung einer doppelten Verlustberücksichtigung im In- und Ausland werden spätere Gewinne der Tochtergesellschaft bis zur Höhe der zuvor abgezogenen Verluste dem Einkommen der Muttergesellschaft hinzugerechnet (,Nachversteuerung“) ${ }^{463}$

Dementsprechend ermöglicht die Nachversteuerungsmethode lediglich eine vertikal aufwärts gerichtete Verlustverrechnung, also eine Verrechnung von Verlusten der Tochter- mit Gewinnen der Muttergesellschaft. ${ }^{464}$ Die Wirkung der Verlustverrechnung bei der Muttergesellschafft ist - mit Ausnahme endgültiger Verluste - temporärer Natur. ${ }^{465}$

Für die Ausgestaltung der Nachversteuerung sind unterschiedliche Ansätze möglich. Cordewener/Dahlberg/Pistone et al. unterscheiden in zeitlicher Hinsicht zwischen einer Nachversteuerung ex tunc und einer Nachversteuerung ex nunc. Während im ersten Fall die Nachversteuerung zu einer nachträglichen Änderung der Steuererklärung für das Jahr der Verlustberücksichtigung führt, erfolgt im zweiten Fall die Berücksichtigung in der Steuererklärung des Jahres, in dem die Nachversteuerung entsteht. ${ }^{466}$ Wird die Nachversteuerungsmethode ohne Verein-

461 Vgl. ähnlich Wittkowski (2008), S. 243 und 285, der diese Methode ebenfalls fur nicht geeignet hält. Er weist ergänzend darauf hin, dass dieses Konzept den Zielen einer entscheidungsneutralen und leistungsgerechten Besteuerung widerspricht.

$462 \mathrm{Vgl}$. Oestreicher/Scheffler/Spengel et al. (2008), S. 218-219.

$463 \mathrm{Vgl}$. Oestreicher/Scheffler/Spengel et al. (2008), S. 105.

$464 \mathrm{Vgl}$. Wittkowski (2008), S. 242. Eine vertikal abwärts gerichtete Verlustverrechnung ist unverändert nur möglich, wenn bei der Muttergesellschaft ausländische Dividenden nach der Anrechnungsmethode besteuert werden.

$465 \mathrm{Vgl}$. Scheffler (2005), S. 157

$466 \mathrm{Vgl}$. Cordewener/Dahlberg/Pistone et al. (2004) S. 138-139. 
heitlichung der Gewinnermittlung eingeführ ${ }^{467}$, könnte der zu berücksichtigende Verlust (und der zur Nachversteuerung führende Gewinn) entweder nach den Vorschriften des Sitzlands der ausländischen Tochtergesellschaft oder der inländischen Muttergesellschaft ermittelt werden. ${ }^{468}$ Schließlich bestehen auch für die Definition des nachversteuerungsauslösenden Tatbestands unterschiedliche Möglichkeiten. So können entweder sämtliche späteren Gewinne der Tochtergesellschaft eine Nachversteuerung auslösen oder die Nachversteuerung auf Gewinne beschränkt werden, die im Land der Tochtergesellschaft bei der Steuerermittlung zu einer Verrechnung mit vorherigen Verlusten führen. Zusätzlich könnte eine gewinnunabhängige Nachversteuerung nach einer bestimmten Zeit vorgesehen werden. ${ }^{469}$

Eine grenzüberschreitende Verlustverrechnung nach Maßgabe der Nachversteuerungsmethode geht grundsätzlich über die Vorgaben des EuGH hinaus und ist dementsprechend europarechtlich unbedenklich. ${ }^{470}$ Etwas anderes gilt lediglich bei Berücksichtigung einer gewinnunabhängigen Nachversteuerung, wie im Richtlinienvorschlag von 1991 vorgesehen. Eine derartige Lösung würde der Mindestvorgabe des EuGH gerade nicht entsprechen, da es für endgültige Verluste im Sinne von „Marks\&Spencer“ im Ergebnis nicht zu einer Verrechnung bei der Muttergesellschaft kommt. Weitere Vorteile der Nachversteuerungsmethode werden insbesondere in ihrer problemlosen Integration in ein auf dem Trennungsprinzip beruhenden System der Konzernbesteuerung gesehen. ${ }^{471}$ Ferner kann bei der Umsetzung auf umfangreiche praktische Erfahrungen zurückgegriffen werden. So kommt diese Methode aktuell im Rahmen der grenzüberschreitenden Gruppenbesteuerung in Österreich sowie der Verrechnung von Verlusten ausländischer Betriebsstätten in insgesamt fünf europäischen Ländern zur Anwendung. ${ }^{472}$ Auch in Deutschland war diese Methode bis 1999 für die steuerliche Behandlung von Verlusten einer ausländischen DBA-Betriebsstätte vorgesehen. ${ }^{473}$ Als weiterer Vorteil vermeidet der fehlende Eingriff in die Besteuerung der Tochtergesellschaft, schließlich, die Notwendigkeit eines Kompensationsmechanismus für Minderheitsgesellschafter. ${ }^{474}$

Das Kernproblem der Nachversteuerungsmethode in praktischer Hinsicht wird in der Sicherstellung der Nachversteuerung gesehen. Zur Vermeidung von

467 Vgl. hierzu auch Kapitel 4.3.4.

468 In der Verlustrichtlinie aus dem Jahr 1991 war eine Ermittlung nach den ausländischen Vorschriften vorgesehen. Vgl. Kommission der Europäischen Gemeinschaften (1991), S. 6.

469 So war im Rahmen des Richtlinienvorschlags aus dem Jahr 1991 eine Nachversteuerung spätestens nach Ablauf von fünf Jahren vorgesehen. Vgl. Kommission der Europäischen Gemeinschaften (1991), S. 6.

470 Vgl. Wittkowski (2008), S. 242.

471 Vgl. Rödder (2007), S. 391.

472 Vgl. Kapitel 3.2.1.2.

473 Vgl. §2a Abs. 3,4 EStG a.F.

$474 \mathrm{Vgl}$. Oestreicher/Scheffler/Spengel et al. (2008), Reifl9 mwN 
Gestaltungsspielräumen für die Unternehmen sind hierfür insbesondere detaillierte Regelungen für den Fall aperiodischer Vorgänge (Verkauf, Liquidation oder Umwandlung der Tochtergesellschaft) erforderlich. ${ }^{475}$ Aus einer betriebswirtschaftlichen und volkswirtschaftlichen Perspektive ist zudem die asymmetrische Behandlung von Totalverlusten (Kapitalexportneutralität) und von Totalgewinnen (Kapitalimportneutralität), die sowohl eine Entscheidungsverzerrung bei der Standortwahl als auch bei der Risikoübernahme bewirken kann, problematisch. ${ }^{476}$ Eine Nachversteuerung ex nunc beinhaltet schließlich das Problem einer möglicherweise erforderlichen Verzinsung der Steuernachzahlung. ${ }^{47}$

Auch die Definition des nachversteuerungsauslösenden Tatbestands kann zu Problemen führen. So kann es bei einer Anknüpfung an die steuerliche Verlustverrechnung im Ausland zu einem unerwünschten ,race-to-the-bottom“ bezüglich der Verlustvortragsdauern kommen. ${ }^{48}$ Abhilfe kann hier eine Harmonisierung der interperiodischen Verlustverrechnung schaffen ${ }^{479}$ Umgekehrt kann eine Nachversteuerung für Gewinne im Inland, für die ein Verlustabzug im Ausland ausgeschlossen ist, eine Verzerrung der Entscheidung zur Unternehmensfortführung bewirken, da es in diesem Fall bei Verlusten zu einer einfachen Verrechnung kommt, für Gewinne aber eine doppelten Besteuerung im In- und Ausland erfolgt. Diese asymmetrischen Besteuerungsfolgen können im Extremfall dazu führen, dass der zu erwartende Zufluss aus der Tochtergesellschaft negativ wird und die Tochtergesellschaft aus diesem Grund nicht fortgefuihrt wird. ${ }^{480}$

\subsubsection{Zurechnungsmethode}

Im Gegensatz zur Nachversteuerungsmethode würde eine Umsetzung der Zurechnungsmethode eine konzeptionelle Neuausrichtung der Konzernbesteuerung bewirken. So sieht diese Methode den Übergang von der international vorherrschenden kapitalimportneutralen Besteuerung auf eine kapitalexportneutrale Besteuerung vor. ${ }^{481}$ Gleichzeitig führt eine Umsetzung dieser Methode zu einer Durchbrechung des Trennungsprinzips und der damit verbundenen steuerlichen Abschirm-

$475 \mathrm{Vgl}$. hierzu ausführlich Oestreicher/Scheffler/Spengel et al. (2008), S. 225-255.

$476 \mathrm{Vgl}$. Oestreicher/Scheffler/Spengel et al. (2008), S. 264; Jacobs (2007), S. 254. Voraussetzung für diese Asymmetrie ist die Anwendung der Freistellungsmethode fur ausländische Dividenden.

$477 \mathrm{Vgl}$. Cordewener/Dahlberg/Pistone et al. (2004), S. 139.

$478 \mathrm{Vgl}$. hierzu Kapitel 4.3.4.

479 Vgl. Oestreicher/Scheffler/Spengel et al. (2008), S. 220; Spengel/Braunagel (2006), S. 41.

480 Dabei wird unterstellt, dass eine Stilllegung der Tochtergesellschaft entsprechend des „Marks\&Spencer"-Urteils des EuGH keine Nachversteuerung auslöst.

481 Vgl. Wittkowski (2008), S. 245; Oestreicher/Scheffler/Spengel et al. (2008), S. 255-257; Rödder (2007), S. 391. 
wirkung der Tochterkapitalgesellschaft. ${ }^{482}$ Wie bei der Nachversteuerungsmethode kommt es auch bei Anwendung der Zurechnungsmethode lediglich zu einem Eingriff in die Besteuerung der Muttergesellschaft, während die Besteuerung der Tochtergesellschaft unverändert bleibt. Die Muttergesellschaft unterliegt nach diesem Konzept unabhängig von der Gewinnverwendung der Tochtergesellschaft mit dem aggregierten Ergebnis aller in- und ausländischen Gruppengesellschaften der Besteuerung. Zur Vermeidung einer Mehrfachbesteuerung von Gewinnen können die auf Ebene der ausländischen Tochtergesellschaften gezahlten Steuern angerechnet werden. Da die Gewinne der Tochtergesellschaft bereits bei Entstehung auf Ebene der Muttergesellschaft einer Besteuerung unterliegen, sind Dividendenzahlungen an die Muttergesellschaft freizustellen. ${ }^{483}$ Aufgrund der Einbeziehung der Gewinne ausländischer Tochterunternehmen in die Besteuerung der Muttergesellschaft erlaubt diese Methode zwar konzeptionell eine Verlustverrechnung sowohl vertikal aufwärts als auch vertikal abwärts, der fehlende Eingriff in die Besteuerung der Auslandstochter führt allerdings dazu, dass die Berücksichtigung der Auslandsgewinne im Ergebnis lediglich zur Nutzung eines inländischen Verlustvortrags, nicht aber auch zu einer Minderung der Konzernsteuerbelastung führt. ${ }^{484}$

Anhaltspunkte für die konkrete Ausgestaltung geben insbesondere Gruppenbesteuerungssysteme im geltenden Steuerrecht, welche dieses Konzept umsetzen (Dänemark, Frankreich und Italien). ${ }^{485}$ In allen drei Ländern wird die Anrechnung auf die Höhe der im Inland auf die ausländischen Einkünfte entfallenden Steuern begrenzt. ${ }^{486}$ Dieses kann bei höheren Steuersätzen im Ausland, Verlusten im Inoder Ausland oder abweichenden Gewinnermittlungsvorschriften im In- und Ausland $z u$ Anrechnungsüberhängen führen. ${ }^{487}$ Der Anrechnungshöchstbetrag wird in den bestehenden Systemen entweder für jeden ausländischen Staat (,per-countrylimitation“: Frankreich, Dänemark) oder für jede Tochtergesellschaft (,per-

482 Vgl. Wittkowski (2008), S. 245; Oestreicher/Scheffler/Spengel et al. (2008), S. 255; Grotherr (1996), S. 360.

$483 \mathrm{Vgl}$. Oestreicher/Scheffler/Spengel et al. (2008), S. 127-128.

484 Wagner und Wittkowski schlagen als mögliche Lösung eine Erstattung der von der ausländischen Tochtergesellschaft gezahlten Steuer vor. Ferner könnte ein wahlweiser Abzug der Auslandssteuer oder der Anrechnungsüberhänge negative Konsequenzen abmildern. Vgl. Wittkowski (2008), S. 234; Oestreicher/Scheffler/Spengel et al. (2008), S. 274; Wagner (2006), S. 193.

485 Frankreich und Italien sehen neben der Zurechnung auch eine Eliminierung konzerninterner Zwischengewinne vor. Für dieses Konzept wird in der Literatur teilweise der Begriff ,eingeschränktes Einheitskonzept“" verwendet. Vgl. Wittkowski (2008), S. 244; Grotherr (1996), S. 360.

$486 \mathrm{Vgl}$. zur europarechtlichen Würdigung einer begrenzten Anrechnung Wittkowski (2008), S. 245; Oestreicher/Scheffler/Spengel et al. (2008), S. 269-270; Cordewener/Schnitger (2006), S. 73; Flick/Wassermeyer/Baumhoff (2006), Vor § 34c EStG, Rn. 32.

$487 \mathrm{Vgl}$. Oestreicher/Scheffler/Spengel et al. (2008), \$ $268-269$ 
company-limitation": Italien) separat ermittelt. ${ }^{488}$ Aufgrund europarechtlicher Bedenken bezüglich einer derartigen Ermittlung des Anrechnungshöchstbetrags, bei dem Anrechnungsüberhänge aus einem Land nicht mit verbleibendem Anrechnungspotential aus anderen Ländern verrechnet werden können, ist aber unter Umständen eine „overall-limitation“ geboten. ${ }^{489}$ Aus der Anwendung des Anrechnungshöchstbetrags resultierende Anrechnungsüberhänge können in allen drei Ländern in künftige Veranlagungszeiträume vorgetragen werden (Dänemark: zeitlich unbegrenzt; Frankreich: fünf Jahre; Italien: acht Jahre) ${ }^{490}$

Die wesentlichen Vor- und Nachteile der Zurechnungsmethode gehen vom Übergang auf eine kapitalexportneutrale Besteuerung aus. Durch die Sicherstellung von Kapitalexportneutralität lässt sich das Ziel einer allokationseffizienten und entscheidungsneutralen Besteuerung für den Binnenmarkt mit der Zurechnungsmethode erreichen. So führt die Zurechnungsmethode zu einer symmetrischen Besteuerung von Gewinnen und Verlusten ausländischer Tochtergesellschaften, wodurch die Entscheidung zwischen risikolosen und risikobehafteten Investitionen nicht zusätzlich verzerrt wird. ${ }^{491}$ Auch Finanzierungsentscheidungen werden durch Steuern weniger stark beeinflusst, da bei Gesellschafterdarlehen und Eigenkapitalüberlassungen derselbe Steuersatz zur Anwendung kommt. Diese Gleichbehandlung der Finanzierungsalternativen stellt sich allerdings nur aus Unternehmenssicht ein, aus Sicht der beteiligten Länder ergibt sich hingegen unverändert eine abweichende Aufkommensverteilung. ${ }^{492}$

Auf der anderen Seite muss berücksichtigt werden, dass eine Umsetzung der Zurechnungsmethode in den Ländern, die in ihren Doppelbesteuerungsabkommen für ausländische Betriebsstätten die Freistellungsmethode vorsehen, ohne deren Anpassung zu einem ,treaty override“ fuhrt. ${ }^{493}$ Ferner läuft eine kapitalexportneutrale Besteuerung der Entwicklungstendenz bei den europäischen Körperschaftsteuersystemen und den Bestrebungen der Kommission zur Einführung einer

$488 \mathrm{Vgl}$. Oestreicher/Scheffler/Spengel et al. (2008), S. 74-75.

$489 \mathrm{Vgl}$. Oestreicher/Scheffler/Spengel et al. (2008), S. 270-272.

$490 \mathrm{Vgl}$. Wittkowski (2008), S. 245; Oestreicher/Scheffler/Spengel et al. (2008), S. 74-75. In Italien ist daruber hinaus ein Rücktrag von Anrechnungsüberhängen in die vorhergehenden acht Perioden möglich. Vgl. Oestreicher/Scheffler/Spengel et al. (2008), S. 272.

$491 \mathrm{Vgl}$. Oestreicher/Scheffler/Spengel et al. (2008), S. 106.

$492 \mathrm{Vgl}$. Oestreicher/Scheffler/Spengel et al. (2008), S. 257. Dieses gilt allerdings nur, solange kein Anrechnungshöchstbetrag zur Anwendung kommt, also das ausländische Steuerniveau geringer ist.

493 Oestreicher/Scheffler/Spengel et al. (2008), S. 264-265; Wittkowski (2008), S. 245-246; Jacobs (2007), S. 255. Allerdings ist es in Frankreich und Italien trotz Durchbrechung der Doppelbesteuerungsabkommen bisher nicht zu negativen Reaktionen von DBA-Vertragspartnern gekommen. Dănemark hat inzwischen eine Überarbeitung der DBA vorgenommen. Bei einer EU-weiten Lösung erübrigt sich eine Neuverhandlung der DBA aufgrund des Vor-

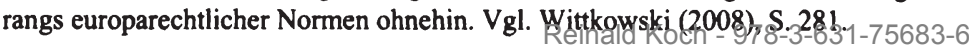


GKKB, welche auf der Grundidee einer kapitalimportneutralen Besteuerung beruht, entgegen. ${ }^{494}$

\subsubsection{Ergebnisaufteilungsmethode}

Im Unterschied zu den beiden zuvor beschriebenen Konzepten führt die Ergebnisaufteilungsmethode - wie die GKKB - zu einem Eingriff in die Besteuerung sowohl der Mutter- als auch der Tochtergesellschaft. Wie die GKKB sieht auch diese Methode eine separate Ermittlung der Ergebnisse für die einzelnen Gruppengesellschaften vor, die im Anschluss auf Gruppenebene zusammengefasst werden. In einem zweiten Schritt wird das so ermittelte Gruppenergebnis auf die beteiligten Gruppengesellschaften aufgeteilt und dort dem landesspezifischen Steuersatz unterworfen. Dabei wird ein positives Gruppenergebnis proportional auf die gewinnerzielenden Gruppengesellschaften verteilt, die verlusterzielenden Gruppengesellschaften erhalten ein Ergebnis von null. Ein Gesamtverlust wird entsprechend proportional auf die verlusterzielenden Gruppengesellschaften verteilt, während die gewinnerzielenden Gesellschaften ein Ergebnis von null zugewiesen bekommen. ${ }^{495}$ Konzeptionell gewährleistet die Ergebnisaufteilungsmethode eine dem Umfang nach weitreichende Verlustverrechnung im Konzern, da diese sowohl vertikal aufwärts und abwärts als auch in horizontaler Richtung möglich ist.

Für den Vortrag und Rücktrag der zugewiesenen Verluste sind zwei verschiedene Ansätze denkbar. Folgt man dem in Dänemark für nationale Gruppen zur Anwendung kommenden Konzept, werden während der Gruppenzugehörigkeit entstandene Verlustvorträge in der Folgeperiode primär mit einem eigenen positiven Einkommen der jeweiligen Gesellschaft verrechnet. Übersteigende Verlustvorträge werden allerdings erneut in das aufzuteilende Gruppeneinkommen einbezogen. ${ }^{496}$ Diese Vorgehensweise gewährleistet eine schnellstmögliche Verlustverrechnung und dient damit dem Ziel der Kommission zur Verbesserung der Wettbewerbsfähigkeit europäischer Unternehmen. Der weiteren Vorgabe der Kommission einer primären Verrechnung auf Ebene der verlusterzielenden Gesellschaft wird allerdings durch die Einbeziehung von Verlustvorträgen in die Aufteilung über das dem Konzept ohnehin inhärente Maß (Übertragung von Verlusten auf andere Gesellschaften) hinaus widersprochen.

Als Alternative wäre eine intertemporale Verlustverrechnung ausschließlich auf Ebene der jeweiligen Gruppengesellschaft möglich. Dieser Ansatz hat den Vorteil, dass zumindest die zugewiesenen Gruppenverluste bei der verlusterzielenden Gesellschaft selbst verrechnet würden. Mögliche negative Folge wäre allerdings ein Einschluss von Verlustvorträgen bei dauerhaft verlusterzielenden

494 Vgl. Rödder (2007), S. 391.

495 Vgl. Grotherr (1996), S. 360.

496 Vgl. Dörr (2008), S. 36-37; Endres (2003), S. 465; Grotherr (1996) S 360 - 36 1-75683-6 
Gruppengesellschaften. So würde diese Ausgestaltung ein eigenes positives Einkommen in zukünftigen Perioden voraussetzen, um einen einmal zugewiesenen Verlust zu verrechnen. Ein positives Konzernergebnis wie beispielsweise bei der GKKB wäre hier nicht ausreichend.

\subsubsection{Begleitende Maßnahmen}

Auch bei Einführung einer Verlustrichtlinie stellt sich die Frage, nach welchen Vorschriften die Ermittlung des zugrunde liegenden Einkommens erfolgen soll. In diesem Zusammenhang kann für die Notwendigkeit harmonisierter Gewinnermittlungsvorschriften im Rahmen der Ergebnisaufteilungsmethode auf die entsprechenden Ausführungen zur GKKB verwiesen werden. Für die Ermittlung des Einkommens der ausländischen Tochtergesellschaft für Zwecke der inländischen Besteuerung im Rahmen der Nachversteuerungsmethode und der Zurechnungsmethode werden in der Literatur insgesamt drei Ansätze unterschieden: erstens könnten die ausländischen Gewinnermittlungsvorschriften zugrundegelegt werden, zweitens auf die inländischen Vorschriften abgestellt werden und drittens eine Harmonisierung der Gewinnermittlungsvorschriften erfolgen. ${ }^{497}$

Ein Abstellen auf die ausländischen Gewinnermittlungsvorschriften erzeugt dabei eine Abhängigkeit des inländischen Steueraufkommens von den ausländischen Gewinnermittlungsvorschriften. So könnten die negativen Aufkommenskonsequenzen steuerlicher Investitionsanreize (z.B. Sonderabschreibungen) zumindest partiell externalisiert werden ${ }^{498}$ Ferner führt eine Gewinnermittlung nach ausländischen Vorschriften gerade nicht zur europarechtlich gebotenen Gleichstellung inländischer und ausländischer Tochtergesellschaften ${ }^{499}$ und stünde zudem im Widerspruch zu einer kapitalexportneutralen Behandlung der Verluste, welche sowohl bei der Nachversteuerungsmethode als auch der Zurechnungsmethode vorgesehen ist. ${ }^{500}$ Aus diesem Grund wird dieser Ansatz in der Literatur überwiegend abgelehnt. ${ }^{501}$

Eine Ermittlung nach dem Steuerrecht der Muttergesellschaft würde hingegen eine zweifache Einkommensermittlung und damit zusätzliche Befolgungskosten mit sich bringen. ${ }^{502}$ Auch wenn nicht davon auszugehen ist, dass hieraus eine europarechtswidrige Diskriminierung ausländischer Tochtergesellschaften

497 Vgl. so Scheunemann (2006), S. 150-151.

$498 \mathrm{Vgl}$. so Oestreicher/Scheffler/Spengel et al. (2008), S. 217; Wittkowski (2008), S. 286; Jacobs (2007), S. 254.

$499 \mathrm{Vgl}$. Scheunemann (2006), S. 150.

$500 \mathrm{Vgl}$. Oestreicher/Scheffler/Spengel et al. (2008), S. 217. Gleiches gilt bei der Zurechnungsmethode auch fur die Ermittlung der ebenfalls kapitalexportneutral besteuerten Gewinne.

$501 \mathrm{Vgl}$. Oestreicher/Scheffler/Spengel et al. (2008), S. 215-218; Wittkowski (2008), S. 286; Herzig/Wagner (2005), S. 7.

$502 \mathrm{Vgl}$. Oestreicher/Scheffler/Spengel et al. (2008) R. 217. 
abgeleitet werden $\mathrm{kann}^{503}$, bringt auch bei Einführung einer Verlustrichtlinie die Harmonisierung der Gewinnermittlung Vorteile mit sich, weshalb sich einige Autoren für diesen Weg aussprechen..$^{50}$

Ferner sollte die Einführung einer Verlustrichtlinie mit einer Harmonisierung der Vorschriften zur intertemporalen Verlustverrechnung sowie zur Verlustverrechnung zwischen nationalen Konzerngesellschaften einhergehen. Andernfalls könnte es zu einer Verzerrung des Steuerwettbewerbs kommen, da eine Einschränkung der Möglichkeiten zur Verlustverrechnung im Ausland zu einer Minderung des inländischen Steueraufkommens führen würde. ${ }^{505}$ Ein derartiges „raceto-the-bottom" bei den Verlustvortragsdauern würde die steuerliche Benachteiligung risikobehafteter Investitionen verstärken. ${ }^{506}$ Schließlich würde eine fehlende Harmonisierung in diesem Bereich Raum für Planungsüberlegungen schaffen und somit zusätzliche Kosten und Entscheidungsverzerrungen hervorrufen. ${ }^{507}$

$503 \mathrm{Vgl}$. Oestreicher/Scheffler/Spengel et al. (2008), S. 218; Scheunemann (2006), S. 150; Herzig/Englisch/Wagner (2005), S. 312.

504 Vgl. Spengel (2008), S. 29; Schön (2007), S. 421.

505 Vgl. Wittkowski (2008), S. 269-270; Oestreicher/Scheffler/Spengel et al. (2008), S. 220; Maiterth (2006), S. 916; Scheunemann (2006), S. 148. Dieses gilt bei der Ergebnisaufteilungsmethode nur für die konzerninterne Verlustverrechnung, wenn die intertemporale Verlustverrechnung ausschließlich auf Ebene der Einzelgesellschaft erfolgt.

506 Vgl. Wittkowski (2008), S. 269-270.

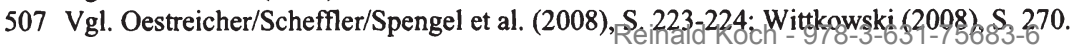




\section{Datenbasis und Methodik der empirischen Analyse}

\section{1 Überblick}

Die Ermittlung der quantitativen Wirkungen, die mit der Einführung einer grenzüberschreitenden Gruppenbesteuerung und konsolidierten Körperschaftsteuerbemessungsgrundlage verbunden sind, erfolgt mittels Mikrosimulation. Diese Methode ist auf die Abbildung der für die Untersuchung relevanten Mikroeinheiten (hier: Unternehmen) ausgerichtet und ermöglicht eine explizite Berücksichtigung der Strukturmerkmale dieser Mikroeinheiten. Insbesondere aufgrund der Heterogenität der Merkmale von Konzernen ist dieses Verfahren für die zu untersuchende Fragestellung gegenüber anderen überlegen..$^{508}$

Streng genommen sollte eine Mikrosimulation entweder für die gesamte Grundgesamtheit (Vollerhebung) oder eine repräsentative Stichprobe durchgeführt werden. ${ }^{509}$ Auch wenn beide Voraussetzungen bei Zugrundelegen der hier verwendeten Datenbank AMADEUS nicht erfullt sind ${ }^{510}$, ist davon auszugehen, dass die Anzahl der erfassten Unternehmen groß genug ist, damit die aus den Berechnungen abgeleiteten Ergebnisse Allgemeingültigkeit beanspruchen können. ${ }^{511}$

Die folgende Darstellung der Datenbasis und Methodik umfasst sechs Unterabschnitte. Nach einem Überblick über die den Berechnungen zugrundeliegende Datenbank AMADEUS (Kapitel 5.2) wird in Kapitel 5.3 das Vorgehen bei der Bereinigung und Aufbereitung der Datenbasis sowie der Abbildung der Konzernkreise skizziert. Die verbleibenden Abschnitte sind dem Vorgehen bei der Ermittlung der Steuerzahlungen de lege lata und de lege ferenda (Kapitel 5.4) sowie der Ermittlung des Steueraufkommens (Kapitel 5.5) und der durchschnittlichen Steuerbelastung (Kapitel 5.6) gewidmet.

$508 \mathrm{Vgl}$. Oestreicher/Scheffler/Spengel et al. (2008), S. 373. Eine Gegenüberstellung verschiedener Simulationsansätze zur Evaluierung von Steuerreformen findet sich bei Peichl (2006).

$509 \mathrm{Vgl}$. Oestreicher/Scheffler/Spengel et al. (2008), S. 373.

510 So enthält die Datenbank weder sämtliche Unternehmen noch erfullt die Auswahl die Voraussetzungen einer Zufallsstichprobe, da über die Aufnahme eines Unternehmens die Verfügbarkeit bestimmter Mindestinformationen entscheidet. Vgl. Oestreicher/Scheffler/Spengel et al. (2008), S. 373-374.

$511 \mathrm{Vgl}$. Oestreicher/Scheffler/Spengel et al. (2008), S. 373-374. 


\subsection{Inhalt und Umfang der verwendeten Datenbasis}

Zur Abschätzung der Wirkungen, die mit der Einführung einer grenzüberschreitenden Gruppenbesteuerung oder GKKB auf die Steuerbelastung der Konzerne sowie das Steueraufkommen der Mitgliedsstaaten verbunden sind, sollte idealerweise auf originär steuerliche Daten zurückgegriffen werden. ${ }^{512}$ Dieses würde für die beabsichtigte Untersuchung einen Zugang zu den Steuerstatistiken sämtlicher Mitgliedsstaaten voraussetzen. Zudem müsste eine Verknüpfung mit externen Datenquellen (z.B. Angaben über Beteiligungsbeziehungen zwischen den Unternehmen) möglich sein. Vor dem Hintergrund der Beschränkungen, welche beim Zugang zu steuerlichen Daten üblicherweise bestehen, wird die EU-weite $\mathrm{Zu}$ sammenführung steuerlicher Daten in einer gemeinsamen Datenbank für nicht realistisch erachtet. ${ }^{513}$ Diese Probleme bei der Beschaffung einer geeigneten Datenbasis erklären auch die geringe Verbreitung von Mikrosimulationsmodellen im Bereich der Unternehmensbesteuerung. ${ }^{514}$ Alternativ bietet sich die Verwendung publizierter Daten aus handelsrechtlichen Jahres- und Konzernabschlüssen an. $\mathrm{Zu}$ diesem Zweck basieren die im Rahmen dieser Arbeit erfolgenden Simulationsrechnungen auf Daten aus der Datenbank AMADEUS. Sie erscheint mit Blick auf die Untersuchungsziele insgesamt am besten geeignet. ${ }^{515}$

AMADEUS ist eine Unternehmensdatenbank, die in dem hier verwendeten Update $^{516}$ Angaben zu mehr als acht Millionen privater und öffentlicher Unternehmen aus 38 europäischen Ländern beinhaltet. Berücksichtigung finden dabei grundsätzlich alle Unternehmen, für die ,ausreichend plausible und zeitnahe Informationen verfügbar sind". ${ }^{517}$ Der durchschnittliche Erfassungsgrad der EUKapitalgesellschaften in AMADEUS beträgt im Vergleich mit der Eurostat-Statistik zur Unternehmensdemografie 74,4\% (vgl. Tabelle 12). ${ }^{518}$ Dabei zeigt sich, dass der Erfassungsgrad im Ländervergleich in einer Bandbreite von 5,9 Prozent (Litauen) und 125,1 Prozent (Estland) deutlich variiert. ${ }^{519}$

$512 \mathrm{Vgl}$. Oestreicher/Scheffler/Spengel et al. (2008), S. 374.

$513 \mathrm{Vgl}$. Oestreicher/Scheffler/Spengel et al. (2008), S. 374.

514 Soweit ersichtlich existieren bisher lediglich die folgenden Modelle: Canada's Corporation Tax Model (Kanada), TaxCoMM (ZEW, Deutschland), Biz Tax (DIW, Deutschland), DIECOFIS (Italien), MATIS (Italien) und CorpSim (Australien und Großbritannien). Diese Modelle basieren überwiegend ebenfalls auf handelsrechtlichen Daten.

$515 \mathrm{Vgl}$. so auch Oestreicher/Scheffler/Spengel et al. (2008), S. 374. Für einen Vergleich mit anderen Datenbanken vgl. Poppe (2008), S. 86-89.

516 Den Berechnungen liegt die Version AMADEUS, Stand: Februar 2005 (Update 125) zugrunde.

517 Oestreicher/Scheffler/Spengel et al. (2008), S. 376. Vgl. Poppe (2008), S. 90 für eine detaillierte Auflistung der Einbeziehungsvoraussetzungen.

518 Vgl. Poppe (2008), S. 91.

519 Werte von mehr als 100 Prozent sollten insbesondere auf zeitliche Differenzen der Beobachtungen sowie Ungenauigkeiten bei der Abgrenzung von Personen- und Kapitalgesellschaften zurückzuführen sein. Vgl. Poppe (2008), S. 91. 
Tabelle 12: Erfassungsgrad europäischer Kapitalgesellschaften in AMADEUS

\begin{tabular}{|c|c|c|c|c|c|}
\hline \multirow[t]{2}{*}{ Land } & \multicolumn{3}{|c|}{ Eurostat $^{520} /$ Destatis } & \multirow{2}{*}{$\begin{array}{c}\text { AMADEUS, } \\
\text { Stand } \\
\text { Feb. } 2005\end{array}$} & \multirow[t]{2}{*}{ Anteil in \% } \\
\hline & Jahr & Anzahl & Erfassungsgrad & & \\
\hline Dänemark & 2001 & 74.816 & $\sim 99 \%$ & 88.331 & $118,1 \%$ \\
\hline Deutschland & 2001 & $650.522^{521}$ & $100 \%$ & 554.902 & $85,3 \%$ \\
\hline Estland & 2002 & 30.740 & $\sim 99 \%$ & 38.468 & $125,1 \%$ \\
\hline Finnland & 2003 & 116.244 & $\sim 95 \%$ & 64.659 & $55,6 \%$ \\
\hline Großbritannien & 2003 & 1.137 .870 & $\begin{array}{c}\sim 750.000 \text { nicht } \\
\text { erfasst }\end{array}$ & 1.324 .558 & $116,4 \%$ \\
\hline Italien & 2002 & 584.093 & sehr gut & 242.261 & $41,5 \%$ \\
\hline Lettland & 2002 & 27.531 & k.A. & 5.015 & $18,2 \%$ \\
\hline Litauen & 2002 & 28.099 & $\sim 80-83 \%$ & 1.923 & $6,8 \%$ \\
\hline Luxemburg & 2002 & 18.343 & $\sim 99 \%$ & 1.084 & $5,9 \%$ \\
\hline Niederlande & 2001 & 221.653 & $\sim 99 \%$ & 189.925 & $85,7 \%$ \\
\hline Portugal & 2002 & 263.107 & k.A. & 55.757 & $21,2 \%$ \\
\hline Schweden & 2002 & 246.260 & $\sim 99 \%$ & 218.698 & $88,8 \%$ \\
\hline Slowakei & 2003 & 65.197 & $\sim 99 \%$ & 3.551 & $5,4 \%$ \\
\hline Slowenien & 2002 & 28.537 & $100 \%$ & 22.063 & $77,3 \%$ \\
\hline Spanien & 2002 & 959.657 & $\sim 99 \%$ & 674.533 & $70,3 \%$ \\
\hline Tsch'e Republik & 2003 & 135.435 & $\sim 99 \%$ & 14.476 & $10,7 \%$ \\
\hline Ungarn & 2002 & 127.789 & $100 \%$ & 80.421 & $62,9 \%$ \\
\hline Summe & & 4.065 .371 & & 3.025 .723 & $74,4 \%$ \\
\hline
\end{tabular}

Quelle: Poppe (2008), S. 91.

In sachlicher Hinsicht umfasst AMADEUS ein Unternehmensprofil, welches neben einer Identifikationsnummer auch aus Angaben über die Rechtsform sowie den Tätigkeitsbereich der Unternehmen besteht. Die Tätigkeit der Unternehmen wird zum einen verbal beschrieben und zum anderen entsprechend nationaler Nomenklaturen sowie der vierstelligen Wirtschaftszweigsystematik der Europäischen Gemeinschaft („NACE 1.1“) klassifiziert. Für eine möglichst genaue Beschreibung der Tätigkeit werden in AMADEUS für die Erfassungen der beiden Hauptgeschäftsfelder eines Unternehmens ein primärer Branchencode (,primary code") und ein sekundärer Branchencode (,secondary code") unterschieden. Ferner werden in AMADEUS die Bilanzen und Gewinn- und Verlustrrechnungen aus

520 Abrufbar unter http://epp.eurostat.ec.europa.eu/.

521 Die Angaben für Deutschland stammen aus der Kërperschaftsteuerstatistik 2091 
den handelsrechtlichen Einzel- und/oder Konzernabschlüssen sowie Informationen zu Anteilseignern und Beteiligungen des Unternehmens ausgewiesen.

\subsection{Aufbereitung der Mikrodaten}

\subsubsection{Jahresabschlussinformationen}

\subsubsection{Angaben in AMADEUS}

Das hier verwendete Update der AMADEUS-Datenbank beinhaltet Finanzinformationen aus Jahresabschlüssen für die Geschäftsjahre 1994 bis 2004. Für die früheren Jahre und das Jahr 2004 ist die Anzahl der enthaltenen Jahresabschlüsse allerdings gering. Mit Blick auf das Jahr 2004 ist dieser geringe Erfassungsgrad darauf zurückzuführen, dass betreffende Jahresabschlüsse zum Zeitpunkt des Updates (Februar 2005) in der Mehrzahl noch nicht veröffentlicht waren. Aus diesem Grund wird der Betrachtungszeitraum für die Simulationsrechnungen auf die Jahre 1994 bis 2003 begrenzt.

Hinsichtlich des verfügbaren Umfangs an Finanzinformationen werden die Unternehmen in AMADEUS in fünf Klassen eingeteilt. Für die Mehrzahl der europäischen Unternehmen (74,41 Prozent) sind ausschließlich unkonsolidierte Abschlüsse hinterlegt, für 0,48 Prozent der Unternehmen ausschließlich konsolidierte Konzernabschlüsse, während für weitere 0,48 Prozent der Unternehmen sowohl konsolidierte als auch unkonsolidierte Abschlüsse verfügbar sind. Für die verbleibenden Unternehmen sind entweder überhaupt keine finanziellen Informationen vorhanden (12,25 Prozent) oder nur in sehr geringem Umfang (12,39 Prozent). ${ }^{522}$ Bei den letztgenannten Unternehmen beschränken sich die Angaben zumeist auf die Umsatzerlöse und die Anzahl der Mitarbeiter. Auch wenn der Erfassungsgrad der Jahresabschlüsse über alle europäischen Unternehmen mit 75 Prozent sehr gut ist, muss bei der weiteren Analyse berücksichtigt werden, dass dieser sich zwischen den einzelnen Mitgliedsstaaten deutlich unterscheidet. ${ }^{523}$

Die Bilanzen und Gewinn- und Verlustrechnungen in AMADEUS werden von nationalen Datenanbietern bereitgestellt und nach Maßgabe fester Überleitungstabellen in ein europaweit einheitlich aggregiertes Format überführt. Diese Standardisierung erleichtert die Weiterverarbeitung für die Erstellung eines europaweiten Mikrosimulationsmodells. Allerdings ist darauf hinzuweisen, dass AMADEUS nicht $\mathrm{zu}$ entnehmen ist, nach welchen Rechnungslegungsstandards diese Abschlüsse erstellt wurden ${ }^{524}$, so dass für die folgende Analyse davon ausgegangen werden muss, dass die Abschlüsse auf nationalen Rechnungslegungsvor-

522 Quelle: Eigene Berechnungen auf Basis der Angaben in AMADEUS.

523 Vgl. hierzu auch Kapitel 5.3.1.3.

524 Vgl. zu diesem Problem auch Poppe (2008), S. 192eilfa3 Koch - 978-3-631-75683-6 
schriften basieren und daher eine geeignete Ausgangsbasis für die Ermittlung des Steuerbilanzgewinns bilden. Auf der anderen Seite führt die zuvor angesprochene Aggregation zu einem Verlust an Detailinformationen. Problematisch für die Steuerberechnung ist hierbei insbesondere die fehlende Trennung von Zinserträgen und Dividendenerträgen. Für Kontrollzwecke wäre ferner eine separate Angabe von Steuerzahlungen und latentem Steueraufwand hilfreich gewesen. Tabelle 13 gibt die Struktur der Jahresabschlussinformationen in AMADEUS wieder.

Tabelle 13: Struktur der Jahresabschlussinformationen in AMADEUS

\begin{tabular}{|c|c|c|c|}
\hline Nummer & $\begin{array}{c}\text { Positionsbezeichnung in AMA- } \\
\text { DEUS }\end{array}$ & Ermittlung & $\begin{array}{c}\text { In dieser Arbeit } \\
\text { verwendete Ab- } \\
\text { kürzung }\end{array}$ \\
\hline \multicolumn{4}{|c|}{ Gewinn- und Verlustrechnung } \\
\hline 1 & Operating Revenue & $\begin{array}{l}\text { Turnover and stock move- } \\
\text { ments as well as other capita- } \\
\text { lised costs }\end{array}$ & opre \\
\hline 2 & Thereof: Sales & & \\
\hline 3 & Costs of goods sold & & \\
\hline 4 & Gross profit & $1-3$ & \\
\hline 5 & Other operating expenses & & \\
\hline 6 & Operating profit/loss & $4-5$ & \\
\hline 7 & Financial Revenue & & fire \\
\hline 8 & Financial Expenses & & \\
\hline 9 & Thereof: interest paid & & \\
\hline 10 & Financial profit/loss & $7-8-9$ & \\
\hline 11 & Profit/loss before tax & $6+10$ & plbt \\
\hline 12 & Taxation & Income taxes and other taxes & \\
\hline 13 & Profit/loss after tax & $11-12$ & \\
\hline 14 & Extraordinary revenue & & \\
\hline 15 & Extraordinary expenses & & \\
\hline 16 & $\begin{array}{l}\text { Extraordinary and other prof- } \\
\text { it/loss }\end{array}$ & $14-15$ & \\
\hline 17 & Profit/loss for period & $13+16$ & plfp \\
\hline \multicolumn{4}{|l|}{ Bilanz } \\
\hline 1 & Fixed assets & $2+3+4$ & \\
\hline 2 & Intangible fixed assets & & ifas \\
\hline 3 & Tangible fixed assets & & tfas \\
\hline 4 & Other fixed assets & & \\
\hline 5 & Current assets & $6+7+8$ & \\
\hline 6 & Stocks & & stocks \\
\hline 7 & Debtors & & \\
\hline 8 & Other current assets & & \\
\hline
\end{tabular}




\begin{tabular}{|l|l|l|l|}
\hline 9 & Total assets & $1+5$ & \\
\hline & & & \\
\hline 10 & Shareholders funds & $11+12$ & E \\
\hline 11 & Capital & & \\
\hline 12 & $\begin{array}{l}\text { Other shareholder funds (incl. } \\
\text { reserves) }\end{array}$ & & \\
\hline 13 & Non-current liabilities & $14+15$ & ltl \\
\hline 14 & Long-term debt & & Itd \\
\hline 15 & $\begin{array}{l}\text { Other non-current liabilities (incl. } \\
\text { provisions) }\end{array}$ & oltl \\
\hline 16 & Current liabilities & $17+18+19$ & \\
\hline 17 & Loans & & \\
\hline 18 & Creditors & & \\
\hline 19 & Other current liabilities & & \\
\hline 20 & $\begin{array}{l}\text { Total shareholder funds and } \\
\text { liabilities }\end{array}$ & $10+13+16$ & \\
\hline
\end{tabular}

Quelle: Bureau van Dijk, Correspondence Table zur Dafne-Datenbank.

\subsubsection{Bereinigung und Aufbereitung}

Für die Berechnung der Steuerzahlungen im System der GKKB werden Informationen aus der Gewinn- und Verlustrechnung sowie der Bilanz benötigt, sodass Jahresabschlüsse nur dann Berücksichtigung finden, wenn Informationen zu beiden Rechnungen in AMADEUS enthalten sind. Zielsetzung bei der Aufbereitung dieser Informationen ist dementsprechend neben einer Beseitigung von Fehlinformationen auch eine Vervollständigung der Datenbasis, wo diese unzweifelhaft möglich ist.

Bei der Aufbereitung der Bilanz wird in drei Schritten vorgegangen. In einem ersten Schritt werden fehlende Werte berechnet, die sich eindeutig aus der Obersumme und/oder den übrigen zur selben Oberposition gehörigen Werten ergeben. Hierbei werden zunächst fehlende Werte für die Zwischensummen („Fixed assets", „Current assets", „Shareholder funds", „Non-current liabilities" und "Current liabilities") mit ihren jeweiligen Unterpositionen und anschließend fehlende Werte für die Bilanzsumme („Total assets“, „Total shareholder funds and liabilities") mit den dazugehörigen Zwischensummen ergänzt. Wurden Änderungen an einer Bilanz vorgenommen, wird Anpassungsschritt I erneut durchlaufen.

In einem zweiten Schritt werden die Bilanzangaben auf ihre Richtigkeit überprüft und entsprechend angepasst. Hierbei wird zunächst die Übereinstimmung der Bilanzsumme auf Aktiv- und Passivseite geprüft. Sich ergebende Abweichungen können nur anhand der Zwischensummen der einen oder der anderen Seite ursächlich zugeordnet werden. Ist eine derartige Anpassung der Bilanzsumme anhand der Zwischensummen nicht möglich, wird die Bilanz vollständig 
gelöscht soweit die Abweichung materiell bedeutsam ist, während der Höhe nach unbedeutsame Abweichungen ignoriert werden (Anpassungsschritt Ila). Dabei wird eine Abweichung als bedeutsam eingestuft, wenn diese zehn Prozent des Mittelwerts der beiden Bilanzsummen übersteigt. Abweichungen zwischen den Zwischensummen und der Bilanzsumme (Anpassungsschritt Ilb) sowie zwischen den Unterpositionen und den Zwischensummen (Anpassungsschritt IIc) werden durch proportionale Erhöhung oder Verminderung der Zwischensummen (IIb) beziehungsweise der Unterpositionen (IIc) beseitigt.

Tabelle 14: Aufbereitung der Jahresabschlussinformationen (Bilanz)

\begin{tabular}{|c|c|c|c|c|c|c|}
\hline \multirow{2}{*}{\multicolumn{2}{|c|}{ Bilanzposition }} & \multicolumn{2}{|c|}{ Anpassungsschritt I } & \multicolumn{2}{|r|}{ Anpassungsschritt II } & \multirow{2}{*}{$\begin{array}{c}\text { Anp.schritt III } \\
\text { neg. Werte } \\
\end{array}$} \\
\hline & & Ia & Ib & $11 \mathbf{a}$ & IIb//Ilc & \\
\hline 1 & Fixed assets & $=2+3+4$ & $=9-5$ & \multirow{20}{*}{ 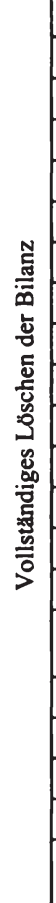 } & $=9 * 1 /(1+5)$ & Löschen: $1-8$ \\
\hline 2 & Intangible fixed assets & $=1-3-4$ & & & $=1 * 2 /(2+3+4)$ & Löschen: 2-4 \\
\hline 3 & Tangible fixed assets & $=1-2-4$ & & & $=1 * 3 /(2+3+4)$ & Loschen: $2-4$ \\
\hline 4 & Other fixed assets & $=1-2-3$ & & & $=1 * 4 /(2+3+4)$ & Loschen: $2-4$ \\
\hline 5 & Current assets & $=6+7+8$ & $=9-1$ & & $=9 * 5 /(1+5)$ & Löschen: 1-8 \\
\hline 6 & Stocks & $=5-7-8$ & & & $=5 * 6 /(6+7+8)$ & Löschen: 6-8 \\
\hline 7 & Debtors & $=5-6-8$ & & & $=5 * 7 /(6+7+8)$ & Löschen: 6-8 \\
\hline 8 & Other current assets & $=5-6-7$ & & & $=5 * 8 /(6+7+8)$ & Löschen: 6-8 \\
\hline 9 & Total assets & & $=1+5$ & & & \\
\hline 10 & Shareholders funds & $=11+12$ & $=20-13-16$ & & $=20 * 10 /(10+13+16)$ & Loschen: $10-19$ \\
\hline 11 & Capital & $=10-12$ & & & $=10 * 11 /(11+12)$ & Löschen: 11-12 \\
\hline 12 & Other shareholder funds & $=10-11$ & & & $=10 * 12 /(11+12)$ & Löschen: 11-12 \\
\hline 13 & Non-current liabilities & $=14+15$ & $=20-10-16$ & & $=20 * 13 /(10+13+16)$ & Loschen: 10-19 \\
\hline 14 & Long-term debt & $=13-15$ & & & $=13 * 14 /(14+15)$ & Losschen: 14-15 \\
\hline 15 & $\begin{array}{l}\text { Other non-current lia- } \\
\text { bilities }\end{array}$ & $=13-14$ & & & $=13 * 15 /(14+15)$ & Loschen: 14-15 \\
\hline 16 & Current liabilities & $=17+18+19$ & $=20-10-13$ & & $=20 * 16 /(10+13+16)$ & Löschen: 10-19 \\
\hline 17 & Loans & $=16-18-19$ & & & $=16 * 17 /(17+18+19)$ & Loschen: 17-19 \\
\hline 18 & Creditors & $=16-17-19$ & & & $=16 * 18 /(17+18+19)$ & Löschen: 17-19 \\
\hline 19 & Other current liabilities & $=16-17-18$ & & & $=16 * 19 /(17+18+19)$ & Löschen: 17-19 \\
\hline 20 & $\begin{array}{l}\text { Total shareholder funds } \\
\text { and liabilities }\end{array}$ & & $=10+13+16$ & & & \\
\hline
\end{tabular}

Quelle: Eigene Darstellung

Abschließend werden negative Werte für Bilanzpositionen gelöscht, soweit diese außerhalb des Eigenkapitals auftreten, da davon auszugehen ist, dass es sich hierbei um fehlerhafte Einträge handelt. Mögliche Ursache hierfür können Fehlzuordnungen innerhalb einer Zwischensumme oder einer Obersumme sein. Aus diesem Grund werden bei negativen Werten einer Unterposition sämtliche Unter- 
positionen derselben Zwischensumme gelöscht, während bei negativen Werten einer Zwischensumme sämtliche Positionen der Bilanzseite mit Ausnahme der Bilanzsumme entfernt werden. Eine Zusammenfassung dieser Anpassungsschritte für die Bilanzpositionen gibt Tabelle 14.

Analog zur Vorgehensweise bei der Bilanz, werden auch bei der Gewinnund Verlustrechnung zunächst fehlende Werte ergänzt, die sich eindeutig aus anderen Werten der Gewinn- und Verlustrechnung ergeben (Anpassungsschritt Ia und Ib). Diese Anpassungsschritte werden erneut durchlaufen, wenn Änderungen an der Gewinn- und Verlustverrechnung vorgenommen wurden. Für wenige Unternehmen sind lediglich die Umsatzerlöse (,Sales“), nicht aber die operativen Erträge („Operating revenue“) verfügbar. In diesem Fall wird den operativen Erträgen als bestmögliche Approximation der Wert der Umsatzerlöse zugewiesen (Anpassungsschritt Ic).

Tabelle 15: Aufbereitung der Jahresabschlussinformationen (Gewinn- und Verlustrechnung)

\begin{tabular}{|c|c|c|c|c|c|c|}
\hline \multicolumn{2}{|r|}{ GuV-Position } & \multicolumn{3}{|c|}{ Anpassungsschritt I } & \multirow[t]{2}{*}{ Anpassungsschritt } & \multirow{2}{*}{$\begin{array}{c}\text { Anpassungsschritt } \\
\text { Negative Werte }\end{array}$} \\
\hline & & Ia & Ib & Ic & & \\
\hline 1 & Operating Revenue & $=4+3$ & & $=2$ & \multirow{16}{*}{ 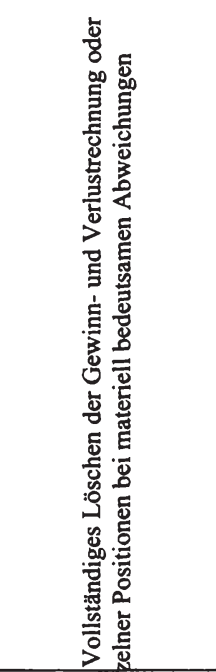 } & $3=3-1$ \\
\hline 2 & Thereof: Sales & & & & & \\
\hline 3 & Costs of goods sold & $=1-4$ & & & & $1=1-3$ \\
\hline 4 & Gross profit & $=1-3$ & & & & \\
\hline 5 & Other operating expenses & $=4-6$ & & & & $1=1-5$ oder $4=4-5$ \\
\hline 6 & Operating profit/loss & $=4-5$ & $=10-9$ & & & \\
\hline 7 & Financial Revenue & $=10+8$ & & & & $8=8-7$ \\
\hline 8 & Financial Expenses & $=7-10$ & & & & $7=7-8$ \\
\hline 9 & Financial profit/loss & $=7.8$ & $=10-6$ & & & \\
\hline 10 & Profit/loss before tax & $=12-11$ & $=6+9$ & & & \\
\hline 11 & Taxation & $=10-12$ & & & & \\
\hline 12 & Profit/loss after tax & $=10-11$ & $=16-15$ & & & \\
\hline 13 & Extraordinary revenue & $=15+1$ & & & & $14=14-13$ \\
\hline 14 & Extraordinary expenses & $=13-15$ & & & & $13=13-14$ \\
\hline 15 & $\begin{array}{l}\text { Extraordinary and other } \\
\text { profit/loss }\end{array}$ & $=13-14$ & $=16-12$ & & & \\
\hline 16 & Profit/loss for period & $=12+1$ & $=12+1$ & & & \\
\hline
\end{tabular}

Quelle: Eigene Darstellung

In einem zweiten Schritt werden auch bei der Gewinn- und Verlustrechnung Datenfehler eliminiert. Im Unterschied zur Bilanz ist eine hierarchische Vorgehensweise hierbei nicht möglich, sodass der Ursprung einer bestehenden Abweichung nicht zweifelsfrei bestimmt werden kann. So kann die Ursache einer Abweichung zwischen der Summe aus „Operating profit/loss“ (6) und „Financial profit/loss“ (9) auf der einen Seite und „Profit/loss before tax“(10) auf der ande- 
ren Seite in Fehlangaben für jede dieser drei Positionen liegen. Aufgrund der zentralen Bedeutung der Position „Profit/Loss before tax“ für die Ermittlung der Steuerzahlungen wird ein Jahresabschluss vollständig gelöscht, wenn diese Position fehlt (und nicht in Anpassungsschritt I ergänzt werden konnte) oder wenn sich eine Abweichung von mehr als 10 Prozent zwischen dieser Position auf der einen Seite und der Summe der Positionen 6 und 9 oder der Differenz der Positionen 12 und 11 auf der anderen Seite ergibt. Sich ergebende Differenzen zwischen Einzelpositionen und anderen Zwischensummen, die mehr als zehn Prozent des Betrags der Zwischensumme ausmachen, führen zur Eliminierung einzelner Positionen und/oder Zwischensummen. Für Abweichungen von weniger als zehn Prozent erfolgen grundsätzlich keine Anpassungen.

In Anpassungsschritt III werden Anpassungen für negative Werte bei Aufwands- und Ertragspositionen vorgenommen. Hierbei wird vorzugsweise der betreffenden Position der Wert null zugewiesen und eine hiermit in Zusammenhang stehende Aufwandsposition (bei negativen Werten einer Ertragsposition), Ertragsposition (bei negativen Werten einer Aufwandsposition) oder Zwischensumme (bei negativen Werten einer Ertragsposition oder Aufwandsposition) entsprechend erhöht. Ist dieses mangels Wertangaben oder bei negativen Wertangaben für die zu erhöhende Position nicht möglich wird der Wert der negativen Position gelöscht.

\subsubsection{Ergänzung fehlender Angaben für die Formelfaktoren}

Für die Ermittlung der Steuerzahlungen nach dem Konzept der GKKB werden neben dem Ergebnis der gewöhnlichen Geschäftstätigkeit auch die Angaben für alle relevanten Formelfaktoren benötigt. Unter Berücksichtigung einer alternativen Definition des Arbeitsfaktors als Mitarbeiteranzahl oder Lohnsumme werden somit vier weitere Größen (Vermögen, Umsatz, Lohnsumme und Mitarbeiteranzahl) für die Einbeziehung eines Unternehmens in die Stichprobe benötigt. ${ }^{525}$ Eine restriktive Vorgehensweise bei der Auswahl der in die Simulation einzubeziehenden Unternehmen würde alle Unternehmen ausschließen, bei denen eine dieser benötigten Größen nicht in AMADEUS enthalten ist. Hiervon könnte allerdings nicht nur eine deutliche Reduktion des Stichprobenumfangs, sondern auch eine Beeinträchtigung der Repräsentativität der Unternehmensstichprobe hinsichtlich der geografischen Verteilung ausgehen, da im Extremfall auch der vollständige Ausschluss eines Landes die Folge sein kann. Alternativ könnte der Erfassungsgrad der einbezogenen Unternehmen zu Lasten der Datengenauigkeit durch Auffüllen fehlender Werte mithilfe eines durchschnittlichen Lohns oder einer geschätzten Produktionsfunktion erhöht werden.

$525 \mathrm{Vgl}$. Kapitel 5.4.2 für die genaue Definition der Aufteilungsfaktoren in den Simulationsrechnungen. 
Tabelle 16: Datenverfügbarkeit bei Unternehmen in der Stichprobe

\begin{tabular}{|c|c|c|c|c|c|c|c|c|c|}
\hline & $\begin{array}{l}\text { (1) } \\
\text { Anzahl Un- } \\
\text { ternehmen } \\
\end{array}$ & $\begin{array}{c}(2) \\
\text { plbt } \\
2002 \\
\end{array}$ & $\begin{array}{c}(3) \\
\text { opre } \\
2002 \\
\end{array}$ & $\begin{array}{c}(4) \\
\text { cempl } \\
2002 \\
\end{array}$ & $\begin{array}{c}(5) \\
\text { empl } \\
2002 \\
\end{array}$ & $\begin{array}{c}(6) \\
\text { tfas }^{526} \\
2002 \\
\end{array}$ & $\begin{array}{l}\text { (7) } \\
\text { alle }\end{array}$ & $\begin{array}{c}(8) \\
\text { cempl// } \\
\text { empl }\end{array}$ & $\begin{array}{r}(9) \\
2 \text { aus } 3 \\
\end{array}$ \\
\hline Belgien & 325.109 & $84,26 \%$ & $85,03 \%$ & $41,70 \%$ & $32,03 \%$ & $78,66 \%$ & $13,61 \%$ & $17,21 \%$ & $53,16 \%$ \\
\hline Dänemark & 144.226 & $79,63 \%$ & $80,10 \%$ & $44,01 \%$ & $38,48 \%$ & $68,95 \%$ & $11,33 \%$ & $13,92 \%$ & $49,59 \%$ \\
\hline Deutschland & 783.196 & $2,42 \%$ & $70,43 \%$ & $2,14 \%$ & $61,90 \%$ & $2,42 \%$ & $0,53 \%$ & $2,04 \%$ & $2,36 \%$ \\
\hline Estland & 50.033 & $82,98 \%$ & $82,65 \%$ & $49,49 \%$ & $50,62 \%$ & $82,98 \%$ & $42,28 \%$ & $57,19 \%$ & $71,36 \%$ \\
\hline Finnland & 69.703 & $90,94 \%$ & $90,94 \%$ & $77,52 \%$ & $54,30 \%$ & $86,72 \%$ & $50,80 \%$ & $75,84 \%$ & $87,12 \%$ \\
\hline Frankreich & 898.961 & $83,28 \%$ & $83,18 \%$ & $71,95 \%$ & $37,54 \%$ & $83,28 \%$ & $37,08 \%$ & $71,93 \%$ & $79,59 \%$ \\
\hline Griechenland & 26.769 & $88,35 \%$ & $88,35 \%$ & $0,00 \%$ & $68,67 \%$ & $88,35 \%$ & $0,00 \%$ & $68,36 \%$ & $86,53 \%$ \\
\hline Großbritannien & 1.743 .515 & $22,26 \%$ & $68,47 \%$ & $12,64 \%$ & $6,04 \%$ & $42,24 \%$ & $3,80 \%$ & $9,47 \%$ & $16,27 \%$ \\
\hline Irland & 118.788 & $13,64 \%$ & $78,13 \%$ & $0,00 \%$ & $15,03 \%$ & $54,99 \%$ & $0,00 \%$ & $1,51 \%$ & $6,07 \%$ \\
\hline Italien & 281.804 & $89,88 \%$ & $89,87 \%$ & $77,89 \%$ & $89,82 \%$ & $89,88 \%$ & $77,75 \%$ & $87,72 \%$ & $89,86 \%$ \\
\hline Lettland & 6.119 & $75,03 \%$ & $75,03 \%$ & $2,08 \%$ & $65,08 \%$ & $75,03 \%$ & $1,85 \%$ & $65,01 \%$ & $74,70 \%$ \\
\hline Litauen & 3.164 & $69,28 \%$ & $71,52 \%$ & $0,00 \%$ & $71,21 \%$ & $70,80 \%$ & $0,00 \%$ & $68,71 \%$ & $69,28 \%$ \\
\hline Luxemburg & 1.444 & $31,44 \%$ & $91,14 \%$ & $23,82 \%$ & $1,94 \%$ & $87,53 \%$ & $1,45 \%$ & $17,59 \%$ & $27,70 \%$ \\
\hline Malta & 1.733 & $66,53 \%$ & $22,85 \%$ & $2,14 \%$ & $2,77 \%$ & $66,53 \%$ & $1,44 \%$ & $3,06 \%$ & $23,20 \%$ \\
\hline Niederlande & 319.766 & $7,19 \%$ & $79,08 \%$ & $4,64 \%$ & $29,00 \%$ & $68,72 \%$ & $1,85 \%$ & $3,01 \%$ & $6,44 \%$ \\
\hline Österreich & 98.707 & $1,06 \%$ & $52,00 \%$ & $0,99 \%$ & $43,52 \%$ & $1,06 \%$ & $0,26 \%$ & $0,85 \%$ & $1,05 \%$ \\
\hline Polen & 25.968 & $64,18 \%$ & $74,17 \%$ & $48,42 \%$ & $59,75 \%$ & $63,46 \%$ & $35,96 \%$ & $59,53 \%$ & $63,55 \%$ \\
\hline Portugal & 60.850 & $46,90 \%$ & $47,10 \%$ & $43,15 \%$ & $2,31 \%$ & $46,18 \%$ & $2,27 \%$ & $42,60 \%$ & $44,99 \%$ \\
\hline Schweden & 247.269 & $87,95 \%$ & $89,75 \%$ & $66,57 \%$ & $68,71 \%$ & $82,87 \%$ & $62,84 \%$ & $66,34 \%$ & $79,94 \%$ \\
\hline Slowakei & 4.070 & $73,37 \%$ & $73,32 \%$ & $65,72 \%$ & $30,20 \%$ & $64,23 \%$ & $22,90 \%$ & $56,86 \%$ & $70,57 \%$ \\
\hline Slowenien & 31.995 & $0,00 \%$ & $99,62 \%$ & $80,25 \%$ & $9,95 \%$ & $20,15 \%$ & $0,00 \%$ & $0,00 \%$ & $0,00 \%$ \\
\hline Spanien & 706.443 & $82,81 \%$ & $84,62 \%$ & $70,88 \%$ & $58,83 \%$ & $84,26 \%$ & $57,91 \%$ & $69,96 \%$ & $77,95 \%$ \\
\hline Tsch. Republik & 15.700 & $69,58 \%$ & $69,53 \%$ & $60,95 \%$ & $65,00 \%$ & $67,43 \%$ & $57,31 \%$ & $61,67 \%$ & $67,96 \%$ \\
\hline Ungarn & 190.648 & $5,90 \%$ & $28,99 \%$ & $5,60 \%$ & $24,52 \%$ & $6,08 \%$ & $3,09 \%$ & $5,55 \%$ & $5,81 \%$ \\
\hline Zypern & 154 & $95,45 \%$ & $76,62 \%$ & $35,06 \%$ & $35,71 \%$ & $95,45 \%$ & $33,77 \%$ & $34,42 \%$ & $76,62 \%$ \\
\hline Gesamtanteil & 6.156 .134 & $46,26 \%$ & $75,39 \%$ & $34,93 \%$ & $36,48 \%$ & $55,38 \%$ & $21,73 \%$ & $32,35 \%$ & $40,42 \%$ \\
\hline Mittelwert & & $56,57 \%$ & $74,10 \%$ & $35,50 \%$ & $40,92 \%$ & $63,13 \%$ & $20,80 \%$ & $38,41 \%$ & $49,27 \%$ \\
\hline Standardabw. & & $33,63 \%$ & $18,27 \%$ & $29,68 \%$ & $24,80 \%$ & $27,68 \%$ & $24,39 \%$ & $29,96 \%$ & $31,78 \%$ \\
\hline Var.koeffizient & & $59,44 \%$ & $24,66 \%$ & $83,60 \%$ & $60,60 \%$ & $43,84 \%$ & $117,2 \%$ & $78,00 \%$ & $64,51 \%$ \\
\hline
\end{tabular}

Quelle: Eigene Berechnungen auf Basis der Angaben in AMADEUS.

526 Aus Vereinfachungsgründen wird (im Unterschied zu den Berechnungen) an dieser Stelle nur die Position "Tangible Fixed Assets" als Repräsentant für den Vermögensfaktor herangezogen. 
Zur Verdeutlichung möglicher Auswirkungen der restriktiven Vorgehensweise bei der Auswahl der Unternehmen gibt Tabelle 16 zunächst den Anteil der europäischen Unternehmen in der AMADEUS-Datenbank wieder, für den die verschiedenen Größen, die für die Berechnungen benötigt werden, einzeln verfügbar sind (Spalten 2 bis 6). Dabei zeigt sich sowohl im Mittelwert der Länder als auch im Gesamtanteil aller Unternehmen eine unterdurchschnittliche Verfügbarkeit der Angaben zum Arbeitsfaktor (Gesamtanteil: 34,93\% (Lohnsumme) und 36,48\% (Mitarbeiter)). Würden alle fünf genannten Positionen als Einbeziehungsvoraussetzung zugrundegelegt, würden für vier Länder (Irland, Griechenland, Litauen, Slowenien) sämtliche Unternehmen aus der Stichprobe herausfallen (vgl. Spalte 7). Insgesamt sind nur für gut ein Fünftel (21,73 Prozent) aller europäischen Unternehmen in AMADEUS alle fünf geforderten Größen verfügbar.

Dieser Anteil ließe sich auf 32,35 Prozent erhöhen, wenn neben den drei anderen Größen nur eine der beiden Angaben für den Arbeitsfaktor für eine Einbeziehung vorausgesetzt würde (vgl. Spalte 8). In diesem Fall wäre lediglich Slowenien ohne Unternehmen in der Stichprobe, was allerdings darauf zurückzuführen

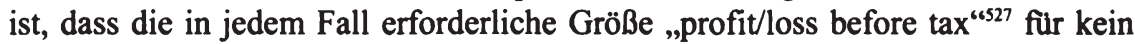
Unternehmen gegeben ist. Würde sogar die Verfügbarkeit von zwei der drei Formelfaktoren ${ }^{528}$ als ausreichend für eine Einbeziehung angesehen, ließe sich der Erfassungsgrad in der Stichprobe auf 40,42 Prozent steigern (Spalte 9). Gleichzeitig sinkt der Variationskoeffizient des Erfassungsgrades in den einzelnen Ländern deutlich $a b$, so dass eine Absenkung der Einbeziehungsvoraussetzungen gleichzeitig zu einer Steigerung der Gleichmäßigkeit und damit der Repräsentativität der Datenbasis führt. Aus diesem Grund wird in dieser Arbeit die Verfügbarkeit von zwei der drei Aufteilungsfaktoren für ausreichend angesehen.

Ausschlaggebend für die Höhe des Fehlers beim Auffüllen fehlender Formelfaktoren sind die Abweichungen zwischen den tatsächlichen Verhältnissen beim aufzufüllenden Unternehmen und den Unternehmen, die für die Ermittlung der Auffüllungsfaktoren herangezogen werden. Soweit bei der Ermittlung der Auffüllungsfaktoren nicht auf Werte aus der AMADEUS-Datenbank zurückgegriffen wird, ist weitere Voraussetzung für ein sinnvolles Auffüllen, dass die Faktoren in AMADEUS und der externen Datenquelle übereinstimmend definiert sind. Dieses kann allerdings insbesondere beim Arbeitsfaktor nicht vollständig

527 Ein Auffullen der Gewinngröße wird insbesondere mit Blick auf die Untersuchungsziele nicht fur sinnvoll erachtet. So sollte die Verwendung einer durchschnittlichen Rendite dazu führen, dass die Ergebnisgröße mit positiven Werten aufgefullt wird. Der Effekt einer Ausdehnung der steuerlichen Verlustverrechnung würde aufgrund dessen unterschătzt werden.

528 Hierbei kann der Arbeitsfaktor erneut entweder als Mitarbeiter oder als Lohnsumme gegeben sein. 
gewährleistet werden ${ }^{529}$, weshalb zum Auffüllen primär Angaben aus AMADEUS herangezogen werden.

Im Einzelnen werden in einem ersten Schritt fehlende Werte für die Lohnsumme und die Mitarbeiteranzahl durch die jeweilige Gegenposition und einen Wert für die durchschnittlichen Arbeitskosten je Mitarbeiter ergänzt. Soweit verfügbar wird hierbei auf die durchschnittlichen Arbeitskosten des Unternehmens in früheren und/oder späteren Perioden zurückgegriffen. Ersatzweise werden Durchschnittswerte verwendet, welche für die Unternehmen desselben Landes und derselben Branche ${ }^{530}$ in AMADEUS in der betreffenden Periode oder - falls nicht verfügbar - in früheren und/oder späteren Perioden ermittelt werden konnten. Fehlen entsprechende Informationen für Unternehmen der betreffenden Branche wird auf Durchschnittswerte über alle Unternehmen eines Landes zurückgegriffen. Für Litauen und Griechenland ist die Berechnung durchschnittlicher Arbeitskosten anhand der in AMADEUS enthaltenen Informationen auch ohne Branchendifferenzierung nicht möglich. Für diese Länder wird aus diesem Grund auf entsprechende Angaben aus der Arbeitskräfteerhebung von Eurostat ${ }^{531}$ zurückge- $^{-}$ griffen, um einen vollständigen Ausschluss der Unternehmen dieser Länder zu vermeiden.

In einem zweiten Schritt werden fehlende Angaben für einen der drei Aufteilungsfaktoren (Umsatz, Lohnsumme/Mitarbeiteranzahl, Vermögen) auf Basis der Angaben für die beiden übrigen Faktoren ergänzt. Zu diesem Zweck werden durchschnittliche Quotienten für die Werte der drei Faktorpaare (Umsatz/Lohnsumme, Umsatz/Vermögen und Lohnsumme/Vermögen) auf Basis der Angaben in AMADEUS ermittelt. Für das Auffüllen wird dabei erneut primär auf Verhältniszahlen zurückgegriffen, die für das Unternehmen selbst in früheren und/oder späteren Periode berechnet werden konnten. Soweit eine Ermittlung unternehmensspezifischer Verhältniszahlen nicht möglich ist, wird ersatzweise auf Durchschnittswerte zurückgegriffen, die sich für Unternehmen desselben Landes und derselben Branche oder desselben Landes ergeben. ${ }^{532}$

529 So kann die Arbeitnehmeranzahl z.B. als Anzahl aller beschäftigten Personen oder als Vollzeitäquivalente angegeben werden, während die Lohnsumme inklusive oder exklusive der Beiträge zur Sozialversicherung gemessen werden kann. Derartige Abweichungen können zwar auch zwischen verschiedenen Unternehmen in der AMADEUS-Datenbank auftreten, allerdings ist davon auszugehen, dass sie sich zumindest innerhalb eines Landes aufgrund einheitlicher Rechnungslegungsvorschriften und der Bereitstellung durch einen einheitlichen Datenprovider in geringerem Ausmaß ergeben.

530 Hierbei werden anhand des NACE-Branchencodes zwölf verschiedene Branchenklassen unterschieden.

531 Abrufbar unter www.eds-destatis.de.

532 Im Unterschied zur Ermittlung der Arbeitskosten wurde an dieser Stelle aus Vereinfachungsgründen auf eine Differenzierung nach Jahren verzichtet. Koch - 978-3-631-75683-6 


\subsubsection{Anteilseignerinformationen}

\subsubsection{Angaben in AMADEUS}

Angaben über Unternehmensbeziehungen werden insbesondere benötigt für die Abbildung der steuerlichen Konzernkreise de lege lata und de lege ferenda. ${ }^{533}$ Ausgangspunkt bilden hierbei die in AMADEUS enthaltenen Angaben über die Anteilseigner eines Unternehmens. Diese liegen sowohl für die direkt beteiligten Anteilseigner als auch für den obersten beherrschenden Gesellschafter („Ultimate owner“'534) vor und sind für 3.377.501 der 6.156.134 europäischen Unternehmen im AMADEUS verfügbar. ${ }^{535}$ Die Angaben umfassen neben dem Namen, der Identifikationsnummer und dem Land des Anteilseigners auch dessen Typus sowie die Höhe der direkt und insgesamt gehaltenen Anteile. Hinsichtlich des Anteilseignertypus werden in AMADEUS elf Klassen unterschieden:

Banks and financial companies, Insurance companies, Industrial companies, Mutual\&Pension funds/Nominees/Trusts/Trustees, Foundations/Research institutes, Public authorities/States/Governments, One or more named individuals or families, Employees/Managers/Directors, Public, Unnamed private shareholders, Other unnamed shareholders

Der Anteilseignertypus wird in dieser Arbeit für die Identifikation konzernrelevanter Anteilseigner zugrundegelegt. Dabei werden als konzernrelevant alle Anteilseigner eingestuft, die nicht einer der folgenden Kategorien zugehörig sind: "public authorities", „one or more named individual or families“, „employees/managers/directors“" und „public“. ${ }^{336}$ Betrachtet man lediglich Unternehmen mit konzernrelevanten Anteilseignern reduziert sich der Kreis potenzieller europäischer Tochterunternehmen auf 1.121.394. Die Gesamtanzahl der relevanten Beteiligungsbeziehungen beträgt 2.252.231, sodass an diesen Unternehmen im Durchschnitt etwa zwei Anteilseigner beteiligt sind. ${ }^{537}$

533 Für die Abgrenzung der Konzernunternehmen, deren Steuerzahlungen und Cashflows bei der Ermittlung der durchschnittlichen Steuerbelastung zusammengefasst werden (vgl. hierzu Kapitel 5.6), wird auf die Konzernkreise de lege ferenda abgestellt.

534 Als „Ultimate owner" werden in AMADEUS Anteilseigner bezeichnet, die zu mehr als 24,9 Prozent an einem Unternehmen beteiligt sind, wenn kein anderer Anteilseigner gleichzeitig mehr Anteile hält und das Kapital des „Ultimate owners“ selbst nicht zu mehr als 24,9 Prozent von einem einzelnen Anteilseigner gehalten wird. Vgl. Bureau van Dijk, Beschreibung zur Ownership Datenbank.

535 Im Unterschied zu den Jahresabschlussinformationen werden Beteiligungsinformationen in AMADEUS nur fur einen Zeitpunkt berichtet. Hierbei wird die letzte verfugbare Information zum Zeitpunkt des Updates zugrundegelegt.

536 Vgl. Poppe (2008), S. 92.

537 Vgl. Poppe (2008), S. 92. 
Als direkte Beteiligung wird die Höhe der vom Anteilseigner unmittelbar in der betreffenden Gesellschaft gehaltenen Anteile angegeben, während die Gesamtbeteiligung sowohl direkt als auch indirekt gehaltene Anteile umfasst. Letztere wird in AMADEUS nur angegeben, wenn sich diese unmittelbar aus einer verwendeten Informationsquelle ergibt. Eine Berechnung durch den Datenbankanbieter auf der Basis der Angaben zu direkten Beteiligungsbeziehungen erfolgt hingegen nicht. ${ }^{538}$ Dementsprechend ist die Ermittlungsmethode der Gesamtbeteiligung (additiv oder multiplikativ) abhängig von der in der Informationsquelle gewählten Vorgehensweise und damit uneinheitlich. Für die Abbildung von Beteiligungsstrukturen wird aus diesem Grund auf die direkten Beteiligungsketten abgestellt. ${ }^{539}$

\subsubsection{Bereinigung und Aufbereitung}

Bei der Aufbereitung der Beteiligungsinformationen und der Abbildung der Konzernkreise kann weitgehend auf die Ergebnisse früherer Arbeiten im Rahmen des in der Einleitung genannten Forschungsprojekts zurückgegriffen werden. ${ }^{540}$ Diese Schritte sind ausführlich erläutert in Poppe (2008), werden hier allerdings aus Gründen der Vollständigkeit kurz beschrieben.

Eine Aufbereitung der Beteiligungsinformationen ist aus zwei Gründen erforderlich. Zum einen dienen Anpassungen der Beseitigung von Fehlinformationen, die unzweifelhaft vorliegen, wenn die Gesamtbeteiligung der Anteilseigner an einem Unternehmen 100 Prozent übersteigt. Zum anderen dienen Anpassungen dem Auffüllen fehlender Informationen über die Beteiligungshöhe sowie der Konkretisierung dieser Informationen, wenn nur der Anteilseigner, nicht aber die Beteiligungshöhe eindeutig bekannt ist. ${ }^{541}$ Die Schritte dieser Aufbereitung sind in Tabelle 17 zusammengefasst. ${ }^{542}$

Für 45,4\% der Unternehmensverbindungen ist die Beteiligungshöhe eindeutig bekannt (X, CQPI, G), für die verbleibenden Unternehmensverbindungen erfolgt die Anpassung in drei Stufen.$^{543}$ In einem ersten Schritt wird jeder Unternehmensverbindung eine exakte Beteiligungshöhe zugewiesen. Dieser zugewiesene Wert beträgt null, wenn die Beteiligungshöhe unbekannt ist. Ist für die Beteiligungshöhe ein Mindest- oder Höchstwert bekannt, wird der Unternehmensverbindung dieser Schwellenwert zugewiesen. In Anpassungsschritt II werden die Unternehmensverbindungen gelöscht, bei denen die Beteiligung aller Anteilseigner

538 Vgl. Bureau van Dijk, Beschreibung zur Ownership Datenbank.

539 Vgl. Poppe (2008), S. 93.

540 Weitere verwendete Vorarbeiten betreffen insbesondere die Ermittlung der Konzernbranche und die Zuordnung von Klassifikation von Rechtsformen als Personen- und Kapitalgesellschaft. Vgl. hierzu ebenfalls Poppe (2008)

541 Vgl. ebenso Poppe (2008), S. 93.

542 Vgl. für eine ausführliche Beschreibung Poppe (2008), S. 92-96.

543 Vgl. Poppe (2008), S. 94.

Reinald Koch - 978-3-631-75683-6 
in Summe 100\% übersteigt. Dieses war bei 25 Unternehmen der Fall. In Anpassungsschritt III werden die der Höhe nach unbekannten (-) oder nicht exakt bekannten $(>X, M O)$ Beteiligungsverbindungen so angepasst, dass sich die Summe der Beteiligungen aller Anteilseigner auf $100 \%$ addiert. $^{544}$

Dabei kommt folgende Formel zur Anwendung: ${ }^{.45}$

(1) $\quad B_{n}=B_{a}+\frac{100-G}{A}$

mit: $\quad B_{n} \quad$ Beteiligungshöhe nach Anpassung

$B_{a} \quad$ Beteiligungshöhe vor Anpassung

G bekannte Gesamtbeteiligung vor Anpassung

A Anzahl der Anteilseigner, die angepasst werden können.

Tabelle 17: Aufbereitung der Anteilseignerinformationen

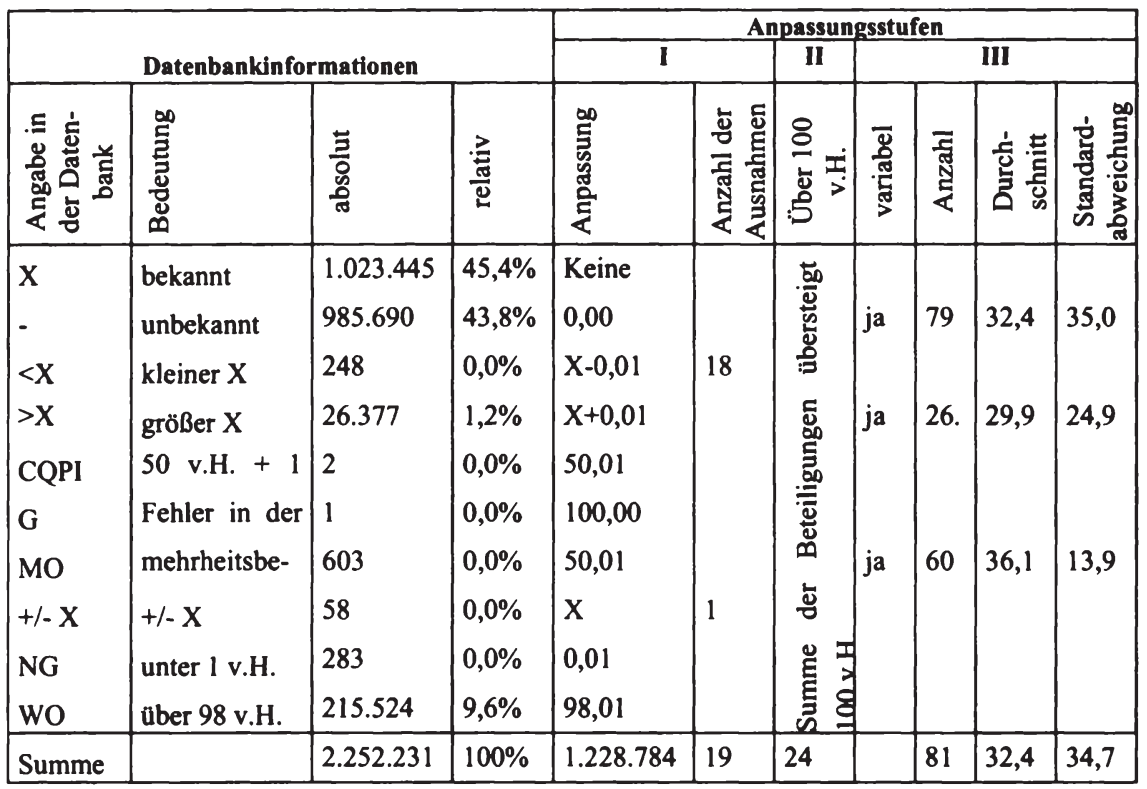

Quelle: Poppe (2008), S. 93. 


\subsubsection{Abbildung der Konzernkreise und Auswahl der Unternehmen}

\subsection{Datenbasis für Zwecke der Aufkommensberechnungen}

Einbezogen in die Analyse werden grundsätzlich alle Kapitalgesellschaften, die als Muttergesellschaft oder als Tochtergesellschaft Bestandteil eines europäischen Konzerns oder Teilkonzerns sind. Nicht-konzernverbundene Unternehmen werden hingegen nicht berücksichtigt, da die betrachteten Reformvorschläge hier keine Änderung der Steuerzahlungen hervorrufen würden. Ebenfalls ausgeschlossen werden Holdinggesellschaften, welche für Zwecke dieser Arbeit definiert werden als Gesellschaften mit einem NACE-Branchencode ${ }^{546}$ von 7415 oder mit Finanzanlagen, deren Höhe mindestens $75 \%$ des gesamten Anlagevermögens ausmacht. Diese Gesellschaften bleiben unberücksichtigt, da zum einen davon auszugehen ist, dass sie aufgrund des hohen Anteils an Beteiligungen im Regelfall über ein nur geringes steuerpflichtiges Einkommen verfügen. Zum anderen besteht die Gefahr, dass es bei diesen Gesellschaften aufgrund von pauschalen Annahmen bei der Ermittlung der (steuerfreien) Dividenden sowie der ebenfalls nicht möglichen Abschätzung von in mehreren Mitgliedsstaaten nicht steuerwirksamen Beteiligungsbuchwertabschreibungen und Erfolgen aus der Veräußerung von Beteiligungen $\mathrm{zu}$ größeren Abweichungen zwischen den geschätzten und den tatsächlichen zu versteuernden Einkommens kommt.

Bei der Abbildung der Konzernkreise ${ }^{57}$ und der Auswahl der in die Berechnungen einzubeziehenden Unternehmen ist den unterschiedlichen Untersuchungszielen der Aufkommens- und Belastungsrechnungen und den hiermit verbundenen Datenanforderungen Rechnung zu tragen. Dementsprechend wird in dieser Arbeit auf die Verwendung einer einheitlichen Datenbasis verzichtet. Vielmehr wird bei der Festlegung der Mindestanforderungen an die verfügbaren Informationen und der Abgrenzung der Konzernkreise von den Anforderungen der jeweiligen Berechnung ausgegangen, um den Aufkommensberechnungen und den Belastungsrechnungen die größtmögliche Datenbasis zugrunde legen zu können. Aus diesem Grund bezieht sich die in diesem Abschnitt erfolgende Beschreibung der Vorgehensweise bei der Abgrenzung der Konzernkreise und der Auswahl der Unternehmen nur auf die Aufkommensberechnungen. Sich ergebende Unterschiede bei der Abgrenzung für Zwecke der Belastungsrechnungen werden in Kapitel 5.3.2.3.2 erläutert.

Die Ermittlung europäischer Konzerne und Teilkonzerne, welche den Aufkommensberechnungen zugrundegelegt werden, erfolgt in drei Schritten. In einem ersten Schritt werden sämtliche europäischen Kapitalgesellschaften, die von einer

546 Klassifikation erfolgt entsprechend NACE $1.1 \mathrm{rev}$.

547 Die folgenden Ausführungen beziehen sich auf die Abbildung der Konzernkreise de lege ferenda. Für die Konzernkreise de lege lata wird eine vergleichbare Vorgehensweise gewählt, allerdings die landesspezifischen Anwendungsveraussetzpngen zygundeggelegt 683-6 
gemeinsamen europäischen Kapitalgesellschaft beherrscht werden mit dieser beherrschenden Kapitalgesellschaft $\mathrm{zu}$ einer Unternehmensgruppe zusammengefasst. Beherrschung wird dabei definiert als eine direkte und/oder indirekte ${ }^{548} \mathrm{Ka}$ pitalbeteiligung von mindestens 50 Prozent an der beherrschten Gesellschaft. Dieser Schritt ist ausführlich beschrieben bei Poppe (2008). ${ }^{549}$

Aufgrund der Begrenzung des Umfangs der AMADEUS Datenbank auf europäische Unternehmen sind nur sehr begrenzte Informationen über die Beteiligung von nicht-europäischen Mutter- oder Zwischengesellschaften verfügbar. Dieses hat zur Folge, dass mit dem in Schritt 1 gewählten Verfahren mehrere europäische Untergruppen einer gemeinsamen nicht-europäischen Muttergesellschaft getrennt erfasst werden. Ebenso kommt es zu einer Unterbrechung der Konzernkette bei Zwischenschaltung einer nicht-europäischen Gesellschaft. Aus diesem Grund werden in einem zweiten Schritt europäische Teilkonzerne zusammengefasst, wenn für die Spitzeneinheiten dieser Teilkonzerne in AMADEUS eine gemeinsame Dachgesellschaft („Ultimate owner") ausgewiesen ist. Dabei muss berücksichtigt werden, dass für die Definition des "Ultimate owners" in AMADEUS eine Beteiligungsschwelle von 25 Prozent zugrundegelegt wird, weshalb dieser nicht notwendigerweise gleichzusetzen ist mit einer Muttergesellschaft entsprechend der in dieser Arbeit unterstellten Konzerndefinition. Aus diesem Grund wird die Zusammenführung von Teilkonzernen manuell auf Fälle begrenzt, in denen die Spitzeneinheiten der Teilkonzerne über einen gemeinsamen „Ultimate owner" verfügen und eine große Ähnlichkeit der Namen der europäischen Spitzeneinheiten und des „Ultimate owners“ besteht.

In einem dritten Schritt werden diejenigen Konzerne ausgeschlossen, bei denen nur für höchstens eine Gesellschaft die erforderlichen Jahresabschlussinformationen verfügbar sind. Mit diesem Schritt werden Unternehmen aus der Datenbasis entfernt, bei denen eine Reform der Konzernbesteuerung sich aufgrund der Zugehörigkeit zu einem Konzern zwar in der Realität auswirken dürfte, bei denen sich diese Wirkungen aufgrund der unvollständigen Datenverfügbarkeit aber in den Berechnungen nicht zeigen können.

Verfügbarkeit der erforderlichen Jahresabschlussinformationen bedeutet in diesem Zusammenhang, dass die für die Berechnungen erforderlichen Finanzinformationen für mindestens ein Jahr des Betrachtungszeitraums (1994 bis 2003) in AMADEUS enthalten sind. Hierzu zählt das Ergebnis der gewöhnlichen Geschäftstätigkeit (plbt), welches als Ausgangsgröße zur Berechnung des steuerlichen Einkommens verwendet wird. Ferner müssen zur Abbildung der Wirkungen, die aus der Einfuhrung einer GKKB resultieren, auch die für die Ermittlung der Formelfaktoren erforderlichen Informationen bekannt sein. Dieses sind im Einzelnen für den Umsatzfaktor die betrieblichen Erträge (opre), für den Arbeitsfaktor die Mitarbeiteranzahl (empl) und die Lohnsumme (cempl) und für den Ver-

548 Direkt und indirekt gehaltene Anteile werden dabei ohne multiplikative Verknüpfung addiert.

549 Vgl. Poppe (2008), S. 116-121. 
mögensfaktor die Positionen Sachanlagevermögen (tfas), immaterielles Anlagevermögen (ifas) und Vorräte (stocks). ${ }^{550}$ Wie in Kapitel 5.3.1.3 erläutert ist dabei allerdings die Verfügbarkeit von Angaben für zwei der drei Formelfaktoren ausreichend. Tabelle 18 fasst die geografische Zusammensetzung der den Aufkommensberechnungen zugrundegelegten Datenbasis zusammen. Nationale Konzerne bezeichnen dabei Konzerne mit ausschließlich inländischen Gesellschaften, während multinationale Konzerne über wenigstens eine ausländische Tochtergesellschaft verfügen.

Tabelle 18: Geografische Zusammensetzung der Stichprobe (Aufkommensberechnungen) ${ }^{551}$

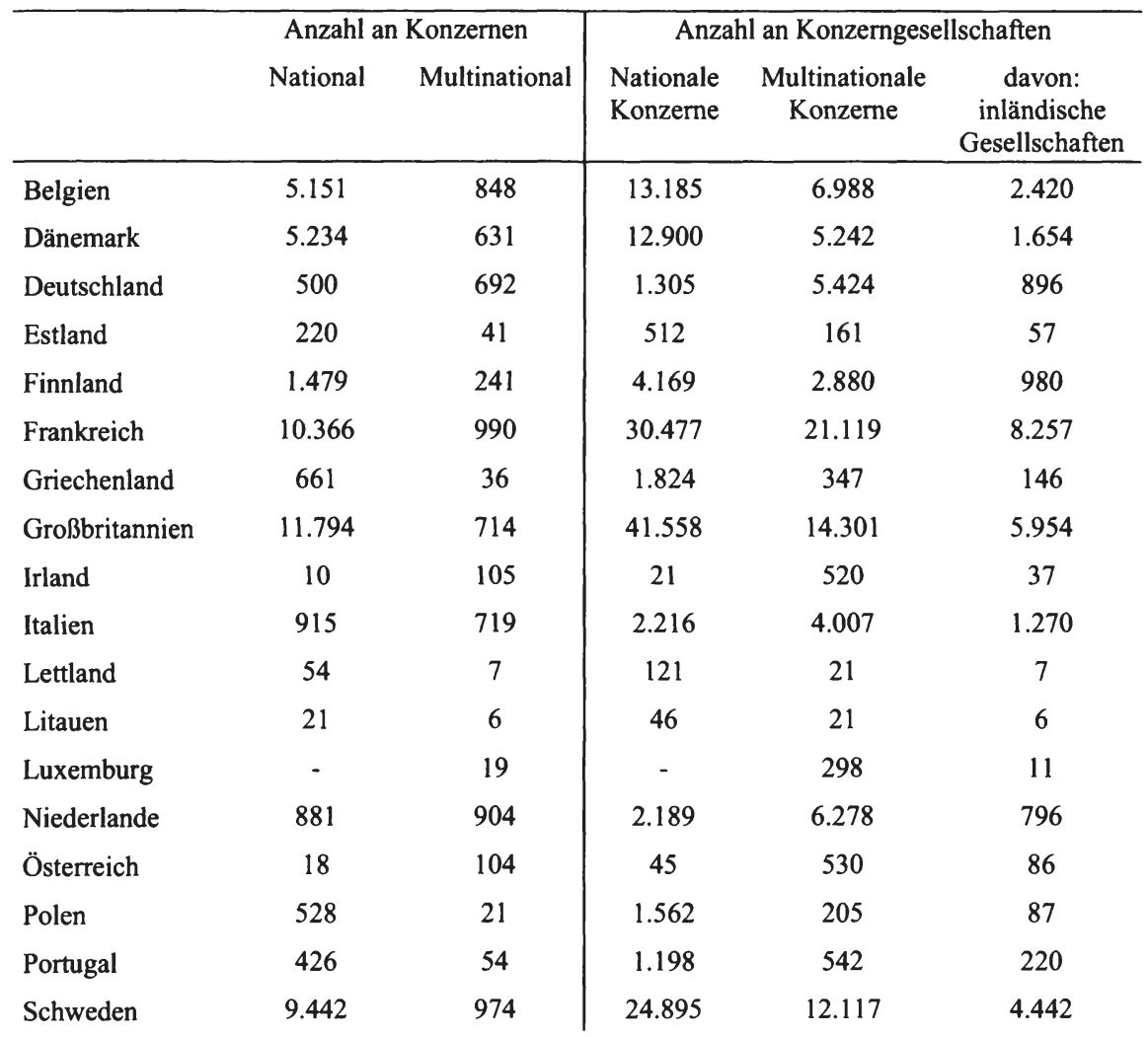

$550 \mathrm{Vgl}$. für die Simulation der Steuerberechnung nach dem System der GKKB auch Kapitel 5.4.2.

551 Nicht berücksichtigt in den Berechnungen wurden Unternehmen aus Bulgarien und Rumänien, da diese zum Zeitpunkt der Berechnungen nicht Bestandteil der EU waren sowie Unternehmen aus Malta und Slowenien, da AMADEUS für diese Länder bei keinem Unternehmen die erforderlichen Mindestinformationen bereitstellt 


\begin{tabular}{lcc|ccc} 
Slowakei & - & 2 & - & 10 & 2 \\
Spanien & 8.561 & 510 & 24.684 & 7.118 & 3.047 \\
Tsch'e Republik & 106 & 30 & 246 & 217 & 90 \\
Ungarn & 409 & 7 & 1.034 & 78 & 31 \\
Zypern & 2 & 5 & 5 & 20 & 3 \\
Nicht-EU & - & 802 & - & 5.277 & - \\
\hline Gesamt & 56.778 & 8.462 & 164.192 & 93.721 & 30.499 \\
\hline
\end{tabular}

Quelle: Eigene Berechnung auf Basis der Angaben in AMADEUS

\subsection{Datenbasis für Zwecke der Belastungsrechnungen}

Die Datenbasis, die den Berechnungen der Belastungswirkungen zugrundegelegt wird, unterscheidet sich von derjenigen für die Aufkommensberechnungen in dreifacher Hinsicht. Erstens wird bei der Abgrenzung der Konzernkreise auf Anpassungsschritt 2 verzichtet. Die Zusammenführung verschiedener europäischer Teilkonzerne führt zu einer Berücksichtigung von Konzernen nicht-europäischer Muttergesellschaften in der Datenbasis (vgl. Tabelle 18). Dieses ist unproblematisch für die Ermittlung von Aufkommenswirkungen, da hier dem Sitzland der Muttergesellschaft keine Bedeutung zukommt. Vielmehr werden die Steuerzahlungen nach dem Sitzland der jeweiligen Konzerngesellschaft zum Steueraufkommen aggregiert. ${ }^{552}$ Die durchschnittliche Steuerbelastung wird allerdings als Durchschnittswert der Steuerbelastungen aller Konzerne eines Landes ermittelt ${ }^{533}$ und soll unter anderem als Maßgröße für die Standortattraktivität dieses Landes dienen. Die Berücksichtigung nicht-europäischer Konzerne erscheint mit Blick auf diese Zielsetzung nicht sinnvoll, insbesondere auch da aufgrund des Fehlens von Jahresabschlussinformationen $\mathrm{zu}$ den nicht-europäischen Unternehmen sich die Steuerbelastung dieser Länder selbst in den Berechnungen nicht widerspiegeln würde.

Zweitens sind bei der Auswahl der einzubeziehenden Unternehmen weitergehende Anforderungen hinsichtlich des Mindestumfangs an Jahresabschlussinformationen $\mathrm{zu}$ berücksichtigen. So umfassen die erforderlichen Informationen neben den Angaben, die für die Ermittlung der Steuerzahlungen benötigt werden, auch die Angaben, die für die indirekte Ermittlung der Cashflows erforderlich sind. ${ }^{554}$ Insbesondere wirkt sich in diesem Zusammenhang einschränkend aus, dass die indirekte Cashflow-Ermittlung die Verfügbarkeit dieser Angaben in mindestens zwei aufeinanderfolgenden Jahren erfordert. 
Drittens wird die Datenbasis für diese Berechnungen schließlich weiter eingeschränkt, da multinationale Konzerne, bei denen keine Informationen zu inländischen Konzernunternehmen in der Datenbasis enthalten sind, ausgeschlossen werden. Bei diesen Konzernen fehlt es an einem sinnvollen Zusammenhang zwischen der ermittelten Konzernsteuerbelastung und dem Steuerniveau im Sitzland der Konzernmutter. Erweitert wird die Datenbasis hingegen, da bei nationalen Konzernen auch Konzerne berücksichtigt werden, die mit nur einer Konzerngesellschaft in die Datenbasis eingehen. Obwohl sich bei diesen Konzernen eine Erweiterung der Möglichkeiten zur konzerninternen Verlustverrechnung nicht auswirken kann, werden diese Konzerne nicht aus der Datenbasis gelöscht, um eine weitere Verkleinerung zu vermeiden. Aufgrund der Vorgehensweise bei der Ermittlung der durchschnittlichen Steuerbelastung ${ }^{555}$ wären Länder vollständig von der Belastungsanalyse auszunehmen, wenn nicht zumindest ein rentabler und ein verlusterzielender nationaler Konzern in der Datenbasis enthalten sind. Dieses wäre - neben einigen anderen Ländern, die ohnehin aus der Analyse herausfallen - auch bei Irland und Österreich der Fall. Da der Schwerpunkt der Analyse ohnehin auf der Analyse der Steuerbelastung multinationaler Konzerne liegt und insbesondere Irland als Niedrigsteuerland hierbei von besonderem Interesse ist, wird die aus der Einbeziehung dieser Konzerne in die Datenbasis resultierende Ungenauigkeit bei der Ermittlung der Belastungswirkungen nationaler Konzerne in Kauf genommen. ${ }^{556}$

Tabelle 19 fasst die geografische Zusammensetzung der den Steuerbelastungsberechnungen zugrundegelegten Datenbasis zusammen.

Tabelle 19: Geografische Zusammensetzung der Stichprobe (Belastungsberechnungen) ${ }^{557}$

\begin{tabular}{lcc|ccc}
\hline & \multicolumn{2}{c|}{ Anzahl an Konzernen } & \multicolumn{3}{c}{ Anzahl an Konzerngesellschaften } \\
National & Multinational & $\begin{array}{c}\text { Nationale } \\
\text { Konzerne }\end{array}$ & $\begin{array}{c}\text { Multination- } \\
\text { ale Konzerne }\end{array}$ & $\begin{array}{c}\text { davon: } \\
\text { inländische } \\
\text { Gesellschaften }\end{array}$ \\
\hline Belgien & 5.367 & 437 & 9.287 & 2.149 & 1.132 \\
Dänemark & 9.897 & 329 & 13.270 & 1.838 & 909 \\
Deutschland & 492 & 166 & 596 & 1.655 & 294 \\
Estland & 150 & 19 & 250 & 50 & 27 \\
Finnland & 1.386 & 170 & 2.938 & 1.260 & 671
\end{tabular}

555 Vgl. hierzu Kapitel 5.6 und 6.1.

556 Zur Verdeutlichung des Einflusses dieser Annahme werden an entscheidenden Stellen in Fußnoten auch die Ergebnisse berichtet, die sich ohne die Einbeziehung dieser Konzerne ergeben hätten.

557 Nicht berücksichtigt in den Berechnungen wurden Unternehmen aus Bulgarien und Rumänien, da diese zum Zeitpunkt der Berechnungen nicht Bestandteil der EU waren sowie Unternehmen aus Malta und Slowenien, da AMADEUS für diese Länder bei keinem Unternehmen die erforderlichen Mindestinformationen bereitstellt 


\begin{tabular}{lcc|ccc} 
Frankreich & 12.813 & 671 & 26.461 & 8.041 & 5.064 \\
Griechenland & 315 & 8 & 728 & 43 & 34 \\
Großbritannien & 17.420 & 489 & 30.495 & 4.773 & 3.210 \\
Irland & 51 & 15 & 51 & 34 & 15 \\
Italien & 814 & 375 & 1.453 & 1.387 & 668 \\
Lettland & 36 & 4 & 65 & 8 & 4 \\
Litauen & 16 & 1 & 27 & 3 & 1 \\
Luxemburg & 4 & 4 & 4 & 198 & 8 \\
Niederlande & 2.512 & 271 & 3.043 & 1.630 & 478 \\
Österreich & 42 & 23 & 48 & 80 & 27 \\
Polen & 354 & 6 & 735 & 20 & 11 \\
Portugal & 300 & 18 & 589 & 83 & 61 \\
Schweden & 11.531 & 607 & 18.342 & 4.524 & 2.567 \\
Slowakei & 1 & 2 & 1 & 8 & 2 \\
Spanien & 5.271 & 255 & 12.136 & 1.819 & 1.346 \\
Tsch'e Republik & 96 & 13 & 155 & 27 & 14 \\
Ungarn & 283 & 1 & 507 & 7 & 4 \\
Zypern & 2 & 1 & 4 & 7 & 1 \\
\hline Gesamt & 69.153 & 3.885 & 121.185 & 29.644 & 16.548 \\
\hline Quelle: Eigene Berech & & $13 n g$ & &
\end{tabular}

Quelle: Eigene Berechnung auf Basis der Angaben in AMADEUS.

\subsection{Ermittlung der Steuerzahlungen}

\subsubsection{Geltendes Steuerrecht}

Die primäre Wirkung einer Reform der Konzernbesteuerung betrifft die Ertragsteuerzahlungen der Konzerngesellschaften. Mittelbare Wirkungen können sich zwar auch für die Ertragsteuerzahlungen natürlicher Personen ergeben, die als Gesellschafter oder Arbeitnehmer an den betroffenen Kapitalgesellschaften beteiligt sind, sowie - aufgrund von Verhaltensreaktionen der Konzerne - für Substanzsteuern, die auf dem Vermögen der Kapitalgesellschaft lasten. Eine Berücksichtigung dieser Steuerarten würde die Komplexität der Berechnungen allerdings deutlich erhöhen. Außerdem sind die für die Ermittlung dieser Steuern erforderlichen Informationen nicht vollständig bekannt. Aus diesen Gründen beschränkt sich die folgende Analyse auf die Ertragsteuerzahlungen von Kapitalgesellschaften, die Bestandteil eines nationalen oder grenzüberschreitend tätigen Konzerns sind. Dabei werden neben der Körperschaftsteuer auch Gemeindesteuern, Zuschlagsteuern und sonstige Ertragsteuern berücksichtigt. Auf eine detail- 
lierte Abbildung dieser weiteren Ertragssteuern wird aus Vereinfachungsgründen allerdings verzichtet. Diese werden vielmehr durch eine entsprechende Erhöhung des nominellen Steuersatzes erfasst, wobei Dependenzen zwischen den verschiedenen Ertragsteuern berücksichtigt werden.

Die Ermittlung der Ertragsteuerzahlungen erfolgt auf Basis der in AMADEUS veröffentlichten unkonsolidierten Gewinn- und Verlustrechnungen für die Jahre 1994 bis 2003. ${ }^{558}$ Ausgangspunkt bildet dabei das Ergebnis der gewöhnlichen Geschäftstätigkeit („Profit/loss before tax“). Auf eine Einbeziehung des außerordentlichen Ergebnisses wird verzichtet, da dieses in einigen Mitgliedsstaaten vermindert um die auf dieses Ergebnis entfallenden Steuern ausgewiesen wird.

Zur Überleitung zum zu versteuernden Einkommen wird das Ergebnis der gewöhnlichen Geschäftstätigkeit in drei Schritten angepasst, mit denen der Abweichung zwischen handelsrechtlichem Ergebnis und steuerlichem Einkommen in drei wichtigen Bereichen Rechnung getragen wird. Dieses sind im Einzelnen die Vorschriften zur Vermeidung der Doppelbesteuerung bei Dividenden sowie den verschiedenen Regelungen zur intertemporalen und konzerninternen Verlustverrechnung. ${ }^{559}$ Hierbei wird einheitlich der Rechtsstand 1.1.2006 zugrundegelegt. ${ }^{560}$ Weiteren Abweichungen, wie sie sich zum Beispiel aus der Behandlung ausländischer Betriebsstätteneinkünfte, den Besonderheiten der steuerlichen Gewinnermittlung oder steuerlichen Unterkapitalisierungsregeln ergeben, wird hingegen nicht Rechnung getragen. Als Gründe sei hier auf das Fehlen entsprechender Angaben in AMADEUS sowie die hohe Komplexität der Erstellung eines europaweiten steuerlichen Mikrosimulationsmodells verwiesen, welche eine Beschränkung auf ausgewählte Vorschriften erforderlich macht.

In einem ersten Schritt werden die Auswirkungen der steuerlichen Behandlung von Dividenden auf das Einkommen der empfangenden Konzerngesellschaft simuliert. Die Höhe der an eine Gesellschaft zugeflossenen Dividenden kann dabei nicht unmittelbar den Angaben in AMADEUS entnommen werden, da Fi-

558 Jahresabschlüsse für das Jahr 2004 werden aufgrund des geringen Erfassungsgrads nicht berücksichtigt.

559 Aus Vereinfachungsgründen unterbleibt eine Abbildung der grenzüberschreitenden Gruppenbesteuerungssysteme in Dänemark, Frankreich, Italien und Österreich. Ebenfalls aus Vereinfachungsgründen wird auf eine Simulation der nachgelagerten Besteuerung in Estland verzichtet und vielmehr ein - entsprechend der Vorgehensweise in den anderen Mitgliedsstaaten ermitteltes - Einkommen zugrundegelegt. Dabei wird von einem zeitlich und betragsmäßig unbegrenzten Verlustvortrag ohne Möglichkeit eines Verlustrücktrags ausgegangen.

560 Ein aktuellerer Rechtsstand konnte nicht zugrundegelegt werden, da europaweite Informationen über aktuelle Steuerrechtsänderungen nur mit zeitlicher Verzögerung verfügbar sind und die hohe Komplexität des Modells eine nachträgliche Aktualisierung nur mit erheblichem Aufwand zulässt. Ein Überblick über diese Rechtsvorschriften findet sich in den Tabellen 50 bis 53 im Anhang. Insgesamt zeigen sich nur geringfügige Abweichungen gegenüber dem in Kapitel 0 beschriebenen aktuellen Rechtsstand. 
nanzerträge hier nur als aggregierte Position (,Financial revenue“), nicht aber nach Zinserträgen und Dividendenerträgen differenziert ausgewiesen werden. Die Höhe der Dividenden muss aus diesem Grund indirekt anhand der Beteiligungsinformationen sowie dem Jahresergebnis und der Eigenkapitalveränderung der ausschüttenden Gesellschaft ermittelt werden.

Hierzu wird zunächst die Höhe der ausgeschütteten Gewinne jedes Unternehmens $\mathrm{j}$ in jeder Periode $t$ ermittelt. Diese ergibt sich als positive Differenz der Eigenkapitalveränderung und des Jahresergebnisses der jeweiligen Periode (vgl. Formel (2)). Den Jahresüberschuss übersteigende Erhöhungen des Eigenkapitals (negative Ausschüttungen) sollten auf Eigenkapitalerhöhungen zurückzuführen sein und werden deshalb nicht berücksichtigt. mit:

$$
d_{j}^{t}=\max \left[p l f p_{j}^{t}-\left(E_{j}^{t}-E_{j}^{t-1}\right) ; 0\right]
$$

$\begin{array}{ll}\mathrm{d}_{\mathrm{j}}^{t} & \text { Gewinnausschüttung von Unternehmen } \mathrm{j} \text { in Periode } t \\ \mathrm{plfp}_{j}^{t} & \text { Jahresüberschuss von Unternehmen } \mathrm{j} \text { in Periode } t \\ \mathrm{E}_{j}^{t} & \text { Bilanzielles Eigenkapital von Unternehmen } \mathrm{j} \text { zum Ende von } \\ & \text { Periode } t\end{array}$

Bei der Zuordnung der Dividendenzahlungen zu den beteiligten Unternehmen wird von einer beteiligungsproportionalen Gewinnausschüttung ausgegangen. Die Höhe der von einem Unternehmen i empfangenen Dividenden ergibt sich daher als Summe der anteiligen Gewinnausschüttungen aller Unternehmen $j$, an denen Unternehmen $\mathrm{i}$ eine Beteiligung hält. Nach oben begrenzt wird die Höhe der Dividendenerträge durch die Höhe der in AMADEUS ausgewiesenen Finanzerträge (vgl. Formel (3)).

$$
\operatorname{div}_{i}^{t}=\min \left(\sum_{j} d_{j}^{t} \cdot q_{i ; j} ; \text { fire } e_{i}^{t}\right)
$$

mit:

$\operatorname{div}_{i}^{t} \quad$ Dividendenerträge von Unternehmen i in Periode $t$

$d_{j}^{t}$ Gewinnausschüttung von Unternehmen $j$ in Periode $t$

$\mathrm{q}_{\mathrm{i} ; \mathrm{j}}$ Beteiligung (in \%) von Unternehmen $i$ an Unternehmen $j$ entfire $_{i}^{t}$ sprechend der Anteilseignerinformationen in AMADEUS Finanzerträge von Unternehmen i in Periode t entsprechend der Angaben in AMADEUS

Für die Behandlung von inländischen und ausländischen Dividenden bei der empfangenden Gesellschaft sehen die meisten Mitgliedsstaaten eine vollständige oder teilweise Freistellung vor, lediglich in fün Mitgliedsstaaten kommt nach dem zugrundegelegten Rechtsstand die Anrechnungsmethode zur Anwendung 
(vgl. Tabelle 50 im Anhang). Eine Simulation der Besteuerungsfolgen der Anrechnungsmethode würde allerdings Kenntnis über den Sitz der ausschüttenden Kapitalgesellschaft voraussetzen. Da bereits die Bestimmung der Höhe der in den Finanzerträgen enthaltenen Dividendenerträge mit Unsicherheit behaftet ist ${ }^{561}$, wird auf eine Simulation der Anrechnungsmethode verzichtet. Anstelle einer Anrechnung wird daher eine vollständige Freistellung unterstellt. Zur Abbildung der (vollständigen oder teilweisen) Dividendenfreistellung wird das Ergebnis der gewöhnlichen Geschäftstätigkeit um den Anteil der freizustellenden Dividenden gekürzt. Die sich ergebende Zwischensumme wird mit inc_1 $1_{i}^{t}$ bezeichnet.

mit:

$$
\text { inc } 1_{-}^{t}=p l b t_{i}^{t}-\operatorname{div}_{i}^{t} \cdot e x_{c_{i}}
$$

\begin{tabular}{|c|c|}
\hline inc_ $1_{i}^{t}$ & $\begin{array}{l}\text { Zwischensumme } 1 \text { bei der Ermittlung des Einkommens von } \\
\text { Unternehmen } \mathrm{i} \text { in Periode } \mathrm{t}\end{array}$ \\
\hline $\mathrm{plbt}_{\mathrm{i}}^{\mathrm{t}}$ & $\begin{array}{l}\text { Ergebnis der gewöhnlichen Geschäftstätigkeit von Unterneh- } \\
\text { men } \mathrm{i} \text { in Periode } t \text { entsprechend der Angaben in AMADEUS }\end{array}$ \\
\hline & Dividendenerträge von Unternehmen $i$ in Periode $t$ \\
\hline & $\begin{array}{l}\text { Prozentual freizustellender Dividendenanteil im Sitzland c von } \\
\text { Unternehmen i }\end{array}$ \\
\hline
\end{tabular}

In einem zweiten Schritt wird inc_1 $1_{i}^{t}$ weiter angepasst, um den steuerlichen Konsequenzen, die sich aus der Anwendung der Vorschriften zur konzerninternen Verlustverrechnung zwischen inländischen Gruppengesellschaften ergeben, Rechnung zu tragen. Zu diesem Zweck wird inc_1 $1_{i}^{t}$ um einen Änderungsbetrag gt $t_{i}^{t}$ erhöht oder vermindert (vgl. Formel (5)). Die sich ergebende neue Zwischensumme wird mit inc_2 $2_{\mathrm{i}}^{\mathrm{t}}$ bezeichnet.

mit:

$$
\text { inc_ } 2_{i}^{t}=i n c_{-} 1_{i}^{t}+g t_{i}^{t}
$$

inc_2 ${ }_{i}^{t}$ Zwischensumme 2 bei der Ermittlung des Einkommens von Unternehmen i in Periode $t$

inc_1 $1_{\mathrm{i}}^{\mathrm{t}}$ Zwischensumme 1 bei der Ermittlung des Einkommens von Unternehmen i in Periode $t$

$\mathrm{gt}_{\mathrm{i}}^{\mathrm{t}} \quad$ Erhöhung/Minderung des Einkommens von Unternehmen $\mathrm{i}$ in Periode $\mathrm{t}$ aufgrund der Anwendung der Vorschriften zur konzerninternen Verlustverrechnung

561 So sind erstens nur aktuelle Informationen über die Beteiligungsbeziehungen, nicht aber historische Angaben verfügbar, zweitens nicht alle ausschüttenden Gesellschaften in AMADEUS enthalten und drittens die Annahme einer beteiligungsproportionalen Ausschüttung mit Ungenauigkeiten verbunden. 
Zur Ermittlung von $\mathrm{gt}_{\mathrm{i}}^{\mathrm{t}}$ werden die steuerlichen Gruppen anhand der in AMADEUS enthaltenen Anteilseignerinformationen unter Berücksichtigung der wesentlichen Anwendungsvoraussetzungen für eine konzerninterne Verlustverrechnung (Mindestbeteiligungsquote, Berücksichtigung indirekter Anteile (ja/nein), konzerninterne Verlustverrechnung zwischen Schwestergesellschaften (ja/nein)) abgebildet. ${ }^{562}$ Für Deutschland und Slowenien wird zusätzlich die Voraussetzung eines Ergebnisabführungsvertrags berücksichtigt. Aufgrund des Ausweises dieser Gewinnabführungen im außerordentlichen Ergebnis wird der Abschluss eines derartigen Vertrags vermutet, wenn das Ergebnis der gewöhnlichen Geschäftstätigkeit größer oder kleiner als Null ist, während der Jahresüberschuss Null beträgt. ${ }^{563}$

Tabelle 20: Simulation der Wirkungen von Gruppenbesteuerungssystemen im geltenden Steuerrecht

\begin{tabular}{|c|c|c|}
\hline & Zusammenveranlagung & $\begin{array}{l}\text { Aufteilung/Group relief/Group con- } \\
\text { tribution }\end{array}$ \\
\hline Länder & $\begin{array}{l}\text { Deutschland, Frankreich, Italien, Luxem- } \\
\text { burg, Malta, Niederlande, Österreich, } \\
\text { Polen, Portugal, Slowenien, Ungarn }\end{array}$ & $\begin{array}{l}\text { Dänemark, Finnland, Großbritannien, } \\
\text { Irland, Lettland, Schweden, Zypern }\end{array}$ \\
\hline $\begin{array}{l}\text { Mutter- } \\
\text { gesellschaft }\end{array}$ & 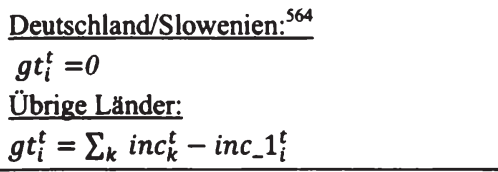 & 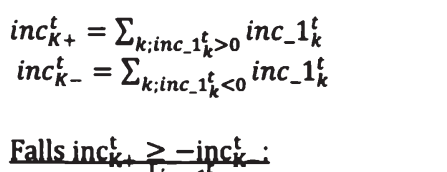 \\
\hline $\begin{array}{l}\text { Tochter- } \\
\text { gesellschaft }\end{array}$ & $g t_{i}^{t}=i n c_{-} 1_{i}^{t} \cdot(-1)$ & 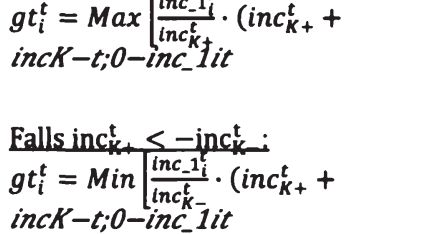 \\
\hline
\end{tabular}

mit:

$\mathrm{gt}_{\mathrm{i}}^{\mathrm{t}}$ Änderungsbetrag aufgrund der Anwendung der konzerninternen Verlustverrechnung bei Unternehmen $i$ in Periode $t$

562 Für eine Übersicht über die Anwendungsvoraussetzungen in den einzelnen Mitgliedsstaaten nach dem hier zugrundegelegten Rechtsstand vgl. Tabelle 51 im Anhang.

563 Auch bei der Ermittlung der steuerlichen Gruppen wurde auf Vorarbeiten im Rahmen des übergeordneten Forschungsprojekts zurückgegriffen. Für Einzelheiten zur Abbildung der steuerlichen Gruppen vgl. Poppe (2008), S. 121-125.

564 In Deutschland und Slowenien werden keine Anpassungen für die Muttergesellschaft vorgenommen, da die bei der Muttergesellschaft zu versteuernden Ergebnisse der Tochtergesellschaft aufgrund des Erfordernisses eines Ergebnisabfuhrungsvertrags bereits im handelsrechtlichen Ergebnis enthalten sind. 


$\begin{array}{ll}\text { inc_1 }_{\mathrm{i}}^{\mathrm{t}} & \begin{array}{l}\text { Zwischensumme } 1 \text { bei der Ermittlung des Einkommens von } \\ \text { Unternehmen } \mathrm{i} \text { in Periode } \mathrm{t}\end{array} \\ \text { inc }_{\mathrm{K}+}^{\mathrm{t}} & \begin{array}{l}\text { Summe der positiven Zwischensummen } 1 \text { (inc_1 } 1_{\mathrm{i}}^{\mathrm{t}} \text { ) aller zu } \\ \text { einer gemeinsamen steuerlichen Gruppe gehörenden Unter- } \\ \text { nehmen } \mathrm{k} \text { in Periode } \mathrm{t}\end{array} \\ \text { inc }_{\mathrm{K}-}^{\mathrm{t}} & \begin{array}{l}\text { Summe der negativen Zwischensummen } 1 \text { (inc_1 } 1_{\mathrm{i}}^{\mathrm{t}} \text { ) aller zu } \\ \text { einer gemeinsamen steuerlichen Gruppe gehörenden Unter- } \\ \text { nehmen } \mathrm{k} \text { in Periode } \mathrm{t}\end{array} \\ \mathrm{k} \quad \begin{array}{l}\text { Index für die Unternehmen, die zu einer gemeinsamen steuerli- } \\ \text { chen Gruppe K gehören }\end{array}\end{array}$

Zur Abbildung der steuerlichen Konsequenzen der konzerninternen Verlustverrechnung werden Gruppenbesteuerungssysteme, die eine Zusammenfassung der steuerlichen Ergebnisse auf Ebene der Muttergesellschaft (Zurechnungskonzept oder Konsolidierung) vorsehen, von Gruppenbesteuerungssystemen unterschieden, die eine proportionale Aufteilung eines positiven Gesamtergebnisses auf die gewinnerzielenden Gesellschaften und umgekehrt eines negativen Gesamtergebnisses auf die verlusterzielenden Gesellschaften bewirken (Dänemark). Einige Länder erlauben in ihrem Gruppenbesteuerungssystem eine flexible Übertragung von Gewinnen oder Verlusten zwischen Gruppengesellschaften. Da die Ausübung dieser Gestaltungsspielräume bei der Verlustverrechnung in den Daten nicht beobachtet werden kann, wird für diese Länder ebenfalls eine proportionale Aufteilung des Gesamtergebnisses unterstellt. Die formale Vorgehensweise bei der Simulation der nationalen Gruppenbesteuerungssysteme ist in Tabelle 20 erläutert.

In einem dritten Schritt werden ausgehend von Zwischensumme 2 (inc_2t ${ }_{i}^{t}$ ) die Wirkungen der länderspezifischen Vorschriften zur intertemporalen Verlustverrechnung simuliert (vgl. Formel (6)) ${ }^{565}$ Hierbei werden die in den Mitgliedsstaaten zur Anwendung kommenden zeitlichen und betragsmäßigen Beschränkungen des Verlustrücktrags $\left(\operatorname{lcb}_{\mathrm{c}_{\mathrm{i}}}\right)$ und Verlustvortrags $\left(\operatorname{lcf}_{\mathrm{c}_{\mathrm{i}}}\right)$ abgebildet. In Ermangelung von Informationen über einen bestehenden Verlustvortrag zu Beginn des Betrachtungszeitraums wird dieser Wert bei allen Gesellschaften mit Null angenommen werden. ${ }^{566}$

$$
\text { inc } c_{i}^{t}=f\left(i n c_{-} 2_{i}^{t} ; l c b_{c_{i}} ; l c f_{c_{i}}\right)
$$

mit:

565 Technisch erfolgt diese Simulation durch ein VBA-Excel-Makro. Hierbei führt ein negativer Wert für $i n c_{-} 2_{i}^{t}$ zu einem negativen $i n c_{i}^{t}$, soweit ein Verlustrücktrag möglich ist. Ohne Verlustrücktrag ergibt sich ein Wert von null. Positive Werte für inc_2 ${ }_{i}^{t}$ werden gemindert, soweit ein Verlustvortrag abgezogen werden kann.

566 Dieses führt im Ergebnis zu einer Unterschätzung der Wirkung, der von einer Ausdehnung der Möglichkeiten zur steuerlichen Verlustverrechnungapsgeht. 978-3-631-75683-6 
inc $_{\mathrm{i}}^{\mathrm{t}} \quad$ ermittelter Näherungwert für das zu versteuernde Einkommen von Unternehmen i in Periode $t$

inc_2 $2_{i}^{t}$ Zwischensumme 2 bei der Ermittlung des Einkommens von Unternehmen i in Periode $\mathrm{t}$

$\operatorname{lcb}_{c_{\mathbf{i}}} \quad$ zeitliche und betragsmäßige Beschränkungen des Verlustrücktrags im Sitzland c von Unternehmen i

$\operatorname{lcf}_{c_{i}} \quad$ zeitliche und betragsmäßige Beschränkungen des Verlustvortrags im Sitzland c von Unternehmen i

inc $_{i}^{t}$ dient als Näherungswert für das zu versteuernde Einkommen von Unternehmen $i$ in Periode t. Zur Ermittlung der Steuerzahlung tax $x_{i}^{t}$ wird dieser Wert mit dem landesspezifischen Steuersatz $\tau_{c_{i}}$ multipliziert, welcher die nominelle Steuerbelastung unter Berücksichtigung der nationalen Körperschaftsteuer, regionaler Ertragsteuern sowie Zuschlagsteuern widerspiegelt. Bei regional unterschiedlichen Gemeindesteuersätzen werden diese mit einem durchschnittlichen Wert berücksichtigt. ${ }^{567}$

Dabei werden für Zwecke der Aufkommensberechnungen die Steuersätze der jeweiligen Jahre (1994 bis 2003) zugrundegelegt (vgl. Formel (7a)), da diese auch für die Entscheidungen der Unternehmen und damit den in den Daten enthaltenen Steuergestaltungen maßgebend waren. Mit der Ermittlung der durchschnittlichen Steuerbelastung wird hingegen das Ziel verbunden, eine Belastungsgröße zu ermitteln, welche die steuerlichen Vorschriften eines Jahres widerspiegelt, um - unter anderem - Vergleiche zu Belastungskennziffern anderer Modelle (zum Beispiel „Devereux/Griffith“) ziehen zu können. Aus diesem Grund wird für Zwecke der Belastungsrechnungen einheitlich der Steuersatz für das Jahr 2006 zugrundegelegt (vgl. Formel (7b)).

$$
\operatorname{tax} x_{i}^{t}=i n c_{i}^{t} \cdot \tau_{c_{i}}^{t}
$$

mit:

$$
\begin{aligned}
& \operatorname{tax}_{i}^{t} \quad \text { ermittelte Steuerzahlung von Unternehmen } i \text { in Periode } t \\
& \text { inc }_{i}^{t} \quad \text { ermittelter Näherungwert für das zu versteuernde Einkommen } \\
& \text { von Unternehmen i in Periode } t \\
& \tau_{c_{i}}^{t} \quad \text { nominelle Ertragsteuerbelastung im Sitzland c von Unterneh- } \\
& \text { men } \mathrm{i} \text { in Periode } \mathbf{t}
\end{aligned}
$$

$$
\operatorname{tax} x_{i}^{t}=i n c_{i}^{t} \cdot \tau_{c_{i}}^{2006}
$$

mit:

$\operatorname{tax}_{i}^{t} \quad$ ermittelte Steuerzahlung von Unternehmen $\mathrm{i}$ in Periode $\mathrm{t}$

567 Für die Höhe der zugrundegelegten Steuersätze in den einzelnen Mitgliedsstaaten vgl. Tabelle 52 im Anhang. 

inc $_{\mathrm{i}}^{\mathrm{t}}$
ermittelter Näherungwert für das zu versteuernde Einkommen von Unternehmen $\mathrm{i}$ in Periode $\mathrm{t}$
$\tau_{c_{i}}^{2006}$
nominelle Ertragsteuerbelastung im Sitzland c von Unterneh- men i nach dem Rechtsstand 1.1.2006

\subsubsection{Gemeinsame konsolidierte Körperschaftsteuerbemessungsgrundlage}

Auch bei Einführung einer GKKB können nicht alle Elemente des (neu gestalteten) Steuersystems mit der hier angewendeten Methodik und auf der Basis der hier zugrundegelegten Daten abgebildet werden. Ausgangspunkt bei der Ermittlung der Steuerzahlungen bildet auch hier das in AMADEUS ausgewiesene handelsrechtliche Ergebnis der gewöhnlichen Geschäftstätigkeit; die steuerliche Gewinnermittlung selbst ist also nicht Gegenstand der Simulation. Folglich können auch die Konsequenzen einer Harmonisierung der steuerlichen Gewinnermittlung als ein Element der GKKB nicht erfasst werden. Gleiches gilt auch für die Auswirkungen einer Zwischenergebniseliminierung für konzerninterne Lieferungsund Leistungsbeziehungen, da Informationen über das Bestehen und die Konditionen derartiger Transaktionen publizierten Jahresabschlüssen nicht zu entnehmen sind. Die Elemente der GKKB, die mithilfe des hier verwendeten Simulationsmodells abgebildet werden können, beschränken sich somit auf (1) die Einführung einer europaweiten Verlustverrechnung in nationalen und grenzüberschreitend tätigen Konzernen in Europa, (2) den Übergang von einer direkten auf eine indirekte Gewinnabgrenzung und (3) die vollständige Steuerfreistellung von konzerninternen Dividenden als Konsequenz des Konsolidierungsmechanismus. Weiterhin soll bereits hier einschränkend darauf hingewiesen werden, dass bei den Berechnungen nicht an jeder Stelle die aktuellsten Vorschläge der Arbeitsgruppe umgesetzt werden konnten. Teilweise ist dieses dem Fehlen erforderlicher Informationen geschuldet, teilweise der schrittweisen Veröffentlichung der Papiere der Arbeitsgruppe. ${ }^{568}$

Erster Schritt bei der Ermittlung des zu versteuernden Einkommens einer GKKB ist die Bestimmung des aufzuteilenden Ergebnisses der einzelnen Konzerngesellschaften. Hierzu wäre nach dem Vorschlag der Arbeitsgruppe der Gewinn nach Maßgabe der harmonisierten Regeln zu ermitteln sowie eine Konsolidierung durchzuführen. In Ermangelung erforderlicher Angaben beschränkt sich dieser Schritt hier auf eine Eliminierung konzerninterner Dividenden als Folge des Konsolidierungsmechanismus. Ferner werden auch konzernexterne Dividenden hier nicht in die Aufteilung einbezogen, sondern den empfangenden Konzernge-

568 Mehrfache Neuberechnungen wären aufgrund der Komplexität des Modells mit erheblichem Aufwand verbunden gewesen. Insgesamt kann allerdings davon ausgegangen werden, dass die hierdurch entstehenden Ungenauigkeiten das Berechnungsergebnis nicht wesentlich beeinflussen. Diese Vermutung wird an einigen Stellen durch entsprechende Variationsrechnungen belegt. 
sellschaften direkt zugerechnet und auf Ebene dieser Gesellschaften in Übereinstimmung mit dem geltenden Steuerrecht behandelt. Die Ermittlung erhaltener Gewinnausschüttungen erfolgt entsprechend der in Formel (2) und (3) dargelegten Weise. Konzerninterne und konzernexterne Dividenden werden anhand der Anteilseignerinformationen aus AMADEUS voneinander abgegrenzt.

$$
\text { inc } \_1_{i}^{t}=p l b t_{i}^{t}-d i v_{i}^{t}
$$

mit:
inc_ $1_{i}^{t}$
Zwischensumme 1 bei der Ermittlung des Einkommens von Unternehmen $\mathrm{i}$ in Periode $\mathrm{t}$
plbt $_{i}^{t} \quad$ Ergebnis der gewöhnlichen Geschäftstätigkeit von Unterneh- men $\mathrm{i}$ in Periode $t$ entsprechend der Angaben in AMADEUS
$\operatorname{div}_{i}^{t} \quad$ Dividendenerträge von Unternehmen $i$ in Periode $t$

In einem zweiten Schritt wird das aufzuteilende Ergebnis aller Gesellschaften j, die Bestandteil eines gemeinsamen Konzerns sind, auf Konzernebene zusammengefasst und anhand einer einheitlich definierten Aufteilungsformel den einzelnen Konzerngesellschaften zugewiesen. Das zu versteuernde Einkommen ergibt sich dann als Summe des zugewiesenen Teils des Konzernergebnisses und des steuerpflichtigen Teils der direkt zugeordneten konzernexternen Dividenden (vgl. Formel (9)).

$$
\text { inc } 2_{i}^{t}=p_{i}^{t} \cdot \sum_{j} i n c_{-} 1_{j}^{t}+d i v_{-} e_{i}^{t} \cdot\left(1-e x_{c_{i}}\right)
$$

mit:

inc_2 $2_{\mathrm{i}}^{\mathrm{t}}$ Zwischensumme 2 bei der Ermittlung des Einkommens von Unternehmen $\mathrm{i}$ in Periode $\mathrm{t}$

inc_1 $1_{i}^{t}$ Zwischensumme 1 bei der Ermittlung des Einkommens von Unternehmen i in Periode $t$

$p_{i}^{t} \quad$ Entsprechend der Aufteilungsformel an Unternehmen $i$ in Periode t zuzuweisender Anteil am Konzerneinkommen

div_e $e_{i}^{t} \quad$ Dividendenerträge von Unternehmen $i$ in Periode $t$ aus Beteiligungen an nicht konzernzugehörigen Gesellschaften

ex $_{c_{\mathbf{i}}} \quad$ Prozentual freizustellender Dividendenanteil im Sitzland c von Unternehmen i

Bei der Abgrenzung der GKKB-Gruppen kommt die in Kapitel 5.3.2.3 beschriebene Vorgehensweise zur Anwendung. Die zugrundegelegte Mindestbeteiligungsquote beträgt dementsprechend 50 Prozent, wobei direkte und indirekte 
Beteiligungen $^{569}$ addiert werden. Europäische Schwestergesellschaften sowie europäische Teilkonzerne einer gemeinsamen nicht-europäischen Muttergesellschaft werden entsprechend dem aktuellen Diskussionstand der Europäischen Kommission zu einer gemeinsamen GKKB-Gruppe zusammengefasst. ${ }^{570}$ Die im Vergleich zum Vorschlag der Arbeitsgruppe von 75 Prozent niedrigere Beteiligungsquote sollte für die Berechnungsergebnisse von untergeordneter Bedeutung sein. So zeigen entsprechende Auswertungen bei vergleichbaren Berechnungen in Poppe (2008) und Oestreicher/Scheffler/Spengel et al. (2008) nur eine geringe Abhängigkeit des Aufkommenseffekts von der Höhe der Mindestbeteiligung auf. Als Erklärung wird angeführt, dass Konzerngesellschaften typischerweise vollständig oder annähernd vollständig von konzernzugehörigen Anteilseignern gehalten werden. ${ }^{571}$

$$
p_{i}^{t}=\left(\frac{O P R E_{i}^{t}}{\sum_{j} O P R E_{j}^{t}}+\frac{C E M P L_{i}^{t}}{\sum_{j} C E M P L_{j}^{t}}+\frac{T F A S_{i}^{t}+I F A S_{i}^{t}+S T O C K S_{i}^{t}}{\sum_{j}\left(T F A S_{j}^{t}+I F A S_{j}^{t}+S T O C K S_{j}^{t}\right)}\right) \cdot \frac{1}{3}
$$

mit:

\begin{tabular}{|c|c|}
\hline$p_{i}^{t}$ & $\begin{array}{l}\text { Entsprechend der Aufteilungsformel an Unternehmen i in Peri- } \\
\text { ode } t \text { zuzuweisender Anteil am Konzerneinkommen }\end{array}$ \\
\hline $\mathrm{OPRE}_{\mathrm{i}}^{\mathrm{t}}$ & Operative Erträge von Unternehmen i in Periode $\mathrm{t}$ \\
\hline$\sum_{\mathrm{j}} \mathrm{OPRE}_{\mathrm{j}}^{\mathrm{t}}$ & $\begin{array}{l}\text { Summe der operativen Erträge aller zum gemeinsamen Kon- } \\
\text { zern gehörenden Unternehmen } \mathrm{j} \text { in Periode } \mathrm{t}\end{array}$ \\
\hline CEMPL $_{\mathrm{i}}^{\mathrm{t}}$ & Lohnsumme von Unternehmen $\mathrm{i}$ in Periode $\mathrm{t}$ \\
\hline TFAS $_{i}^{t}$ & Materielles Anlagevermögen von Unternehmen $\mathrm{i}$ in Periode $\mathrm{t}$ \\
\hline IFAS $_{\mathrm{i}}^{\mathrm{t}}$ & $\begin{array}{l}\text { Immaterielles Anlagevermögen von Unternehmen i in Periode } \\
\mathrm{t}\end{array}$ \\
\hline STOCKS $\mathrm{i}_{\mathrm{t}}^{\mathrm{t}}$ & Vorratsvermögen von Unternehmen $\mathrm{i}$ in Periode $\mathrm{t}^{572}$ \\
\hline
\end{tabular}

Die Aufteilung erfolgt grundsätzlich anhand der in Gleichung (10) ausgewiesenen Formel. Im Rahmen der Untersuchung der Aufkommenswirkungen werden allerdings in Variationsrechnungen zusätzlich die Effekte alternativer $\mathrm{Zu}$ sammensetzungen der Aufteilungsformel simuliert. Dabei werden unterschiedliche Gewichtungen und unterschiedliche Definitionen (z.B. Verwendung der Mitarbeiteranzahl anstelle der Lohnsumme zur Definition des Arbeitsfaktors) der oben genannten Faktoren sowie die Effekte einer Aufteilung nach Maßgabe einzelner Aufteilungsfaktoren verwendet. Mit der in Gleichung (10) ausgewiesenen

569 Indirekt gehaltene Anteile werden in voller Höhe berücksichtigt bei einer Beteiligung von mindestens 50 Prozent an der vermittelnden Gesellschaft.

570 Aufgrund der abweichenden Konzerndefinition für Zwecke der Belastungsrechnungen erfolgt dieses allerdings nur bei den Aufkommensberechnungen.

$571 \mathrm{Vgl}$. Oestreicher/Scheffler/Spengel et al. (2008), S. 392.

572 Die Definitionen von $\sum_{j}$ CEMPL $_{j}^{t}$ und $\sum_{j}\left(\right.$ TFAS $_{j}^{t}+$ IFAS $_{j}^{t}+$ STOCKS $\left.S_{j}^{t}\right)$ sind analog zur Definition von $\sum_{\mathrm{j}} \mathrm{OPRE}_{\mathrm{j}}^{\mathrm{t}}$. 
Aufteilungsformel wird insoweit den Vorschlägen der Arbeitsgruppe gefolgt, dass in gleicher Gewichtung die Umsätze, ein Arbeitsfaktor und ein Vermögensfaktor Berücksichtigung finden. Abweichungen ergeben sich allerdings bei der Definition dieser Größen. Während der Vorschlag der Arbeitsgruppe in Bezug auf den Umsatzfaktor auf die nach dem Bestimmungsortprinzip zugeordneten Außenumsätze abstellt, muss hier in Ermangelung anderweitiger Informationen in AMADEUS auf die nach dem Ursprungsortprinzip zugeordneten Gesamtumsätze zurückgegriffen werden. ${ }^{573} \mathrm{Da}$ die Umsätze in AMADEUS bei einer Vielzahl an Unternehmen lediglich als operative Erträge („Operating revenue“) und nicht als Umsatzerlöse aus dem Kerngeschäftsfeld (,Sales") angegeben sind, wird in dieser Arbeit entgegen des Vorschlags der Arbeitsgruppe auf die erstgenannte Größe zurückgegriffen. Für den Arbeitsfaktor spricht sich die Arbeitsgruppe - wohl insbesondere aus politischen Erwägungen - für eine gleichgewichtete Einbeziehung der Lohnsumme und der Mitarbeiteranzahl aus. In dieser Arbeit erfolgt im Ausgangsfall eine ausschließliche Einbeziehung der Lohnsumme, welche aufgrund der gleichzeitigen Berücksichtigung einer Mengen- und einer Preiskomponente insbesondere aus Gründen der Vergleichbarkeit mit den übrigen Aufteilungsfaktoren geeigneter erscheint. In Variationsrechnungen werden allerdings auch die Aufkommenswirkungen bei Aufteilung nach der Mitarbeiteranzahl sowie einer Einbeziehung beider Definitionen des Arbeitsfaktors untersucht. Bei der Definition des Vermögensfaktors finden in dieser Arbeit trotz anderslautender Vorschläge der Arbeitsgruppe das materielle und immaterielle Anlagevermögen sowie die Vorräte Berücksichtigung. Dieses erscheint insofern sachlich gerechtfertigt, da auch in analytischen Literaturbeiträgen grundsätzlich darauf verwiesen wird, dass eine Einbeziehung des immateriellen Anlagevermögens und Vorratsvermögens lediglich aus Praktikabilitätsgründen unterbleiben soll. ${ }^{574}$

Gruppenverluste werden entsprechend der Behandlung von Gruppengewinnen auf die Konzerngesellschaften aufgeteilt und hier nach Maßgabe der geltenden landesspezifischen Vorschriften zur intertemporalen Verlustverrechnung behandelt (vgl. Formel (6)). Eine Harmonisierung dieser Vorschriften wird somit in der Simulation nicht berücksichtigt. Die Aufkommenswirkungen des aktuellen Vorschlags der GKKB-Arbeitsgruppe, der einen Verlustvortrag auf Konzernebene vorsieht, werden in einer Variationsrechnung abgebildet. Auch hier kommen allerdings nicht harmonisierte Verlustverrechnungsbestimmungen zur Anwendung, sondern die Vorschriften im Sitzland der Konzernmutter. Auf das so ermittelte Einkommen wird entsprechend des Vorschlags der Arbeitsgruppe der Steuersatz des jeweiligen Mitgliedsstaats angewendet (vgl. Formel (7a) und (7b)).

573 In Variationsrechnungen werden für die Ermittlung der Aufkommenswirkungen alternativ Außenumsätze zugrundegelegt, die unter vereinfachenden Annahmen aus den veröffentlichten handelsrechtlichen Daten geschätzt werden.

574 Vgl. zu dieser Diskussion Kapitel 4.2.3.3.3. 
Die Aufkommens- und Belastungswirkungen einer GKKB werden sowohl bei verpflichtender Einführung als auch bei optionaler Einführung simuliert. Dabei wird eine Ausübung des Optionsrechts unterstellt, wenn dieses zu einer Absenkung des Nettosteuerbarwerts ${ }^{575}$ des Gesamtkonzerns über den gesamten Betrachtungszeitraums führt. Mit dieser Vorgehensweise bei der Ausübung des Optionsrechts sind mehrere implizite Annahmen verbunden. Zum einen wird nur die Vorteilhaftigkeit einer vollständigen Einbeziehung sämtlicher Konzerngesellschaften in die GKKB geprüft und somit in Übereinstimmung mit dem Vorschlag der Europäischen Kommission von einer „All-in/all-out“-Option ausgegangen. Des Weiteren wird eine Bindungswirkung der Optionsrechtsausübung für den gesamten Betrachtungszeitraum unterstellt, also bis zu zehn Perioden. Diese Prämisse ist restriktiver als die von der Europäischen Kommission vorgeschlagene Bindung für einen Zeitraum von fünf Jahren. Hieraus resultiert tendenziell eine leichte Unterschätzung des Effekts einer optionalen Einführung. Diesem steht allerdings aufgrund der Unterstellung perfekter Voraussicht ein gegenläufiger Effekt gegenüber.

\subsubsection{Alternative Konzepte für eine Verlustrichtlinie}

\subsubsection{Allgemeines}

Unabhängig von dem Verfahren für eine grenzüberschreitende Verlustverrechnung im Konzern, ergeben sich bei der Abgrenzung der steuerlichen Gruppe für Zwecke einer Verlustrichtlinie Unterschiede gegenüber der GKKB. So führen die Nachversteuerungsmethode und die Zurechnungsmethode - im Gegensatz zur GKKB - ausschließlich zu Änderungen bei der Besteuerung der Gruppenmutter. Die Anwendung dieser Methoden setzt daher zwingend die Existenz einer europäischen Muttergesellschaft voraus, weshalb hier weder europäische Schwestergesellschaften noch verschiedene europäische Teilkonzerne einer gemeinsamen nicht-europäischen Muttergesellschaft zu einer Gruppe zusammengefasst werden. Aus Gründen der Einheitlichkeit wird diese - im Vergleich zur GKKB - engere Definition der steuerlichen Gruppe auch für Zwecke der Ergebnisaufteilungsmethode unterstellt.

Bei der Diskussion der möglichen Ausgestaltung einer Verlustrichtlinie in Kapitel 4.3 wurden die Vorteile einer gleichzeitigen Harmonisierung der Vorschriften zur steuerlichen Gewinnermittlung, zur konzerninternen Verlustverrechnung zwischen inländischen Gruppengesellschaften sowie zur intertemporalen Verlustverrechnung herausgestellt. Während eine Simulation harmonisierter Gewinnermittlungsregeln sowie Verlustvortrags- und -rücktragsregeln in Ermangelung hierzu erforderlicher Informationen beziehungsweise aus Vereinfachungsgründen unterbleibt, wird für die Verlustverrechnung zwischen inländischen 
Gruppengesellschaften - unabhängig vom Bestehen eines Gruppenbesteuerungssystems im geltenden Recht - eine Zusammenveranlagung auf Ebene der Inlandsmutter oder Landesholding unterstellt. Zu diesem Zweck werden die Ergebnisse aller inländischen Gesellschaften einer Steuergruppe (im Folgenden als „nationale Teilgruppe" bezeichnet) nach Anpassung für die steuerliche Behandlung zugeflossener Dividenden (vgl. hierzu Formel (4) $)^{576}$ zusammengefasst (vgl. Formel (11)). Die sich ergebende Zwischensumme 2 (inc_2t ${ }_{\text {g;a }}^{t}$ ) bildet den Ausgangspunkt für die in den folgenden Abschnitten erläuterte Anwendung der verschiedenen Konzepte einer grenzüberschreitenden Verlustverrechnung.

$$
\text { inc__ } 2_{g ; a}^{t}=\sum_{k ; c_{k}=a} i n c_{-} 1_{k}^{t}
$$

mit:

inc_2 ${ }_{\text {g; }}^{\mathrm{t}} \quad$ Zwischensumme 2 bei der Ermittlung des Einkommens von der in Land a ansässigen nationalen Teilgruppe von Gruppe $\mathbf{g}$ in Periode $t$

inc_1 $1_{k}^{t}$ Zwischensumme 1 bei der Ermittlung des Einkommens von Unternehmen $\mathrm{k}$ in Periode $\mathrm{t}$

a Länderindex

$c_{k} \quad$ Sitzland c von Unternehmen $k$

$\mathrm{g} \quad$ Index für die (nationalen und grenzüberschreitenden) Steuergruppen

k Index für alle Unternehmen einer gemeinsamen Gruppe $\mathbf{g}$

Hinsichtlich der zugrundegelegten Steuersätze bei Aufkommens- und Belastungsrechnungen sowie der Ausübung des Optionsrechts gilt das beim geltenden Steuerrecht und der GKKB gesagte.

\subsubsection{Nachversteuerungsmethode}

Entsprechend der zugrunde liegenden Konzeption erfolgt bei der Nachversteuerungsmethode eine Berücksichtigung von Verlusten ausländischer Tochtergesellschaften bei der steuerlichen Einkommensermittlung des inländischen Konzernteils, sofern diese nicht im Wege der konzerninternen Verlustverrechnung oder des Verlustrücktrags im Ausland phasengleich verrechnet werden können. Die grenzüberschreitende Verlustverrechnung kommt dementsprechend gegenüber der nationalen Verlustverrechnung im Ausland subsidiär zur Anwendung. Umgekehrt kommt es zu einer Einkommenserhöhung im Inland (Nachversteuerung ex nunc),

576 Abweichend werden bei der Zurechnungsmethode nur konzemexterne Dividenden anteilig in die Besteuerung einbezogen, während konzerninterne Dividenden vollständig aus dem Ergebnis gekürzt werden. Diese sind aufgrund des Zurechnungsmechanismus nicht einer erneuten Besteuerung zu unterwerfen. 
wenn im Ausland zu einem späteren Zeitpunkt steuerliche Gewinne anfallen, die zum Ausgleich von zuvor im Inland verrechneten Verlusten verwendet werden können.

$\mathrm{Zu}$ diesem Zweck wird in einem ersten Schritt das zu versteuernde Einkommen der ausländischen Teilgruppen durch Anwendung der im Ausland geltenden Vorschriften zur intertemporalen Verlustverrechnung ermittelt (vgl. Formel (12)).

$$
\text { inctg;a<>c } c_{g}^{t}=f\left(\text { inc_2 } 2_{g ; a}^{t} ; l c b_{a} ; l c f_{a}\right)
$$

mit:

\begin{tabular}{|c|c|}
\hline$c_{g ; a}^{t}$ & $\begin{array}{l}\text { ermittelter Näherungswert für das zu versteuernde Einkommen } \\
\text { der in Land a ansässigen nationalen Teilgruppe von Gruppe } g \\
\text { in Periode } t\end{array}$ \\
\hline & Sitzland der Muttergesellschaft von Gruppe g \\
\hline & $\begin{array}{l}\text { Zwischensumme } 2 \text { bei der Ermittlung des Einkommens von } \\
\text { der in Land a ansässigen nationalen Teilgruppe von Gruppe } g \\
\text { in Periode } t\end{array}$ \\
\hline & zeitliche und betragsmäßige Beschränkungen des \\
\hline & $\begin{array}{l}\text { Verlustrücktrags in Land a } \\
\text { zeitliche und betragsmäßige Beschränkungen des Verlus } \\
\text { trags in Land a }\end{array}$ \\
\hline
\end{tabular}

In einem zweiten Schritt sind die im Inland zu berücksichtigen Verluste oder Nachversteuerungsbeträge zu berechnen. Diese ergeben sich technisch als Differenz zwischen dem ausländischen Einkommen vor Anwendung der intertemporalen Verlustverrechnung (inc_ $2_{\text {g; }}^{\mathrm{t}}<>\mathrm{c}_{\mathrm{g}}$ ) und dem entsprechenden Wert nach Anwendung dieser Vorschriften (inc $c_{g ; a}<>c_{g}$ ). Dabei sind negative Differenzen

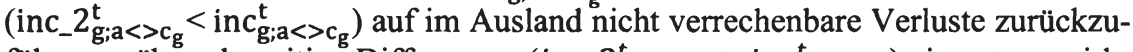
führen, während positive Differenzen (inc_2 $2_{\mathrm{g} ; a<>c_{\mathrm{g}}}>i n c_{\mathrm{g} ; \mathrm{a}<>\mathrm{c}_{\mathrm{g}}}^{t}$ ) eine steuerwirksame Nutzung von Verlustvorträgen im Ausland anzeigen. ${ }^{577}$

$$
N V_{g ; a<>c_{g}}^{t}=i n c_{-} 2_{g ; a}^{t}-i n c_{g ; a}^{t}
$$

mit:

$N V_{g ; a}^{t}$

Einkommensänderung bei der inländischen Teilgruppe von Gruppe g aufgrund der Anwendung der Nachversteuerungsmethode für die nationale Teilgruppe in Land a

577 Hiermit wird eine Nachversteuerung auf solche Auslandsgewinne beschränkt, die im Ausland einen Verlustausgleich bewirken. Diese Beschränkung beinhaltet zwar für die ausländischen Steuerhoheiten einen Anreiz zur Begrenzung des Verlustvortrags, ist aber ein realistisches Reformszenario für den Fall, dass zusätzlich auch die Vorschriften zur intertemporalen Verlustverrechnung harmonisiert werden. 
$\mathrm{c}_{\mathrm{g}} \quad$ Sitzland der Muttergesellschaft von Gruppe $\mathrm{g}$

inc $_{\text {g;a }}^{t} \quad$ ermittelter Näherungwert für das zu versteuernde Einkommen der in Land a ansässigen nationalen Teilgruppe von Gruppe $g$ in Periode $\mathrm{t}$

inc_2 $2_{\text {g;a }}^{t} \quad$ Zwischensumme 2 bei der Ermittlung des Einkommens von der in Land a ansässigen nationalen Teilgruppe von Gruppe $\mathbf{g}$ in Periode $t$

Das steuerliche Gesamtergebnis des inländischen Konzernteils (inc_3 $3_{\mathbf{g} ; \mathbf{c}_{\mathbf{g}}}^{\mathrm{t}}$ ) ergibt sich als Summe des eigenen Ergebnisses (inc_2 $2_{\mathbf{g} ; \mathbf{c}_{\mathbf{g}}}^{\mathrm{t}}$ ) und der aus dem Ausland $\mathrm{zu}$ berücksichtigenden positiven oder negativen Ergebnisbeiträge $\left(\mathrm{NV}_{\mathrm{g} ; \mathrm{a}<>\mathrm{c}_{\mathrm{g}}}^{\mathrm{t}}\right)$. Dieses wird durch Anwendung der nationalen Vorschriften zur intertemporalen Verlustverrechnung zum zu versteuernden Einkommen (inc $\mathrm{g}_{\mathrm{g}}^{\mathrm{t}} \mathrm{c}_{\mathrm{g}}$ ) überführt (vgl. Formel (14) und (15)).

$$
\text { inc_ } 3_{g ; c_{g}}^{t}=i n c_{-} 2_{g ; c_{g}}^{t}+\sum_{a} N V_{g ; a<>c_{g}}^{t}
$$

mit:

inc_3 $3_{g ; c_{g}}^{t}$ Zwischensumme 3 bei der Ermittlung des Einkommens von der inländischen nationalen Teilgruppe von Gruppe $\mathrm{g}$ in Periode $\mathrm{t}$

$c_{g} \quad$ Sitzland der Muttergesellschaft von Gruppe $g$

inc_2 $2_{\text {g; }}^{t} c_{g}$ Zwischensumme 2 bei der Ermittlung des Einkommens von der inländischen nationalen Teilgruppe von Gruppe $g$ in Periode $\mathrm{t}$

$\mathrm{NV}_{\mathrm{g} ; \mathrm{a}}^{\mathrm{t}} \quad$ Einkommensänderung bei der inländischen Teilgruppe von Gruppe g aufgrund der Anwendung der Nachversteuerungsmethode für die nationale Teilgruppe in Land a

$$
\text { inc } c_{g ; c_{g}}^{t}=f\left(i n c_{-} 3_{g ; c_{g}}^{t} ; l c b_{c_{g}} ; l c f_{c_{g}}\right)
$$

mit:

inc $_{\mathbf{g} ; c_{\mathbf{g}}}^{\mathrm{t}} \quad$ ermittelter Näherungwert für das zu versteuernde Einkommen der inländischen nationalen Teilgruppe von Gruppe $\mathrm{g}$ in Periode $\mathrm{t}$

$c_{g} \quad$ Sitzland der Muttergesellschaft von Gruppe $g$

inc_3 $3_{\mathbf{g} ; c_{\mathbf{g}}}^{\mathrm{t}}$ Zwischensumme 3 bei der Ermittlung des Einkommens von der in inländischen nationalen Teilgruppe von Gruppe $\mathrm{g}$ in Periode $\mathrm{t}$

$\mathrm{lcb}_{\mathrm{c}_{\mathbf{g}}} \quad$ zeitliche und betragsmäßige Beschränkungen des Verlustrücktrags im Sitzland der Muttergesellschaft von Gruppe $g$ 
$\operatorname{lcf}_{c_{g}} \quad$ zeitliche und betragsmäßige Beschränkungen des Verlustvortrags im Sitzland der Muttergesellschaft von Gruppe $g$

Aus Vereinfachungsgründen unterbleibt eine separate Fortführung getrennter Verrechnungstöpfe für die Verluste aus inländischer und ausländischer Geschäftstätigkeit. Die steuerwirksame Nachversteuerung wird somit nicht daran geknüpft, dass zuvor verrechnete Auslandsverluste zu einer Steuerminderung im Inland geführt haben. Dieses kann insbesondere in Fällen einer zeitlichen oder betragsmäßigen Beschränkung des Verlustvortrags im Inland zu einer aus Unternehmenssicht nachteilhaften Wirkung der Nachversteuerungsmethode führen.

Die Steuerzahlungen der inländischen und ausländischen Konzernteile ergeben sich durch Anwendung der nationalen Steuersätze auf das so ermittelte zu versteuernde Einkommen. Hierbei ist bei inländischen Konzernteilen multinationaler Konzerne zu berücksichtigen, dass auf die (positiven oder negativen) ausländischen Einkommensbestandteile nur der nationale Körperschaftsteuersatz erhöht um gegebenenfalls anfallende Zuschlagsteuern angewendet wird. Eine Erhöhung des Steuersatzes um die Ertragsteuern regionaler Gebietskörperschaften erfolgt hier nicht, da diese regelmäßig als Inlandssteuern nur auf inländische Einkommensbestandteile angewendet werden. ${ }^{578}$ Technisch wird hierzu das Einkommen ermittelt, welches sich ohne Anwendung der Nachversteuerungsmethode ergeben hätte (Formel (16)) und dieses inländische Einkommen mit dem nominellen Steuersatz in voller Höhe multipliziert. Das Mehr- oder Mindereinkommen aufgrund der Nachversteuerungsmethode wird mit dem um regionale Ertragsteuern verminderten Steuerniveau belastet (Formel (17)).

$$
\begin{aligned}
& \text { inc_nat }{ }_{g ; c_{g}}^{t}=f\left(\text { inc_2 } 2_{g ; c_{g}}^{t} ; l c b_{c_{g}} ; l c f_{c_{g}}\right) \\
& \text { tax }_{g ; c_{g}}^{t}=i n c_{-} n a t_{g ; c_{g}}^{t} \cdot \tau_{c_{g}}^{t}+\left(i n c_{g ; c_{g}}^{t}-i n c_{-} n a t_{g ; c_{g}}^{t}\right) \cdot \tau_{c_{g} ; \text { Ausland }}^{t}
\end{aligned}
$$

mit:

inc_nat ${ }_{g ;}^{\mathrm{t}} \mathrm{c}_{\mathrm{g}}$ ermittelter Näherungwert für das zu versteuernde Einkommen der inländischen nationalen Teilgruppe von Gruppe $\mathrm{g}$ in Periode t ohne Anwendung der Nachversteuerungsmethode

$\mathrm{inc}_{\mathrm{g}}^{\mathrm{c}} 2_{\mathrm{g} ; \mathrm{c}_{\mathrm{g}}}^{\mathrm{t}}$ Sitzland der Muttergesellschaft von Gruppe g Zwischensumme 2 bei der Ermittlung des Einkommens von der in inländischen nationalen Teilgruppe von Gruppe $\mathrm{g}$ in Periode $\mathrm{t}$

$\mathrm{lcb}_{\mathrm{c}_{\mathrm{g}}}$ zeitliche und betragsmäßige Beschränkungen des Verlustrücktrags im Sitzland der Muttergesellschaft von Gruppe $g$ 


\begin{tabular}{|c|c|}
\hline $\mathrm{cf}_{\mathrm{cg}_{\mathrm{g}}}$ & \\
\hline $\operatorname{inc}_{\mathrm{g} ; \mathrm{c}_{\mathrm{g}}}^{\mathrm{t}}$ & $\begin{array}{l}\text { ermittelter Näherungswert für das zu versteuernde Einkommen } \\
\text { der inländischen nationalen Teilgruppe von Gruppe } \mathrm{g} \text { in Peri- } \\
\text { ode t mit Anwendung der Nachversteuerungsmethode }\end{array}$ \\
\hline & $\begin{array}{l}\text { nominelle Ertragsteuerbelastung im Sitzland } \mathrm{c} \text { von Gruppe } \mathrm{g} \text { in } \\
\text { Periode } \mathrm{t}\end{array}$ \\
\hline ;Ausland & $\begin{array}{l}\text { nominelle Ertragsteuerbelastung auf ausländische Einkom- } \\
\text { mensbestandteile im Sitzland } \mathrm{c} \text { von Gruppe } \mathrm{g} \text { in Periode } \mathrm{t}\end{array}$ \\
\hline
\end{tabular}

\subsubsection{Zurechnungsmethode}

Bei der Zurechnungsmethode ergibt sich das steuerliche Ergebnis der inländischen Teilgruppe durch Zusammenfassung des eigenen Ergebnisses mit dem Ergebnis aller ausländischen Teilgruppen (vgl. Formel (18)). Hiermit wird implizit eine Ermittlung nach ausländischen Vorschriften für diese Ergebnisbestandteile unterstellt.

$$
\text { inc } 3_{g ; c_{g}}^{t}=\sum_{a} i n c_{-} 2_{g ; a}^{t}
$$

mit:

inc_ $3_{\mathrm{g} ; c_{g}}^{\mathrm{t}}$ Zwischensumme 3 bei der Ermittlung des Einkommens von der inländischen nationalen Teilgruppe von Gruppe $g$ in Periode $t$

$c_{\mathrm{g}} \quad$ Sitzland der Muttergesellschaft von Gruppe $\mathrm{g}$

inc_2t ${ }_{g}^{t} a$ Zwischensumme 2 bei der Ermittlung des Einkommens von der nationalen Teilgruppe von Gruppe $g$ in Land $a$ in Periode $t$

Die Zwischenergebnisse der ausländischen Teilgruppen (inc_2 $2_{\mathrm{g} ; \mathrm{a}<>c_{\mathrm{g}}}$ ) und der inländischen Konzernteile (inc_3 $3_{\mathbf{g} ; \mathbf{c}_{g}}$ ) werden durch Anwendung der nationalen Vorschriften zur intertemporalen Verlustverrechnung zum zu versteuernden Einkommen transformiert, auf welches im Anschluss die nationalen Steuersätze angewendet werden. Bei der Steuerermittlung für die inländische Teilgruppe ist hierbei - wie bei der Nachversteuerungsmethode - zwischen inländischen und ausländischen Einkommensbestandteilen $\mathrm{zu}$ unterscheiden. Auch hier wird für Zwecke der Trennung der inländischen und ausländischen Einkünfte das steuerliche Einkommen herangezogen, das sich im Inland ohne die Anwendung der $\mathrm{Zu}$ rechnungsmethode ergeben hätte (inc_nat ${ }_{g ; c_{g}}^{t}$ ). Während die inländischen Einkommensbestandteile der Körperschaftsteuer, Zuschlagsteuern und regionalen Ertragsteuern unterliegen, wird auf die ausländischen Einkommensbestandteile auch hier nur ein Ertragsteuersatz angewendet, welcher die Steuerbelastung mit Körperschaftsteuer und Zuschlagsteuern widerspiegelt. Bei der Ermittlung der Steuerzahlungen im Inland können die im Ausland anfallenden Ertragsteuern an- 
gerechnet werden. Auch die Anrechnung ist allerdings auf die nationale Körperschaftsteuer und Zuschlagsteuern beschränkt. Der Anrechnungshöchstbetrag wird dabei länderspezifisch ermittelt (,per-country-limitation“). Des Weiteren wird die Höhe der anrechenbaren Auslandssteuern insgesamt auf die im Inland anfallenden Ertragsteuern begrenzt. Ein Vortrag nicht anrechenbarer Auslandssteuern wird aus Vereinfachungsgründen nicht simuliert. Das Vorgehen bei der Ermittlung der Ertragsteuern der inländischen Teilgruppe ist in Formel (19) dargestellt:

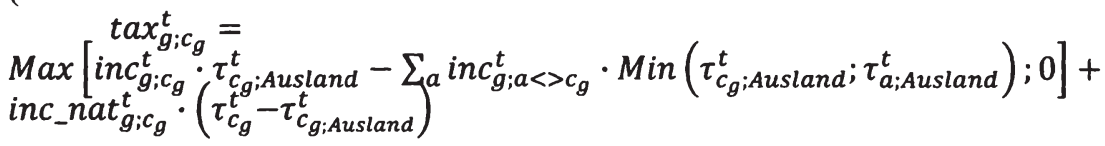

mit:

\begin{tabular}{|c|c|}
\hline$x_{g ; c_{g}}^{t}$ & $\begin{array}{l}\text { Steuerzahlung der inländischen nationalen Teilgruppe von } \\
\text { Gruppe g in Periode } t\end{array}$ \\
\hline & $\begin{array}{l}\text { Sitzland der Muttergesellschaft von Gruppe } g \\
\text { ermittelter Näherungwert für das zu versteuernde Einkommen } \\
\text { der inländischen nationalen Teilgruppe von Gruppe } g \text { in Peri- } \\
\text { ode }\end{array}$ \\
\hline & $\begin{array}{l}\text { ermittelter Näherungswert für das zu versteuernde Einkommen } \\
\text { der nationalen Teilgruppe von Gruppe } g \text { in Land a in Periode } t\end{array}$ \\
\hline & $\begin{array}{l}\text { ermittelter Näherungwert für das zu versteuernde Einkommen } \\
\text { der inländischen nationalen Teilgruppe von Gruppe } g \text { in Peri- } \\
\text { ode t ohne Anwendung der Zurechnungsmethode }\end{array}$ \\
\hline & $\begin{array}{l}\text { nominelle Ertragsteuerbelastung im Sitzland c von Gruppe } g \text { in } \\
\text { Periode } t\end{array}$ \\
\hline ;Ausland & $\begin{array}{l}\text { nominelle Ertragsteuerbelastung auf ausländische Einkom- } \\
\text { mensbestandteile im Sitzland c von Gruppe } \mathrm{g} \text { in Periode } \mathrm{t}\end{array}$ \\
\hline & $\begin{array}{l}\text { nominelle Ertragsteuerbelastung auf ausländische Einkom- } \\
\text { mensbestandteile in Land a in Periode } t\end{array}$ \\
\hline
\end{tabular}

\subsubsection{Ergebnisaufteilungsmethode}

Bei der Ermittlung der steuerlichen Einkommen nach der Ergebnisaufteilungsmethode werden in einem ersten Schritt die für die nationalen Teilgruppen ermittelten Zwischensummen auf Konzernebene zusammengefasst (vgl. Formel (20)). 
(20) inc_ $2_{g}^{t}=\sum_{a} i n c_{-} 2_{g ; a}^{t}$

mit:

inc_2t Zusammengefasstes Einkommen der nationalen Teilgruppen von Gruppe $\mathrm{g}$

inc_2 $2_{\text {g; } a}$ Zwischensumme 2 bei der Ermittlung des Einkommens von der nationalen Teilgruppe von Gruppe $g$ in Land $a$ in Periode $t$

Ist das Gesamtergebnis der Gruppe positiv, wird es proportional unter den nationalen Teilgruppen mit eigenem positivem Ergebnis aufgeteilt, während die nationalen Teilgruppen mit negativem Ergebnis ein Ergebnis von null erhalten. Im Fall eines negativen Gesamteinkommens wird entsprechend umgekehrt verfahren. Hierzu werden zunächst die Summen der positiven und negativen Ergebnisse der nationalen Teilgruppen ermittelt (vgl. Formel (21) und (22)).

(21) inc_2 $2_{g+}^{t}=\sum_{a ; i n c_{-} 2_{g ; a}^{t}>0}$ inc_- $2_{g ; a}^{t}$

(22) inc_2tg- $2_{g-i n c_{-} 2_{g ; a}^{t}<0} i n c_{-} 2_{g ; a}^{t}$

mit:

inc_2 $2_{g+}^{t} \quad$ Summe der positiven Zwischensummen 2 der nationalen Teilgruppen von Gruppe $\mathrm{g}$ in Periode $\mathrm{t}$

inc_2 $2_{g-}^{t} \quad$ Summe der negativen Zwischensummen 2 der nationalen Teilgruppen von Gruppe $\mathrm{g}$ in Periode $\mathrm{t}$

inc_2 $2_{g ; a}^{t}$ Zwischensumme 2 bei der Ermittlung des Einkommens von der nationalen Teilgruppe von Gruppe $\mathbf{g}$ in Land $\mathbf{a}$ in Periode $t$

Die Ergebnisaufteilung ergibt sich dann nach Formel (23).

(23)

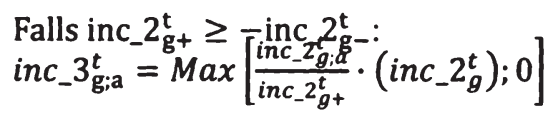

Falls inc_2 $2_{\mathbf{g}_{+}}^{\mathrm{t}}<-$ inc_2 $_{-}^{\mathrm{t}} \mathbf{g}_{-}$:

inc_ $3_{\mathrm{g} ; \mathrm{a}}^{t}=\operatorname{Min}\left[\frac{i n c_{-} g_{g ; a}^{t}}{i n c_{-} 2_{g-}^{t}} \cdot\left(i n c_{-} 2_{g}^{t}\right) ; 0\right]$

mit:

inc_3 $3_{\text {g;a }}^{t}$ Zwischensumme 3 bei der Ermittlung des Einkommens von der nationalen Teilgruppe von Gruppe $\mathrm{g}$ in Land $\mathrm{a}$ in Periode $\mathrm{t}$

inc_2 $2_{g^{+}}$Summe der positiven Zwischensummen 2 der nationalen Teilgruppen von Gruppe $\mathrm{g}$ in Periode $\mathrm{t}$

inc_2t ${ }_{g}^{t}$ Summe der negativen Zwischensummen 2 der nationalen Teilgruppen von Gruppe $\mathrm{g}$ in Periode $\mathrm{t}$ 
inc_2 $2_{\mathrm{g} ; a}^{\mathrm{a} a}$ Zwischensumme 2 bei der Ermittlung des Einkommens von der nationalen Teilgruppe von Gruppe $g$ in Land $a$ in Periode $t$

inc_ $2_{g}^{t} \quad$ Zusammengefasstes Einkommen der nationalen Teilgruppen von Gruppe $g$

Nach der Ergebnisaufteilung zugewiesene Verluste werden auf Ebene der jeweiligen nationalen Teilgruppe nach Maßgabe der nationalen Vorschriften zur intertemporalen Verlustverrechnung behandelt (vgl. Formel (24)). Verlustvorträge werden in zukünftigen Perioden nicht erneut in die Ergebnisaufteilung einbezogen.

$$
\text { inc } c_{g ; a}^{t}=f\left(\text { inc_ } 3_{g ; a}^{t} ; l c b_{a} ; l c f_{a}\right)
$$

mit:

\begin{tabular}{|c|c|}
\hline inc $_{g ; a}^{t}$ & $\begin{array}{l}\text { ermittelter Näherungswert für das zu versteuernde Einkommen } \\
\text { der in Land a ansässigen nationalen Teilgruppe von Gruppe } g \\
\text { in Periode } t\end{array}$ \\
\hline c__3 $3_{\text {; } ; a}^{t}$ & $\begin{array}{l}\text { Zwischensumme } 3 \text { bei der Ermittlung des Einkommens von } \\
\text { der in Land a ansässigen nationalen Teilgruppe von Gruppe } g \\
\text { in Periode } t\end{array}$ \\
\hline & $\begin{array}{l}\text { zeitliche und betragsmäßige Beschränkungen des } \\
\text { Verlustrücktrags in Land a }\end{array}$ \\
\hline & $\begin{array}{l}\text { zeitliche und betragsmäßige Beschränkungen des Verlustvor- } \\
\text { trags in Land a }\end{array}$ \\
\hline
\end{tabular}

Bei der Ermittlung der Steuerzahlungen muss erneut berücksichtigt werden, dass regionale Ertragsteuern regelmäßig nur auf inländische Einkommensbestandteile erhoben werden. Deshalb werden die aufgrund der Ergebnisaufteilung zugewiesenen ausländischen Einkommensbestandteile erneut nur der Körperschaftsteuer und möglichen nationalen Zuschlagsteuern unterworfen. Technisch wird hierzu das Einkommen ermittelt, welches sich ohne Ergebnisaufteilung ergeben hätte (vgl. Formel (16)) und auf dieses inländische Einkommen der nominelle Steuersatz in voller Höhe angewendet. Das Mehr- oder Mindereinkommen aufgrund der Ergebnisaufteilung wird mit dem um regionale Ertragsteuern verminderten Steuerniveau belastet (vgl. Formel (17)).

\subsection{Ermittlung des Steueraufkommens}

Die aggregierte Steuerbemessungsgrundlage und das aggregierte Steueraufkommen der Mitgliedsstaaten a werden sowohl als Bruttogröße als auch als Nettogröße ermittelt. Bruttobemessungsgrundlage und Bruttosteueraufkommen geben dabei die Summe der zu versteuernden Einkommen (inct ${ }_{\text {Relnald }}^{\text {) }}$ ) beziehungsweise der 
Steuerzahlungen $\left(\operatorname{tax}_{\mathrm{i}}^{\mathrm{t}}\right)$ aller Konzerngesellschaften $\mathrm{i}$ mit Sitz in dem jeweiligen Mitgliedsstaat a $\left(=c_{i}\right)$ während des Betrachtungszeitraums 1994 bis 2003 an; verbleibende Verlustvorträge zum Ende des Betrachtungszeitraums werden für diese Größen ignoriert. Nettobemessungsgrundlage und Nettosteueraufkommen werden demgegenüber definiert als der entsprechende Bruttowert abzüglich des Betrags beziehungsweise des steuerlichen Werts von Verlustvorträgen zum Ende des $\mathrm{Be}$ trachtungszeitraums $\left(\mathrm{l}_{1}^{10}\right)$. Der Nettobarwert wird ermittelt, indem das zu versteuernde Einkommen beziehungsweise die Steuerzahlungen mit einem einheitlichen Zinssatz von sechs Prozent diskontiert werden. Für die Verlustvorträge zum Ende des Betrachtungszeitraums wird eine Nutzung in der dem Betrachtungszeitraum unmittelbar folgenden Periode unterstellt.

Tabelle 21: Ermittlung des Steueraufkommens

\begin{tabular}{|l|c|c|}
\hline & Steueraufkommen & Steuerbemessungsgrundlage \\
\hline Brutto & $R_{a}^{g r o s s}=\sum_{i ; c_{i}=a} \sum_{t=1}^{10} t a x_{i}^{t}$ & $T B_{a}^{g r o s s}=\sum_{i_{i}=a} \sum_{t=1}^{10} i n c_{t}^{t}$ \\
\hline Netto & $R_{a}^{\text {net }}=R_{a}^{g r o s s}-\sum_{i: c_{i}=a} l_{i}^{10} \cdot \tau_{a}^{10}$ & $T B_{a}^{\text {net }}=T B_{a}^{g r o s s}-\sum_{i_{i} c_{i}=a} l_{i}^{10}$ \\
\hline $\begin{array}{l}\text { Netto- } \\
\text { Barwert }\end{array}$ & $R_{a}^{N P V}=\sum_{i: c_{i}=a} \sum_{t=1}^{10} \frac{\operatorname{tax} x_{i}^{t}}{1,06^{t}}-\frac{l_{i}^{10} \cdot \tau_{a}^{10}}{1,06^{11}}$ & $T B_{a}^{N P V}=\sum_{i: c_{i}=a} \sum_{t=1}^{10} \frac{i n c_{i}^{t}}{1,06^{t}}-\frac{l_{i}^{10}}{1,06^{11}}$ \\
\hline
\end{tabular}

Quelle: Eigene Darstellung

mit:

$\begin{array}{ll}\operatorname{tax}_{\mathrm{i}}^{\mathrm{t}} & \text { Steuerzahlung von Unternehmen } \mathrm{i} \text { in Periode } \mathrm{t} \\ \operatorname{inc}_{\mathrm{i}}^{\mathrm{t}} & \text { Einkommen von Unternehmen } \mathrm{i} \text { in Periode } \mathrm{t} \\ \mathrm{c}_{\mathrm{i}} & \text { Sitzland } \mathrm{c} \text { von Unternehmen } \mathrm{i} \\ \mathrm{a} & \text { Länderindex } \\ \mathrm{l}_{\mathrm{i}}^{10} & \text { Verlustvortrag von Unternehmen } \mathrm{i} \text { zum Ende des Betrach- } \\ & \text { tungszeitraums (Periode 10) } \\ \tau_{\mathrm{a}}^{10} & \text { nominelle Ertragsteuerbelastung in Land a in Periode } 10\end{array}$

Änderungen in der Bemessungsgrundlage und dem Steueraufkommen werden sowohl als aggregierter Wert für die Gesamt-EU als auch für die einzelnen Mitgliedsstaaten ermittelt. Mit Blick auf die Änderungen für die einzelnen Mitgliedsstaaten wird zusätzlich auch die durchschnittliche Höhe der Änderung des Steueraufkommens beziehungsweise der Bemessungsgrundlage angegeben. Diese Größe lässt die Stärke des Einflusses erkennen, der von den untersuchten Steuerreformen auf die Höhe des Steueraufkommens beziehungsweise der Bemessungsgrundlage der Mitgliedsstaaten (unabhängig von der Richtung dieser Änderung) 
ausgeht. Damit sollte dieser Wert nicht unbedeutend für die Aussicht auf die politische Umsetzbarkeit des jeweiligen Reformvorschlags sein, wenn unterstellt werden kann, dass die Bereitschaft sämtlicher Mitgliedsstaaten zur Teilnahme an einer Reform nicht nur von der Änderung des Gesamtaufkommens sondern auch vom Ausmaß der Verschiebung von Steueraufkommen zwischen den Mitgliedsstaaten abhängt. Eine stärkere Verschiebung ist dabei gleichzusetzen mit einem höheren Risiko hinsichtlich des zu erwartenden Steueraufkommens. Zur Verdeutlichung der Ermittlung dieser Größe wird in Formel (25) die Berechnung der durchschnittlichen/betragsmäßigen Höhe der Änderung des Brutto-Steueraufkommens bei Einführung einer GKKB gezeigt.

$$
\overline{\left|\Delta R^{\text {gross }}\right|}=\sum_{a=1}^{A} \sqrt{\left(\frac{R_{a}^{\text {gross } ; G K K^{\prime}}-R_{a}^{\text {gross } ; \text { geltSR }}}{R_{a}^{\text {gross; } ; \text { geltSR }}}\right)^{2}} / A
$$

mit:

$\overline{\left|\Delta R_{a}^{\text {gross }}\right|}$ durchschnittliche Höhe der Änderung des Bruttoaufkommens

$$
\text { a Länderindex }
$$

A Anzahl an Ländern ${ }^{579}$

$R_{a}^{\text {gross;GKKB }}$ Bruttosteueraufkommen in Land a bei Besteuerung nach dem Konzept der GKKB

$R_{a}^{\text {gross;geltSR }}$ Bruttosteueraufkommen in Land a bei Besteuerung nach dem geltenden Steuerrecht

\subsection{Ermittlung der durchschnittlichen Steuerbelastung}

Bezüglich der Definition der durchschnittlichen Steuerbelastung hat sich in den bestehenden Untersuchungen, die wie in dieser Arbeit ein vergangenheitsbezogenes $\mathrm{Ma} ß$ auf Basis empirischer Mikrodaten zugrunde legen, kein einheitlicher Standard etabliert. Unterschiede ergeben sich hinsichtlich der zugrundegelegten Mikroeinheit, des Betrachtungszeitraums und der Definition der durchschnittlichen Steuerbelastung. Während einige Studien auf den Konzern als relevante Mikroeinheit für die Ermittlung der Steuerbelastung abstellen ${ }^{580}$, werden in anderen Studien einzelne Unternehmen ${ }^{581}$ zugrundegelegt. ${ }^{52}$ Dabei beinhaltet ein $\mathrm{Ab}$ stellen auf Konzernabschlüsse das Problem der Einbeziehung ausländischer Toch-

579 In die Ermittlung der Werte für Zypern, Litauen, Lettland, Luxemburg und der Slowakei ging nur eine geringe Anzahl von Unternehmen, die Bestandteil eines multinationalen Konzerns sind, ein (vgl. Tabelle 18). Aus diesem Grund werden diese Mitgliedsstaaten bei der Ermittlung von Durchschnittswerten nicht berücksichtigt, um mögliche Verzerrungen zu vermeiden.

$580 \mathrm{Vgl}$. unter anderem Buijink/Jannsen/Schols (2000).

581 Vgl. z.B. Nicodeme (2001) und Zimmerman (1983).

582 Vgl. so auch Schratzenstaller (2004), S. 52-59. Reinald Koch - 978-3-631-75683-6 
terunternehmen, was eine Unterscheidung der Steuerbelastung nationaler und grenzüberschreitend tätiger Konzerne erforderlich macht. ${ }^{583} \mathrm{Da}$ das Ziel der hier erfolgenden Untersuchung die Beurteilung der Folgen einer Reform der Konzernbesteuerung ist, besteht kein Zweifel daran, dass in dieser Arbeit auf den Konzern als relevante Untersuchungseinheit abzustellen ist.

Hinsichtlich des Betrachtungszeitraums stellen einige Studien auf eine einzelne Periode $\mathrm{ab}^{584}$, während andere einen längeren Zeitraum betrachten. Durchschnittliche Steuerbelastungen, die auf Basis von einperiodigen Daten ermittelt werden, unterliegen oftmals Verzerrungen durch die in dieser Periode vorherrschenden gesamtwirtschaftlichen Rahmenbedingungen und können die Wirkungen der steuerlichen Verlustverrechnungsvorschriften nicht adäquat abbilden. ${ }^{585}$ In dieser Arbeit wird ein Zeitraum von zehn Jahren zugrundelegt, da ein kürzerer Zeitraum mit Blick auf die Zielsetzung einer Simulation von Änderungen der steuerlichen Verlustverrechnung nicht sinnvoll erscheint.

Ökonomischen Kalkülen folgend sollte die durchschnittliche Steuerbelastung definiert werden als das Verhältnis der Barwerte zukünftiger Steuerzahlungen und zukünftiger ökonomischer Gewinne. ${ }^{586}$ Ökonomische Gewinne können aus den handelsrechtlichen Jahresabschlüssen allerdings nicht abgeleitet werden und müssen daher näherungsweise geschätzt werden. ${ }^{587}$

In den bestehenden Studien werden üblicherweise die Steuerzahlungen (oder Steueraufwendungen) in Relation zu einer Gewinngröße, den Umsatzerlösen oder dem operativen Cashflow gesetzt. ${ }^{588}$ Dabei ist aus ökonomischer Sicht allerdings jede Definition des Nenners, die zu einer Abhängigkeit von den zugrundeliegenden Bilanzierungsnormen führt, abzulehnen, da als Konsequenz eine rechtliche Steuerbelastung und nicht eine wirtschaftliche Steuerbelastung gemessen wird. Außerdem werden internationale Steuerbelastungsvergleiche verzerrt, wenn die Bezugsgröße für die zugrunde liegende Steuerbelastung abhängig ist von den landesspezifischen Bilanzierungsnormen. ${ }^{589}$ Eine Bezugnahme auf die Umsatzerlöse führt zu verzerrten Ergebnissen bei internationalen Profitabilitätsunterschieden. Die Verwendung des operativen Cashflows schließlich fuhrt zur Messung einer wirtschaftlichen Steuerbelastung durch das Abstellen auf eine wirtschaftliche Zielgröße und sichert dadurch auch internationale Vergleichbarkeit. Allerdings wird eine Abhängigkeit der resultierenden Steuerbelastung von der Kapitalintensität der Unternehmen erzeugt. Ceteris paribus führt bei dieser Definition eine höhe-

583 Vgl. Schratzenstaller (2004), S. 54; Nicodeme (2001), S. 8; Gorter/de Mooij (2001), S. 18; Collins/Shackelford (1995), S. 57.

584 Vgl. z.B. Plesko (2003).

$585 \mathrm{Vgl}$. Shevlin (1999), S. 5-6.

$586 \mathrm{Vgl}$. Collins/Shackelford (1995), S. 58.

587 Vgl. Schratzenstaller (2004), S. 52-59; Collins/Shackelford (1995), S. 58.

588 Vgl. Plesko (2003), S. 205; Buijink/Jannsen/Schols (2000), S. 10-11.

$589 \mathrm{Vgl}$. Nicodeme (2001), S. 18; Buijink/Jannsen/Schols (2000) S. S. $1 p_{8-3-631-75683-6}$ 
re Kapitalintensität zu einer geringeren Steuerbelastung ${ }^{590}$, da bilanzielle Abschreibungen zwar die Zählergröße, nicht aber die Nennergröße mindern.

Unter Berücksichtigung der oben dargestellten Probleme erscheint die Definition der durchschnittlichen Steuerbelastung als Minderung des Cashflow to Equity (CF) am zielführendsten. Die Cashflows werden dabei indirekt aus den handelsrechtlichen Jahresabschlüssen abgeleitet und ermittelt als Summe von Operativem Cashflow (OCF), Cashflow aus Fremdfinanzierung (DCF) und Investitionscashflow (ICF).

$$
C F_{i}^{t}=O C F_{i}^{t}+D C F_{i}^{t}+I C F_{i}^{t}
$$

mit:
$\mathrm{CF}_{\mathrm{i}}^{\mathrm{t}}$
$O C F_{i}^{t}$
Cashflow von Unternehmen i in Periode $t$
$D C F_{i}^{t}$
Operativer Cashflow von Unternehmen i in Periode $t$
Cashflow aus Fremdfinanzierung von Unternehmen i in Perio- de $t$
ICF $\mathrm{i}_{\mathrm{i}}^{\mathrm{I}}$ Investitionscashflow von Unternehmen $\mathrm{i}$ in Periode $\mathrm{t}$

Dabei ergibt sich der operative Cashflow nach der hier zugrundegelegten indirekten Ermittlungsform als Summe des Ergebnisses vor Zinsen und Steuern $\left(\mathrm{plbt}_{\mathrm{i}}^{\mathrm{t}}+\right.$ int $_{\mathrm{i}}^{\mathrm{t}}$ ) und der nicht-zahlungswirksamen Aufwendungen (Abschreibungen und Zuführungen zu Rückstellungen). Als Näherung für den Buchwert der Rückstellungen wird hier die AMADEUS-Position "sonstige langfristige Schulden“ $\left(\right.$ olt $_{\mathrm{i}}^{\mathrm{t}}$ ) herangezogen. Der Cashflow aus Fremdfinanzierung erfasst in der hier zugrundegelegten Form die Aufnahme und Tilgung langfristiger Darlehen sowie die Zinszahlungen, während der Investitionscashflow sich aus den Zahlungsvorgängen aus der Anschaffung und Veräußerung von Anlagevermögen zusammensetzt. Diese werden indirekt ermittelt als Änderung des Buchwerts des Anlagevermögens zuzüglich der Abschreibungen der Periode. Zusammenfassend ergibt sich der hier zugrundegelegte Cashflow nach Formel (27) beziehungsweise nach Umformulierung entsprechend Formel (28). deprit

$$
C F_{i}^{t}=p l b t_{i}^{t}+\operatorname{depr}_{i}^{t}+\Delta o l t l_{i}^{t}+i n t_{i}^{t}+\Delta l t d_{i}^{t}-i n t_{i}^{t}-\left(\Delta \text { fias }_{i}^{t}+\right.
$$

$$
C F_{i}^{t}=p l b t_{i}^{t}+\Delta l t l_{i}^{t}-\Delta \text { fias }_{i}^{t}
$$

mit:

$$
\begin{array}{ll}
\mathrm{CF}_{\mathrm{i}}^{\mathrm{t}} & \text { Cashflow von Unternehmen i in Periode } \mathrm{t} \\
\mathrm{plbt}_{\mathrm{i}}^{\mathrm{t}} & \text { Ergebnis der gewöhnlichen Geschäftstätigkeit (,Profit/loss } \\
& \text { before tax“) von Unternehmen i in Periode } \mathrm{t}
\end{array}
$$




\begin{tabular}{|c|c|}
\hline $\operatorname{depr}_{i}^{t}$ & $\begin{array}{l}\text { Abschreibungen ("Depreciation“) von Unternehmen i in Perio- } \\
\text { de } t\end{array}$ \\
\hline$\Delta$ olttl $\mathrm{i}_{\mathrm{i}}$ & $\begin{array}{l}\text { Änderung des Buchwerts der sonstigen Verbindlichkeiten } \\
\text { („Other long-term liabilities“) von Unternehmen i in Periode } t\end{array}$ \\
\hline & Zinsaufwand („Interest") von Unternehmen $\mathrm{i}$ in Periode $\mathrm{t}$ \\
\hline & $\begin{array}{l}\text { Änderung des Buchwerts der langfristigen Verbindlichkeiten } \\
\text { (,Long-term debt") von Unternehmen i in Periode } t\end{array}$ \\
\hline$\Delta$ fias $_{\mathbf{i}}^{t}$ & $\begin{array}{l}\text { Änderung des Buchwerts des Anlagevermögens (,Fixed } \\
\text { assets") von Unternehmen } \mathrm{i} \text { in Periode } t\end{array}$ \\
\hline & $\begin{array}{l}\text { Änderung des Buchwerts der langfristigen Schulden (,Long- } \\
\text { term liabilities“) von Unternehmen } \mathrm{i} \text { in Periode } t\end{array}$ \\
\hline
\end{tabular}

Für die Bestimmung der Durchschnittssteuerbelastung werden Steuerzahlungen und Cashflows mit einem einheitlichen Satz von sechs Prozent diskontiert. ${ }^{591}$ Für die am Ende der Simulationsperiode verbleibenden Verlustvorträge wird die kontinuierliche Nutzung in den zehn folgenden Perioden zu einem festen Prozentsatz (degressiv) unterstellt. ${ }^{52}$ Die Verlustnutzungsrate wird länderabhängig, in Abhängigkeit von der Gruppengröße und separat für jedes der simulierten Steuerszenarien auf der Basis der Simulationsrechnungen ermittelt. Um die Auswirkungen der verschiedenen Reformszenarien auf die Verlustnutzung genauer abbilden zu können, wird für die Ermittlung der Rate zusätzlich zwischen Unternehmen, die zu einem nationalen Konzern gehören, und Unternehmen, die als InboundInvestition bzw. Outbound-Investition zu einem multinationalen Konzern gehören, unterschieden. Bei einer Besteuerung nach geltendem Steuerrecht beträgt die durchschnittliche Verlustnutzungsrate über alle Länder etwa 38 Prozent. Für die verschiedenen Reformszenarien, die grundsätzlich zu einer Ausweitung der Verlustverrechnungsmöglichkeiten führen, liegt die Rate entsprechend höher. Der ökonomische Wert der steuerlichen Verlustvorträge zum Ende der Simulationsperiode (LCF) wird ermittelt als Barwert der von diesen Verlustvorträgen ausgehenden Minderungen künftiger Steuerzahlungen, also als Barwert der mit dem maßgebenden Steuersatz multiplizierten genutzten Verluste.

$$
A T R_{g}=\frac{\sum_{t=1}^{10} t a x_{g}^{t} / 1,06^{t}-L C F_{g}}{\sum_{t=1}^{10} C F_{g}^{t} / 1,06^{t}}
$$

mit:
$\mathrm{ATR}_{\mathrm{g}}$
Durchschnittliche Steuerbelastung von Gruppe $\mathrm{g}$

591 Eine Differenzierung in Vor- und Nachsteuerzins wird nicht berücksichtigt, da die Diskontierung lediglich der Verdichtung der Zahlungsströme dient, nicht aber als Alternativinvestition im finanzwirtschaftlichen Sinne.

592 Aufgrund der Unterstellung einer degressiven Verlustnutzung wird der Verlustvortrag nicht in voller Hơhe wirksam. Bei den hier zugrundegelegten Verlustnutzungsraten fallt dieser Restverlust allerdings nicht ins Gewicht. 

$\operatorname{tax} x_{g}^{t}$
Summe der Steuerzahlungen von Gruppe $\mathrm{g}$ in Periode $\mathrm{t}$
$\mathrm{LCF}_{\mathrm{g}}$
Barwert der Steuervorteile aus der Nutzung des Verlustvor- trags zum Ende des Betrachtungszeitraums von Gruppe $\mathrm{g}$
$\mathrm{CF}_{\mathrm{g}}^{\mathrm{t}}$ Cashflow von Gruppe $\mathrm{g}$ in Periode $\mathrm{t}$

Die beschriebene Cashflow-Definition vermeidet ein gerichtetes Missverhältnis zwischen Zähler und Nenner der durchschnittlichen Steuerbelastung solange sich die Summe der Abschreibungen und die Summe der Investitionszahlungen innerhalb der Simulationsperiode sowie die Aufnahme und die Rückzahlung von langfristigen Verbindlichkeiten in der Simulationsperiode entsprechen. Diese Prämissen sollten im Mittel allerdings nur gelten, solange Unternehmen nicht wachsen. Bei wachsenden Unternehmen ist hingegen eine leichte Unterschätzung der durchschnittlichen Steuerbelastung zu erwarten.

Zudem kann es bei einzelnen Konzernen aufgrund der individuellen Investitions- und Finanzierungspolitik im Simulationszeitraum zu stärkeren Verzerrungen kommen. Um diese zu vermeiden, werden Konzerne ausgeschlossen, deren Ergebnisse der gewöhnlichen Geschäftstätigkeit während der Simulationsperiode in Summe um mehr als 100 Prozent von der Summe der Cashflows abweichen. Um Verzerrungen aufgrund von Ausreißerwerten zu vermeiden bleiben ferner Konzerne, deren durchschnittliche Steuerbelastung um mehr als 100 Prozent vom Median der durchschnittlichen Steuerbelastung aller Konzerne des Sitzlandes der Muttergesellschaft abweicht, unberücksichtigt. ${ }^{593}$

Die Interpretation der so ermittelten Steuerbelastungen als Maß für die Standortattraktivität beziehungsweise Wettbewerbsfähigkeit sowie der Effizienzwirkungen der Besteuerung verlangt die Ermittlung von Durchschnittswerten und Schwankungswerten. ${ }^{594}$ Hierbei werden zwei weitere Differenzierungen vorgenommen. Zum einen wird die Steuerbelastung von Konzernen mit positivem $\left(\mathrm{ATR}^{+}\right)$und negativem Cashflowbarwert (ATR') getrennt betrachtet. Diese Unterscheidung ist erforderlich, da die ermittelten Steuerbelastungen in diesen beiden Fällen eine unterschiedliche Interpretation erfordern. Während $\mathrm{ATR}^{+}$als Steuerlast interpretiert werden muss, ist $\mathrm{ATR}^{-}$als durch die Verluste induzierte Steuerentlastung zu verstehen. Dementsprechend ist steuerliche Standortattraktivität durch niedrige Werte für $\mathrm{ATR}^{+}$und hohe Werte für $\mathrm{ATR}^{-}$gekennzeichnet. Zur Ermittlung der durchschnittlichen Steuerbelastung eines Landes sind daher in einem ersten Schritt die Durchschnittswerte für die Steuerbelastung der nach dem

593 Bei der Ermittlung des Medians wird differenziert zwischen nationalen und multinationalen Konzernen sowie Konzernen, die innerhalb des Betrachtungszeitraums einen positiven Cashflow-Barwert erzielt haben und Konzernen mit negativem Cashflow-Barwert im Betrachtungszeitraum.

594 Bei der Ermittlung derartiger Maßgrößen kann entweder auf die Steuerbelastungen der einzelnen Konzerne oder auf die für einzelne Mitgliedsstaaten zurückgegriffen werden. Vgl. hierzu Kapitel 6.1. 
beschriebenen Ausschluss bestimmter Konzerne in der Stichprobe verbleibenden Konzerne dieses Landes mit positivem beziehungsweise negativem Cashflowbarwert zu ermitteln.

$$
\begin{aligned}
& A T R_{a}^{+}=\overline{A T R_{g ; c_{g}=a ; B W\left(C F_{g}\right)>0}} \\
& A T R_{a}^{-}=\overline{A T R_{g ; c_{g}=a ; B W\left(C F_{g}\right)<0}}
\end{aligned}
$$

mit:
$\mathrm{ATR}_{\mathbf{a}}^{+}$ Durchschnitt der Steuerbelastung rentabler Konzerne in Land a ATR $_{\mathbf{a}}^{-}$ Durchschnitt der Steuerbelastung verlusterzielender Konzerne in Land a
$\operatorname{ATR}_{\mathrm{g}}$ Durchschnittliche Steuerbelastung von Gruppe g
$\mathrm{C}_{\mathrm{g}}$ Sitzland (der Muttergesellschaft) von Gruppe $g$
$\mathrm{BW}\left(\mathrm{CF}_{\mathrm{g}}\right)$ Barwert der Cashflows von Gruppe $\mathrm{g}$ im Betrachtungszeitraum

Unter Berücksichtigung ihrer unterschiedlichen Interpretation lassen sich die beiden Größen (ATR $\mathrm{A}_{\mathrm{a}}^{+}$und $\mathrm{ATR}_{\mathrm{a}}^{-}$) zu einer kombinierten Steuerbelastung (ATR) zusammenfassen. Dabei wird eine positive Differenz zwischen ATR $_{a}^{+}$und ATR AT $_{a}^{-}$ als Zusatzbelastung gewichtet mit dem Anteil von Unternehmen mit Totalverlust $\left(\mathrm{n}_{\mathrm{a}}^{-} /\left(\mathrm{n}_{\mathrm{a}}^{-}+\mathrm{n}_{\mathrm{a}}^{+}\right)\right)$auf die Steuerbelastung von Unternehmen mit Totalgewinn $\left(\mathrm{ATR}_{\mathrm{a}}^{+}\right)$addiert. Eine negative Differenz wird entsprechend als Steuerentlastung von ATR $\mathrm{a}_{\mathrm{a}}^{+}$abgezogen. Die folgende Formel für die kombinierte ATR eines Landes resultiert:

$$
A T R_{a}=A T R_{a}^{+} \cdot \frac{n_{a}^{+}}{n_{a}^{+}+n_{a}^{-}}+\left(A T R_{a}^{+}+A T R_{a}^{+}-A T R_{a}^{-}\right) \cdot \frac{n_{\bar{a}}^{-}}{n_{a<}^{+}+n_{a}^{-}}
$$

mit:

ATR $_{\mathrm{a}} \quad$ Durchschnittliche Steuerbelastung in Land a

ATR $_{a}^{+} \quad$ Durchschnitt der Steuerbelastung rentabler Konzerne in Land a ATR $_{\mathbf{a}}^{-} \quad$ Durchschnitt der Steuerbelastung verlusterzielender Konzerne in Land a

$\mathrm{n}_{\mathrm{a}}^{+} \quad$ Anzahl rentabler Konzerne in Land a

$\mathrm{n}_{\mathrm{a}}^{-} \quad$ Anzahl verlusterzielender Konzerne in Land a

Ferner ist bei der Ermittlung der durchschnittlichen Steuerbelastung zwischen der Steuerbelastung nationaler Konzerne und multinationaler Konzerne mit mindestens einer ausländischen Tochtergesellschaft zu unterschieden. Diese Differenzierung ist erforderlich, da nur die ATR für nationale Konzerne ausschließlich vom Steuersystem eines Landes abhängig ist und damit unmittelbar als Maß für die Standortattraktivität dieses Landes betrachtet werden kann. Die ATR für multinationale Konzerne ist hingegen abhängig von den Steuersystemen aller be- 
teiligten Länder. Außerdem ist diese Unterscheidung sinnvoll, da sich die zu untersuchenden Steuerreformen unterschiedlich auf die Steuerbelastung nationaler und multinationaler Konzerne auswirken. 


\section{Empirische Ergebnisse: Auswirkungen auf die Steu- erbelastung der Konzerne}

\section{1 Überblick}

Die Wirkungen, welche von den verschiedenen Vorschlägen zur Reform der Konzermbesteuerung in Europa auf die Steuerbelastung der betroffenen Konzerne ausgehen, sind Gegenstand der Ausführungen in Kapitel 6. ${ }^{595}$ Mit der Analyse dieser Belastungswirkungen werden zwei Zielsetzungen verbunden.

Ein erstes Ziel liegt in der Untersuchung der Auswirkungen der verschiedenen Reformvorschläge auf die Wettbewerbsfähigkeit europäischer Konzerne und die Attraktivität der Mitgliedsstaaten als Steuerstandort. Zu diesem Zweck erfolgt in Kapitel 6.2 eine Analyse der Änderungen, die sich als Folge der Umsetzung dieser Reformvorschläge bei der Höhe der durchschnittlichen Konzernsteuerbelastung ergeben.

Als zweite Zielsetzung werden in Kapitel 6.3 die Auswirkungen der verschiedenen Reformvorschläge auf das Ausmaß steuerbedingter Wettbewerbs- und Entscheidungsverzerrungen innerhalb des Binnenmarktes untersucht. Wettbewerb und Unternehmensentscheidungen können dabei auf zwei Arten durch die Besteuerung beeinflusst werden. Erstens wird der Wettbewerb zwischen Marktteilnehmern, die in unterschiedlichen Mitgliedsstaaten ansässig sind, verzerrt, wenn diese aufgrund des Nebeneinanders eigenständiger Steuersysteme einer unterschiedlichen Steuerbelastung unterliegen (Verstoß gegen die interjurisdiktionale Neutralität). Das Ausmaß dieser Verzerrung kann erfasst werden über die Schwankung der durchschnittlichen Steuerbelastungen, die für die verschiedenen Mitgliedsstaaten ermittelt werden. Die Messung dieser Schwankung erfolgt hier als Variationskoeffizient (definiert als Quotient von Standardabweichung und Mittelwert der Steuerbelastung), um eine Abhängigkeit von der Höhe der durchschnittlichen Steuerbelastung, wie sie sich bei der Verwendung der Standardabweichung als Schwankungsmaß ergeben würde, zu vermeiden. Die Ergebnisse dieser Berechnungen sind Gegenstand von Kapitel 6.3.1.

Neben der Wettbewerbsverzerrung, welche ihre Ursache im Nebeneinander selbständiger Besteuerungshoheiten in Europa und damit im Wesentlichen im internationalen Steuergefälle hat, werden Entscheidungen und Wettbewerb im Binnenmarkt zweitens aber auch dadurch verzerrt, dass die Steuerbelastung in Abhängigkeit vom Konzerntypus variiert. ${ }^{596}$ Dieses kann insbesondere daran liegen, dass bestimmte begünstigende oder belastende Steuervorschriften sich in

595 Die Ergebnisse der Steuerbelastungsrechnungen sind in ähnlicher Form veröffentlicht in Oestreicher/Koch (2008).

$596 \mathrm{Vgl}$. so auch Nicodeme (2001), S. 14. 
unterschiedlichem Maße auswirken. In diesem Zusammenhang werden hier die durchschnittlichen Steuerbelastungen der folgenden Gruppen von Konzernen verglichen:

(1) Vergleich der durchschnittlichen Steuerbelastung von nationalen Konzernen und multinationalen Konzernen (Kapitel 6.3.2): Dieser Vergleich erlaubt Rückschlüsse, inwiefern die Entscheidung zwischen der Begründung einer inländischen und einer ausländischen Tochtergesellschaft durch die Besteuerung verzerrt wird (internationale Neutralität). Dabei muss beachtet werden, dass die Steuerbelastung nationaler und multinationaler Gruppen nicht direkt verglichen werden kann. So bezieht sich die Steuerbelastung einer nationalen Gruppe auf ein einzelnes Steuersystem, während die Steuerbelastung einer multinationalen Gruppe das Steuersystem von mindestens zwei Mitgliedsstaaten widerspiegelt. Da die Steuernachteile für multinationale Konzerne - zumindest in dem hier gewählten Untersuchungsrahmen - insbesondere in ungünstigeren Verlustverrechnungsmöglichkeiten manifestieren, erfolgt der Vergleich der Steuerbelastung gegen den Maßstab einer entscheidungsneutralen Verlustverrechnungsvorschrift, also einem Steuersystem mit sofortiger Steuererstattung (,Negativsteuer"). Dabei wird die Steuerbelastung bei Negativsteuer sowohl für eine Gewinnabgrenzung nach der direkten Methode als auch einer Gewinnabgrenzung nach der indirekten Methode ermittelt.

(2) Vergleich der durchschnittlichen Steuerbelastung von rentablen Konzernen und verlusterzielenden Konzernen (Kapitel 6.3.3) ${ }^{597}$ : Dieser Vergleich erlaubt Rückschlüsse, in welchem Ausmaß die Entscheidung zwischen risikobehafteten und weniger risikobehafteten Investitionen durch das Besteuerungssystem verzerrt wird. Ein Steuersystem trägt zur Attraktivität risikobehafteter Investitionen bei, solange $\mathrm{ATR}^{-}$größer als $\mathrm{ATR}^{+}$ist, während risikolose Investitionen im umgekehrten Fall begünstigt werden.

(3) Vergleich der durchschnittlichen Steuerbelastung von großen Konzernen und kleinen/mittelgroßen Konzernen (Kapitel 6.3.4): Steuerliche Vorschriften für Kapitalgesellschaften, die nach der Größe des Konzerns, also der Anzahl der Konzerngesellschaften differenzieren, existieren im geltenden Steuerrecht kaum. ${ }^{598}$ Signifikant abweichende Steuerbelastungen für kleine und große Konzerne kön-

597 Rentable Konzerne sind definiert als Konzerne mit einem positiven Cashflow-Barwert (vor Steuern) innerhalb des Betrachtungszeitraums, während verlusterzielende Konzerne dementsprechend einen negativen Cashflow-Barwert (vor Steuern) aufweisen.

598 Ein - hier nicht simuliertes - Beispiel sind De-minimis-Regeln bei den Vorschriften zur Unterkapitalisierung. 
nen sich daher nur daraus ergeben, dass faktische Bedingungen in kleinen Konzernen dazu führen, dass sie stärker oder weniger stark von bestimmten Vorschriften betroffen sind. Ferner ist zu vermuten, dass kleine Unternehmen/Konzerne in geringerem Umfang Steuerplanung betreiben. Dieses lässt erwarten, dass diese Konzerne in weniger starkem Ausmaß vom Übergang auf eine indirekte Gewinnabgrenzung (negativ) betroffen sind.

Bei der Ermittlung der verschiedenen Maßgrößen (Mittelwert, Variationskoeffizient) kann auf die Steuerbelastung der einzelnen Konzerne oder die Steuerbelastung, die sich im Durchschnitt für die einzelnen Mitgliedsstaaten ergibt, Bezug genommen werden. Dabei hat der zweite Ansatz den Vorteil, dass die sich ergebenden Mittelwerte nicht durch Unterschiede in der geografischen Struktur zu vergleichender Unterstichproben (z.B. beim Vergleich der Steuerbelastung nationaler und multinationaler Konzerne oder dem Vergleich der Steuerbelastung rentabler und verlusterzielender Konzerne) beeinflusst werden. Auch wenn diese Vorgehensweise aus diesem Grund sinnvoll erscheint, setzt sie die Verfügbarkeit ausreichender Beobachtungen in sämtlichen relevanten Unterstichproben voraus. In den folgenden Berechnungen wird diese Vorgehensweise deshalb mit der Einschränkung gewählt, dass Zypern, Estland, Litauen, Luxemburg, die Slowakei und Ungarn aufgrund des Fehlens von Beobachtungen in mindestens einer Unterstichprobe aus den Betrachtungen ausgeschlossen werden. Abweichend wird der erste Ansatz (Mittelwert der Steuerbelastung der einzelnen Konzerne) für den Vergleich der Steuerbelastung kleiner/mittelgroßer und großer Konzerne gewählt. Hier hätte andernfalls die geringe Anzahl großer Konzerne in der Datenbasis, also Konzernen mit mehr als 50 Konzerngesellschaften, zum Ausschluss zahlreicher weiterer Länder geführt.

\subsection{Auswirkungen auf die Attraktivität der Mitgliedsstaaten als Steuerstandort und die Wettbewerbsfähigkeit europäischer Konzerne}

\subsubsection{Einführung einer GKKB}

Zur Beurteilung der steuerlichen Standortattraktivität der EU-Mitgliedsländer sowie der Wettbewerbsfähigkeit europäischer Konzerne de lege lata und de lege ferenda wird der Mittelwert der durchschnittlichen Steuerbelastungen nationaler und multinationaler Konzerne ermittelt, die sich für die einzelnen Mitgliedsstaaten ergeben. Dabei werden hier und im Folgenden als multinational alle Konzerne angesehen, die mit mindestens einer ausländischen Tochtergesellschaft in die Berechnungen eingehen. Die Ergebnisse, die sich unter den Vorschriften des geltenden Steuerrechts und bei Simulation einer GKKB ergeben, sind in Tabelle $22 \mathrm{zu}-$ 
sammengefasst. Bei der Simulation der GKKB werden die Annahmen zugrundegelegt, die in Kapitel 5.4.2 als Standardfall bezeichnet wurden. ${ }^{599}$

Tabelle 22: Durchschnittliche Steuerbelastung bei Einführung einer GKKB

\begin{tabular}{lcccc}
\hline & \multicolumn{2}{c}{ Nationale Konzerne } & \multicolumn{2}{c}{ Multinationale Konzerne } \\
& Pflicht & Option & Pflicht & Option \\
\hline Geltendes Recht & \multicolumn{2}{c}{0,2996} & \multicolumn{2}{c}{0,3028} \\
\hline GKKB & 0,2973 & 0,2970 & 0,2964 & 0,2934 \\
Relative Änderung & $-0,0077$ & $-0,0087$ & $-0,0211$ & $-0,0310$ \\
\hline davon: Verlustverrechnung & $-0,0077$ & $-0,0087$ & $-0,0270$ & $-0,0243$ \\
davon: Formelaufteilung & 0,0000 & 0,0000 & $+0,0059$ & $-0,0067$ \\
\hline
\end{tabular}

Quelle: Eigene Berechnungen

Die Ergebnisse in Tabelle 22 verdeutlichen die unterschiedliche Wirkung, die von der Einführung einer GKKB auf die Steuerbelastung nationaler und multinationaler Konzerne ausgeht. So zeigt sich, dass die Einführung einer GKKB nur zu einer geringfügigen Verbesserung der Wettbewerbsfähigkeit europäischer Konzerne beitragen würde, deren Ansässigkeit auf einen Mitgliedsstaat begrenzt ist. So sinkt die Steuerbelastung nationaler Konzerne von 29,96 Prozent auf 29,73 Prozent (-0,77 Prozent) bei verpflichtender Einführung. Bei optionaler Einführung ergibt sich ein nur geringfügig stärkerer Rückgang der Steuerbelastung auf 29,70 Prozent.

Zur weiteren Analyse der Ursachen der Belastungsänderung wird dieser Gesamteffekt in einen Verlustverrechnungseffekt („Verlustverrechnung"), welcher die Ausweitung der Möglichkeiten zur konzerninternen Verlustverrechnung innerhalb eines Landes und über die Grenze widerspiegelt, und einen Formelaufteilungseffekt („Formelaufteilung"), welcher den Übergang von der direkten zur indirekten Gewinnabgrenzung erfasst, zerlegt. ${ }^{600}$ Hierbei zeigt sich, dass bei nationalen Gruppen der Gesamteffekt vollständig auf die Ausweitung der Verlustver-

599 Die Aufteilungsformel setzt sich zu gleichen Teilen aus dem Vermögen, der Lohnsumme und dem Umsatz zusammen. Ein Verlustvortrag erfolgt auf Ebene der Einzelgesellschaft. Zudem wird von der Teilnahme sämtlicher Mitgliedsstaaten an der GKKB ausgegangen.

600 Dabei wird der Effekt des Übergangs auf die indirekte Methode zur Gewinnabgrenzung durch den Vergleich der Steuerbelastungen bei direkter und indirekter Gewinnabgrenzung und sofortiger Verlustverrechnung ermittelt. Die Minderung der Steuerbelastung, die auf die Ausdehnung der konzerninternen Verlustverrechnung zurückgeht, ergibt sich als Residuum. Die Änderungen bei der Dividendenbesteuerung als weitere Konsequenz der Einführung einer GKKB betrifft nur vier Länder und ist von untergeordneter Bedeutung $(-0,0002$ bei nati-

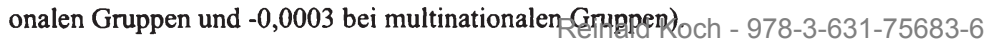


rechnungsmöglichkeiten zurückzuführen ist. Aufgrund der Ansässigkeit sämtlicher Gesellschaften in einem Mitgliedsstaat ist die Umverteilung der Gesamtbemessungsgrundlage im Konzern hier ohne Wirkung.

Der verhältnismäßig geringe Rückgang der durchschnittlichen Steuerbelastung nationaler Gruppen ist auf den Umstand zurückzuführen, dass die Mehrzahl der Mitgliedsstaaten eine nationale Verlustverrechnung im Konzern auch im geltenden Steuerrecht erlaubt. ${ }^{601}$ Vergleicht man die Änderung der Steuerbelastung, die sich bei Einführung einer GKBB in den Mitgliedsstaaten mit und ohne Gruppenbesteuerungssystem im geltenden Steuerrecht ergibt (Tabelle 23), zeigt sich erwartungsgemäß ein höherer Rückgang in den Mitgliedsstaaten, die im geltenden Steuerrecht die konzerninterne Verlustverrechnung versagen (-1,47 Prozent im Vergleich zu -0,60 Prozent). Die - wenn auch kleine - Minderung der Steuerbelastung in Mitgliedsstaaten mit konzerninterner Verlustverrechnung ist auf die teilweise strengeren Anwendungsvoraussetzungen im geltenden Steuerrecht, welche sich in einer höheren Mindestbeteiligungsquote oder zusätzlichen Anforderungen, wie dem Abschluss eines Ergebnisabführungsvertrags, manifestieren, zurückzuführen.

Tabelle 23: Zusammenhang von Steuerbelastungsänderung (GKKB) und relevanten Steuerparametern bei nationalen Konzernen

Nationale Konzerne: Änderung der durchschnittlichen Steuerbelastung

\begin{tabular}{|c|c|c|c|c|}
\hline & \multicolumn{2}{|c|}{ Gruppenbesteuerungssystem } & \multicolumn{2}{|c|}{ Nominelle Steuerbelastung } \\
\hline & $\mathrm{Ja}$ & Nein & Hochsteuerländer & Niedrigsteuerländer \\
\hline Verpflichtende & $-0,0060$ & $-0,0147$ & $-0,0080$ & $-0,0063$ \\
\hline GKKB & & & & \\
\hline
\end{tabular}

Bei multinationalen Konzernen, also Konzernen, die mit mindestens einer ausländischen Tochtergesellschaft in die Berechnungen eingehen, ergibt sich ein höherer Rückgang der durchschnittlichen Steuerbelastung. So sinkt die Steuerbelastung in diesen Gruppen bei verpflichtender Einführung von 30,28 Prozent auf

601 Daneben ist der nur geringe Rückgang zum Teil auch auf die Einbeziehung von Konzernen mit nur einem Konzernunternehmen in der Datenbasis zurückzufuhren. Blieben diese unberücksichtigt, würde der Effekt mit -1,58 Prozent in etwa doppelt so hoch ausfallen, allerdings unverändert deutlich unterhalb des entsprechenden Rückgangs bei multinationalen Konzernen liegen. Werden bei den multinationalen Konzernen die Länder vernachlässigt, die auch bei den nationalen Konzemen unter dieser Annahme vollständig herausfallen (Österreich, Irland), würde der Belastungsrückgang aufgrund der Einfürung der GKKB insgesamt (Verlustverrechnung+Formelaufteilung) sich hier auf -2.59 Prozent belaufen $31-75683-6$ 
29,64 Prozent (-2,11 Prozent). Bei optionaler Einführung verringert sich die Steuerbelastung sogar auf 29,34 Prozent (-3,10 Prozent). Die Einführung einer GKKB kann somit insbesondere einen Beitrag zur Steigerung der Wettbewerbsfähigkeit grenzüberschreitend tätiger Konzerne leisten. Gleichzeitig wird die Attraktivität der EU als Investitionsstandort für nichteuropäische Investoren insbesondere dann verbessert, wenn diese die Investition in mehr als einem Mitgliedsstaat beabsichtigen.

Tabelle 24: Zusammenhang von Steuerbelastungsänderung (GKKB) und relevanten Steuerparametern bei multinationalen Konzernen

Multinationale Konzerne: Änderung der durchschnittlichen Steuerbelastung

\begin{tabular}{llllc}
\hline & Gruppenbesteuerungssystem & \multicolumn{2}{c}{ Nominelle Steuerbelastung } \\
& Ja & Nein & $\begin{array}{c}\text { Hochsteuer- } \\
\text { länder }\end{array}$ & $\begin{array}{c}\text { Niedrig- } \\
\text { steuerländer }\end{array}$ \\
\hline Verpflichtende GKKB & $-0,0101$ & $-0,0503$ & $-0,0267$ & 0,0055 \\
\hline $\begin{array}{l}\text { davon: } \\
\text { Verlustverrechnung }\end{array}$ & $-0,0247$ & $-0,0334$ & $-0,0291$ & $-0,0194$ \\
$\begin{array}{l}\text { davon: } \\
\text { Formelaufteilung }\end{array}$ & 0,0146 & $-0,0169$ & 0,0024 & 0,0249 \\
\hline Optionale GKKB & $-0,0251$ & $-0,0515$ & $-0,0317$ & $-0,0253$ \\
\hline
\end{tabular}

Quelle: Eigene Berechnungen. Hierbei werden als Hochsteuerländer (Niedrigsteuerländer) die Mitgliedsstaaten bezeichnet, deren nominelle Steuerbelastung im Jahr 2006 oberhalb (unterhalb) des EU-Durchschnitts lag.

Eine Zerlegung dieses Gesamteffekts zeigt, dass sich bei multinationalen Konzernen sowohl die Ausdehnung der Möglichkeiten zur Verlustverrechnung als auch der Übergang auf eine indirekte Gewinnabgrenzung auswirken. Eine Ausdehnung der Möglichkeiten zur konzerninternen Verlustverrechnung führt zu einer Minderung der durchschnittlichen Steuerbelastung um 2,70 Prozent und hat damit einen deutlich stärkeren Effekt, als dieses bei nationalen Gruppen der Fall ist (-0,77 Prozent). Dieses Ergebnis ist auf den Umstand zurückzuführen, dass die Anwendung der Gruppenbesteuerungssysteme im geltenden Steuerrecht in den meisten Mitgliedsstaaten auf inländische Tochtergesellschaften beschränkt ist. ${ }^{602}$ Auch hier zeigt sich, dass die Höhe des Rückgangs der Steuerbelastung maßgeblich von der Möglichkeit zur konzerninternen Verlustverrechnung im geltenden

602 Aktuell erlauben nur sechs Mitgliedsstaaten in gewissen Grenzen die Berücksichtigung der Verluste ausländischer Tochterunternehmen. Aus Vereinfachungsgründen werden diese grenzüberschreitenden Gruppenbesteuerungssysteme im Rahmen der hier erfolgenden Simulationsrechnungen nicht abgebildet. 
Steuerrecht abhängt, auch wenn die relative Bedeutung dieser Möglichkeit für die Höhe des Verlustverrechnungseffekts im Vergleich zu nationalen Gruppen geringer ist (vgl. Tabelle 24). So führt die Ausdehnung der Verlustverrechnungsmöglichkeiten in multinationalen Konzernen, deren Muttergesellschaft in einem Mitgliedsstaat mit Gruppenbesteuerungssystem ansässig ist, zu einem Rückgang der Steuerbelastung um 2,47 Prozent, während der entsprechende Effekt -3,34 Prozent beträgt, wenn im Sitzland der Muttergesellschaft kein Gruppenbesteuerungssystem angeboten wird. Die im Vergleich zu nationalen Konzernen ${ }^{603}$ geringeren Bedeutung der Verfügbarkeit eines Gruppenbesteuerungssystems im geltenden Steuerrecht ist neben der überwiegend nationalen Ausrichtung dieser Systeme auch auf den Umstand zurückzuführen, dass die Möglichkeit zur Verlustverrechnung innerhalb der Grenzen eines Landes bei der Klassifikation in Tabelle 24 nur für die inländischen Gesellschaften und damit einen Teil des Konzerns erfasst wird.

Der Übergang von einer direkten zu einer indirekten Gewinnabgrenzung führt - isoliert betrachtet - bei verpflichtender Einführung der GKKB zu einem Anstieg der Steuerbelastung von 0,59 Prozent (vgl. Tabelle 22). Dieser Anstieg ist darauf zurückzuführen, dass bei Besteuerung nach GKKB ein größerer Anteil der Gesamtbemessungsgrundlage in Hochsteuerländern der Besteuerung unterworfen wird, was auf die empirisch belegte Existenz von Steuerplanung im geltenden Steuerrecht zurückzuführen sein sollte. ${ }^{604}$ Wird bei der Analyse danach differenziert, ob die Konzernmuttergesellschaft ihren Sitz in einem Hochsteuerland oder Niedrigsteuerland hat, zeigt sich dass der Übergang auf eine indirekte Gewinnabgrenzung insbesondere zu einem Anstieg der Steuerbelastung führt, wenn der inländische Konzernteil niedrig besteuert wird (+2,49 Prozent, vgl. Tabelle 24). Dieses Ergebnis stellt sich ein, wenn inländische Konzerngesellschaften - unabhängig von der Existenz von Steuersatzunterschieden - im Durchschnitt eine höhere Rendite aufweisen als Tochtergesellschaften, die im Ausland ansässig sind. Diese konzerninterne Renditeverteilung spiegelt sich in der diesen Berechnungen zugrundeliegenden Datenbasis wider. So zeigt ein Vergleich der Umsatzrendite der in- und ausländischen Konzerngesellschaften multinationaler Konzerne, dass inländische Konzerngesellschaften im Durchschnitt über eine höhere Umsatzrendite (2,95 Prozent) verfügen als ausländische Tochtergesellschaften $(2,01$ Pro-

603 Hier fält der Rückgang der durchschnittlichen Steuerbelastung bei Fehlen der Möglichkeit zur konzerninternen Verlustverrechnung im geltenden Steuerrecht mehr als doppelt so hoch $(-1,47$ Prozent) aus, als dieses in Mitgliedsstaaten der Fall ist, die im geltenden Steuerrecht eine entsprechende Vorschrift vorsehen (-0,60 Prozent). Vgl. Tabelle 23.

$604 \mathrm{Vgl}$. für empirische Belege des Zusammenhangs von Profitabilität ausländischer Tochtergesellschaften und dem im Ausland vorherrschenden Steuemiveau u.a. Weichenrieder (2007), Huizinga/Laeven (2005), Clausing (2003), Bartelsmann/Beetsma (2003), Froot/Hines (1995), Hines/Rice (1994), Collins/Shackelford (1992), Grubert/Mutti (1991), Hines/Hubbard (1990). 
zent) ${ }^{605}$ Ursächlich hierfür kann sein, dass bei Begründung eines Auslandsengagements ein Großteil des unternehmerischen Risikos im Inland verbleibt oder dass häufig selbsterstellte und damit nicht bilanzierte - Immaterialwerte der Konzernmutter zugeordnet werden.

Auch hinsichtlich der Wirkung einer optionalen Einführung ergeben sich deutliche Unterschiede zwischen nationalen und multinationalen Konzernen. Während bei nationalen Konzernen einem Optionsrecht nur geringe Bedeutung zukommt (Rückgang der Steuerbelastung von 29,73 Prozent auf 29,70 Prozent, vgl. Tabelle 22), führt es zu einer weiteren Steigerung der Wettbewerbsfähigkeit multinationaler Konzerne (Rückgang der Steuerbelastung von 29,64 Prozent auf 29,34 Prozent, vgl. Tabelle 22).

Bei nationalen Konzernen führt die Einführung einer GKKB ausschließlich zu Änderungen der Möglichkeiten zur konzerninternen Verlustverrechnung. Fehlt es an Verlustsituationen im Betrachtungszeitraum ist die Reform somit wirkungslos. In Verlustsituationen kann die Einführung der GKKB in der hier umgesetzten Form sowohl Vorteile als auch Nachteile mit sich bringen. Vorteilhaft kann sich insbesondere die Ermöglichung einer konzerninternen Verlustverrechnung erweisen, welche im geltenden Recht teilweise ausgeschlossen ist oder nur unter restriktiveren Anwendungsvoraussetzungen gewährt wird. Als nachteilhaft kann sich der hier unterstellte Verlustvortrag auf Ebene der einzelnen Gruppengesellschaft erweisen, da die Gruppenbesteuerungssysteme im geltenden Recht teilweise eine Zusammenveranlagung der Gruppengesellschaften und damit einen Verlustvortrag auf Gruppenebene vorsehen. Insgesamt zeigt sich allerdings in den Berechnungen, dass die GKKB sich nur für 9,79 Prozent der nationalen Konzerne als nachteilhaft erweist, weshalb dem Optionsrecht hier nur geringe Bedeutung zukommt.

Bei multinationalen Konzernen hat die Einführung einer GKKB neben der Ausdehnung der Verlustverrechnungsmöglichkeiten den zusätzlichen Effekt einer Umverteilung der Bemessungsgrundlage, welche sich aus Konzernperspektive sowohl als vorteilhaft als auch als nachteilhaft erweisen kann. Dieser Umstand ist ursächlich für die größere Bedeutung, die einem Optionsrecht bei multinationalen Konzernen zukommt. Zum einen erweist sich die GKKB hier in mehr Fällen (26,80 Prozent) als nachteilhaft, zum anderen führt die Reallokation der Bemessungsgrundlage dazu, dass die sich ergebenden Belastungsunterschiede über einen Zinseffekt aufgrund einer späteren Verlustverrechnung hinausgehen. Als Konsequenz der Optionsrechtsausübung zeigt sich, dass die Minderung der Steuerbelastung aufgrund der Verlustverrechnung leicht zurückgeht, während der Formelaufteilungseffekt bei optionaler Einführung negativ wird (vgl. Tabelle 22).

Bezüglich der tatsächlichen Bedeutung eines Optionsrechts sei einschränkend darauf hingewiesen, dass in den hier erfolgenden Berechnungen vollständige

605 Quelle: Eigene Berechnungen auf Basis der Angaben in AMADEUS. Die angegebenen Umsatzrenditen beziehen sich auf Einzelabschlüsse aus dem Jahr 2092 2 -8-3-631-75683-6 
Voraussicht der Unternehmen unterstellt wird. Der tatsächliche Einfluss des Optionsrechts sollte dementsprechend in der Realität geringer ausfallen. Umgekehrt sieht der Vorschlag der Arbeitsgruppe lediglich eine Bindung von fünf Jahren bei der Optionsrechtsausübung vor. Gegenüber der hier unterstellten einmaligen Ausübung der Option innerhalb des Zehnjahreszeitraums würde eine derartige Regelung mehr Flexibilität bei der Optionsrechtsausübung erlauben.

\subsubsection{Einführung einer Verlustrichtlinie}

Die verschiedenen Konzepte für eine Verlustrichtlinie erlauben eine konzerninterne Verlustverrechnung nicht im selben Umfang, wie dieses bei Besteuerung nach GKKB der Fall wäre. Dementsprechend sinkt die durchschnittliche Steuerbelastung bei diesen Konzepten nicht im selben Ausmaß, die steuerliche Standortattraktivität der Mitgliedsstaaten sowie die Wettbewerbsfähigkeit multinationaler Konzerne würden also in geringerem Ausmaß verbessert. Die Ergebnisse, die sich für die durchschnittliche Steuerbelastung multinationaler Konzerne bei den verschiedenen Konzepten ergeben, sind in Tabelle 25 zusammengefasst. ${ }^{606}$

Tabelle 25: Durchschnittliche Steuerbelastung multinationaler Konzerne bei Einführung einer Verlustrichtlinie

\begin{tabular}{|c|c|c|}
\hline & Pflicht & Option \\
\hline Geltendes Recht & \multicolumn{2}{|c|}{0,3028} \\
\hline Nachversteuerungsmethode & 0,2962 & 0,2956 \\
\hline Relative Änderung & $-0,0218$ & $-0,0238$ \\
\hline Zurechnungsmethode & 0,3043 & 0,2968 \\
\hline Relative Änderung & $+0,0050$ & $-0,0198$ \\
\hline Ergebnisaufteilungsmethode & 0,2965 & 0,2961 \\
\hline Relative Änderung & $-0,0208$ & $-0,0221$ \\
\hline
\end{tabular}

Quelle: Eigene Berechnungen

Während die Ausdehnung der Verlustverrechnungsmöglichkeiten multinationaler Konzerne im Rahmen der verpflichtenden Einführung einer GKKB zu einer Minderung der durchschnittlichen Steuerbelastung um 2,70 Prozent führt (vgl. Tabelle 22, Zeile „davon: Verlustverrechnung“), ergibt sich bei Umsetzung der Nachversteuerungsmethode eine Minderung lediglich um 2,18 Prozent (29,62 Prozent), während die Ergebnisaufteilungsmethode eine Minderung der durchschnittlichen Steuerbelastung um 2,08 Prozent (29,65 Prozent) bewirkt. Grund für

606 Bei nationalen Konzernen ergeben sich bei Einfuhrung einer Verlustrichtlinie nur unwesentliche Abweichungen gegenüber der Einfuhrung einer GKKB. Diese Ergebnisse werden aus diesem Grund nicht berichtet. 
den geringeren Effekt ist bei der Nachversteuerungsmethode, dass diese eine Verlustverrechnung lediglich vertikal aufwärts, nicht aber vertikal abwärts oder horizontal (zwischen Schwestergesellschaften) zulässt. Die Ergebnisaufteilungsmethode hingegen führt nicht zu einer vollständigen Verlustverrechnung bei anhaltenden Verlusten einzelner Konzerngesellschaften, da bei dieser Methode eigene Gewinne Voraussetzung für den Zuweis eines positiven Einkommens sind. Ein positives Konzernergebnis - wie bei der GKKB - ist hingegen hier nicht ausreichend. Bei beiden Konzepten führt Optionalität nur zu einer geringfügigen zusätzlichen Minderung der Steuerbelastung, da sich die Anwendung dieser Methoden in der weit überwiegenden Anzahl der Fälle als vorteilhaft erweist.

Eine Besteuerung nach dem Zurechnungskonzept bewirkt neben einer Ausdehnung der Möglichkeiten zur konzerninternen Verlustverrechnung, die im Ergebnis allerdings ebenfalls nur vertikal aufwärts möglich ist, dass die Gewinne der Auslandstöchter dem höheren Steuerniveau im Inland oder Ausland unterliegen. Eine verpflichtende Einführung dieses Konzepts würde aus diesem Grund einen Anstieg der Steuerbelastung auf 30,43 Prozent bewirken. Aufgrund der von diesem Konzept ausgehenden Steuernachteile insbesondere bei Konzernen mit Sitz in Hochsteuerländern, kommt dem Optionsrecht beim Zurechnungskonzept entscheidende Bedeutung zu. So würde bei optionaler Einführung die Steuerbelastung auf 29,68 Prozent sinken.

Tabelle 26: Zusammenhang von Steuerbelastungsänderung (Verlustrichtlinie) und relevanten Steuerparametern bei multinationalen Konzernen

\begin{tabular}{lcccc}
\hline \multicolumn{4}{c}{ Multinationale Konzerne: Änderung der durchschnittlichen Steuerbelastung } \\
\hline & $\begin{array}{c}\text { Gruppenbesteuerung } \\
\text { Ja }\end{array}$ & Nein & $\begin{array}{c}\text { Nominelle Steuerbelastung } \\
\text { Hochsteuer- } \\
\text { länder }\end{array}$ & $\begin{array}{c}\text { Niedrig- } \\
\text { steuerländer }\end{array}$ \\
\hline Nachversteuerungsmethode & & & & \\
Pflicht & $-0,0167$ & $-0,0344$ & $-0,0250$ & $-0,0029$ \\
Option & $-0,0202$ & $-0,0345$ & $-0,0253$ & $-0,0144$ \\
\hline Zurechnungsmethode & & & & \\
Pflicht & $+0,0070$ & $-0,0057$ & $+0,0057$ & $+0,0016$ \\
Option & $-0,0177$ & $-0,0261$ & $-0,0200$ & $-0,0165$ \\
\hline Ergebnisaufteilungsmethode & & & & \\
Pflicht & $-0,0177$ & $-0,0286$ & $-0,0235$ & $-0,0070$ \\
Option & $-0,0186$ & $-0,0316$ & $-0,0239$ & $-0,0110$ \\
\hline
\end{tabular}

Quelle: Eigene Berechnungen. Hierbei werden als Hochsteuerländer (Niedrigsteuerländer) die Mitgliedsstaaten bezeichnet, deren nominelle Steuerbelastung im Jahr 2006 oberhalb (unterhalb) des EU-Durchschnitts lag. 
Auch bei Einführung einer Verlustrichtlinie hängt die Höhe des Belastungsrückgangs maßgebend von den Möglichkeiten zur konzerninternen Verlustverrechnung im geltenden Steuerrecht und der Höhe der tariflichen Steuerbelastung ab. Unabhängig vom Verlustverrechnungskonzept fällt die Minderung der Steuerbelastung in Ländern ohne Gruppenbesteuerungssystem um ein bis zwei Prozent höher aus. Bei Unterteilung der Mitgliedsstaaten nach der nominellen Steuerbelastung lässt sich in den Hochsteuerländern eine überdurchschnittlich hohe Abnahme der Steuerbelastung bei Implementierung der Nachversteuerungsmethode und Ergebnisaufteilungsmethode beobachten. Ein entgegengesetztes Ergebnis zeigt sich bei verpflichtender Umsetzung der Zurechnungsmethode.

\subsection{Auswirkungen auf steuerliche Wettbewerbsverzerrungen im Binnenmarkt}

\subsubsection{Auswirkungen auf die Schwankung der Steuerbelastung in Europa}

Die Besteuerung kann unter anderem dann wettbewerbsverzerrend wirken, wenn miteinander im Wettbewerb stehende Unternehmen in Abhängigkeit von ihrer Ansässigkeit einer unterschiedlichen Steuerbelastung unterliegen. Zur Beurteilung dieser Verzerrung wird in Tabelle 27 die Schwankung der durchschnittlichen Steuerbelastung der Mitgliedsstaaten, gemessen als Variationskoeffizient, für die unterschiedlichen Reformszenarien widergegeben. Die Zeilen (1) bis (5) dienen dabei als Referenzpunkte für die Beurteilung.

Tabelle 27: Variationskoeffizient der durchschnittlichen Steuerbelastung im Vergleich

\begin{tabular}{|c|c|c|c|c|c|}
\hline \multirow{2}{*}{\multicolumn{2}{|c|}{$\frac{S t a b w(A T R)}{M W(A T R)}$}} & \multicolumn{2}{|c|}{ Nationale Konzerne } & \multicolumn{2}{|c|}{ Multinationale Konzerne } \\
\hline & & Pflicht & Option & Pflicht & Option \\
\hline (1) & Nominalsteuersätze & \multicolumn{2}{|c|}{0,2655} & \multicolumn{2}{|c|}{0,2655} \\
\hline (2) & Negativsteuer (direkte GA) & \multicolumn{2}{|c|}{0,2612} & \multicolumn{2}{|c|}{0,2052} \\
\hline (3) & Negativsteuer (indirekte GA) & \multicolumn{2}{|c|}{0,2612} & \multicolumn{2}{|c|}{0,1945} \\
\hline (4) & Geltendes Recht & \multicolumn{2}{|c|}{0,2632} & \multicolumn{2}{|c|}{0,2078} \\
\hline (5) & $\begin{array}{l}\text { Einheitliche nationale Grup- } \\
\text { penbesteuenung }\end{array}$ & 0,2610 & 0,2611 & 0,2062 & 0,2062 \\
\hline (6) & Nachversteuerungsmethode & & & 0,2013 & 0,2042 \\
\hline (7) & Zurechnungsmethode & & & 0,2094 & 0,2058 \\
\hline (8) & Ergebnisaufteilungsmethode & & & 0,2026 & 0,2031 \\
\hline (9) & GKKB & 0,2625 & 0,2621 & 0,1932 & 0,2028 \\
\hline
\end{tabular}

Quelle: Eigene Berechnungen. Beim Szenario „Einheitliche nationale Gruppenbesteuerung “ wird eine Zusammenveranlagung auf Ebene der nationalen Teilgruppen ohne Verlustverrechmung über die Grenze unterstellt. 
Die Zeilen (1) bis (5) der obigen Tabelle verdeutlichen die verzerrenden Einflüsse der Vorschriften zur Einkommensermittlung im geltenden Steuerrecht. Bei nationalen Konzernen liegt der Variationskoeffizient mit 26,32 Prozent unterhalb des entsprechenden Wertes für die nominellen Steuersätze (26,55 Prozent). Der letztgenannte Wert repräsentiert das Szenario einer vollständigen Harmonisierung der Vorschriften zur Einkommensermittlung, also der Gewinnermittlungsvorschriften sowie der Vorschriften zur intertemporalen und konzerninternen Verlustverrechnung. Das Verhältnis dieser beiden Werte deutet darauf hin, dass die Vorschriften zur Einkommensermittlung in Summe einen kompensatorischen Effekt haben, also dass in Mitgliedsstaaten mit hohen tariflichen Steuerbelastungen tendenziell günstigere Vorschriften zur Einkommensermittlung vorherrschen. Vereinheitlicht man die Vorschriften zur nationalen konzerninternen Verlustverrechnung $^{607}$ (Zeile (5)) oder der steuerlichen Verlustverrechnung insgesamt (Zeile (2)) sinkt der Variationskoeffizient noch stärker auf 26,10 Prozent beziehungsweise 26,12 Prozent ab. Hieraus lässt sich ersehen, dass der kompensatorische Effekt offensichtlich von den Vorschriften zur Gewinnermittlung ausgeht, während die Vorschriften zur konzerninternen Verlustverrechnung die Besteuerungsunterschiede verstärken. Vergleicht man die Verfügbarkeit von Gruppenbesteuerungssystemen in Hoch- und Niedrigsteuerländern, so zeigt sich, dass zwar 83,33 Prozent der Hochsteuerländer über Gruppenbesteuerungssysteme verfügen, während der entsprechende Anteil in Niedrigsteuerländern lediglich 80 Prozent beträgt. ${ }^{608}$ Allerdings führen tendenziell restriktivere Anwendungsvoraussetzungen in den Hochsteuerländern dazu, dass in diesen die Anzahl der Steuergruppen bei Einführung einer einheitlichen Gruppenbesteuerung mit einer Mindestbeteiligungsquote von 50 Prozent um 26,17 Prozent sinkt, während der entsprechende Rückgang in den Niedrigsteuerländern 25,41 Prozent beträgt. Aus diesem Grund führt eine Harmonisierung der konzerninternen Verlustverrechnung oder der Verlustverrechnung insgesamt zu einer Minderung der Schwankung der durchschnittlichen Steuerbelastungen bei nationalen Konzernen. Die Einführung einer konzerninternen Verlustverrechnung über die Grenze hat hingegen keinen weitergehenden Effekt für die Besteuerung nationaler Konzerne, da hier unterstellt wird, dass unabhängig vom konkreten Konzept der Verlustrichtlinie für nationale Konzerne oder Teilkonzerne eine Zusammenveranlagung auf Ebene der Muttergesellschaft oder Landesholding erfolgt. Der sich in Tabelle 27 zeigende Unterschied für die Einführung einer GKKB ist auf die Annahme zurückzuführen, dass der Verlustvortrag im Konzept der GKKB nicht auf Konzernebene, sondern auf Ebene der Einzelgesellschaft erfolgt.

607 Beim Szenario „Einheitliche nationale Gruppenbesteuerung“ wird eine Zusammenveranlagung auf Ebene der nationalen Teilgruppen ohne Verlustverrechnung über die Grenze unterstellt.

608 Prozentangaben beziehen sich nur auf die Länder, die bei der Ermittlung der Variationskoeffizienten Berücksichtigung finden. 
Bei multinationalen Konzernen stellt die durchschnittliche Steuerbelastung eine Mischsteuerbelastung dar, welche das Steuerniveau von mindestens zwei Mitgliedsstaaten widerspiegelt. Dieses erklärt, warum der Variationskoeffizient der durchschnittlichen Steuerbelastung hier unabhängig vom simulierten Steuersystem deutlich unterhalb des Variationskoeffizienten der nominellen Steuersätze liegt. Auch bei multinationalen Konzernen zeigt sich, dass eine Harmonisierung der nationalen konzerninternen Verlustverrechnung (Zeile (5)) oder der Verlustverrechnungsvorschriften insgesamt (Zeile (2)) zu einem Rückgang der Schwankung in den durchschnittlichen Steuerbelastungen führt. Während bereits die Einführung einer einheitlichen nationalen konzerninternen Verlustverrechnung (Zeile (5)) die Schwankung der durchschnittlichen Steuerbelastungen annähernd auf das Niveau bei sofortiger Verlustverrechnung (Zeile (2)) senkt, wird dieses bei Umsetzung der Nachversteuerungsmethode oder der Ergebnisaufteilungsmethode sogar unterschritten.

Der Übergang von einer direkten zu einer indirekten Gewinnabgrenzung bei Einführung einer GKKB führt zu einer signifikanten Reduktion der Streuung in den durchschnittlichen Steuerbelastungen. Dieses gilt unabhängig davon, ob eine sofortige Verlustverrechnung (Variationskoeffizient: 19,45 Prozent; Zeile (3)) oder eine Verlustverrechnung entsprechend der geltenden Vorschriften auf Ebene der Konzerngesellschaften (Variationskoeffizient: 19,32 Prozent; Zeile (9)) unterstellt wird. Dementsprechend verringert der Übergang auf eine formelhafte Gewinnaufteilung die steuerinduzierten Verzerrungen des Wettbewerbs im Binnenmarkt, da die durchschnittliche Steuerbelastung von Konzernen in Niedrigsteuerländern stärker erhöht wird, als dieses für Konzerne in Hochsteuerländern der Fall ist. $^{609}$

\subsubsection{Auswirkungen auf Belastungsunterschiede zwischen nationalen und multinationalen Konzernen}

Neben standortabhängigen Besteuerungsunterschieden ergeben sich steuerliche Wettbewerbs- und Entscheidungsverzerrungen auch dann, wenn unterschiedliche Arten von Konzernen einer unterschiedlich hohen Steuerbelastung ausgesetzt sind. Zur Untersuchung derartiger Besteuerungsunterschiede wird in diesem Abschnitt zunächst die Steuerbelastung nationaler und grenzüberschreitend tätiger Konzerne - gemessen als relative Abweichung dieser beiden Belastungsmaße ermittelt. Dieser Vergleich kann allerdings nicht direkt erfolgen, da die Steuerbelastung inländischer Konzerne ausschließlich von einem Steuersystem bestimmt wird, während die Steuerbelastung grenzüberschreitend tätiger Konzerne die Wirkungen der Steuersysteme von wenigstens zwei Mitgliedsstaaten widerspiegelt. Ist die Mehrzahl der Auslandsgesellschaften eines multinationalen Konzerns in Hochsteuerländern ansässig, so ist davon auszugehen, dass die durchschnittliche 
Steuerbelastung das Steuerniveau eines vergleichbaren Inlandskonzerns übersteigt. Entsprechend sollte die Steuerbelastung eines Inlandskonzerns höher sein, wenn die Mehrzahl der Auslandsgesellschaften eines multinationalen Konzerns in Niedrigsteuerländern domiziliert.

Eine vergleichende Beurteilung der Wirkungen alternativer Verlustverrechnungssysteme auf nationale und grenzüberschreitend tätige Konzerne erfolgt aus diesem Grund gegen den Maßstab einer neutralen Verlustverrechnungsvorschrift. $\mathrm{Zu}$ diesem $\mathrm{Zweck}$ werden die Steuerbelastungen von inländischen und multinationalen Konzernen verglichen, die sich bei sofortiger Verlustverrechnung im Wege einer Negativsteuer entweder bei direkter oder bei indirekter Gewinnabgrenzung einstellen. Dabei zeigt sich, dass inländische Konzerne bei direkter Gewinnabgrenzung im Durchschnitt einer um 0,77 Prozent höheren Steuerbelastung unterliegen, während die Mehrbelastung bei indirekter Gewinnabgrenzung 0,22 Prozent beträgt. Eine gleichmäßige Belastung von inländischen und multinationalen Konzernen durch die Vorschriften zur Verlustverrechnung und zur Konzernbesteuerung im Allgemeinen stellt sich dementsprechend bei einer Mehrbelastung nationaler Konzerne in dieser Größenordnung ein.

Tabelle 28: Relative Abweichung der Steuerbelastung bei nationalen und multinationalen Konzernen

\begin{tabular}{llll}
\hline \multicolumn{1}{c}{$A T R^{\text {mult }}-A T R^{\text {nat }}$} \\
$A T R^{\text {dom }}$ & Pflicht & Option \\
\hline Negativsteuer (direkte GA) & & $-0,0077$ & \\
Negativsteuer (indirekte GA) & $-0,0022$ & \\
\hline Geltendes Recht & 0,0107 & \\
\hline Einheitliche nationale Gruppen- & & 0,0094 \\
besteuerung & 0,0091 & $-0,0030$ \\
Nachversteuerungsmethode & $-0,0013$ & 0,0010 \\
Zurechnungsmethode & 0,0260 & & $-0,0013$ \\
Ergebnisaufteilungsmethode & $-0,0003$ & $-0,0121$ \\
\hline GKKB & $-0,0030$ & & \\
\hline
\end{tabular}

Quelle: Eigene Berechnungen

Die Ergebnisse in Tabelle 28 verdeutlichen die Diskriminierung multinationaler Konzerne im geltenden Steuerrecht, welche in den Simulationsrechnungen auf das Fehlen einer konzerninternen Verlustverrechnung über die Grenze zurückzuführen ist. ${ }^{610}$ Verglichen mit dem Vergleichsmaßstab einer Negativsteuer

610 Andere steuerliche Benachteiligungen multinationaler Konzerne finden in den Berechnungen keine Berücksichtigung. Vgl. hierzu Kapitel 3.5.2einald Koch - 978-3-631-75683-6 
mit direkter Gewinnabgrenzung beträgt die relative Benachteiligung multinationaler Konzerne 1,84 Prozent (+1,07 Prozent gegenüber $-0,77$ Prozent).

Die verschiedenen Konzepte einer grenzüberschreitenden Gruppenbesteuerung, mindern die Benachteiligung multinationaler Konzerne deutlich, beseitigen diese allerdings nicht vollständig. So liegen die Mehrbelastungen nationaler Konzerne bei der Nachversteuerungsmethode mit 0,13 Prozent bei verpflichtender Einführung und 0,30 Prozent bei optionaler Einführung sowie die korrespondierenden Werte bei der Ergebnisaufteilungsmethode mit 0,03 Prozent und 0,13 Prozent unterhalb des Wertes, der sich bei sofortiger Verlustverrechnung und direkter Gewinnabgrenzung einstellt (0,77 Prozent). Auch hier wird deutlich, dass diese Konzepte keine vollständige Verlustverrechnung sicherstellen, da es im Falle der Nachversteuerungsmethode zwischen Schwestergesellschaften und von der Muttergesellschaft zur Tochtergesellschaft nicht zu einer Verlustverrechnung kommt, während bei der Ergebnisaufteilungsmethode eine Verlustverrechnung bei dauerhaften Verlusten einzelner Tochtergesellschaften unterbleibt. Die Zurechnungsmethode führt bei verpflichtender Einführung zu einer deutlichen Verstärkung der Diskriminierung multinationaler Konzerne (Mehrbelastung in Höhe von $+2,60$ Prozent). Während dieses Konzept bei nationalen Konzernen eine vollständige Verlustverrechnung sicherstellt, führt es bei grenzüberschreitenden Konzernen zu einer Belastung mit dem höheren Steuerniveau von In- und Ausland. Dieser Nachteil wird durch Einräumung eines Optionsrechts zwar deutlich gemindert, dennoch kann die Zurechnungsmethode auch in diesem Fall die Diskriminierung multinationaler Konzerne von den betrachteten Konzepten in geringstem Ausmaß beseitigen.

Relevanter Vergleichsmaßstab für die Beurteilung der Wirkungen einer GKKB ist das Ergebnis für das Szenario einer sofortigen Verlustverrechnung bei indirekter Gewinnabgrenzung. Für dieses Szenario ergibt sich eine Mehrbelastung der nationalen Konzerne von 0,22 Prozent, welche dementsprechend verglichen mit dem Fall einer sofortigen Verlustverrechnung und direkter Gewinnabgrenzung um etwa ein halbes Prozent niedriger ausfällt. Grund hierfür sollte die Möglichkeit zur Steuergestaltung durch Gewinnverlagerung sein, welche bei der direkten Methode zur Gewinnabgrenzung bei multinationalen Konzernen besteht. Die Ergebnisse für die Einführung einer GKKB verdeutlichen, dass dieses Konzept bei verpflichtender Einführung die Benachteiligung multinationaler Konzerne im geltenden Steuerrecht, welche aus der Untersagung der konzerninternen Verlustverrechnung über die Grenze resultiert, vollständig beseitigt. Die Mehrbelastung nationaler Konzerne beträgt hier 0,30 Prozent. Eine optionale GKKB führt hingegen zu einem Anstieg der Mehrbelastung nationaler Konzerne auf 1,21 Prozent, beinhaltet also eine signifikante Steuerbegünstigung multinationaler Konzerne. Dieses Ergebnis ist darauf zurückzuführen, dass mit einer optionalen GKKB für multinationale Konzerne ein äußerst wirksames Instrument zur Steuerplanung eingeführt würde, welches in dem Wahlrecht zwischen zwei potenziell vorteil- 
haften Steuersystemen besteht. Bei Inlandskonzernen erweist sich die GKKB hingegen in der überwiegenden Anzahl der Fälle als vorteilhaft. Die Implementierung einer optionalen GKKB würde somit einen Anreiz zur Durchführung von Auslandsinvestitionen in das Steuersystem einführen, die Standortwahl multinationaler Konzerne also in entgegengesetzter Richtung verzerren. ${ }^{611}$

\subsubsection{Auswirkungen auf intersektorale Belastungsunterschiede}

Neben der Standortentscheidung kann von den steuerlichen Verlustverrechnungsvorschriften insbesondere auch eine Verzerrung der Entscheidung bezüglich des Grads der Risikoübernahme ausgehen. Dabei zeigen modelltheoretische Untersuchungen, dass die Einführung einer Steuer mit sofortigem Verlustausgleich aufgrund der Beteiligung des Staates an Rendite und Risiko zu einer Ausweitung des Investitionsrisikos führt, während die Bereitschaft zur Risikoübernahme mit zunehmender Einschränkung der Verlustverrechnung, also zunehmender steuerlicher Benachteiligung risikobehafteter Investitionen, abnimmt. ${ }^{612}$ Dieses Ergebnis einer verminderten Risikoübernahme kann zumindest mit Blick auf den Unternehmenssektor politisch und ökonomisch nicht gewünscht sein, da eine Risikoübernahme (mit Ausnahme von Spekulationsrisiken) einen Beitrag zur Steigerung von Innovation und Schaffung von Arbeitsplätzen leisten kann.

Der hier zugrundegelegte Ansatz zur Messung durchschnittlicher Steuerbelastungen erlaubt eine Messung des Grads der Diskriminierung unrentabler Investitionen durch den Vergleich der Steuerbelastung rentabler Unternehmen (ATR ${ }^{+}$) und der Steuerentlastung verlusterzielender Unternehmen (ATR'). Hierbei sollten stärker negative Werte für die in Tabelle 29 angegebene relative Abweichung dieser beiden Größen mit einer stärkeren Minderung der Bereitschaft von Unternehmen zur Übernahme von Risiken korrespondieren.

Die Ergebnisse zeigen, dass im geltenden Steuerrecht bei nationalen Konzernen die Steuerentlastung bei Verlustunternehmen um durchschnittlich 37,62 Prozent unterhalb der Steuerbelastung von Gewinnunternehmen liegt. Bei multinationalen Konzernen liegt der entsprechende Wert mit 42,83 Prozent höher, was erneut auf die fehlende Möglichkeit zur konzerninternen Verlustverrechnung über die Grenze zurückzuführen sein sollte.

611 Bleiben die nationalen Konzerne mir nur einem Konzernunternehmen in der Datenbasis und damit Österreich und Irland vollständig unberücksichtigt, beträgt die Mehrbelastung nationaler Konzerne bei verpflichtender GKKB 0,26 Prozent, bei optionaler GKKB 0,76 Prozent. Der ,faire Wert“ bei sofortiger Verlustverrechnung und indirekter Gewinnabgrenzung ist hier 0,50 Prozent, sodass sich die oben beschriebenen Effekte hier nur mit Abstrichen zeigen. Hierbei ist allerdings zu berücksichtigen, dass dieses Ergebnis insofern verfälscht, als die Wirkung des Optionsrechts bei multinationalen Konzernen ohne Irland und Österreich geringer ausfällt.

612 Vgl. grundlegend Domar/Musgrave (1994). Vgl. hierzu auch die Ausfuhrungen in Kapitel 8.2.2. 
Tabelle 29: Relative Abweichung der Steuerbelastung bei profitablen und verlusterzielenden Konzernen

\begin{tabular}{lcccc}
\hline \multicolumn{1}{c}{$\frac{A T R^{-}-A T R^{+}}{A T R^{+}}$} & \multicolumn{2}{c}{ Nationale Konzerne } & \multicolumn{2}{c}{ Multinationale Konzerne } \\
& Pflicht & Option & Pflicht & Option \\
\hline Geltendes Recht & \multicolumn{2}{c}{$-0,3762$} & \multicolumn{2}{c}{$-0,4283$} \\
\hline Einheitliche nationale Grup- & \multirow{2}{*}{$-0,3548$} & $-0,3524$ & $-0,3933$ & $-0,3929$ \\
penbesteuerung & & & $-0,3773$ & $-0,3629$ \\
Nachversteuerungsmethode & & & $-0,4158$ & $-0,3721$ \\
Zurechnungsmethode & & & $-0,3820$ & $-0,3798$ \\
Ergebnisaufteilungsmethode & & & $-0,3658$ & $-0,3571$ \\
\hline GKKB & $-0,3569$ & $-0,3538$ & & \\
\hline
\end{tabular}

Quelle: Eigene Berechnungen

Eine Beseitigung der Beschränkungen der nationalen konzerninternen Verlustverrechnung („Einheitliche nationale Gruppenbesteuerung“) führt bei nationalen Konzernen zu einer Reduzierung der Benachteiligung von Verlustkonzernen auf 35,48 Prozent (bei verpflichtender Einführung) und 35,24 Prozent (bei optionaler Einführung). Die Werte, die sich bei Einführung einer GKKB ergeben, liegen in einem vergleichbaren Bereich. Bei grenzüberschreitend tätigen Konzernen führt das Zurechnungskonzept bei verpflichtender Einführung nur zu einer geringfügigen Reduzierung der steuerlichen Diskriminierung von Verlustkonzernen (41,58 Prozent). Grund hierfür ist, dass die Ausweitung der Verlustverrechnungsmöglichkeiten zwar einen Anstieg von ATR bewirkt, gleichzeitig sich allerdings auch $\mathrm{ATR}^{+}$aufgrund des Anrechnungsverfahrens erhöht. Die beiden anderen Konzepte einer grenzüberschreitenden Gruppenbesteuerung reduzieren die steuerliche Benachteiligung der multinationalen Verlustkonzerne gegenüber dem geltenden Steuerrecht zwar stärker (-37,73 Prozent bei Nachversteuerungsmethode, 38,20 Prozent bei Ergebnisaufteilungsmethode) ohne allerdings den entsprechenden Wert zu erreichen, der sich bei einheitlicher nationaler Gruppenbesteuerung oder GKKB für die Inlandskonzerne einstellt. Dieses ist erneut auf die unvollständige Verlustverrechnung zurückzuführen, die sich bei diesen Konzepten in bestimmten Situationen einstellt.

Die Ergebnisse, die sich für das Reformszenario einer GKKB bei multinationalen Konzernen ergeben, sind im Vergleich zu den übrigen Konzepten aus zweierlei Gründen hervorzuheben. Zum einen führt die GKKB von allen betrachteten Konzepten zur geringsten Diskriminierung (-36,58 Prozent bei verpflichtender Einführung; 35,71 Prozent bei optionaler Einführung). Zum anderen stellt dieses Konzept zumindest bei optionaler Einführung sicher, dass multinationale Ver- 
lustkonzerne in etwa im gleichen Ausmaß diskriminiert werden, wie dieses bei nationalen Konzernen der Fall ist. ${ }^{613}$

Des Weiteren bleibt festzuhalten, dass unabhängig vom betrachteten Reformszenario die Benachteiligung der Verlustkonzerne abnimmt, wenn die Reform optional durchgeführt wird. Dieses Ergebnis deutet darauf hin, dass Verlustkonzerne in stärkerem Ausmaß von einem Optionsrecht profitieren. Eine optionale Einführung sollte demnach einen Beitrag zur Steigerung der Risikoübernahme leisten.

\subsubsection{Auswirkungen auf größenabhängige Belastungsunterschiede}

Schließlich kommt es auch dann zu einer steuerlichen Wettbewerbsverzerrung, wenn Konzerne in Abhängigkeit von ihrer Größe einer unterschiedlichen Steuerbelastung unterliegen. Hierzu werden in Tabelle 30 die Steuerbelastungsänderungen gegenüber gestellt, die von der Einführung einer GKKB bei großen und kleinen/mittelgroßen multinationalen Konzernen ausgeht. ${ }^{614}$

Tabelle 30: Abhängigkeit der Steuerbelastung multinationaler Konzerne von der Konzerngröße (GKKB)

\begin{tabular}{lcc|cc} 
& \multicolumn{2}{c|}{$\begin{array}{c}\text { Große Konzerne } \\
\text { Pflicht }\end{array}$} & Option & Pfline/mittelgroße Konzerne \\
\hline Geltendes Recht & \multicolumn{2}{c|}{0,3074} & \multicolumn{2}{c}{0,3389} \\
\hline GKKB & 0,2947 & 0,2935 & 0,3296 & 0,3277 \\
Relative Änderung & $-0,0413$ & $-0,0452$ & $-0,0274$ & $-0,0330$ \\
\hline davon: Verlustverrechnung & $-0,0452$ & $-0,0414$ & $-0,0297$ & $-0,0277$ \\
davon: Formelaufteilung & $+0,0039$ & $-0,0037$ & $+0,0023$ & $-0,0053$ \\
\hline
\end{tabular}

Quelle: Eigene Berechnungen

Dabei zeigt sich, dass insbesondere der Effekt, welcher sich aus der Ausdehnung der Möglichkeiten zur nationalen und grenzüberschreitenden konzerninternen Verlustverrechnung („Verlustverrechnung“) ergibt, bei großen Konzernen

613 Dieses gilt auch, wenn bei der Ermittlung der Steuerbelastung nationaler Konzerne die Konzerne mit nur einem Unternehmen unberücksichtigt bleiben. In diesem Fall beträgt die durchschnittliche Diskriminierung von nationalen Konzernen bei der GKKB 35,18 Prozent (Pflicht) bzw. 34,69 Prozent (Option). Werden aus Gründen der Vergleichbarkeit auch bei den multinationalen Konzernen Österreich und Irland ausgeschlossen, würde die entsprechende Diskriminierung 35,52 Prozent bei verpflichtender Einführung und 34,76 Prozent bei optionaler Einführung betragen.

614 Diese Ergebnisse sind nicht direkt mit vorherigen zu vergleichen, da sich die durchschnittliche Steuerbelastung hier als Durchschnitt aller Konzerne und nicht als Länderdurchschnitt ergibt. 
deutlich größer ausfällt. Soweit davon ausgegangen werden kann, dass der Internationalisierungsgrad (also der Anteil ausländischer Konzerngesellschaften) bei großen Konzernen im Durchschnitt höher ist, profitieren große Konzerne entsprechend stärker von der Einführung einer konzerninternen Verlustverrechnung über die Grenze. Dementsprechend fällt auch der Rückgang der Steuerbelastung bei Einführung einer grenzüberschreitenden Verlustverrechnung nach dem Konzept der Nachversteuerungsmethode und der Ergebnisaufteilungsmethode bei großen Konzernen entsprechend höher aus (vgl. Tabelle 31). Dabei fällt auf, dass die entlastende Wirkung, die von der Nachversteuerungsmethode bei großen Konzernen ausgeht, im Vergleich zu kleinen/mittelgroßen Konzernen in deutlich stärkerem Ausmaß unterhalb der entsprechenden Wirkung einer GKKB liegt. Hier könnte die größere Anzahl an Auslandsgesellschaften bei großen Konzernen dafür verantwortlich sein, dass die Auslandsverluste bei der Nachversteuerungsmethode mangels ausreichender inländischer Bemessungsgrundlage in einer höheren Anzahl von Fällen nicht vollständig steuerwirksam werden können. Ebenfalls auf den höheren Internationalisierungsgrad großer Konzerne sollte die stärker ausfallende Zunahme der Steuerbelastung bei der Zurechnungsmethode zurückzuführen sein.

Tabelle 31: Abhängigkeit der Steuerbelastung multinationaler Konzerne von der Konzerngröße (Verlustrichtlinie)

\begin{tabular}{lcccc}
\hline & \multicolumn{2}{c}{ Große Konzerne } & \multicolumn{2}{c}{ Kleine/mittelgroße Konzeme } \\
& Pflicht & Option & Pflicht & Option \\
\hline Geltendes Recht & \multicolumn{2}{c}{0,3074} & \multicolumn{2}{c}{0,3389} \\
\hline Nachversteuerungsmethode & 0,2970 & 0,2970 & 0,3298 & 0,3296 \\
Relative Änderung & $-0,0338$ & $-0,0338$ & $-0,0269$ & $-0,0274$ \\
\hline Zurechnungsmethode & 0,3118 & 0,3023 & 0,3419 & 0,3312 \\
Relative Änderung & $+0,0143$ & $-0,0166$ & $+0,0089$ & $-0,0227$ \\
\hline Ergebnisaufteilungsmethode & 0,2943 & 0,2943 & 0,3304 & 0,3302 \\
Relative Änderung & $-0,0426$ & $-0,0426$ & $-0,0251$ & $-0,0257$ \\
\hline
\end{tabular}

Quelle: Eigene Berechnungen

Der Übergang von einer direkten hin zu einer indirekten Gewinnabgrenzung (siehe Tabelle 30, Zeile „Formelaufteilung") im Rahmen der Einführung einer GKKB führt unabhängig von der Größe zu einem Anstieg der Steuerbelastung, was auf das Bestehen von Steuerplanung im geltenden Steuerrecht zurückzuführen sein sollte. Dabei zeigt sich allerdings, dass der entsprechende Effekt bei großen Konzernen ( $+0,39$ Prozent) im Vergleich zu kleinen Konzernen ( $+0,23$ Prozent) zumindest geringfügig höher ausfällt. Dieses kann zumindest als schwacher Beleg für die verbreitete Vermutung verstanden werden, dass insbesondere große 
Konzerne steuerplanerisch tätig werden, während kleine und mittelgroße Konzerne aus Kostengründen in geringerem Ausmaß von dieser Möglichkeit Gebrauch machen.

\subsection{Zwischenfazit}

Insgesamt zeigen die in Kapitel 6 enthaltenen Ergebnisse zu den Auswirkungen der Einführung einer GKKB und einer Verlustrichtlinie auf die Steuerbelastung nationaler und multinationaler Konzerne, dass eine derartige Reform einen Beitrag zur Verbesserung der steuerlichen Standortattraktivität der EU sowie der Wettbewerbsfähigkeit europäischer Konzerne leisten würde, wobei die steuerlichen Rahmenbedingungen insbesondere für grenzüberschreitend tätige Konzerne verbessert würden. Dabei zeigen sich bei verpflichtender Einführung mit Ausnahme der Zurechnungsmethode nur geringfügige Unterschiede zwischen den verschiedenen Konzepten. Bei der Zurechnungsmethode kommt es aufgrund der zusätzlichen Belastung von Gewinnen ausländischer Tochtergesellschaften hingegen sogar zu einem Anstieg der Steuerbelastung. Bei optionaler Einführung bringt die GKKB die mit Abstand deutlichste Verbesserung der Standortattraktivität mit sich.

Bei der Analyse der Wettbewerbsverzerrungen, die von den verschiedenen Konzepten ausgehen zeigt sich, dass die Schwankung der Steuerbelastung multinationaler Konzerne durch die Einführung einer Verlustrichtlinie oder einer GKKB deutlich gesenkt werden könnte. Dieser Effekt fällt bei Einführung einer GKKB mit Abstand am stärksten aus, was insbesondere darauf zurückzuführen sein sollte, dass der Übergang auf eine GKKB dazu führt, dass mehr Bemessungsgrundlage den ausländischen Tochtergesellschaften als dem inländischen Konzernteil zugewiesen wird. Dieses führt zu einem Absinken der Steuerbelastung von Konzernen mit Sitz in Hochsteuerländern, während die Steuerbelastung von Konzernen, die ihren Sitz in einem Niedrigsteuerland haben, ansteigt.

Auch hinsichtlich der intersektoralen Neutralität zeigt die GKKB gegenüber der Verlustrichtlinie Vorteile. So führt die GKKB dazu, dass die steuerliche Benachteiligung verlusterzielender multinationaler Konzerne gegenüber gewinnerzielenden Konzernen im stärksten Maße abgemildert wird. Von den verschiedenen Konzepten einer Verlustrichtlinie geht hingegen eine weniger starke Verbesserung der steuerlichen Rahmenbedingungen verlusterzielender multinationaler Konzerne aus.

Ein Vergleich der Steuerbelastung nationaler und grenzüberschreitend tätiger Konzerne ergibt, dass die verpflichtende Einführung einer GKKB sowie - mit Abstrichen - eine optionale Einführung der Nachversteuerungsmethode zu einer fairen Behandlung von nationalen und multinationalen Konzernen führen, während insbesondere die Umsetzung der Zurechnungsmethode die Nachteile multinationaler Konzerne nicht in vollem Ausmaß beseitigen kann ${ }_{3}$ Die optionale Ein- 
führung einer GKKB würde hingegen aufgrund der durch diese Reform zusätzlich geschaffenen Gestaltungsspielräume eine Bevorteilung grenzüberschreitend tätiger Konzerne bewirken.

Ein Vergleich der Belastungswirkungen, die sich bei großen und kleinen/mittelgroßen multinationalen Konzernen einstellen, ergibt, dass die Minderung der Steuerbelastung großer Konzerne bei der GKKB, der Nachversteuerungsmethode und der Ergebnisaufteilungsmethode ebenso wie die Zunahme der Steuerbelastung bei der Zurechnungsmethode höher ausfällt. Dieses könnte als Indiz dafür gewertet werden, dass große Konzerne in stärkerem Maße über ausländische Tochtergesellschaften verfügen und somit von der Einführung einer grenzüberschreitenden Verlustverrechnung stärker profitieren beziehungsweise von der zusätzlichen Besteuerung der Auslandsgewinne bei der Zurechnungsmethode stärker belastet werden. Der Vergleich zwischen den verschiedenen Methoden lässt erkennen, dass die Belastungsminderung großer Konzerne bei der Nachversteuerungsmethode deutlich geringer ausfallt, als dieses bei der GKKB oder der Ergebnisaufteilungsmethode der Fall ist. Auch hierfür kann ein größerer Anteil ausländischer Tochtergesellschaften ursächlich sein. So können Auslandsverluste in diesem System nur bis zur Höhe der Gewinne des inländischen Konzernteils verrechnet werden. Eine Verrechnung gegen die Gewinne anderer Auslandsgesellschaften ist hingegen im Unterschied zur GKKB oder der Ergebnisaufteilungsmethode hier nicht möglich. 
Reinald Koch - 978-3-631-75683-6

Downloaded from PubFactory at 01/11/2019 03:01:46AM

via free access 


\section{Empirische Ergebnisse: Auswirkungen auf das Steu- eraufkommen der Mitgliedsstaaten}

\section{1 Überblick}

Mit der in diesem Kapitel erfolgenden Analyse der Aufkommenswirkungen, die von der Einführung einer GKKB sowie einer Verlustrichtlinie ausgehen ${ }^{615}$, werden zwei Zielsetzungen verfolgt.

Auf der einen Seite, sollen die möglichen Aufkommenswirkungen anhand des Mikrosimulationsmodells ermittelt werden. Hierzu beinhaltet Kapitel 7.2 die Simulationsergebnisse, die sich für einen Basisfall der GKKB ergeben. Für diesen Basisfall wird von einer Teilnahme sämtlicher Mitgliedsstaaten, einem Verlustvortrag auf Ebene der einzelnen Konzerngesellschaft sowie einer Definition der Aufteilungsformel entsprechend der Massachusetts-Formel, d.h. einer gleichgewichteten Aufteilung nach Vermögen, Lohnsumme und Umsatzerlösen, ausgegangen. Zur Verdeutlichung des Einflusses einzelner Ausgestaltungsmerkmale werden in Kapitel 7.3 die Aufkommenswirkungen für verschiedene Alternativszenarien analysiert. Hierbei werden alternative Ausgestaltungen der Aufteilungsformel, ein Verlustvortrag auf Konzernebene sowie die Einführung der GKKB im Wege der verstärkten Zusammenarbeit betrachtet. Die Aufkommensänderungen, die sich bei Einführung einer Verlustrichtlinie nach den verschiedenen hier betrachteten Konzepten ergeben würden, sind Gegenstand von Kapitel 7.4.

Auf der anderen Seite sollen die Ursachen der Aufkommenswirkungen, die sich in den Berechnungen für die einzelnen Mitgliedsstaaten ergeben, erklärt werden. Zu diesem Zweck erfolgt in 7.5 eine Regressionsanalyse, mit deren Hilfe der Aufkommenseffekt der Mitgliedsstaaten unter Rückgriff auf verschiedene steuerliche und außersteuerliche Parameter erklärt wird.

\subsection{Einführung einer GKKB}

\subsubsection{Basisszenario: Verpflichtende GKKB}

Die Ergebnisse der komparativ-statischen Mikrosimulation ergeben, dass die EUGesamtbemessungsgrundlage durch Einführung einer verpflichtenden GKKB deutlich gesenkt werden würde (vgl. Tabelle 32). Während die Bruttobemessungsgrundlage um 5,17 Prozent sinken würde, fiele der Nettobarwert der EUBemessungsgrundlage nur um 1,56 Prozent. Für diese Differenz ist ursächlich, dass eine Ausweitung der Möglichkeiten zur Verlustverrechnung einen absoluten

615 Die Ergebnisse der Aufkommensberechnungen zur Einführung einer GKKB sind in ahnlicher Form veröffentlicht in Oestreicher/Koch (2007). 
Effekt auf die Bruttobemessungsgrundlage haben kann, während sie für den Nettobarwert lediglich einen Zinseffekt bewirkt.

Tabelle 32: Wirkung der verpflichtenden Einführung einer GKKB auf das EU-Gesamtsteueraufkommen

\begin{tabular}{lccccc}
\hline & \multicolumn{2}{c}{ EU-Bemessungsgrundlage } & \multicolumn{2}{c}{ EU-Steueraufkommen } \\
& Brutto & Nettobarwert & V'vortrag & Brutto & Nettobarwert \\
\hline $\begin{array}{l}\text { Geltendes Recht } \\
\text { (MEUR) }\end{array}$ & 1.903 .264 & 1.099 .102 & 412.552 & 654.309 & 386.921 \\
\hline GKKB (MEUR) & 1.804 .840 & 1.081 .940 & 319.587 & 622.694 & 381.148 \\
Relative Änderung & $-0,0517$ & $-0,0156$ & $-0,2253$ & $-0,0483$ & $-0,0149$ \\
\hline davon: & & & & & \\
$\begin{array}{l}\text { Inländische Verlustver- } \\
\text { rechnung }\end{array}$ & $-0,0277$ & $-0,0072$ & $-0,1263$ & $-0,0267$ & $-0,0081$ \\
Formelaufteilung & $-0,0227$ & $-0,0068$ & $-0,1000$ & $-0,0201$ & $-0,0050$ \\
Dividendenbesteuerung & $-0,0013$ & $-0,0017$ & $+0,0009$ & $-0,0015$ & $-0,0018$ \\
\hline
\end{tabular}

Quelle: Eigene Berechnungen

Der Effekt auf das Steueraufkommen ist kleiner als der korrespondierende Effekt auf die Bemessungsgrundlage. So sinkt das Bruttoaufkommen um 4,83 Prozent, während der Nettobarwert des Steueraufkommens um 1,49 Prozent sinkt. Der Unterschied zwischen der Minderung des Steueraufkommens und der Minderung der Bemessungsgrundlage ist darauf zurückzuführen, dass bei Besteuerung nach dem Konzept der GKKB offensichtlich ein größerer Anteil der Bemessungsgrundlage in Hochsteuerländern besteuert wird als dieses im geltenden Steuerrecht der Fall ist. Auf der anderen Seite ist zu berücksichtigen, dass die nominellen Steuersätze im Betrachtungszeitraum in der überwiegenden Anzahl der Mitgliedsstaaten gefallen sind. Eine Ausweitung der Verlustverrechnungsmöglichkeiten führt bei im Zeitablauf fallenden Steuersätzen dazu, dass Verluste zu höheren Steuersätzen verrechnet werden können, was einen gegenläufigen Effekt auf das Bruttoaufkommen bewirkt. ${ }^{616}$

Der mittels Mikrosimulation ermittelte Gesamteffekt kann in verschiedene Teileffekte zerlegt werden. In der hier umgesetzten Form können der Effekt der Ausweitung der nationalen Gruppenbesteuerung (,inländische Verlustverrechnung"), die Auswirkungen des Übergangs von der direkten auf die indirekte Gewinnabgrenzung in Verbindung mit der Einführung einer grenzüberschreitenden Verlustverrechnung („Formelaufteilung“) und die Auswirkungen der Änderungen

616 Allerdings ist fraglich, inwiefern dieser Effekt aufgrund der bereits weitreichenden Steuersatzsenkungen der Vergangenheit in Zukunft in vergleichbarem Maße zu beobachten sein wird. 
bei der Dividendenbesteuerung („Dividendenbesteuerung“) separiert werden. ${ }^{617}$ Hierbei ergibt sich die Aufkommensänderung aufgrund der Ausdehnung der Möglichkeiten zur inländischen Verlustverrechnung durch einen Vergleich des Steueraufkommens im geltenden Steuerrecht gegenüber einer konzernweiten $\mathrm{Zu}$ sammenveranlagung aller Gruppengesellschaften eines Landes. Der Effekt der Dividendenbesteuerung erfasst die Aufkommensänderung bei Wegfall der Besteuerung der fünfprozentigen Pauschale auf konzerninterne Dividendenausschüttungen in Belgien, Deutschland, Frankreich und Italien. Der Effekt der grenzüberschreitenden Verlustverrechnung und Formelaufteilung ergibt sich als Residuum.

Mit Blick auf die EU-Gesamtbemessungsgrundlage ist der kombinierte Effekt aus Einführung einer grenzüberschreitenden Verlustverrechnung und Übergang von direkter zu indirekter Gewinnabgrenzung vollständig der Ausweitung der Verlustverrechnung zuzurechnen, da die Einführung einer formelhaften Gewinnaufteilung nur die zwischenstaatliche Aufteilung der Bemessungsgrundlage, nicht aber deren Höhe betrifft. Demnach ist der Effekt der Einführung einer GKKB auf die Gesamtbemessungsgrundlage der EU-25 zu mehr als 97 Prozent (5,04 Prozent) des Gesamteffekts (-5,17 Prozent) mit der Ausweitung der Verlustverrechnung zu begründen. Der verbleibende Effekt ( $-0,13$ Prozent) geht auf den durch die Konsolidierung hervorgerufenen Wegfall der Dividendenbesteuerung zurück. Bei Bezugnahme auf den Nettobarwert der Gesamtbemessungsgrundlage ist der Anteil, der auf die Dividendenbesteuerung zurückzuführen ist, deutlich höher, da in diesem Fall die Ausweitung der Verlustverrechnung lediglich einen Zinseffekt bewirkt. Ein Vergleich der Einzeleffekte auf die EU-Gesamtbemessungsgrundlage und das EU-Steueraufkommen verdeutlicht ferner, dass der Effekt des Übergangs von direkter zu indirekter Gewinnabgrenzung („Formelaufteilung") auf das Aufkommen unter dem korrespondierenden Effekt auf die Bemessungsgrundlage liegt. Dieses gilt aufgrund des geringeren Einflusses der Verlustverrechnung umso mehr, wenn auf den Nettobarwert abgestellt wird.

Die relative Bedeutung der Einzeleffekte ändert sich, wenn auf die Änderung der Bemessungsgrundlage der einzelnen Mitgliedsstaaten abgestellt wird (vgl. Tabelle 33). Während die EU-weite Aufkommensänderung maßgeblich von der Ausweitung der Verlustverrechnungsmöglichkeiten bestimmt wird, wird der Änderung bei den einzelnen Mitgliedsstaaten dominiert vom Wechsel der Methode zur Einkünfteabgrenzung. So beträgt die durchschnittliche Höhe der Aufkommensänderung durch Einführung einer grenzüberschreitenden Verlustverrechnung und Übergang auf eine indirekte Gewinnabgrenzung 7,48 Prozent (vgl. Tabelle 33, Spalte „Formelaufteilung"), während der entsprechende Wert für die Aus-

617 Die Aufkommensänderungen, die sich aus der Einführung vereinheitlichter Gewinnermittlungsvorschriften ergeben würden, sowie die Wirkungen der Einführung einer Zwischenergebniseliminierung und anderer Konsolidierungsmechanismen können mit der zugrundeliegenden Datenbasis hingegen nicht abgebildet werden 
weitung der nationalen Verlustverrechnung lediglich 2,27 Prozent beträgt (vgl. Tabelle 33, Spalte „Verlustverrechnung“). Der Wegfall der Dividendenbesteuerung betrifft lediglich die vier Mitgliedsstaaten (Belgien, Deutschland, Italien, Frankreich), die eine fünf-Prozent Pauschale der Dividenden als nicht-abziehbare Betriebsausgaben der Besteuerung unterwerfen, und ist daher auch hier von untergeordneter Bedeutung.

Die Ergebnisse der Mikrosimulation ergeben, dass bei Einführung der GKKB lediglich drei Mitgliedsstaaten (Italien, Lettland und Slowakei) an Steueraufkommen gewinnen. Estland, Finnland, Irland, Litauen, die Niederlande, Österreich und Ungarn würden die höchsten Aufkommensverluste hinzunehmen haben. Die Ergebnisse zeigen ferner, dass die Mitgliedsstaaten mit der höchsten nominellen Steuerbelastung im Betrachtungszeitraum (Deutschland, Italien und Belgien) unterproportional an Bemessungsgrundlage verlieren (durchschnittlicher Aufkommensverlust in Höhe von -1,99 Prozent), während die Niedrigsteuerländer Irland und Ungarn eine überproportional hohe Aufkommensminderung zu verzeichnen haben (-16,14 Prozent Aufkommensverlust im Durchschnitt). Dasselbe gilt auch für Länder mit speziellen Besteuerungsvergünstigungen für bestimmte Funktionen. So verlieren die Niederlande (Holding Privileg), Irland (IFSC Besteuerungsregime), Belgien (Coordination Center) und Dänemark (grenzüberschreitende Gruppenbesteuerung) durchschnittlich 10,63 Prozent an Bemessungsgrundlage.

Tabelle 33: Wirkung der verpflichtenden Einführung einer GKKB auf das Steueraufkommen der einzelnen Mitgliedsstaaten

\begin{tabular}{lcccccc}
\hline & \multicolumn{2}{c}{ Relative Aufkommensänderung } & \multicolumn{3}{c}{$\begin{array}{c}\text { Relative Bruttoänderung: } \\
\text { Einzeleffekte }\end{array}$} \\
& Brutto & $\begin{array}{c}\text { Netto-bar- } \\
\text { wert }\end{array}$ & $\begin{array}{c}\text { Rang } \\
\text { (Brutto) }\end{array}$ & $\begin{array}{c}\text { Verlustver- } \\
\text { rechnung }\end{array}$ & $\begin{array}{c}\text { Formel- } \\
\text { aufteilung }\end{array}$ & $\begin{array}{c}\text { Div'best } \\
\text { euerung }\end{array}$ \\
\hline Belgien & $-0,0137$ & 0,0323 & 4 & $-0,0479$ & 0,0424 & $-0,0082$ \\
Dänemark & $-0,0341$ & 0,0175 & 7 & $-0,0116$ & $-0,0226$ & 0,0000 \\
Deutschland & $-0,0775$ & $-0,0394$ & 12 & $-0,0137$ & $-0,0610$ & $-0,0028$ \\
Estland & $-0,1906$ & $-0,2261$ & 21 & $-0,0224$ & $-0,1683$ & 0,0000 \\
Finnland & $-0,1483$ & $-0,1384$ & 17 & $-0,0084$ & $-0,1399$ & 0,0000 \\
Frankreich & $-0,0220$ & 0,0139 & 6 & $-0,0261$ & 0,0077 & $-0,0036$ \\
Griechenland & $-0,0145$ & 0,0027 & 5 & $-0,0210$ & 0,0065 & 0,0000 \\
Großbritannien & $-0,0530$ & $-0,0151$ & 10 & $-0,0398$ & $-0,0132$ & 0,0000 \\
Irland & $-0,1745$ & $-0,0615$ & 20 & $-0,0015$ & $-0,1730$ & 0,0000 \\
Italien & 0,0315 & 0,0625 & 3 & $-0,0025$ & 0,0359 & $-0,0020$ \\
Lettland & 0,1055 & 0,1693 & 2 & $-0,0117$ & 0,1172 & 0,0000 \\
Litauen & $-0,1707$ & $-0,1270$ & 19 & $-0,0060$ & $-0,1647$ & 0,0000 \\
& & & Reinald & Koch - 978-3-631-75683-6
\end{tabular}




\begin{tabular}{lcccccc} 
Luxemburg & $-0,1172$ & $-0,1089$ & 16 & $-0,0010$ & $-0,1162$ & 0,0000 \\
Niederlande & $-0,2029$ & $-0,1794$ & 22 & $-0,0251$ & $-0,1778$ & 0,0000 \\
Österreich & $-0,2079$ & $-0,2482$ & 23 & 0,0000 & $-0,2079$ & 0,0000 \\
Polen & $-0,0455$ & $-0,0374$ & 9 & $-0,0473$ & 0,0018 & 0,0000 \\
Portugal & $-0,0555$ & $-0,0224$ & 11 & $-0,0306$ & $-0,0249$ & 0,0000 \\
Schweden & $-0,1014$ & $-0,0638$ & 15 & $-0,0192$ & $-0,0822$ & 0,0000 \\
Slowakei & 0,1613 & 0,1806 & 1 & $-0,0004$ & 0,1617 & 0,0000 \\
Spanien & $-0,0453$ & $-0,0239$ & 8 & $-0,0198$ & $-0,0255$ & 0,0000 \\
Tsch'e Republik & $-0,0778$ & $-0,0325$ & 13 & $-0,0605$ & $-0,0173$ & 0,0000 \\
Ungarn & $-0,1483$ & $-0,1420$ & 17 & $-0,0106$ & $-0,1377$ & 0,0000 \\
Zypern & $-0,0801$ & $-0,1855$ & 14 & 0,0000 & $-0,0801$ & 0,0000 \\
\hline Durchschnittliche & & & & & & \\
absolute Änderung & 0,0914 & 0,0755 & & 0,0227 & 0,0748 & 0,0009 \\
\hline
\end{tabular}

Quelle: Eigene Berechnungen

\subsubsection{Basisszenario: Optionale GKKB}

Tabelle 34 fasst die EU-weiten Aufkommenswirkungen zusammen, die sich bei optionaler Einführung einer GKKB ergeben. Für die Ausübung der Option wird unterstellt, dass Konzerne das Besteuerungskonzept wählen, das den Nettobarwert der Steuerzahlungen während des Simulationszeitraums minimiert. Der Nettobarwert wird dabei ermittelt durch Abzinsung der Steuerzahlungen mit einem Zinssatz von sechs Prozent. Für zum Ende des Simulationszeitraums bestehende Verlustvorträge wird eine Nutzung in der ersten dem Simulationszeitraum folgenden Periode unterstellt. Der steuerliche Vorteil aus der Nutzung dieser Verlustvorträge wird ebenfalls barwertig bei der Ermittlung des Nettobarwerts berücksichtigt.

Die Ergebnisse in Tabelle 34 verdeutlichen, dass der negative Aufkommenseffekt aus der Implementierung einer GKKB durch die Einführung des Optionsrechts verstärkt wird. Verglichen mit der Besteuerung nach geltendem Recht, sinkt das EU-weite Steueraufkommen um 4,99 Prozent, während der negative Effekt auf den Steuerbarwert auf -2,12 Prozent erhöht wird.

618 Kursiv gedruckte Länder werden aufgrund einer nur kleinen Anzahl von Unternehmen $(<20)$, die Bestandteil eines multinationalen Konzerns sind, nicht für die Ermittlung des Durchschnittswertes berilcksichtigt. 
Tabelle 34: Wirkung der optionalen Einführung einer GKKB auf das EU-Gesamtsteueraufkommen

\begin{tabular}{lccccc}
\hline & \multicolumn{2}{c}{ EU-Bemessungsgrundlage } & \multicolumn{2}{c}{ EU-Steueraufkommen } \\
& Brutto & Nettobarwert & V'vortrag & Brutto & Nettobarwert \\
\hline Geltendes Recht & 1.903 .264 & 1.099 .102 & 412.552 & 654.309 & 386.921 \\
\hline $\begin{array}{l}\text { Verpflichtende GKKB } \\
\text { (MEUR) }\end{array}$ & 1.804 .840 & 1.081 .940 & 319.587 & 622.694 & 381.148 \\
Relative Änderung & $-0,0517$ & $-0,0156$ & $-0,2253$ & $-0,0483$ & $-0,0149$ \\
\hline $\begin{array}{l}\text { Optionale GKKB } \\
\text { (MEUR) }\end{array}$ & 1.813 .218 & 1.083 .061 & 327.978 & 621.629 & 378.712 \\
Relative Änderung & $-0,0473$ & $-0,0146$ & $-0,2050$ & $-0,0499$ & $-0,0212$ \\
\hline
\end{tabular}

Quelle: Eigene Berechnungen

Die Ergebnisse in Tabelle 35 zeigen, dass auch das den einzelnen Mitgliedsstaaten zugewiesene Aufkommen durch die Einführung des Optionsrechts wesentlich beeinflusst wird.

Tabelle 35: Wirkung der optionalen Einführung einer GKKB auf das Steueraufkommen (brutto) der einzelnen Mitgliedsstaaten

\begin{tabular}{lcccc}
\hline & \multicolumn{2}{c}{ Verpflichtende GKKB } & \multicolumn{2}{c}{ Optionale GKKB } \\
& Änderung & Rang & Änderung & Rang \\
\hline Belgien & $-0,0137$ & 4 & $-0,0981$ & 18 \\
Dänemark & $-0,0341$ & 7 & $-0,0312$ & 7 \\
Deutschland & $-0,0775$ & 12 & $-0,0990$ & 19 \\
Estland & $-0,1906$ & 21 & $-0,1877$ & 23 \\
Finnland & $-0,1483$ & 17 & $-0,0947$ & 17 \\
Frankreich & $-0,0220$ & 6 & $-0,0689$ & 14 \\
Griechenland & $-0,0145$ & 5 & $-0,0493$ & 10 \\
Großbritannien & $-0,0530$ & 10 & $-0,0185$ & 5 \\
Irland & $-0,1745$ & 20 & 0,0171 & 3 \\
Italien & 0,0315 & 3 & $-0,0392$ & 8 \\
Lettland & 0,1055 & 2 & 0,0407 & 1 \\
Litauen & $-0,1707$ & 19 & $-0,1408$ & 22 \\
Luxemburg & $-0,1172$ & 16 & $-0,1063$ & 20 \\
Niederlande & $-0,2029$ & 22 & $-0,0610$ & 12 \\
Österreich & $-0,2079$ & 23 & $-0,1300$ & 21 \\
Polen & $-0,0455$ & 9 & $-0,0655$ & 13 \\
& & Reinald Koch $-978-3-631-75683-6$ \\
& Downloaded from PubFactory at $01 / 11 / 2019$ & $03: 01: 46 \mathrm{AM}$ \\
& & & via free access
\end{tabular}




\begin{tabular}{lcccc} 
Portugal & $-0,0555$ & 11 & $-0,0799$ & 15 \\
Schweden & $-0,1014$ & 15 & $-0,0208$ & 6 \\
Slowakei & 0,1613 & 1 & 0,0181 & 2 \\
Spanien & $-0,0453$ & 8 & $-0,0461$ & 9 \\
Tsch'e Republik & $-0,0778$ & 13 & $-0,0874$ & 16 \\
Ungarn & $-0,1483$ & 17 & $-0,0555$ & 11 \\
Zypern & $-0,0801$ & 14 & $-0,0028$ & 4 \\
\hline Durchschnittliche & 0,0914 & & 0,0694 & \\
absolute Änderung & & & &
\end{tabular}

Quelle: Eigene Berechnungen

Dabei wirkt sich die Einführung des Optionsrechts insbesondere auf das Aufkommen von Niedrigsteuerländern wie Irland und Ungarn (durchschnittlicher Aufkommensverlust: -1,92 Prozent gegenüber -16,14 Prozent bei verpflichtender GKKB) positiv aus. Hochsteuerländer (Deutschland, Italien und Belgien) hingegen würden Aufkommen durch die Einführung des Optionsrechts verlieren (durchschnittlicher Aufkommensverlust: -7,88 Prozent gegenüber -1,99 Prozent bei verpflichtender GKKB). Diese Ergebnisse verdeutlichen, dass die Einführung des Optionsrechts einen negativen Zusammenhang zwischen Aufkommenseffekt und nomineller Steuerbelastung erzeugt, da multinationale Konzerne insbesondere dann für die GKKB optieren werden, wenn die den Niedrigsteuerländern zugewiesene Bemessungsgrundlage gegenüber dem geltenden Steuerrecht erhöht wird. Außerdem zeigen die Ergebnisse, dass die durchschnittliche Höhe der Aufkommensänderung bei optionaler Anwendung (6,94 Prozent) unterhalb des entsprechenden Wertes bei verpflichtender GKKB liegt (9,14 Prozent). Dieses Ergebnis ist plausibel, da es bei Option für weniger Unternehmen zu Änderungen der Steuerzahlungen kommt.

\subsection{Einführung einer GKKB: Alternativszenarien}

\subsection{1 Überblick}

Die konkrete Ausgestaltung des möglichen Aufteilungsmechanismus ist noch nicht endgültig festgelegt. Zwar ist davon auszugehen, dass die Aufteilungsformel die Faktoren Vermögen, Arbeit und Umsatz beinhaltet. Allerdings bestehen unterschiedliche Vorschläge bezüglich der konkreten Ausgestaltung dieser Faktoren

619 Kursiv gedruckte Lănder werden aufgrund einer nur kleinen Anzahl von Unternehmen $(<20)$, die Bestandteil eines multinationalen Konzems sind, nicht für die Ermittlung des Durchschnittswertes berücksichtigt. 
sowie deren Gewichtung. Aus diesem Grund werden in diesem Abschnitt die unterschiedlichen Wirkungen alternativer Zusammensetzungen der Aufteilungsformel untersucht. Zu diesem Zweck wird auf die Faktoren Vermögen, Lohnsumme, Anzahl an Arbeitnehmern und Umsatz abgestellt. Der letztgenannte Faktor wird sowohl in seiner Definition als Gesamtumsätze als auch als Außenumsätze berücksichtigt. Dabei wird unterstellt, dass reine Produktionsgesellschaften ausschließlich konzernintern verkaufen und damit ausschließlich Innenumsätze erzielen, während alle übrigen Konzerngesellschaften ausschließlich an Konzernfremde verkaufen. ${ }^{620}$ Außerdem werden die Aufkommenswirkungen sowohl für Einzel-Faktor-Formeln (Kapitel 7.3.2.1) und Mehr-Faktor-Formeln (Kapitel 7.3.2.2) untersucht. Hierbei ist insbesondere zu untersuchen, wie sich die Wahl der Aufteilungsfaktoren auf die durchschnittliche Höhe der Aufkommensänderung der einzelnen Mitgliedsstaaten auswirkt. Ferner wird für den Arbeitsfaktor analysiert, welche der beiden möglichen Definitionen (Mitarbeiteranzahl oder Lohnsumme) die Arbeitsproduktivität besser erfasst. Beide Auswertungen erlauben Rückschlüsse für die Eignung der einzelnen Faktoren.

Ebenfalls ist zum heutigen Zeitpunkt unklar, auf welcher Ebene Gesamtverluste des Konzerns im Rahmen einer GKKB vorzutragen sind. Während in den bisherigen Berechnungen hierzu ein Vortrag auf Ebene der Einzelgesellschaft vorgesehen war, bevorzugt die Arbeitsgruppe eher einen Vortrag auf Konzernebene. Aus diesem Grund werden in Kapitel 7.3.3 die Aufkommenswirkungen dargestellt, die sich bei dieser Ausgestaltung ergeben würden. Schließlich wird in der Literatur bezweifelt, dass die Einführung einer GKKB unter Teilnahme sämtlicher Mitgliedsstaaten ein realistisches Politikszenario darstellt. ${ }^{621}$ Als mögliche Alternative wird vielfach die Einführung im Wege der verstärkten Zusammenarbeit unter Teilnahme von mindestens acht Mitgliedsstaaten angeführt. ${ }^{622}$ Die Aufkommenswirkungen dieses Szenarios werden in Kapitel 7.3.4 analysiert.

\subsubsection{Alternative Aufteilungsfaktoren}

\subsubsection{Einzel-Faktor-Formeln}

Die Berechnungsergebnisse für alternative Einzel-Faktor-Formeln zeigen, dass die Wahl des Aufteilungsfaktors nur einen geringfügigen Einfluss auf die Höhe des EU-weiten Gesamtsteueraufkommens hat. Die Minderung dieses Gesamtaufkommens liegt für die verschiedenen Aufteilungsfaktoren zwischen -4,26 Prozent und -5,29 Prozent. Der hohe Aufkommensrückgang bei Aufteilung nach der Mit-

620 Als reine Produktionsgesellschaften werden dabei alle Unternehmen angesehen, deren primärer Branchencode zwischen 15 und 37 liegt (Verarbeitendes Gewerbe) und für die kein sekundärer Branchencode verfügbar ist oder dieser nicht zwischen 50 und 52 (Handel) liegt.

621 Vgl. so Sorenson (2002), S. 35.

622 Vgl. den Kommentar von Lang in Rädler (2008), S. 65; McLure (2008), S. 46; Schön (2007), S. 443. 
arbeiteranzahl ist damit zu begründen, dass aufgrund von bestehenden Lohnniveauunterschieden innerhalb des Binnenmarktes dieser Aufteilungsfaktor einen höheren Anteil der Bemessungsgrundlage den Niedrigsteuerländern zuweisen würde.

Tabelle 36: Relative Änderung des EU-Gesamtsteueraufkommens (brutto) bei alternativen Aufteilungsfaktoren (verpflichtende Einfuihrung)

\begin{tabular}{lccccc}
\hline & Vermögen & $\begin{array}{c}\text { Lohn- } \\
\text { summe }\end{array}$ & Mitarbeiter & Umsätze & $\begin{array}{c}\text { Außen- } \\
\text { umsătze }\end{array}$ \\
\hline Relative Änderung & $-0,0503$ & $-0,0455$ & $-0,0529$ & $-0,0475$ & $-0,0426$ \\
\hline Quelle: Eigene Berechnungen & & & &
\end{tabular}

Deutlich höhere Unterschiede ergeben sich hingegen für die Verteilung der Gesamtbemessungsgrundlage auf die Mitgliedsstaaten. Die relativen Änderungen der Nettobemessungsgrundlagen der Mitgliedsstaaten bei Aufteilung nach den alternativen Einzel-Faktor-Formeln sind in Tabelle 37 zusammengefasst. ${ }^{623}$ Die Ergebnisse in Tabelle 37 verdeutlichen insbesondere den Einfluss der Definition des Arbeitsfaktors auf die Bemessungsgrundlage der Beitrittsstaaten. Aufgrund des durchgehend niedrigen Lohnniveaus ist für diese Mitgliedsstaaten grundsätzlich eine Aufteilung nach der Anzahl der Arbeitnehmer von allen untersuchten Aufteilungsfaktoren am günstigsten (Ausnahme: Slowakei, Zypern), während eine Aufteilung nach der Lohnsumme diesen Mitgliedsstaaten die geringste Bemessungsgrundlage zuordnet (Ausnahme: Ungarn). Vergleichbares gilt grundsätzlich auch für die Länder der EU-15 mit geringem Lohnniveau (Griechenland und Portugal). In Griechenland ist lediglich eine Aufteilung nach Vermögen und AuBenumsätzen noch leicht günstiger als die Aufteilung nach der Anzahl der Arbeitnehmer.

Zusätzlich wird für jeden Aufteilungsfaktor die durchschnittliche absolute Bemessungsgrundlagenänderung ermittelt (vgl. Tabelle 37, letzte Zeile). Diese Kennzahl verdeutlicht, wie hoch die Verzerrung gegenüber dem geltenden Steuerrecht für die jeweiligen Aufteilungsfaktoren ist. Dabei zeigt sich, dass der Aufteilungsfaktor „Umsätze“ die geringste durchschnittliche Höhe der Bemessungsgrundlagenänderung (9,06 Prozent) verursacht. Eine mögliche Erklärung für dieses Ergebnis ist, dass eine Aufteilung nach diesem Faktor am wenigsten durch internationale Produktivitätsdifferenzen beeinflusst wird. Ebenso führen „Vermögen“ und „Lohnsumme“ nur zu einer geringen durchschnittlichen Höhe der Bemessungsgrundlagenänderung, da beide Faktoren neben einer Mengenkompo-

623 An dieser Stelle wird auf die Nettobemessungsgnundlage abgestellt, damit der Effekt der Formelaufteilung und damit der einzelnen Aufteilungsfaktoren unabhăngig von der Entwicklung der Steuersätze im Zeitablauf und den Auswirkungen der erweiterten Verlustverrechnungsmöglichkeiten untersucht werden kann 
nente (Arbeits-/Kapitalintensität) auch eine Preiskomponente (Arbeits/Kapitalkosten) beinhalten. Im Gegensatz dazu erscheint „Mitarbeiter“ mit einer durchschnittlichen Höhe der Bemessungsgrundlagenänderung von 22,50 Prozent als Aufteilungsfaktor ungeeignet, da Produktivitätsdifferenzen durch diesen Faktor nur unzureichend reflektiert werden.

Tabelle 37: Relative Änderung der Bemessungsgrundlage (netto) der einzelnen Mitgliedsstaaten bei alternativen Aufteilungsfaktoren (verpflichtende Einführung)

\begin{tabular}{|c|c|c|c|c|c|}
\hline Land & Vermögen & Lohnsumme & Mitarbeiter & Umsätze & $\begin{array}{l}\text { Außen-um- } \\
\text { sätze }\end{array}$ \\
\hline Belgien & 0,0155 & 0,0855 & $-0,0651$ & 0,1020 & 0,2211 \\
\hline Dänemark & 0,0336 & 0,0803 & 0,0025 & $-0,0322$ & 0,0369 \\
\hline Deutschland & $-0,0537$ & 0,0338 & $-0,0899$ & $-0,0142$ & $-0,1216$ \\
\hline Estland & $-0,1391$ & $-0,3529$ & 0,6913 & $-0,2252$ & 0,1000 \\
\hline Finnland & $-0,1281$ & $-0,1320$ & $-0,1638$ & $-0,1711$ & $-0,1390$ \\
\hline Frankreich & $-0,0453$ & 0,0743 & 0,0069 & 0,0426 & $-0,0405$ \\
\hline Griechenland & 0,0567 & $-0,0461$ & 0,0555 & 0,0119 & 0,3363 \\
\hline Großbritannien & 0,0358 & $-0,0042$ & 0,0018 & $-0,0125$ & 0,0108 \\
\hline Irland & $-0,1688$ & $-0,1109$ & 0,1434 & 0,1315 & $-0,9191$ \\
\hline Italien & 0,0802 & 0,0882 & 0,0864 & 0,0914 & 0,2123 \\
\hline Lettland & 0,3008 & 0,2277 & 1,0279 & 0,3317 & 1,4083 \\
\hline Litauen & $-0,0299$ & $-0,3509$ & 0,0542 & $-0,1460$ & $-0,2321$ \\
\hline Luxemburg & $-0,0740$ & $-0,1343$ & $-0,1492$ & $-0,0943$ & $-0,0353$ \\
\hline Niederlande & $-0,1768$ & $-0,2620$ & $-0,2974$ & $-0,1198$ & 0,0613 \\
\hline Österreich & $-0,2963$ & $-0,1778$ & $-0,2423$ & $-0,3498$ & $-0,4128$ \\
\hline Polen & 0,3767 & $-0,1993$ & 1,1312 & 0,1045 & $-0,0303$ \\
\hline Portugal & 0,0024 & $-0,0184$ & 0,1282 & 0,0073 & 0,0128 \\
\hline Schweden & $-0,0095$ & $-0,0947$ & $-0,0710$ & $-0,0469$ & $-0,1760$ \\
\hline Slowakei & 0,6754 & $-0,0388$ & 0,2579 & $-0,0444$ & $-0,5923$ \\
\hline Spanien & $-0,0120$ & $-0,0294$ & $-0,0198$ & $-0,0169$ & 0,0039 \\
\hline Tsch'e Republik & 0,1421 & $-0,2021$ & 0,6579 & $-0,0469$ & $-0,0441$ \\
\hline Ungarn & $-0,1313$ & $-0,2180$ & 0,1947 & $-0,1033$ & $-0,2370$ \\
\hline Zypern & $-1,2132$ & $-0,3163$ & $-0,9466$ & $-0,4840$ & $-0,4840$ \\
\hline $\begin{array}{l}\text { Durchschnittliche } \\
\text { absolute Änderung }\end{array}$ & 0,1058 & 0,1228 & 0,2250 & 0,0906 & 0,1731 \\
\hline
\end{tabular}

Quelle: Eigene Berechnungen

624 Kursiv gedruckte Länder wurden aufgrund einer nur kleinen Anzahl von Unternehmen in der Stichprobe nicht für die Ermittlung des Durchschnittswertes berügkssichtigt 
Insbesondere die Definition des Arbeitsfaktors hat somit offensichtlich einen gewichtigen Einfluss auf die Verteilung des Steueraufkommens unter einer GKKB. Die GKKB-Arbeitsgruppe der Europäischen Kommission spricht sich in diesem Zusammenhang für eine Kombination aus Lohnsumme und Mitarbeiteranzahl aus. ${ }^{625}$ Die obigen Ergebnisse deuten allerdings darauf hin, dass eine Einbeziehung der Mitarbeiter zu stärkeren Verschiebungen von Bemessungsgrundlage zwischen den Mitgliedsstaaten führt. Eine Variation der Gewichtung bei der Definition des Arbeitsfaktors innerhalb der Massachusetts-Formel ergibt, dass die geringste durchschnittliche Höhe der Änderung der Nettobemessungsgrundlage bei einer Gewichtung von Lohnsumme und Mitarbeiteranzahl im Verhältnis 90:10 erzielt werden kann. Eine höhere Gewichtung des Arbeitsfaktors führt zu einer deutlichen Erhöhung der durchschnittlichen Nettobemessungsgrundlagenänderung (vgl. Abbildung 4).

Abbildung 4: Durchschnittliche Höhe der Bemessungsgrundlagenänderung in Abhängigkeit von der Gewichtung des Arbeitsfaktors

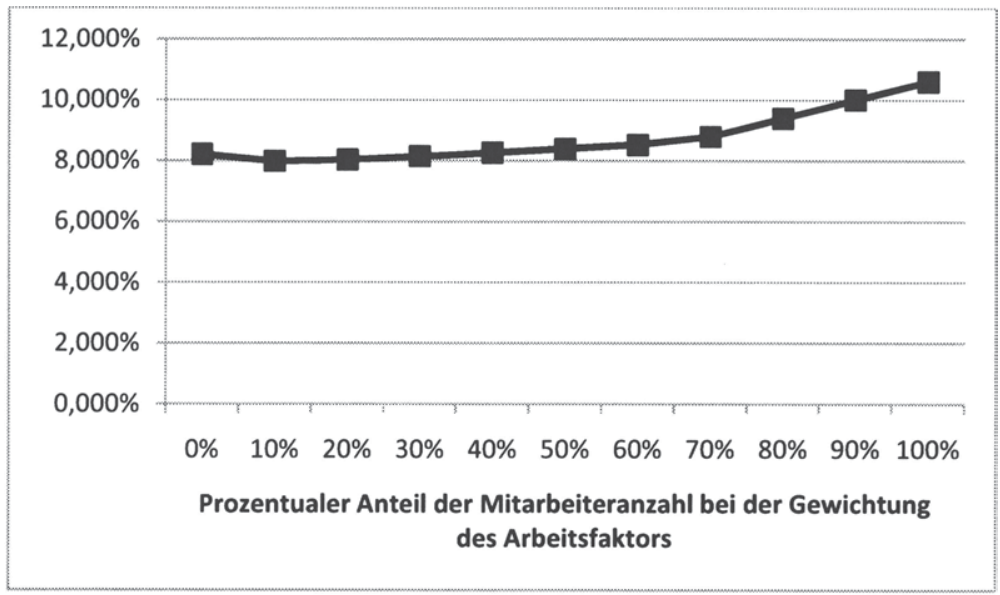

Quelle: Eigene Berechnungen

Dabei muss allerdings berücksichtigt werden, dass der Änderung des Steueraufkommens im Vergleich zum geltenden Recht zwar politische Bedeutung zukommt, aus einer theoretischen Perspektive die Aufteilungsformel allerdings so ausgestaltet sein sollte, dass sie den Beitrag einzelner Faktoren zur Einkommenserzielung bestmöglich widerspiegelt. Aufschluss darüber, ob der Beitrag des Arbeitsfaktors zur Einkommenserzielung besser durch die Mitarbeiteranzahl oder die Lohnsumme erfasst wird, gibt eine Analyse des Zusammenhangs der Bruttounter- 
nehmensgewinne und Arbeitnehmeranzahl bzw. Lohnsumme auf gesamtwirtschaftlicher Basis. ${ }^{626}$ Dabei stellt sich eine bestmögliche Erfassung der Arbeitsproduktivität bei einer Korrelation zwischen Arbeitsfaktor und Unternehmensgewinn in Höhe von 1 ein. Die Analyse der gesamtwirtschaftlichen Daten für das Jahr 2004 ergibt eine Korrelation von 0,988 für die Lohnsumme und 0,924 für die Mitarbeiteranzahl (vgl. Abbildung 5 für die Punktstreudiagramme; um größenabhängige Verzerrungen zu vermeiden werden sämtliche Werte im natürlichen Logarithmus herangezogen). Es bleibt somit festzuhalten, dass eine Definition des Arbeitsfaktors anhand der Lohnsumme nicht nur die durchschnittliche Höhe der Aufkommensänderung reduziert, sondern nach diesen Ergebnissen auch eine bessere Erfassung der Arbeitsproduktivität gewährleistet.

Abbildung 5: Zusammenhang von Bruttobetriebsgewinnen (gesamtwirtschaftlich, 2004) und dem Arbeitsfaktor (gesamtwirtschaftlich, 2004) in den Mitgliedsstaaten
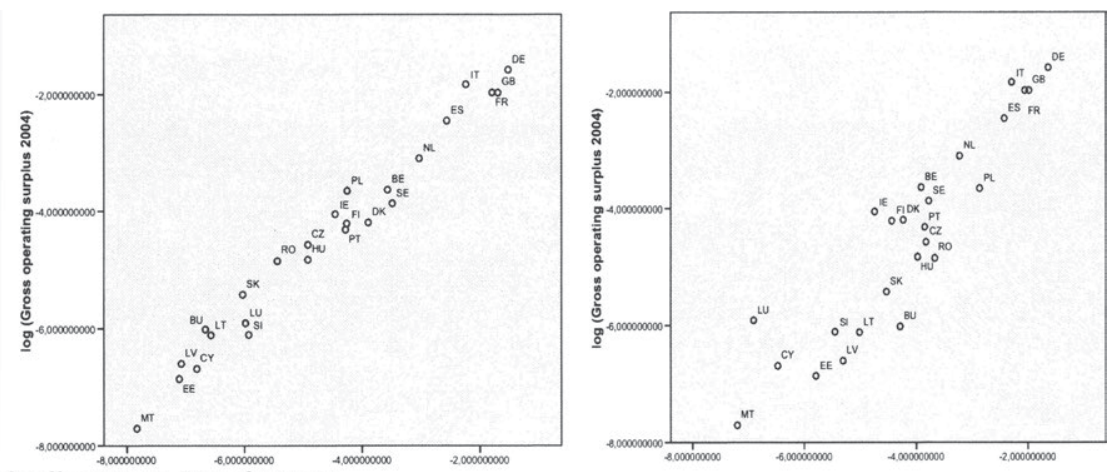

Quelle: Eigene Berechnungen

Die Entwicklung der Korrelation zwischen den Bruttounternehmensgewinnnen und den Ausprägungen für die beiden Definitionen des Faktors „Arbeit“ im Zeitablauf (1995 bis 2006) verdeutlicht einen konstant starken Zusammenhang zur Lohnsumme (Korrelation nahe 1), während die entsprechenden Werte für die Mitarbeiteranzahl von 0,86 auf 0,93 gestiegen sind, damit aber unverändert eine niedrigere Korrelation als die Lohnsumme aufweisen (vgl. Abbildung 6). Dieses Ergebnis sollte auf eine Angleichung des Lohnniveaus innerhalb von Europa zurückzuführen sein. Hält dieser Trend an, sollte die Bedeutung der Gewichtung des Arbeitsfaktors für die Aufkommenswirkungen der GKKB in Zukunft abnehmen. 
Abbildung 6: Korrelation zwischen den Bruttounternehmensgewinnen (gesamtwirtschaftlich) und dem Arbeitsfaktor (gesamtwirtschafilich) im Zeitablauf

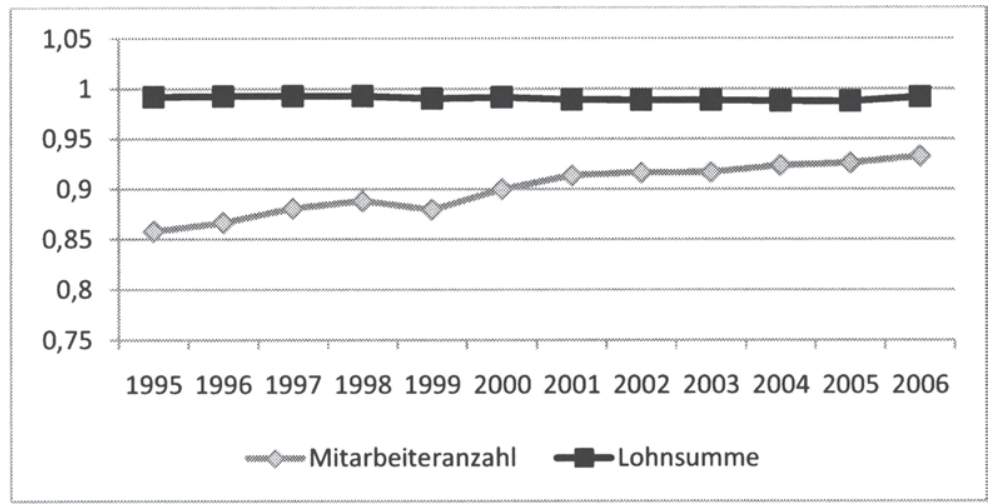

Quelle: Eigene Berechnungen

Die obigen Ergebnisse für die Verzerrung der alternativen Aufteilungsfaktoren werden bestätigt für den Fall einer optionalen Einführung der GKKB (vgl. Tabelle 38). Erneut ergibt sich die kleinste durchschnittliche Höhe der Bemessungsgrundlagenänderung für den Faktor "Umsätze" (7,60 Prozent).

Tabelle 38: Relative Änderung der Bemessungsgrundlage (netto) der einzelnen Mitgliedsstaaten bei alternativen Aufteilungsfaktoren (optionale Einführung)

\begin{tabular}{|c|c|c|c|c|c|}
\hline & Vermögen & Lohnsumme & Mitarbeiter & Umsätze & $\begin{array}{l}\text { Außen- } \\
\text { umsätze }\end{array}$ \\
\hline Belgien & $-0,0656$ & $-0,0460$ & $-0,1466$ & $-0,0596$ & $-0,1535$ \\
\hline Dänemark & 0,0090 & 0,0541 & 0,0125 & 0,0112 & 0,0823 \\
\hline Deutschland & $-0,1042$ & $-0,0415$ & $-0,1314$ & $-0,0573$ & $-0,2069$ \\
\hline Estland & $-0,1100$ & $-0,2947$ & 0,4833 & $-0,2441$ & $-0,0522$ \\
\hline Finnland & $-0,0755$ & $-0,0758$ & $-0,0855$ & $-0,0955$ & $-0,0814$ \\
\hline Frankreich & $-0,0865$ & $-0,0104$ & $-0,0694$ & $-0,0341$ & $-0,0822$ \\
\hline Griechenland & 0,0104 & $-0,0761$ & $-0,0121$ & $-0,0449$ & 0,0701 \\
\hline Großbritannien & 0,0702 & 0,0511 & 0,0600 & 0,0527 & 0,1100 \\
\hline Irland & 0,2575 & 0,2428 & 0,3186 & 0,3344 & 0,3088 \\
\hline Italien & $-0,0138$ & $-0,0446$ & $-0,0466$ & $-0,0125$ & $-0,1477$ \\
\hline Lettland & 0,2713 & 0,0617 & 0,7055 & 0,1725 & 1,1336 \\
\hline Litauen & $-0,0131$ & $-0,2340$ & 0,0267 & $-0,0949$ & 0,0115 \\
\hline Luxemburg & $-0,0674$ & $-0,1129$ & $-0,1401$ & $-0,0725$ & $-0,0046$ \\
\hline Niederlande & $-0,0448$ & $-0,0790$ & ald'koch. & $\begin{array}{l}-0,1408 \\
3-631-75\end{array}$ & -6,0005 \\
\hline
\end{tabular}




\begin{tabular}{llllll} 
Österreich & $-0,1483$ & $-0,1576$ & $-0,1346$ & $-0,1023$ & $-0,2834$ \\
Polen & 0,3158 & $-0,2162$ & 0,8938 & 0,0066 & 0,0328 \\
Portugal & $-0,0406$ & $-0,0206$ & 0,0402 & $-0,0362$ & $-0,0488$ \\
Schweden & 0,0623 & 0,0555 & 0,0385 & 0,0663 & 0,0978 \\
Slowakei & 0,1871 & 0,0637 & 0,1565 & 0,0042 & 0,0339 \\
Spanien & $-0,0153$ & $-0,0263$ & $-0,0178$ & $-0,0203$ & 0,0020 \\
Tsch'e Republik & 0,1237 & $-0,1790$ & 0,6001 & 0,0133 & $-0,0149$ \\
Ungarn & $-0,0076$ & $-0,1051$ & 0,1753 & $-0,0361$ & 0,1434 \\
Zypern & $-0,3783$ & $-0,3163$ & $-0,3620$ & $-0,4840$ & $-0,4840$ \\
\hline $\begin{array}{l}\text { Durchschnittliche } \\
\text { absolute Änderung }\end{array}$ & 0,0867 & 0,0987 & 0,1885 & 0,0760 & 0,1066 \\
\hline
\end{tabular}

Quelle: Eigene Berechnungen

Auch für die Höhe der Minderung des EU-Gesamtsteueraufkommens (Tabelle 39) ergeben sich grundsätzlich nur geringe Abweichungen gegenüber der verpflichtenden Einführung. Diese Minderung fällt für jeden Aufteilungsfaktor erwartungsgemäß höher aus, als dieses bei der verpflichtenden Einführung der Fall war. Auffallig ist allerdings die hohe Differenz, die sich für den Aufteilungsfaktor „Außenumsätze“ zwischen einer verpflichtenden Einführung (-4,26 Prozent; vgl. Tabelle 36) und optionaler Einführung (-5,56 Prozent; vgl. Tabelle 39) ergibt. Dieses Ergebnis deutet darauf hin, dass dem Optionsrecht bei diesem Faktor im Vergleich zu den übrigen Faktoren eine deutlich größere Bedeutung zukommt. Mögliche Ursache hierfür ist eine deutlich stärkere Umverteilung der Bemessungsgrundlage innerhalb der einzelnen Konzerne.

Tabelle 39: Relative Änderung des EU-Gesamtsteueraufkommens (brutto) bei alternativen Aufteilungsfaktoren (optionale Einführung)

\begin{tabular}{lccccc}
\hline & Vermögen & $\begin{array}{c}\text { Lohn- } \\
\text { summe }\end{array}$ & Mitarbeiter & Umsätze & $\begin{array}{c}\text { Außen- } \\
\text { umsätze }\end{array}$ \\
\hline Relative Änderung & $-0,0520$ & $-0,0483$ & $-0,0542$ & $-0,0500$ & $-0,0556$ \\
\hline
\end{tabular}

Quelle: Eigene Berechnungen

\subsubsection{Multi-Faktor-Formeln}

Die Ergebnisse für die Einführung einer GKKB können verbessert werden im Sinne einer geringeren durchschnittlichen Höhe der Bemessungsgrundlagenände-

627 Kursiv gedruckte Länder werden aufgrund einer nur kleinen Anzahl von Unternehmen in der Stichprobe nicht für die Ermittlung des Durchschnittswertes berüglksichtight -75683-6 
rung durch Kombination mehrerer Aufteilungsfaktoren. Hierdurch wird dem Umstand Rechnung getragen, dass auch die Produktionsfunktion aus zumindest zwei Faktoren (Arbeit und Kapital) besteht und zudem gleichzeitig die Produktionsseite (Arbeit, Kapital) und die Absatzseite (Umsatz) in der Formel reflektiert werden kann.

Die Ergebnisse für verschiedene Mehr-Faktor-Formeln sind zusammengefasst in Tabelle 40 für den Fall der verpflichtenden GKKB. MAS_1 und MAS_2 stehen für zwei verschiedene Ausprägungen der Massachusetts-Formel, die die drei Faktoren Vermögen, Lohnsumme und Umsatz gleichgewichtet berücksichtigt. MAS 1 definiert den Umsatzfaktor dabei - wie im Basisfall - als Gesamtumsätze, MAS_ 2 als Außenumsätze. Die Formeln OPT_1 und OPT_2 sind so definiert, dass die durchschnittliche Höhe der Bemessungsgrundlagenänderung der Mitgliedsstaaten minimiert wird (,minimum impact formulae“). Diese Formeln könnten einen Anhaltspunkt bieten, soweit die EU-Kommission eine Lösung anstrebt, die nur eine geringe Änderung des Steueraufkommens der Mitgliedsstaaten hervorruft. Die Einigung auf eine gemeinsame Formel im politischen Prozess könnte auf diesem Wege gegebenenfalls erleichtert werden. Nach Musgrave ist diese Zielsetzung einer Minimierung der Aufkommensverschiebung auch aus theoretischer Sicht gerechtfertigt, wenn die Motivation für die Einführung der GKKB nicht in der unzutreffenden Erfolgszuordnung bei direkter Gewinnabgrenzung liegt, sondern vielmehr in der administrativen Unmöglichkeit der Angemessenheitsprüfung für Verrechnungspreise. ${ }^{628}$

Tabelle 40: Wirkung alternativer einheitlicher Multi-Faktor-Formeln auf die durchschnittliche Höhe der Änderung der Bemessungsgrundlage (netto) der einzelnen Mitgliedsstaaten

\begin{tabular}{lccccc|c}
\hline & $\begin{array}{c}\text { Ver- } \\
\text { mögen }\end{array}$ & $\begin{array}{c}\text { Lohn- } \\
\text { summe }\end{array}$ & $\begin{array}{c}\text { Mit- } \\
\text { arbeiter }\end{array}$ & $\begin{array}{c}\text { Gesamt- } \\
\text { umsätze }\end{array}$ & $\begin{array}{c}\text { Außen- } \\
\text { umsätze }\end{array}$ & $\begin{array}{c}\text { Durchschnittliche } \\
\text { absolute Änderung }\end{array}$ \\
\hline MAS_1 & 0,3333 & 0,3333 & & 0,3333 & & 0,0821 \\
MAS_2 & 0,3333 & 0,3333 & & & 0,3333 & 0,1058 \\
\hline OPT_1 & 0,0000 & 0,5500 & 0,2000 & 0,2500 & & 0,0757 \\
OPT_2 & 0,0000 & 0,7500 & 0,2500 & & 0,0000 & 0,0789 \\
\hline
\end{tabular}

Quelle: Eigene Berechnungen

Die Anwendung der Massachusetts-Formel verringert die durchschnittliche Höhe der Bemessungsgrundlagenänderung gegenüber der Einzel-Faktor-Formel mit der geringsten durchschnittlichen Änderung für den Fall, dass der Umsatzfaktor als Gesamtumsätze definiert wird. Werden die Außenumsätze zugrundege- 
legt, erhöht sich dieser Wert hingegen. ${ }^{629}$ Die „minimum impact formulae“ (OPT_1 und OPT_2) führen definitionsgemäß zu einer weiteren Reduktion dieses Wertes. Die Ergebnisse zeigen, dass in diesen Formeln dem Faktor "Lohnsumme" ein Gewicht von 55 Prozent zugeordnet wird, wenn der Umsatzfaktor als Gesamtumsätze definiert wird, und 75 Prozent bei Definition als Außenumsätze.

Tabelle 41: Wirkung branchenspezifischer Multi-Faktor-Formeln

\begin{tabular}{|c|c|c|c|c|c|c|c|}
\hline Branche & $\begin{array}{l}\text { Ver- } \\
\text { mögen }\end{array}$ & $\begin{array}{l}\text { Lohn- } \\
\text { summe }\end{array}$ & $\begin{array}{l}\text { Mitar- } \\
\text { beiter }\end{array}$ & $\begin{array}{l}\text { Um- } \\
\text { sätze }\end{array}$ & $\begin{array}{l}\varnothing \text { absolute } \\
\text { Änderung }\end{array}$ & $\begin{array}{l}\text { Vergleich: } \\
\text { MAS_1 }\end{array}$ & $\Delta_{\text {rel }}$ \\
\hline Baugewerbe & 0,9000 & 0,1000 & 0,0000 & 0,0000 & 0,0779 & 0,1079 & $-0,2780$ \\
\hline $\begin{array}{l}\text { Energieversor- } \\
\text { gung }\end{array}$ & 0,0000 & 0,0000 & 0,0000 & 1,0000 & 0,0771 & 0,1300 & $-0,4069$ \\
\hline $\begin{array}{l}\text { Finanzdienst- } \\
\text { leistungen }\end{array}$ & 0,3000 & 0,0000 & 0,0000 & 0,7000 & 0,1242 & 0,1884 & $-0,3408$ \\
\hline $\begin{array}{l}\text { Gesundheitswe- } \\
\text { sen }\end{array}$ & 0,0000 & 0,0000 & 1,0000 & 0,0000 & 0,1621 & 0,1900 & $-0,1468$ \\
\hline $\begin{array}{l}\text { Verarbeitendes } \\
\text { Gewerbe }\end{array}$ & 0,4000 & 0,0500 & 0,0000 & 0,5500 & 0,1267 & 0,1344 & $-0,0573$ \\
\hline Bergbau & 1,0000 & 0,0000 & 0,0000 & 0,0000 & 0,4492 & 0,5623 & $-0,2011$ \\
\hline $\begin{array}{l}\text { Dienstleistun- } \\
\text { gen }\end{array}$ & 0,6000 & 0,0000 & 0,1500 & 0,2500 & 0,1003 & 0,1233 & $-0,1865$ \\
\hline Handel & 0,0000 & 0,5500 & 0,0000 & 0,4500 & 0,2499 & 0,3202 & $-0,2196$ \\
\hline $\begin{array}{l}\text { Verkehr/Nach- } \\
\text { richtenüberm. }\end{array}$ & 0,0000 & 0,0000 & 0,7000 & 0,3000 & 0,1564 & 0,2492 & $-0,3724$ \\
\hline Alle Branchen & 0,0000 & 0,5500 & 0,2000 & 0,2500 & 0,0757 & 0,0821 & $-0,0780$ \\
\hline
\end{tabular}

Quelle: Eigene Berechnungen

Die GKKB-Arbeitsgruppe weist darauf hin, dass die Verwendung einer standardisierten Aufteilungsformel nicht für alle Branchen zu angemessenen Ergebnissen führen muss. In diesem Zusammenhang wird insbesondere auf die Branchen „Finanzdienstleistungen, Transportdienstleistungen...sowie Fernsehund Rundfunkdienstleistungen" Bezug genommen. ${ }^{630}$ Die von der Arbeitsgruppe vorgeschlagene Lösung einer abweichenden Definition oder Bewertung der Aufteilungsfaktoren ${ }^{631}$ kann auf Basis der verfügbaren Daten nicht sinnvoll abgebildet werden. Ein gewisser Aufschluss über die Eignung der Massachusetts-Formel für die Aufteilung der Bemessungsgrundlage in diesen Branchen kann allerdings durch die Ermittlung branchenabhängiger ,minimum impact formulae“ gewonnen werden. Dabei zeigt ein Vergleich der durchschnittlichen Änderungen, die sich für

629 Hierbei muss allerdings berücksichtigt werden, dass die hier erfolgende Schätzung der AuBenumsätze auf stark vereinfachenden Annahmen beruht.

630 Arbeitsgruppe GKKB (2007a), S. 20.

631 Vgl. Arbeitsgruppe GKKB (2007a), S. 20. 
diese branchenspezifischen „minimum impact formulae“ ergeben, mit denen, die sich bei Verwendung der Massachusetts-Formel (MAS_1) für die einzelnen Branchen einstellen, dass eine Verwendung der Massachusetts-Formel überwiegend nur in geringem Ausmaß zu höheren Verzerrungen führt. Insbesondere in den auch von der Arbeitsgruppe genannten Branchen "Finanzdienstleistungen" und "Verkehr/Nachrichtenübermittlung" führt eine Verwendung der MassachusettsFormel allerdings nicht zu akzeptablen Ergebnissen (vgl. Tabelle 41). Hier kann die durchschnittliche Höhe der Bemessungsgrundlagenänderung bei Aufteilung nach den branchenspezifischen „minimum impact formulae“ um 34,08 Prozent bzw. 37,54 Prozent gegenüber einer Aufteilung nach der Massachusetts-Formel gesenkt werden.

\subsubsection{Einfluss der Ebene des Verlustvortrags}

Ein Vortrag von Verlusten auf Konzernebene kann im Vergleich zu einer Verrechnung auf Ebene der Einzelgesellschaft grundsätzlich zu einem höheren oder einem geringeren Steueraufkommen führen. Während bei einer Verlustverrechnung auf Ebene der Einzelgesellschaft eine Aufteilung dieser Verluste nach den Verhältnissen im Jahr der Verlustentstehung erfolgt, sind bei Vortrag auf Konzernebene die Verhältnisse im Jahr der Verlustnutzung maßgebend. ${ }^{632}$ Dieses kann in Abhängigkeit von den jeweiligen Umständen zu einer früheren oder späteren Verlustverrechnung führen.

Tabelle 42: Wirkung der verpflichtenden Einführung einer GKKB mit Verlustvortrag auf Konzernebene auf das EU-Gesamtsteueraufkommen

\begin{tabular}{lccccc}
\hline & \multicolumn{2}{c}{ EU-Bemessungsgrundlage } & \multicolumn{2}{c}{ EU-Steueraufkommen } \\
& Brutto & $\begin{array}{c}\text { Nettobar- } \\
\text { wert }\end{array}$ & $\begin{array}{l}\text { Verlust- } \\
\text { vortrag }\end{array}$ & Brutto & $\begin{array}{c}\text { Netto- } \\
\text { barwert }\end{array}$ \\
\hline Geltendes Recht (MEUR) & 1.903 .264 & 1.099 .102 & 412.552 & 654.309 & 386.921 \\
\hline $\begin{array}{l}\text { GKKB (Pflicht) mit Ver- } \\
\text { lustvortrag bei K`Umen }\end{array}$ & 1.804 .840 & 1.081 .940 & 319.587 & 622.694 & 381.148 \\
Relative Änderung & $-0,0517$ & $-0,0156$ & $-0,2253$ & $-0,0483$ & $-0,0149$ \\
\hline GKKB (Pflicht) mit Ver- & & & & & \\
lustvortrag bei Konzern & & & & & \\
(MEUR) (MEUR) & 1.801 .619 & 1.080 .883 & 317.705 & 621.132 & 380.504 \\
Relative Änderung & $-0,0534$ & $-0,0166$ & $-0,2299$ & $-0,0507$ & $-0,0166$ \\
\hline Quelle: Eigene Berechnungen & & & & &
\end{tabular}


Systematische Ungleichbehandlungen ergeben sich allerdings bei Auflösung von Konzerngesellschaften. ${ }^{633}$ Hier führt der Verlustvortrag auf Ebene der Einzelgesellschaft zu einem endgültigen Verlustuntergang, während bei Vortrag auf Konzernebene eine Nutzung durch die übrigen Konzerngesellschaften möglich bleibt. Die hieraus abzuleitende Erwartung, dass ein Verlustvortrag auf Ebene der Konzernmutter tendenziell zugunsten der Unternehmen wirkt, wird durch die Ergebnisse in Tabelle 42 bestätigt. So fällt der Rückgang des Bruttogesamtsteueraufkommens mit -5,07 Prozent geringfügig höher aus, als dieses bei einem Vortrag auf Ebene der Einzelgesellschaft der Fall ist (-4,83 Prozent). Insgesamt fallen die sich ergebenden Unterschiede allerdings nicht stark ins Gewicht.

\subsubsection{Verstärkte Zusammenarbeit mehrerer Mitgliedsstaaten}

Die Zustimmung aller Mitgliedsstaaten für die EU-weite Einführung einer GKKB ist zum jetzigen Zeitpunkt kaum vorstellbar, da einige Mitgliedsstaaten sich entschieden gegen eine derartige Reform aussprechen. ${ }^{634}$ Aus diesem Grund wird das alternative Szenario der Einführung im Wege einer verstärkten Zusammenarbeit von mindestens acht Mitgliedsstaaten immer mehr ins Zentrum der Überlegungen gestellt. ${ }^{635}$ Dabei werden hier die folgenden neun Mitgliedsstaaten als mögliche Kandidaten für eine verstärkte Zusammenarbeit angesehen: Österreich, Belgien, Deutschland, Dänemark, Spanien, Frankreich, Italien, Luxemburg und Schweden. Tabelle 43 fasst die Auswirkungen der Einführung einer verpflichtenden oder optionalen Einführung einer GKKB zusammen, wenn diese auf die genannten neun Mitgliedsstaaten beschränkt bliebe.

Die Ergebnisse dieser Berechnungen verdeutlichen, dass der prozentuale Rückgang des Steueraufkommens dieser Mitgliedsstaaten mit 4,12 Prozent zwar leicht unterhalb des Rückgangs des Gesamtsteueraufkommens bei EU-weiter Einführung liegt, im Vergleich zur Änderung, die eine EU-weite Einführung für das Steueraufkommen dieser neun Mitgliedstaaten bewirken würde (-3,53 Prozent), fällt diese allerdings leicht höher aus. Bei optionaler Einführung beträgt die Aufkommensänderung lediglich -4,28 Prozent, während sich der entsprechende Rückgang bei einer EU-weiten Einführung in diesen Mitgliedsstaaten auf -6,24 Prozent belaufen würde. Diese Differenz sollte insbesondere auf geringere Unter-

633 Diesem ist in den Berechnungen der Fall gleichzustellen, dass für ein Konzernunternehmen in späteren Jahren keine Daten mehr vorliegen.

$634 \mathrm{Vgl}$. McLure (2008), S. 47. Hier werden explizit Irland, Großbritannien, Lettland, Litauen, Malta, die Slowakei und Zypern genannt. Schön sieht die Bereitschaft zur Einführung einer GKKB lediglich in einer „Kerngruppe europäischer Mitgliedsstaaten“ um Deutschland, Frankreich, Österreich und die Niederlande gegeben. Vgl. Schön (2007), S. 443. Plasschaert hält die Teilnahme insbesondere der großen Mitgliedsstaaten für bedeutsam. Vgl. Plasschaert (2002), S. 16.

635 Vgl. so den Kommentar von Lang in Rädler (2008), S. 65; McLure (2008), S. 46; Schön (2007), S. 443. 
schiede in den nominellen Steuerbelastungen innerhalb dieser Ländergruppe zurückzuführen sein.

Tabelle 43: Wirkung der Einführung einer GKKB im Wege der verstärkten Zusammenarbeit

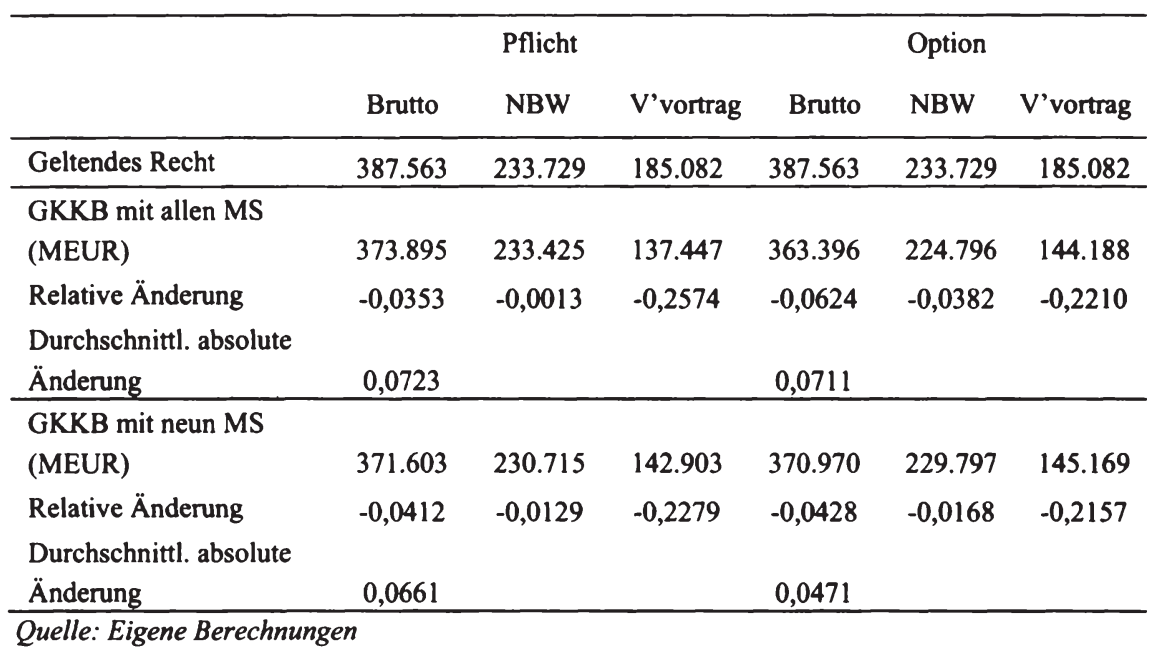

Sowohl bei verpflichtender als auch bei optionaler Einführung geht die durchschnittliche Höhe der Aufkommensänderung zurück. Der Grund hierfür kann in geringeren Unterschieden hinsichtlich der ökonomischen Rahmenbedingungen innerhalb dieser Gruppe von Mitgliedsstaaten gesehen werden. Das geringere Ausmaß von zwischenstaatlichen Aufkommensverschiebungen und das hiermit verbundene geringere Risiko von Aufkommensverlusten könnte die Entscheidung der Mitgliedsstaaten zur Teilnahme an einer GKKB erleichtern.

\subsection{Einführung einer Verlustrichtlinie}

\subsubsection{Verpflichtende Einführung}

Die Ergebnisse der vorherigen Abschnitte haben einen Rückgang des EU-Gesamtsteueraufkommens von 4,83 Prozent bei verpflichtender Einführung einer GKKB und 4,99 Prozent bei einer optionalen Einführung aufgezeigt. Die durchschnittliche Höhe der Bemessungsgrundlagenänderung, die sich für die einzelnen Mitgliedsstaaten einstellt, beträgt 9,14 Prozent (verpflichtende Einführung) und 6,94 Prozent (optionale Einführung). Diesen Ergebnissen werden in Kapitel 7.4 die Aufkommensänderungen gegenübergestellt, die sich bei der verpflichtenden (Kapitel 7.4.1) und optionalen Einführung (Kapitel 7.4.2) einer Verlustrichtlinie 
ergeben. Die Auswirkungen einer verpflichtenden Verlustrichtlinie auf das EUGesamtsteueraufkommen sind hierzu in Tabelle 44 zusammengefasst.

Tabelle 44: Wirkung der verpflichtenden Einführung einer Verlustrichtlinie auf das EUGesamtsteueraufkommen

\begin{tabular}{|c|c|c|c|}
\hline & \multicolumn{2}{|c|}{ EU-Steueraufkommen } & \multirow[t]{2}{*}{ Verlustvortrag } \\
\hline & Brutto & Nettobarwert & \\
\hline Geltendes Recht (MEUR) & 654.309 & 386.921 & 412.552 \\
\hline Nachversteuerungsmethode (MEUR) & 628.306 & 381.945 & 337.497 \\
\hline Relative Änderung & $-0,0397$ & $-0,0129$ & $-0,1819$ \\
\hline Zurechnungsmethode (MEUR) & 653.249 & 402.224 & 317.705 \\
\hline Relative Änderung & $-0,0016$ & 0,0395 & $-0,2299$ \\
\hline Ergebnisaufteilungsmethode (MEUR) & 624.592 & 381.624 & 325.239 \\
\hline Relative Änderung & $-0,0454$ & $-0,0137$ & $-0,2116$ \\
\hline GKKB mit Verlustvortrag bei K'umen & 622.694 & 381.148 & 319.587 \\
\hline Relative Änderung & $-0,0483$ & $-0,0149$ & $-0,2253$ \\
\hline
\end{tabular}

Quelle: Eigene Berechnungen

Die Ergebnisse in Tabelle 44 zeigen, dass die verpflichtende Einführung einer Verlustrichtlinie aufgrund der Ausdehnung steuerlicher Verlustverrechnungsmöglichkeiten zu einem Rückgang des EU-weiten Bruttosteueraufkommens führen würde. Die Höhe dieses Aufkommensrückgangs liegt zwar unabhängig vom zugrundeliegenden Konzept zur Verlustverrechnung über die Grenze unter dem entsprechenden Wert, der sich für die verpflichtende Einführung einer GKKB ergibt, ist aber dennoch wesentlich von diesem abhängig. Eine erste Begründung für die im Vergleich zur GKKB durchweg geringere Aufkommensminderung liegt in der abweichenden Definition der steuerlichen Gruppe. So schließt das Erfordernis einer europäischen Gruppenmuttergesellschaft die Verlustverrechnung zwischen europäischen Schwestergesellschaften und Teilkonzernen einer gemeinsamen nicht-europäischen Muttergesellschaft bei Einführung der Verlustrichtlinie aus. ${ }^{636}$

Die Nachversteuerungsmethode und die Ergebnisaufteilungsmethode begründen ausschließlich oder im Wesentlichen eine Ausdehnung der Möglichkeiten zur konzerninternen Verlustverrechnung, deren Ausmaß allerdings hinter dem zurückbleibt, das von der Einführung einer GKKB ausgehen würde. So können bei Anwendung der Nachversteuerungsmethode ausschließlich Verluste der Auslandstöchter mit Gewinnen des inländischen Konzernteils verrechnet werden. Eine Verlustverrechnung in umgekehrter Richtung (Verrechnung von Verlusten des 
inländischen Konzernteils mit Auslandsgewinnen) ist hingegen konzeptionell ausgeschlossen. Dementsprechend sinken die kumulierten Verlustvorträge gegenüber dem geltenden Steuerrecht nur um 18,19 Prozent, das EU-Gesamtaufkommen sinkt um lediglich 3,97 Prozent.

Auch die Ergebnisaufteilungsmethode gewährleistet im Vergleich zur GKKB eine Verlustverrechnung nur in begrenztem Ausmaß. Zwar unterscheidet sich dieses Konzept von der GKKB nicht darin, dass eine Verlustverrechnung zwischen Konzerngesellschaften vertikal aufwärts, vertikal abwärts und in horizontaler Richtung möglich ist und dass in einer Periode nicht ausgleichsfähige Verluste auf Ebene der einzelnen Konzerngesellschaft vorgetragen werden. Die Art der Ergebnisaufteilung bei dieser Methode sollte allerdings dazu führen, dass im Vergleich zur GKKB Verlustvorträge einzelner Gesellschaften ungenutzt bleiben oder erst zu einem späteren Zeitpunkt genutzt werden können. Wie bereits in Kapitel 6.2.2 erläutert setzt bei dieser Methode die Zuweisung eines positiven Einkommens, welche Voraussetzung für die Nutzung von Verlustvorträgen ist, ein eigenes positives Ergebnis voraus, während bei der GKKB hierfür ein positives Konzernergebnis ausreichend ist. Die kumulierten Verlustvorträge liegen dementsprechend bei der Ergebnisaufteilungsmethode mit 325.239 MEUR deutlich über dem entsprechenden Wert, der sich für die GKKB ergibt. Auch der Aufkommensrückgang fällt mit 4,54 Prozent entsprechend geringer aus. Dennoch verdeutlichen die Ergebnisse, dass die Einschränkung der Verlustverrechnungsmöglichkeiten bei der Ergebnisaufteilungsmethode weniger stark ins Gewicht fällt, als dieses bei der Nachversteuerungsmethode der Fall ist, weshalb größere Aufkommensrückgänge bei dieser Methode zu erwarten sind.

Die Einführung einer Verlustrichtlinie, welche die grenzüberschreitende Verlustverrechnung entsprechend der Zurechnungsmethode gewährleistet, wäre gemessen am Bruttosteueraufkommen aller Mitgliedsstaaten - weitgehend aufkommensneutral möglich (Aufkommensrückgang von -0,16 Prozent). Hierfür ist insbesondere ausschlaggebend, dass die Zurechnungsmethode neben einer Ausdehnung der Verlustverrechnungsmöglichkeiten ${ }^{637}$ auch dazu führen würde, dass Auslandsgewinne im Ergebnis dem höheren Steuerniveau im Inland oder Ausland unterliegen. Mit Blick auf die Aufkommenswirkung einer Verlustrichtlinie wäre diese Methode aus Sicht der Mitgliedsstaaten allerdings mit einem weiteren wesentlichen Vorteil verbunden. So führt die Besteuerung der Auslandsgewinne dazu, dass diese Methode ebenso wie die GKKB grundsätzlich auch zu einer Verrechnung von Verlusten der Muttergesellschaft mit Gewinnen ausländischer Tochterunternehmen führt, allerdings ohne dass es hierdurch im Fall der Zurechnungsmethode zu einer Minderung der Steuerbelastung des Konzerns kommt. Da

637 Diese Ausdehnung der Verlustverrechnungsmöglichkeiten fallt gemessen am verbleibenden Verlustvortrag im Vergleich zur GKKB höher aus. Vorteilhaft bei der Zurechnungsmethode wirkt sich dabei der Verlustvortrag auf Konzernebene anstelle auf Ebene der Konzerngesellschaft, wie hier bei der GKKB unterstellt, aus. 
diese Methode im Unterschied zur GKKB nicht in die Besteuerung der Auslandsgesellschaften eingreift, führt diese Verlustverrechnung lediglich zu einem Wegfall der Verlustvorträge im Inland. Dieser Effekt spiegelt sich insbesondere in der Änderung des Nettobarwerts des Steueraufkommens wider, der mit einem Anstieg von 3,95 Prozent bei der Zurechnungsmethode sogar deutlich positiv ausfallen würde.

Bei der Auswertung der Bruttoaufkommensänderungen (Tabelle 45), die sich für die einzelnen Mitgliedsstaaten ergeben, zeigt sich, dass bei der Nachversteuerungsmethode 22 der 23 in den Berechnungen berücksichtigten Mitgliedsstaaten Aufkommen verlieren. Dieses Ergebnis ist wenig überraschend, da die Nachversteuerungsmethode auf eine Ausweitung der Verlustverrechnungsmöglichkeiten beschränkt ist und somit grundsätzlich aufkommensmindernde Wirkung haben sollte. Eine aufkommenserhöhende Wirkung kann in den Berechnungen allerdings dadurch entstehen, dass inländische und ausländische Verluste nicht separat behandelt werden und die Nachversteuerung nicht an eine tatsächliche steuerliche Entlastung durch die Auslandsverluste im Inland geknüpft wird. Bewirkt die Berücksichtigung der Auslandsverluste nur die Erhöhung eines inländischen Verlustvortrags kann unter Umständen eine steuerwirksame Nachversteuerung erfolgen, obwohl die Auslandsverluste im Inland zwar Berücksichtigung gefunden haben, sich allerdings auf die Höhe der Steuerzahlungen nicht ausgewirkt haben. Mögliche Ursachen sind eine im Vergleich zum ausländischen Steuerrecht kürzere Verlustvortragsdauer oder stärkere betragsmäßige Beschränkung des Verlustvortrags im Inland. In dieser Weise erklärt sich der Aufkommenszuwachs, der sich bei Umsetzung der Nachversteuerungsmethode in Österreich, wo der Verlustvortrag in Form einer Mindestbesteuerungsregel betragsmäßig beschränkt ist, ergibt.

Demgegenüber ist die Anzahl der Mitgliedsstaaten, die an Aufkommen gewinnen, bei Anwendung der Zurechnungsmethode erwartungsgemäß höher. Hier können mit Österreich, Deutschland, Irland, Italien, Luxemburg und den Niederlanden insgesamt sechs Mitgliedsstaaten Zuwächse verzeichnen. Dabei ist zwar zu beobachten, dass unter diesen Mitgliedsstaaten mit Deutschland, Luxemburg, Italien, Österreich und den Niederlanden Mitgliedsstaaten sind, die mit Blick auf den Gesamtzeitraum als Hochsteuerländer einzustufen sind oder im EU-Vergleich zumindest im oberen Mittelfeld anzusiedeln sind. Gleichzeitig gewinnt mit Irland allerdings auch der Mitgliedsstaat mit der im Durchschnitt niedrigsten Steuerbelastung. Dadurch wird deutlich, dass das inländische Steuerniveau zwar einen wesentlichen Parameter für die Höhe des Aufkommenseffekts darstellt, dass dieser allerdings auch von der Ergebnisverteilung im Konzern maßgeblich bestimmt wird. So führt der Wegfall steuerlicher Verlustvorträge bei Verlusten der Muttergesellschaft und Gewinnen der Tochter unabhängig vom Steuerniveau im Inland zu einem Aufkommensanstieg. Ein Blick auf die Verlierer dieses Reformvorschlags lässt feststellen, dass mit Belgien, Zypern und Tschechien lediglich drei 
Mitgliedsstaaten einen Aufkommensrückgang von mehr als fünf Prozent zu verzeichnen haben.

Tabelle 45: Wirkung der verpflichtenden Einführung einer Verlustrichtlinie auf das Bruttosteueraufkommen der einzelnen Mitgliedsstaaten

\begin{tabular}{|c|c|c|c|c|c|c|}
\hline & \multicolumn{2}{|c|}{$\begin{array}{l}\text { Nachversteuerungs- } \\
\text { methode }\end{array}$} & \multicolumn{2}{|c|}{ Zurechnungsmethode } & \multicolumn{2}{|c|}{$\begin{array}{l}\text { Ergebnisauftei- } \\
\text { lungsmethode }\end{array}$} \\
\hline & Änderung & Rang & Änderung & Rang & Änderung & Rang \\
\hline Belgien & $-0,0654$ & 22 & $-0,0551$ & 21 & $-0,0788$ & 19 \\
\hline Dänemark & $-0,0370$ & 15 & $-0,0088$ & 10 & $-0,0422$ & 11 \\
\hline Deutschland & $-0,0293$ & 13 & 0,0705 & 2 & $-0,0235$ & 4 \\
\hline Estland & $-0,0243$ & 10 & $-0,0219$ & 16 & $-0,1340$ & 23 \\
\hline Finnland & $-0,0292$ & 12 & $-0,0216$ & 15 & $-0,0509$ & 13 \\
\hline Frankreich & $-0,0620$ & 19 & $-0,0019$ & 9 & $-0,0582$ & 16 \\
\hline Griechenland & $-0,0203$ & 9 & $-0,0200$ & 14 & $-0,0413$ & 8 \\
\hline Großbritannien & $-0,0405$ & 17 & $-0,0095$ & 11 & $-0,0414$ & 9 \\
\hline Irland & $-0,0025$ & 2 & 0,0049 & 5 & $-0,0173$ & 2 \\
\hline Italien & $-0,0100$ & 6 & 0,0005 & 6 & $-0,0227$ & 3 \\
\hline Lettland & $-0,0118$ & 8 & $-0,0118$ & 13 & $-0,0364$ & 7 \\
\hline Litauen & $-0,0089$ & 5 & 0,0000 & 7 & $-0,0675$ & 17 \\
\hline Luxemburg & $-0,0039$ & 3 & 0,0313 & 4 & $-0,0262$ & 5 \\
\hline Niederlande & $-0,0622$ & 20 & 0,2301 & 1 & $-0,0545$ & 14 \\
\hline Österreich & 0,0099 & 1 & 0,0474 & 3 & $-0,0293$ & 6 \\
\hline Polen & $-0,0474$ & 18 & $-0,0469$ & 20 & $-0,0893$ & 21 \\
\hline Portugal & $-0,0327$ & 14 & $-0,0286$ & 18 & $-0,0547$ & 15 \\
\hline Schweden & $-0,0384$ & 16 & $-0,0306$ & 19 & $-0,0417$ & 10 \\
\hline Slowakei & $-0,0073$ & 4 & $-0,0014$ & 8 & 0,0797 & 1 \\
\hline Spanien & $-0,0278$ & 11 & $-0,0253$ & 17 & $-0,0440$ & 12 \\
\hline Tsch'e Republik & $-0,0625$ & 21 & $-0,0623$ & 23 & $-0,1089$ & 22 \\
\hline Ungarn & $-0,0117$ & 7 & $-0,0117$ & 12 & $-0,0872$ & 20 \\
\hline Zypern & $-0,0774$ & 23 & $-0,0577$ & 22 & $-0,0774$ & 18 \\
\hline $\begin{array}{l}\text { Durchschnittliche } \\
\text { absolute Änderung } \\
{ }^{638}\end{array}$ & 0,0341 & & 0,0388 & & 0,0567 & \\
\hline
\end{tabular}

Quelle: Eigene Berechnungen

638 Kursiv gedruckte Länder wurden aufgrund einer nur kleinen Anzahl von Unternehmen in der Stichprobe nicht fur die Ermittlung des Durchschnittswertes perigcksiçhtigt. 
Die Ergebnisaufteilungsmethode beinhaltet zwar wie die GKKB neben einer konzerninternen Verlustverrechnung auch eine zwischenstaatliche Umverteilung von Bemessungsgrundlage. Dennoch zeigt sich, dass mit Ausnahme der Slowakei bei diesem Vorschlag kein Mitgliedsstaat einen Aufkommenszuwachs zu verzeichnen hätte. Bei einem Blick auf die größten Verlierer fällt auf, dass diese mit Estland, Tschechien, Ungarn und Polen ausnahmslos aus den Beitrittsstaaten stammen.

Die durchschnittliche Höhe der Aufkommensänderung fällt bei allen drei Reformvorschlägen deutlich niedriger aus, als dieses bei der GKKB $(9,41$ Prozent; vgl. Tabelle 33) der Fall ist. Hierfür dürften insbesondere zwei Umstände ursächlich sein. Zum einen sind die Auswirkungen der Nachversteuerungsmethode und der Zurechnungsmethode auf die Steuerzahlungen der Muttergesellschaften grenzüberschreitend tätiger Konzern begrenzt und damit auf einen - im Vergleich zur GKKB - deutlich geringeren Anteil der in der Datenbasis enthaltenen Unternehmen. Aus diesem Grund ist es wenig überraschend, dass die durchschnittliche Höhe der Aufkommensänderung hier geringer ausfällt. Zum anderen steht bei der Ergebnisaufteilungsmethode und insbesondere der Nachversteuerungsmethode die Verlustverrechnung als maßgebender Effekt im Vordergrund, während bei der GKKB auch der zwischenstaatlichen Reallokation der Bemessungsgrundlage große Bedeutung zukommt. Diese Reallokation führt in den Berechnungen zu einer Abschwächung der Aufkommensminderung in den Hochsteuerländern und einer Verstärkung der Aufkommensminderung in den Niedrigsteuerländern. Kann davon ausgegangen werden, dass die großen Mitgliedsstaaten tendenziell höhere Steuersätze aufweisen, sollte der letztgenannte Effekt bei einer prozentualen Betrachtung stärker ins Gewicht fallen. Eine stärkere Streuung bei den Aufkommensänderungen ist die Folge.

\subsubsection{Optionale Einführung}

Die Auswirkungen der Einführung einer optionalen Verlustrichtlinie auf das EUweite Steueraufkommen sind in Tabelle 46 zusammengefasst. Dabei zeigt sich, dass die Einräumung eines Optionsrechts für die Unternehmen bei der Nachversteuerungsmethode und der Ergebnisaufteilungsmethode nur geringfügige Wirkung entfaltet. So sinkt der für die Unternehmensentscheidung maßgebende Nettosteuerbarwert bei der optionalen Nachversteuerungsmethode gegenüber einer verpflichtenden Einführung um 112 MEUR (381,833 MEUR ggü. 381.945 MEUR), während der entsprechende Unterschied bei der Ergebnisaufteilungsmethode 564 MEUR (381.060 MEUR ggü. 381.624 MEUR) beträgt. Das Bruttosteueraufkommen steigt bei beiden Konzepten gegenüber einer verpflichtenden Einführung leicht an, was allerdings durch den geringeren Rückgang der am Ende des Betrachtungszeitraums bestehenden Verlustvorträge kompensiert wird. 
Tabelle 46: Wirkung der optionalen Einführung einer Verlustrichtlinie auf das EUGesamtsteueraufkommen

\begin{tabular}{lccc}
\hline & \multicolumn{2}{l}{ EU-Steueraufkommen } & \multirow{2}{*}{ Verlustvortrag } \\
& Brutto & Nettobarwert & \\
\hline Geltendes Recht (MEUR) & 654.309 & 386.921 & 412.563 \\
\hline Nachversteuerungsmethode (MEUR) & 628.383 & 381.833 & 338.485 \\
Relative Änderung & $-0,0396$ & $-0,0132$ & $-0,1795$ \\
\hline Zurechnungsmethode (MEUR) & 642.700 & 384.697 & 379.147 \\
Relative Änderung & $-0,0177$ & $-0,0057$ & $-0,0810$ \\
\hline Ergebnisaufteilungsmethode (MEUR) & 625.263 & 381.060 & 327.883 \\
Relative Änderung & $-0,0444$ & $-0,0151$ & $-0,2052$ \\
\hline GKKB mit Verlustvortrag bei KU (MEUR) & 621.629 & 378.712 & 327.978 \\
Relative Änderung & $-0,0499$ & $-0,0212$ & $-0,2050$ \\
\hline
\end{tabular}

Quelle: Eigene Berechnungen

Demgegenüber ist der Effekt des Optionsrechts im Rahmen der Zurechnungsmethode bedeutsam. Diese Methode ist aus Unternehmenssicht nur bei Verlusten der Tochtergesellschaft vorteilhaft, während bei Gewinnen der Tochterunternehmen die Anwendung dieser Methode in vielen Fällen zu Nachteilen führt. Dieses gilt umso mehr, wenn die Muttergesellschaft in einem Hochsteuerland domiziliert. Der Rückgang des Bruttoaufkommens fällt aus diesem Grund mit 1,77 Prozent deutlich höher aus, als dieses bei verpflichtender Einführung der Fall ist $(-0,16$ Prozent). Dieser Effekt wird zusätzlich durch einen deutlich höheren Verlustvortrag zum Ende des Betrachtungszeitraums verstärkt.

Die Auswirkungen einer optionalen Verlustrichtlinie auf das Steueraufkommen der einzelnen Mitgliedsstaaten sind in Tabelle 47 zusammengefasst. Auch hier sind die Unterschiede, die sich bei der Nachversteuerungsmethode und der Ergebnisaufteilungsmethode gegenüber der verpflichtenden Einführung ergeben, nur gering. Allerdings ist zu beobachten, dass bei der Nachversteuerungsmethode nun sämtliche Mitgliedsstaaten an Aufkommen verlieren. Dieses geht auf den Umstand zurück, dass bei der Anwendung dieser Methode nur in die Besteuerung einer Gesellschaft jeder Gruppe und damit das Steueraufkommen nur eines Mitgliedsstaates eingegriffen wird. Positive Aufkommensänderungen sind aus diesem Grund - zumindest unter der Annahme vollständiger Voraussicht - weitgehend ausgeschlossen. ${ }^{639}$

639 In Ausnahmefallen kann es zu Aufkommenssteigerungen bei gleichzeitigem Rackgang des fur die Entscheidung maßgebenden Steuerbarwerts kommen. 
Tabelle 47: Wirkung der optionalen Einführung einer Verlustrichtlinie auf das Bruttosteueraufkommen der einzelnen Mitgliedsstaaten

\begin{tabular}{|c|c|c|c|c|c|c|}
\hline & \multicolumn{2}{|c|}{$\begin{array}{l}\text { Nachversteuerungs- } \\
\text { methode }\end{array}$} & \multicolumn{2}{|c|}{ Zurechnungsmethode } & \multicolumn{2}{|c|}{$\begin{array}{l}\text { Ergebnisaufteilungs- } \\
\text { methode }\end{array}$} \\
\hline & Änderung & Rang & Änderung & Rang & Änderung & Rang \\
\hline Belgien & $-0,0652$ & 22 & $-0,0515$ & 21 & $-0,0793$ & 20 \\
\hline Dänemark & $-0,0369$ & 15 & $-0,0179$ & 12 & $-0,0409$ & 13 \\
\hline Deutschland & $-0,0301$ & 13 & $-0,0085$ & 9 & $-0,0223$ & 4 \\
\hline Estland & $-0,0243$ & 10 & $-0,0202$ & 16 & $-0,1071$ & 23 \\
\hline Finnland & $-0,0292$ & 12 & $-0,0184$ & 13 & $-0,0461$ & 15 \\
\hline Frankreich & $-0,0619$ & 19 & $-0,0184$ & 13 & $-0,0594$ & 19 \\
\hline Griechenland & $-0,0201$ & 9 & $-0,0185$ & 15 & $-0,0365$ & 9 \\
\hline Großbritannien & $-0,0403$ & 17 & $-0,0139$ & 11 & $-0,0403$ & 12 \\
\hline Irland & $-0,0020$ & 1 & 0,0000 & 1 & $-0,0077$ & 3 \\
\hline Italien & $-0,0083$ & 4 & $-0,0054$ & 6 & $-0,0224$ & 5 \\
\hline Lettland & $-0,0119$ & 8 & $-0,0106$ & 10 & $-0,0285$ & 8 \\
\hline Litauen & $-0,0089$ & 5 & $-0,0064$ & 7 & $-0,0558$ & 18 \\
\hline Luxemburg & $-0,0039$ & 2 & $-0,0003$ & 3 & $-0,0260$ & 6 \\
\hline Niederlande & $-0,0622$ & 20 & $-0,0036$ & 5 & $-0,0539$ & 16 \\
\hline Österreich & $-0,0097$ & 6 & $-0,0030$ & 4 & $-0,0283$ & 7 \\
\hline Polen & $-0,0471$ & 18 & $-0,0203$ & 17 & $-0,0874$ & 21 \\
\hline Portugal & $-0,0327$ & 14 & $-0,0283$ & 20 & $-0,0555$ & 17 \\
\hline Schweden & $-0,0384$ & 16 & $-0,0228$ & 18 & $-0,0379$ & 10 \\
\hline Slowakei & $-0,0073$ & 3 & 0,0000 & 1 & 0,0043 & 1 \\
\hline Spanien & $-0,0278$ & 11 & $-0,0246$ & 19 & $-0,0435$ & 14 \\
\hline Tsch'e Republik & $-0,0624$ & 21 & $-0,0575$ & 22 & $-0,1062$ & 22 \\
\hline Ungarn & $-0,0117$ & 7 & $-0,0069$ & 8 & $-0,0394$ & 11 \\
\hline Zypern & $-0,0774$ & 23 & $-0,0585$ & 23 & 0,0006 & 2 \\
\hline $\begin{array}{l}\text { Durchschnittliche } \\
\text { absolute Änderung }\end{array}$ & 0,0339 & & 0,0189 & & 0,0508 & \\
\hline
\end{tabular}

Quelle: Eigene Berechnungen

Aus dem gleichen Grund sind auch bei der Zurechnungsmethode bei optionaler Einführung keine Gewinner mehr vorhanden. Insbesondere die Niederlande

640 Kursiv gedruckte Länder wurden aufgrund einer nur kleinen Anzahl von Unternehmen in der Stichprobe nicht für die Ermittlung des Durchschnittswertes berügksichtigt 
und das Hochsteuerland Deutschland werden bei Optionalität im Vergleich zur verpflichtenden Einführung schlechter gestellt.

Die durchschnittliche Höhe der Abweichung gegenüber dem geltenden Steuerrecht fällt bei allen drei Konzepten geringer aus, als dieses bei der verpflichtenden Einführung der Fall ist. Nur bei der Zurechnungsmethode ist dieser Unterschied allerdings substanziell. Dieser deutliche Rückgang ist wenig überraschend. Da sich aufgrund der hohen Steuersätze in einigen Ländern die Zurechnungsmethode für Konzerne dieser Länder nur selten als vorteilhaft erweist, fällt die Aufkommensänderung bei optionaler Einführung in diesen Ländern entsprechend geringer aus.

\subsection{Analyse der Einflussfaktoren der Aufkommenseffekte}

\subsubsection{Regressionsmodell}

Die in den vorherigen Abschnitten dargestellten Ergebnisse der Simulationsrechnungen haben für die unterschiedlichen Reformvorschläge einen Aufkommensrückgang von bis zu fünf Prozent ergeben. Insbesondere die verpflichtende Einführung einer GKKB würde ferner deutliche Aufkommensverschiebungen zwischen den einzelnen Mitgliedsstaaten mit sich bringen. Vor diesem Hintergrund ist es die Zielsetzung dieses Abschnitts, mögliche Einflussfaktoren für die Aufkommensänderungen der einzelnen Mitgliedsstaaten mittels Regressionsanalyse auf ihre empirische Signifikanz hin zu überprüfen. Abhängige Variable ist hierbei die von den verschiedenen Reformvorschlägen ausgehende Änderung des Bruttosteueraufkommens der einzelnen Mitgliedsstaaten im Vergleich zum geltenden Recht.

Als erklärende Variablen für die Höhe dieser Aufkommensänderungen werden Konzernstrukturvariablen sowie steuerliche und gesamtwirtschaftliche Einflussgrößen einbezogen. Die Berücksichtigung von Konzernstrukturvariablen erlaubt zunächst eine Identifikation von Strukturen mit überdurchschnittlich hoher Verlustwahrscheinlichkeit. Treten derartige Strukturen gehäuft in einem Mitgliedsstaat auf, ist davon auszugehen, dass die Ausdehnung der Verlustverrechnungsmöglichkeiten bei Umsetzung der verschiedenen Reformkonzepte überdurchschnittlich starke Wirkung entfaltet, also überdurchschnittlich hohe Aufkommensrückgänge auslöst. Von einer erhöhten Verlustwahrscheinlichkeit ist insbesondere bei einer Neugründung ausländischer Tochterunternehmen auszugehen. Hier sollte es aufgrund des erforderlichen Aufbaus neuer Unternehmensstrukturen sowie der Betätigung in einem unbekannten Markt vielfach zu Anlaufverlusten kommen. Dieser Effekt wird in der Regression durch die Variablen NIB und NOB erfasst. Da die Einführung einer GKKB neben der Ausdehnung der konzerninternen Verlustverrechnung auch den Übergang auf eine indirekte Gewinnabgrenzung vorsieht, ist für die Aufkommensänderung der einzelnen Mit- 
gliedsstaaten auch von Bedeutung, ob die Anlaufverluste bei einer im Inland neugegründeten Tochtergesellschaft selbst oder aufgrund einer entsprechenden Ausgestaltung konzerninterner Lieferungs- und Leistungsbeziehungen bei der investierenden Konzerngesellschaft im Ausland anfallen. Während im ersten Fall das Sitzland der neugegründeten Tochtergesellschaft zu Lasten des Sitzlandes der investierenden Konzerngesellschaft vom Übergang auf die indirekte Gewinnabgrenzung profitieren sollte, ist für den zweiten Fall ein umgekehrter Effekt zu erwarten. Um diesem Umstand Rechnung zu tragen, geht die Anzahl derartiger Investitionen sowohl als Anteil der inländischen Muttergesellschaft mit neugegründeten Auslandstöchtern (NOB) als auch als Anteil der im Inland neugegründeten Tochtergesellschaften mit Auslandsmüttern (NIB) - beides gemessen im Verhältnis zur Gesamtzahl inländischer Gesellschaften, die Bestandteil eines multinationalen Konzerns sind - in die Regression ein. Soweit davon ausgegangen werden kann, dass die Anlaufverluste teils auf Ebene der neugegründeten Gesellschaft und teils auf Ebene der investierenden Gesellschaft anfallen, lassen aufgrund des Verlustverrechnungseffekts hohe Werte für beide Variablen eine stärkere Aufkommensminderung bei Einführung der GKKB erwarten.

Daneben sollte bei der Nachversteuerungsmethode und der Zurechnungsmethode der Anteil an Outboundinvestitionen Einfluss auf die Höhe der Aufkommensänderung nehmen. Beide Methoden sind so ausgestaltet, dass sich positive oder negative Aufkommensänderungen ausschließlich im Sitzland der Muttergesellschaft ergeben. OB_M und OB_T messen diesen Anteil an Outboundinvestitionen. Dabei steht eine hohe Anzahl ausländischer Tochterunternehmen (OB_T) bei der Nachversteuerungsmethode dafür, dass mehr Verluste ausländischer Tochterunternehmen zu berücksichtigen sind, was ceteris paribus zu höheren Aufkommensverlusten führen sollte. Bei der Zurechnungsmethode führt eine hohe Anzahl ausländischer Tochterunternehmen ferner dazu, dass auch der Umfang im Inland der Besteuerung zu unterwerfender Auslandsgewinne zunimmt. Wird davon ausgegangen, dass die überwiegende Anzahl der Auslandsgesellschaften rentabel ist, ist ein positiver Zusammenhang von OB_T und der Aufkommensänderung bei der Zurechnungsmethode zu erwarten, während der $\mathrm{Zu}$ sammenhang von OB_T und der Aufkommensänderung bei der Nachversteuerungsmethode negativ sein sollte. Für die Höhe der Aufkommensminderung, die von der Berücksichtigung ausländischer Verluste auf das inländische Steueraufkommen bei Umsetzung der Nachversteuerungs- und der Zurechnungsmethode ausgehen, ist neben der Anzahl ausländischer Tochterunternehmen auch die Anzahl inländischer Muttergesellschaften mit Auslandstöchtern von Bedeutung. So ist Voraussetzung für eine Aufkommensminderung im Inland, dass entsprechend hohe positive inländische Einkünfte zur Verrechnung vorliegen. Zur Erfassung des Anteils inländischer Mutterunternehmen mit Auslandstochter wird die Variable OB_M für die beiden genannten Methoden in die Regressionsfunktion einbezogen. $\mathrm{OB}_{-} \mathrm{M}$ und $\mathrm{OB}$ - $\mathrm{T}$ sind ebenfalls im Verhältnis zur Gesamtheit aller inländi- 
schen Konzerngesellschaften, die Bestandteil eines multinationalen Konzerns sind, definiert. Um bei diesen Variablen zusätzlich die Unternehmensgröße berücksichtigen zu können, wird bei der Ermittlung des Anteils hier nicht auf die Anzahl, sondern die Umsatzerlöse der jeweiligen Unternehmen Bezug genommen.

Für die Aufkommenswirkung der Ergebnisaufteilungsmethode sind insbesondere Konstellationen von Bedeutung, bei denen im Inland Gewinne bei gleichzeitigen Verlusten in ausländischen Konzerneinheiten anfallen oder umgekehrt. Nur bei derartigen Ergebnisverteilungen im Konzern entfaltet die Ergebnisaufteilung Wirkung. Sie können gehäuft bei Investitionen von EU15-Konzernen in den Beitrittsstaaten vermutet werden. Diese Investitionen in den Beitrittsstaaten sollten zum einen aufgrund der fehlenden Marktkenntnis mit einem überdurchschnittlich hohen Risiko verbunden sein, zum anderen ist davon auszugehen, dass dieses Risiko - zumindest in den Anfangsjahren - regelmäßig nicht auf die Auslandsgesellschaften übertragen wird, sondern diese vielmehr - im Fall von Produktionsgesellschaften - häufig die Funktion eines Lohnfertigers einnehmen. ${ }^{641}$ Zur Erfassung der Investitionen von EU-15 Konzernen in den Beitrittsstaaten dient die Variable E10. Bei der Definition dieser Variablen werden für die EU-15 Mitgliedsstaaten die Umsatzerlöse der ausländischen Tochtergesellschaften in den Beitrittsstaaten ins Verhältnis zu den Umsatzerlösen aller inländischen Konzernunternehmen gesetzt, während für die Beitrittsstaaten das Verhältnis von Umsatzerlösen der inländischen Konzerngesellschaften mit Muttergesellschaft in einem EU15Land und Umsatzerlösen aller inländischen Gesellschaften gebildet wird. Zur Kennzeichnung der entgegengesetzten Investitionsrichtung werden die Werte der EU-15-Länder mit positivem Vorzeichen versehen, während die Variablenwerte der Beitrittsstaaten negativ sind. Treffen die oben formulierten Erwartungen bezüglich der Ausgestaltung dieser Investitionen zu, sollten positive Werte dieser Variablen bei Umsetzung der Ergebnisaufteilungsmethode mit Aufkommenszuwächsen verbunden sein.

Als steuerlicher Einflussfaktor sind in besonderem Maße die Bedingungen zur steuerlichen Verlustverrechnung im geltenden Steuerrecht von Bedeutung. Jeder der betrachteten Reformschläge sieht neben der Einführung einer Verlustverrechnung über die Grenze auch eine Vereinheitlichung der Vorschriften zur konzerninternen Verlustverrechnung im nationalen Bereich vor. Hiervon sollte eine Aufkommensminderung ausgehen, wenn im geltenden Recht eine konzerninterne Verlustverrechnung nicht oder nur unter restriktiveren Anwendungsvoraussetzungen gewährt wird. Die Verfügbarkeit und Restriktivität der Anwendungsvoraussetzungen des im geltenden Steuerrecht eines Landes verankerten nationalen Gruppenbesteuerungssystems wird durch GTS erfasst. Dazu ist die Variable defi-

641 Als Grund für diese Annahme sei beispielsweise auf die fehlende Kenntnis dieses Wirtschaftsraums sowie die zu Beginn des Betrachtungszeitraums noch nicht vollständig gefestigten wirtschaftlichen und politischen Verhältnisse in diesen Lăndern verwiesen 
niert als die Abnahme der Anzahl nationaler Steuergruppen eines Landes bei Einführung einer GKKB oder Verlustrichtlinie, welche als die Relation der Anzahl nationaler Steuergruppen nach geltendem Recht und der entsprechenden Anzahl nach der Reform gemessen wird. Unternehmen, die vor der Reform einzeln besteuert wurden, werden dabei als eigene steuerliche Gruppe behandelt. Gemäß dieser Definition nimmt GTS den Wert 1 an, wenn (1) im geltenden Steuerrecht ein Gruppenbesteuerungssystem verfügbar ist, (2) die Mindestbeteiligungsquote ebenfalls 50 Prozent beträgt und (3) keine weiteren Anwendungsvoraussetzungen bestehen. Ein niedriger Wert deutet hingegen auf das Fehlen eines Gruppenbesteuerungssystems oder das Bestehen restriktiverer Anwendungsvoraussetzungen hin. Aus diesem Grund ist von einem positiven Zusammenhang zwischen dem Wert dieser Variablen und der Aufkommensänderung bei Einführung einer GKKB oder Verlustrichtlinie auszugehen.

In gleicher Weise sollte die Aufkommensminderung aufgrund der Ausweitung der Verlustverrechnungsmöglichkeiten umso höher ausfallen, je stärker die intertemporale Verlustverrechnung im geltenden Recht beschränkt ist. In diesem Zusammenhang ist bei der Nachversteuerungsmethode und der Zurechnungsmethode allerdings zu berücksichtigen, dass bei diesen Konzepten Beschränkungen der intertemporalen Verlustverrechnung im Sitzland der Muttergesellschaft auch dazu führen, dass im Inland zu berücksichtigende Auslandsverluste in geringerem Ausmaß steuerwirksam werden. Die Einflussrichtung derartiger Beschränkungen ist bei diesen Methoden also unklar. Um dem Einfluss der Vorschriften zur intertemporalen Verlustverrechnung im geltenden Recht Rechnung zu tragen, kommt die Variable LC zur Anwendung. Da sich bei einem Betrachtungszeitraum von zehn Jahren zeitliche Beschränkungen des Verlustvortrags in den Simulationsrechnungen kaum auswirken können, wird bei der Definition dieser Variablen nur auf die Möglichkeit eines Verlustrücktrags sowie betragsmäßige Beschränkungen des Verlustvortrags abgestellt. Im Einzelnen entspricht der Wert dieser Variablen der Dauer des nach Maßgabe des jeweiligen Steuerrechts zulässigen Verlustrücktrags. Bei betragsmäßigen Beschränkungen des Verlustvortrags wird der Variablenwert pauschal um eins herabgesetzt. Dementsprechend stehen hohe Werte dieser Variablen für großzügige Vorschriften zur intertemporalen Verlustverrechnung, während ein Wert von null oder negative Werte auf restriktive Regelungen in diesem Bereich hindeuten.

Ferner ist bei der Nachversteuerungsmethode und der Zurechnungsmethode zu berücksichtigen, dass die konzerninterne Verlustverrechnung über die Grenze nachrangig gegenüber der intertemporalen Verlustverrechnung im Ausland zur Anwendung kommt. Der Aufkommensrückgang aufgrund des Bestehens derartiger Verluste sollte also umso höher ausfallen, je stärker die Verlustverrechnung im Ausland beschränkt ist. Zur Berücksichtigung dieses Zusammenhangs wird für diese Methoden die Variable LC_T in die Regressionsfunktion einbezogen, welche definiert ist als Mittelwert der Werte der Variablen LC über alle ausländi- 
schen Tochterunternehmen der inländischen Mutterunternehmen eines Landes. Dementsprechend ist von einem positiven Zusammenhang zwischen dieser Variablen und der für die Zurechnungsmethode und Nachversteuerungsmethode gemessenen Aufkommensänderung eines Landes auszugehen.

Neben den steuerlichen Verlustverrechnungsvorschriften sollten auch die Höhe und die Entwicklung der Steuersätze im Zeitablauf Einfluss auf die Höhe der Aufkommensänderung haben. Ein Zusammenhang zwischen dem Steuerniveau und der Aufkommensänderung ist dabei für die GKKB und die Zurechnungsmethode zu erwarten. Wird davon ausgegangen, dass vor der Reform Bemessungsgrundlage gezielt in Niedrigsteuerländer verlagert wurde und derartige Gestaltungen bei der GKKB unwirksam sind, sollten Hochsteuerländer durch die Einführung einer verpflichtenden GKKB an Bemessungsgrundlage gewinnen. Bei optionaler Einführung ist hingegen von einem gegenläufigen Einfluss auszugehen. So wird die Option unter der Annahme vollständiger Voraussicht der Besteuerungsfolgen nur ausgeübt werden, wenn sie im Ergebnis zu einer geringeren Steuerbelastung des Konzerns führt. Sollte diese Absenkung der Steuerbelastung nicht auf eine schnellere Verlustberücksichtigung zurückgeführt werden können, ist Voraussetzung für die Ausübung der Option, dass aufgrund des Übergangs zur indirekten Gewinnabgrenzung mehr Bemessungsgrundlage in Niedrigsteuerländern besteuert wird. Dabei ist allerdings in beiden Fällen weniger die absolute Höhe des Steuerniveaus relevant, sondern vielmehr die Differenz zwischen der inländischen Steuerbelastung und der Steuerbelastung, der im Durchschnitt die ausländischen Gesellschaften in gemeinsamen multinationalen Konzernen ausgesetzt sind ${ }^{642}$ Aus diesem Grund wird für die Ermittlung der Variablen TR für jede Gesellschaft, die Bestandteil eines multinationalen Konzerns ist, die Differenz zwischen der eigenen Steuerbelastung und der durchschnittlichen nominellen Steuerbelastung des Konzerns gebildet. Die Werte dieser Variable ergeben sich dann als Mittelwert der Differenzen für alle Gesellschaften eines Landes, die Bestandteil eines multinationalen Konzerns sind.

Die Zurechnungsmethode führt neben der Einführung einer Verlustverrechnung über die Grenze auch dazu, dass die Gewinne ausländischer Tochtergesellschaften bei der inländischen Muttergesellschaft einer zusätzlichen Besteuerung unterworfen werden, soweit das inländische Steuerniveau über dem ausländischen liegt. Insofern ist - zumindest bei verpflichtender Einführung - von einem positiven Aufkommenseffekt auszugehen, je höher der inländische Steuersatz über dem durchschnittlichen Steuerniveau der ausländischen Tochtergesellschaften liegt. Bei optionaler Einführung kann ein höherer inländischer Steuersatz multinationale Konzerne zumindest von der Option für das Gruppenbesteuerungssystem abhalten. Hierbei ist allerdings im Unterschied zur GKKB lediglich die Steuersatzdifferenz, die sich zwischen den in- und den ausländischen Konzernteilen in

642 Hierbei wird auf den Mittelwert der nominellen Steuerbelastung im Betrachtungszeitraum (1994 bis 2003) abgestellt. 
Outbound-Konzernen ergibt, von Relevanz. Aus diesem Grund wird bei der $\mathrm{Zu}$ rechnungsmethode die Variable TR_OB einbezogen, die analog zu TR definiert ist, allerdings die Steuersatzdifferenz nur für die Outbound-Konzerne eines Landes erfasst.

Schließlich ist auch die Veränderung der Steuersätze innerhalb des Betrachtungszeitraums (TR_red). von Bedeutung. Kommt es zu einem Absinken der Steuersätze, wie in den meisten Mitgliedsstaaten zwischen 1994 und 2003 der Fall, dann bewirkt eine von der Ausweitung der Verlustverrechnungsmöglichkeiten im Konzern ausgehende schnellere Verlustverrechnung nicht nur einen Zinsvorteil, sondern gleichzeitig, dass die Verluste zu einem höheren Steuersatz wirksam werden. Im Zeitablauf sinkende Steuersätze sollten dementsprechend den Aufkommensrückgang im Durchschnitt verstärken.

Als gesamtwirtschaftlicher Einflussfaktor findet schließlich die durchschnittliche Profitabilität der in den Mitgliedsstaaten ansässigen Unternehmen Berücksichtigung (PRO). Wird davon ausgegangen, dass zwischenstaatliche Renditeunterschiede, deren Ursache zum Beispiel in der unterschiedlichen Bereitstellung öffentlicher Güter oder unterschiedlichen Qualifikation der Mitarbeiter liegen kann, auch konzernintern bestehen, sollten diese neben gezielten Steuerplanungen den Umverteilungseffekt einer GKKB maßgeblich bestimmen. So sollten Mitgliedsstaaten mit hoher durchschnittlicher Rentabilität beim Übergang von einer direkten zu einer indirekten Aufteilung tendenziell an Bemessungsgrundlage verlieren, während Länder mit geringer Profitabilität umgekehrt profitieren sollten. Gleichzeitig ist zu erwarten, dass es in Mitgliedsstaaten mit niedriger Profitabilität häufiger $\mathrm{zu}$ Verlustsituationen kommt. ${ }^{643}$ Zumindest soweit diese in nationalen Konzernen auftreten, wirkt sich eine Ausweitung der Verlustverrechnungsmöglichkeiten - wie von der GKKB und den Konzepten für eine Verlustrichtlinie vorgesehen - hier besonders aufkommensbelastend aus. Für die Erfassung der gesamtwirtschaftlichen Profitabilität wird hier auf das Verhältnis von Nettobetriebsüberschuss und kumulierten Umsatzerlösen eines Landes abgestellt. PRO ist definiert als der Mittelwert der Werte dieser Größe, die sich für den Zeitraum von 1994 bis 2003 für die jeweiligen Länder ergeben und wird normiert auf den Mittelwert aller Länder.

Tabelle 48 fasst die in die Regression einbezogenen unabhängigen Variablen zusammen.

643 Diesem steht nicht entgegen, dass in der Finanzwirtschaft hohe Kapitalkosten mit hohem Investitionsrisiko einhergehen. Im Gegensatz zu diesen Theorien wird hier nicht auf Renditeerwartungen, sondern auf realisierte Ergebnisse der Vergangenheit_Bezuggengmmen. 
Tabelle 48: Unabhängige Variablen - Deskriptive Statistik

\begin{tabular}{|c|c|c|c|c|}
\hline Name & Beschreibung & Quelle ${ }^{644}$ & MW & Stabw \\
\hline NIB & $\begin{array}{l}\text { Anteil (Anzahl) neu gegründeter inländischer } \\
\text { Tochterunternehmen mit ausländischer Mutterge- } \\
\text { sellschaft }\end{array}$ & $\begin{array}{l}\text { Eigene } \quad \text { Berech- } \\
\text { nungen }\end{array}$ & ,221 &, 133 \\
\hline NOB & $\begin{array}{l}\text { Anteil (Anzahl) inlăndischer Muttergesellschaften } \\
\text { mit neu gegründeten Auslandstöchtern }\end{array}$ & $\begin{array}{l}\text { Eigene } \quad \text { Berech- } \\
\text { nungen }\end{array}$ & ,073 & ,072 \\
\hline OB_T & $\begin{array}{l}\text { Anteil (Umsatz) von Auslandstöchtem inländischer } \\
\text { Muttergesellschaften }\end{array}$ & $\begin{array}{l}\text { Eigene Berechnun- } \\
\text { gen }\end{array}$ &, 432 &, 648 \\
\hline OB_M & $\begin{array}{l}\text { Anteil (Umsatz) von inländischen Muttergesell- } \\
\text { schaften mit Auslandstöchtem }\end{array}$ & $\begin{array}{l}\text { Eigene Berechnun- } \\
\text { gen }\end{array}$ &, 306 &, 176 \\
\hline E10 & $\begin{array}{l}\text { Anteil (Umsatz) der grenzuberschreitenden Investi- } \\
\text { tionen von EU15-Konzemen in den Beitrittsstaaten } \\
\text { unter Berilcksichtigung der Inv.richtung }\end{array}$ & $\begin{array}{l}\text { Eigene Berechnun- } \\
\text { gen }\end{array}$ &,- 077 &, 210 \\
\hline TR & $\begin{array}{l}\text { Durchschnittliche nominelle Steuerbelastung in- } \\
\text { lăndischer Konzernunternehmen (1994 bis 2003) } \\
\text { relativ zum Konzemdurchschnitt }\end{array}$ & $\begin{array}{l}\text { Tabelle 52; eigene } \\
\text { Berechnungen }\end{array}$ &,- 913 & 6,13 \\
\hline TR_OB & $\begin{array}{l}\text { Durchschnittliche nominelle Steuerbelastung in- } \\
\text { låndischer Konzernunternehmen mit Auslandstoch- } \\
\text { tem (1994 bis 2003) relativ zum Konzerndurch- } \\
\text { schnitt }\end{array}$ & $\begin{array}{l}\text { Tabelle 52; eigene } \\
\text { Berechnungen }\end{array}$ & ,038 & 4,01 \\
\hline TR_red & $\begin{array}{l}\text { Relative Änderung des nominellen Steuersatzes } \\
\text { innerhalb des Betrachtungszeitraums }\end{array}$ & Tabelle 52 &,- 097 &, 181 \\
\hline GTS & $\begin{array}{l}\text { Anzahl inländischer Steuergruppen de lege ferenda } \\
\text { / Anzahl inländischer Steuergruppen de lege lata }\end{array}$ & $\begin{array}{l}\text { Eigene Berechnun- } \\
\text { gen }\end{array}$ &, 711 &, 212 \\
\hline LC & $\begin{array}{l}\text { Verlustrlicktragsdauer im geltenden Steuerrecht } \\
\text { (2006); betragsmäßige Beschränkungen des Ver- } \\
\text { lustvortragsmindern LC um } 1 \text {. }\end{array}$ & Kesti (2006) &, 353 & 1,12 \\
\hline LC_T & $\begin{array}{l}\text { Durchschnittlicher Wert für LC der Auslandstöch- } \\
\text { ter inländischer Muttergesellschaften }\end{array}$ & $\begin{array}{l}\text { Kesti (2006); eige- } \\
\text { ne Berechnungen }\end{array}$ & ,869 &, 560 \\
\hline PRO & $\begin{array}{l}\text { Durchschnittliche gesamtwirtschaftliche Profitabi- } \\
\text { lität (1994 bis 2003), zentralisiert auf einen Mit- } \\
\text { telwert von null }\end{array}$ & Eurostat &, 000 &, 025 \\
\hline
\end{tabular}

Quelle: Eigene Berechnungen

Als Regressionsmodell kommt für jeden Reformvorschlag ein lineares OLSModell zur Anwendung, wobei zusätzlich zwischen einer verpflichtenden und optionalen Einführung unterschieden wird. Die theoretischen Vorüberlegungen in diesem Kapitel haben gezeigt, dass für die verschiedenen Reformvorschläge unterschiedliche Einflussfaktoren relevant sind, weshalb auf die Verwendung einer einheitlichen Regressionsfunktion für alle Reformvorschläge verzichtet wird. Um die Vergleichbarkeit der Regressionsergebnisse, die sich für die verpflichtende und optionale Einführung desselben Reformvorschlags ergeben, zu gewährleisten, werden diese Modelle hingegen einheitlich definiert.

Bei der Definition der Regressionsfunktion werden nicht sämtliche Variablen berücksichtigt, die im Rahmen der theoretischen Vorüberlegungen als relevant 
identifiziert wurden. Dieses ist zum einen auf die geringe Anzahl an Beobachtungen zurückzuführen. Grundsätzlich wurden mithilfe des Simulationsmodells Aufkommensänderungen für nur 23 Mitgliedsstaaten ermittelt. Für fünf dieser Mitgliedsstaaten (Zypern, Lettland, Litauen, Luxemburg, Slowakei) geht nur eine geringe Anzahl an Unternehmen in die Simulationsrechnungen ein, weshalb die Ergebnisse für diese Mitgliedsstaaten als nicht hinreichend substantiiert angesehen werden und diese daher als Beobachtungen von der Regressionsuntersuchung ausgeschlossen werden. Aufgrund der geringen Anzahl an Freiheitsgraden, muss die Auswahl der unabhängigen Variablen daher auf einige wenige signifikante Einflussfaktoren beschränkt werden. Zum anderen werden Variablen aufgrund von hoher Korrelation zu anderen unabhängigen Variablen ausgeschlossen (vgl. Tabelle 54 im Anhang für eine Korrelationstabelle). Um Verzerrungen zu vermeiden, werden Variablen nicht berücksichtigt, wenn Korrelation von mehr als $0.6 \mathrm{zu}$ mindestens einer anderen Variablen besteht.

\subsubsection{Regressionsergebnisse}

Tabelle 49 fasst die Ergebnisse der Regressionsanalyse bezüglich der Einflussfaktoren auf die Aufkommensänderungen, die von der Einführung einer verpflichtenden oder optionalen GKKB beziehungsweise Verlustrichtlinie ausgehen, zusammen. Dabei ergibt sich für die verschiedenen Reformszenarien ein korrigiertes $\mathbf{R}^{2}$ zwischen .395 und .743, was darauf hindeutet, dass die gemessenen Aufkommensänderungen in nicht unerheblichem Ausmaß auf die einbezogenen unabhängigen Variablen zurückgeführt werden können. Zudem sind die Modelle trotz der nur geringen Anzahl an Beobachtungen signifikant.

Eine Analyse der Koeffizienten, die für die verschiedenen Variablen geschätzt werden, zeigt, dass sowohl bei optionaler als auch bei verpflichtender Einführung einer GKKB sich ein hoher Anteil neugegründeter Auslandsgesellschaften signifikant negativ auf die Änderung des Steueraufkommens auswirkt. Keinen Unterschied macht es dabei, ob die Tochtergesellschaften als Inbound-Investition im eigenen Land (NIB) oder als Outbound-Investition in einem anderen Mitgliedsstaat (NOB) gegründet werden. Dieses kann zum einen darauf zurückzuführen sein, dass hohe Werte beider Variablen auf einen hohen Internationalisierungsgrad der Konzerne eines Landes hinweisen. Die im Rahmen der GKKB erfolgende Einführung einer grenzüberschreitenden Verlustverrechnung entfaltet in diesem Fall besonders starke Wirkung. Zum anderen sollte die Neugründung ausländischer Tochtergesellschaften in verstärktem Maße mit (Anlauf-)Verlusten verbunden sein, was die Wirkung der Ausdehnung der Verlustverrechnungsmöglichkeiten weiter verstärkt. Entstehen diese Verluste teilweise auf Ebene der neu gegründeten Gesellschaft selbst und teilweise auf Ebene der investierenden Gesellschaft, können sich negative Koeffizienten für beide Variablen auch unter Berücksichtigung des Übergangs von einer direkten auf eine indirekte Gewinnabgrenzung ergeben. 
Tabelle 49: Regressionsergebnisse

\begin{tabular}{|c|c|c|c|c|c|c|c|c|}
\hline & (1) & (2) & (3) & (4) & (5) & (6) & (7) & (8) \\
\hline $\begin{array}{l}\text { Reformkonzept } \\
\text { Einfuhrung }\end{array}$ & $\begin{array}{l}\text { GKKB } \\
\text { Pflicht }\end{array}$ & $\begin{array}{l}\text { GKKB } \\
\text { Option }\end{array}$ & $\begin{array}{l}\text { NVM } \\
\text { Pflicht }\end{array}$ & $\begin{array}{l}\text { NVM } \\
\text { Option }\end{array}$ & $\begin{array}{l}\text { ZUM } \\
\text { Pflicht }\end{array}$ & $\begin{array}{l}\text { ZUM } \\
\text { Option }\end{array}$ & $\begin{array}{l}\text { EAM } \\
\text { Pflicht }\end{array}$ & $\begin{array}{c}\text { EAM } \\
\text { Option }\end{array}$ \\
\hline NIB & $\begin{array}{c}-.259^{* *} \\
(.102) \\
\end{array}$ & $\begin{array}{c}-.510^{* * *} \\
(.135) \\
\end{array}$ & & & & & & \\
\hline NOB & $\begin{array}{c}-.632^{* * * *} \\
(.162) \\
\end{array}$ & $\begin{array}{l}-.149 \\
(.214)\end{array}$ & & & & & & \\
\hline OB_M & & & & & $\begin{array}{l}-.106 \\
(.068)\end{array}$ & $\begin{array}{l}-.009 \\
(.018)\end{array}$ & & \\
\hline OB_T & & & & & $\begin{array}{l}.057^{* * *} \\
(.018) \\
\end{array}$ & $\begin{array}{l}.009^{*} \\
(.005) \\
\end{array}$ & & \\
\hline E10 & & & & & & & $\begin{array}{c}.121 * * * \\
(.020) \\
\end{array}$ & $\begin{array}{l}.077^{* *} \\
(.027)\end{array}$ \\
\hline TR & $\begin{array}{l}.006^{* *} \\
(.002)\end{array}$ & $\begin{array}{c}-.010^{* * *} \\
(.003) \\
\end{array}$ & & & & & & \\
\hline TR_OB & & & & & $\begin{array}{l}.010^{* * *} \\
(.003) \\
\end{array}$ & $\begin{array}{r}.001 \\
(.001) \\
\end{array}$ & & \\
\hline TR_red & $\begin{array}{l}-.088 \\
(.073) \\
\end{array}$ & $\begin{array}{l}-.107 \\
(.096) \\
\end{array}$ & & & & & & \\
\hline GTS & & & $\begin{array}{l}.035^{*} \\
(.017) \\
\end{array}$ & $\begin{array}{r}.028 \\
(.016) \\
\end{array}$ & $\begin{array}{l}.125^{* *} \\
(.052) \\
\end{array}$ & $\begin{array}{l}.041^{* *} \\
(.014) \\
\end{array}$ & $\begin{array}{r}.025 \\
(.020) \\
\end{array}$ & $\begin{array}{r}.028 \\
(.027) \\
\end{array}$ \\
\hline LC & & & & & $\begin{array}{l}.024^{* *} \\
(.010) \\
\end{array}$ & $\begin{array}{c}.000 \\
(.003)\end{array}$ & & \\
\hline LC*OB_M & & & $\begin{array}{c}-.028^{* *} \\
(.010)\end{array}$ & $\begin{array}{c}-.023^{* *} \\
(.009) \\
\end{array}$ & & & & \\
\hline LC_T & & & $\begin{array}{c}-.019^{* *} \\
(.006) \\
\end{array}$ & $\begin{array}{l}-.017^{* *} \\
(.006) \\
\end{array}$ & $\begin{array}{r}-.020 \\
(.017) \\
\end{array}$ & $\begin{array}{c}-.016^{* * *} \\
(.004) \\
\end{array}$ & & \\
\hline PRO & $\begin{array}{c}1.226^{* *} \\
(.557)\end{array}$ & $\begin{array}{c}1.605^{* *} \\
(.737)\end{array}$ & $\begin{array}{c}.192 \\
(.144)\end{array}$ & $\begin{array}{r}.234 \\
(.137) \\
\end{array}$ & & & $\begin{array}{r}.207 \\
(.158) \\
\end{array}$ & $\begin{array}{r}.227 \\
(.215) \\
\end{array}$ \\
\hline Anzahl an & 18 & 18 & 18 & 18 & 18 & 18 & 18 & 18 \\
\hline Korrigiertes $\mathbf{R}^{2}$ & .630 & .576 & .564 & .538 & .667 & .584 & .743 & .395 \\
\hline F-Statistik & 6.778 & 5.626 & 6.507 & 5.943 & 6.663 & 4.984 & 17.386 & 4.703 \\
\hline Signifikanz & .003 & .007 & .004 & .006 & .004 & .011 & .000 & .018 \\
\hline
\end{tabular}

Quelle: Eigene Berechnungen. Eine Konstante wurde berücksichtigt, aber nicht berichtet; Standardfehler werden in Klammern berichtet; ***, ** und * kennzeichnen Signifikanzniveaus von 1\%, 5\% und 10\%. Alle Variablen sind überwiegend unkorreliert (Korrelationskoeffizienz < 0.6). Eine Korrelationsmatrix ist im Anhang enthalten.

Bezüglich des Einflusses der Höhe der nominellen Steuerbelastung zeigt sich - entsprechend der Erwartungen - ein signifikant positiver Zusammenhang zwischen TR und der Aufkommensänderung bei einer verpflichtenden GKKB und ein signifikant negativer Koeffizient für den Fall der Einführung einer optionalen GKKB. Dabei sollte das positive Vorzeichen bei verpflichtender Einfuihrung auf Steuerplanungsaktivitäten im geltenden Steuerrecht zurückzuführen sein, die bei 
Übergang auf eine GKKB wirkungslos werden. Demgegenüber wird bei optionaler Einführung die Besteuerung nach GKKB insbesondere durch die multinationalen Konzerne gewählt, bei denen - verglichen mit dem geltenden Steuerrecht Hochsteuerländern ein geringerer Bemessungsgrundlagenanteil zugewiesen wird. Für die gesamtwirtschaftliche Rentabilität (PRO) wird sowohl bei optionaler als auch bei verpflichtender Einführung ein positiver Koeffizient gemessen. Somit scheint eine hohe Profitabilität mit einer geringeren Verlustwahrscheinlichkeit einherzugehen, welches sich sowohl bei verpflichtender als auch bei optionaler Reform positiv auf das Aufkommen auswirkt. Demgegenüber scheint ein hoher Wert für PRO kein Indikator für positive konzerninterne Renditeunterschiede zu sein. Entgegen der Erwartungen negativ, aber nicht signifikant, ist der Einfluss einer Reduktion der nominellen Steuerbelastung während des Betrachtungszeitraums.

Für die betrachteten Szenarien der Einführung einer Verlustrichtlinie ergibt sich ein positiver Koeffizient für GTS, der zumindest bei der Nachversteuerungsmethode und der Zurechnungsmethode signifikant ist. Dieses Ergebnis entspricht den Erwartungen, da niedrige Werte bei GTS für das Fehlen eines Gruppenbesteuerungssystems im geltenden Recht oder restriktive Anwendungsvoraussetzungen eines derartigen Systems stehen. Die Ausdehnung der Verlustverrechnung zwischen nationalen Konzerngesellschaften hat unter diesen Voraussetzungen stärkeren Einfluss. Auch das positive Vorzeichen des Koeffizienten für PRO bei der Nachversteuerungsmethode und der Ergebnisaufteilungsmethode entspricht den Erwartungen. Hier sollte - analog zur Argumentation für die GKKB - ein hoher Wert auf eine geringere Verlustwahrscheinlichkeit und damit eine geringere Wirksamkeit der Ausdehnung der Verlustverrechnungsmöglichkeiten hindeuten.

Für die Vorschriften zur intertemporalen Verlustverrechnung im Inland ergeben sich bei der Zurechnungsmethode und der Nachversteuerungsmethode unterschiedliche Wirkungsrichtungen, welche aber unter Umständen auch auf die abweichende Variablendefinition zurückzuführen sein können. So ergibt sich bei der Nachversteuerungsmethode ein signifikant negativer Koeffizient für den Interaktionsterm LC*OB_M, welcher zur Erfassung des steuerwirksamen Anteils an Auslandsverlusten sowohl auf die Verfügbarkeit einer positiven Bemessungsgrundlage im Inland (OB_M) als auch die Möglichkeiten zur intertemporalen Verlustverrechnung (LC) abstellt. Ein hoher Wert für diesen Interaktionsterm führt erwartungsgemäß zu einer signifikant stärkeren Aufkommensminderung. Demgegenüber deutet der signifikant positive Koeffizient für LC bei der Zurechnungsmethode darauf hin, dass bei dieser Variablendefinition ${ }^{645}$ die weniger starke Wirkung einer Ausdehnung der nationalen konzerninternen Verlustverrechnung, welche bei hohen Werten für diese Variable zu erwarten ist, dominiert.

645 Bei der Zurechnungsmethode wird OB_M separat und nicht als Interaktionsterm mit LC einbezogen, da sich ein hoher Anteil an Outbound-Investitionen hier gleichzeitig aufgrund der zusätzlichen Besteuerung der Auslandsgewinne positiv auswisken kann 
Nicht in Einklang mit den Erwartungen sind hingegen die sowohl für die Nachversteuerungsmethode als auch die Zurechnungsmethode gemessenen signifikant negativen Koeffizienten für LC_T. So wäre grundsätzlich davon auszugehen, dass eine Folge großzügiger Möglichkeiten zur intertemporalen Verlustverrechnung im Ausland ist, dass weniger Auslandsverluste im Inland steuerwirksam werden. Eine mögliche Erklärung liefern hier Gestaltungsanreize großzügiger Verlustverrechnungsvorschriften. Führen diese dazu, dass riskante Investitionen und damit Investitionen mit hohem Verlustrisiko eher durchgeführt werden, erscheint der negative Koeffizient nachvollziehbar.

Weitere signifikante Einflussfaktoren für den Aufkommenseffekt einer verpflichtenden Einführung der Zurechnungsmethode sind der Anteil ausländischer Tochterunternehmen (OB_T) sowie die durchschnittliche Steuersatzdifferenz zwischen dem inländischen Steuersatz und dem durchschnittlichen Steuerniveau ausländischer Tochtergesellschaften (TR_OB). In Übereinstimmung mit den theoretischen Vorüberlegungen ergeben sich für beide Variablen bei verpflichtender Einführung signifikant positive Koeffizienten. Bei optionaler Einführung fällt der Wert beider Koeffizienten geringer, aber unverändert positiv aus und ist in geringerem Ausmaß oder nicht signifikant. Dieses Ergebnis kann damit begründet werden, dass bei Optionalität die Zurechnungsmethode zwar selten in Anspruch genommen wird, wenn diese zu einer zusätzlichen Besteuerung der Auslandsgewinne bei der Muttergesellschaft führt, positive Steuersatzdifferenzen bei optionaler Einführung der Zurechnungsmethode aber zumindest dazu führen, dass die Ausdehnung der Verlustverrechnung sich in weniger starkem Ausmaß aufkommensmindernd auswirkt.

Bei der Ergebnisaufteilungsmethode zeigt sich schließlich, dass sowohl bei verpflichtender als auch bei optionaler Einführung von einem hohen Anteil von Investitionen aus einem EU-15-Land in einem Beitrittsstaat ein signifikanter Aufkommensanstieg im EU-15-Land und ein signifikanter Aufkommensrückgang im Beitrittsstaat ausgehen. Dieses Ergebnis kann - in Übereinstimmung mit den theoretischen Vorüberlegungen - darauf zurückgeführt werden, dass das Risiko derartiger Investitionen in den ersten Jahren vermehrt vom investierenden Unternehmen getragen wird, während das Investitionsobjekt für die Übernahme bestimmter Funktionen mit einer festen Vergütung entlohnt wird.

\subsection{Zwischenfazit}

Die Ergebnisse der Aufkommensberechnungen zeigen, dass die verpflichtende Einführung einer GKKB einen Rückgang des EU-Gesamtsteueraufkommens von 4,83 Prozent bewirken würde. Bei optionaler Einführung fällt der entsprechende Effekt unwesentlich höher aus (-4,99 Prozent). Diese Ergebnisse liegen in der 
Mitte der Ergebnisse vergleichbarer Studien. ${ }^{646}$ Poppe (2008) basiert seine Untersuchung auf europaweiten, aber einperiodigen Daten, die ebenfalls der Datenbank AMADEUS entstammen. Er findet einen Rückgang der EUGesamtbemessungsgrundlage von 14,1 Prozent bei verpflichtender Einführung der GKKB. Werden zukünftige Steuerminderungen aufgrund von in der Periode nicht verrechneten Verlusten pauschal berücksichtigt, stellt sich eine Aufkommensminderung von 10,8 Prozent ein. ${ }^{647}$ Demgegenüber messen Devereux/Loretz (2008) auf Basis der Jahresabschlüsse europäischer Unternehmen für die Jahre 2001 bis 2005 einen Anstieg des EU-Gesamtsteueraufkommens von 2 Prozent für die verpflichtende Einführung einer GKKB, während die optionale Einführung in ihren Berechnungen einen Aufkommensrückgang von etwa 2,5 Prozent hervorruft..$^{648}$

Wird eine Verlustrichtlinie mit dem Konzept der Nachversteuerungsmethode oder der Ergebnisaufteilungsmethode eingeführt, fällt der entsprechende Effekt etwas geringer aus (-3,97 Prozent und -4,54 Prozent bei verpflichtender Einführung). Demgegenüber wäre eine verpflichtende Einführung der Zurechnungsmethode nahezu aufkommensneutral möglich (-0,16 Prozent). Insgesamt bleibt festzuhalten, dass - wie nicht anders zu erwarten - die Reformvorschläge, welche die größte Minderung der durchschnittlichen Steuerbelastung mit sich bringen würden, auch die stärksten Aufkommensverluste verursachen. Die Abwägung zwischen einer Verbesserung der Standortattraktivität auf der einen Seite und einer Begrenzung der Aufkommensverluste auf der anderen kann nur auf politischem Wege erfolgen.

Wird davon ausgegangen, dass die Bereitschaft der Mitgliedsstaaten für die Teilnahme an einer derartigen Reform neben der erwarteten Minderung des EUGesamtsteueraufkommens insbesondere auch vom Risiko des länderbezogenen Aufkommensverlusts abhängig ist, sollten Reformvorschläge mit einer hohen durchschnittlichen Aufkommensänderung deutlich schlechtere Aussichten auf Umsetzung haben. In diesem Zusammenhang zeigt sich, dass von der Einführung einer GKKB mit einer durchschnittlichen Aufkommensänderung von 9,14 Prozent bei verpflichtender Einführung und 6,94 Prozent bei optionaler Einführung ein hohes Risiko für die Mitgliedsstaaten ausgeht, während die entsprechenden Werte für jedes der drei Konzepte einer Verlustrichtlinie deutlich niedriger liegen. Mit Blick auf die GKKB ist ferner erwähnenswert, dass sowohl die Aufkommensminderung als auch die durchschnittliche Höhe der Aufkommensänderung bei einer optionalen Einführung im Wege der verstärkten Zusammenarbeit deutlich gesenkt werden könnten. Der Grund hierfür sollte in der weitaus höheren Homogenität der

646 Als nicht vergleichbar eingestuft werden die Ergebnisse in Oestreicher/Scheffler/Spengel et al. (2008), Fuest/Hemmelgarn/Ramb (2007) und Fuest/Hemmelgarn/Ramb (2005), da diese Studien sich auf deutsche Outbound-Konzerne beschränken.

647 Vgl. Poppe (2008), S. 209-210.

648 Vgl. Devereux/Loretz (2008), S. 1. 
teilnehmenden Mitgliedsstaaten hinsichtlich der steuerlichen und wirtschaftlichen Rahmenbedingungen liegen. Ein sinnvoller Weg der Implementierung könnte aus diesem Grund die zunächst auf einige Mitgliedsstaaten beschränkte Einführung sein. Weitere Mitgliedsstaaten könnten der GKKB beitreten, sobald sich die wirtschaftlichen und steuerlichen Rahmenbedingungen dieser Länder an die Verhältnisse der übrigen teilnehmenden Mitgliedsstaaten angeglichen haben.

Des Weiteren ist festzuhalten, dass die durchschnittliche Höhe der Aufkommensänderung wesentlich von der Ausgestaltung der Aufteilungsformel abhängt. Dabei ergibt sich die geringste Aufkommensänderung bei der Aufteilung anhand des Vermögensfaktors sowie des Umsatzfaktors, während sich die größten Änderungen bei der Mitarbeiteranzahl ergeben. $\mathrm{Zu}$ dem letztgenannten Aufteilungsfaktor bleibt anzumerken, dass die von der Kommission vorgeschlagene gleichgewichtete Einbeziehung von Lohnsumme und Mitarbeiteranzahl mit Blick auf eine zutreffende Erfassung des Arbeitsfaktors nicht optimal erscheint. So geben gesamtwirtschaftliche Auswertungen Anlass zu der Annahme, dass bei Übergewichtung der Lohnsumme eine bessere Erfassung der Arbeitsproduktivität gewährleistet werden kann.

Die Analyse der Einflussfaktoren auf die Höhe der länderspezifischen Aufkommensänderungen bestätigt im Wesentlichen die aus theoretischen Überlegungen abgeleiteten Vermutungen. So zeigt sich, dass die - in jedem Vorschlag vorgesehene - Ausweitung der Verlustverrechnung zwischen den Gesellschaften eines nationalen Konzerns zu Aufkommensrückgängen insbesondere in den Mitgliedsstaaten führt, die aktuell über kein Gruppenbesteuerungssystem verfügen oder dieses an restriktive Anwendungsvoraussetzungen knüpfen. Auch eine hohe gesamtwirtschaftliche Rentabilität mindert die negative Aufkommenswirkung, da in diesen Fällen von einem geringeren Verlustrisiko ausgegangen werden kann. In besonderem Maße ist allerdings der Zusammenhang von Aufkommenseffekt und Steuersatz hervorzuheben. Dieser (positive) Zusammenhang erscheint bei der $\mathrm{Zu}$ rechnungsmethode noch vertretbar, da hier ein niedriger Steuersatz zwar mit größeren Aufkommensverlusten einhergeht, gleichzeitig sich die Standortattraktivität dieser Länder - im Verhältnis zu den Hochsteuerländern - aber in weniger starkem Ausmaß verschlechtert (vgl. Tabelle 26). Bei der GKKB hingegen könnte er für Probleme bei der Umsetzung sorgen. So besteht auch bei der verpflichtenden GKKB ein positiver Zusammenhang von Aufkommenseffekt und Steuersatz. Im Unterschied zur Zurechnungsmethode führt hier die Umverteilung der Bemessungsgrundlage dazu, dass Niedrigsteuerländer zum einen an Steueraufkommen verlieren, dieses aber auf der anderen Seite zu Lasten der durchschnittlichen Steuerbelastung und damit der Standortattraktivität dieses Landes erfolgt. Im Unterschied zu den Hochsteuerländern kommt es hier zu einem Anstieg der durchschnittlichen Steuerbelastung (vgl. Tabelle 24). Ebenfalls problematisch ist der signifikant negative Zusammenhang, der sich bei der optionalen GKKB ergibt. Dieser führt - wie auch im geltenden Steuerrecht - dazu, dass eine beabsichtigte 
Steigerung des inländischen Körperschaftsteueraufkommen mit Problemen verbunden ist, da eine Erhöhung des Körperschaftsteuersatzes einen Verlust an Steueraufkommen aufgrund der Optionsrechtsausübung bewirkt. Verglichen mit dem geltenden Steuerrecht sollte dieser Effekt im Rahmen einer GKKB noch schwerer wiegen, da hier die Gestaltung des Steuersatzes mithin die einzige Möglichkeit zur Beeinflussung des inländischen Steueraufkommens darstellt. 


\section{Unternehmerische Verhaltensreaktionen als Folge ei- ner Reform der Konzernbesteuerung}

\section{1 Überblick}

Die Simulationsrechnungen in dieser Arbeit beruhen auf der Annahme, dass die betroffenen Unternehmen auf die Reform nicht reagieren. Eine Einbeziehung derartiger Verhaltensreaktionen in das Mikrosimulationsmodell ist für den zweiten Abschnitt des in der Einleitung genannten Forschungsprojekts angedacht. Über die Stärke des Einflusses dieser Reaktionen auf die Höhe der Aufkommens- und Belastungswirkungen können daher zum heutigen Zeitpunkt nur Vermutungen angestellt werden. Als Ausblick soll allerdings in diesem Kapitel ein kurzer Überblick über mögliche Verhaltenswirkungen gegeben werden. Hierbei kann für die GKKB auf bestehende analytische, modelltheoretische und empirische Literatur Bezug genommen werden. ${ }^{649}$ Für die Einführung einer Verlustrichtlinie - werden soweit nicht anders kenntlich gemacht - einige kurze eigene Überlegungen angestellt.

\subsection{Unternehmerische Verhaltensreaktionen bei Einführung ei- ner GKKB}

\subsubsection{Steuerplanungsgestaltungen}

Steuerreformen können in zwei Bereichen Einfluss auf unternehmerische Entscheidungen nehmen. Zum einen können Anpassungen bei rein steuerlich motivierten Gestaltungen (Steuerplanung) hervorgerufen werden, zum anderen kann die Steuerreform realwirtschaftliche Entscheidungen beeinflussen. ${ }^{650}$ Wie an früherer Stelle angefürt, wird das große Ausmaß an Steuerplanung und die daher nur eingeschränkte Möglichkeit einer effektiven Steuererhebung in einem auf der Trennungstheorie beruhenden Steuersystem als eine der wesentlichen Rechtfertigungen für eine Reform der Konzernbesteuerung in Europa angesehen. ${ }^{651}$ So erlaubt das geltende System die Verlagerung von Einkünften in Niedrigsteuerländer durch gezielte Gestaltung konzerninterner Verrechnungspreise sowie konzerninterner Finanzierungsbeziehungen. Eine weitere Möglichkeit besteht in der Zuord-

649 Für einen ausfürlichen Überblick uber die Literatur zu Verhaltenswirkungen im Rahmen einer GKKB vgl. Agundez-Garcia (2006), S. 59-69 und Weiner (2005), S. 38-50.

$650 \mathrm{Vgl}$. Creedy/Gemmel (2007), S. 1.

651 Vgl. Kapitel 3.5 
nung von immateriellen Wirtschaftsgütern in niedrig besteuerte Holdinggesellschaften. ${ }^{652}$

Allerdings ist fraglich, inwiefern der mit der Einführung einer GKKB verbundene Übergang von einer direkten auf eine indirekte Gewinnabgrenzung die Möglichkeiten zur Steuergestaltung tatsächlich einschränken würde. Richtig ist, dass konzerninterne Finanzierungsunterschiede innerhalb der GKKB-Gruppe die Höhe der Steuerbelastung des Konzerns nicht beeinflussen würden und die Verlagerung immaterieller Wirtschaftsgüter - zumindest soweit diese nicht in die Aufteilungsformel einbezogen werden - wirkungslos wäre. ${ }^{653}$ Mintz/Smart (2004) zeigen für die Besteuerung auf Ebene der kanadischen Provinzen entsprechende Unterschiede bei der Steuergestaltung mittels konzerninterner Finanzierung zwischen Gruppeneinheiten, die konsolidiert werden und Gruppeneinheiten, die nicht konsolidiert werden, auf. ${ }^{654}$ Allerdings ist zu berücksichtigen, dass die traditionelle Steuergestaltung mittels Verrechnungspreisen und Finanzierungsgestaltungen durch die Einführung einer GKKB nicht vollständig ausgeschlossen wäre. Derartige Gestaltungen müssten vielmehr an die geografische Grenze der GKKBGruppe verlagert werden. ${ }^{655}$ In dieser Hinsicht erscheint insbesondere die Einführung einer GKKB unter Beteiligung nicht sämtlicher Mitgliedsstaaten problematisch. ${ }^{656}$

Das Ausmaß, zu dem Gewinnverlagerungen auch innerhalb der GKKBGruppe möglich sind, sollte entscheidend von der Definition der Aufteilungsformel abhängen. Schreiber verweist darauf, dass die örtliche Zuordnung von Löhnen und Kapital ${ }^{657}$ relativ wenig Gestaltungsspielraum lässt. Aus diesem Grund sollten sich Möglichkeiten zur Steuergestaltung insbesondere beim Umsatzfaktor ergeben. ${ }^{658}$ In diesem Zusammenhang werden in der Literatur Probleme bei der Lokalisierung von Verkäufen über das Internet besonders betont. ${ }^{659}$ Ferner könnte bei einer ursprungsortbezogenen Definition des Umsatzfaktors Bemessungsgrundlage durch den Einsatz von Vertriebsgesellschaften verlagert werden, während bei einer bestimmungsortbezogenen Definition Verrechnungspreisgestaltungen möglich sind. ${ }^{660}$ Die steueroptimale Gestaltung von Umsatzerlösen im Rahmen der

652 Vgl. Weiner (2005), S. 45; Schreiber (2004), S. 218-219.

$653 \mathrm{Vgl}$. Oestreicher/Scheffler/Spengel et al. (2008), S. 364.

654 Vgl. Mintz/Smart (2004), S. 1149; so auch Weiner (2005), S. 43-44.

655 Vgl. Blumenberg (2008), S. 16-17; Schön (2007), S. 437; Agundez-Garcia (2006), S. 67; Schreiber (2004), S. 219.

656 Vgl. Schön (2007), S. 438-439.

657 Scheffler zeigt mögliche Gestaltungsansätze bei Aufteilung anhand eines Vermögensfaktors auf. Vgl. Scheffler (2005a) S. 326-327. Derartige Ansätze, wie beispielsweise in der strategischen Entscheidung für einen kreditfinanzierten Kauf oder Leasing von Anlagevermögen, können allerdings durch geeignete Definition des Aufteilungsfaktors vermieden werden.

658 Vgl. Schreiber (2004), S. 222.

659 Vgl. Hellerstein/McLure (2004), S. 213; Mintz (1999), S. 408

$660 \mathrm{Vgl}$. Scheffler (2005a), S. 317 und 327-328. 
formelhaften Gewinnaufteilung in den USA und Kanada konnte von Klassen/Shackelford (1998) empirisch nachgewiesen werden. ${ }^{661}$

Abschließend ist darauf hinzuweisen, dass bei optionaler Einführung einer GKKB diese selbst ein weitreichendes Instrument zur Steuergestaltung bildet, welches neben die im geltenden Recht bestehenden Planungsmöglichkeiten tritt. ${ }^{662}$

\subsubsection{Realwirtschaftliche Entscheidungen}

Neben dem Einfluss der GKKB auf die unternehmerische Steuerplanung sollte ihre Einführung auch Einfluss auf realwirtschaftliche Entscheidungen nehmen. Verhaltenswirkungen in diesem Bereich können sich insbesondere bei der Faktorallokation sowie der unternehmerischen Risikoübernahme ergeben.

Ein Einfluss der Einführung einer GKKB auf die Lokalisierung betrieblicher Funktionen kann sich aus dem Übergang von einer direkten zu einer indirekten Gewinnabgrenzung ergeben. Zwar werden Investitions- und Standortentscheidungen auch im geltenden Steuerrecht steuerlich verzerrt ${ }^{663}$, allerdings gibt es Grund zu der Annahme, dass der steuerliche Einfluss auf derartige Entscheidungen bei Einführung einer GKKB verstärkt würde. So führt der Übergang auf die Formelaufteilung zum einen dazu, dass sich durch Verlagerung von Produktionsfaktoren ein größerer Bemessungsgrundlagenanteil verschieben lässt ${ }^{664}$, zum anderen wird die Verlagerung bestehender Funktionen unter der GKKB dadurch erleichtert, dass eine Aufdeckung stiller Reserven bei Verlagerung hier nicht erfolgt. ${ }^{665}$

Das Ausmaß des steuerlichen Einflusses auf die Faktorallokation sollte ebenso wie die Möglichkeiten zur Steuergestaltung - maßgebend von der Definition der Aufteilungsformel abhängen. Im Allgemeinen wird davon ausgegangen, dass der (bestimmungsortbezogen definierte) Umsatzfaktor derartige Gestaltungen in nur geringem Ausmaß zulässt, da dieser nicht im alleinigen Entscheidungsbereich des Unternehmens liegt. ${ }^{666}$ Scheffler verweist allerdings darauf, dass Unternehmen versuchen könnten, durch gezielte Absatzpreisdifferenzierungen die Abnehmer zur Gründung von Einkaufsgesellschaften in Niedrigsteuerländern zu bewegen $^{667}$. Der Einfluss der Formelaufteilung auf Investitions- und Beschäftigungsentscheidungen konnte wiederholt gezeigt werden. So zeigen Gupta/Hofmann (2003) und Weiner (1994) für die USA bestehende Investitionswirkungen einer Erhöhung beziehungsweise Verminderung des Faktorgewichts auf Vermögen auf, während Goolsbee/Maydew (2000) eine vergleichbare Wirkung für den Arbeits-

661 Vgl. Agundez-Garcia (2006), S. 66; Weiner (2005), S. 43-44.

662 Vgl. hierzu Blumenberg (2008), S. 16.

663 Vgl. Mintz/Weiner (2003), S. 699-700.

$664 \mathrm{Vgl}$. Oestreicher/Scheffler/Spengel et al. (2008), S. 365; Spengel (2008), S. 46.

$665 \mathrm{Vgl}$. Oestreicher/Scheffler/Spengel et al. (2008), S. 365; Spengel (2008), S. 46; Scheffler (2005a), 322-324.

$666 \mathrm{Vgl} \mathrm{stellvertretend} \mathrm{Mors/Rautenstrauch} \mathrm{(2008),} \mathrm{S.} \mathrm{102;} \mathrm{Agundez-Garcia} \mathrm{(2006),} \mathrm{S.} 52$.

$667 \mathrm{Vgl}$. Scheffler (2005a), S. 328. 
faktor nachweisen. ${ }^{668}$ Weiner (1994) findet entsprechende Investitionswirkungen auf Basis kanadischer Daten auch bei einheitlicher Formel aber abweichenden Steuersätzen. ${ }^{669}$

Insgesamt bleibt festzuhalten, dass auch eine Konzernbesteuerung mit indirekter Gewinnabgrenzung zu Verzerrungen führt, solange die Körperschaftsteuersätze variieren. Inwiefern die Entscheidungsverzerrungen von größerer Bedeutung als bei direkter Gewinnabgrenzung sind oder nicht, ist allerdings unklar. ${ }^{670}$ In jedem Fall ist aber davon auszugehen, dass die Komplexität der Steuerplanung zunimmt, da im multinationalen Konzern die Planung für alle Konzerngesellschaften simultan erfolgen muss. Ursächlich hierfür sind Rückwirkungen der Verlagerung auch auf nicht unmittelbar beteiligte Unternehmen. ${ }^{671}$

Neben dem Einfluss auf die Allokation von Produktionsfaktoren ist davon auszugehen, dass von der mit der Einführung einer GKKB verbundenen Ausweitung der steuerlichen Verlustverrechnungsmöglichkeiten Einfluss auf die unternehmerische Risikoübernahme genommen werden würde. Der Zusammenhang zwischen der steuerlichen Verlustverrechnung und dem Grad der Risikoübernahme wurde bisher insbesondere in der modelltheoretischen Literatur intensiv diskutiert. Grundlegend ist hierbei der Beitrag von Domar/Musgrave (1944), welcher die Entscheidung zwischen einer risikobehafteten und einer risikolosen Investition auf der Basis eines einperiodigen Modells für einen risikoaversen Investor untersucht. Domar/Musgrave kommen auf der Basis graphisch abgeleiteter Indifferenzkurven und des erwarteten Verlusts als Risikoma $ß \mathrm{zu}$ dem generellen Ergebnis, dass die Beteiligung des Staates an Investitionsverlusten die Bereitschaft zur Risikoübernahme beeinflusst. Verglichen mit einer Welt ohne Steuern ist die Richtung dieses Einflusses maßgeblich abhängig von der Höhe der tariflichen Steuerbelastung und der Beschränkung der Verlustverrechnung. Restriktivere Verlustverrechnungsvorschriften sollten dabei allerdings ceteris paribus regelmäßig zu geringerer Risikoübernahme führen. Ausnahmen davon könnten nur bei einer außergewöhnlichen Präferenzordnung des Investors eintreten. Weiterführende Modelle (Mossin (1968), Stiglitz (1969) und Haegert/Kramm (1975)) integrieren insbesondere abweichende Risikodefinitionen sowie formale Beschreibungen des Risikonutzens und der Risikoaversion, bestätigen allerdings die von Domar/Musgrave gewonnenen Erkenntnisse. Empirisch wurde dieser Zusammenhang für unternehmerische Investitionen bisher - soweit erkennbar - lediglich von Koch/Prassel (2009) untersucht. Dieser Beitrag weist auf eine gesteigerte Risikoübernahme belgischer Unternehmen als Folge der Abschaffung der Mindestbesteuerung im Jahr 1998 hin. Bei Übertragung dieser Ergebnisse sollte entspre-

$668 \mathrm{Vgl}$. Weiner (2005), S. 38-39.

669 Vgl. Weiner (2005), S. 38-39.

670 Vgl. Mintz/Weiner (2003), S. 700.

671 Vgl. Scheffler (2005a), S. 324-326. 
chend auch die Einführung einer GKKB zu einer Zunahme der Risikobereitschaft führen.

\subsection{Unternehmerische Verhaltensreaktionen bei Einführung ei- ner Verlustrichtlinie}

Unabhängig vom Konzept zur grenzüberschreitenden Verlustverrechnung würde die Einführung einer Verlustrichtlinie eine Ausweitung der Möglichkeiten zur Verlustverrechnung bewirken. Auch für diesen Reformvorschlag ist daher von einer Ausdehnung der unternehmerischen Risikoübernahme auszugehen. Demgegenüber sollte sich die Wirkung einer Verlustrichtlinie auf Steuergestaltung und den steuerlichen Einfluss auf die Faktorallokation für die verschiedenen Konzepte zur Verlustverrechnung unterscheiden.

So führt die Nachversteuerungsmethode nur in Verlustsituationen zu einer partiellen Durchbrechung des Trennungsprinzips, welches bei Gewinnen der Tochtergesellschaft unverändert Berücksichtigung findet. Dementsprechend ist auch Steuergestaltung in unverändertem Maße möglich. Gleiches gilt grundsätzlich auch für die Ergebnisaufteilungsmethode. Für dieses Konzept könnte es sogar zu einer Zunahme an Steuergestaltung kommen, da Konzerne bemüht sein werden, die Ergebnisaufteilung für Gestaltungszwecke zu nutzen. Ein möglicher Ansatz hierzu könnte die zeitliche Gestaltung des Anfalls von Gewinnen und Verlusten sein, da deren steuerliche Behandlung insbesondere vom Konzerneinkommen in der jeweiligen Periode abhängt.

Demgegenüber führt die Zurechnungsmethode zu einer Durchbrechung des Trennungsprinzips und zum Übergang von einer kapitalimportneutralen auf eine kapitalexportneutrale Besteuerung. Diese führt grundsätzlich dazu, dass Gewinnverlagerungen und Verlagerungen von Funktionen nicht zu einer Minderung der Steuerbelastung führen können. ${ }^{672}$ Gestaltungsanreize können allerdings auch bei dieser Methode durch die Beschränkung der Anrechnung auf einen Anrechnungshöchstbetrag ausgehen. ${ }^{673}$ Darüber hinaus muss berücksichtigt werden, dass sowohl bei der Zurechnungsmethode als auch bei der Nachversteuerungsmethode den steuerlichen Rahmenbedingungen im Sitzland der Muttergesellschaft zentrale Bedeutung für die Höhe der Konzernsteuerbelastung zukommt. Konzerne könnten daher bemüht sein, ihre Muttergesellschaft in Mitgliedsstaaten mit großzügigen Vorschriften zur intertemporalen Verlustverrechnung sowie - bei der Zurechnungsmethode - in Mitgliedsstaaten mit niedrigen Steuersätzen zu verlagerm. ${ }^{674}$

$672 \mathrm{Vgl}$. Oestreicher/Scheffler/Spengel et al. (2008), S. 368.

$673 \mathrm{Vgl}$. Oestreicher/Scheffler/Spengel et al. (2008), S. 368.

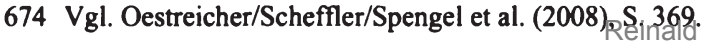


Reinald Koch - 978-3-631-75683-6

Downloaded from PubFactory at 01/11/2019 03:01:46AM

via free access 


\section{Zusammenfassende Beurteilung der Reformvorschläge}

Die Zielsetzung der vorliegenden Arbeit lag in der empirischen Analyse der Steuerlast- und Steueraufkommenswirkungen verschiedener Vorschläge für die Reform der Konzernbesteuerung in Europa. Auf Basis dieser Berechnungsergebnisse sollten auf der einen Seite Schlussfolgerungen für die Bedeutung dieser Vorschläge für die Standortattraktivität und die Effizienz des Steuersystems gezogen werden, zum anderen gibt die Analyse der Aufkommenswirkungen Aufschluss über die mögliche Umsetzbarkeit dieser Reformvorschläge.

Es wurde gezeigt, dass das bestehende Konzernbesteuerungssystem aus Unternehmenssicht mit Hemmnissen für die grenzüberschreitende Geschäftstätigkeit verbunden ist, die zu einem Großteil, aber nicht ausschließlich, auf das Nebeneinander separater Besteuerungssysteme im Binnenmarkt zurückzuführen sind. Für die Mitgliedsstaaten besteht das Problem, dass die Möglichkeit zur Steuergestaltung einer effektiven Steuererhebung entgegensteht. Dieses Problem wird durch die zunehmend einschränkende Wirkung der EuGH-Rechtsprechung auf die Steuerrechtsausgestaltung verstärkt.

Mögliche Reformvorschläge für die Konzernbesteuerung reichen von einer von der EuGH-Rechtsprechung getriebenen unilateralen Fortentwicklung des nationalen Rechts (passive Harmonisierung) über gezielte Harmonisierungsmaßnahmen bis hin zu einer weitgehenden oder vollständigen Vereinheitlichung der Regelungen im Wege der aktiven Harmonisierung. Dabei wurde gezeigt, dass eine passive Harmonisierung den europäischen Anforderungen nur in unzureichendem Maße gerecht wird, weshalb dieser Harmonisierungsweg in den weiteren Überlegungen nicht berücksichtigt wurde. Bestgeeignet für eine systematische Beseitigung der steuerlichen Probleme im Binnenmarkt ist die Einführung einer „Gemeinsamen konsolidierten Körperschaftsteuerbemessungsgrundlage“ (GKKB), weshalb dieses Reformszenario in das Zentrum der weiteren Ausführungen gestellt wurde. Dabei ist zum heutigen Zeitpunkt allerdings unklar, inwiefern die für eine Einführung erforderliche Zustimmung sämtlicher Mitgliedsstaaten erreicht werden kann. Aus diesem Grund wurden auch gezielte Maßnahmen, von denen ein weniger starker Eingriff in die Steuersouveränität der Mitgliedsstaat ausgeht, einbezogen. Dabei lässt sich allerdings feststellen, dass durch gezielte Maßnahmen von den verbleibenden Problemen lediglich das Fehlen einer grenzüberschreitenden Verlustverrechnung im Konzern beseitigt werden kann. Aus diesem Grund wird die Einführung einer Verlustrichtlinie als zweites Reformszenario berücksichtigt, wobei für die Ausgestaltung der Verlustverrechnung alternativ die Nachversteuerungsmethode, die Zurechnungsmethode und die Ergebnisaufteilungsmethode in Frage kommen.

Als Ergebnis der Berechnungen zu den Auswirkungen der Einführung einer GKKB und einer Verlustrichtlinie auf die Steuerbelastung nationaler und grenz- 
überschreitend tätiger Konzerne bleibt festzuhalten, dass eine derartige Reform unabhängig vom zugrundegelegten Konzept einen Beitrag zur Verbesserung der steuerlichen Standortattraktivität der EU sowie der Wettbewerbsfähigkeit europäischer Konzerne leisten würde, wobei die steuerlichen Rahmenbedingungen insbesondere für grenzüberschreitend tätige Konzerne verbessert würden. Hinsichtlich der Effizienzwirkungen der Reformvorschläge ergeben sich allerdings deutliche Unterschiede. So konnte gezeigt werden, dass lediglich eine verpflichtende GKKB und - mit Abstrichen - eine optionale Einführung der Nachversteuerungsmethode eine faire steuerliche Behandlung von nationalen und multinationalen Konzernen gewährleistet. Während die Ergebnisaufteilungsmethode und die Zurechnungsmethode die Nachteile multinationaler Konzerne bei der grenzüberschreitenden Verlustverrechnung nur in unzureichendem Maße beseitigen können, eröffnet die optionale Einführung einer GKKB zusätzliche Gestaltungsmöglichkeiten und damit steuerliche Vorteile für multinationale Konzerne. Dieses Ergebnis spricht dafür, ein Optionsrecht nicht vorzusehen oder dieses auf nationale Konzerne zu beschränken. Für die Einführung einer zumindest für multinationale Konzerne verpflichtenden GKKB spricht ferner, dass der Übergang auf die indirekte Gewinnabgrenzung eine deutliche Reduzierung der Schwankung der durchschnittlichen Steuerbelastung bewirken würde.

Auch wenn die verpflichtende GKKB hinsichtlich der Effizienzwirkungen gegenüber den übrigen Reformvorschlägen Vorteile aufweist, so zeigen die Aufkommensberechnungen, dass dieses Szenario mit erheblichen Aufkommensrisiken für die Mitgliedsstaaten verbunden ist. Dabei erscheint weniger der Rückgang des Gesamtsteueraufkommens, welcher mit -5,17 Prozent nur unwesentlich über der Aufkommensänderung der übrigen Reformvorschläge liegt, als vielmehr die hohe durchschnittliche Aufkommensänderung (9,14 Prozent) problematisch. Insbesondere Niedrigsteuerländer müssen dabei mit hohen Aufkommensverlusten rechnen. Abgeschwächt werden kann dieses Problem, wenn nicht sämtliche Mitgliedsstaaten, sondern anfänglich nur eine Gruppe ausgewählter Mitgliedsstaaten an der Einführung der GKKB teilnehmen würde. Dieses würde zwar andere Vorteile, die zum Beispiel in einem Rückgang der Befolgungskosten oder der vollständigen Beseitigung des Verrechnungspreisproblems bestehen, einschränken und zu einer höheren Absenkung des Gesamtaufkommens dieser Mitgliedsstaaten führen, die durchschnittliche Höhe der Aufkommensänderung und damit das Aufkommensrisiko für die Mitgliedsstaaten könnte auf diesem Weg allerdings deutlich reduziert werden. Hierfür sollte insbesondere die größere wirtschaftliche Ähnlichkeit dieser Mitgliedsstaaten ursächlich sein. Weitere Mitgliedsstaaten könnten - ähnlich zur Vorgehensweise bei der Euro-Einführung - bei weitgehender Angleichung der wirtschaftlichen Rahmenbedingungen später beitreten. Auch die Einführung branchenspezifischer Aufteilungsformeln könnte einen Beitrag zu einer weniger starken Schwankung der Aufkommensänderung leisten. 
Abschließend soll einschränkend darauf hingewiesen werden, dass die Ergebnisse dieser Arbeit auf der Annahme beruhen, dass unternehmerische Verhaltensreaktionen auf die durchgeführte Steuerreform unterbleiben. Dieses gilt vor allem für die Wirkung des Übergangs von einer direkten auf eine indirekte Gewinnabgrenzung, bei der die bisher erfolgenden Gestaltungsmaßnahmen überwiegend unwirksam werden. Bestehende Studien insbesondere für den nordamerikanischen Raum stellen diese Annahme allerdings in Frage. Es bleibt somit abzuwarten, inwiefern auch bei Einbeziehung von Verhaltensanpassungen in das Simulationsmodell die hier gewonnenen Erkenntnisse bestätigt werden können. 
Reinald Koch - 978-3-631-75683-6

Downloaded from PubFactory at 01/11/2019 03:01:46AM

via free access 


\section{Anhang}

Tabelle 50: Steuerrechtliche Grundlage der Simulationsberechnungen (1)

\begin{tabular}{|c|c|c|c|c|}
\hline & \multicolumn{2}{|c|}{ Verlustabzug } & \multicolumn{2}{|c|}{$\begin{array}{l}\text { Behandlung von Dividenden einer Kapital- } \\
\text { gesellschaft }\end{array}$} \\
\hline & Rücktrag & Vortrag & inländische & ausländische \\
\hline Belgien & - & unbegrenzt & Freistellung (95\%) & Freistellung (95\%) \\
\hline Dänemark & - & unbegrenzt & Freistellung (100\%) & Freistellung (100\%) \\
\hline Deutschland & $1 \mathrm{Jahr}^{675}$ & unbegrenzt ${ }^{676}$ & Freistellung (95\%) & Freistellung (95\%) \\
\hline Estland $^{677}$ & n.a. & n.a. & Freistellung (100\%) & Freistellung (100\%) \\
\hline Finnland & - & 10 Jahre & Freistellung (100\%) & Freistellung (100\%) \\
\hline Frankreich & 3 Jahre & unbegrenzt & Freistellung (95\%) & Freistellung (95\%) \\
\hline Griechenland & - & 5 Jahre & Freistellung (100\%) & Anrechnung \\
\hline Großbritannien & $1 \mathrm{Jahr}$ & unbegrenzt & Freistellung $(100 \%)$ & Anrechnung \\
\hline Irland & $1 \mathrm{Jahr}$ & unbegrenzt & Freistellung $(100 \%)$ & Anrechnung \\
\hline Italien & - & 5 Jahre & Freistellung (95\%) & Freistellung (95\%) \\
\hline Lettland & - & 5 Jahre & Freistellung (100\%) & Freistellung ( $100 \%)$ \\
\hline Litauen & - & 5 Jahre & Freistellung (100\%) & Freistellung (100\%) \\
\hline Luxemburg & - & unbegrenzt & Freistellung (100\%) & Freistellung (100\%) \\
\hline Niederlande & 3 Jahre & unbegrenzt & Freistellung (100\%) & Freistellung ( $100 \%)$ \\
\hline Österreich & - & unbegrenzt $^{678}$ & Freistellung ( $100 \%)$ & Freistellung (100\%) \\
\hline Polen & - & 5 Jahre $^{679}$ & Freistellung $(100 \%)$ & Anrechnung \\
\hline Portugal & - & 6 Jahre & Freistellung $(100 \%)$ & Freistellung (100\%) \\
\hline Schweden & - & unbegrenzt & Freistellung (100\%) & Freistellung ( $100 \%)$ \\
\hline Slowakei & - & 5 Jahre & Freistellung (100\%) & Freistellung ( $100 \%)$ \\
\hline Spanien & - & 15 Jahre & Anrechnung & Freistellung ( $100 \%)$ \\
\hline Tsch'e Republik & - & 5 Jahre & Freistellung (100\%) & Freistellung ( $100 \%)$ \\
\hline Ungam & - & unbegrenzt & Freistellung ( $100 \%)$ & Freistellung ( $100 \%)$ \\
\hline Zypern & - & unbegrenzt & Freistellung ( $100 \%)$ & Freistellung ( $100 \%)$ \\
\hline
\end{tabular}

Quelle: Oestreicher/Scheffler/Spengel et al. (2008); Endres/Oestreicher/Scheffler (2007) et al.; Kesti (2006)

675 Maximal 511.500€ verrechenbar.

676 Maximal $1.000 .000 €$ zuzüglich $60 \%$ des verbleibenden positiven Einkommens einer Periode verrechenbar.

677 Aus Vereinfachungsgründen wird auf eine Abbildung der nachgelagerten Besteuerung in Estland verzichtet. Für die Simulation der Einkommensermittlung wird hier von einem betragsmäßig und zeitlich unbegrenzten Verlustvortrag ohne Verlustrücktrag ausgegangen.

678 Maximal 75\% des Einkommens einer Periode verrechenbar.

679 Maximal 50\% des Verlustvortrags mit dem Einkommen einer Periode verrechenbar. 
Tabelle 51: Steuerrechtliche Grundlage der Simulationsberechnungen (2)

\begin{tabular}{|c|c|c|c|c|c|}
\hline & \multicolumn{5}{|c|}{ Anwendungsvoraussetzungen der Gruppenbesteuerungssysteme } \\
\hline & $\mathrm{ja} /$ nein & $\begin{array}{l}\text { Mindestbeteili- } \\
\text { gung }\end{array}$ & indirekte Anteile & $\begin{array}{l}\text { Schwestergesell- } \\
\text { schaften }\end{array}$ & $\begin{array}{l}\text { sonstige } \\
\text { Anforde- }\end{array}$ \\
\hline Belgien & nein & - & - & - & \\
\hline Dänemark & ja & mind. $51 \%$ & ja, additiv & ja & \\
\hline Deutschland & ja & $>60 \%$ & ja, additiv & nein & $\begin{array}{l}\text { Ergebnisab- } \\
\text { fuhrungs- } \\
\text { vertrag }\end{array}$ \\
\hline Estland & nein & - & - & - & \\
\hline Finnland & ja & mind. $90 \%$ & ja, additiv & nein & \\
\hline Frankreich & ja & mind. $95 \%$ & ja, additiv & nein & \\
\hline Griechenland & nein & - & - & - & \\
\hline Großbritannien & $\mathrm{ja}$ & mind. $75 \%$ & ja, additiv & ja & \\
\hline Irland & ja & mind. $75 \%$ & ja, additiv & ja & \\
\hline Italien & ja & $>50 \%$ & ja, additiv & $\mathrm{ja}$ & \\
\hline Lettland & ja & mind. $90 \%$ & nein & $\mathrm{ja}$ & \\
\hline Litauen & nein & - & - & - & \\
\hline Luxemburg & ja & mind. $95 \%$ & ja, additiv & nein & \\
\hline Niederlande & ja & mind. $95 \%$ & ja, additiv & nein & \\
\hline Österreich & ja & $>50 \%$ & ja, additiv & nein & \\
\hline Polen & ja & mind. $95 \%$ & nein & nein & \\
\hline Portugal & ja & mind. $90 \%$ & ja, multiplikativ & nein & \\
\hline Schweden & ja & $>90 \%$ & ja, additiv & ja & \\
\hline Slowakei & nein & - & - & - & \\
\hline Spanien & $\mathrm{ja}$ & mind. $75 \%$ & ja, multiplikativ & nein & \\
\hline Tsch'e Republik & nein & - & - & - & \\
\hline Ungarn & nein & - & - & - & \\
\hline Zypern & ja & mind. $75 \%$ & $\mathrm{ja}$, additiv & ja & \\
\hline
\end{tabular}


Tabelle 52: Steuerrechtliche Grundlage der Simulationsberechnungen (3)

\begin{tabular}{|c|c|c|c|c|c|c|c|c|c|c|c|c|}
\hline & \multicolumn{12}{|c|}{ Nominelle Ertragsteuerbelastung für inländische Einkünfte } \\
\hline & 1994 & 1995 & 1996 & 1997 & 1998 & 1999 & 2000 & 2001 & 2002 & 2003 & 2004 & 2006 \\
\hline Belgien & 40,17 & 40,17 & 40,17 & 40,17 & 40,17 & 40,17 & 40,17 & 40,17 & 40,17 & 33,99 & 33,99 & 33,99 \\
\hline Dänemark & 34,00 & 34,00 & 34,00 & 34,00 & 34,00 & 32,00 & 32,00 & 30,00 & 30,00 & 30,00 & 30,00 & 28,00 \\
\hline Deutschland & 55,64 & 59,00 & 59,00 & 57,50 & 56,60 & 52,30 & 51,60 & 38,36 & 38,36 & 39,58 & 38,29 & 38,34 \\
\hline Estland & 26,00 & 26,00 & 26,00 & 26,00 & 26,00 & 26,00 & 26,00 & 26,00 & 26,00 & 26,00 & 26,00 & 23,00 \\
\hline Finnland & 25,00 & 25,00 & 28,00 & 28,00 & 28,00 & 28,00 & 29,00 & 29,00 & 29,00 & 29,00 & 29,00 & 26,00 \\
\hline Frankreich & 33,33 & 36,66 & 36,66 & 36,66 & 41,66 & 40,00 & 36,66 & 35,33 & 34,33 & 34,33 & 34,33 & 33,33 \\
\hline G'britannien & 33,00 & 33,00 & 33,00 & 31,00 & 31,00 & 31,00 & 30,00 & 30,00 & 30,00 & 30,00 & 30,00 & 30,00 \\
\hline Griechenland & 35,00 & 35,00 & 35,00 & 40,00 & 40,00 & 40,00 & 40,00 & 37,50 & 35,00 & 35,00 & 35,00 & 29,00 \\
\hline Irland & 10,00 & 10,00 & 10,00 & 10,00 & 10,00 & 10,00 & 10,00 & 10,00 & 10,00 & 12,50 & 12,50 & 12,50 \\
\hline Italien & 52,20 & 53,20 & 53,20 & 53,20 & 41,25 & 41,25 & 41,25 & 40,25 & 40,25 & 38,25 & 37,25 & 37,25 \\
\hline Lettland & 25,00 & 25,00 & 25,00 & 25,00 & 25,00 & 25,00 & 25,00 & 25,00 & 22,00 & 19,00 & 15,00 & 15,00 \\
\hline Litauen & 29,00 & 29,00 & 29,00 & 29,00 & 29,00 & 29,00 & 24,00 & 24,00 & 15,00 & 15,00 & 15,00 & 15,00 \\
\hline Luxemburg & 39,39 & 40,29 & 40,29 & 39,35 & 37,45 & 37,45 & 37,45 & 37,45 & 30,38 & 30,38 & 30,38 & 29,63 \\
\hline Niederlande & 35,00 & 35,00 & 35,00 & 35,00 & 35,00 & 35,00 & 35,00 & 35,00 & 34,50 & 34,50 & 34,50 & 29,60 \\
\hline Österreich & 34,00 & 34,00 & 34,00 & 34,00 & 34,00 & 34,00 & 34,00 & 34,00 & 34,00 & 34,00 & 34,00 & 25,00 \\
\hline Polen & 40,00 & 40,00 & 40,00 & 38,00 & 36,00 & 34,00 & 30,00 & 28,00 & 28,00 & 27,00 & 19,00 & 19,00 \\
\hline Portugal & 39,60 & 39,60 & 39,60 & 39,60 & 37,40 & 37,40 & 37,40 & 35,20 & 33,00 & 33,00 & 27,50 & 27,50 \\
\hline Schweden & 28,00 & 28,00 & 28,00 & 28,00 & 28,00 & 28,00 & 28,00 & 28,00 & 28,00 & 28,00 & 28,00 & 28,00 \\
\hline Slowakei & 40,00 & 40,00 & 40,00 & 40,00 & 40,00 & 40,00 & 40,00 & 29,00 & 25,00 & 25,00 & 19,00 & 19,00 \\
\hline Spanien & 35,00 & 35,00 & 35,00 & 35,00 & 35,00 & 35,00 & 35,00 & 35,00 & 35,00 & 35,00 & 35,00 & 35,00 \\
\hline Tsch'e Rep. & 45,00 & 41,00 & 39,00 & 25,00 & 25,00 & 35,00 & 31,00 & 31,00 & 31,00 & 31,00 & 28,00 & 24,00 \\
\hline Ungam & 33,00 & 33,00 & 33,00 & 18,00 & 18,00 & 18,00 & 18,00 & 18,00 & 18,00 & 18,00 & 16,00 & 16,00 \\
\hline Zypern & 25,00 & 25,00 & 25,00 & 25,00 & 29,00 & 29,00 & 29,00 & 28,00 & 28,00 & 15,00 & 15,00 & 10,00 \\
\hline
\end{tabular}

Quelle: KPMG (2006); Angaben aus den European Tax Handbooks der jeweiligen Jahrgänge 
Tabelle 53: Steuerrechtliche Grundlage der Simulationsberechnungen (4)

\begin{tabular}{|c|c|c|c|c|c|c|c|c|c|c|c|c|}
\hline & \multicolumn{12}{|c|}{ Nominelle Ertragsteuerbelastung für ausländische Einkünfte } \\
\hline & 1994 & 1995 & 1996 & 1997 & 1998 & 1999 & 2000 & 2001 & 2002 & 2003 & 2004 & 2006 \\
\hline Belgien & 40,17 & 40,17 & 40,17 & 40,17 & 40,17 & 40,17 & 40,17 & 40,17 & 40,17 & 33,99 & 33,99 & 33,99 \\
\hline Dänemark & 34,00 & 34,00 & 34,00 & 34,00 & 34,00 & 32,00 & 32,00 & 30,00 & 30,00 & 30,00 & 30,00 & 28,00 \\
\hline Deutschland & 45,00 & 48,38 & 48,38 & 48,38 & 47,48 & 42,20 & 42,20 & 26,38 & 26,38 & 27,96 & 26,38 & 26,38 \\
\hline Estland & 26,00 & 26,00 & 26,00 & 26,00 & 26,00 & 26,00 & 26,00 & 26,00 & 26,00 & 26,00 & 26,00 & 23,00 \\
\hline Finnland & 25,00 & 25,00 & 28,00 & 28,00 & 28,00 & 28,00 & 29,00 & 29,00 & 29,00 & 29,00 & 29,00 & 26,00 \\
\hline Frankreich & 33,33 & 36,66 & 36,66 & 36,66 & 41,66 & 40,00 & 36,66 & 35,33 & 34,33 & 34,33 & 34,33 & 33,33 \\
\hline G'britannien & 33,00 & 33,00 & 33,00 & 31,00 & 31,00 & 31,00 & 30,00 & 30,00 & 30,00 & 30,00 & 30,00 & 30,00 \\
\hline Griechenland & 35,00 & 35,00 & 35,00 & 40,00 & 40,00 & 40,00 & 40,00 & 37,50 & 35,00 & 35,00 & 35,00 & 29,00 \\
\hline Irland & 10,00 & 10,00 & 10,00 & 10,00 & 10,00 & 10,00 & 10,00 & 10,00 & 10,00 & 12,50 & 12,50 & 12,50 \\
\hline Italien & 36,00 & 37,00 & 37,00 & 37,00 & 37,00 & 37,00 & 37,00 & 36,00 & 36,00 & 34,00 & 33,00 & 33,00 \\
\hline Lettland & 25,00 & 25,00 & 25,00 & 25,00 & 25,00 & 25,00 & 25,00 & 25,00 & 22,00 & 19,00 & 15,00 & 15,00 \\
\hline Litauen & 29,00 & 29,00 & 29,00 & 29,00 & 29,00 & 29,00 & 24,00 & 24,00 & 15,00 & 15,00 & 15,00 & 15,00 \\
\hline Luxemburg & 33,33 & 34,32 & 34,32 & 33,28 & 31,20 & 31,20 & 31,20 & 31,20 & 22,88 & 22,88 & 22,88 & 23,10 \\
\hline Niederlande & 35,00 & 35,00 & 35,00 & 35,00 & 35,00 & 35,00 & 35,00 & 35,00 & 34,50 & 34,50 & 34,50 & 29,60 \\
\hline Österreich & 34,00 & 34,00 & 34,00 & 34,00 & 34,00 & 34,00 & 34,00 & 34,00 & 34,00 & 34,00 & 34,00 & 25,00 \\
\hline Polen & 40,00 & 40,00 & 40,00 & 38,00 & 36,00 & 34,00 & 30,00 & 28,00 & 28,00 & 27,00 & 19,00 & 19,00 \\
\hline Portugal & 39,60 & 39,60 & 39,60 & 39,60 & 37,40 & 37,40 & 37,40 & 35,20 & 33,00 & 33,00 & 27,50 & 27,50 \\
\hline Schweden & 28,00 & 28,00 & 28,00 & 28,00 & 28,00 & 28,00 & 28,00 & 28,00 & 28,00 & 28,00 & 28,00 & 28,00 \\
\hline Slowakei & 40,00 & 40,00 & 40,00 & 40,00 & 40,00 & 40,00 & 40,00 & 29,00 & 25,00 & 25,00 & 19,00 & 19,00 \\
\hline Spanien & 35,00 & 35,00 & 35,00 & 35,00 & 35,00 & 35,00 & 35,00 & 35,00 & 35,00 & 35,00 & 35,00 & 35,00 \\
\hline Tsch'e Rep. & 45,00 & 41,00 & 39,00 & 25,00 & 25,00 & 35,00 & 31,00 & 31,00 & 31,00 & 31,00 & 28,00 & 24,00 \\
\hline Ungarn & 33,00 & 33,00 & 33,00 & 18,00 & 18,00 & 18,00 & 18,00 & 18,00 & 18,00 & 18,00 & 16,00 & 16,00 \\
\hline Zypern & 25,00 & 25,00 & 25,00 & 25,00 & 29,00 & 29,00 & 29,00 & 28,00 & 28,00 & 15,00 & 15,00 & 10,00 \\
\hline
\end{tabular}

Quelle: KPMG (2006); Angaben aus den European Tax Handbooks der jeweiligen Jahrgänge 
Tabelle 54: Korrelationen zwischen den unabhängigen Variablen

\begin{tabular}{|c|c|c|c|c|c|c|c|c|c|c|c|}
\hline & NOB & OB_M & OB_T & E10 & TR & $\begin{array}{l}\mathrm{TR}_{-} \\
\mathrm{OB}\end{array}$ & $\begin{array}{l}\mathrm{TR}_{-} \\
\text {red }\end{array}$ & GTS & LC & LC_T & PRO \\
\hline NIB & -.335 & $-.690^{* *}$ & -.249 & $-.837 * *$ & -.357 & -.245 & -.154 & -.283 & -.252 & -.248 & .382 \\
\hline NOB & & .322 & $.761^{* *}$ & .419 & .040 & .114 & .233 & .424 & .101 & .054 & -.035 \\
\hline OB_M & & & .104 & $.547^{*}$ & $.533^{*}$ & $.552^{*}$ & .060 & .110 & .024 & .123 & $.620 * *$ \\
\hline OB_T & & & & .393 & & -.204 & .409 & .336 & .458 & -.009 & .186 \\
\hline E10 & & & & & .305 & .105 & $.493^{*}$ & .332 &. .211 & .153 & -.060 \\
\hline TR & & & & & & $.901^{* *}$ & -.385 & -.295 & .121 & .155 & $-.546^{*}$ \\
\hline TR_OB & & & & & & & $-.562 *$ & -.373 & -.173 & .002 & $-.582^{*}$ \\
\hline TR_red & & & & & & & & .346 & .327 & -.093 & .340 \\
\hline GTS & & & & & & & & & .006 & .156 & .130 \\
\hline LC & & & & & & & & & & -.021 & -.163 \\
\hline LC_T & & & & & & & & & & & -.008 \\
\hline
\end{tabular}

Quelle: eigene Berechnungen 
Reinald Koch - 978-3-631-75683-6

Downloaded from PubFactory at 01/11/2019 03:01:46AM

via free access 


\section{Literaturverzeichnis}

Agundez-Garcia, A. (2006), The Delineation and Apportionment of an EU Consolidated Tax Base for Multi-jurisdictional Corporate Income Taxation: A Review of Issues and Options, Working Paper No. 9/2006 TAXUD/2006/3202, Luxembourg 2006

Anand, B./Sansing, R. (2000), The Weighting Game: Formula Apportionment as an Instrument of Public Policy, in: National Tax Journal 2000, S. 183-199

Arbeitsgruppe GKKB (2006), Bisherige Fortschritte und künftiges Programm für die GKKB, CCCTB/ WP046, Brüssel 2006

Arbeitsgruppe GKKB (2006a), Fragen zur Konzernbesteuerung, CCCTB/WP035, Brüssel 2006

Arbeitsgruppe GKKB (2007), GKKB: mögliche Elemente der technischen Ausgestaltung, CCCTB/WP057, Brüssel 2007

Arbeitsgruppe GKKB (2007a), GKKB: mögliche Elemente des Aufteilungsmechanismus, CCCTB/WP060, Brüssel 2007

Arbeitsgruppe GKKB (2007b), Input from national tax administrations for the Impact Assessment of the reforms at the EU level of corporate tax systems, CCCTB/WP058, Brüssel 2007

Baetge, J./Kirsch, H.-J./Thiele, S. (2004), Konzernbilanzen, 7. Auflage, Düsseldorf 2004

Bartelsmann, E./Beetsma, R. (2003), Why pay more? Corporate tax avoidance through transfer pricing in OECD countries, in: Journal of Public Economics 2003, S. 2225-2252

Blaschka, B. (1982), Profit centers in gesellschaftsrechtlicher Form, in: Zeitschrift für Betriebswirtschaft 1982, S. 397-403

Blumenberg, J. (2008), Unsere Steuern 2033 aus der Sicht von Jens Blumenberg, Frankfurt, in: Rädler, A. J. (Hrsg.), Tax Science Fiction, Wie sieht unser Steuerrecht in 25 Jahren aus?, München 2008, S. 13-19

Breuninger, G.E./Ernst, M. (2009), Abschied vom Abzug endgültig gewordener ausländischer Betriebsstättenverluste im Inland? Kein „Import-Stopp" nach 
der EuGH-Entscheidung Wannsee!, in: Deutsches Steuerrecht 2009, S. 1981-1985

Büttner, T./Overesch, M./Schreiber, U. et al. (2008), The Impact of ThinCapitalization Rules on Multinationals' Financing and Investment Decision, Bundesbank Discussion Paper 03/2008

Buijink, W./Jannsen, B./Schols, Y. (2000), Evidence of the effect of domicile on corporate average effective tax rates in the European Union, MARC Working Paper WP/3/2000-11

Bundesverband der deutschen Industrie/PriceWaterhouseCoopers (2006), Verlustberücksichtigung über Grenzen hinweg, Frankfurt am Main 2006

Center for European Policy Studies (2005) (Hrsg.), Achieving a Common Consolidated Corporate Tax Base in the EU, Brüssel 2005

Chennels, L./Griffith, R. (1997), Taxing profits in a changing world, IFS Working Paper, 1997

Clarebout, X./Dhaene, M. (2005), Country Reports - EU Member States - Belgium, in: European Taxation 2005, S. 372-377

Clausing, K. (2003), Tax-motivated transfer pricing and US intrafirm trade prices, in: Journal of Public Economics 2003, S. 2207-2223

Cnossen, S. (2004), Reform and Coordination of Corporation Taxes in the European Union: An Alternative Agenda, in: Bulletin for international fiscal documents 2004, S. 134-150

Collins, J. H./Shackelford, D. A. (1992), Foreign Tax Credit Limitations and Preferred Stock Issuances, in: Journal of Accounting Research 1992, Supplement, S. 103-124

Collins, J. H./Shackelford, D. A. (1995), Corporate Domicile and Average Effective Tax Rates: The Cases of Canada, Japan, the United Kingdom, and the United States, in: International Tax and Public Finance 1995, S. 55-83

Cordewener, A./Dahlberg, M./Pistone, P. et al. (2004), The Tax Treatment of Foreign Losses: Ritter, M \& S, and the Way Ahead (Part One), in: European Taxation 2004, S. 135-142 
Cordewener, A./Schnitger, A. (2006), Europarechtliche Vorgaben für die Vermeidung der internationalen Doppelbesteuerung im Wege der Anrechnungsmethode, in: Steuer und Wirtschaft 2006, S. 50-79

Creedy, J./Gemmel, N. (2007), Modelling Behavioural Responses to Profit taxation: The Case of the UK Corporation Tax, The University of Melbourne Department of Economics Research paper Number 998, Melbourne 2007

Czakert, E. (2006), Der Stand der Arbeiten an einer gemeinsamen konsolidierten Körperschaftsteuer-Bemessungsgrundlage in der Europäischen Union, in: Internationales Steuerrecht 2006, S. 561-566

Czakert, E. (2008), Ansatz der CCCTB working group zur Harmonisierung der Steuerbemessungsgrundlage in Europa, in: Betriebswirtschaftliche Forschung und Praxis 2008, S. 433-438

De Mooij, R. A./Ederveen, S. (2008), Corporate tax elasticities: a reader's guide to empirical findings, in: Oxford Review of Economic Policy 2008, S. 680697

Devereux, M. P. (2004), Debating proposed reforms of the taxation of corporate income in the European Union, in: International Tax and Public Finance 2004, S. $71-90$

Devereux, M. P./Elschner, C./Endres, D. et al. (2008), Project for the EU Commission TAXUD/2005/DE/3 10 - Final Report, Mannheim/Oxford 2008, abrufbar unter: www.zew.de

Devereux, M. P./Griffith, R. (1999), The Taxation of Discrete Investment Choices, Institute for Fiscal Studies Working Paper Series W98/16, 1999

Devereux, M.P./Loretz, S. (2008), The Effects of EU Formula Apportionment on Corporate Tax Revenues, in: Fiscal Studies 2008, S. 1-34

Devereux, M.P./Loretz; S. (2008a), Increased efficiency through consolidation and formula apportionment in the European Union?, Oxford University Centre for Business Taxation WP 08/12, Oxford 2008

Domar, E. D. / Musgrave, R.A. (1944), Proportional Income Taxation and RiskTaking, in: The Quarterly Journal of Economics 1944, S. 388-422.

Doralt, P./Feyl , P. (2005), Country Reports - EU Member States -Austria, in: European Taxation 2005, S. 370-371 
Dörr, I. (2008), Verlustbehandlung im Rahmen der Konzernbesteuerung in Europa, Frankfurt/Main u.a. 2008

Drumm, H.-J. (1982), Rechtlich selbständige Geschäftsbereiche - ein allgemeines Modell?, in: Zeitschrift für Betriebswirtschaft 1982, S. 404-407

Eibelshäuser, M. (2002), Wirtschaftliche Betrachtungsweise im Steuerrecht Herkunft und Bedeutung, in: Deutsches Steuerrecht 2002, S. 1426-1431

Elschner, C./Lammersen, L./Overesch, M. et al. (2006), The effective tax burden of companies and on highly skilled manpower: Tax policy strategies in a globalised economy, in: Fiscal Studies 2006, S. 513-534

Elschner, C./Schwager, R. (2007), A simulation method to measure the effective tax rate on highly skilled labour, in: Finanzarchiv 2007, S. 563-582

Emmerich, V./Habersack, M. (2005), Konzernrecht, München 2005

Endres, D. (2003), Konzernbesteuerung in wichtigen Industriestaaten, in: Herzig, N. (Hrsg.), Organschaft, Stuttgart, S. 461-482

Endres, D./Oestreicher, A./Scheffler, W. et al. (2007), The Determination of Corporate Taxable Income in the EU Member States, Alphen aan den Rijn 2007

Endres, D./Oestreicher, A./Scheffler, W. et al. (Hrsg.), Die internationale Unternehmensbesteuerung im Wandel, München 2005

Ernst\&Young (2007), 2007-2008 Global Transfer Pricing Survey: Global Transfer Pricing Trends, Practices and Analyses, 2007

Feldstein, M./Hines, J. R./Hubbard, R. (Hrsg.), The Effects of Taxation on Multinational Corporations, Chicago 1995

Flick, H./Wassermeyer, F./Baumhoff, H. (2006),§§ 1-6 AStG, in: Wassermeyer, F./Piltz, D. J. (Hrsg.), Außensteuerrecht: Kommentar, Köln 2006

Fox, W. F./Luna, L. (2002), State Corporate Tax Revenue Trends: Causes and Possible Solutions, in: National Tax Journal 2002, S. 491-508

Fox, W. F./Murray, M. N./Luna, L. (2005), How Should a Subnational Corporate Income Tax on Multistate Business Be Structured?, in: National Tax Journal 2005, S. $139-159$

Froot, K. A./Hines , J. R. (1995), Interest allocation rules, financing patterns and the operations of US multinationals, in: Feldstein, M./Hines, J. R./Hubbard, 
R. (Hrsg.), The Effects of Taxation on Multinational Corporations, Chicago: University of Chicago Press, S. 277-307

Frotscher, G./Oestreicher, A. (2009), The German Approach to Taxing Business Restructurings: An Arm's Length Ahead?, in: Intertax 2009, S. 375-381

Fuest, C./Hemmelgarn, T./Ramb, F. (2005), Wirkungen einer EU-weiten Verlustverrechnung auf das Steueraufkommen, in: Wirtschaftsdienst 2005, S. 365369

Fuest, C./Hemmelgarn, T./Ramb, F. (2007), How would the introduction of an EU-wide formula apportionment affect the distribution and size of the corporate tax base? : An analysis based on German multinationals, in: International tax and public finance 2007, S.605-626

Gangemi, B. (2005), Country Reports - EU Member States - Italy, in: European Taxation 2005, S. 404-407

Goolsbee, A./Maydew, E.L. (2000), Coveting thy neighbor's manufacturing: the dilemma of state income apportionment, in: Journal of Public Economics 2000 , S. $125-144$

Gorter, J./De Mooij, R. (2001), Capital Income Taxation in Europe: Trends and Trade-Offs, Den Haag 2001

Gouthière, B. (2005), A Comparative Study of the Thin Capitalization Rules in the Member States of the European Union and Certain Other States - Special Issue - Introduction, in: European Taxation 2005, S. 367-368

Grochla, E. (Hrsg.), Handwörterbuch der Organisation, 2. Aufl., Stuttgart 1980

Grotherr, S. (1993), Die Eignung des körperschaftsteuerlichen Vollanrechnungssystems als Modell für den Europäischen Binnenmarkt unter internationalem Blickwinkel, in: Internationales Steuerrecht 1993, Beihefter zu Heft 5, S. $1-8$

Grotherr, S. (1996), Die unterschiedlichen Konzernbesteuerungssysteme in den Mitgliedstaaten der Europäischen Union, in: Steuer und Wirtschaft 1996, S. 356-378

Grubert, H./Mutti, J. (1991), Taxes, tariffs and transfer pricing in multinational corporate decision making, in: Review of Economcis and Statistics 1991, S. 285-293 
Grunewald, B. (2005), Gesellschaftsrecht, 6. Aufl., Tübingen 2005

Gupta, S./Hofmann, M.A. (2003), The effect of state income tax apportionment and tax incentives on new capital expenditures, in: The journal of the American Taxation Association 2003, Beilage, S. 1-25

Gupta, S./Mills, L. (2003), Does Disconformity in State Corporate Income Tax Systems Affect Compliance Cost Burdens?, in: National Tax Journal 2003, S. $355-371$

Gutenberg, E. (1983), Grundlagen der Betriebswirtschaftlehre, Erster Band: Produktion, 24. Auflage, Berlin/Heidelberg/New York 1983

Haegert, L. / Kramm, R. (1975), Der Einfluss von Ertragsteuern auf die Vorteilhaftigkeit von Investitionen mit unterschiedlichem Risiko, in: Zeitschrift für betriebswirtschaftliche Forschung 1975, S. 69-83

Haussmann, F. (1926), Grundlegung des Rechts der Unternehmenszusammenfassungen, Mannheim/Berlin/Leipzig 1926

Hellerstein, J. R. (1982), Allocation and Apportionment of Dividends and the delineation of the Unitary Business, in: Tax Notes 1982, Special Report

Hellerstein, J. R./Hellerstein, W. (1998), State Taxation, 3. Aufl., Boston 1998

Hellerstein, W. (2001), The Business/Non-Business Income Distinction and The Case for Its Abolition, in: State Tax Notes 2001, S. 725-739

Hellerstein, W./McLure, C. E. (2004), The European Commission's Report on Company Income Taxation: What the EU Can Learn from the Experience of the US States, in: International Tax and Public Finance 2004, S. 199-220

Herzig, N. (2003), Einführung, in: Herzig, N. (Hrsg.), Organschaft, Stuttgart 2003, S. $1-35$

Herzig, N. (2008), Tax Harmonization in Europe: Methods of consolidation, in: Lang, M./Pistone, P./Schuch, J. et al. (Hrsg.), Common Consolidated Corporate Tax Base, Wien 2008, S. 547-572

Herzig, N. (Hrsg.), Besteuerung der Europäischen Aktiengesellschaft, Köln 2004

Herzig, N. (Hrsg.), Organschaft, Stuttgart 2003 
Herzig, N./ Englisch, J./ Wagner, T. (2005), Steuerliche Berücksichtigung von Verlusten ausländischer Konzerntöchter, in: Der Konzern 2005, S. 298-318

Herzig, N./Wagner, T. (2005), Zukunft der Organschaft im EUG-Binnenmarkt, in: Der Betrieb 2005, S. 1-9

Hey, J. (2005), Erosion nationaler Besteuerungsprinzipien im Binnenmarkt?- zugleich zu den Rechtfertigungsgründen der „Europatauglichkeit“ und „Wettbewerbsfähigkeit" des Steuersystems, in: Steuer und Wirtschaft 2005, S. 317-326

Hey, J. (2008), Gestaltungsspielraum des Gesetzgebers und Sicherung des Steueraufkommens, in: Finanz-Rundschau 2008, S. 1033-1080

Hickson, J. H. (2005), Country Reports - EU Member States - Ireland, in: European Taxation 2005, S. 402-404

Hines, J. R./ Rice, E. (1994), Fiscal Paradise: Foreign Tax Havens and American Business, in: The quarterly journal of economics 1994, S. 149-182

Hines, J. R./Hubbard, R. G. (1990), Coming home to America: dividend repatriations by US multinationals, in: Razin, A. (Hrsg.), Taxation in the Global Economy, Chicago 1990, S. 161-200

Homburg, S. (2007), Allgemeine Steuerlehre, München 2007

Hübner, H. (1980), Recht und Organisation, in: Grochla, E. (Hrsg.), Handwörterbuch der Organisation, 2. Aufl., Stuttgart 1980

Huizinga, H./Laeven, L. (2005), International Profit Shifting within European Multinationals, Tilburg University Working Paper, 2005

Isay, R. (1910), Das Recht am Unternehmen, Berlin 1910

Jacobs, O.H. ( 2007), Internationale Unternehmensbesteuerung, 6. Auflage, München 2007

Jacobs, O.H. (2005), Harmonisierung der Unternehmensbesteuerung in Europa, in: Der Schweizer Treuhänder 2005, S. 133-146

Jacobs, O.H. (2009), Unternehmensbesteuerung und Rechtsform, 4. Auflage, München 2009

Jacobs, O.H./Spengel, C. (1996), European Tax Analyzer, Baden-Baden 1996 
Jurkat, W. (1975), Die Organschaft im Körperschaftsteuerrecht, Heidelberg 1975

Kersting, C. (2005), Das Verhältnis zwischen handelsrechtlicher und steuerrechtlicher Rechnungslegung in Großbritannien, in: Schön, W. (Hrsg.), Steuerliche Maßgeblichkeit in Deutschland und Europa, Köln 2005, S. 281-363

Kessler, W. (2004), Rahmenbedingungen der Konzernbesteuerung in Deutschland, in: Kessler, W./Kröner, M./Köhler, S. (Hrsg.), Konzernsteuerrecht, München 2004, S. 1-28

Kessler, W./Kröner, M./Köhler, S. (Hrsg.), Konzernsteuerrecht, München 2004, S. $1-28$

Kesti, J. (2006), European Tax Handbook 2006, Amsterdam 2006

Kesti, J. (2008), European Tax Handbook 2008, Amsterdam 2008

Kirchner, C. (1984), Ansätze zu einer ökonomischen Analyse des Konzernrechts, in: Jahrbuch für Neue Politische Ökonomie 1984, S. 223-251

Klassen, K.J./Shackelford, D.A. (1998), State and provincial corporate tax planning: income shifting and sales apportionment factor management, in: Journal of accounting and economics 1998, S. 385-406

Koch, R./Prassel, J. (2009), Die Auswirkungen einer Reform der steuerlichen Verlustverrechnung auf die unternehmerische Risikoübernahme, unveröffentlichtes Working Paper, Göttingen 2009

Kommission der Europäischen Gemeinschaften (1991), Vorschlag für eine Richtlinie des Rates über eine Regelung für Unternehmen zur Berücksichtigung der Verluste ihrer in anderen Mitgliedsstaaten belegenen Betriebsstätten und Tochtergesellschaften, KOM (90) 595 endg., Brüssel 1991

Kommission der Europäischen Gemeinschaften (1996), Bericht der Kommission an den Rat: "Die Steuern in der Europäischen Union - Bericht über Entwicklung der Steuersysteme", KOM(96) 546 endg., Brüssel 1996

Kommission der Europäischen Gemeinschaften (2001), Mitteilung der Kommission an den Rat, das europäische Parlament und an den europäischen Wirtschafts- und Sozialausschuss vom 23.5.2001: „Steuerpolitik in der Europäischen Union - Prioritäten für die nächsten Jahre“, KOM (2001) 260 endg., Brüssel 2001 
Kommission der Europäischen Gemeinschaften (2001a), Mitteilung der Kommission an den Rat, das europäische Parlament und an den europäischen Wirtschafts- und Sozialausschuss vom 23.10.2001: „Ein Binnenmarkt ohne steuerliche Hindernisse - Strategie zur Schaffung einer konsolidierten Körperschaftsteuer-Bemessungsgrundlage für die grenzüberschreitende Unternehmenstätigkeit in der EU“, KOM (2001) 582 endg., Brüssel 2001

Kommission der Europäischen Gemeinschaften (2002), Arbeitsdokument der Dienststellen der Europäischen Kommission: „Unternehmensbesteuerung im Binnenmarkt“, KOM(2001) 582 endg., Luxemburg 2002

Kommission der Europäischen Gemeinschaften (2003), Mitteilung der Kommission an den Rat, das europäische Parlament und an den europäischen Wirtschafts- und Sozialausschuss vom 24.11.2003: „Ein Binnenmarkt ohne unternehmenssteuerlich Hindernisse - Ergebnisse, Initiativen, Herausforderungen, KOM (2003) 726 endg., Brüssel 2003

Kommission der Europäischen Gemeinschaften (2004), European Tax Survey, SEC (2004), 1128/2, Brüssel 2004

Kommission der Europäischen Gemeinschaften (2005), Mitteilung der Kommission an den Rat, das europäische Parlament und den europäischen Wirtschafts- und Sozialausschuss vom 25.10.2005: „Der Beitrag der Steuer- und Zollpolitik zur Lissabon-Strategie“, KOM (2005) 532 endg., Brüssel 2005

Kommission der Europäischen Gemeinschaften (2006), Mitteilung der Kommission an den Rat, das europäische Parlament und den europäischen Wirtschafts- und Sozialausschuss vom 19.10.2006: „Steuerliche Behandlung von Verlusten bei grenzübergreifenden Sachverhalten", KOM (2006) 824 endg., Brüssel 2006

Kommission der Europäischen Gemeinschaften (2006a), Mitteilung der Kommission an den Rat, das europäische Parlament und an den europäischen Wirtschafts- und Sozialausschuss vom 19.12.2006: „Koordinierung der Regelungen der Mitgliedstaaten zu den direkten Steuern im Binnenmarkt", KOM (2006) 823 endg., Brüssel 2006

Kommission der Europäischen Gemeinschaften (2006b), Mitteilung der Kommission an den Rat, das europäische Parlament und den europäischen Wirtschafts- und Sozialausschuss vom 5.4.2006: „Umsetzung des LissabonProgramms der Gemeinschaft: Bisherige Fortschritte und weitere Schritte zu einer gemeinsamen konsolidierten Körperschaftsteuer-Bemessungsgrundlage (GKKB)“, KOM (2006) 157 endg., Brüssel 2006 
Kommission der Europäischen Gemeinschaften (2007), Mitteilung der Kommission an den Rat, das europäische Parlament und den europäischen Wirtschafts- und Sozialausschuss, „Anwendung von Maßnahmen zur Missbrauchsbekämpfung im Bereich der direkten Steuern (innerhalb der EU und im Hinblick auf Drittländer), KOM (2007) 785 endg., Brüssel 2007

Kommission der Europäischen Gemeinschaften (2007a), Mitteilung der Kommission an den Rat, das europäische Parlament und den europäischen Wirtschafts- und Sozialausschuss vom 2.5.2007: „Umsetzung des Programms der Gemeinschaft für mehr Wachstum und Beschäftigung und eine Steigerung der Wettbewerbsfähigkeit von EU-Unternehmen: Weitere Fortschritte im Jahr 2006 und nächste Schritte zu einem Vorschlag einer gemeinsamen konsolidierten Körperschaftsteuer-Bemessungsgrundlage (GKKB)“, KOM (2007) 223 endg., Brüssel 2007

Kosiol, E. (1956), Unternehmung, in: Seischab, H./Schwantag, K. (Hrsg.), Handwörterbuch der Betriebswirtschaft, Band 1, 3. Auflage, Stuttgart 1956, Sp. 5540-5545

KPMG (2006), Corporate Tax Rate Survey: An international analysis of corporate tax rates from 1993 to 2006, abrufbar unter: http://www.kpmg.com

KPMG (2007), EU business wants single, pan-European tax system, says KPMG International study, abrufbar unter: http://www.kpmg.co.uk/news/detail. $\mathrm{cfm}$ ?pr $=2943$

Krebühl, H.-H. (2003), Konzernbesteuerung de lege ferenda, in: Herzig, N. (Hrsg.), Organschaft, Stuttgart 2003, S. 595-611

Küting, K. (1990), Ein erneutes Plädoyer für eine Einheitsbesteuerung, in: Der Betrieb 1990, S. 489-497

Lamprecht, P. (2008), Betriebsstättenverluste, Verlustvortragsrecht und Aufteilung der Besteuerungsbefugnisse nach dem Urteil des EuGH in der Rs. KR Wannsee, in: Internationales Steuerrecht 2008, S. 766-769

Lang, M. (2008), Unsere Steuern 2033 aus der Sicht von Michael Lang, Wien, in: Rädler, A. J. (Hrsg.), Tax Science Fiction, Wie sieht unser Steuerrecht in 25 Jahren aus?, München 2008, S. 21-26

Lang, M./Aigner, H.-J./Scheuerle, U. et al. (2004), CFC Legislation, Tax Treaties and EC Law, Wien 2004 
Lang,M./Pistone,P/Schuch, J. et al. (Hrsg.), Common Consolidated Corporate Tax Base, Wien 2008

Leffson, U. (1976), Die Grundsätze ordnungsmäßiger Buchführung, 4. Aufl., Düsseldorf 1976

Lodin, S. O./Gammie, M. (2001), Home State Taxation, Amsterdam 2001

Maisto, G./Pistone, P. (2008), A European Model for Member States' Legislation on the Taxation of Controlled Foreign Subsidiaries (CFCs) - Part 2, in: European Taxation 2008, S. 554-570

Maiterth, R. (2006), Das EuGH-Urteil „Marks\&Spencer“ und die grenzüberschreitende Verlustverrechnung aus ökonomischer Sicht, in: Deutsches Steuerrecht 2006, S. 915-919

McLure, C. E. (1984), Defining a Unitary Business: An Economist's View, in: McLure, C. E. (Hrsg.), The State Corporation Income Tax: Issues in Worldwide Unitary Combination, Stanford 1984, S. 89-124

McLure, C. E. (2000), Implementing State Corporate Income Taxes in the Digital Age, in: National Tax Journal 2000, S. 1287-1305

McLure, C. E. (2004), Corporate Tax Harmonization in the European Union: The Commission's Proposals, in: Tax Notes International 2004, S. 45-69

McLure, C. E. (2005), The European Commission's Proposal for Corporate Tax Harmonization, in: CESifo Forum 2005, S. 32-41

McLure, C. E. (2008), Harmonizing Corporate Income Taxes in the US and the EU: Legislative, Judicial, Soft Law and Cooperative Approaches, in: CESifo Forum 2008, S. 46-52

McLure, C. E. (Hrsg.), The State Corporation Income Tax - Issues in Worldwide Unitary Combination, Stanford 1984, S. 228-246

Meussen, G. (2003), The Marks\&Spencer case: reaching the boundaries of the EC Treaty, in: EC Tax Review 2003, S. 144-148

Mintz, J. (1999), Globalization of the corporate Income Tax: The Role of Allocation, in: Finanzarchiv N. F., Bd. 56, S. 389-423

Mintz, J. (2002), Company Taxation and the International Market, in: CESifo Forum 2002, S. 3-9 
Mintz, J. (2004), Corporate tax harmonization in Europe: It's all about compliance, in: International Tax and Public Finance 2004, S. 221-234

Mintz, J./Smart, M. (2004), Income shifting, investment, and tax competition: theory and evidence from provincial taxation in Canada, in: Journal of Public Economics 2004, S. 1149-1168

Mintz, J./Weichenrieder, A. (2005), Taxation and the financial structure of German Outbound FDI, CESifo Wokring Paper No. 1612, 2005

Mintz, J./Weiner, J. M. (2003), Exploring formula allocation for the European Union, in: International Tax and Public Finance 2003, S. 695-711

Mintz, J./Weiner, J.M. (2003), Exploring Formula Allocation for the European Union, in: International Tax and Public Finance 2003, S. 695-711

Moreno Gonzalez, S. Sanz Diaz-Palacios, J. A., The Common Consolidated Corporate Tax Base: Treatment of Losses, in: Lang, M./Pistone, P./Schuch, J. et al. (Hrsg.), Common Consolidated Corporate Tax Base, Wien 2008, S. 441464

Mors, M./Rautenstrauch, G. (2008), Die gemeinsame konsolidierte Körperschaftsteuer-Bemessungsgrundlage (GKKB) als harmonisiertes europäisches Körperschaftsteuerrecht der Zukunft?, in: Die Unternehmensbesteuerung 2008, S. 97-104

Mossin, J. (1968), Taxation and Risk-Taking: An Expected Utility Approach, in: Economica 1968, S. 74-82

Musgrave, P. (1972), International tax base division and the multinational corporation, in: Public Finance 1972, S. 394-413

Musgrave, P. (1984), Principles for Dividing the State Corporate Tax Base, in: McLure, C. E. (Hrsg.), The State Corporation Income Tax - Issues in Worldwide Unitary Combination, Stanford 1984, S. 228-246

Neumark, F. (1970), Grundsätze gerechter und ökonomisch rationaler Steuerpolitik, Tübingen 1970

Nias, P./Purcell, N. (2005), Country Reports - EU Member States -United Kingdom, in: European Taxation 2005, S. 435-441 
Nicodème, G. (2001), Computing effective corporate tax rates: comparison and results, in: Economic Papers, European Commission, Directorate-General for Economic and Financial Affairs, Brüssel u.a. 2001

OECD (1995), Transfer Pricing Guidelines for Multinational Enterprises and Tax Administrations, Paris 1995, Stand: 1999

OECD (2006), Report on the Attribution of Profits to Permanent Establishments, Part I: General Considerations, Paris 2006

Oestreicher, A. (2000), Konzern-Gewinnabgrenzung, München 2000

Oestreicher, A. (2002), Konzernbesteuerung in Europa- Zum Vorschlag einer konsolidierten körperschaftsteuerlichen Bemessungsgrundlage für die grenzüberschreitende Unternehmenstätigkeit in der EU, in: Steuer und Wirtschaft 2002, S. 342-356

Oestreicher, A. (2005a), Gewinnaufteilung, in: Endres, D. et al. (Hrsg.), Die internationale Unternehmensbesteuerung im Wandel, München 2005, S. 73-91

Oestreicher, A. (2005b), Konzernbesteuerung im Spannungsfeld zwischen wirtschaftlicher Einheit und rechtlicher Vielheit, in: Oestreicher, A. (Hrsg.), Konzernbesteuerung: Beiträge zu einer Ringveranstaltung an der GeorgAugust-Universität Göttingen zum Sommer 2004, Herne/Berlin 2005, S. 131

Oestreicher, A. (2007), Zukunft des Steuerbilanzrechts aus deutscher Sicht, in: Die Wirtschaftsprüfung 2007, S. 572-582

Oestreicher, A. (2008), Methods of Consolidation, in: Lang, M./Pistone, P./Schuch, J. et al. (Hrsg.), Common Consolidated Corporate Tax Base, Wien 2008, S. 517-546

Oestreicher, A. (2009), Die (reformbedürftigen) Regelungen zur Ermittlung der Verrechnungspreise in Fällen der Funktionsverlagerung, in: Die Unternehmensbesteuerung 2009, S. 80-95

Oestreicher, A. (Hrsg.), Konzernbesteuerung: Beiträge zu einer Ringveranstaltung an der Georg-August-Universität Göttingen zum Sommer 2004, Herne/Berlin 2005

Oestreicher, A./Hundeshagen, C. (2008), Bewertung von Transferpaketen bei Funktionsverlagerungen, in: Der Betrieb 2008, S. 1637-1643 und 1693-1700 
Oestreicher, A./Hundeshagen, C. (2009), Weder Wirtschaftsgut noch Unternehmen - die Bewertung von Transferpakten anlässlich der grenzüberschreitenden Verlagerung von Unternehmensfunktionen, in: Internationales Steuerrecht 2009, S. 145-151

Oestreicher, A./Koch, R. (2007), The Revenue Consequences of Using CCCTB to Determine Taxable Income in EU Member States, FAT Working Paper No. 07-001, Göttingen 2007

Oestreicher, A./Koch, R. (2008), Corporate average tax rates under the CCCTB and possible Methods for international loss-offset, FAT Working Paper No. 08-001, Göttingen 2008

Oestreicher, A./Reister, T./Spengel, C. (2009), Common Corporate Tax Base and Effective Tax Burdens in the EU Member States, in: World Tax Journal 2009, S. 46-66

Oestreicher, A./Scheffler, W./Spengel, C. et al.. (2008): Modelle einer Konzernbesteuerung für Deutschland und Europa, Baden-Baden 2008

Oestreicher, A./Spengel, C. (2003), Steuerliche Abschreibung und Standortattraktivität, Baden-Baden 2003

Oestreicher, A./Spengel, C. (2001), Anwendung von IAS in der EU - Zukunft des Maßgeblichkeitsprinzips und Steuerbelastung in: Recht der internationalen Wirtschaft 2001, S. 889-902

Oestreicher, A./Spengel, C. (2007), Tax Harmonization in Europe: The Determination of Corporate Taxable Income in the Member States, in: European Taxation 2007, S. 437-451

Ordelheide, D. (1986), Der Konzern als Gegenstand betriebswirtschaftlicher Forschung, in: Betriebswirtschaftliche Forschung und Praxis 1986, S. 293-312

Ordelheide, D. (1986a), Konzern und Konzernerfolg, in: Wirtschaftswissenschaftliches Studium 1986, S. 495-502

Peichl, A. (2006), Die Evaluation von Steuerreformen durch Simulationsmodelle, Finanzwissenschaftliche Diskussionsbeiträge 05-1, Köln 2006

Plasschaert, S. R. F . (2002), Comprehensive Approaches to EU Company Taxation: To Which Companies Should They Apply?, in: European Taxation 2002, S. 7-17 
Plasschaert, S. R. F. (1997), An EU Tax on the Consolidated Profits of Multinational Enterprises, in: European Taxation 1997, S. 2-13

Plesko, G. A. (2003), An Evaluation of alternative Measure of Corporate Tax Rates, Journal of Accounting and Economics 2003, S. 201-226.

Poppe, A. (2008), Auswirkungen der Einführung einer konsolidierten Körperschaftsteuer-Bemessungsgrundlage in der Europäischen Union, Frankfurt am Main/Berlin/Bern u.a. 2008

Rädler, A. J. (Hrsg.), Tax Science Fiction, Wie sieht unser Steuerrecht in 25 Jahren aus?, München 2008

Razin, A. (Hrsg.), Taxation in the Global Economy, Chicago 1990

Richter, C. (2005), Das Verhältnis zwischen handelsrechtlicher und steuerrechtlicher Rechnungslegung in Österreich, in: Schön, W. (Hrsg.), Steuerliche Maßgeblichkeit in Deutschland und Europa, Köln 2005, S. 443-511

Rödder, T. (2007), Perspektiven der Konzernbesteuerung, in: Zeitschrift für das gesamte Handels- und Wirtschaftsrecht 2007, S. 380-408

Ruding, O. (2005), The past and the future of EU corporate tax, in: EC Tax Review 2005 , S. $2-4$

Rupp, R. (1983), Die Ertragsbesteuerung nationaler Konzerne - Konzernsteuerbilanz oder Weiterentwicklung der körperschaftsteuerlichen Organschaft?, Frankfurt am Main/Bern/New York 1983

Russo, A. (2005), Formulary Apportionment for Europe: An Analysis and A Proposal, in: Intertax 2005, S. 2-31

Rust, A. (2008), CFC Legislation and EC Law, in: Intertax 2008, S. 492-501

Scheffler, W. (2005), Grenzüberschreitende Verlustverrechnung Nachversteuerungsmethode im Vergleich zum geltenden Recht und zur konsolidierten Körperschaftsteuer-Bemessungsgrundlage, in: Betriebswirtschaftliche Forschung und Praxis 2005, S. 156-175

Scheffler, W. (2005a), Gemeinsame konsolidierte Steuerbemessungsgrundlage (CCTB) in der EU - Das Ende der Steuerplanung mit Verrechnungspreisen?, in: Oestreicher, A. (Hrsg.), Konzernbesteuerung: Beiträge zu einer Ringveranstaltung an der Georg-August-Universität Göttingen zum Sommer 2004, Herne/Berlin 2005, S. 305-331 
Scheuchzer, M. (1994), Konzernbesteuerung in der Europäischen Union, Bielefeld 1994

Scheunemann, M. (2006): Praktische Anforderungen einer grenzüberschreitenden Verlustberücksichtigung im Konzern in Inbound- und Outboundfällen nach der Entscheidung Marks\&Spencer, in: Internationales Steuerrecht 2006, S. 145-180

Schneider, D. (2002), Steuerlast und Steuerwirkung, München 2002

Schneider, D. (2003), Wider Marktpreise als steuerliche Verrechnungspreise, in: Der Betrieb 2003, S. 53-58

Schön, W. (2004), International Accounting Standards: A "Starting Point" for a Common European Tax Base?, in: European Taxation 2004, S. 426-440

Schön, W. (2007), Perspektiven der Konzernbesteuerung, in: Zeitschrift für das gesamte Handelsrecht und Wirtschaftsrecht 2007, S. 409-445

Schön, W. (Hrsg.), Steuerliche Maßgeblichkeit in Deutschland und Europa, Köln 2005

Schön, W./Spengel, C./Schreiber, U. (Hrsg.), A Common Consolidated Corporate Tax Base for Europe - Eine einheitliche Körperschaftsteuerbemessungsgrundlage für Europa, Berlin/Heidelberg 2008

Schratzenstaller, M. (2004), Zur Ermittlung der faktischen effektiven Unternehmensteuerlast, in: Schratzenstaller, M./Truger, A. (Hrsg.), Perspektiven der Unternehmensbesteuerung, Marburg 2004, S. 43-76

Schratzenstaller, M./Truger, A. (Hrsg.), Perspektiven der Unternehmensbesteuerung, Marburg 2004

Schreiber, U. (2004), Unternehmensbesteuerung im Binnenmarkt. Angleichung der Gewinnermittlung und des Satzes der Körperschaftsteuer?, in: Steuer und Wirtschaft 2004, S. 212-226

Schreiber, U. (2004), Unternehmensbesteuerung im Binnenmarkt. Angleichung der Gewinnermittlung und des Satzes der Körperschaftsteuer, in: Steuer und Wirtschaft 2004, S. 212-226

Seischab, H./Schwantag, K. (Hrsg.), Handwörterbuch der Betriebswirtschaft, Band 1, 3. Auflage, Stuttgart 1956 
Shevlin, T. (1999), A Critique of Plesko's „An Evaluation of Alternative Measures Corporate Tax Rates“, University of Washington 1999

Sieker, S, (1998), Verfassungsmäßigkeit des §15a EStG im Falle nachträglicher Einlageleistungen des Kommanditisten, in: Finanz-Rundschau 1988, S. 453467

Sievert, E. (2006), Konzernbesteuerung in Deutschland und Europa, Düsseldorf 2006

Sorenson, P. B. (2004), Company tax reform in the European Union, in: International Tax and Public Finance 2004, S. 91-115

Sorenson, P.B. (2002), To harmonise or not to harmonise?, in: CESifo Forum $1 / 2002$, S. 31-35

Spengel, C. (1998), Wettbewerbswirkungen der Körperschaftsteuer in Europa Analyse und Reformvorschläge, in: Die Betriebswirtschaft 1998, S. 348-368

Spengel, C. (2008), A Concept and Necessity of a Common Tax Base - an academic introduction, in: Schön, W./Spengel, C./Schreiber, U. (Hrsg.), A Common Consolidated Corporate Tax Base for Europe - Eine einheitliche Körperschaftsteuerbemessungsgrundlage für Europa, Berlin/Heidelberg 2008, S. $1-47$

Spengel, C./Braunagel, R. U. (2006), EU-Recht und Harmonisierung der Konzernbesteuerung in Europa, in: Steuern und Wirtschaft 2006, S. 34-49

Spengel, C./Oestreicher, A. (2009), Gemeinsame (konsolidierte) Körperschaftsteuerbemessungsgrundlage in der EU und Umsetzungsfragen, in: Deutsches Steuerrecht 2009, S. 773-781

Steuer- und Finanzausschuss (1962), Bericht des Steuer- und Finanzausschusses (Neumark-Bericht), Brüssel 1962

Steuerreformkommission (1971), Gutachten der Steuerreformkommission 1971, Schriftenreihe des Bundesministeriums der Finanzen, Heft 17, Bonn 1971

Stiglitz, J.E. (1969), The Effects of Income, Wealth, and Capital Gains Taxation on risk-taking, in: The Quarterly Journal of Economics 1969, S. 263-283

Theisen, M. R. (2000), Der Konzern, 2. Auflage, Stuttgart 2000 
Thiel, J. (2004), Der fortschreitende Einfluss des EuGH auf die Ertragsbesteuerung der Unternehmen - Aktuelle Urteile und anhängige Verfahren, in: Der Betrieb 2004, S. 2603-2609

Tipke, K./Lang, J. (2002), Steuerrecht, 17. Auflage, Köln 2002

Unabhängiger Sachverständigenausschuss Ruding (1992), Bericht des unabhängigen Sachverständigenausschusses zur Unternehmensbesteuerung, 1992

UNICE (1990), Position paper on the consolidation of losses, Brüssel 1990

UNICE (2000), Memorandum on cross-border company taxation obstacles in the Single Market, Brüssel 2000

Vanistendael, F. (1996), The European Tax Paradox: How less begets more, in: IBFD-Bulletin 1996, S. 531-534

Wagner, T. (2006), Konzeption einer Gruppenbesteuerung, Lohmar 2006

Wassermeyer, F. (2005), Verrechnungspreise, in: Endres, D./Oestreicher, A./Scheffler, W. et al. (Hrsg.), Die internationale Unternehmensbesteuerung im Wandel, München 2005, S. 63-72

Wassermeyer, F./Piltz, D. J. (Hrsg.), Außensteuerrecht: Kommentar, Köln 2006

Watrin, C./Sievert, E./Strohm, C. (2004), Ertragsteuerrecht, in: Finanz-Rundschau 2004, S. $1-52$

Weichenrieder, A. J. (2007), Profit Shifting in the EU: Evidence from Germany, CESifo Working Paper Nr. 2043, 2007

Weiner, J. M. (2001), The European Union and Formula Apportionment: Caveat Emptor, in: European Taxation 2001, S. 380-388

Weiner, J. M. (2005), Formulary Apportionment and group taxation in the European Union: Insights from the United States and Canada, Working Paper No. 8/2005 TAXUD/2005/2601, Luxembourg 2005

Weiner, J.M. (1994), Company Taxation for the European Community. How SubNational Tax Variation Affects Business Investment in the United States and Canada, Harvard 1994

Wendt, C. (2009), A Common Tax Base for Multinational Enterprises in the European Union, Wiesbaden 2009 
Westberg, B. (2002), Consolidated Corporate Tax Bases for EU-Wide Activities: Evaluation of Four Proposals Presented by the European Commission, in: European Taxation 2002, S. 322-330

Wittkowski, A. (2008), Grenzüberschreitende Verlustverrechnung in Deutschland und Europa, Wiesbaden 2008

Zimmerman, J. L. (1983), Taxes and Firm Size, in: Journal of Accounting and Economics 1983, S.119-149 
Reinald Koch - 978-3-631-75683-6

Downloaded from PubFactory at 01/11/2019 03:01:46AM

via free access 


\section{Quellenverzeichnis}

Abkommen zwischen der Bundesrepublik Deutschland und der Volksrepublik Bulgarien zur Vermeidung der Doppelbesteuerung auf dem Gebiet der Steuern vom Einkommen und vom Vermögen vom 2. Juni 1987, BGBl. 1988 II, S. 771

Abkommen zwischen der Bundesrepublik Deutschland und Rumänien zur Vermeidung der Doppelbesteuerung auf dem Gebiet der Steuern vom Einkommen und vom Vermögen vom 4. Juli 2001, BGBl. 2003 II, S. 1595

Richtlinie 2003/123/EG des Rates vom 22. Dezember 2003 zur Änderung der Richtlinie 90/435/EWG über das gemeinsame Steuersystem der Mutter- und Tochtergesellschaften verschiedener Mitgliedstaaten, Abl. EG 2004 Nr. L 7, S. 41

Richtlinie 2003/49/EG des Rates vom 3. Juni 2003 über eine gemeinsame Steuerregelung für Zahlungen von Zinsen und Lizenzgebühren zwischen verbundenen Unternehmen verschiedener Mitgliedsstaaten, Abl. EG 2003 Nr. L 157 , S. 49

Richtlinie 2005/19/EG des Rates v. 17. Februar 2005 zur Änderung der Richtlinie 90/434/EWG über das gemeinsame Steuersystem für Fusionen, Spaltungen, die Einbringung von Unternehmensteilen und den Austausch von Anteilen, die Gesellschaften verschiedener Mitgliedsstaaten betreffen, Abl. EG 2005 Nr. L 58, S. 19 
Reinald Koch - 978-3-631-75683-6

Downloaded from PubFactory at 01/11/2019 03:01:46AM

via free access 
Rechtsprechungsverzeichnis

EuGH v. 12.12.2002 Rs. C-324/00 (Lankhorst- EuGHE 2002, S. I-11779 Hohorst)

EuGH v.13.12.2005 Rs. C-446/03

EuGHE 2005, S. I-10837

(Marks\&Spencer)

EuGH v. 12.9.2006 Rs. C-196/04 (Cadbury EuGHE 2006, S. I-7995

Schweppes)

EuGH v. 19. 9. 2006 Rs. C-356/04 (Lidl Bel- Abl. EU 2006 Nr. C 281, gium)

S. 7

EuGH v. 18. 7. 2007 Rs. C-231/05 (Oy AA)

Abl. EU 2007 Nr. C 235,

S. 3

EuGH, v. 23.04.2008 C-201/05 (Test Claimants ABI. EU 2008 Nr. C 209, in the CFC and Dividend S. 13

Group Litigation)

EuGH, v. 23.10.2008 C-157/07 (Krankenheim ABI. EU 2008 Nr. C 313, Ruhesitz am Wannsee- S. 5

Seniorenheimstatt) 


\section{cege-Schriften}

Das cege - Centrum für Europa-, Governance- und Entwicklungsforschung - wurde 1999 von der Wirtschaftswissenschaftlichen Fakultät der Georg-August-Universität gegründet. Das cege dient als Forum zur internationalen und interdisziplinären Forschungszusammenarbeit in den Themenbereichen Europäische Integration, Governance und Entwicklungsökonomik. In den cege-Schriften werden Forschungsergebnisse aus Dissertationen, Habilitationen oder anderen Forschungsprojekten des cege veröffentlicht.

Band 1 Axel Gerloff: Wechselkurspolitik in Mittel- und Osteuropa. Eine theoretische Analyse unter besonderer Berūcksichtigung der Erfahrungen der zehn Kandidaten für eine EU-Osterweiterung. 2001.

Band 2 Ingo Konrad: Zur Integration ausgewählter mittel- und osteuropäischer Länder in die wãhrungspolitische Ordnung Europas. 2002.

Band 3 Axel Hennighausen: Wegekostenfinanzierung und Lenkung im deregulierten europäischen Verkehrsmarkt. 2002.

Band 4 Wolfgang Mūnch: Effects of EU Enlargement to the Central European Countries on Agricultural Markets. 2002.

Band 5 Holger Niermann: Welchen Finanzausgleich braucht Europa? Ein konkreter Vorschlag zur Neugestaltung der europäischen Finanzbeziehungen mit Simulationsmodell. 2002.

Band 6 Jens Südekum: Agglomeration and Regional Unemployment Disparities. A Theoretical Analysis with Reference to the European Union. 2003.

Band 7 Christoph Schinke: Der Geldmarkt im Euro-Währungsraum. Geldmarktgeschäfte, Zinsbildung und die Taylor Rule. 2004.

Band 8 Kai Stukenbrock: The Stability of Currency Boards. 2004.

Band 9 Harald Grethe: Effects of Including Agricultural Products in the Customs Union between Turkey and the EU. A Partial Equilibrium Analysis for Turkey. 2004.

Band 10 Götz Zeddies: Perspektiven der Gemeinschaftspolitiken in der erweiterten EU. 2005.

Band 11 Ingmar Kumpmann: Systemwettbewerb und Umverteilung. Gefährdet die Globalisierung den Sozialstaat? 2005.

Band 12 Katarzyna Haverkamp: Bestimmungsfaktoren der Vertragsforschung in Deutschland. Eine theoretische und ökonometrische Analyse. 2007.

Band 13 Peter Schwarz: Kapitalsteuerwettbewerb zwischen Nationalstaaten unter besonderer Berūcksichtigung Deutschlands. Eine empirische Analyse. 2007.

Band 14 Andreas Poppe: Auswirkungen der Einführung einer konsolidierten KörperschaftsteuerBemessungsgrundlage in der Europäischen Union. Eine empirische Analyse der Konzernstrukturen und des Steueraufkommens. 2008.

Band 15 Sebastian Hess: Meta-Analyse angewandter Gleichgewichtsmodelle des internationalen Agrarhandels. 2008.

Band 16 Mareike Köller: Ausländische Direktinvestitionen und regionale Integration - das Beispiel Irland. 2009.

Band 17 Verena Mertins: Institutionenökonomische Analyse von Innovationsförderung. Eine theoretische und empirische Betrachtung am Beispiel Niedersachsens. 2009.

Band 18 Reinald Koch: Die Aufkommens- und Belastungswirkungen alternativer Vorschläge zur Reform der Konzernbesteuerung in Europa. 2010.

www.peterlang.de 
Reinald Koch - 978-3-631-75683-6

Downloaded from PubFactory at 01/11/2019 03:01:46AM

via free access 
Reinald Koch - 978-3-631-75683-6

Downloaded from PubFactory at 01/11/2019 03:01:46AM

via free access 